\title{
Fracturing Fluid Cleanup by Controlled Release of Enzymes from Polyelectrolyte Complex Nanoparticles
}

\author{
By \\ Reza Barati Ghahfarokhi \\ B.Sc. Petroleum Engineering, Petroleum University of Technology, Ahwaz, Iran, 2002 \\ M.Sc. Petroleum Engineering, University of Kansas, USA, 2005 \\ Submitted to the graduate degree program in Department of Chemical and Petroleum \\ Engineering and the Graduate Faculty of the University of Kansas in partial fulfillment of the \\ requirements for the degree of Doctor of Philosophy. \\ Committee members:
}

Jenn-Tai Liang

(Chairperson)

D. W. Green
(Co-chairperson)

G. P. Willhite

S. J. Johnson

C. S. $\mathrm{McCool}$

A. W. Walton

Date defended:

12-09-2010 
The Dissertation Committee for Reza Barati Ghahfarokhi certifies that this is the approved version of the following dissertation:

\section{Fracturing Fluid Cleanup by Controlled Release of Enzymes from Polyelectrolyte Complex Nanoparticles}

Committee members:

$\begin{array}{r}\begin{array}{r}\text { Jenn-Tai Liang } \\ \text { (Chairperson) }\end{array} \\ \hline \begin{array}{r}\text { D. W. Green } \\ \text { (Co-chairperson) }\end{array} \\ \hline \text { G. P. Willhite } \\ \hline \text { S. J. Johnson } \\ \hline \text { C. S. McCool } \\ \hline\end{array}$

A. W. Walton

Date approved:

12-09-2010 


\section{Abstract}

Guar-based polymer gels are used in the oil and gas industry to viscosify fluids used in hydraulic fracturing of production wells, in order to reduce leak-off of fluids and pressure, and improve the transport of proppants. After fracturing, the gel and associated filter cake must be degraded to very low viscosities using breakers to recover the hydraulic conductivity of the well. Enzymes are widely used to achieve this but injecting high concentrations of enzyme may result in premature degradation, or failure to gel; denaturation of enzymes at alkaline $\mathrm{pH}$ and high temperature conditions can also limit their applicability.

In this study, application of polyelectrolyte nanoparticles for entrapping, carrying, releasing and protecting enzymes for fracturing fluids was examined. The objective of this research is to develop nano-sized carriers capable of carrying the enzymes to the filter cake, delaying the release of enzyme and protecting the enzyme against $\mathrm{pH}$ and temperature conditions inhospitable to native enzyme.

Polyethylenimine-dextran sulfate (PEI-DS) polyelectrolyte complexes (PECs) were used to entrap two enzymes commonly used in the oil industry in order to obtain delayed release and to protect the enzyme from conditions inhospitable to native enzyme. Stability and reproducibility of PEC nanoparticles was assured over time.

An activity measurement method was used to measure the entrapment efficiency of enzyme using PEC nanoparticles. This method was confirmed using a concentration measurement method (SDS-PAGE). Entrapment efficiencies of pectinase and a commercial high-temperature enzyme mixture in polyelectrolyte complex nanoparticles were maximized. Degradation, as revealed by reduction in viscoelastic moduli of borate-crosslinked hydroxypropyl guar (HPG) gel by commercial enzyme loaded in polyelectrolyte nanoparticles, was delayed, compared to equivalent systems where the enzyme mixture was not entrapped. This indicates that PEC nanoparticles delay the activity of enzymes by entrapping them. It was also observed that control PEC nanoparticles decreased both viscoelastic moduli, but with a slower rate compared to the PEC nanoparticles loaded with enzyme.

Preparation shear and applied shear showed no significant effect on activity of enzyme-loaded PEC nanoparticles mixed with HPG solutions. However, fast addition of chemicals during the 
preparations showed smaller particle size compared to the drop-wise method. PEC nanoparticles (PECNPs) also protected both enzymes from denaturation at elevated temperature and $\mathrm{pH}$.

Following preparation, enzyme-loaded PEC nanoparticles were mixed with borate crosslinked HPG and the mixture was injected through a shear loop. Pectinase-loaded nanoparticles mixed with gelled HPG showed no sensitivity to shear applied along the shear loop at $25^{\circ} \mathrm{C}$. However, EL2X-loaded PEC nanoparticles showed sensitivity to shear applied along the shear loop at $40{ }^{\circ} \mathrm{C}$.

Filter cake was formed and degraded in a fluid loss cell for borate crosslinked HPG solutions mixed with either enzymes or enzyme-loaded PEC nanoparticles. Cleanup slopes of filter cake degraded using enzyme-loaded PEC nanoparticles and systems with enzymes mixed with HPG gel were significantly higher than for the filter cake formed with HPG gel mixed with no enzyme.

In a different application, enzyme-loaded PEC nanoparticles showed significantly slower reduction in viscosity of HPG solution over time compared to the HPG systems mixed with enzyme. Increasing the viscosity of low concentration HPG, used as slick-water, decreases the proppant settling velocity. This is of specific interest in fracturing fluids used for unconventional reservoirs. 


\section{Acknowledgement}

First and foremost, I would like to express my gratitude to Prof. Jenn-Tai Liang and Prof. Don W. Green for their continuous motivation and support during the entire period of this study. This work would have not been accomplished without their guidance and support.

Deep appreciation is also extended to Dr. Stephen J. Johnson for being so generous in his time and all his thoughtful comments which greatly improved the whole research. I gratefully acknowledge him for his comments regarding my writing as well.

I would also like to thank Prof. G. P. Willhite and Dr. S. C. McCool for their enlightening and insightful comments which greatly improved the whole research.

I would also like to extend my appreciation to Prof. Anthony W. Walton for serving in my committee.

My deep appreciation is also extended to Mr. Richard D. Hutchins, reservoir stimulation fluid adviser in Schlumberger, for his valuable comments and for recommending the donation of the fluid loss cell used in this study by Schlumberger.

I greatly appreciate Dr. Karen Peltier, Mr. Scott Ramskill and Mr. Jim Pilch for all their assistance with construction and operating laboratory equipment and procedures.

I would also like to extend my gratitude to Dr. Cory Berkland and his group for nicely providing me with their lab equipment. Specifically, I would like to thank Dr. Sheng-Xue Xie and Chuda Chitasopha for giving me the training required for some analytical techniques.

I also thank all the other Tertiary Oil Recovery Project (TORP) staff members and my fellow students for their help and friendship.

I affectionately dedicate this thesis to my parents (Parvin Haghighatfard and Hosein Barati) and my wife (Hajar Aghababa) without whom none of this would have been possible. 


\section{Table of Contents}

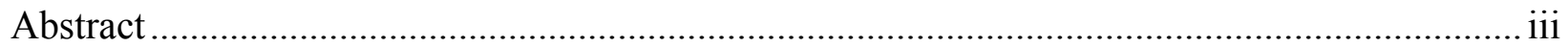

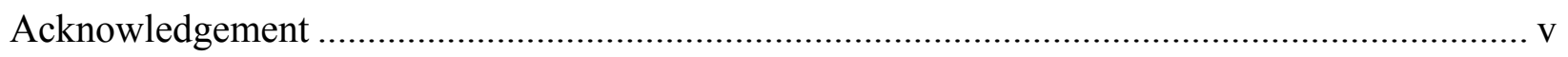

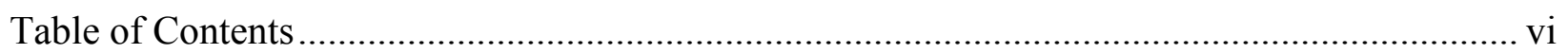

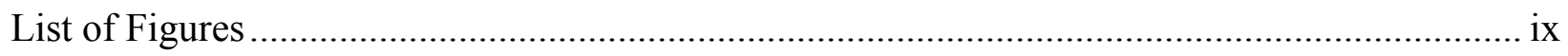

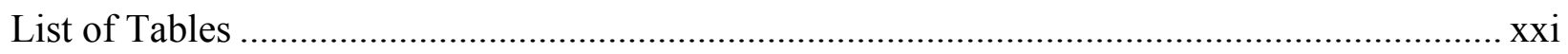

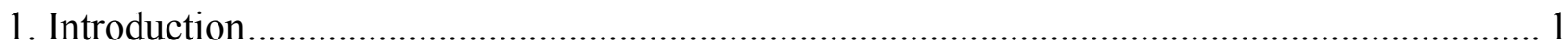

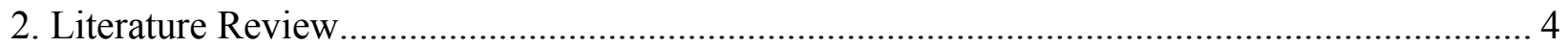

2.1 Hydraulic Fractures, Propagation and Cleanup .......................................................... 4

2.1-1 Hydraulic Fracturing in Conventional Reservoirs ............................................... 4

2.1-2 Hydraulic Fracturing in Unconventional Reservoirs ......................................... 6

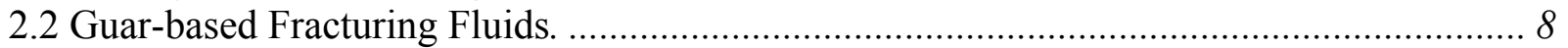

2.2-1 Guar and Its Derivatives ............................................................................... 8

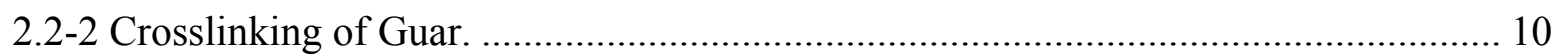

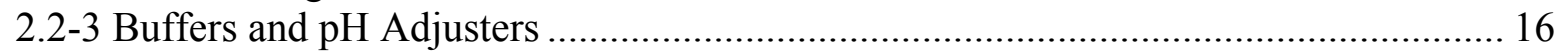

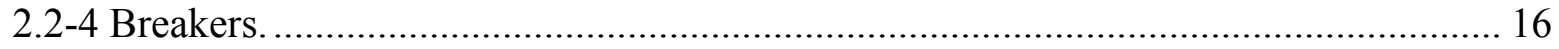

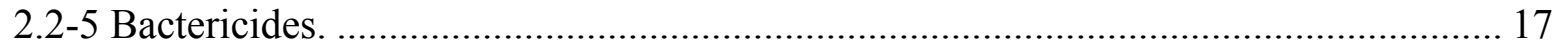

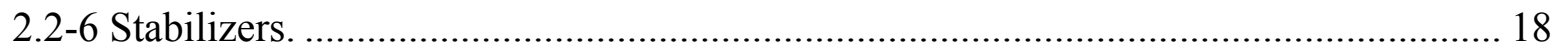

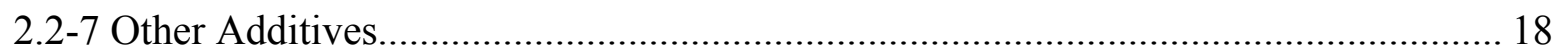

2.3 Nanoparticles, Polyelectrolytes and PEI/DS System................................................. 18

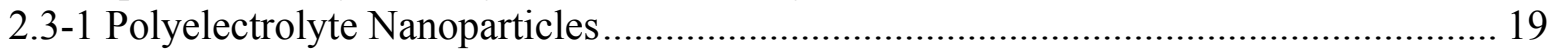

2.3-2 Assembly Mechanism of Polyelectrolyte Nanoparticles.......................................... 24

2.3-3 Polyethylenimine/Dextran Sulfate System of Tiyaboonchai.................................. 25

2.3-4 Application of Polyethylenimine/Dextran Sulfate System In Petroleum Engineering 28

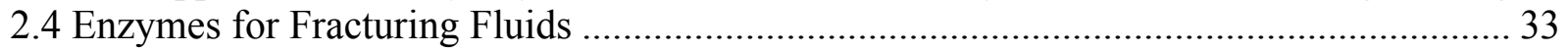

2.4-1 Amino Acids, Proteins, Enzymes and Polysaccharides ......................................... 33

2.4-2 Michaelis-Menten Kinetics of Enzymes........................................................... 40

2.4-3 Viscosity-average Molecular Weight of Guar during Degradation........................... 41

2.4-4 Viscometric Assays for Determining Concentration of Enzyme from Activity

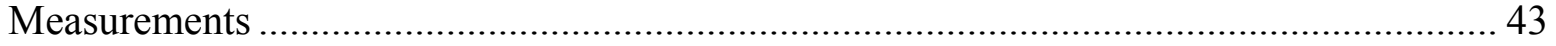

2.4-5 Methods for Determining Concentration of Enzymes.......................................... 44

2.4-6 Enzymatic Degradation of Guar Solutions and Guar-Borate Gels ........................... 45

2.4.7 Enzymes Used to Break Guar Gum ................................................................. 47

2.5 Pre-conditioning the Fracturing Fluids, Considering the Effect of Shear and Temperature

on Rheology of Fracturing Fluids during the Injection ................................................ 50

2.6 Fluid Loss during the Formation of Filter Cake ..................................................... 57

2.6.1 Classical Carter Model for Filtration .................................................................. 57

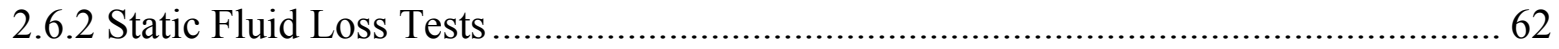

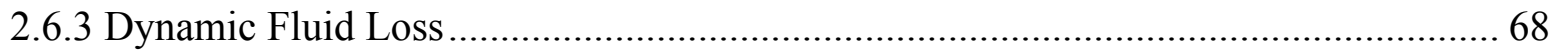


2.7 Laboratory Measurement of Fracture Conductivity ........................................................... 73

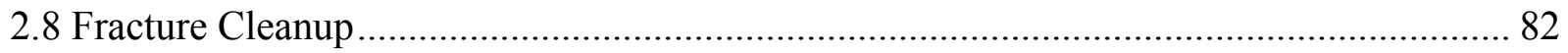

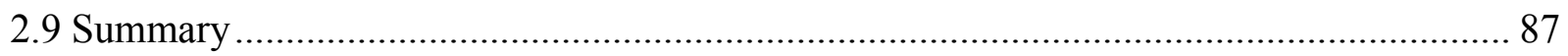

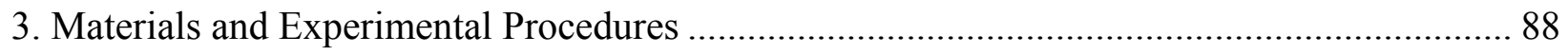

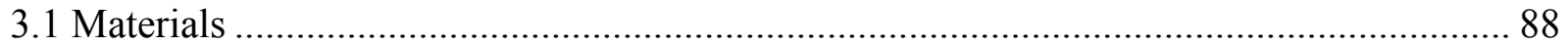

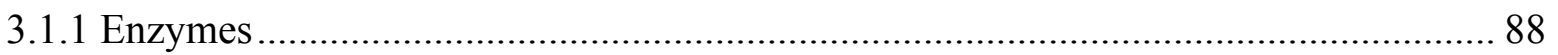

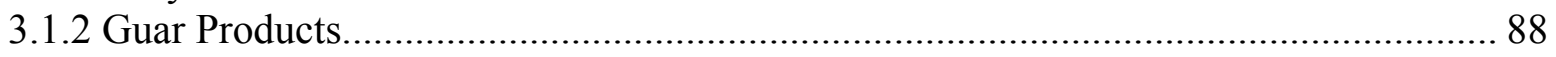

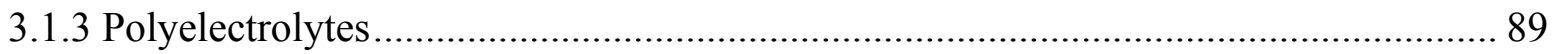

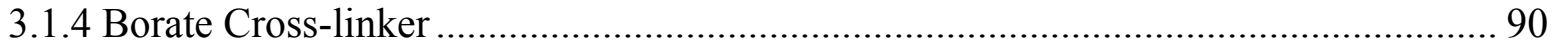

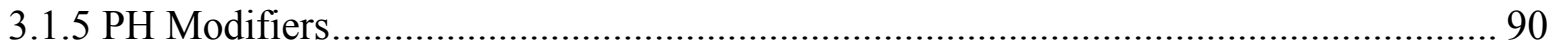

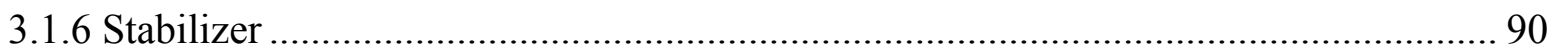

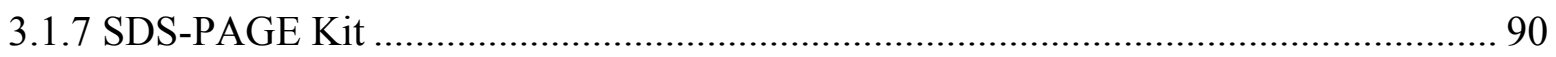

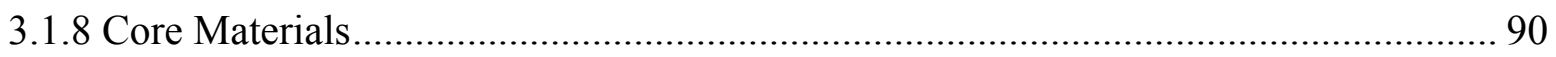

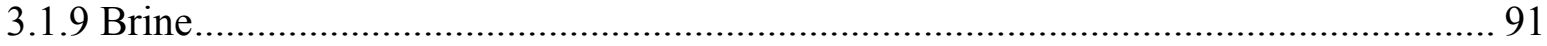

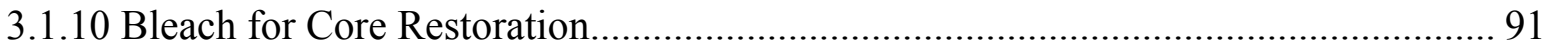

3.2 Equipment and Experimental Procedures.................................................................... 92

3.2.1 Preparation of Polyelectrolyte Complex Nanoparticles............................................. 92

3.2.2 Size and Zeta Potential Measurement of Polyelectrolyte Complex Nanoparticles ..... 94

3.2.3 Separation of Nanoparticles ...................................................................................... 94

3.2.4 Determination of Enzyme Activity for Nanoparticles and their Supernatants ............ 94

3.2.5 Determination of Enzyme Concentration using SDS-PAGE...................................... 95

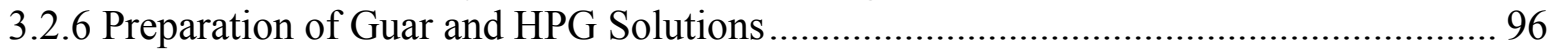

3.2.7 Preparation of Borate-Crosslinked Guar/HPG............................................................ 97

3.2.8 Measurement of Viscosity and Viscoelastic Moduli ................................................... 97

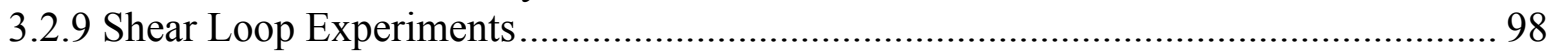

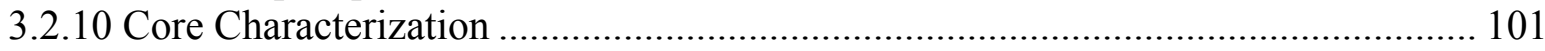

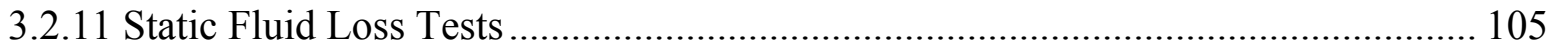

3.2.12 Filtration of Polymeric Solutions or Gelled HPG................................................. 114

3.2.13 Core Restoration after Tracer and Fluid Loss Tests ............................................ 116

3.2.14 Chlorine Measurement in Effluent During Core Cleanup after Restoration ........... 116

3.2.15 Measurement of $\mathrm{pH}$ for Solutions and Gelled Polymers ....................................... 117

3.2.16 Total Organic Carbon and Nitrogen Measurement.................................................. 117

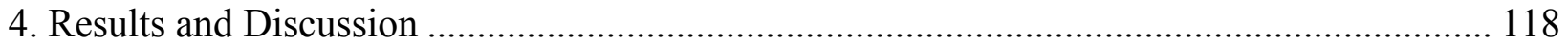

4.1 Preparation of Guar/HPG Solutions and Borate Cross-linked Guar/HPG ....................... 118

4.1-1 Centrifuged Guar Solutions ............................................................................... 118

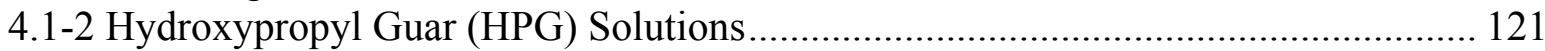

4.1-3 Borate Cross-linked Guar/HPG ...................................................................... 123

4.2 Characterization and Activity Measurement of Two Enzymes Used In this Study ......... 125

4.2-1 Ultraviolet-visible (UV-vis) Spectrometry ............................................................ 125

4.2-2 Sodium Dodecyl Sulfate Polyacrylamide Gel Electrophoresis (SDS-PAGE) .......... 128

4.2-3 Viscometric Assay ............................................................................................. 132

4.3 Preparation and Optimization of Enzyme-Loaded Nanoparticles ................................... 139 
4.3-1 Preparation and Optimization of Enzyme-Loaded PEC Nanoparticles.

4.3-2 Effect of Shear During Particle Preparation and Effect of Applied Shear on Enzyme-

Loaded Nanoparticles ..... 148

4.4 Degradation of Borate Cross-linked Guar/HPG Using Enzymes and Enzyme-Loaded

Nanoparticles 151

4.4-1 Degradation of Borate Cross-Linked Guar/HPG Using Pectinase and PectinaseLoaded Nanoparticles ..... 151

4.4-2 Degradation of Borate Cross-Linked HPG Using EL2X and EL2X-Loaded PEC Nanoparticles 155

4.5 Protective Effect of Nanoparticles on Enzymes 161

4.6 Effect of Simulated Wellbore Shear on Enzyme-loaded Nanoparticles: Shear Loop Experiments 163

4.6-1 Effect of Simulated Wellbore Shear on Pectinase-Loaded Nanoparticles 163

4.6-2 Effect of Simulated Wellbore Shear on EL2X-loaded Nanoparticles 167

4.7 Filter-cake Formation and Cleanup Using Static Fluid-Loss Tests 172

4.7-1 Permeability Measurement and Tracer Tests for Cores Used in Fluid-Loss Tests ... 172

4.7-2 Fluid-Loss Tests and Filter-Cake Cleanup .. 179

4.7-3 Permeability of Cores After the Fluid-Loss Tests ............................................... 187

4.8 Effect of Enzyme-Loaded Nanoparticles on Low Concentration HPG For Slick-water

Applications ..... 189

4.8-1 Pectinase-Loaded PEC Nanoparticles Used for Breaking Low Concentration HPG 189 4.8-2 EL2X-Loaded PEC Nanoparticles Used for Breaking Low Concentration HPG .... 191 4.9 Summary of Results 194

5. Conclusions 197

5.1 Preparation of Guar/HPG Solutions and Borate Cross-linked Guar/HPG 197

5.2 Characterization and Activity Measurement of Pectinase and EL2X 197

5.3 Preparation and Optimization of Enzyme-Loaded PEC Nanoparticles. 197

5.4 Degradation of Borate Cross-linked Guar/HPG Using Enzymes and Enzyme-Loaded

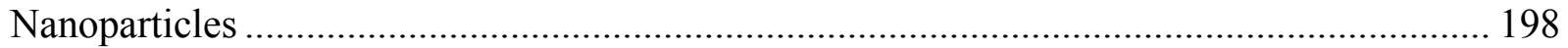

5.5 Protective Effect of Nanoparticles on Enzymes .......................................................... 198

5.6 Effect of Simulated Wellbore Shear on Enzyme-loaded Nanoparticles: Shear Loop Experiments 198

5.7 Filter-Cake Formation and Cleanup Using Static Fluid-Loss Tests 199

5.8 Enzyme-Loaded PEC Nanoparticles Used for Breaking Low Concentration HPG......... 199

6. Recommendations for Future Work............................................................................ 200

6.1 Use of Guar Specific and High Temperature Enzymes ............................................. 200 
6.2 Improving Enzyme-Loaded Polyelectrolyte Complex Nanoparticles 200

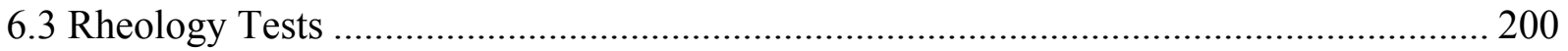

6.4 Protective Effect of Nanoparticles on Enzymes .............................................................. 201

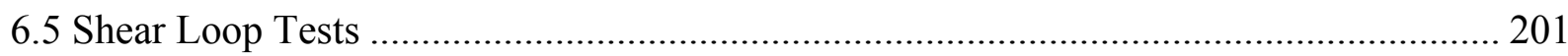

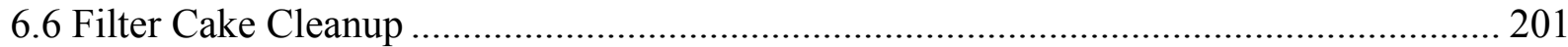

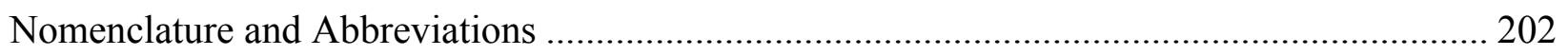

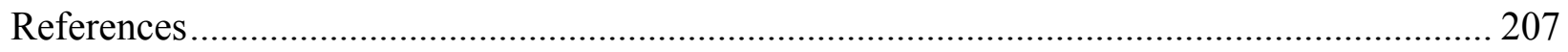

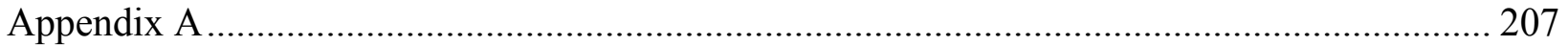

A-1 Permeability Measurements and Tracer Tests for Cores Used in Fluid Loss Tests ........ 215

A-2 Permeability Measurements for Cores Resaturated After Tracer Tests and Before Being

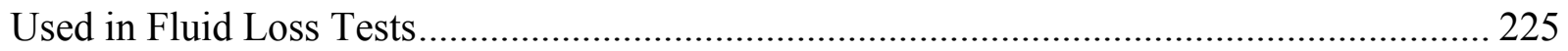

A-3 Permeability Measurements for Cores Restored After Fluid Loss Tests and Before Being Reused for Fluid Loss Tests.......................................................................................... 230

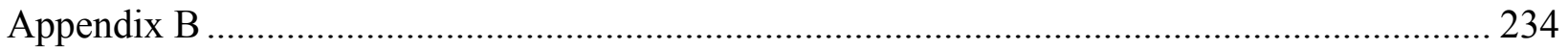

\section{List of Figures}

Figure 1 Schematic picture of one side of a fracture after closure (Barati et al. ${ }^{[7]}$........................ 6

Figure 2 Chemical structure of guar showing mechanism of crosslinking by borate, and ether bond vulnerable to cleavage by enzyme (-R denotes another guar molecule). ${ }^{[23]}$......................... 9 Figure $3 \mathrm{pH}$ range for different crosslinkers, Rae et al. ${ }^{[27]}$...................................................... 12

Figure 4 Temperature range for different crosslinkers, Rae et al. ${ }^{[27]}$........................................ 13

Figure 5 Dimensionless concentration of monoborate ions vs. $\mathrm{pH}$ for a range of temperature,

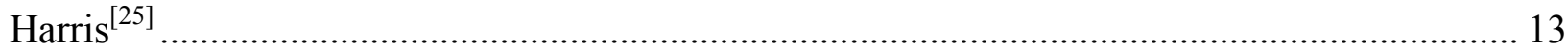

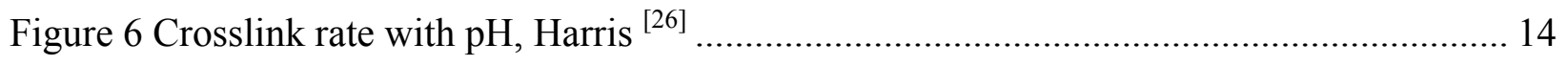

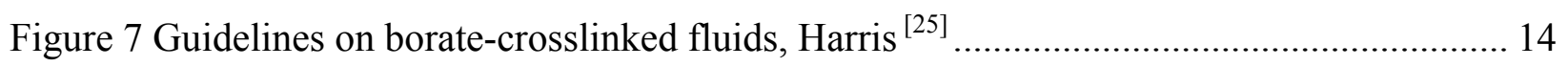

Figure 8 Borate-crosslinked gel guideline, Harris et al. ${ }^{[26]}$................................................... 15

Figure 9 Classification of polyelectrolytes in terms of their charge, Koets and Kosmella ${ }^{[36]}$.... 19

Figure 10 Simplified illustration of the surface and zeta potential for a charged suspension drop dispersed in high (saline water) and low (fresh water) electrolyte concentration aqueous

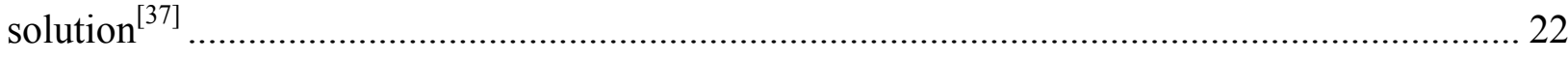


Figure 11 Schematic representation of ladder (a) and scrambled egg (b) structures. Black represents the negative polyelectrolyte (polyanion) while gray represents the positive

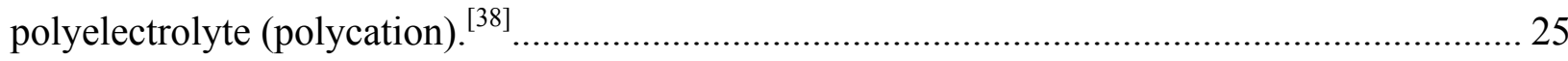

Figure 12 Chemical structure of PEI (Drawn by Stephen J. Johnson, used by permission). ....... 30

Figure 13 Chemical structure of DS (Drawn by Stephen J. Johnson, used by permission). ........ 30

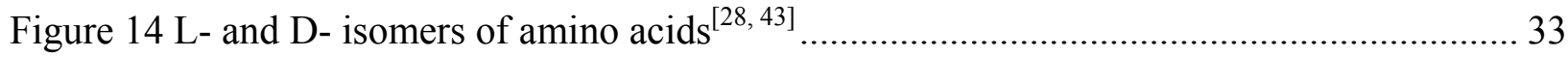

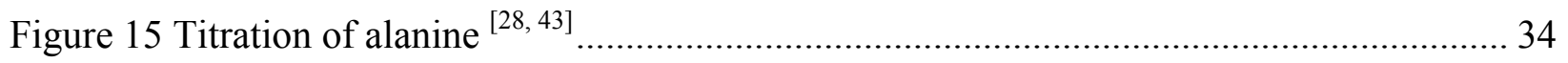

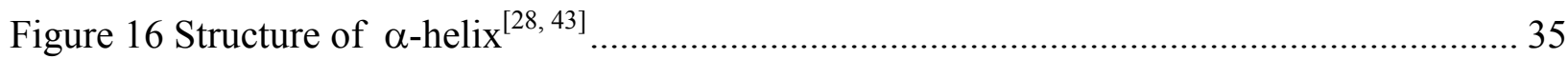

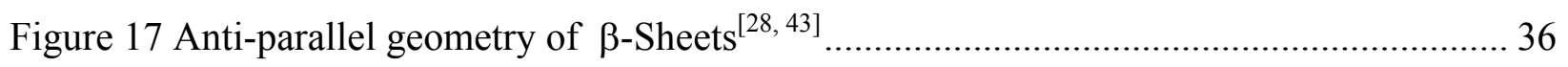

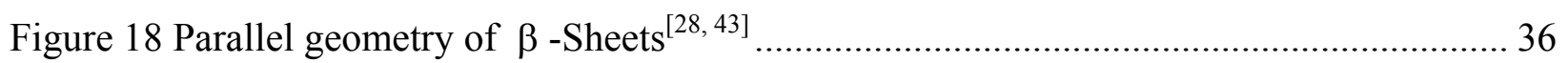

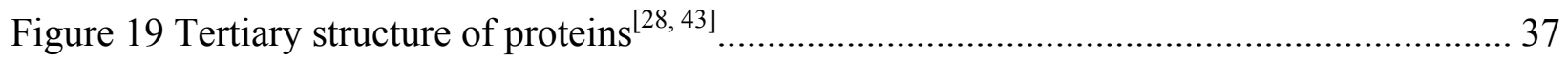

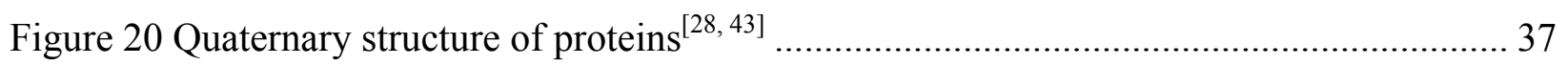

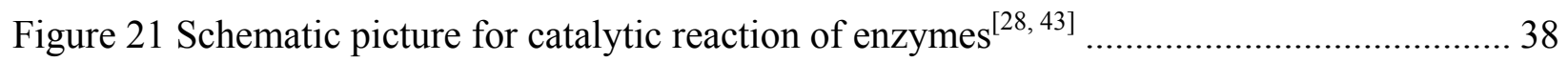

Figure 22 Formation of polysaccharides from simple sugar units ${ }^{[28,43]}$.................................. 40

Figure 23 Structure of a guar molecule and different enzymes specified for different bonds

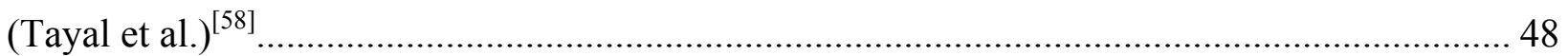

Figure 24 Zero shear viscosity of $0.7 \% \mathrm{w} / \mathrm{v}$ guar solution as a function of enzyme concentration at $25^{\circ} \mathrm{C}$ and $\mathrm{pH}=5$ (Tayal et al.) ${ }^{[58]}$ 49

Figure 25 Comparing the viscosity reduction obtained by a commercial enzyme and a hightemperature enzyme (Tayal et al.) ${ }^{[58]}$

Figure 26 Shear rate profile during a hydraulic fracturing job, fracture height $=300 \mathrm{ft}$, injection

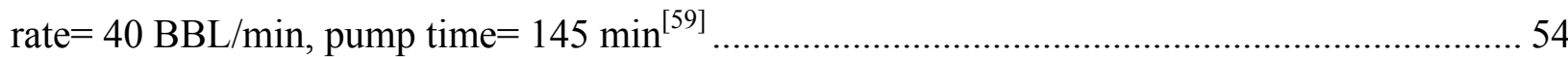

Figure 27 Shear rate history over a rock segment $50 \mathrm{ft}$ away from the wellbore ${ }^{[59]}$................. 54

Figure 28 Temperature profile during a hydraulic fracturing job ${ }^{[63]}$........................................ 55

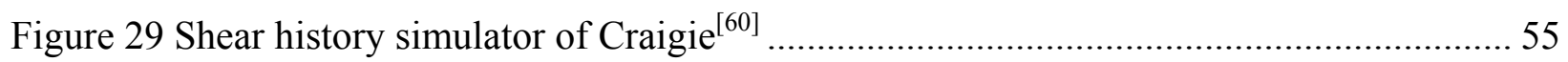

Figure 30 Flow diagram of reciprocating Schlumberger Dowell dynamic fluid loss cell published

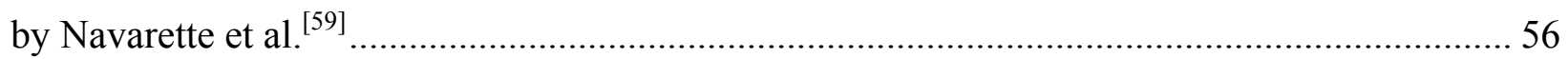

Figure 31 Schematic picture of the laboratory setup used by McGowen and Vitthal ${ }^{[61]}$............. 56

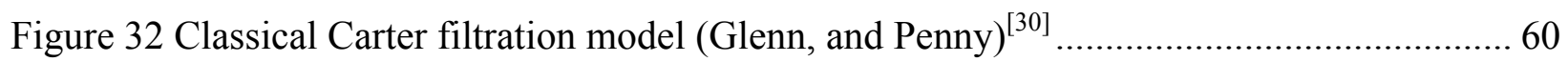

Figure 33 Schematic picture of a static fluid loss cell (Constien et al. ${ }^{[1]}$.................................. 65

Figure 34 Modified Baroid high-pressure static fluid loss cell (From API RP 39) ${ }^{[68]}$................ 65 
Figure 35 Halliburton high pressure static fluid loss cell (From API RP 39) ${ }^{[68]}$ 66

Figure 36 Schematic figure of high pressure high temperature cell used by McGowen and Vitthal ${ }^{[61,62]}$

Figure 37 Schematic picture of static fluid loss cell proposed by Asadi et al. ${ }^{[69]}$ See page 64 for key. 67

Figure 38 Schematic of a disassembled $175 \mathrm{~mL}$ static fluid loss cell presented by Asadi et al. ${ }^{\text {[69] }}$

See page 64 for key. 67

Figure 39 Schematic picture of dynamic fluid loss process, Vitthal et al ${ }^{[61]}$................................ 69

Figure 40 Schematic picture of dynamic fluid loss cells used by Navarette et al ${ }^{[59]}{ }^{[. . . \ldots \ldots \ldots \ldots \ldots . . . . . . . . . ~} 71$

Figure 41 Dynamic fluid loss cell of McGowen and Vitthal ${ }^{[61,62]}$............................................. 73

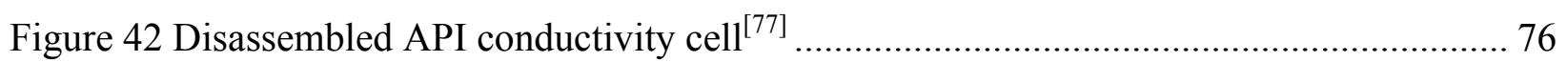

Figure 43 Schematic of flow paths through the API conductivity cell ${ }^{[77]}$.................................... 76

Figure 44 Body of conductivity cell published by Penny ${ }^{[78]}$.................................................... 78

Figure 45 Modified core slab and piston of Penny to allow fluid leak off ${ }^{[78]}$............................. 79

Figure 46 Flow effects on filter cake using different dynamic fluid loss cells, McGowen and Vitthal ${ }^{[62]}$ 80

Figure 47 Schematic picture of pumping setup for dynamic conductivity cell (Marpaung et al.) ${ }^{[80]}$

Figure 48 Schematic picture of conductivity measurement (Marpaung et al.) ${ }^{[80]}$.. 81

Figure 49 Residual gel damage remain after breaking a Zr X-linked CMHPG (35 ppt) in a long

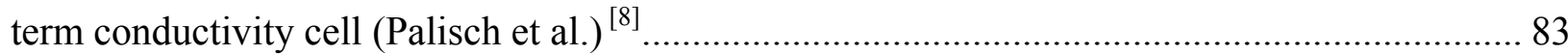

Figure 50 Filter cake build up of 35 ppt Zr X-linked CMHPG (Palisch et al. ${ }^{[8]}$........................ 83

Figure 51 Simulated incomplete cleanup of fracture with a Herschel-Bulkley fluid as fracturing fluid in a tight gas formation (Barati et al. $)^{[7]}$ 84

Figure 52 Experimental apparatus for measuring flow initiation pressure across the filter cake

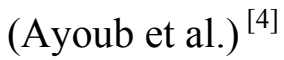
86

Figure 53 Chemical structures of guar showing mechanism of crosslinking by borate, and ether bond cleaved by enzymes. $\mathrm{R}$ is another guar molecule (Drawn by Stephen J. Johnson, used by permission). 89

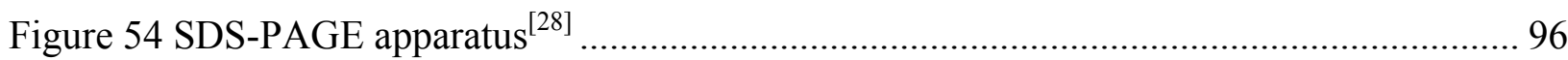


Figure 56 Shear loop set up including three Constametric pumps, temperature controlled air bath, differential pressure transducers and the computer with Labview software used to monitor and

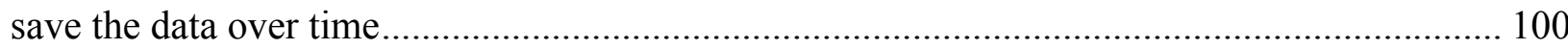

Figure 55 Schematic picture of the shear loop............................................................... 100

Figure 57 Schematic figure of the core saturation setup................................................ 101

Figure 58 Schematic of the permeability measurement and tracer test setup........................ 102

Figure 59 Solution of the convection-dispersion equation for a Peclet Number of 100 at $t_{D}=0.50$ pore volume injected ${ }^{[93]} . \mathrm{X}_{\mathrm{D}}$ is the dimensionless distance. .............................................. 105

Figure 60 Fluid loss cell flow schematic (Drawn by Richard Hutchins) ${ }^{[94]}$............................. 108

Figure 61 Fluid loss cell placed inside a pressure control cabinet supplied with a nitrogen tank

Figure 62 Cell heater with thermocouple inserted inside for preheating. A thermocouple is placed inside the heating jacket to control its temperature.

Figure 63 Fluid loss cell with thermocouple inserted (photo by Richard Hutchins, used by permission) ${ }^{[94]}$

Figure 64 Various endcap assemblies for short cell. The assembled core endcap is shown at the bottom of the picture ${ }^{[94]}$ The top two are the endcap used for disk or filter paper supported by a disk. The third and fourth from the top show the assembly of the setup for cores. (photo by

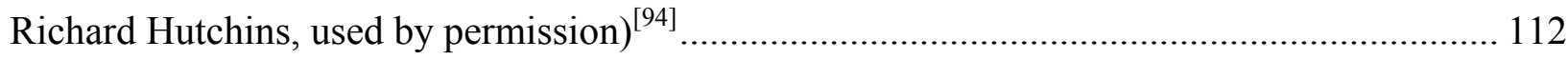

Figure 65 Assembled core endcap with core in place...................................................... 112

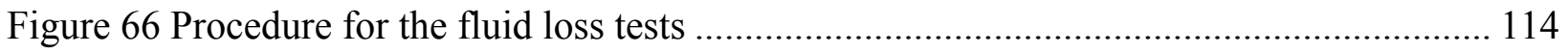

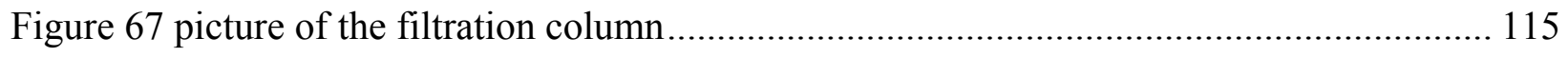

Figure 68 Chlorine test kit, ppm concentrations shown by different colors .......................... 116 Figure 69 Effect of hydration time on guar viscosity. Viscosity values were measured at $11.25 \mathrm{~s}^{-1}$

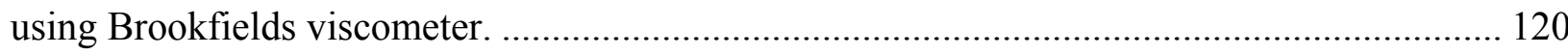

Figure 70 Viscosity versus shear rate for three different 5000 ppm guar solutions in $2 \% \mathrm{KCl} .120$ Figure 71 Viscosity $\left(90 \mathrm{~s}^{-1}\right)$ versus temperature measured for HPG solutions with different $\mathrm{pH}$ values 121

Figure 72 Flow behavior index calculated for HPG solutions with three different $\mathrm{pH}$ values versus temperature 
Figure 73 Flow consistency index calculated for HPG solutions with three different $\mathrm{pH}$ values versus temperature.

Figure 74 Viscoelastic parameters measured for borate-crosslinked HPG versus different concentrations of borax added at $25^{\circ} \mathrm{C}$ and $0.5 \mathrm{~Hz}$.

Figure 75 Viscoelastic parameters measured for borate-crosslinked HPG versus different concentrations of borax added at $40{ }^{\circ} \mathrm{C}$ and $0.5 \mathrm{~Hz}$.

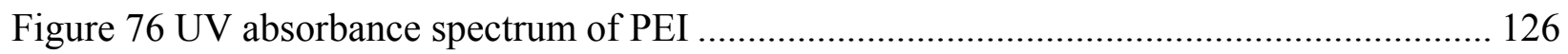

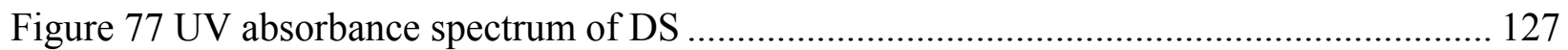

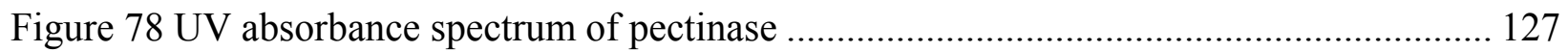

Figure 79 SDS-PAGE gel. Contents of each lane are described in Table 18. Numbers on left are molecular weight of marker proteins in Lane 1.

Figure 80 SDS-PAGE run for different concentrations of pectinase reported in Table 19 (lanes 17), the supernatant of PEC nanoparticle system A (Lanes 8-9) and the supernatant of PEC nanoparticle system B (Lane 10)

Figure 81 Calibration curve for the area under the intensity peak at $116 \mathrm{kDa}$ versus the concentration of pectinase. Supernatants of PECNP systems $A$ and $B$ reported in Lanes 8 and 10 of Table 19 are also shown.

Figure 82 SDS-PAGE run for different concentrations of EL2X reported in Table 20 (lanes 1-7) and the supernatant of PEC nanoparticle system $H^{\prime}$ (Lanes 8-10).

Figure 83 Viscometric assay performed for different concentrations of pectinase mixed with HPG solutions at $\mathrm{pH}$ of 8.4 and $25^{\circ} \mathrm{C}$

Figure 84 Calibration curve measured for different concentrations of pectinase mixed with HPG solutions at $\mathrm{pH}$ of 8.4 and $25^{\circ} \mathrm{C}$. 134 Figure 85 Calibration curve measured for different concentrations of EL2X mixed with HPG solutions at $\mathrm{pH}$ of 8.4 and $25^{\circ} \mathrm{C}$.

Figure 86 Activity versus temperature of pectinase for different $\mathrm{pH}$ values. Final concentration of pectinase in the HPG solutions is $0.02 \% \mathrm{w} / \mathrm{w}$. 135 Figure 87 Activity versus temperature and $\mathrm{pH}$ of pectinase. Final concentration of pectinase in the HPG solutions is $0.02 \% \mathrm{w} / \mathrm{w}$. 136 Figure 88 Activity versus temperature of EL2X for different $\mathrm{pH}$ values. Final concentration of EL2X in the HPG solutions is $0.08 \% \mathrm{w} / \mathrm{w}$. 136 
Figure 89 Activity versus temperature and $\mathrm{pH}$ of EL2X. Final concentration of EL2X in the HPG solutions is $0.08 \% \mathrm{w} / \mathrm{W}$.

Figure 90 Denaturation of different concentrations of pectinase at $\mathrm{pH}$ of 9.1 and $40^{\circ} \mathrm{C}$. Final concentrations of pectinase in the solution are used as labels. 138 Figure 91 Viscometric assay for guar solutions mixed with PECNP D, supernatant of PECNP D or different concentrations of pectinase at $25 \mathrm{C}$ and $\mathrm{pH} 8.7$. 144

Figure 92 Mean size and zeta potential versus $\mathrm{pH}$ for pectinase-loaded PECNPs $\left(A^{\prime}\right)$. 145 Figure $93 \mathrm{pH}$ of $1 \% \mathrm{w} / \mathrm{w}$ PEI $(1 \mathrm{~L})$ versus volume of $4 \mathrm{~N} \mathrm{HC}$ added at $25{ }^{\circ} \mathrm{C}$ 146 Figure 94 Entrapment efficiency $\left(\mathrm{EE}_{\mathrm{A}}\right)$ vs. $\mathrm{pH}$ for pectinase $\left(\mathrm{A}^{\prime}\right)$ loaded PECNPs at $25^{\circ} \mathrm{C} \ldots . . .146$ Figure 95 Mean size and zeta potential versus $\mathrm{pH}$ for EL2X-loaded PECNPs $\left(H^{\prime}, I^{\prime}, J^{\prime}\right)$. Error bars $=1 \mathrm{SE}, \mathrm{n}=3$. 147

Figure 96 Entrapment efficiency $\left(\mathrm{EE}_{\mathrm{A}}\right)$ vs. $\mathrm{pH}$ for EL2X-loaded PECNPs at $40{ }^{\circ} \mathrm{C}$. Error bars $=1$ $\mathrm{SD}, \mathrm{n}=3$ 148

Figure 97 Comparison of effect of shear on the activity of $0.1 \%$ pectinase solution and pectinaseloaded nanoparticles (PECNP System A) mixed with HPG solution at $25^{\circ} \mathrm{C}$ and $\mathrm{pH} 8.8$. Activity $=1 /$ time taken to degrade $5000 \mathrm{ppm}$ HPG solution to $50 \%$ of its initial viscosity. Error bars $=1$ $\mathrm{SD}, \mathrm{n}=3$ 150

Figure 98 Comparison of effect of shear on the activity of $0.4 \%$ EL2X solution and EL2X-loaded nanoparticles (PECNP System $H$ ) mixed with HPG solution at $40{ }^{\circ} \mathrm{C}$ and $\mathrm{pH} 9$. Activity $=1 /$ time taken to degrade $5000 \mathrm{ppm}$ HPG solution to $50 \%$ of its initial viscosity. 150 Figure 99 Viscosity of borate (1000 ppm) cross-linked guar (5000 ppm) gel mixed with $0.1 \%$ pectinase or pectinase-loaded PEC nanoparticles vs. time at $25^{\circ} \mathrm{C}$ and $\mathrm{pH} 9$. N.B. the viscometer is only able to measure viscosity up to $1028 \mathrm{cP}$ so graph indicates the latest measurement for which viscosity exceeded this value. 152 Figure $100 \mathrm{G}^{\prime}$ and $\mathrm{G}^{\prime \prime}$ vs. time for $5000 \mathrm{ppm}$ HPG solution, and borate (2000 ppm)-crosslinked HPG gel degraded using either $0.1 \%$ pectinase or nanoparticle-entrapped pectinase (PEC nanoparticle System $A$ ) at $25^{\circ} \mathrm{C}$ and $\mathrm{pH} 9$. 153 Figure 101 Reproducible degradation of elastic modulus versus time for 5000 ppm HPG solution, and borate (2000 ppm)-crosslinked HPG gel degraded using either $0.1 \%$ pectinase or nanoparticle-entrapped pectinase (PECNP System $A$ ) at $25^{\circ} \mathrm{C}$ and $\mathrm{pH} 9$ 154 
Figure 102 Reproducible degradation of viscous modulus versus time for 5000 ppm HPG solution, and borate (2000 ppm)-crosslinked HPG gel degraded using either $0.1 \%$ pectinase or nanoparticle-entrapped pectinase (PECNP System $A$ ) at $25^{\circ} \mathrm{C}$ and $\mathrm{pH} 9$.

Figure 103 Viscosity of borate (2000 ppm) cross-linked HPG (5000 ppm) gel mixed with 0.4\% EL2X or EL2X-loaded PEC nanoparticles vs. time at $40^{\circ} \mathrm{C}$ and $\mathrm{pH}$ 9.2. N.B. the viscometer is only able to measure viscosity up to $1028 \mathrm{cP}$ so graph indicates the latest............................ 156 Figure $104 \mathrm{G}^{\prime}$ vs. time for borate (2000 ppm)-crosslinked HPG gel degraded using either $0.4 \mathrm{wt}$ $\%$ EL2X or nanoparticle-entrapped EL2X (PECNP systems $H, K$ and $L$ ) at $40{ }^{\circ} \mathrm{C}$ and $\mathrm{pH} 9.2$.

Figure $105 \mathrm{G}^{\prime \prime}$ vs. time for borate (2000 ppm)-crosslinked HPG gel degraded using either $0.4 \mathrm{wt}$ $\%$ EL2X or nanoparticle-entrapped EL2X (PECNP systems $H, K$ and $L$ ) at $40{ }^{\circ} \mathrm{C}$ and $\mathrm{pH} 9.2$.

Figure $106 \mathrm{G}^{\prime}$ and $\mathrm{G}^{\prime \prime}$ vs. time for borate (2000 ppm)-crosslinked HPG gel degraded using either $0.4 \mathrm{wt} \%$ EL2X or nanoparticle-entrapped EL2X (PECNP system $I^{\prime}$ ) at $40{ }^{\circ} \mathrm{C}$ and $\mathrm{pH} 9.2 \ldots . .158$ Figure 107 Reproducible degradation of elastic modulus versus time for 5000 ppm HPG solution, and borate (2000 ppm)-crosslinked HPG gel degraded using either $0.4 \mathrm{wt} \%$ EL2X or PECNP System $I^{\prime}$ at $40{ }^{\circ} \mathrm{C}$ and $\mathrm{pH} 9.2$. 158

Figure 108 Reproducible degradation of viscous modulus versus time for 5000 ppm HPG solution, and borate (2000 ppm)-crosslinked HPG gel degraded using either $0.4 \mathrm{wt} \%$ EL2X or PECNP System $\mathrm{I}^{\prime}$ at $40^{\circ} \mathrm{C}$ and $\mathrm{pH} 9.2$. 159

Figure $109 \mathrm{G}^{\prime}$ and $\mathrm{G}^{\prime \prime}$ vs. time for borate (2000 ppm)-crosslinked HPG gel and HPG gel mixed with control nanoparticles system $H$ at $40{ }^{\circ} \mathrm{C}$ and $\mathrm{pH} 9.2$.

Figure $110 \mathrm{G}^{\prime}$ vs. time for borate (2000 ppm)-crosslinked HPG gel, HPG gel mixed with control PEC nanoparticles, HPG gel mixed with PEI and HPG gel mixed with DS at $40{ }^{\circ} \mathrm{C}$ and $\mathrm{pH} 9.2$.

Figure $111 \mathrm{G}^{\prime \prime}$ vs. time for borate (2000 ppm)-crosslinked HPG gel, HPG gel mixed with control PEC nanoparticles, HPG gel mixed with PEI and HPG gel mixed with DS at $40{ }^{\circ} \mathrm{C}$ and $\mathrm{pH} 9.2$.

Figure 112 Entrapment of enzymes in PEC nanoparticles $A^{\prime}$ protects activity of pectinase (pH $\left.9.1,40{ }^{\circ} \mathrm{C}\right)$ 162 
Figure 113 Entrapment of enzymes in PEC nanoparticles $H^{\prime}$ protects activity of EL2X (pH 9.75, $\left.50{ }^{\circ} \mathrm{C}\right)$. 163

Figure 114 Pressure drop across the first section of shear loop for HPG gel, (HPG gel + PECNP A) and (HPG gel $+0.1 \% \mathrm{w} / \mathrm{w}$ pectinase) at $\mathrm{pH} 9$ and $25^{\circ} \mathrm{C}$ 164

Figure 115 Pressure drop across the second section of shear loop for HPG gel, (HPG gel + PECNP $A$ ) and (HPG gel $+0.1 \% \mathrm{w} / \mathrm{w}$ pectinase) at $\mathrm{pH} 9$ and $25{ }^{\circ} \mathrm{C}$ 165

Figure 116 Pressure drop across the third section of shear loop for HPG gel, (HPG gel + PECNP A) and (HPG gel $+0.1 \% \mathrm{w} / \mathrm{w}$ pectinase) at $\mathrm{pH} 9$ and $25^{\circ} \mathrm{C}$ 165

Figure 117 Elastic modulus measured at $0.5 \mathrm{~Hz}$ for samples taken from different sections of shear loop for HPG gel, (HPG gel + PECNP $A$ ) and (HPG gel $+0.1 \%$ w/w pectinase) at $\mathrm{pH} 9$ and $25{ }^{\circ} \mathrm{C}$ 166

Figure 118 Viscous modulus measured at $0.5 \mathrm{~Hz}$ for samples taken from different sections of shear loop for HPG gel, (HPG gel + PECNP $A$ ) and (HPG gel $+0.1 \%$ w/w pectinase) at $\mathrm{pH} 9$ and $25{ }^{\circ} \mathrm{C}$ 166

Figure 119 Elastic modulus measured for HPG gel, (HPG gel + PECNP $H$ ) and (HPG gel +0.4 wt $\%$ EL2X) at the end of different sections of shear loop $\left(\mathrm{pH}=9.2\right.$ and $\left.\mathrm{T}=40{ }^{\circ} \mathrm{C}\right)$. HPG gel was

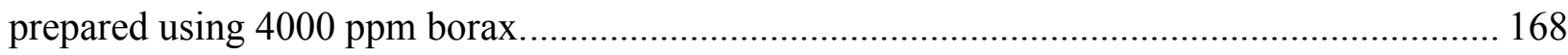
Figure 120 Viscous modulus measured for HPG gel, (HPG gel + PECNP $H)$ and (HPG gel + 0.4 wt $\%$ EL2X) at the end of different sections of shear loop $\left(\mathrm{pH}=9.2\right.$ and $\left.\mathrm{T}=40{ }^{\circ} \mathrm{C}\right)$. HPG gel was prepared using $4000 \mathrm{ppm}$ borax. 169

Figure 121 Pressure drop across the first section of shear loop for HPG gel, (HPG gel + PECNP $\left.\mathrm{I}^{\prime}\right)$ and (HPG gel $+0.4 \% \mathrm{w} / \mathrm{w}$ EL2X) at $\mathrm{pH} 9.2$ and $40{ }^{\circ} \mathrm{C}$ 170

Figure 122 Pressure drop across the second section of shear loop for HPG gel, (HPG gel + PECNP I') and (HPG gel $+0.4 \% \mathrm{w} / \mathrm{w}$ EL2X) at $\mathrm{pH} 9.2$ and $40{ }^{\circ} \mathrm{C}$ 171

Figure 123 Pressure drop across the third section of shear loop for (HPG gel + PECNP I') and (HPG gel $+0.4 \% \mathrm{w} / \mathrm{w}$ EL2X) at $\mathrm{pH} 9.2$ and $40{ }^{\circ} \mathrm{C}$ 171

Figure 124 Flow rate, pressure drop, UV absorbance and permeability of core\#6 before fluid-loss tests at $25{ }^{\circ} \mathrm{C}$.

Figure 125 Flow rate, pressure drop and permeability versus time for core\# 6 resaturated after tracer test 
Figure 126 Tracer injection UV absorbance versus time curves for different cores used in fluidloss tests. UV absorbance was measured at $302 \mathrm{~nm}$ while tracer was injected and cleaned up with $1 \mathrm{~mL} /$ minutes flow rate.

Figure 127 Tracer cleanup UV absorbance versus time curves for different cores used in fluidloss tests. UV absorbance was measured at $302 \mathrm{~nm}$ while $2 \% \mathrm{w} / \mathrm{w} \mathrm{KCl}$ was injected and cleaned up with $1 \mathrm{~mL} /$ minutes flow rate. 175

Figure 128 Flow rate, pressure drop and permeability versus time for core\# 6 after restoration 176 Figure 129 Flow rate, pressure drop and permeability of core\#17 before fluid-loss tests at $40{ }^{\circ} \mathrm{C}$.

Figure 130 Flow rate, pressure drop, UV absorbance and permeability of core\#12 before fluidloss tests at $40{ }^{\circ} \mathrm{C}$. Pressure drop was not recorded above 10 psi thus permeability was not calculated since the pressure transmitters were set for this range. 178 Figure 131 Flow rate, pressure drop and permeability versus time for core\# 12 after resaturation. Core was dried and resaturated after the tracer test was performed. 178 Figure 132 Fluid-loss versus $\mathrm{t}^{1 / 2}$ for HPG gel, (HPG gel + PECNP control), (HPG gel +PECNP $A)$ and (HPG gel $+0.1 \% \mathrm{w} / \mathrm{w}$ pectinase) at $25{ }^{\circ} \mathrm{C}$. 182

Figure 133 Filter-cake cleanup versus time for HPG gel, (HPG gel + PECNP control), (HPG gel $+\mathrm{PECNP} A$ ) and (HPG gel $+0.1 \% \mathrm{w} / \mathrm{w}$ pectinase) at $25^{\circ} \mathrm{C}$ 182

Figure 134 Fluid-loss versus $\mathrm{t}^{1 / 2}$ for HPG gel, (HPG gel + PECNP control), (HPG gel +PECNP $\left.I^{\prime}\right)$ and (HPG gel $+0.4 \% \mathrm{w} / \mathrm{w}$ EL2X) at $40{ }^{\circ} \mathrm{C}$. 186 Figure 135 Filter-cake cleanup versus time for HPG gel, (HPG gel + PECNP control), (HPG gel + PECNP $\left.I^{\prime}\right)$ and (HPG gel $+0.4 \% \mathrm{w} / \mathrm{w}$ EL2X) at $40{ }^{\circ} \mathrm{C}$

Figure 136 Permeability measurement for core 4 (restored) after the fluid-loss and cleanup tests reported in Table 35 . 188

Figure 137 Permeability measurement for core 24 after the fluid-loss and cleanup tests reported in Table 39 ...... 188

Figure 138 Viscosity at $90 \mathrm{~s}^{-1}$ versus time for $1000 \mathrm{ppm}$ HPG samples mixed with pectinase, PECNP $A^{\prime}$ and controlled PECNPs diluted $8 \times$ compared with a control HPG system at $25^{\circ} \mathrm{C} .191$ Figure 139 Viscosity at $90 \mathrm{~s}^{-1}$ versus time for 1200 ppm HPG samples mixed with EL2X, PECNP I' and controlled PECNPs diluted 4× compared with a control HPG system at $40{ }^{\circ} \mathrm{C} .193$ 
Figure 140 Flow rate, pressure drop, UV absorbance and permeability of core\#1 before fluid loss tests at $25{ }^{\circ} \mathrm{C}$ including tracer test results. 215

Figure 141 Flow rate, pressure drop, UV absorbance and permeability of core\#3 before fluid loss

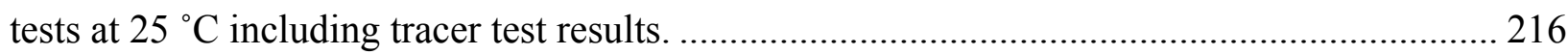
Figure 142 Flow rate, pressure drop, UV absorbance and permeability of core\#4 before fluid loss tests at $25{ }^{\circ} \mathrm{C}$ including tracer test results. 216 Figure 143 Flow rate, pressure drop, UV absorbance and permeability of core\#5 before fluid loss tests at $25{ }^{\circ} \mathrm{C}$ including tracer test results. 217

Figure 144 Flow rate, pressure drop, UV absorbance and permeability of core\#6 before fluid loss tests at $25{ }^{\circ} \mathrm{C}$ including tracer test results. 217 Figure 145 Flow rate, pressure drop, UV absorbance and permeability of core\#8 before fluid loss tests at $25{ }^{\circ} \mathrm{C}$ including tracer test results. 218 Figure 146 Flow rate, pressure drop, UV absorbance and permeability of core\#9 before fluid loss tests at $25{ }^{\circ} \mathrm{C}$ including tracer test results. 218 Figure 147 Flow rate, pressure drop, UV absorbance and permeability of core\#10 before fluid loss tests at $25^{\circ} \mathrm{C}$ including tracer test results.

Figure 148 Flow rate, pressure drop, UV absorbance and permeability of core\#13 before fluid loss tests at $40{ }^{\circ} \mathrm{C}$ including tracer test results. 219 Figure 149 Flow rate, pressure drop and permeability of core\#14 before fluid loss tests at $40{ }^{\circ} \mathrm{C}$. 220

Figure 150 Flow rate, pressure drop and permeability of core\#15 before fluid loss tests at $40{ }^{\circ} \mathrm{C}$. 220

Figure 151 Flow rate, pressure drop and permeability of core\#16 before fluid loss tests at $40{ }^{\circ} \mathrm{C}$.

Figure 152 Flow rate, pressure drop and permeability of core\#18 before fluid loss tests at $40{ }^{\circ} \mathrm{C}$. 221

Figure 153 Flow rate, pressure drop and permeability of core\#19 before fluid loss tests at $40{ }^{\circ} \mathrm{C}$. 222

Figure 154 Flow rate, pressure drop and permeability of core $\# 20$ before fluid loss tests at $40{ }^{\circ} \mathrm{C}$. 222 
Figure 155 Flow rate, pressure drop and permeability of core\#21 before fluid loss tests at $40{ }^{\circ} \mathrm{C}$. 223

Figure 156 Flow rate, pressure drop and permeability of core\#22 before fluid loss tests at $40{ }^{\circ} \mathrm{C}$. 223

Figure 157 Flow rate, pressure drop and permeability of core\#23 before fluid loss tests at $40{ }^{\circ} \mathrm{C}$.

Figure 158 Flow rate, pressure drop and permeability of core\#24 before fluid loss tests at $40{ }^{\circ} \mathrm{C}$.

Figure 159 Flow rate, pressure drop and permeability of core\#1 resaturated after tracer test and before fluid loss tests at $25{ }^{\circ} \mathrm{C}$. 225

Figure 160 Flow rate, pressure drop and permeability of core\#2 resaturated after tracer test and before fluid loss tests at $25{ }^{\circ} \mathrm{C}$ 226

Figure 161 Flow rate, pressure drop and permeability of core\#3 resaturated after tracer test and before fluid loss tests at $25^{\circ} \mathrm{C}$. 226 Figure 162 Flow rate, pressure drop and permeability of core\#4 resaturated after tracer test and before fluid loss tests at $25{ }^{\circ} \mathrm{C}$.

Figure 163 Flow rate, pressure drop and permeability of core\#5 resaturated after tracer test and before fluid loss tests at $25^{\circ} \mathrm{C}$. 227

Figure 164 Flow rate, pressure drop and permeability of core\#8 resaturated after tracer test and before fluid loss tests at $25{ }^{\circ} \mathrm{C}$...... 228 Figure 165 Flow rate, pressure drop and permeability of core\#9 resaturated after tracer test and before fluid loss tests at $25{ }^{\circ} \mathrm{C}$. 228

Figure 166 Flow rate, pressure drop and permeability of core\#10 resaturated after tracer test and before fluid loss tests at $25{ }^{\circ} \mathrm{C}$. 229 Figure 167 Flow rate, pressure drop and permeability of core\#13 resaturated after tracer test and before fluid loss tests at $40{ }^{\circ} \mathrm{C}$. 229 Figure 168 Flow rate, pressure drop and permeability of core\#1 restored after fluid loss test and before being reused for fluid loss tests at $25^{\circ} \mathrm{C}$...... 230 Figure 169 Flow rate, pressure drop and permeability of core\#4 restored after fluid loss test and before being reused for fluid loss tests at $25^{\circ} \mathrm{C}$. 231 
Figure 170 Flow rate, pressure drop and permeability of core\#5 restored after fluid loss test and

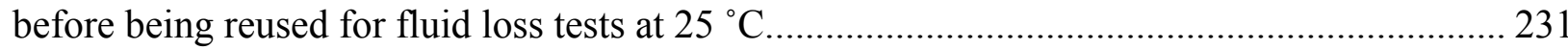
Figure 171 Flow rate, pressure drop and permeability of core\#6 restored after fluid loss test and

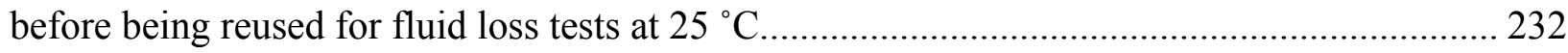
Figure 172 Flow rate, pressure drop and permeability of core\#9 restored after fluid loss test and before being reused for fluid loss tests at $25^{\circ} \mathrm{C}$. 232

Figure 173 Flow rate, pressure drop and permeability of core\#10 restored after fluid loss test and

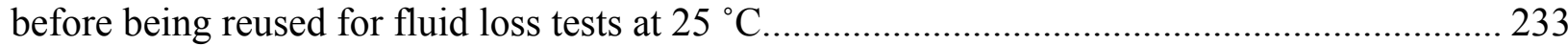
Figure 174 Flow rate, pressure drop and permeability of core\#10 after fluid loss test at $25{ }^{\circ} \mathrm{C} .234$ Figure 175 Flow rate, pressure drop and permeability of core $\# 9$ after fluid loss test at $25^{\circ} \mathrm{C}$. 235 Figure 176 Flow rate, pressure drop and permeability of core\#1 after fluid loss test at $25^{\circ} \mathrm{C}$. 235 Figure 177 Flow rate, pressure drop and permeability of core $\# 4$ after fluid loss test at $25^{\circ} \mathrm{C}$. . 236 Figure 178 Flow rate, pressure drop and permeability of core $\# 8$ after fluid loss test at $25^{\circ} \mathrm{C}$. 236 Figure 179 Flow rate, pressure drop and permeability of core\#6 after fluid loss test at $25^{\circ} \mathrm{C}$. . 237 Figure 180 Flow rate, pressure drop and permeability of core $\# 5$ after fluid loss test at $25^{\circ} \mathrm{C}$. . 237 Figure 181 Flow rate, pressure drop and permeability of restored core\#1 after fluid loss test at 25 ${ }^{\circ} \mathrm{C}$. 238

Figure 182 Flow rate, pressure drop and permeability of restored core\#10 after fluid loss test at $25^{\circ} \mathrm{C}$ 238

Figure 183 Flow rate, pressure drop and permeability of restored core $\# 9$ after fluid loss test at 25 ${ }^{\circ} \mathrm{C}$. 239

Figure 184 Flow rate, pressure drop and permeability of restored core\#6 after fluid loss test at 25 ${ }^{\circ} \mathrm{C}$. 239

Figure 185 Flow rate, pressure drop and permeability of restored core $\# 5$ after fluid loss test at 25 ${ }^{\circ} \mathrm{C}$. 240

Figure 186 Flow rate, pressure drop and permeability of core\#15 after fluid loss test at $40{ }^{\circ} \mathrm{C} .240$ Figure 187 Flow rate, pressure drop and permeability of core\#18 after fluid loss test at $40{ }^{\circ} \mathrm{C} .241$ Figure 188 Flow rate, pressure drop and permeability of core\#19 after fluid loss test at $40{ }^{\circ} \mathrm{C} .241$ Figure 189 Flow rate, pressure drop and permeability of core\#21 after fluid loss test at $40{ }^{\circ} \mathrm{C} .242$ Figure 190 Flow rate, pressure drop and permeability of core\#22 after fluid loss test at $40{ }^{\circ} \mathrm{C} .242$ Figure 191 Flow rate, pressure drop and permeability of core\#23 after fluid loss test at $40{ }^{\circ}$ C. 243 


\section{List of Tables}

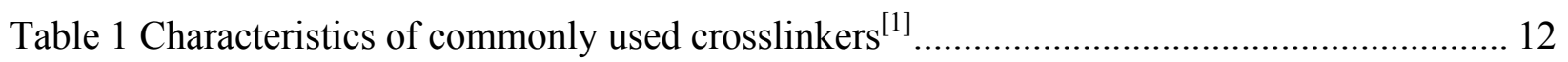

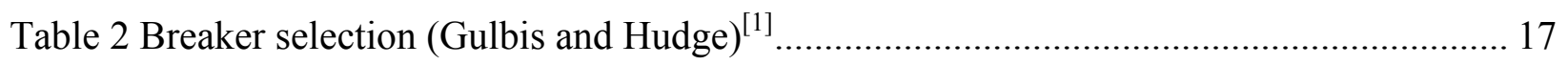

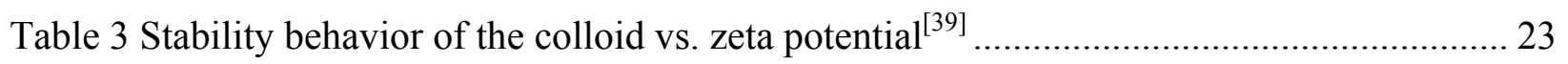

Table 4 Effect of polymer ratio on the mean particle size and polydispersity of Amphotericin B

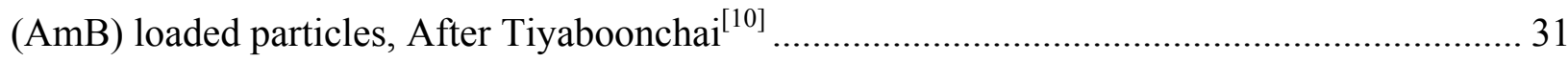

Table 5 Effect of the $\mathrm{pH}$ of PEI solutions on the mean particle size and polydispersity of

Amphotericin B (AmB) loaded particles, After Tiyaboonchai ${ }^{[10]}$.............................................. 31

Table 6 Effect of zinc sulfate concentration on the mean particle size and polydispersity of

Amphotericin B (AmB) loaded particles, After Tiyabooncha ${ }^{[10]}$................................................ 32

Table 7 Effect of dextran sulfate concentration on the mean particle size and polydispersity of Amphotericin B (AmB) loaded particles, After Tiyaboonchai ${ }^{[10]}$............................................. 32

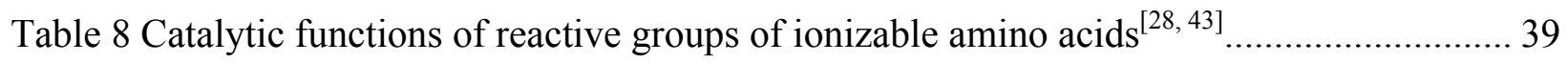

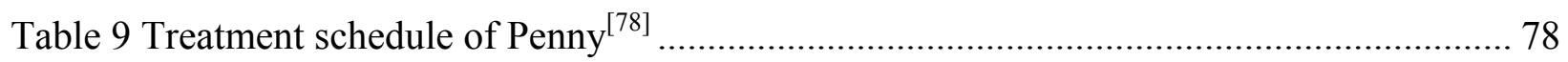

Table 10 Calculations related to porosity measurement for six different cores before the tracer

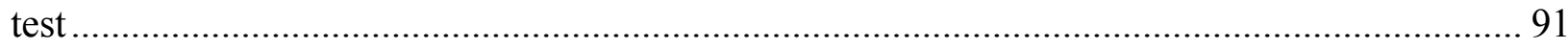

Table 11 Nanoparticle systems differed in the ratio of PEI, DS, and pectinase and order of addition.

Table 12 Shear rate calculated for different RPM values of Brookfield viscometer fitted with SP-

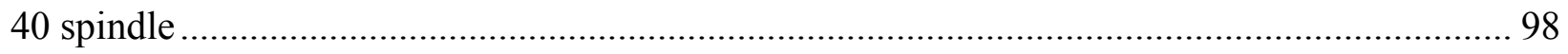

Table 13 Mixing flow rates used for different shear loop experiments ........................................ 99

Table 14 Shear calculations for three sections of shear loop ........................................................ 99

Table 15 Weight percent of residues taken from 5000 ppm guar solution versus time............... 119

Table 16 Viscosity $\left(90 \mathrm{~s}^{-1}\right)$ versus temperature measured for HPG solutions with different $\mathrm{pH}$

values

Table 17 Average flow behavior ( $\left.\mathrm{n}^{\prime}\right)$ and flow consistency $\left(\mathrm{K}^{\prime}\right)$ indices versus temperature measured for different samples ............................................................................................... 123

Table 18 Samples $(\mathrm{mL})$ in SDS-PAGE lanes shown in Figure 79 ......................................... 130

Table 19 Samples in SDS-PAGE lanes shown in Figure 80 ................................................... 130

Table 20 Samples in SDS-PAGE lanes shown in Figure 82 ................................................... 131 
Table 21 Activity versus temperature of pectinase for different $\mathrm{pH}$ values. Final concentration of pectinase in the HPG solutions is $0.02 \% \mathrm{w} / \mathrm{w}$.

Table 22 Activity versus temperature of EL2X for different $\mathrm{pH}$ values. Final concentration of EL2X in the HPG solutions is $0.08 \% \mathrm{w} / \mathrm{w}$. 138

Table 23 Nanoparticle systems differed in the ratio of PEI, DS, and pectinase and order of addition.

Table 24 Mean diameter vs. time for 10 samples of PEC nanoparticles $A$. 142

Table 25 Mean particle diameter and zeta potential $(\zeta)$ vs. time for nanoparticles, $\mathrm{pH}=8.7 \ldots \ldots .142$ Table 26 Activity and entrapment efficiency of pectinase-loaded nanoparticles and their supernatants using viscometric assay $\left(\mathrm{T}=25{ }^{\circ} \mathrm{C}, \mathrm{pH}=8.8\right)$. Centrifuged $5000 \mathrm{ppm}$ guar prepared in de-ionized water was used for this assay. 143

Table 27 Entrapment efficiencies and enzyme concentrations for different supernatants using the first intensity peak.

Table 28 Effect of preparation shear on size and zeta potential of PECNP $A$ ' system at $\mathrm{pH} 8.8$ and $25^{\circ} \mathrm{C}$

Table 29 Final concentrations of HPG/guar, borax and enzymes in the solutions for all gel degradation studies reported in this research.....

Table 30 Percentage of viscoelastic moduli for HPG gel with PECNP $A$ and $0.1 \% \mathrm{w} / \mathrm{w}$ pectinase over that of HPG gel at $\mathrm{pH} 9$ and $25^{\circ} \mathrm{C}$ 167

Table 31 Pressure drops of HPG gel (4000 ppm borax), (HPG gel + PECNP H) and (HPG gel + $0.4 \mathrm{wt} \%$ EL2X) across the shear loop at $\mathrm{pH} 9.2$ and $40{ }^{\circ} \mathrm{C}$. HPG gel was prepared using 4000 ppm borax. 168

Table 32 Pressure drops of HPG gel (2000 ppm borax), (HPG gel + PECNP $\left.I^{\prime}\right)$ and (HPG gel + $0.4 \mathrm{wt} \%$ EL2X) across the shear loop at $9.2 \mathrm{pH}$ and $40{ }^{\circ} \mathrm{C}$. HPG gel was prepared using 2000 ppm borax. 170

Table 33 Permeability and porosity measurements for cores used at $25^{\circ} \mathrm{C}$. Porosity using tracer test and porosity and permeability after restoration is also reported. 173 Table 34 Permeability and porosity measurements for cores used at $40{ }^{\circ} \mathrm{C}$. Permeability after resaturation is also reported. 177 Table 35 Fluid-loss parameters for different fluids run at $25^{\circ} \mathrm{C}$. 181 Table 36 Cleanup slope calculated for different fluid-loss tests reported in Table 35 183 
Table 37 Size measurement for PEC nanoparticles used in fluid-loss tests reported in Table 35 $(\mathrm{pH}=8.7)$ 183

Table 38 TOC and ICP measured by Dr. Karen Peltier for filtrates remain after the fluid-loss tests ....... 184

Table 39 Fluid-loss parameters for different fluids run at $40{ }^{\circ} \mathrm{C}$ 185 Table 40 Size measurement for PEC nanoparticles used in fluid-loss tests reported in Table 39 $(\mathrm{pH}=9.4)$. 187

Table 41 Mixing ratios for different HPG samples mixed with R.O. water, control PECNPs and PECNP $A^{\prime}$ at $25^{\circ} \mathrm{C}$. 190

Table 42 Particle size report for pectinase-loaded nanoparticles used for slick-water applications 190

Table 43 Mixing ratios for different HPG samples mixed with R.O. water, control PECNPs and PECNP $I^{\prime}$ at $40^{\circ} \mathrm{C}$. 192

Table 44 Particle size report for EL2X-loaded nanoparticles used for slick water applications 192 


\section{Introduction}

Hydraulic fracturing is a successful technology to increase conductivity and effective well-sand interfacial area during production of hydrocarbons. To propagate a fracture into a reservoir, fracturing fluids are used with two main functions of opening the fracture and transporting propping agents along the fracture. ${ }^{[1]}$ Incorporation of salts, like potassium or calcium chloride, in the treating fluids to protect water-sensitive formations, and considering the fact that waterbased fluids are cheaper and safer, directed companies towards using water-based fracturing fluids more than other types of fracturing fluid. Guar gum is one of the oldest examples of waterbased polymers used to viscosify water for fracturing purposes. Even though fracturing jobs are shifting towards slick-water treatments ${ }^{[2]}$ for unconventional reservoirs, guar gum and its derivatives are commonly used to viscosify water in fracturing fluids for conventional treatments.

The fracturing fluid must be viscous during the injection and be broken easily after the injection to maintain high conductivity during production. In order to do this, cross-linkers (like borate) and breakers (either oxidizers or enzymes) are added to the fluid. However the viscosity development during injection should not occur too early thereby increasing the friction pressure in the tubulars, leading to higher surface pressure and horsepower requirements.

Injecting the viscous fracturing fluid by itself causes problems, like filter-cake formation and fluid loss to the matrix. Filter cakes with high polymer concentration form on the two faces of the fracture during injection, but normally a small path in the middle of the fracture has the properties of the injected polymer unless the fracture is totally plugged by filter cakes. According to the latest research ${ }^{[3,4]}$, filter cakes do not form with uniform thickness and concentration along the fracture. Maximum pressure drop between a fracture and the reservoir during the production occurs through the filter cake. ${ }^{[1]}$ This makes the filter cake the main target for the breakers. Using high concentrations of enzymes as the breaker causes premature degradation while encapsulated breakers (EB) break the filter cake only if they are delivered directly into and uniformly along the filter cake. Even though a mixture of enzymes and $\mathrm{EB}^{[5]}$ is normally used to break the filter cake and fracturing fluid, filter cake is reportedly ${ }^{[3,6,7]}$ broken in a non-uniform manner. Barati et al ${ }^{[7]}$ reported that yield stress of the filter cake and fracturing fluid, and formation damage as a 
result of fracturing fluid invasion into the matrix have significant effects on production of hydrocarbons especially in low permeability formations.

Incomplete cleanup of hydraulic fractures caused by gel residues ${ }^{[8]}$, width loss caused by filter cake $^{[3,4]}$ and fracture length loss due to unbroken fluid near the tip of the fracture ${ }^{[7]}$ decreases the effective conductivity of hydraulic fractures compared to their designed conductivity. Delivering sufficient concentrations of breakers directly to the filter cake and distributing the breakers uniformly results in better cleanup of fractures. ${ }^{[3,4]}$

Enzymes have been used successfully as breakers for fracturing fluids for many years. ${ }^{[1]}$ Enzymes are polymer specific, environmentally benign, easy to handle, miscible in the fluid, equipment friendly and not consumed since they act as catalysts. ${ }^{[9]}$ Oxidizers on the other hand, are not environmentally nor equipment friendly. Oxidizers affect the activity of enzymes when mixed. ${ }^{[5]}$ The main limitation of enzymes is their denaturation at high temperature and alkaline $\mathrm{pH}$ environments.

Polyelectrolyte (PEC) complex nanoparticles used in this research were first introduced for drug delivery applications. ${ }^{[10-12]}$ Polyelectrolyte complex (PEC) nanoparticles have been also used successfully to entrap and release Cr(III) in a controlled manner for water control applications. ${ }^{[13,}$

14] It was hypothesized that such nanoparticles will be capable of releasing enzymes in a controlled manner with the potential for application in breaking fracturing fluids. Use of such a carrier for breakers in fracturing fluids would require high entrapment efficiency of the breaker, homogeneous distribution and flexible release time.

In this study, application of polyelectrolyte nanoparticles for entrapping, carrying, releasing and protecting enzymes for fracturing fluids has been examined. The objective of this research is to develop nano-sized carriers capable of carrying enzymes to the filter cake, delaying the release of enzyme and protecting the enzyme against the $\mathrm{pH}$ and temperature conditions inhospitable to native enzyme. The approach taken towards the objective of this research is threefold. First, charged nanoparticles are developed which have high enzyme entrapment efficiency and are capable of carrying breakers for fracturing fluids and have flexible release time. In this part, we present a proof of concept for the application of PEC nanoparticles to entrap and release enzyme breakers for fracturing fluids. Positively charged PEC nanoparticles were made by varying the total concentration and charge of a polycation (polyethylenimine) and a polyanion (dextran sulfate). Two enzymes typically used in the petroleum industry to break the fracturing fluids 
were added to a polyethylenimine (PEI) solution, either before or after the addition of dextran sulfate (DS) and were entrapped in the PEC nanoparticles. Entrapment efficiency (EE) was calculated for the nanoparticles loaded with enzymes using a viscometric assay and confirmed using sodium dodecyl sulfate polyacrylamide gel electrophoresis (SDS-PAGE). Nanoparticles were then used to degrade borate-cross-linked guar solutions at $25{ }^{\circ} \mathrm{C}$ and $40{ }^{\circ} \mathrm{C}$. Retardation of enzyme activity was observed by measuring the viscosity of the gelled guar and by monitoring the viscoelastic moduli of the gel. Delay times were compared with equivalent systems using unentrapped enzymes. Nanoparticles were also studied for their protective effect on both enzymes at temperature and $\mathrm{pH}$ values inhospitable to native enzyme. Activity of nanoparticles loaded with enzymes was compared to activity of unentrapped enzymes at these harsh $\mathrm{pH}$ and temperature conditions.

Second, a shear loop in a temperature control cabinet was used to study the effect of shear on release of enzymes from nanoparticles. Borate-cross-linked HPG mixed with enzyme or nanoparticles before gelation was injected through a $617 \mathrm{ft}$ shear loop under a shear of $190 \mathrm{~s}^{-1}$ at 25 and $40{ }^{\circ} \mathrm{C}$. The pressure drop required to displace the gel along each section was compared for the gels with no breaker, nanoparticle and free enzyme.

Third, enzyme-loaded nanoparticles were applied in order to break the filter-cake formed by guar based fracturing fluids. Static fluid-loss cells were used to filter borate-cross-linked gels with enzyme or nanoparticles under 500 psi differential pressure at $25{ }^{\circ} \mathrm{C}$ and $40{ }^{\circ} \mathrm{C}$. After the formation of filter-cake the setup was shut-in for 12 hours with the remaining fluid setting on top of the filter cake. After 12 hours the fluid was replaced with $2 \% \mathrm{KCl}$ and the test was started again to study the effectiveness of enzymes or nanoparticles in breaking the filter-cake.

The result of this research provides a product which is potentially capable of distributing the breakers uniformly along the fracture, including the filter cake. This decreases the yield stress and viscosity of the fracturing fluid and filter cake uniformly along the fracture. This system can potentially be applied to direct the breakers to some specific region of the fracture or even reservoir for high permeability applications. 


\section{Literature Review}

In this section, literature pertinent to hydraulic fracturing process, fracturing fluids, polyelectrolyte nanoparticles, enzymes applied in the fracturing industry and their behavior, and setups simulating the shear history, fluid loss process, and damage occurred to proppant packs are reviewed. In section 2.1 a general introduction to propagation and cleanup of hydraulic fractures is presented. In section 2.2 chemistry of fracturing fluids and specifically guar-based fracturing fluids is explained. In section 2.3 polyelectrolyte nanoparticles used in this project are introduced. Section 2.4 presents a detailed review of the enzymes used in the fracturing industry and their properties. Section 2.5 explains the setups used in industry to simulate the shear, fluid loss and degradation conditions fracturing fluids experience during a typical fracturing job. In section 2.6 dynamic and static fluid loss cells used in the hydraulic fracturing industry and their advantages and limitations are presented. Section 2.7 describes different conductivity cells used in the fracturing industry. The fracture cleanup process and mechanisms which result in damage to the proppant pack are reviewed in section 2.8. Section 2.9 summarizes the literature review.

\subsection{Hydraulic Fractures, Propagation and Cleanup}

\section{1-1 Hydraulic Fracturing in Conventional Reservoirs}

Hydraulic fracturing is a successful technology to increase conductivity and available sand surface during production. To propagate an open fracture into a reservoir, fracturing fluids have been used with two main functions of initiating the fracture and transporting propping agents along the fracture. ${ }^{[1]}$ Guar gum is the oldest example of aqueous, viscous fluids used during the injection. The fracturing fluid must be viscous to allow transport of the proppant, which is required to keep the fracture open, during the injection. Fracturing fluid must be broken fully after the injection to maintain high conductivity during the production phase. To accomplish these tasks, crosslinkers (like borate and zirconate) and delayed breakers (either oxidizers or enzymes) are added to the fluid. ${ }^{[1]}$

During injection of the viscous fracturing fluid, fluid loss to the matrix occurs and filter cake forms. Filter cakes with high polymer concentration form on the two faces of the fracture during the injection (Figure 1) but normally a small path in the middle of the fracture has the properties

of the injected polymer unless the fracture is totally plugged by filter cakes from both faces. ${ }^{[3]}$ 
Different exposure times to fracturing fluid, ${ }^{[15]}$ and different proppant concentrations along the fracture cause local polymer concentration changes along the fracture. Thus encapsulated breakers are seldom uniformly distributed to break the concentrated fluid completely.

At the end of a fracture treatment, normally there is a shut-in period to allow fracture closure. Fluid continues to leak off into the reservoir during this stage. The fluid which leaks off causes hydraulic and physical damage to the reservoir (Figure 1). Hydraulic damage is caused by shifted capillary pressure and relative permeability curves in the invaded area. Physical damage is caused by processes like clay swelling, and/or invasion of fracturing fluid into the formation. Hydraulic fractures contain partially broken fracturing fluid, and residues remain after the breaker acts on the guar. It has been postulated that fracturing fluids need a minimum pressure gradient to start flowing back through the proppant pack. ${ }^{[4]}$

Different studies have shown incomplete cleanup of fractures after proppagation. May et $\mathrm{al}^{[16]}$ using a 2-D, fully implicit, compositional model capable of modeling the flow of HerschelBulkley fluids in proppant pack showed significant damage to the oil production as a result of incomplete cleanup of fracturing fluids. The significance of damage to fracture conductivity caused by partially broken fracturing fluid and filter cake was also shown by other researchers. ${ }^{[7,}$ 17] 


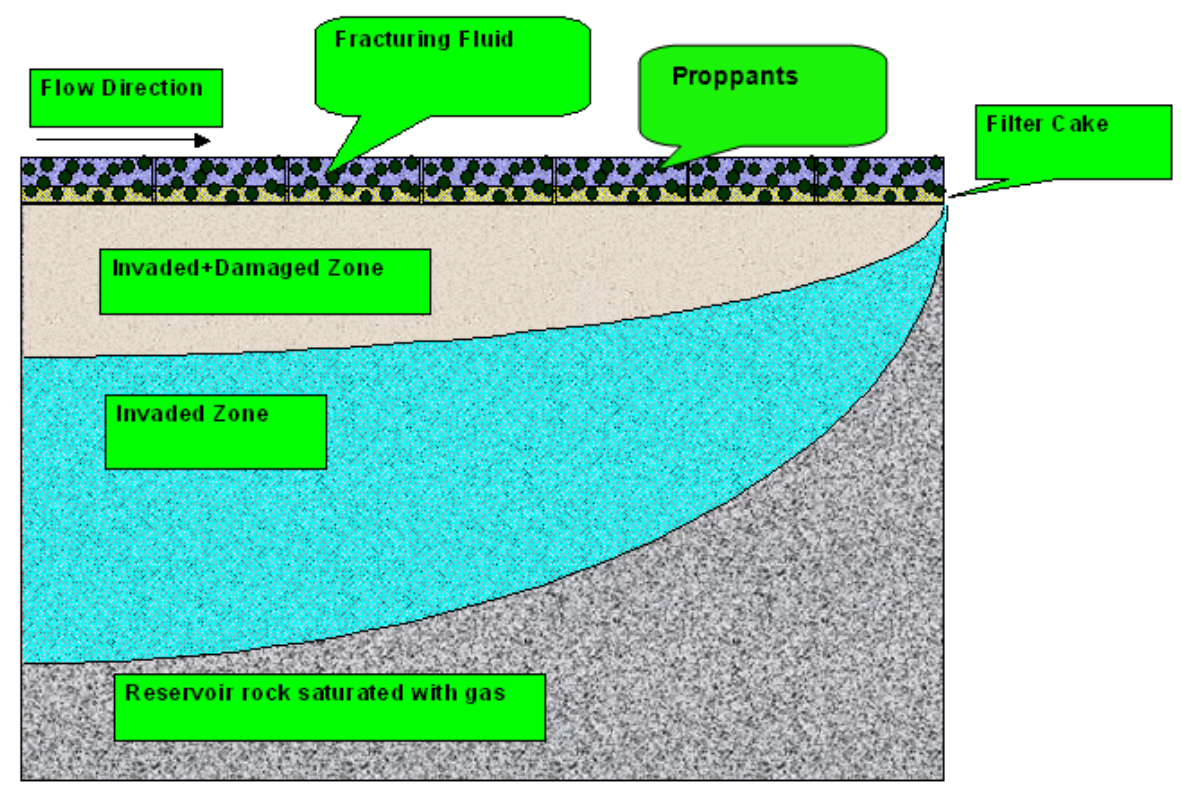

Figure 1 Schematic picture of one side of a fracture after closure (Barati et al.) ${ }^{[7]}$

\section{1-2 Hydraulic Fracturing in Unconventional Reservoirs}

Development of unconventional (e.g. tight gas) reservoirs has increased the application of proppant transport using slick-water or hybrid treatments. Slick-water treatment, also known as waterfrac or riverfrac, is defined as "a fracture treatment that utilizes a large volume of water to create an adequate fracture geometry and conductivity to obtain commercial production from low permeability, large net-pay reservoirs". ${ }^{[18]}$ The purpose of using the slickening agents, like polyacrylamide or low concentration $(\sim 10 \mathrm{pptg}$ or $1.2 \mathrm{~g} / \mathrm{L})$ guar solutions, is to reduce the fluid friction. ${ }^{[19]}$

The following are some advantages of slick-water treatments named in the literature ${ }^{[18,19]}$ :

1. Cost cutting as a result of less proppants and polymers used

2. Reduced gel damage within the fracture. A typical crosslinked fracturing fluid includes between 20-40 pptg polymers while a typical slick-water job includes 5-10 pptg.

3. Reduced fracture height growth as a result of lower viscosity.

However, the following disadvantages were found with the slick-water system ${ }^{[18,19]}$ : 
1. Since high volumes of fluid are used as a result of higher rates (used to make up for concerns like poor proppant transport and narrower pumping width) the cost is not really lower unless the job is near a large water source.

2. Poor proppant transport and suspendability. Lightweight proppants must be used. However, crushing is severe for the light proppant systems.

3. Complex fracture geometry

4. Higher leakoff as a result of minimal wall building capability. This results in physical (permeability reduction) and hydraulic (capillary pressure shift) damage of formation especially in super tight reservoirs.

Despite above-mentioned disadvantages, slick-water jobs were $30 \%$ of the stimulation fracturing jobs pumped in 2004. ${ }^{[19]}$

Viscosity of the fracturing fluid, slick-water or crosslinked, is an important factor in proppant settling. Proppant settling velocity in a vertical fracture is calculated using Stoke's law as follows: ${ }^{[18]}$

$v_{S}=\frac{\left(\rho_{p}-\rho_{f}\right) g d^{2}}{18 \mu}$

Equation 1

Where:

$\rho_{p}=$ density of proppants $\left(\mathrm{kg} / \mathrm{m}^{3}\right)$

$\rho_{S}=$ density of fluids $\left(\mathrm{kg} / \mathrm{m}^{3}\right)$

$d=$ proppant diameter $(\mathrm{m})$

$\mu=$ viscosity of fluid $\left(\mathrm{kg} \mathrm{m}^{-1} \mathrm{~s}^{-1}\right)$

$g=$ acceleration due to gravity $\left(\mathrm{m} / \mathrm{s}^{2}\right)$

This equation indicates that increasing the viscosity of the fracturing fluid decreases the proppant settling velocity. Note that increasing the velocity of the fracturing fluid, the fluid loss volume decreases as well. 


\subsection{Guar-based Fracturing Fluids}

Guar-based fluids have the advantages of being cheap, easy to handle, and performing well under the shear and temperature conditions encountered. Since for polymer solutions thinning increases significantly with increasing temperature and increasing the concentration of polymer damages the proppant pack and reservoir as well as being expensive, crosslinked fluids are used in the majority of fracturing jobs.

\section{2-1 Guar and Its Derivatives}

Guar, a long-chain, high-molecular-weight polymer, composed of mannose and galactose sugars ${ }^{[1]}$ has been widely used to viscosify water for fracturing applications. Guar concentrations of $0.12-0.96 \% \mathrm{w} / \mathrm{w}$ are reportedly used for fracturing different formations. ${ }^{[2]}$ Guar structure is shown in Figure 2. The polymannose backbone of guar is not soluble in water while the galactose branches cause solubility in water. The ratio of mannose to galactose sugars may range from 1.6:1, to $1.8: 1 .^{[1]}$ However, the distribution is not uniform. Weaver et al. ${ }^{[21]}$ reported that as few as 6 contigous un-branched mannose units can form a helix of polymannose which is insoluble. As much as $6-10 \%$ by weight insoluble residue is expected from guar. ${ }^{[1]}$ This initial insoluble residue causes damage to the proppant pack. In addition to the residue made during the preparation, the breakers also generate more residues. Experiments using enzyme breakers have shown that giving more than enough time to the breaker causes more residues as a result of helices made by inappropriate breaking of the polymer's backbone. ${ }^{[21]}$ These generated residues reduce the conductivity of the proppant pack. It takes precipitates a couple of hours to a few days to develop, which is known to be smaller than the flow back time for fracturing fluids. ${ }^{[21]}$

Guar derivatives are made by exposing the guar powder to high $\mathrm{pH}$ water at high temperature for a period of time to swell the powder. This process breaks up the helices and exposes the backbone polymer to reaction with a derivatizing agent such as propylene oxide. Derivatizing the guar with propylene oxide generates hydroxypropyl guar (HPG), which contains about 2-4 wt \% insoluble residue. ${ }^{[1,21]}$ Although, Brannon and Pulsinelli ${ }^{[22]}$ reported the same degree of proppant pack damage for guar and HPG, HPG is reportedly more stable at higher temperatures than guar. $^{[1]}$

A "double-derivatized" guar named carboxymethyl hydroxypropyl guar (CMHPG) and cellulose 
derivatives like carboxymethylhydroxyethylcellulose (CMHEC) have also been used for different temperatures using appropriate crosslinkers. ${ }^{[1]}$

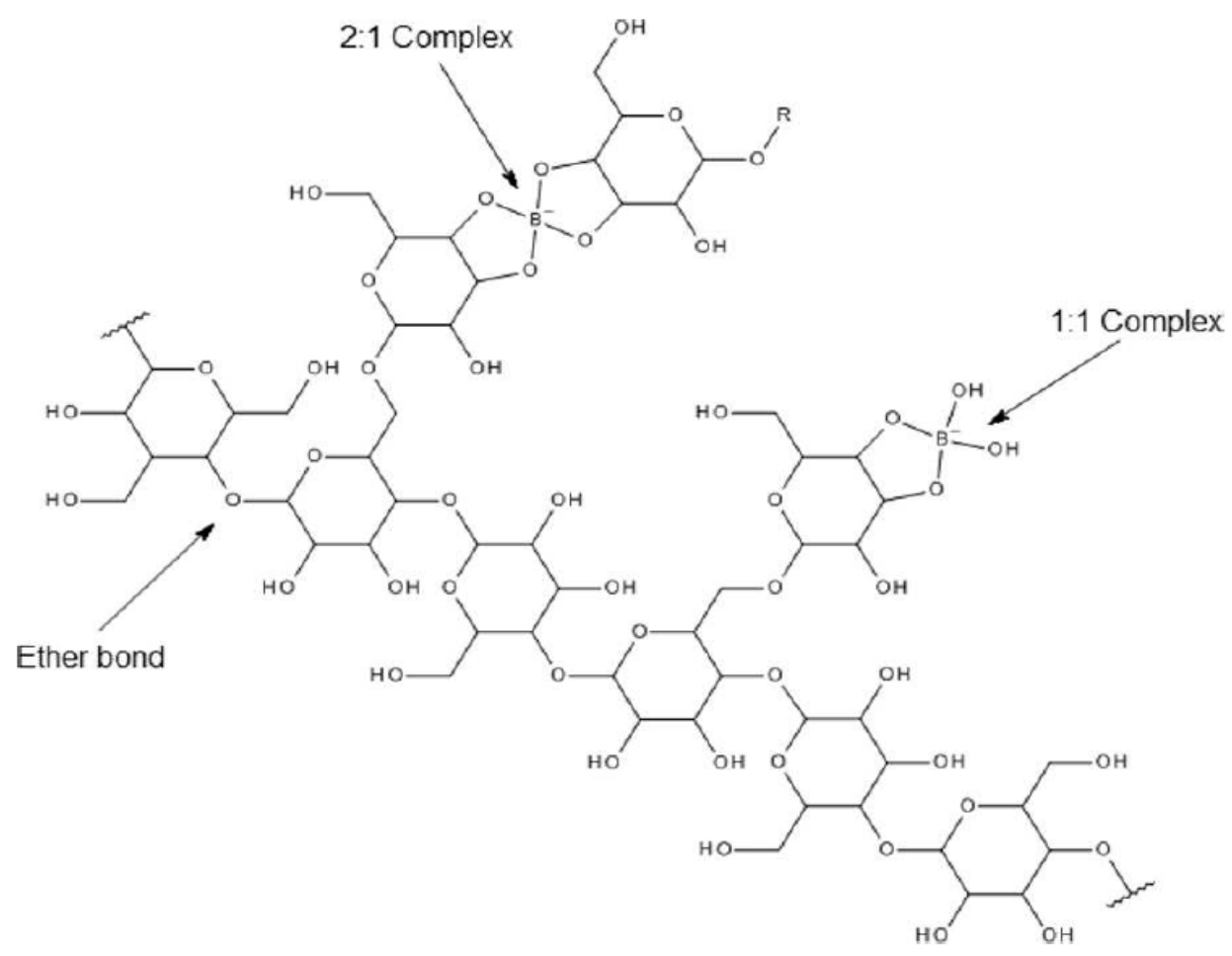

Figure 2 Chemical structure of guar showing mechanism of crosslinking by borate, and ether bond vulnerable to cleavage by enzyme (-R denotes another guar molecule). ${ }^{[23]}$ 


\section{2-2 Crosslinking of Guar}

Metal ions like borate, $\mathrm{Ti}(\mathrm{IV}), \mathrm{Zr}(\mathrm{IV})$, and $\mathrm{Al}(\mathrm{III})$ are used to crosslink water soluble polymers. Crosslinking occurs by reacting through cis-OH pairs on the galactose side chains of guar. Characteristics of these crosslinkers are compared in Table 1. Crosslinking makes the rheological properties of the polymers more favorable for fracturing purposes. Crosslinking agents are restricted within certain $\mathrm{pH}$, and temperature ranges as well as the type of polymer they can crosslink.

Crosslinking is delayed for some polymers in order to minimize the surface pressure during the fracturing job. Increasing $\mathrm{pH}$ is one way of delaying the crosslinking process. Increasing the $\mathrm{pH}$ for example from 9 to 11 results in polymer being attacked more through neutrally charged boric acid, which makes a 1:1 complex. After 1:1 complexes are formed the boric acid hydrolyzes to monoborate ion, which is able to form 1:2 complexes. This effect is shown in Figure 5 and

\section{Figure 6.}

Borax (sodium tetraborate decahydrate) and boric acid (plus caustic soda) were used by Robert et al. ${ }^{[20]}$ to crosslink guar. Typical concentrations of $0.024-0.18 \% \mathrm{w} / \mathrm{w}$ are reported for different fracturing jobs. ${ }^{[20]}$ Colemanite and ulexite, which consist of mainly low solubility calcium or calcium/sodium borate, have been applied for high temperature applications or when a delayed crosslink is required. Organoborates can also be used for crosslinking guar chains. Irrespective of the boron form added to the fluid, monoborate is the crosslinking species that interacts by hydrogen bonding or perhaps by ionic bonding, with the cis-hydroxyls on the guar to provide either inter- or intra-molecular crosslinking, or both. Availability of monoborate ions is totally $\mathrm{pH}$ and temperature dependent (Figure 5, Figure 6 and Figure 7). Too much monoborate causes excessive intra-molecular crosslinking or syneresis. Syneresis is a reversible process in which polymer chains clump-together and exclude liquid from their structure causing nonhomogeneous fluid. On the other hand, too few inter-molecular crosslinks make the fluid very thin. Neither of the two mentioned fluids is able to carry the proppants. ${ }^{[2]}$

Borate ions form mostly 1:1 complexes with cis-diol pairs but only a minor number of 2:1 complexes which are the crosslinks (Figure 2). The number of 2:1 complexes is proportional to the number of interchain contacts, which is a function of polymer concentration to the power of 2.6. The number of interchain contacts decline exponentially with temperature weakening the base polymer at high temperatures. ${ }^{[25]}$ 
Guar-borate gels are not permanently degraded by shear since the polymer interaction time is about 1 millisecond. Breaking and reforming of borate complexes in a continuous process causes borate gels to reheal quickly. This is caused by rapid exchange equilibrium of borate acid and monoborate ion. This is why when the temperature in Figure $\mathbf{5}$ is increased; the pH must be increased as well to compensate the reduction of borate ion concentration and exponential decrease in interchain contacts. ${ }^{[25]}$

As temperature increases, more polymer is needed to maintain sufficient interchain contacts and hence enough viscosity for the fracturing fluid. Fluid $\mathrm{pH}$ needs to increase with increasing temperature as well, to provide sufficient active borate ion (Figure 5, Figure 6, Figure 7 and Figure 8). The guidelines reported by Harris ${ }^{[25]}$ in Figure 7 were later adjusted by Harris and Heath $^{[26]}$ to account for low polymer concentrations at low temperatures (Figure 8).

Alternative crosslinkers like titanium (IV) and zirconium (IV) compounds are used typically as titanium acetylacetonate, titanium mono-triethanolamine chelate, zirconium ammonium lactate and zirconium tetra-acetate, in temperature and $\mathrm{pH}$ ranges for which borate cannot be used. However, shear-sensitivity is the major problem for such crosslinkers. ${ }^{[1]}$ Titanium and zirconium crosslinked fluids have shown lower fracture conductivity and more face damage comparing to the borate crosslinked fluids. ${ }^{[24]}$ 
Table 1 Characteristics of commonly used crosslinkers ${ }^{[1]}$

\begin{tabular}{|c|c|c|c|c|}
\hline Cossinker & Borate & Thinate & Zironate & Alurinumin \\
\hline \multirow[t]{2}{*}{ Crossinkhable polyners } & Guar, $\mathrm{HPG}$, & Guar, $H P G$, & Gus: HPG: & CVHPG, ONHEC \\
\hline & CMHPG & OMHPG, OWHEO & CMHPG, CMHEC & \\
\hline pH range & $8-12$ & $3-11$ & $3-11$ & 3 \\
\hline Upper temperature linit( (F) & 325 & 325 & 400 & 150 \\
\hline Shear depraded & No & Yes & Yes & $Y_{e s}$ \\
\hline 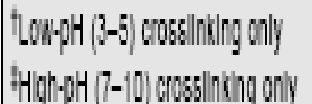 & & & & \\
\hline
\end{tabular}

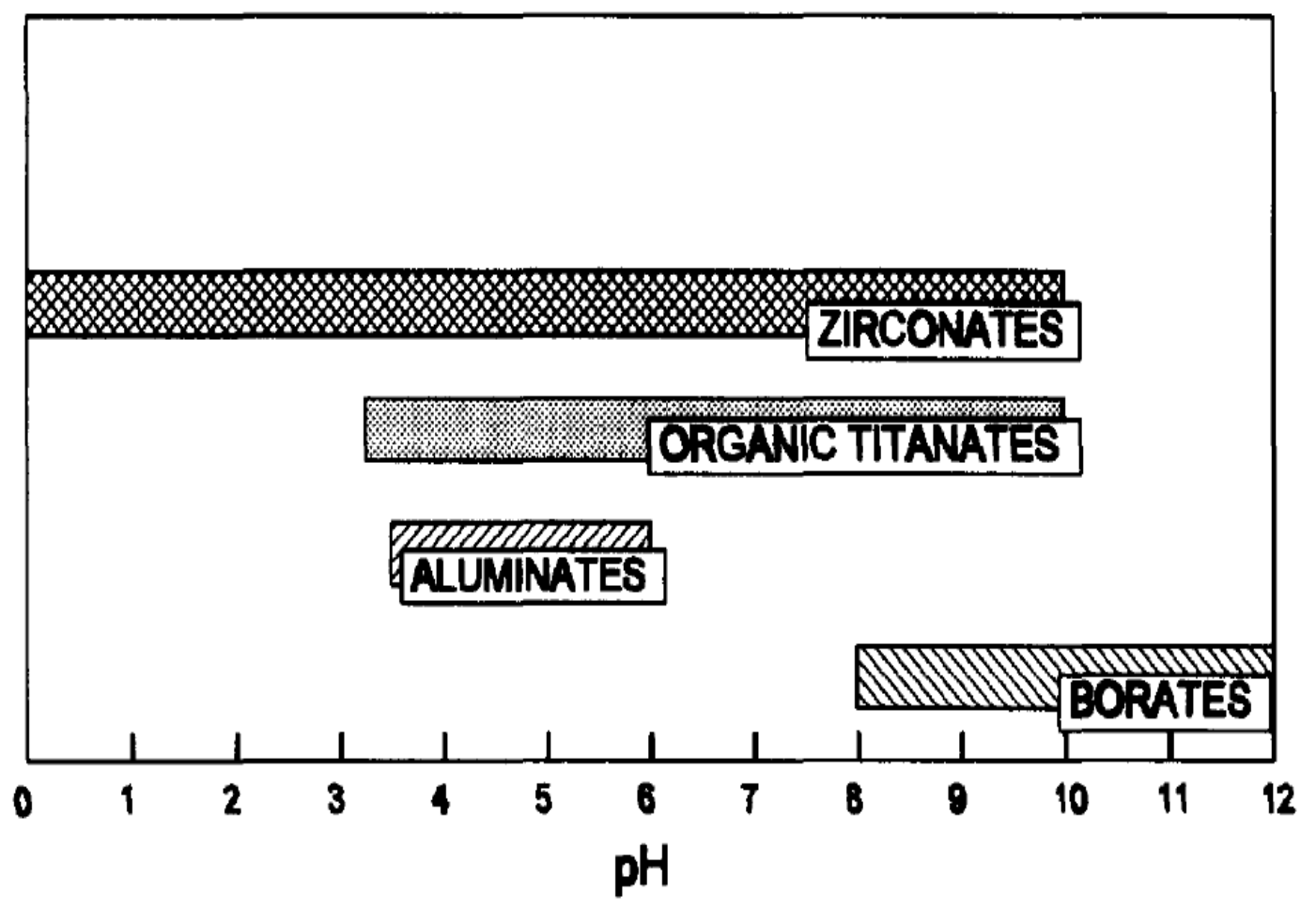

Figure 3 pH range for different crosslinkers, Rae et al. ${ }^{[27]}$ 


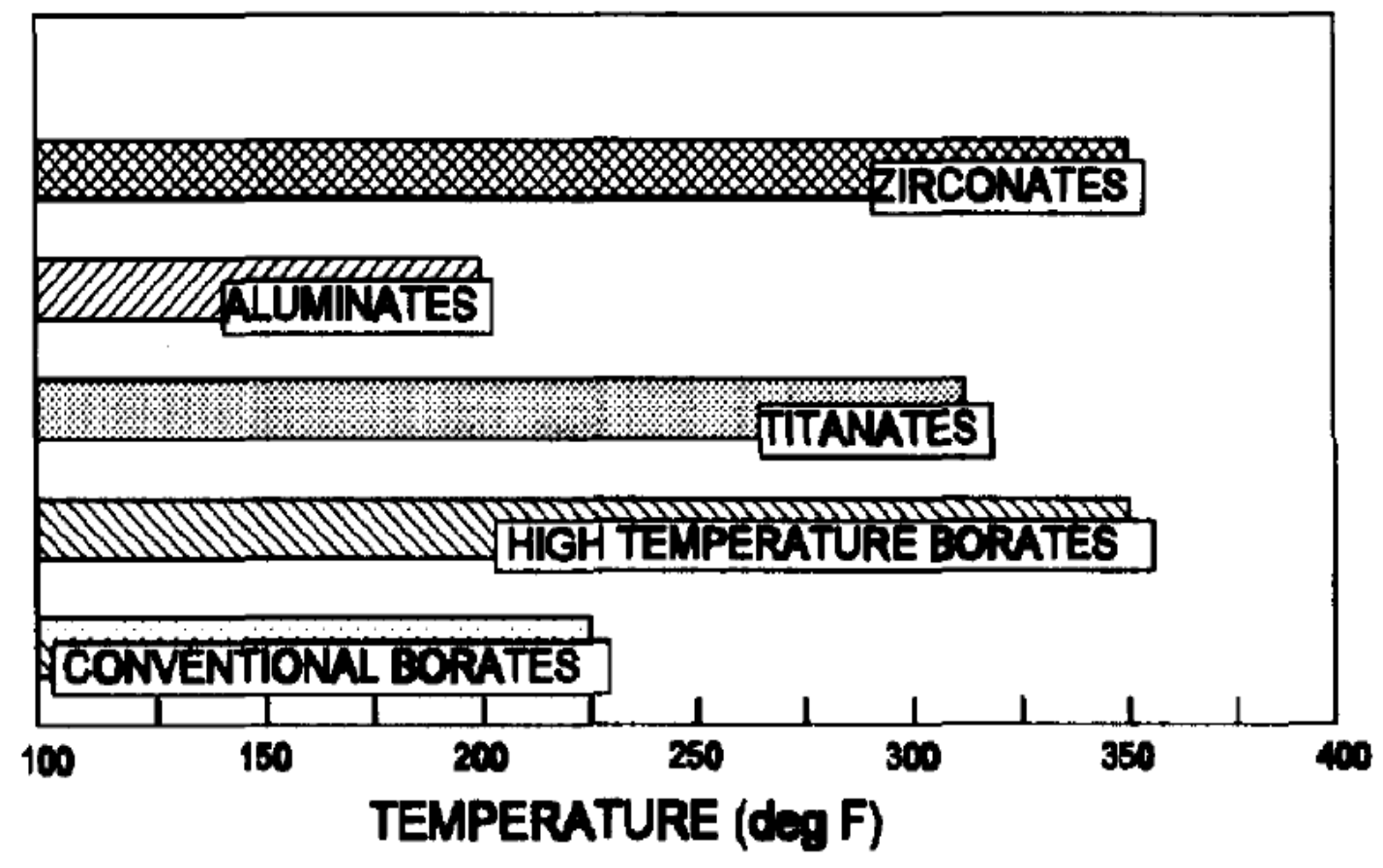

Figure 4 Temperature range for different crosslinkers, Rae et al. ${ }^{[27]}$

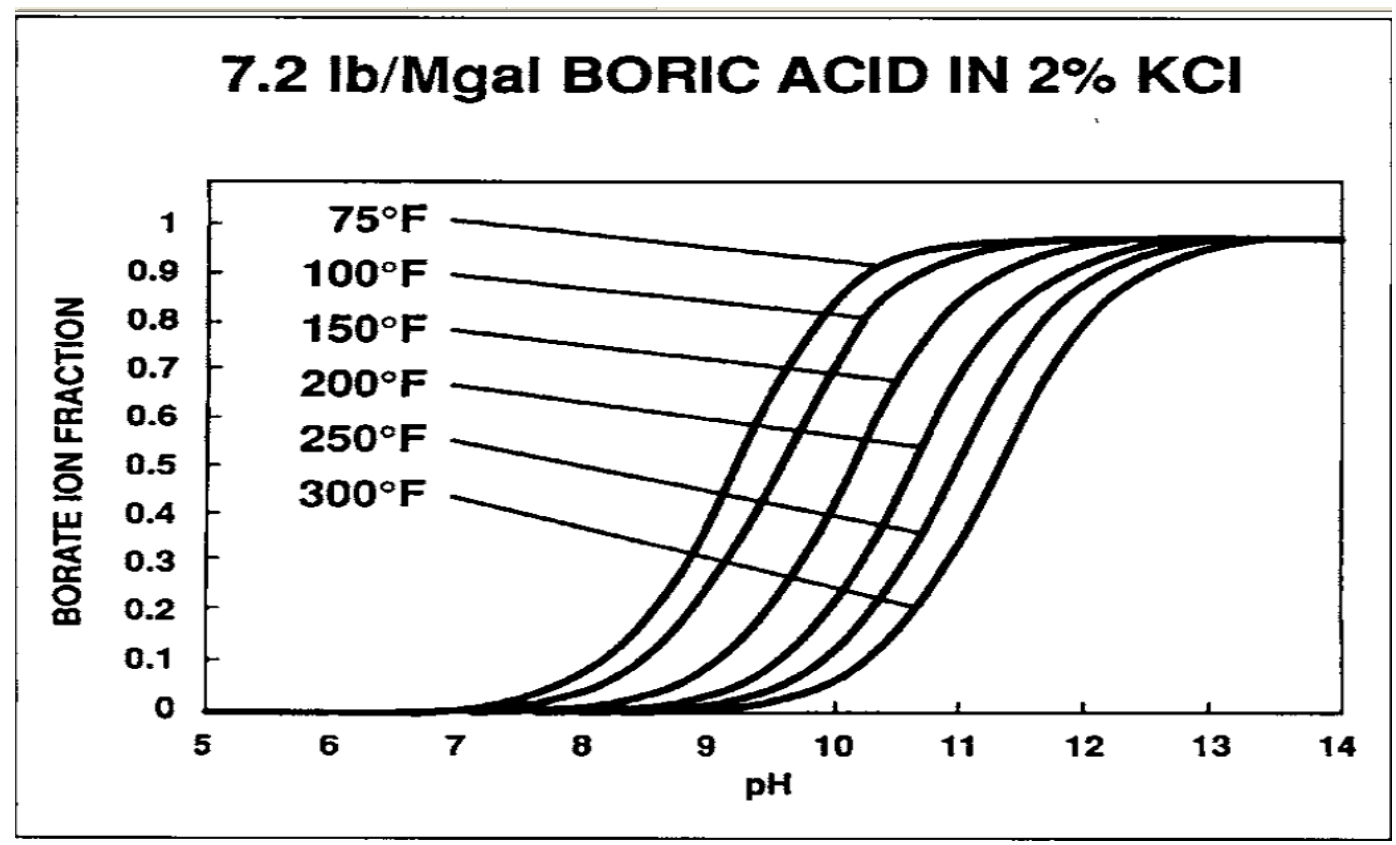

Figure 5 Dimensionless concentration of monoborate ions vs. pH for a range of temperature, Harris ${ }^{[25]}$ 


\section{Ib/Mgal GUAR: BORATE}

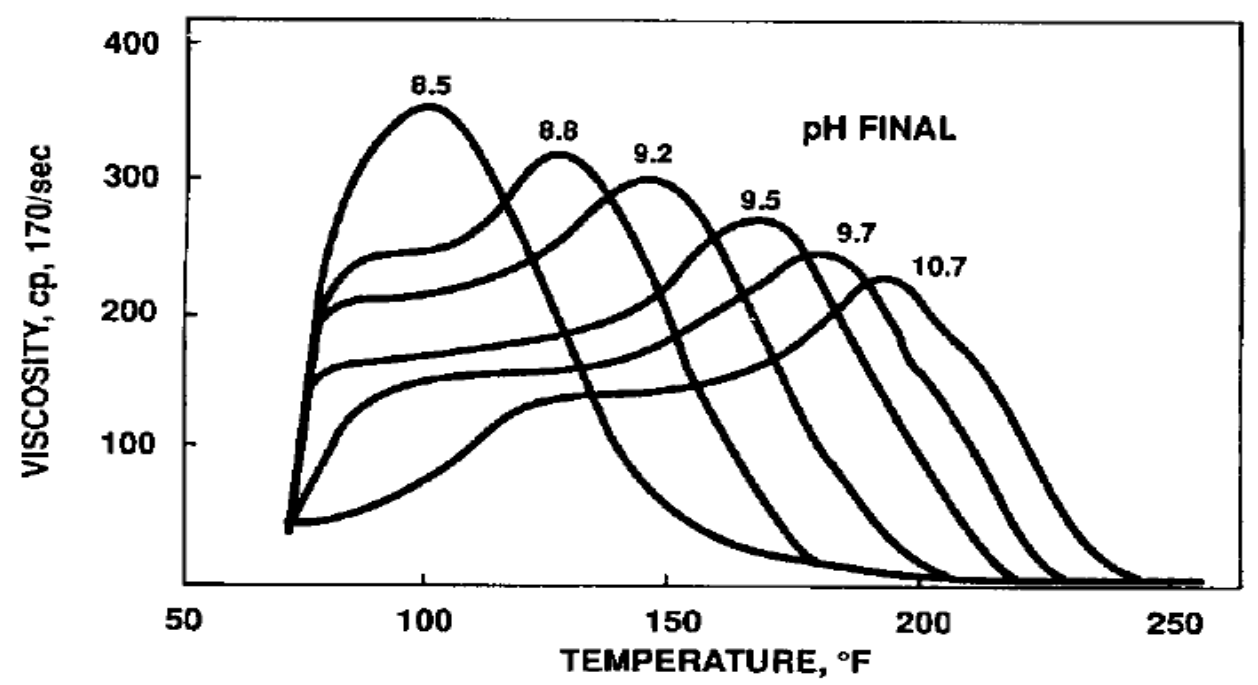

Figure 6 Crosslink rate with pH, Harris ${ }^{[26]}$

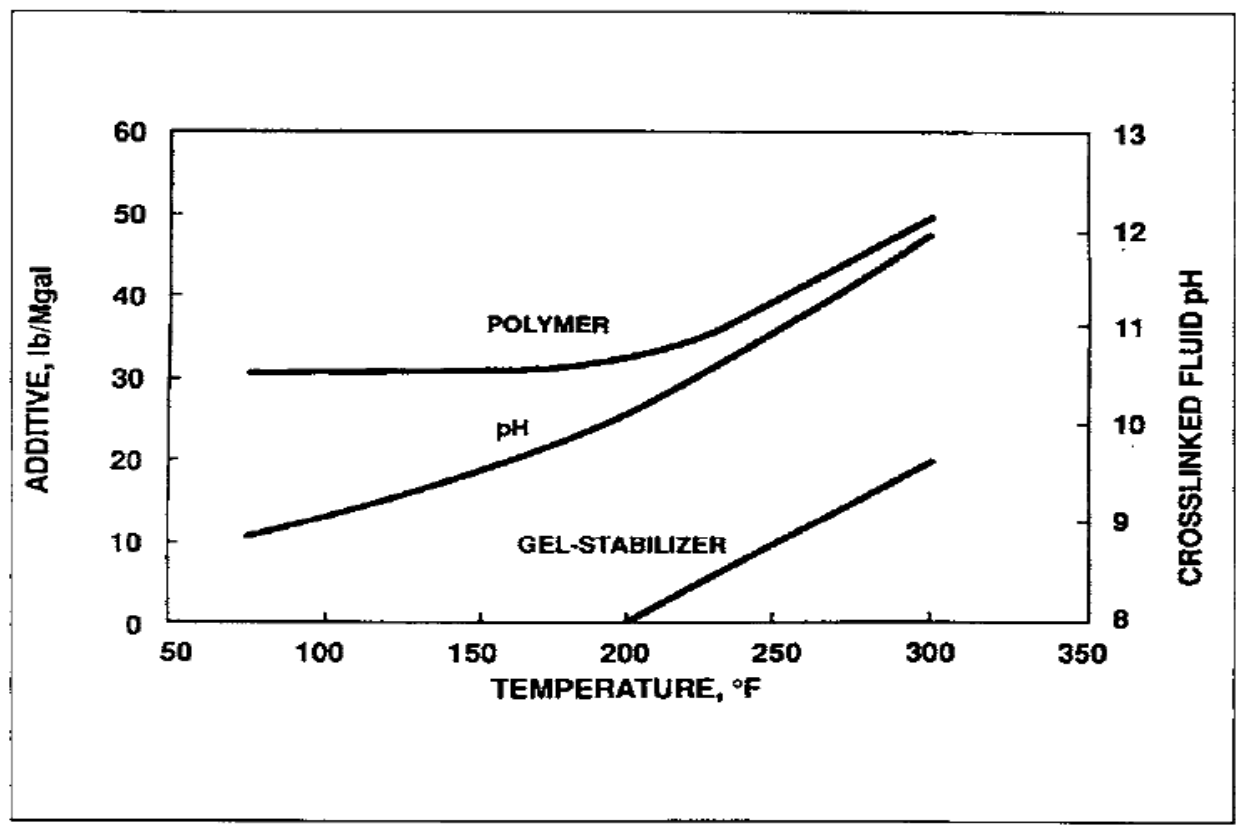

Figure 7 Guidelines on borate-crosslinked fluids, Harris ${ }^{[25]}$ 


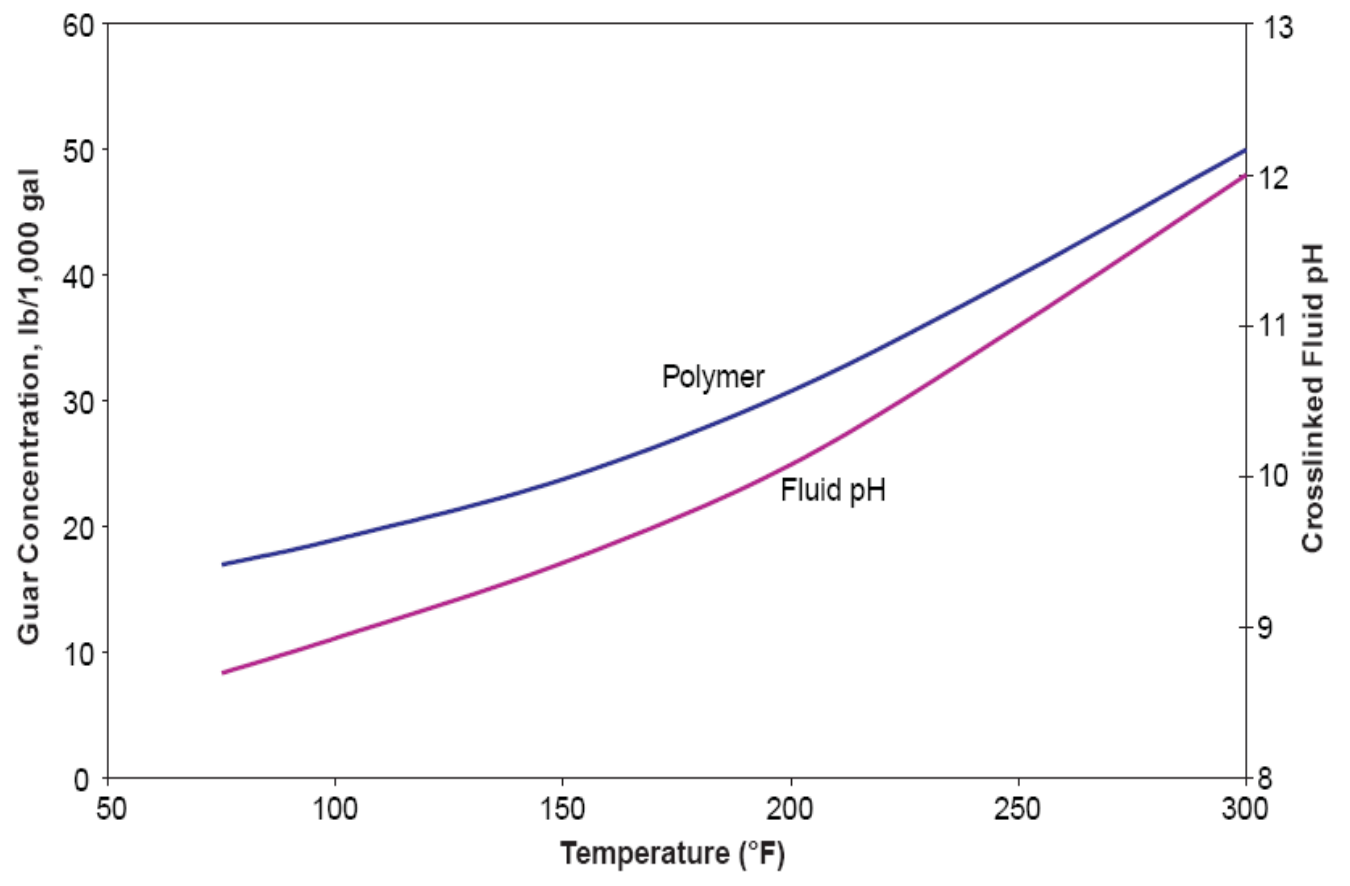

Figure 8 Borate-crosslinked gel guideline, Harris et al. ${ }^{[26]}$ 


\section{2-3 Buffers and pH Adjusters}

In order to adjust the $\mathrm{pH}$ to an appropriate range for crosslinkers and breakers, $\mathrm{pH}$ adjusters like caustic soda or hydrochloric acid are used. However, when the $\mathrm{pH}$ is supposed to be kept in a specific range buffers must be used. Buffers are usually salts of weak acids with strong bases. ${ }^{1}$ Typical buffers used in hydraulic fracturing are sodium and potassium carbonates for high $\mathrm{pH}$ and organic acids and their salts like acetic acid and sodium diacetate for low $\mathrm{pH}$. Organic bases like amines do not cause precipitation with sea-water and their use is recommended if using seawater is necessary. ${ }^{[1,21]}$

\section{2-4 Breakers}

The viscous fluid, either crosslinked or linear, and the filter cake made on the face of the rock must be broken in order to have high conductivity in the proppant pack. Polymers are cleaved into small molecular weight fragments by breakers. Oxidizers, enzymes and acids are typical breakers used depending on different fracturing conditions.

Oxidizers are the most common type of breakers. Ammonium, potassium, and sodium salts of peroxydisulfate (persulfate) are common oxidizers. Since the generation of free radicals is based on the thermal decomposition of persulfates, the reaction is not fast at temperatures below 125 ${ }^{\circ} \mathrm{F} .{ }^{[1]}$ At high temperatures, on the other hand, the oxidizers may degrade quicker. Encapsulating the breakers delays the breaking of fluid for high temperature applications. However, there is no perfect encapsulation. ${ }^{[24]}$

Enzymes are breakers of the class hemicellulase, which cause reduction of the guar molecular weight. However, unlike the oxidizing agents, they are not consumed after reaction. They denature at elevated temperatures or extreme $\mathrm{pH}$ values. Enzymes could have increased lifetime at high pressure. Enzymes are very cheap but sensitive to $\mathrm{pH}$ and temperature. ${ }^{[1,28]}$

Table 2 represents a comparison between the two classes of breakers and their application for different temperature and $\mathrm{pH}$.

Breaking a linkage between two mannose groups by breakers leads to an immediate reduction in the average molecular weight, and hence viscosity. Breaking a galactose-mannose linkage dos not change the viscosity significantly. However, removing more than 6 galactoses causes precipitation due to helix formation. This happens by extended exposure to breaker. ${ }^{[21]}$ The most 
effective breakers are the ones that break the backbone and side chain of the polymers simultaneously. ${ }^{[29]}$

Introduction of encapsulated breakers allowed high concentrations of breakers to be used. A film of a crushable material like polymer acts as a barrier between the active breaker and fracturing fluid. Release of breaker is caused by crushing, osmotic rupture, or diffusion of the breaker chemical. Stronger coating is necessary at high temperatures. A mixture of dissolved and encapsulated breakers is usually used to achieve a better performance. ${ }^{[1]}$

Table 2 Breaker selection (Gulbis and Hudge) ${ }^{[1]}$

\begin{tabular}{|c|c|c|c|}
\hline Selection Criterion & Oxidizers & Enzymes & Comments \\
\hline Performance at high temperature & + & & $\begin{array}{l}\text { Oxidizers have been identified for high-temperature applications. } \\
\text { Current enzymes have some activity up to } 225^{\circ} \mathrm{F}\left[105^{\circ} \mathrm{C}\right] \text {. } \\
\text { Higher temperature versions will most likely be identified in the } \\
\text { future. }\end{array}$ \\
\hline Completeness of break & & + & $\begin{array}{l}\text { Theoretically, enzymes should have the advantage because of } \\
\text { their catalytic nature. However, enzyme sensitivity to tempera- } \\
\text { ture, pH and other chemicals can significantly shorten the life- } \\
\text { ime of the enzyme. Under ideal conditions (less than approxi- } \\
\text { mately } 180^{\circ} \mathrm{F}\left[80^{\circ} \mathrm{C}\right] \text {, pH 5-8), enzymes break the polymer into } \\
\text { smaller fraglments than oxidizers do, but there is no information } \\
\text { in the literature to document that significant production of simple } \\
\text { sugars results. }\end{array}$ \\
\hline Duration of breaking & & + & $\begin{array}{l}\text { Enzymes, unless exposed to extremes of pH or temperature, } \\
\text { react with the polymer over a more extended time period (days) } \\
\text { than oxidizers (hours). }\end{array}$ \\
\hline Fast break & + & & $\begin{array}{l}\text { A fast break to allow quick tumaround of the well is accomplished } \\
\text { much better with oxidizers. }\end{array}$ \\
\hline Chemical sensitivity & + & + & $\begin{array}{l}\text { Enzymes are highly sensitive to } \mathrm{pH} \text {, so control of } \mathrm{pH} \text { within } \\
\text { a limited range is necessary for predictable performance. } \\
\text { Oxidizers are affected by the presence of curable-resin-coated } \\
\text { proppants, whereas enzymes are not. }\end{array}$ \\
\hline
\end{tabular}

\section{2-5 Bactericides}

Since polysaccharides are an excellent food source for bacteria adding bactericides to such fluids is recommended to prevent bacterial degradation. ${ }^{[30]}$ Not only can bacteria reduce the molecular weight of polymers, some of them can also sour the reservoir fluid by reducing sulfate ions to hydrogen sulfide. ${ }^{[30]}$ Adding bactericides to the polymer kills the bacteria but it is not able to inactivate the enzymes already produced by the bacteria. That is why it is always recommended 
to add bactericides to fracture tanks before adding water. ${ }^{[1,31]}$ Using deionized (D.I.) water in the lab "where the enzymes are denatured and hopefully removed from the arena" is always recommended. ${ }^{[1,31]}$ Cheng and Prud'homme ${ }^{[32]}$ recommended adding 100 ppm of sodium azide to the solution after adding guar to D.I. water and being stirred for $1 \mathrm{hr}$.

\section{2-6 Stabilizers}

Degradation of guar gels is prevented at high temperatures by using stabilizers. Using 10-20 $\mathrm{lbm} / 1000$ gal of sodium thiosulfate is recommended as the most effective stabilizer. ${ }^{[33,34]}$ Gulbis et al. ${ }^{[1]}$ reported that even though the mechanism for the stabilizers is not fully understood, it is believed that they prevent the degradation of gel caused by dissolved oxygen by acting as oxygen scavenger. They also stated that high $\mathrm{pH}$ fluids $(\mathrm{pH}=9-11)$ must be used if a long-term fluid stability is required since guar and its derivatives are hydrolyzed at low pH especially at temperatures higher than $200^{\circ} \mathrm{F}$.

\section{2-7 Other Additives}

Surfactants are used in order to reduce the surface tension between hydrocarbon and water, which results in better cleanup of formation and fracture. Use of $1-3 \% \mathrm{KCl}$ solutions or solutions containing tetramethyl ammonium chloride as base fluid is also recommended for high $\mathrm{pH}$ fracturing fluids. ${ }^{[1]}$

\subsection{Nanoparticles, Polyelectrolytes and PEI/DS System}

Tiyaboonchai ${ }^{[10-12]}$ introduced polyelectrolyte nanoparticles (PECNPs) used in drug delivery as solid colloidal particles with diameters ranging from 1-100 nm. She divided the nanoparticles into two categories of nano-spheres, in which drugs are adsorbed or dispersed onto their surface, and nanocapsules, which entrap drugs in the core or adsorb on their exterior, depend on their preparation process. 


\section{3-1 Polyelectrolyte Nanoparticles}

Polymers carrying multiple ionic groups are called polyelectrolytes. They exhibit a dual character of highly charged electrolytes and macromolecular chain molecules at the same time. Their ionic groups will dissociate in aqueous phase making the polymer charged ${ }^{[35,36]}$ Koetz and Kosmella $^{[36]}$ classified the polyelectrolytes into natural, modified natural, and synthetic. The polyethylenemine/dextran sulfate system introduced in the next section is a synthetic system. Chargewise they divided the polyelectrolytes into polyanions, polycations, and polyampholytes (Figure 9).

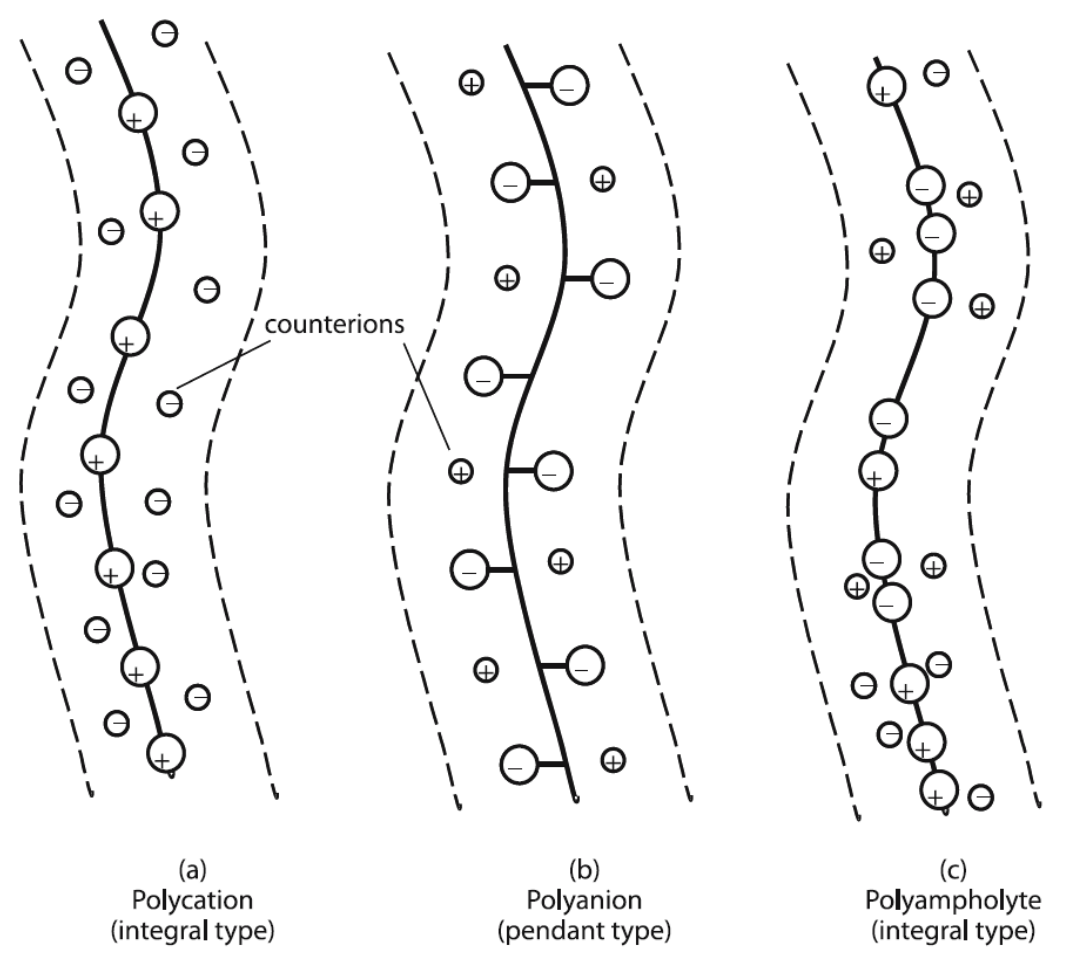

Figure 9 Classification of polyelectrolytes in terms of their charge, Koets and Kosmella ${ }^{[36]}$

Polyelectrolytes that have a broad range of size, shape and mass characteristics are called "polydisperse". Polydispersity index is defined as the measure of distribution of molecular mass in a given sample of polymer. Polydispersity index (PDI) is calculated as weight average molecular weight divided by number average molecular weight of the polymer, which is always smaller than one. 
Where number average molecular weight is defined as:

$M_{n}=\frac{\sum M_{i} N_{i}}{\sum N_{i}}$

Equation 3

and weight average molecular weight is defined as:

$M_{w}=\frac{\sum M_{i}^{2} N_{i}}{\sum M_{i} N_{i}}$

Equation 4

in which $\mathrm{N}_{\mathrm{i}}$ is the number of molecules of $\mathrm{M}_{\mathrm{i}}$ molecular weight.

Polyelectrolyte nanoparticle solutions are essentially colloidal particles with at least one dimension between $\sim 1$ and $1000 \mathrm{~nm}$. Colloidal dispersions have been categorized into two broad types $^{[37]}$ :

- Lyophilic colloids: formed spontaneously since the dispersion is thermodynamically stable.

- Lyophobic colloids: include all petroleum suspensions. Need mechanical energy like agitation to form, since they are thermodynamically unstable.

The term "stability" is usually considered against sedimentation which results from a density difference between two liquid phases and aggregation in which some particles clump together, touching at certain points and with virtually no change in the total surface area. Aggregation can be twofold for suspensions: coagulation which refers to the formation of compact aggregates, and flocculation which refers to the formation of a loose network of particles. The identity of species is retained in aggregation, while they lose their kinetic independence.

Suspension stability is not necessarily a function of particle size. In some suspensions, a particle size distribution heavily weighted toward the smaller sizes represents the most stable suspension. For such cases, smaller particles and narrower particle size distribution yield a more viscous 
suspension. Other parameters like particle charge and density also affect the stability of suspension. ${ }^{[38]}$ The stability of the dispersion depends on how particles interact. Electrostatic repulsion is the main cause of repulsive forces between like charged particles. Van der Waals forces are the main attractive forces.

Light scattering can yield particle size information for suspensions with small suspension concentration and small particles. A beam of light might be absorbed, scattered, or transmitted after entering a suspension. Intensity of light that is scattered by each particle is related to $\mathrm{r}^{6}$. Electric Double Layer (EDL): Consists of a charged surface and a distribution of neutralizing excess of counter-ions over co-ions near the surface (Figure 10). EDL contains two layers: an inner layer of adsorbed ions (Stern layer) and a diffuse layer, in which ions are distributed according to the influence of electrical forces and thermal motion. This whole area is electrically neutral. The diffuse double layer was modeled like Equation 5.

$\psi=\psi_{0} \exp (-k x)$ Equation 5

In which $\psi$ is the potential at distance $\mathrm{x}, \psi_{0}$ is the surface potential, and $1 / \mathrm{k}$ is called the EDL thickness. 


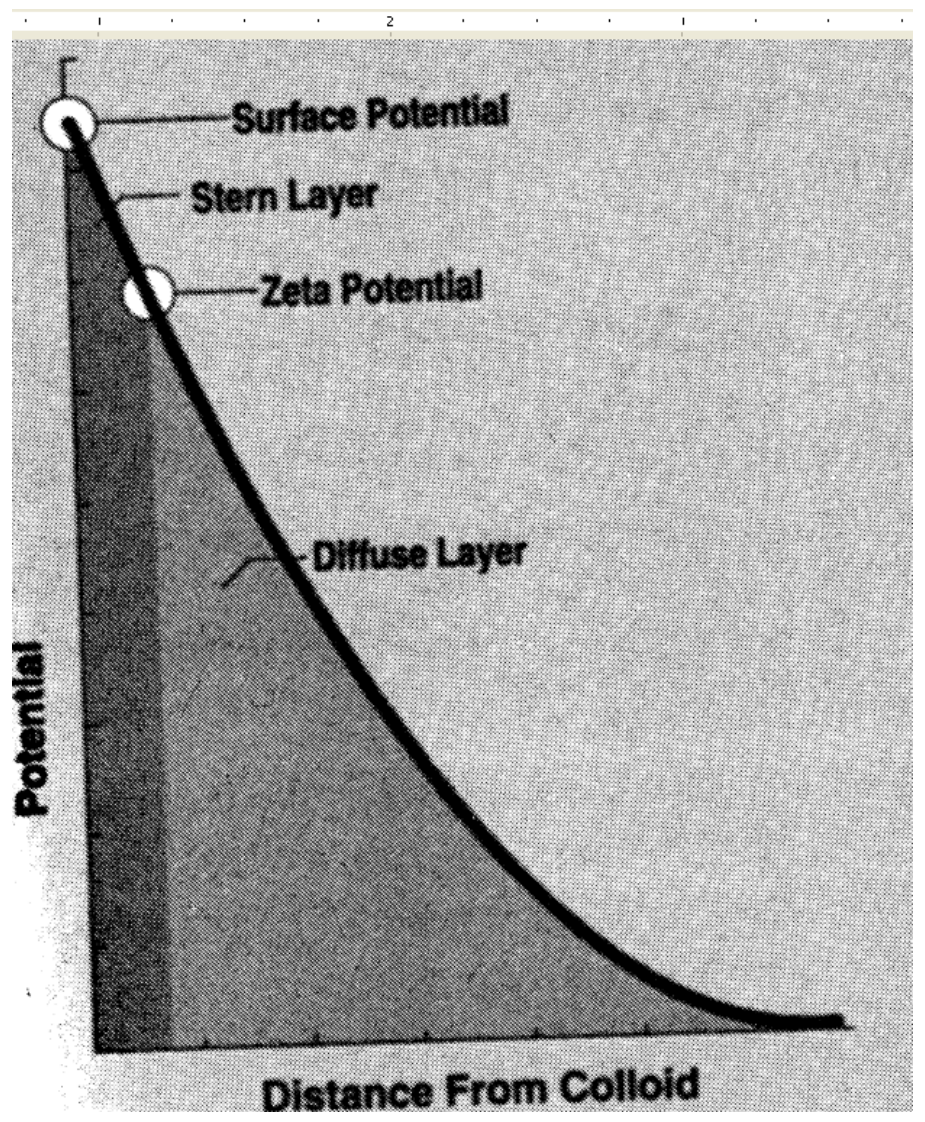

Figure 10 Simplified illustration of the surface and zeta potential for a charged suspension drop dispersed in high (saline water) and low (fresh water) electrolyte concentration aqueous solution ${ }^{[37]}$

Electrokinetic motion occurs when the mobile part of the EDL is sheared away from the inner layer. Electrophoresis, in which an electric field causes charged particles to move toward the oppositely charged electrode, is used to measure the zeta potential. The result of this method is charge density $(\sigma)$ or potential $(\psi)$ at the plane of shear, which is called zeta potential.

Measuring the electrophoretic velocity at a location in a sample cell under some electric field gradient, the electrophoretic mobility $\left(\mu_{\mathrm{E}}\right)$ is defined as the electrophoretic velocity divided by the electric field gradient at the location where velocity was measured. This mobility can be related to the zeta potential by simple relations like Huckel Theory and Smoluchowski Theory. ${ }^{[37]}$

Huckel Theory: For small particles with thick EDLs, i.e. $\mathrm{ka}<1$ : 
$\mu_{E}=\frac{\xi \varepsilon}{1.5 \eta}$

Equation 6

In which $\eta$ is the viscosity of suspension, and $\varepsilon$ is dielectric constant.

Smoluchowski Theory: For large particles with thin EDLs, i.e. ka>100:

$\mu_{E}=\frac{\xi \varepsilon}{\eta}$

Equation 7

Thus, zeta potential is the electric potential in the interfacial double layer at the slipping plane vs. a point in the bulk fluid. Zeta potential is a marker of nanoparticle stability. ${ }^{[38]}$ It is a function of excess polymer. Charge repulsion caused by the presence of significantly positive or negative charge prevents further aggregation. ${ }^{[38]}$ As the zeta potential approaches zero, particles tend to aggregate (Table 3). Colloids with high zeta potential, negative or positive, are electrically stabilized while colloids with low zeta potential tend to coagulate or flocculate. ${ }^{[37]}$

Table 3 Stability behavior of the colloid vs. zeta potential ${ }^{[39]}$

\begin{tabular}{|c|c|}
\hline Zeta Potential $(\mathbf{m V})$ & Stability behavior of the colloid \\
\hline 0 to \pm 5 & Rapid coagulation or flocculation \\
\hline \pm 10 to \pm 30 & Incipient instability \\
\hline \pm 30 to \pm 40 & Moderate stability \\
\hline \pm 40 to \pm 60 & Good stability \\
\hline more than \pm 61 & Excellent stability \\
\hline
\end{tabular}




\section{3-2 Assembly Mechanism of Polyelectrolyte Nanoparticles}

Polyelectrolyte complexes (PECs) have been used as carriers for vaccines and anticancer drugs to limit their off-target tissue toxicity. ${ }^{[10-12,38,40]}$ PECs result from strong electrostatic interaction between two oppositely charged polyelectrolytes. Therapeutic agents have been incorporated by encapsulation, covalent attachment, or surface adsorption. ${ }^{[38]}$

Although electrostatic interactions are the main molecular forces for PECs, hydrogen bonding, hydrophobic interactions and van der Waals forces complete their formation. Hartig et al. ${ }^{[38]}$ named two major steps involved in complexation of PECs:

1. Kinetic diffusion process of mutual entanglement between polymers at very short times.

2. Thermodynamic rearrangement of the already formed aggregates due to conformational changes and disentanglement at long times. This step causes instability in the PECs.

Webster et al. ${ }^{[41]}$ reported three types of prepared PECs:

- Soluble PECs, i.e. macroscopically homogeneous systems containing small PEC aggregates.

- Turbid colloidal systems with suspended PEC particles in the transition range to phase separation.

- Two-phase systems of supernatant liquid and precipitated PEC, which are readily separated as a solid after washing and drying. This system is not desirable.

Hartig et al. ${ }^{[38]}$ reported two structural models discussed in the literature based on the characteristics of the polyion groups, stoichiometry, and molecular weights:

- Ladder-like structure: They have a complex formation which on a molecular level, occurs via conformational adaptation. This structure consists of hydrophilic single-stranded and hydrophobic double-stranded segments. This structure results from the mixing of polyelectrolytes with weak ionic groups and large differences in molecular dimensions. The complex of oppositely charged ions occurs via "zip" mechanism where there is insufficient ion pairing. A high MW polyion with a weak charge density is titrated into a shorter, smaller MW counterion non-stoichiometrically to form PECs (Figure 11).

- Scrambled-egg structure: This structure is made by complexes made by combination of polyions with strong ionic groups and comparable molar masses. This structure makes insoluble, highly aggregated complexes under a 1:1 stiochiometry (Figure 11). 
(a)

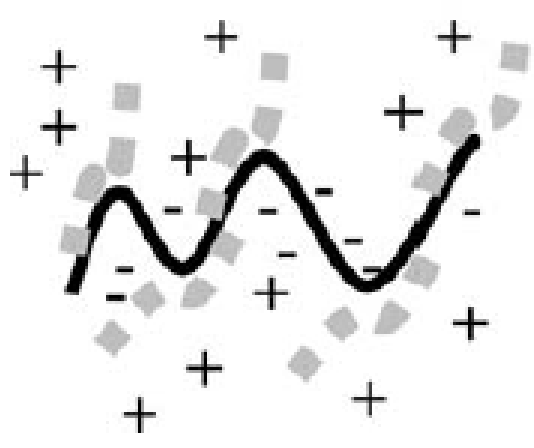

(b)

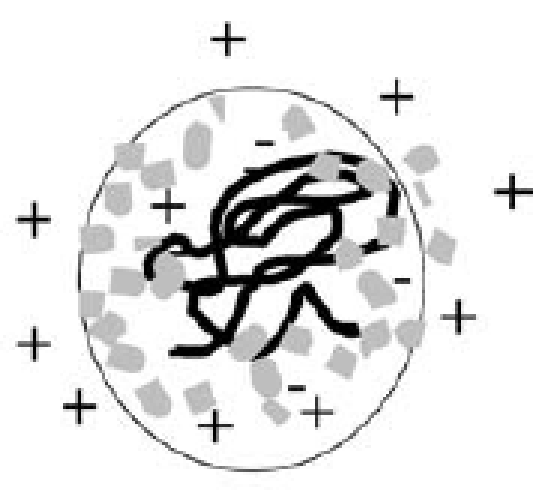

Figure 11 Schematic representation of ladder (a) and scrambled egg (b) structures. Black represents the negative polyelectrolyte (polyanion) while gray represents the positive polyelectrolyte (polycation). ${ }^{[38]}$

Polyelectrolyte complexes have been used in the drug industry to protect proteins via encapsulation from the environments with inhospitable conditions. Drug release from polymeric matrices occurs as a function of $\mathrm{pH}$, electric field, temperature, ultrasound, or light. ${ }^{[38]}$

\section{3-3 Polyethylenimine/Dextran Sulfate System of Tiyaboonchai}

Tiyaboonchai ${ }^{[10]}$ introduced a system of oppositely charged nanoparticles consist of polyethylenimine (PEI) and dextran sulfate (DS), with zinc sulfate as a stabilizing agent. Her system works as a delivery vehicle for pharmaceutical applications. She used a mild preparation technique, which does not use organic solvents, heat or high shear forces.

She listed the following reasons for choosing PEI:

- PEI is a cationic water-soluble polymer.

- PEI offers the highest transfection efficiency among other cationic polymers used in the pharmaceutical industry.

- Availability of PEI in wide range of molecular weights and structures (linear and branched).

PEI, shown in Figure 12, can be found in variety of molecular weights (a few hundred Daltons$1500 \mathrm{KDa}$ ). Branched PEI is water-soluble at room temperature while linear PEI is only soluble in hot water. ${ }^{[10]}$ 
Toxicity of PEI caused by its strong cationic nature was indicated as a major concern. However, DS, which is the anionic polymer, is potentially capable of decreasing the toxicity.

She also indicated that cationic density of PEI is totally $\mathrm{pH}$ dependent and the highest buffering capacity of this weak base exhibits itself within a $\mathrm{pH}$ range of 8-9.5. Moghimi ${ }^{[42]}$ also indicated that PEI exhibits a high buffering capacity above $\mathrm{pH} 7$.

Dextran sulfate, which is available in a wide molecular range of 5-500 $\mathrm{kDa}$, is used as anionic polymer. It is reportedly soluble in water and insoluble in ethanol and ether. Figure 13 shows the structure of DS. Both PEI and DS are biodegradable and biocompatible. ${ }^{[10-12,42]}$

Lyophilization, subliming the water directly from a frozen material after reducing its surrounding pressure, of nanoparticles was recommended by Tiyaboonchai ${ }^{[10-12]}$ for the sake of safe handling and stability issues. Mannitol was recommended as a redispersing agent after lyophilization.

Loading procedure of Tiyaboonchai: She added $20 \mu \mathrm{L}$ of the loading particle, Amphotericin B $(\mathrm{AmB})$, solution in dimethylsulfoxide $(10 \mathrm{mg} / \mathrm{mL})$ to $0.2 \mathrm{~mL}$ of a DS aqueous solution $(1 \% \mathrm{w} / \mathrm{v})$ which was continuously stirred at $600 \mathrm{rpm}$. After stirring for 30 minutes she added $0.44 \mathrm{~mL}$ of aqueous PEI solution $(1 \% \mathrm{w} / \mathrm{v})$ and stirred the resulting nanoparticles for 5 minutes. After adding $20 \mu \mathrm{L}$ of a $1 \mathrm{M}$ zinc sulfate solution she washed the resulting nanoparticles for $24 \mathrm{~h}$ in the dark by dialysis against a 5\% mannitol solution. She mentioned that dialyzing the unloaded nanoparticles in water causes agglomeration while dialyzing in 5\% mannitol solution uniformly distributes the spherical shape nanoparticles. She lyophilized the resulting nanoparticles at 1272 mtorr $(1$ torr $=133.32 \mathrm{~Pa})$ and $-46{ }^{\circ} \mathrm{C}$ for 24 hours and finally stored them in a desiccator at 2$8{ }^{\circ} \mathrm{C}$. She used scanning electron microscopy to investigate the surface and shape of both loaded and unloaded nanoparticles.

Dynamic light scattering was used by Tiyaboonchai ${ }^{[10-12]}$ to measure the mean particle size, size distribution and polydispersity of the nanoparticles before and after the lyophilyzation. She mentioned that the ratio of PEI to DS was controlling the particles size of the nanoparticles. Increasing that ratio decreased the mean particle size. She assumed this behavior is because of PEI acting as a colloidal protective agent, which prevents aggregation of the nanoparticles. The cationic to anionic ratio of above 2:1 generated the reasonably small mean particle size at $\mathrm{pH}$ of 7. Decreasing the $\mathrm{pH}$ of PEI solution caused larger particle size and polydispersity. The optimal system with no aggregation was made using PEI solution with $\mathrm{pH}$ of 8 . Table 4, generated by 
Tiyaboonchai, ${ }^{[12]}$ reports the mean particle sizes and polydispersity indices before and after lyophilization at $\mathrm{pH}=7$ and $25 \mu \mathrm{M} \mathrm{ZS}$ for $\mathrm{AmB}$ loaded nanoparticles.

Table 5, generated by Tiyaboonchai, ${ }^{[12]}$ reports the mean particles sizes and polydispersity indices before and after lyophilization for using PEI solutions with different $\mathrm{pH}$, mass ratio of 2:1 and $25 \mu \mathrm{M}$ ZS for AmB loaded nanoparticles. Note that reasonable mean particle size and zeta potential were achieved at $\mathrm{pH}$ of 9 . Increasing the $\mathrm{pH}$ of PEI from 5 to 9 and increasing the PEI:DS ratio, she reported smaller nanoparticles.

She reported that formulations with zinc sulfate (ZS) are more stable before and after the lyophilization. Optimal amount of ZS was found to be $15-25 \mu \mathrm{M}$ since smaller and similar particle sizes were generated using this amount before and after lyophilization (Table 6).

She also applied phase analysis light scattering using ZetaPALS instrument (Smoluchowski approximation) to determine the zeta potential of nanoparticles. Positive zeta potential for the formulations with ZS is believed to be caused partially by zinc crosslinking of the particle surface. While positive charge of formulations with no ZS suggests that PEI is concentrated on the surface of particle.

Increasing the amount of DS increased the mean particle size of Tiyaboonchai's AmB loaded system. She found the optimal amount of DS to be in the range of $1.5-2.5 \mathrm{mg} / \mathrm{mL}$ when $\mathrm{pH}$ of PEI solution was 8 (Table 7).

Decreasing the concentration of mannitol during the lyophilization was shown to increase the mean particle size and polydispersity index after lyophilization. 5\% mannitol was found to be the optimal concentration, which produces the same particle size as nanoparticles before lyophilization.

PEI solution was reported to have stable fluid with no precipitation at $\mathrm{pH}$ range of 6-9 while $\mathrm{pH}$ of 9 exposed the lowest turbidity. Higher concentrations of DS at constant mass ratio showed higher turbidity as well.

Entrapment Efficiency (EE): The content of drug in loaded nanoparticles was determined by centrifugation before lyophilization. She centrifuged $0.5 \mathrm{~mL}$ of nanoparticle suspension before lyophilization at $12000 \mathrm{~g}$ for 20 minutes. Dissolved pellet in $0.5 \mathrm{~mL}$ of dimethylsulfoxide was centrifuged at $12000 \mathrm{~g}$ for 20 minutes. She then mixed the supernatant with $0.98 \mathrm{~mL}$ of methanol:water (1:1) solution. The amount of drug was determined using its absorption at 408 $\mathrm{nm}$. The percentage of entrapped drug was calculated as: 
Drug entrapment $(\%)=($ Amount of drug in particle $) \times($ Volume tested $) \times(100 \%) \div[($ Total sample volume $) \times($ Initial amount of drug)]

Increased amount of ZS added to the solution increased the EE. PH of 8 showed the best EE.

Drug Recovery: Dissolving $10 \mathrm{mg}$ of lyophilized nanoparticles in $0.2 \mathrm{~mL}$ DMSO and centrifuging the solution at $12000 \mathrm{~g}$ for 20 minutes then mixing $20 \mu \mathrm{L}$ of the supernatant with $0.98 \mathrm{~mL}$ of methanol:water (1:1) solution she defined the amount of drug using its absorption at $408 \mathrm{~nm}$. She defined the percent of drug recovery as:

Drug Recovery $(\%)=($ Amount of drug in particle $\times$ Total particles mass $) \times(100 \%) \div($ Particle mass tested $\times \%$ Drug entrapment $\times$ Initial amount of drug)

In order to determine the final PEI:DS mass ratio in the centrifuged solution Tiyaboonchai ${ }^{[10]}$ subtracted the amount of PEI and DS in the supernatant from the initial PEI/DS present in the solution. She used ninhydrin assay to determine the amount of PEI and spectrophotometer to determine the amount of DS.

She studied the release of drugs from the loaded nanoparticles by placing them in the dissolution medium while it was stirred under $200 \mathrm{rpm}$ and specific temperature. Samples of the dissolution fluid were taken by time and amount of drug was measured.

Explaining the peroral use of drugs delivered by nanoparticles she mentioned that limiting the diameter of the nano-sized particles to less than $500 \mathrm{~nm}$ might protect labile drugs from enzymatic degradation. She mentioned that drug must be entrapped inside the particle to avoid enzymatic degradation.

\section{3-4 Application of Polyethylenimine/Dextran Sulfate System In Petroleum Engineering}

Cordova et al. ${ }^{[14]}$ modified the system presented by Tiyaboonchai ${ }^{[10]}$ to delay hydrolyzed polyacrylamide (HPAM) gelation by sequestering the chromium (III) crosslinker in PEC nanopartciles. Cordova et al. ${ }^{[14]}$ hampered the rapid release of $\mathrm{Cr}$ (III) by generating transient electrostatic binding events. Cordova et al. ${ }^{[14]}$ formed $<200 \mathrm{~nm}$ PEC nanoparticles by adjusting the concentration of PEI and DS and also PEI:DS ratio. They mentioned that mixing ratios above $1: 1 \mathrm{v} / \mathrm{v}$ are needed to generate positively charged nanoparticles. They generated the negatively charged particles by decreasing the PEI weight ratios. They added 1\% DS $\left(\mathrm{M}_{\mathrm{w}}=500 \mathrm{KDa}\right.$ Fisher Scientific) solution to $1 \%$ PEI $\left(\mathrm{M}_{\mathrm{w}}=25 \mathrm{KDa}\right.$ Aldrich $)$ solution with PEI: DS ratio of 2.22 and 
stirred for 15 minutes at $600 \mathrm{rpm}$. Then, they added $1 \mathrm{ml}$ of $\mathrm{Cr}$ stock solution $(1.95 \% \mathrm{w} / \mathrm{w})$ to $49.58 \mathrm{~g}$ of PEI/DS solution and stirred for another 30 minutes. They dialyzed the nanoparticles against D.I. water for $48 \mathrm{~h}$ using dialysis membrane with a MWCO of $100 \mathrm{KDa}$. They determined the concentration of $\mathrm{Cr}$ (III) in the solutions by oxidizing $\mathrm{Cr}(\mathrm{III})$ to $\mathrm{Cr}(\mathrm{IV})$ and then concentration of $\mathrm{Cr}(\mathrm{IV})$ using the UV-vis absorbance at wavelength of $373 \mathrm{~nm}$.

They reported that PEI by itself crosslinks HPAM. They also indicated that in a PEI/HPAM system increasing the concentration of PEI makes the gelation faster. However they reported no significant changes in gelation time using dextran sulfate.

They reported the $\mathrm{pH}$ of the assembled nanoparticle systems around 9 because of the buffering capacity of PEI. They reported that increasing the concentration of PECs generates higher viscosity during the pre-gelled phase. However, the cases with lower concentration of PECs showed faster increase in viscosity after reaching the gelation point. Increasing the temperature, higher viscosities of gelant were reported recommending that PEI release is faster from the PEI/DS bonds at higher temperatures forming the PEI:HPAM interaction. Unloaded PECs were not able to produce gel when low concentrations of HPAM (2000 ppm) were used.

Cordova et al. ${ }^{[14]}$ showed that the Cr-loaded nanoparticles were capable of delaying the gelation time for about 4.5 days at $40{ }^{\circ} \mathrm{C}$ while the controlled system with no PECs showed gelation time of around 30 minutes. They also reported that increasing the temperature reduces the gelation time significantly. They discussed that PEI is primarily responsible for entrapment of $\mathrm{Cr}$ ions in the PECs since the count rate of nanoparticles for the PEI mixture with $\mathrm{Cr}$ is significantly higher than in for the DS mixture with Cr. They further proved this claim by showing fast gelation of negatively charged particles (PEI:DS ratio of 0.45 ) with low $\mathrm{pH}$ of $\sim 6.6$. They also recommended that calcium delays gelation time by occupying $\mathrm{Cr}$ binding sites on HPAM. 


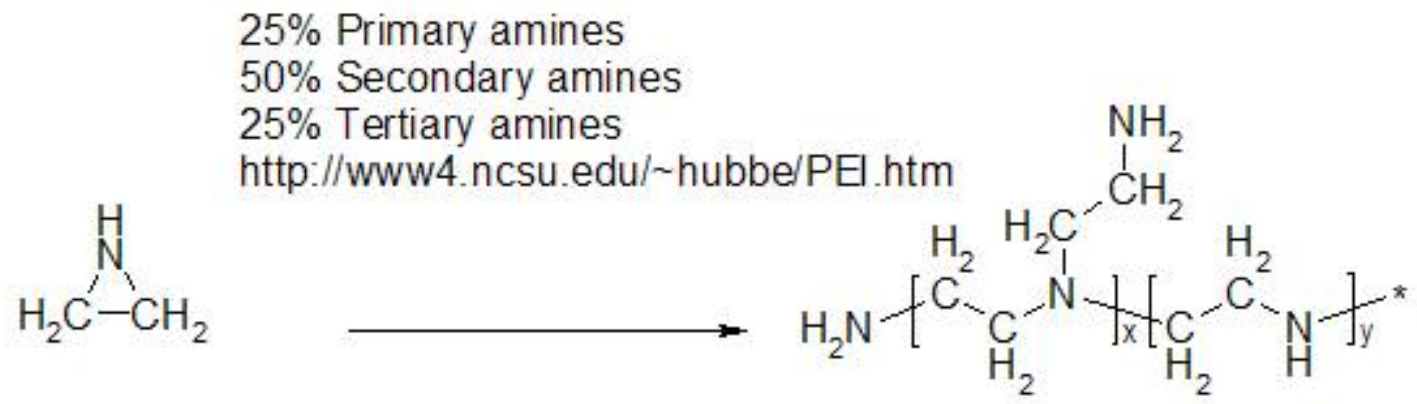

Assumes PEI made by polymerizing ethylimine monomers:

Figure 12 Chemical structure of PEI (Drawn by Stephen J. Johnson, used by permission).<smiles>CC1OC(O)C(S(=O)(=O)O)C(S(=O)(=O)O)C1S(=O)(=O)O</smiles>

Figure 13 Chemical structure of DS (Drawn by Stephen J. Johnson, used by permission). 
Table 4 Effect of polymer ratio on the mean particle size and polydispersity of Amphotericin B (AmB) loaded particles, After Tiyaboonchai ${ }^{[10]}$

\begin{tabular}{ccccc}
\hline $\begin{array}{c}\text { Polymer } \\
\text { ratios } \\
(\text { PEI :DS) }\end{array}$ & \multicolumn{2}{c}{ Before lyophilization } & \multicolumn{2}{c}{ After lyophilization } \\
\cline { 2 - 5 } & $\begin{array}{c}\text { Mean (nm) } \\
\pm \text { s.d. }\end{array}$ & $\begin{array}{c}\text { Polydispersity } \\
\text { index }\end{array}$ & $\begin{array}{c}\text { Mean (nm) } \\
\pm \text { s.d. }\end{array}$ & $\begin{array}{c}\text { Polydispersity } \\
\text { index }\end{array}$ \\
\hline $0.74: 1$ & $\mathrm{NM}^{*}$ & - & $\mathrm{NM}^{*}$ & - \\
$1: 1$ & $525 \pm 20$ & 0.27 & $\mathrm{NM}^{*}$ & - \\
$1.5: 1$ & $330 \pm 5$ & 0.26 & $\mathrm{NM}^{*}$ & - \\
$2: 1$ & $279 \pm 11$ & 0.24 & $438 \pm 2$ & 0.30 \\
$3: 1$ & $224 \pm 3$ & 0.17 & $249 \pm 3$ & 0.20 \\
\hline
\end{tabular}

$\mathrm{NM}^{*}=$ Micron size particles which could not be measured by DLS

$a^{a}=$ the PEI to DS mass ratio is roughly equivalent to the PEI to DS charge ratio.

Preparation conditions: PEI solution at $\mathrm{pH} 7$ and $25 \mu \mathrm{M}$ zinc sulfate.

Table 5 Effect of the pH of PEI solutions on the mean particle size and polydispersity of Amphotericin B

(AmB) loaded particles, After Tiyaboonchai ${ }^{[10]}$

\begin{tabular}{ccccc}
\hline $\begin{array}{c}\text { pH of PEI } \\
\text { solutions }\end{array}$ & \multicolumn{2}{c}{ Before lyophilization } & \multicolumn{2}{c}{ After lyophilization } \\
\cline { 2 - 5 } & $\begin{array}{c}\text { Mean }(\mathrm{nm}) \\
\pm \text { s.d. }\end{array}$ & $\begin{array}{c}\text { Polydispersity } \\
\text { index }\end{array}$ & $\begin{array}{c}\text { Mean }(\mathrm{nm}) \\
\pm \text { s.d. }\end{array}$ & $\begin{array}{c}\text { Polydispersity } \\
\text { index }\end{array}$ \\
\hline $5(4: 1)^{\mathrm{a}}$ & $1310 \pm 52$ & 0.37 & $\mathrm{NM}^{*}$ & - \\
$6(3: 1)^{\mathrm{a}}$ & $471 \pm 49$ & 0.26 & $\mathrm{NM}^{*}$ & - \\
$7(2: 1)^{\mathrm{a}}$ & $279 \pm 11$ & 0.24 & $438 \pm 2$ & 0.30 \\
$8(1.4: 1)^{\mathrm{a}}$ & $276 \pm 1$ & 0.20 & $263 \pm 2$ & 0.22 \\
$9(1: 1)^{\mathrm{a}}$ & $373 \pm 5$ & 0.23 & $441 \pm 2$ & 0.27 \\
\hline
\end{tabular}

$\mathrm{NM}^{*}=$ Micron size particles which could not be measured by DLS

()$^{a}=$ the numbers in parenthesis indicates the charge ratio of the polymers (PEI:DS)

Preparation conditions: PEI:DS mass ratio of 2:1 with $25 \mu \mathrm{M}$ zinc sulfate. 
Table 6 Effect of zinc sulfate concentration on the mean particle size and polydispersity of Amphotericin B (AmB) loaded particles, After Tiyaboonchai ${ }^{[10]}$

\begin{tabular}{ccccc}
$\begin{array}{c}\text { Zinc Sulfate } \\
(\mu \mathrm{M})\end{array}$ & \multicolumn{2}{c}{ Before lyophilization } & \multicolumn{2}{c}{ After lyophilization } \\
\cline { 2 - 5 } & $\begin{array}{c}\text { Mean }(\mathrm{nm}) \\
\pm \text { s.d. }\end{array}$ & $\begin{array}{c}\text { Polydispersity } \\
\text { index }\end{array}$ & $\begin{array}{c}\text { Mean }(\mathrm{nm}) \\
\pm \text { s.d. }\end{array}$ & $\begin{array}{c}\text { Polydispersity } \\
\text { index }\end{array}$ \\
\hline 0 & $805 \pm 2$ & 0.23 & $\mathrm{NM}^{*}$ & - \\
12.5 & $353 \pm 1$ & 0.24 & $410 \pm 7$ & 0.25 \\
25 & $286 \pm 1$ & 0.16 & $267 \pm 3$ & 0.19 \\
50 & $351 \pm 2$ & 0.17 & $317 \pm 31$ & 0.20 \\
75 & $386 \pm 2$ & 0.18 & $431 \pm 1$ & 0.20 \\
\hline
\end{tabular}

$\mathrm{NM}^{*}=$ Micron size particles which could not be measured by DLS

Preparation conditions: PEI solution at $\mathrm{pH} 8 ; \mathrm{PEI}$ :DS mass ratio of 2:1

Table 7 Effect of dextran sulfate concentration on the mean particle size and polydispersity of Amphotericin B (AmB) loaded particles, After Tiyaboonchai ${ }^{[10]}$

\begin{tabular}{ccccc}
\hline $\begin{array}{c}\text { Dextran sulfate } \\
(\mathrm{mg} / \mathrm{mL})\end{array}$ & \multicolumn{2}{c}{ Before lyophilization } & \multicolumn{2}{c}{ After lyophilization } \\
\cline { 2 - 5 } & $\begin{array}{c}\text { Mean }(\mathrm{nm}) \\
\pm \mathrm{s.d} \text {. }\end{array}$ & $\begin{array}{c}\text { Polydispersity } \\
\text { index }\end{array}$ & $\begin{array}{c}\text { Mean }(\mathrm{nm}) \\
\pm \text { s.d. }\end{array}$ & $\begin{array}{c}\text { Polydispersity } \\
\text { index }\end{array}$ \\
\hline 1.5 & $229 \pm 1$ & 0.26 & $268 \pm 5$ & 0.26 \\
2 & $276 \pm 1$ & 0.20 & $263 \pm 2$ & 0.22 \\
2.5 & $230 \pm 2$ & 0.22 & $260 \pm 5$ & 0.23 \\
3 & $321 \pm 3$ & 0.20 & $337 \pm 3$ & 0.26 \\
\hline
\end{tabular}

Preparation conditions: PEI solution at $\mathrm{pH} 8$; PEI:DS mass ratio of $2: 1 ; 25 \mu \mathrm{M}$ zinc sulfate 


\subsection{Enzymes for Fracturing Fluids}

Enzymes as proteins, amino acids as building blocks of proteins, polysaccharides (like guar) and hydrolysis of polysaccharides are explained in this section. Basics of catalytic reactions speeded up with enzymes are also covered in this section.

\section{4-1 Amino Acids, Proteins, Enzymes and Polysaccharides}

Amino acids: Amino acids are building blocks of protein molecules. Amino acids consist of an amino group $\left(-\mathrm{NH}_{3}^{+}\right)$protonated at neutral $\mathrm{pH}$ and a carboxyl group $\left(-\mathrm{COO}^{-}\right)$ionized at neutral $\mathrm{pH}$. The amino group and carboxyl group are both attached to an $\alpha$-carbon $(\mathrm{C}-2)$ in addition to a hydrogen atom and a side chain ( $\mathrm{R}$ group) which is a characteristic of each amino acid. There are 20 different naturally-occuring amino acids categorized based on their different side chains. The 20 common amino acids are organized into 6 different groups: Aliphatics (5), Aromatics (3), Sulfur/Alcohols (4), Basics (3), Acid/Amides (4) and "Oddball” (1). ${ }^{[28,43]}$

Chiral stereoisomers (molecules with the same set of atoms bonded in different spatial configurations) of amino acids are named enantiomers (Figure 14). In nature only the L-isomers amino acids are involved as components of proteins. ${ }^{[28,43]}$

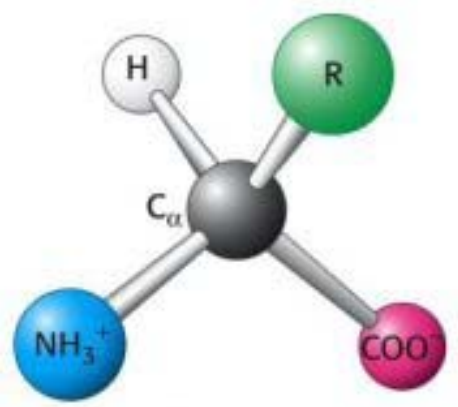

$\mathrm{L}$ isomer

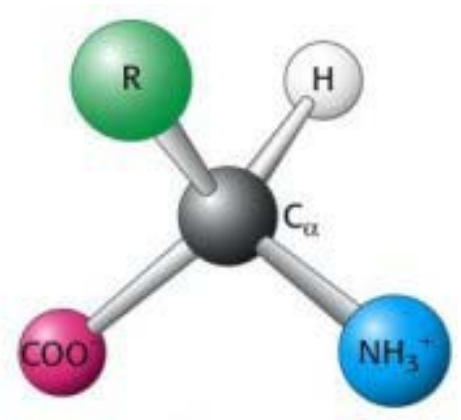

D isomer

Figure 14 L- and D- isomers of amino acids ${ }^{[28,43]}$

Some amino acids such as basics and the acid/amides have hydrophilic side chains. Some other amino acids like aliphatics and aromatics have hydrophobic side chains.

Individual amino acids can be ionized at least twice: once for the $\alpha$-carboxyl group and once for the $\alpha$-amino group. Determining the $\mathrm{pK}_{\mathrm{a}}$ values of amino acids from its titration curve the $\mathrm{pH}$ value at which ionization occurs will be identified. The $\mathrm{pH}$ value at which the net charge is 
zero is called the isoelectric point (pI) which is 6.15 for alanine (Figure 15). All the 20 standard amino acids have $\alpha$-carboxyl $\mathrm{pK}_{\mathrm{a}}$ values less than 3 and $\alpha$-amino $\mathrm{pK}_{\mathrm{a}}$ values less than 11 . Ionization of side chains will cause more inflection points in the titration curve of amino acids. Changes in isoelectric points of amino acids depend on the charge carried by their side chains.

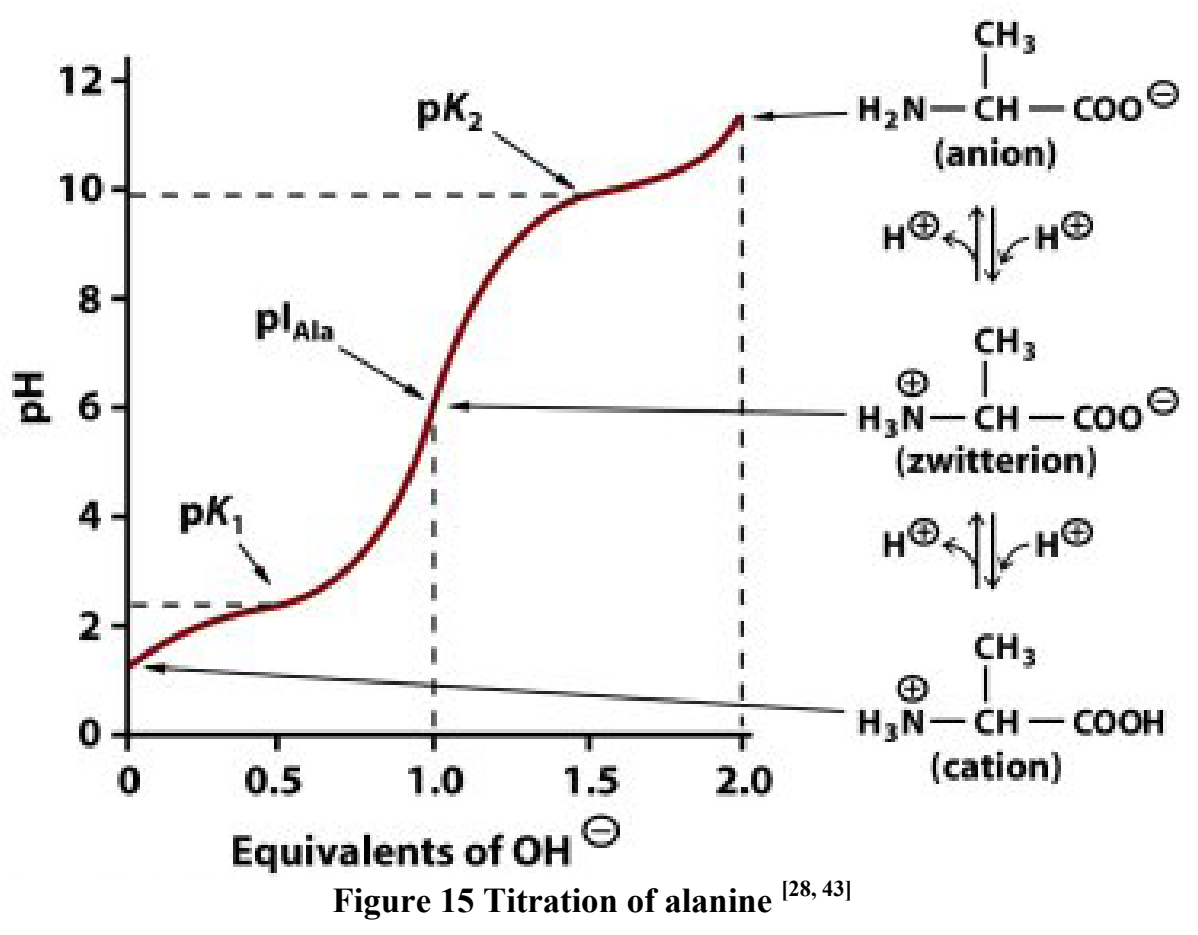

Proteins are polymers formed by condensation of individual amino acids. An amide linkage (peptide bond) forms as a result of a reaction between an amino group from one amino acid and a carboxylate group from the other one. The direction in which the amino acids are added to a growing chain is from the $\mathrm{N}$-terminal to the $\mathrm{C}$-terminal.<smiles>[R]C([N])C(=O)NC([R])C(=O)O</smiles>

Structure of Proteins: Folding of the linear chain in 3D dimensional space makes a functional structure. There are four levels of structure that are used in describing the proteins ${ }^{[28,43]}$ :

1. Primary structure (amino acid sequence): sequence of different amino acids in a directional way from $\mathrm{N}$-terminal to $\mathrm{C}$-terminal makes different primary structures for a variety of proteins. 
2. Secondary structure ( $\alpha$-helices and $\beta$-sheets): Refers to regularities in local conformations, maintained by hydrogen bonds from the peptide backbone. The most important types of secondary structure are $\alpha$-helices and $\beta$-sheets. Loops and turns are additional types of secondary structure.

- $\alpha$-Helix is the most common kind of secondary structure found in proteins shown in Figure 16. The $\alpha$-helix is stabilized by hydrogen bonds formed between the carbonyl of a residue and the amino of another residue.
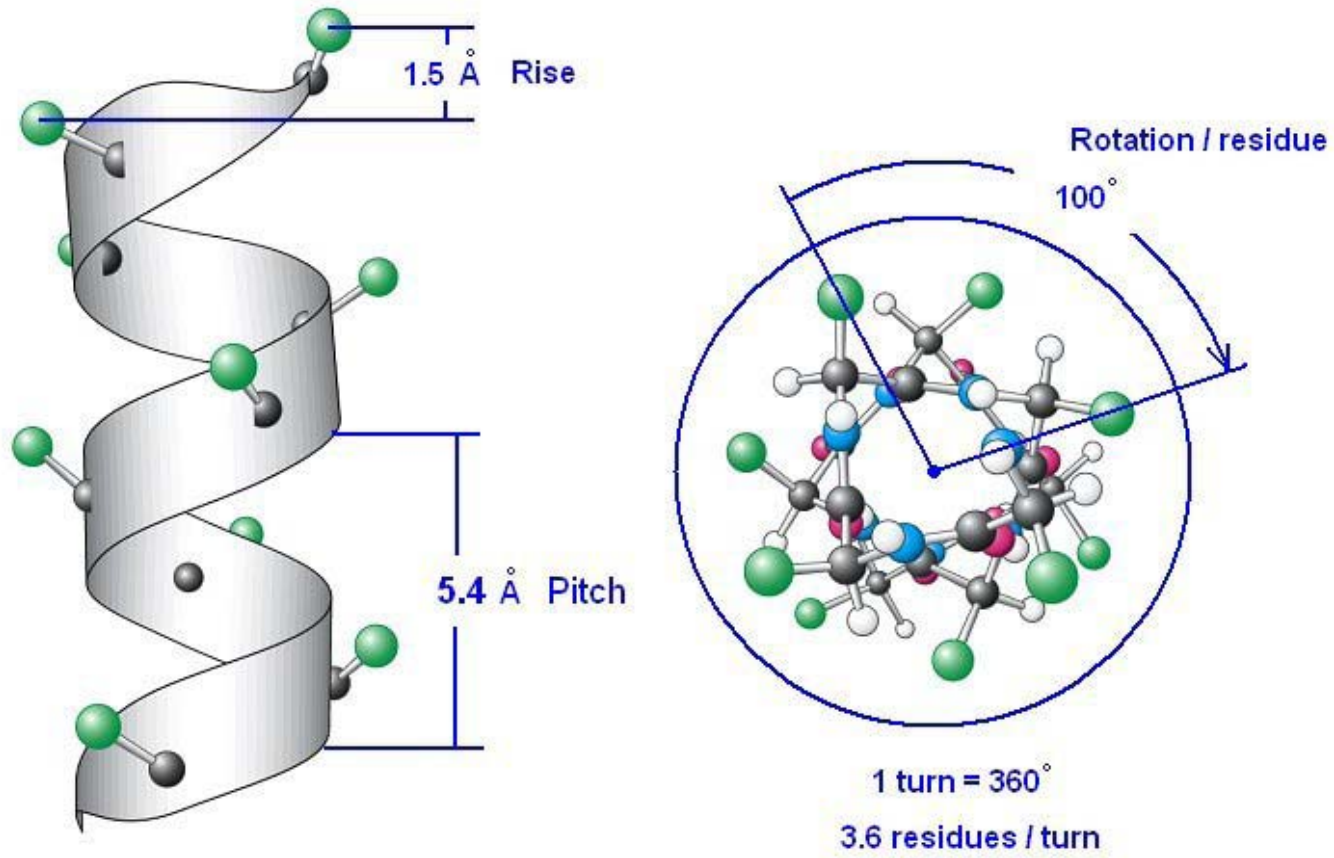

Figure 16 Structure of $\alpha$-helix ${ }^{[28,43]}$

- $\quad \beta$-Sheets: are the second most significant types of protein secondary structure. They are made of two or more individual strands that form hydrogen bonds with each other. The individual strands can also be aligned in two different orientations: parallel and anti-parallel (Figure 17 and Figure 18). However, $\beta$-sheets can also be mixed in structure with strands running in both parallel and anti-parallel directions. 


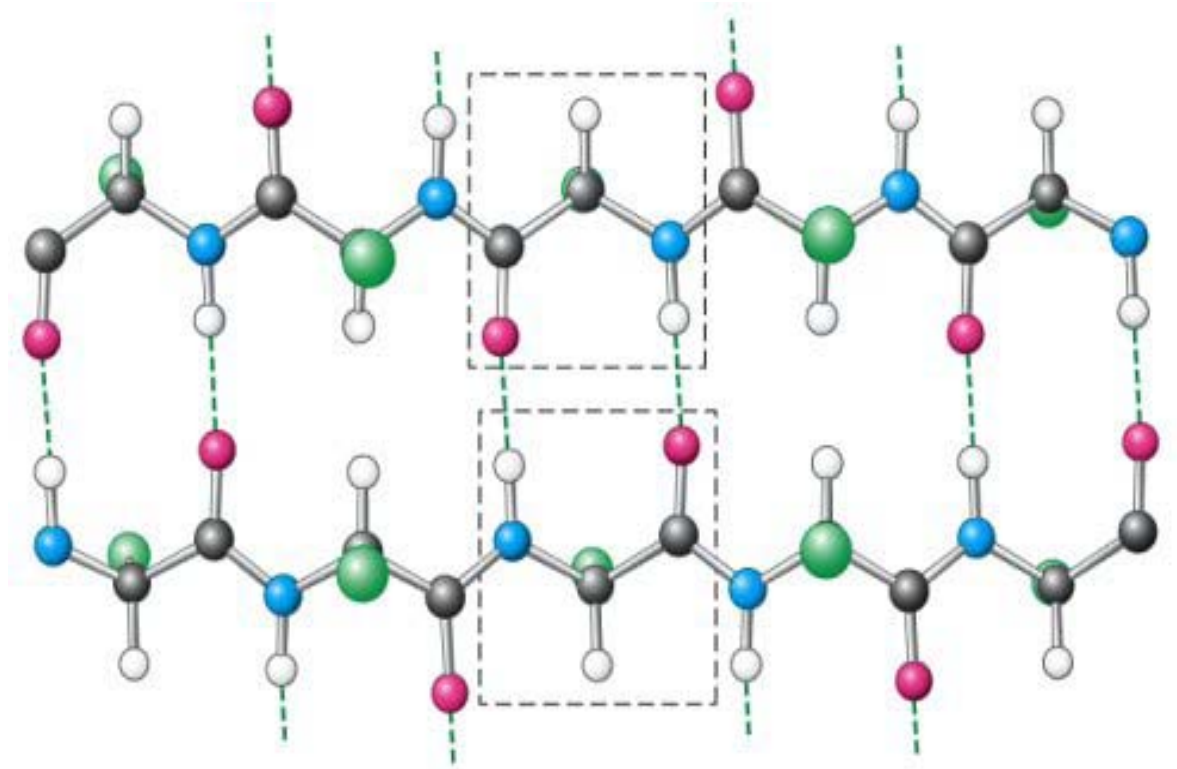

Figure 17 Anti-parallel geometry of $\beta$-Sheets ${ }^{[28,43]}$

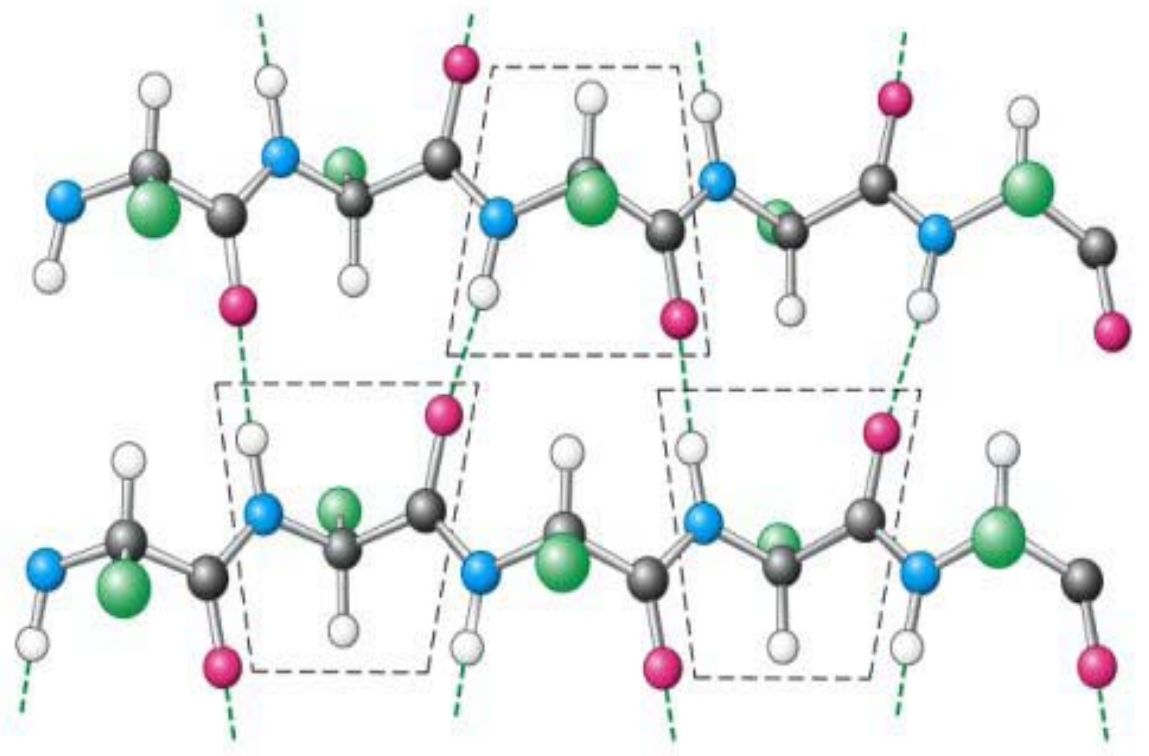

Figure 18 Parallel geometry of $\beta$-Sheets ${ }^{[28,43]}$

3. Tertiary structure (structure of an entire polypeptide chain): Refers to the spatial arrangement of an entire polypeptide chain with compact globular units called domains. In addition to hydrogen bonding of local secondary structure, tertiary structure is determined by noncovalent interactions between surfaces of adjacent domains (Figure 19). 


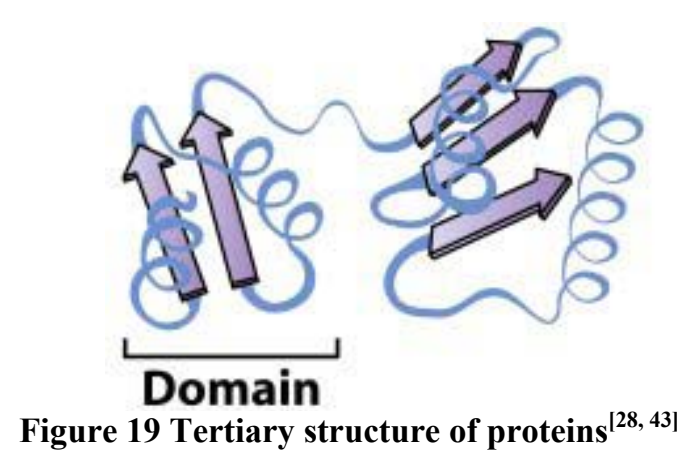

4. Quaternary structure (arrangement of multiple subunits): Describes proteins formed by the noncovalent association of distinct polypeptide chains called subunits. Subunits can be either identical or distinct from each other.

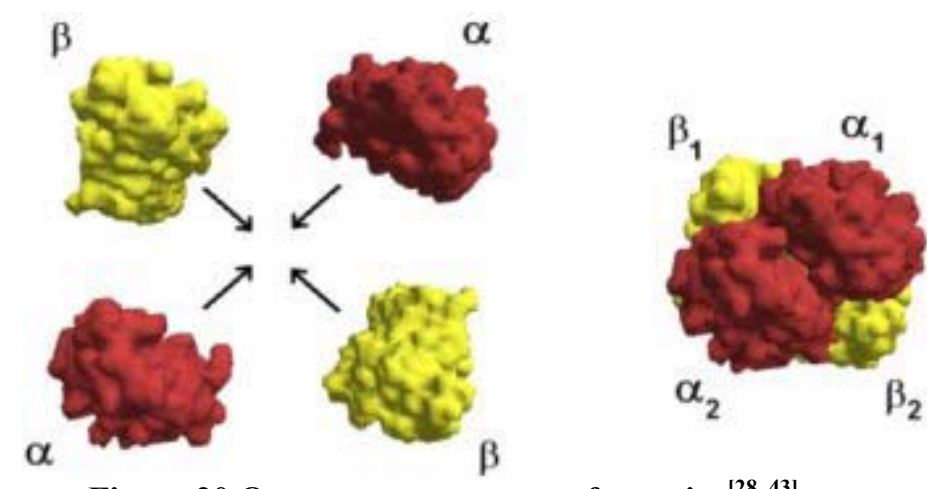

Figure 20 Quaternary structure of proteins ${ }^{[28,43]}$

3D structure of proteins can be determined using x-ray crystallography or NMR.

Denaturation and renaturation of proteins: demonstrates how protein function can be lost and regained upon changes in structure. Denaturation is the disruption of the 3D structure of a protein while renaturation is the process in which the native conformation of a protein is reacquired. Harsh conditions like raising or lowering the $\mathrm{pH}$ or heating can irreversibly denature proteins by disrupting weak interactions or covalent changes in the structure of enzyme. Denaturation of proteins by temperature occurs in very small temperature ranges. The melting temperature of enzyme is defined as the midpoint of the transition from native to denatured states.

Denaturation of enzymes under less harsh conditions and by chemicals that do not cleave covalent bonds (chaotropic agents and detergents) result only in disruption of secondary, tertiary, and quaternary structures which may be reversible. 
Enzymes: are specific catalysts of biochemical reactions. Enzymes are mostly proteins and only affect the rate and cannot change the overall equilibrium of a reaction. However, they can couple two reactions by using the energy from one reaction to affect the equilibrium of the other reaction.

Enzymes are organized into 6 main groups based on the kind of reaction that they catalyze:

1. Oxidoreductases: catalyze oxidation-reduction reactions.

2. Transferases: catalyze transfer of a group from one molecule to another.

3. Hydrolases: catalyze cleavage of a bond by water.

4. Lyases: catalyze addition/removal of a double bond.

5. Isomerases: catalyze intermolecular rearrangement.

6. Ligases: catalyze joining of substrates.

Enzymes catalyze reactions by introducing an intermediate step in which an enzyme-substrate complex is formed (Figure 21). Next the enzyme accelerates the conversion of substrate into product:

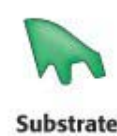

[S]

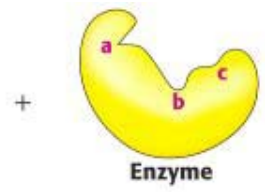

[E]

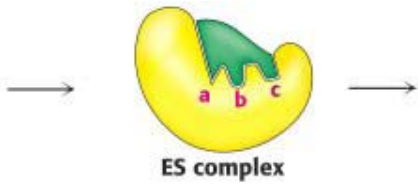

[ES]

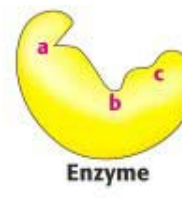

[E]

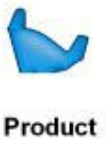

[P]

Figure 21 Schematic picture for catalytic reaction of enzymes ${ }^{[28,43]}$

Association and dissociation rates of enzyme and substrate are very rapid since only noncovalent interactions occur. Bond breaking and formation occur in the second reaction and that is why $\mathrm{k}_{2}$ is very slow and is the rate-limiting constant in the whole reaction.

Two major aspects of enzyme catalysis are chemical effects and binding effects. Chemical effects include acid-base catalysis and covalent catalysis. Both of these effects are caused through the polar and ionizable side chains in the enzyme. Table 8 shows the side chains that act to provide the majority of reactive groups in enzyme catalysis.

- Acid-base catalysis uses the transfer of a proton to accelerate bond-breaking and bond formation in reactions. In enzymes the ionizable groups of amino acid side chains act as general acids and bases that can donate and receive protons. 
- Covalent catalysis consists of covalent bond formation between the enzyme and substrate to produce reactive intermediates followed by bond-breaking to release the final products.

Increasing the $\mathrm{pH}$ from acidic to basic for an enzyme usually results in a bell-shaped activity curve caused by the ionization of key residues some protonated and some deprotonated at different $\mathrm{pH}$ values. ${ }^{[44]}$

Table 8 Catalytic functions of reactive groups of ionizable amino acids ${ }^{[28,43]}$

\begin{tabular}{llcl} 
Amino acid & Reactive group & Net charge at pH 7 & Principal functions \\
\hline Aspartate & $-\mathrm{COO}^{\ominus}$ & -1 & Cation binding; proton transfer \\
Glutamate & $-\mathrm{COO}^{\ominus}$ & -1 & Cation binding; proton transfer \\
Histidine & Imidazole & $\mathrm{Near} 0$ & Proton transfer \\
Cysteine & $-\mathrm{CH}_{2} \mathrm{SH}$ & $\mathrm{Near} 0$ & Covalent binding of acyl groups \\
Tyrosine & $\mathrm{Phenol}^{\text {Lysine }}$ & 0 & Hydrogen bonding to ligands \\
Arginine & $\mathrm{NH}_{3}^{\oplus}$ & +1 & Anion binding; proton transfer \\
Serine & $\mathrm{Guanidinium}^{-\mathrm{CH}_{2} \mathrm{OH}}$ & +1 & Anion binding \\
& & 0 & Covalent binding of acyl groups
\end{tabular}

Binding effects include reduction of entropy and transition state stabilization. For a molecule to move from one stable arrangement to another, it must cross intermediate barriers with higher energy levels. Enzymes are capable of lowering the activation energy (the energy barrier to the progress of the reaction) of reactions in different ways. ${ }^{[28,43]}$

Polysaccharides are carbohydrates formed from simple sugars by reacting between the C-1 carbon of one sugar with a hydroxyl $(\mathrm{OH})$ group of the other one to form a glycosidic bond (the functional group that joins one sugar molecule to another). For glucose ring drawn as below $\alpha-$ and $\beta$ - glycosidic bonds can be distinguished by location of $\mathrm{C}-1$ hydroxyl being below or above the plane of the ring. Numbers 1, 4, and 6 are applied in the nomination to determine the carbon atoms at each end of the glycosidic bond (Figure 22). 


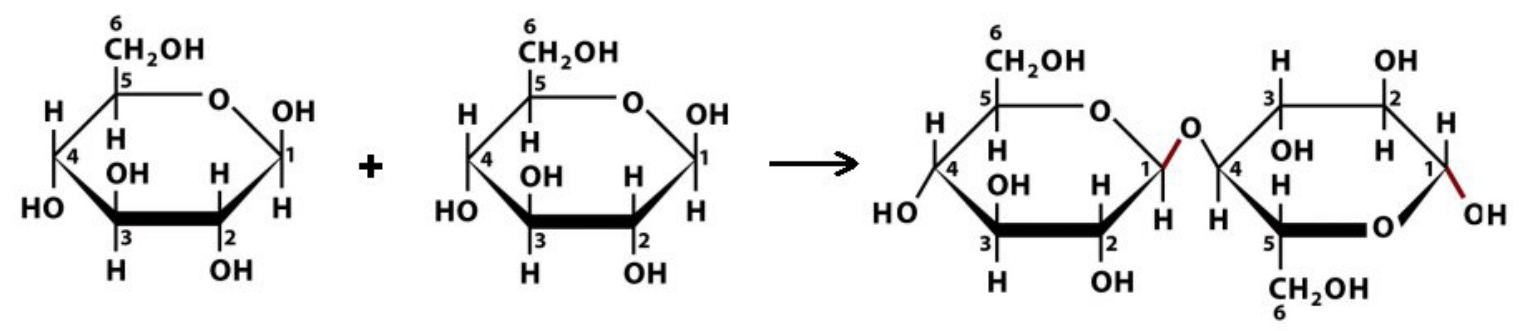

Figure 22 Formation of polysaccharides from simple sugar units ${ }^{[28,43]}$

Condensation of monomers may be a reversible reaction (hydrolysis). Hydrolysis of polysaccharides can be speeded up by a family of enzymes named glycoside hydrolases. Glycoside hydrolases attack the glycosidic bond of polysaccharides. ${ }^{[28,43]}$

The net dipole characteristic of water caused by strong attraction of electrons by oxygen (more electronegativity of oxygen compared to hydrogen) gives water an important nucleophilic property. Nucleophiles are attracted to positively charged or electron deficient species. Ionization of water is an example of this property in which water oxygen attacks the hydrogen of other water molecules. Hydrogen and hydroxide ions made by ionization of water may participate in further reactions like hydrolysis of polysaccharides. ${ }^{[28,43]}$

\section{4-2 Michaelis-Menten Kinetics of Enzymes}

Enzymes catalyze the reactions. The products and reactants in an enzymatic reaction are the same as the uncatalyzed reactions. An essential step during the enzyme reaction is the formation of a complex between the enzyme and substrates. Reaction rate will be independent of [S] if [S] is significantly larger than [E] and assuming that the equilibrium between the enzyme and substrate happens rapidly. Thus, the rate of the second reaction can determine the rate of an enzymatic reaction.

$E+S \stackrel{k_{1}}{\longrightarrow} E S \stackrel{k_{2}}{\longrightarrow} E+P$ Equation 8

For low concentrations of substrate there is a linear relation between the reaction rate and substrate concentration. However, this curve approaches a maximum asymptotically because of enzyme active sites being saturated at high substrate concentrations (Figure 21). 
Considering that enzymes can be saturated at high concentrations of substrate, Michaelis and Menten defined their reaction constant as $K_{s}=\frac{[E][S]}{[E S]}$ and the rate of the reaction as $v=\frac{k_{2}\left[E_{0}\right][S]}{K_{s}+[S]}$. This velocity increases, increasing the substrate concentration till enzymes will be saturated and velocity stays constant. Modifying the velocity as $v=\frac{k_{2}\left[E_{0}\right][S]}{K_{m}+[S]}$, in which $K_{m}=\frac{\left(k_{2}+k_{-1}\right)}{k_{1}}$, Lineweaver-Burk (1934) equation was defined as below. In these plots velocity becomes half when $K_{m}=[S]$. So, $\mathrm{K}_{\mathrm{m}}$ is that concentration of substrate, which gives half of the maximum velocity.

$$
\frac{1}{v}=\frac{1}{v_{\max }}+\left(\frac{K_{m}}{v_{\max }}\right)\left(\frac{1}{S}\right)
$$

Equation 9

Changing the concentration of substrate and measuring data accurately one can find the slope of concentration vs. time near the time zero. Plotting the reciprocal of such a velocity vs. reciprocal of substrate concentration one might calculate $\mathrm{K}_{\mathrm{m}}$ and $\mathrm{v}_{\max }$ from the slope and intercept, respectively. ${ }^{[45,46]}$

\section{4-3 Viscosity-average Molecular Weight of Guar during Degradation}

Cheng et al. ${ }^{[47]}$ measured the zero shear viscosity of a polymeric solution and the solvent in order to calculate the relative viscosity $\left(\eta_{r}=\frac{\eta}{\eta_{0}}\right)$. Calculating the specific viscosity from this relative viscosity $\left(\eta_{s p}=\eta_{r}-1\right)$ they defined the reduced viscosity as the ratio of specific viscosity over the concentration $\left(\eta_{r e d}=\frac{\eta_{s p}}{C}\right)$. Extrapolating the plot of reduced viscosity vs. concentration to $C=0$, they determined the intrinsic viscosity ( $[\eta])$. They reported that viscosity of a polymer solution is related to its concentration based on the following relationship in which $\mathrm{K}$ is known as Huggins constant (near 0.35 for flexible polymer molecules in a solvent). 
Reddy and Tammishetti ${ }^{[48]}$ reported the following equation to relate the intrinsic viscosity to concentration and viscosity of the guar solutions.

$[\eta]=\left[\left(1+1.14 \eta_{s p}\right)^{0.5}-1\right] / 0.7 C$

Equation 11

Viscosity-average molecular weight $\left(M_{v}\right)$ of the polymers can be calculated from the intrinsic viscosity applying the Houwink equation. $k=3.67 \times 10^{-4}-3.8 \times 10^{-4}$ and $\alpha=0.723-0.884$ are recommended for guar solutions. ${ }^{[1]}$

$[\eta]=k M_{v}^{\alpha}$

Equation 12

Since breakers break the backbone of the polymers, the molecular weight of the polymers decreases vs. time after being introduced to breakers. This relation between intrinsic viscosity and molecular weight makes it possible to track the molecular weight size vs. time using the zero shear viscosity of the polymeric solution. A very good alternative for this method is sizeexclusion chromatography (SEC) ${ }^{[47]}$, using which may be used to measure the molecular weight distribution of a polymer sample. Using these techniques is useful in the lab to measure the MW of polymer while being degraded by breakers.

Another useful definition is the critical overlap concentration $\left(\mathrm{C}^{*}\right)$ which is a concentration above which the polymer coils begin to interact. Plotting the specific viscosity vs. multiplication of concentration and intrinsic viscosity, $\mathrm{C}^{*}$ is defined as point at which a sharp increase in the slope of the curve occurs. $\mathrm{C}^{*}$ is the minimum concentration at which intermolecular crosslinking is possible. ${ }^{[1]}$ 


\section{4-4 Viscometric Assays for Determining Concentration of Enzyme from Activity Measurements}

Bell's viscometric method ${ }^{[44]}$ : This method was proposed as a measurement of pectinase activity in order to study its effect on softening the brine solution in cucumber salt-stock. Sodium polypectate (pectate) solution was made by adding $1.2 \%$ of sodium polypectate in a sodium hydroxide-citric acid buffer $\left(\mathrm{pH}=5 @ 30{ }^{\circ} \mathrm{C}\right)$ in a Waring blender at $\mathrm{T}=50-60{ }^{\circ} \mathrm{C}$. The solution was cooled down and then filtered. Toluene was added to the samples that were made to be stored for a long time. The pectinol (pectinase) solution was diluted in distilled water before use. Brine, which included pectinase, was dialyzed before being used to remove its salt contaminants and prevent their gel reaction with the pectate solution.

Zero shear viscosity measurements were done using Ostwald-Fenske viscosity pipettes after adding different concentrations of dialyzed brine samples to pectate solution, Zero shear viscosity was measured immediately, 20 hours, and 44 hours after adding pectinase. Percent loss in viscosity for a given length of time represented the softening activity and it was calculated as $\frac{\mu_{0 i}-\mu_{0 t}}{\mu_{0 i}-\mu_{0 w}} \times 100$. In which "i", “t”, and "w" subscripts refer to initial time, given time, and water respectively. A $50 \%$ loss in viscosity of $1 \%$ pectate-pectinolytic enzyme solution at $30{ }^{\circ} \mathrm{C}$, and pH 5 for a 20-hour period was defined as 100 units of activity. Plotting the loss in zero shear viscosity values against the $\log$ of the concentration or log of the time to reach specific loss in zero shear viscosity value must be nearly linear except below the $10 \%$ values. The last reading was recommended to be when there is $75 \%$ loss in viscosity of the solution. The same information can be given in a plot of concentration of pectinase against reaction time periods.

Mutlu et al. ${ }^{[49]}$ used Michaelis-Menten method to determine enzyme activity using the viscometric assay. They found a correlation between concentration of pectin in the baseline solution and its viscosity. That equation was then applied to calculate concentration of pectin in the solutions affected by pectinase and relate loss in viscosity to loss in concentration, assuming that only pectin concentration causes the viscosity loss of the fluid. They measured the viscosity vs. time for different concentrations of pectin and constant concentration of pectinase. After translating the viscosity values to concentration of pectin and measuring the initial velocity, they plotted the reciprocal of initial reaction velocity vs. reciprocal of substrate concentration. Plotting concentration of pectinase vs. initial enzyme concentration they found that initial rate of 
enzymatic reaction changes linearly at low concentration but from a certain level the increasing trend of rate diminishes.

\section{4-5 Methods for Determining Concentration of Enzymes}

There are several other methods capable of measuring the concentration of enzymes. Titrimetric methods, ${ }^{[50]}$ spectrophotometric methods ${ }^{[51]}$ and total organic nitrogen $(\mathrm{TON})^{[52]}$ were named in the literature as methods of measuring enzyme concentration. In this section basics of spectrophotometric assay and TON are explained.

Beer-Lambert Law and Spectrophotometric Assays: The Beer-Lambert law is an empirical relationship between the absorption of light and properties of material through which the light is traveling. This law states that there is a logarithmic relation between transmissivity of light through a substance and the product of absorption coefficient of the substance $(\alpha)$ and the distance the light travels through the material.

$T=10^{-\alpha l}=10^{-\varepsilon c l}$

Equation 13

$A=-\log (T)=\alpha l=\varepsilon c l$

Equation 14

In which $\varepsilon$ is the molar absorptivity of the substance, $\mathrm{c}$ is the concentration of absorbing species, and 1 is the path length. This law fails at high concentrations because of overlapping of molecules at higher concentrations.

The composition of a mixture of $\mathrm{n}$ components is found by measuring the absorbance at $\mathrm{N}$ different wavelengths. The value of molar absorptivity must be known at that specific wavelength. The wavelengths at the maximum absorptions for individual components are the best wavelengths to choose. Measuring the absorbance for each individual component at low enough concentrations that produce a linear relation between concentration and absorbance one can calculate the absorptivity of different substances. ${ }^{[53]}$
$A\left(\lambda_{i}\right)=l \sum_{j=1}^{n} \varepsilon_{j}\left(\lambda_{i}\right) c_{j}$
Equation 15 
Using the elimination method to solve the resulting matrix requires a wavelength where the first component does not overlap the second, the second component does not overlap the third and further, and so on. However, using the matrix inversion to solve the matrix is applicable for mutually overlapping spectra. ${ }^{[54]}$ Absorbance values higher than 2 are reported to be unreliable because of very low light levels applied during their measurements. It is also recommended not to use wavelengths for which molar absorptivities are identical for two different species. ${ }^{[54]}$ Sigma ${ }^{[51]}$ published a spectrophotometric enzymatic assay for pectinase in presence of pectin. They applied pectinase (test) and buffer (blank) to a $0.5 \%$ pectin solution in buffer separately and monitored the light absorbance at $235 \mathrm{~nm}$ vs. time. Comparing the absorbance of test and blank samples with the absorbance curve from the base fluid ( $0.5 \%$ pectin solution made in buffer) and defining the increase in the absorbance of the base fluid at $40{ }^{\circ} \mathrm{C}$ and $\mathrm{pH}=5$ to be one over a minute, they defined the activity of enzyme.

Total Organic Nitrogen (TON): ${ }^{[52]}$ The amino-acid groups in the enzymes contain nitrogen. Measuring the total organic nitrogen may be needed to measure concentration of an enzyme. The Apollo 9000 unit measures the total nitrogen and carbon by converting all forms of nitrogen to $\mathrm{NO}$ and all forms of carbon to $\mathrm{CO}_{2}$. After being converted, $\mathrm{NO}$ and $\mathrm{CO}_{2}$ are swept into the nondispersive infrared detector to measure the concentration of $\mathrm{CO}_{2}$ in the sample gas. Next, excited $\mathrm{NO}_{2}$ or $\mathrm{NO}_{2}{ }^{*}$ is formed by mixing $\mathrm{NO}$ with ozone. $\mathrm{NO}_{2}{ }^{*}$ gives off extra energy as light while returning to its ground state (chemiluminescence). The light signal is then converted to an electronic signal. The amount of light detected is proportional to the amount of NO in the sample gas. ${ }^{[52]}$ Plotting the area under the curve of detected voltage $(\mathrm{mV})$ vs. time (in seconds) generates a calibration curve that can be helpful in finding the concentration of enzyme in any unknown solution. Note that one must ensure that the amount of nitrogen in other additives of the fluid is known.

\section{4-6 Enzymatic Degradation of Guar Solutions and Guar-Borate Gels}

Tayal et al. ${ }^{[55,56]}$ studied the enzymatic degradation of guar polymer solutions using Gammanase. Gammanase is a commercial extract of Aspergillus niger consisting primarily of a mixture of endo- $\beta$-mannanase and $\alpha$-galactosidase (Figure 23). They reported that $\beta$-mannanase is the main cause of polymer degradation using such a commercial gammanase. They reported that 
enzymatic degradation was of zeroth order in guar concentration. However, they indicated that $\frac{1}{M_{w}} \propto k t$ is respected regardless of the reaction order.

Tayal et al. ${ }^{[55,56]}$ applied gel permeation chromatography (GPC) to measure the molecular weight of the polymers during the degradation process. The GPC showed that average molecular weight decreases significantly during the degradation of guar while the molecular weight distribution broadens significantly. Measuring the viscosity of guar and calculating the zero shear viscosity they reported that rate of viscosity reduction decreases significantly when increasing the concentration of guar. Similar shapes of viscosity profiles gave them the idea of superimposing the viscosity reduction profiles by plotting them versus a reduced time variable. The coefficient that related the time to the reduced time was reported to be a linear function of enzyme concentration. This superposition gave them ability to predict the viscosity of guar solutions vs. time for different enzyme concentrations.

Studying the enzymatic degradation of guar-borate hydrogel using $\beta$-mannanase, $\alpha$-galactosidase and combination of both, Tayal et al. ${ }^{[55-57]}$ recognized three different degradation regimes in plots of rheological moduli $\left(G^{\prime}\right.$ and $\left.G^{\prime \prime}\right)$ and the complex viscosity vs. frequency. They made their guar samples in water containing $0.5 \mathrm{M}$ sodium chloride and $0.05 \mathrm{M}$ sodium thiosulfate. After centrifuging the guar for $1.5 \mathrm{~h}$ under $20000 \mathrm{~g}$ they made the gel by adding borax and adjusting the $\mathrm{pH}$ at $25{ }^{\circ} \mathrm{C}$. They heated up the gel up to $65^{\circ} \mathrm{C}$ for $15-30$ minutes then and cooled it down to room temperature to ensure the homogeneity of the gel. They also set the gel on a horizontal shaker over night to mix well before being used.

Measuring the rheological properties of the guar solution itself they found that the loss modulus $\left(G^{\prime \prime}\right)$ dominates the response over most of the frequency domain. However, upon crosslinking of the guar both moduli increased significantly while the elastic modulus $\left(G^{\prime}\right)$ is dominant. $G^{\prime}$ reaches a plateau at intermediate frequencies where $G^{\prime \prime}$ reaches a maximum. These features are characteristics of a formed network structure.

Taking a sample of guar-borate gel at different times after adding enzyme and increasing the temperature of the sample to around $80^{\circ} \mathrm{C}$, in order to deactivate the enzymes, they measured the rehological properties. They indicated that both moduli curves vs. frequency shift down significantly by time. Tayal et al. ${ }^{[55-57]}$ also stated that the plateau region decreases over the time and disappears after the network is completely broken. After this point, $G^{\prime \prime}$ is higher than $G^{\prime}$ and 
the system behaves as a solution. They applied the time-temperature superposition concept to generate a master curve that makes rheological behavior of guar-borate gels predictable. They interpreted the three observed stages of viscosity reduction as follows:

1. Since the crosslinking of polymer chains is interpreted as an increase in the effective molecular weight, they interpreted the initial reduction in moduli as chain scission. They measured the molecular weight using GPC after removing the crosslinks by decreasing the $\mathrm{pH}$.

2. During the intermediate times significant drop in moduli is prevented by sufficient chain length, which causes enough connectivity between chains and slow rate of reduction in moduli at a constant frequency.

3. At long degradation times the chains are very small so they cannot overlap with other chains. Even if the borate ions crosslink chains locally, when only $\beta$-mannanase is used, the chains are not capable of maintaining any long range connectivity. This causes fast rate of reduction in moduli at a constant frequency.

Comparing $\beta$-mannanase, $\alpha$-galactosidase and combination of both enzymes they demonstrated that $\alpha$-galactosidase showed insignificant effects on the properties of gel. However, combination of both enzymes degrades the gel significantly faster. In addition to decreasing the crosslinking capacity of guar they also indicated the increase in the activity of $\beta$-mannanase as a result of $\alpha$ galactosidase as a reason for this significant decrease in rheological properties.

\subsubsection{Enzymes Used to Break Guar Gum}

Typically a guar molecule is made of a linear backbone of $\beta-1,4$ mannose units with $\alpha-1,6$ galactoseside chains. Different bonds of guar that can be attacked by enzymes are the endo- and exo- $\beta-1,4$ bonds between the D-mannose sugar units on the backbone and the $\alpha-1,6$ bonds between the backbone and the galactose side chains. The mentioned sites can be cleaved by endo- and exo- $\beta$-mannanase and $\alpha$-galactosidase enzymes respectively. Tayal et al. ${ }^{[55-58]}$ indicated that insufficient $\alpha$-D-galactosidase in an enzyme package results in short mannose chains, which cannot be degraded further because of steric hindrances from the galactose side chains. On the other hand, insufficient endo- $\beta$-mannanase results in unsubstituted mannose chains thus forming insoluble residues. 
Tayal et al. ${ }^{[58]}$ studied the effect of each of the aforementioned enzymes on a $0.7 \% \mathrm{w} / \mathrm{v}$ guar solution at $25{ }^{\circ} \mathrm{C}$ at $\mathrm{pH}=5$ (Figure 24). Endo-mannanase was shown to be the most effective enzyme in reducing the viscosity of guar solutions.

Tayal et al. ${ }^{[58]}$ introduced an enzyme package made of two hemicellulases (an $\alpha$-galactosidase and a $\beta$-mannanase), which is stable at high temperatures. They stated that $\beta$-D-mannanase which is the main component of traditional enzyme systems has a half-life time of 5-6 minutes at $85{ }^{\circ} \mathrm{C}$ while their high-temperature enzyme shows better thermostability with half-life time of 530 minutes at the same temperature. Figure 25 is a plot presented by Tayal et al. ${ }^{[58]}$ showing the viscosity reduction as a function of temperature for a commercial enzyme, the hightemperature enzyme and a control system (no enzyme). Unlike the commercial enzyme, the high-temperature enzyme is less active at low temperatures and very active at high temperatures. Gammanase (Novo Nordisk Bioindustrial Inc.) has been used as a preferred and popular enzyme by several researchers for typical $\mathrm{pH}$ ranges of 2-11 and temperature ranges of $50-180^{\circ} \mathrm{F} .{ }^{[20,55-58]}$

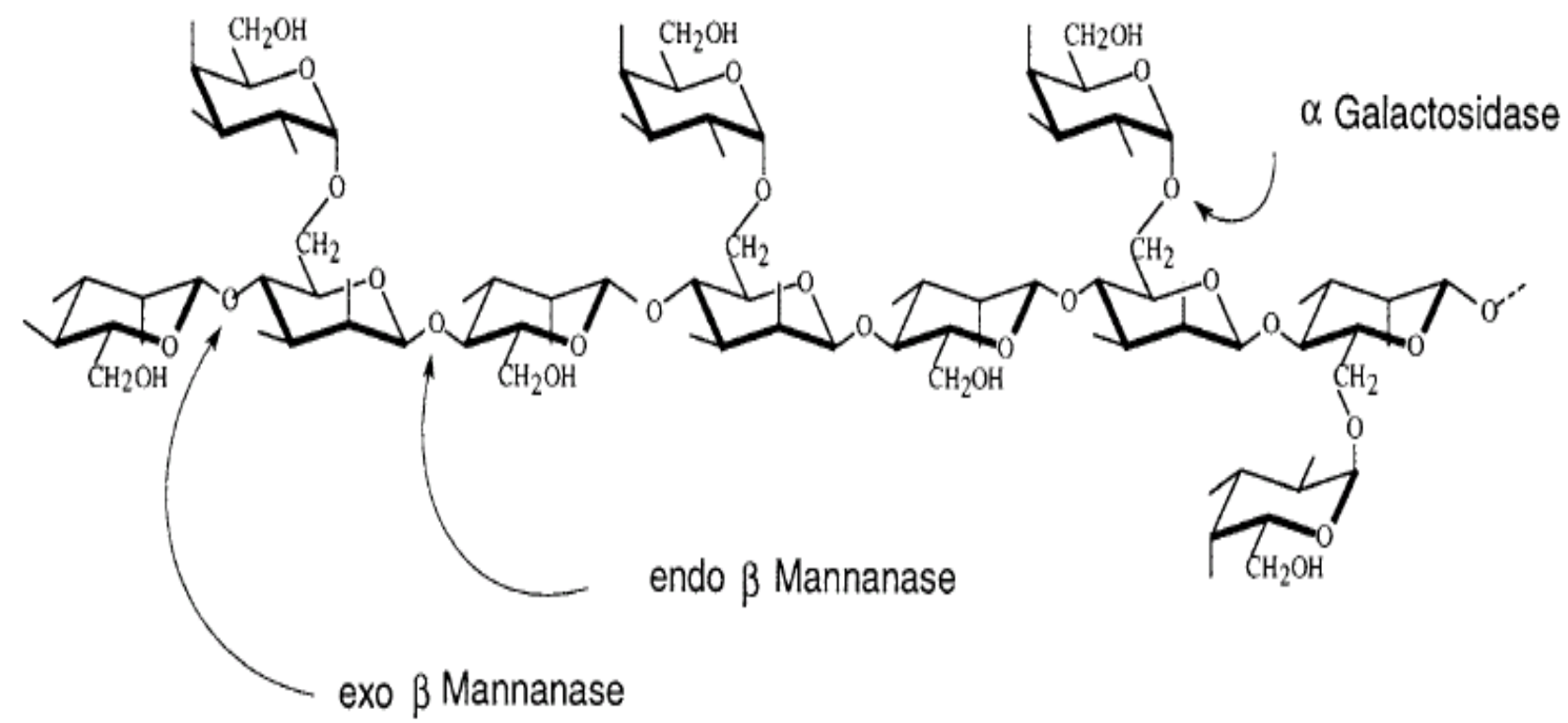

Figure 23 Structure of a guar molecule and different enzymes specified for different bonds (Tayal et al.) ${ }^{[58]}$ 


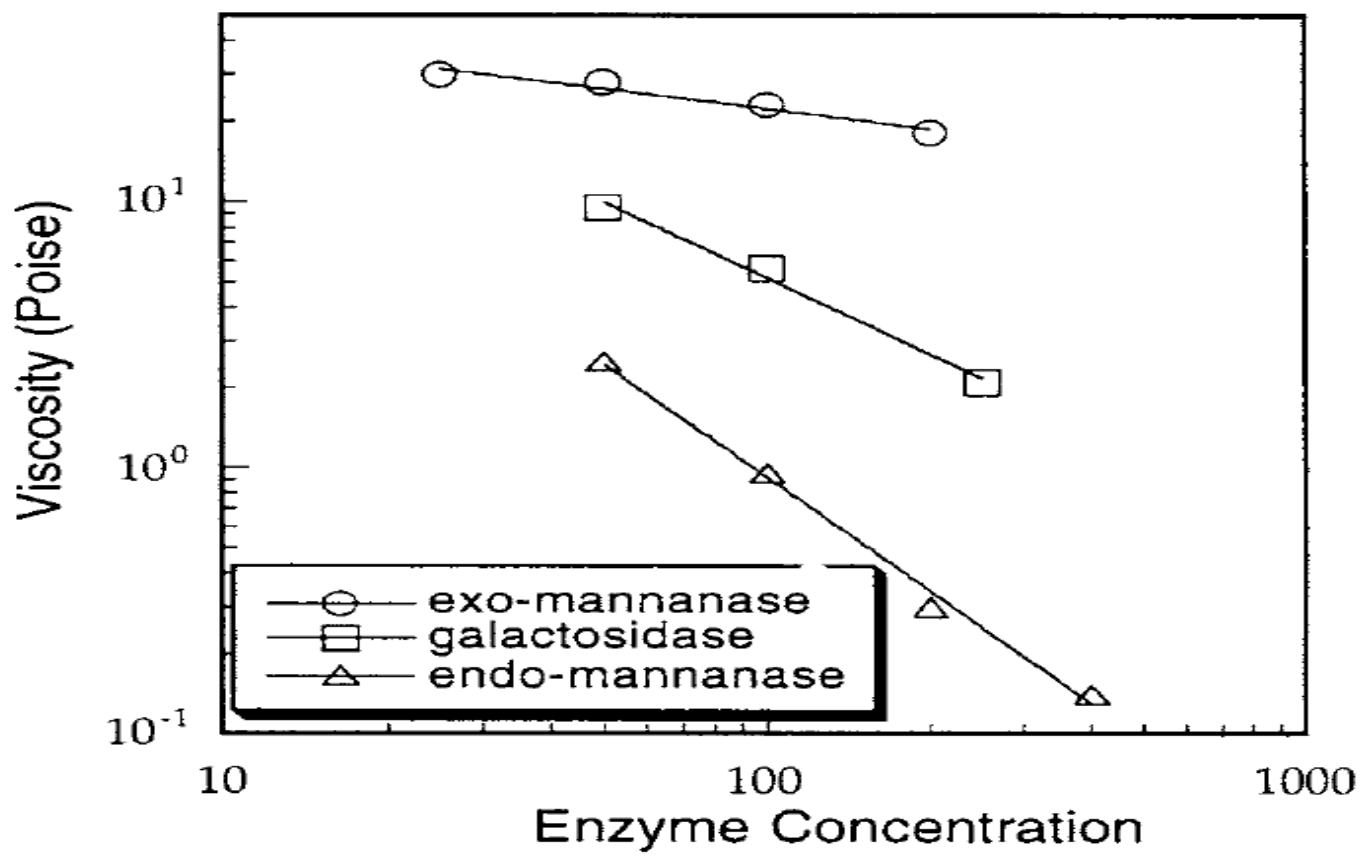

Figure 24 Zero shear viscosity of $0.7 \%$ w/v guar solution as a function of enzyme concentration at $25{ }^{\circ} \mathrm{C}$ and $\mathrm{pH}=5$ (Tayal et al.) ${ }^{[58]}$

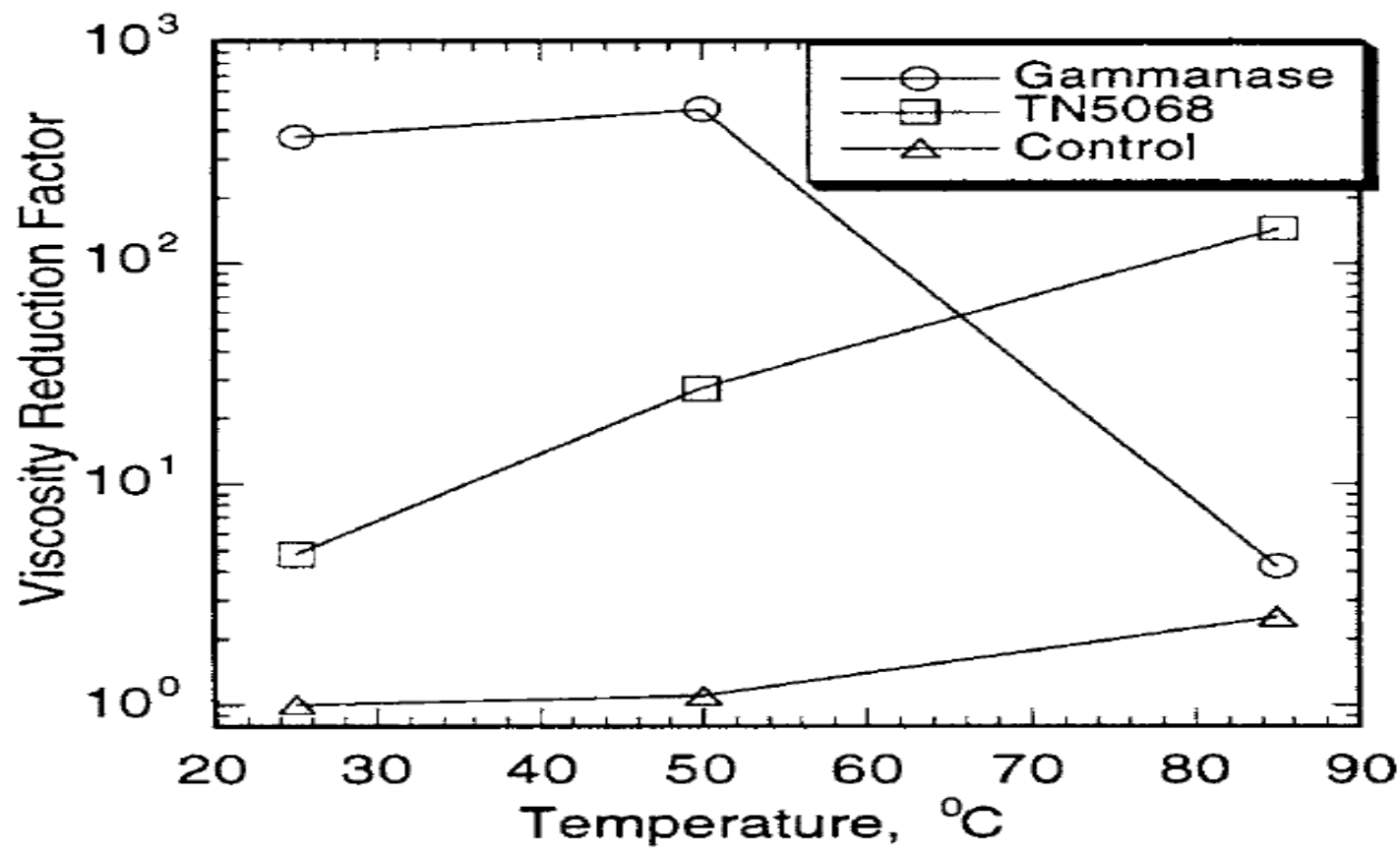

Figure 25 Comparing the viscosity reduction obtained by a commercial enzyme and a high-temperature enzyme (Tayal et al.) ${ }^{[58]}$ 


\subsection{Pre-conditioning the Fracturing Fluids, Considering the Effect of Shear and Temperature on Rheology of Fracturing Fluids during the Injection}

Continuous shear applied to fracturing fluids while traveling through the pipelines at temperatures close to surface temperature and propagating the fracture at temperatures close to reservoir temperature causes changes in the rheology of fracturing fluids. Breakers designed for real fracturing conditions must tolerate these changes in temperature and shear in addition to changes in $\mathrm{pH}$ from surface to reservoir. This is especially important during the design of release mechanisms for breakers in this project. Early release of breakers causes degradation of fluid during the fracturing job, resulting in small fractures, significant leakoff, and sedimented proppants. Late release of breakers or lack of breaker release causes late or poor proppant pack cleanup resulting in significant reduction in cumulative production. ${ }^{[3,6,7,31]}$ High shear values overcoming the attraction force between the polyelectrolytes are theoretically capable of unbinding the nanoparticles and releasing the breaker earlier than the expected time.

Understanding the rheology of fracturing fluids is critical while studying their injection (high shear region) and cleanup (low shear region). Considering the non-Newtonian behavior of fracturing fluids (Equation 16), their rheology data are usually determined under laminar flow conditions using a rotational concentric cylinder viscometer. ${ }^{[1]}$ Equation 16 shows the power law model for non-Newtonian fluids in which apparent viscosity is related to shear rate. $\mathrm{K}^{\prime}$ is the consistency index $\left(\mathrm{kPa} \cdot \mathrm{s}^{\mathrm{n}^{\prime}}\right)$ and $\mathrm{n}^{\prime}$ is the flow behavior index.

$$
\mu_{a}=K^{\prime} \gamma^{n^{\prime}-1}
$$

Equation 16

The highest amount of shear applied to the fluid is while it is traveling through the pipelines. Shear rate decreases significantly in the fracture (Figure 26). Taking a rock segment $50 \mathrm{ft}$ from the well bore, Navarette et al. ${ }^{[59]}$ plotted the shear rate vs. time that this block experiences (Figure 27). Shear rate decreases for a constant position in a fracture since the fracture width is increasing over time. The temperature of the fluid, on the other hand, increases until it reaches the formation temperature (Figure 28). 
Shear has significant effect on shear sensitive crosslinked fracturing fluids. Borate makes reversible crosslinks when added to guar. This reversibility causes shear effects on the gel to be insignificant. ${ }^{[1]}$ This, however, is only true as long as the concentration of guar stays above the overlap concentration $\left(\mathrm{C}^{*}\right)$. Guar concentrations in the range of $20 \mathrm{lbm} / 1000 \mathrm{gal}(2.4 \mathrm{~g} / \mathrm{L})$ are close to $C^{*}$. The guar borate gel is sensitive to shear in this range. ${ }^{[1]}$

Titanate and and zirconate crosslink guar polymers irreversibly. This means that shear degrades such a crosslink easily cutting the gel into small fragments and reducing the viscosity significantly. Even though using delayed crosslink is recommended for such systems to avoid high shear regions (e.g. pipelines) there is still degradation in the low shear region (e.g. fracture). [1]

Craigie $^{[60]}$ presented a shear history simulator consisting of a triplex pump displacing base fluid (polymer solution) from a cylinder using oil, two high pressure syringe pumps and a static mixer to add crosslinking agent and other additives into the fluid. The tubular was simulated using a capillary viscometer in which pressure drop was measured along the capillary tubes. The fluid exiting the capillary viscometer flowed into a concentric cylinder viscometer next to simulate the fracture shear history and temperature. A schematic picture of Craigie's setup is shown in Figure 29. The nominal shear rate in the capillaries was controlled after being calculated using Equation 17. Knowing the length of the capillaries and measuring the differential pressure along the capillaries, the shear stress applied on the fluid was calculated using Equation 18. Tubular shear rates in the range of 300-1400 $\mathrm{sec}^{-1}$ and fracture shear rates in the range of 30-300 $\mathrm{sec}^{-1}$ were applied to simulate different shear histories fracturing fluids experience in a real fracturing job. The time under shear was varied from 1 to 20 minutes. This condition corresponds to pump rates of 3-12 BPM in 2-7/8 in. tubing and 12-50 BPM in 4-1/2 in. casing. The fluid was heated to test temperature in the concentric cylinder viscometer at a rate of 8 . Observing the pressure drop along each of the capillaries, he studied the viscosity changes. The model was then compared with up-scaled model made using coil tubing and satisfactory results were published. Significant impact of shear rate and time at shear on the rheological properties of shear sensitive fracturing fluids were observed. ${ }^{[60]}$

Craigie $^{[60]}$ indicated that in a real fracturing case when a Newtonian fluid is flowing in the volume between casing and tubing in the annular flow regime, pressure drop and shear rate as a result of friction are defined as in Equation 20 and Equation 21. Delaying the crosslinking 
process, either chemically or physically, until it exits the wellbore was recommended in order to prevent high pump rates. ${ }^{[60]}$

$$
\begin{aligned}
& \dot{\gamma}_{w}=\frac{4 Q}{\pi r^{3}} \\
& \tau_{w}=\frac{D \Delta P_{f}}{4 L} \\
& \bar{v}=\frac{Q}{\pi\left(r_{2}^{2}-r_{1}^{2}\right)} \\
& \Delta P_{f}=\frac{12 \mu \bar{v} L}{\left(r_{2}-r_{1}\right)^{2}} \\
& \dot{\gamma}_{w}=\frac{6 \bar{v}}{r_{2}-r_{1}}
\end{aligned}
$$

Navarette et al. ${ }^{[59]}$ studied the effect of shear rate on the dynamic fluid loss behavior of linear and crosslinked guar gels. Assuming that a power law model represents a simple and accurate approximation of gelled fluid in small intervals they used Equation 22 to calculate the shear rate at the walls of a fracture.

$$
\dot{\gamma}_{w f}=\frac{(4+2 / n) Q}{h^{2} W}=\frac{(2+1 / n)}{3 \dot{\gamma}_{a}}
$$

Equation 22

Before studying the dynamic fluid loss process, Navarette et al. ${ }^{[59]}$ preconditioned the fluid using a Tubing Shear History Simulator (TSHS) and a Fracture Shear History Simulator (FSHS).

Their TSHS consists of a $1 / 4$ in. static mixer, and $\sim 800 \mathrm{ft}$ of $1 / 4$ in. stainless steel (SS) 316 tubing (ID=0.194 in.) with a tubing capacity of $5.73 \mathrm{~mL} / \mathrm{ft}$. The crosslinker was injected in line before the static mixer. They reported the nominal shear rate at $1120 \mathrm{~mL} / \mathrm{min}$ to be $380 \mathrm{~s}^{-1}$. They measured the pressure drop across the TSHS using a differential pressure transducer. Their 
fracture simulator consists of two $2700 \mathrm{~mL}$ floating piston accumulators and two $32 \mathrm{ft}$ coils of $3 / 8$ in. SS tubing (ID=0.31 in. equivalent to tubing capacity of $14.8 \mathrm{~mL} / \mathrm{ft}$ ) submerged in a temperature controlled oil bath. Changing the pump rate from 110 to $1120 \mathrm{~mL} / \mathrm{min}$ the shear rate in their fracture simulator changed from 40 to $380 \mathrm{~s}^{-1}$. Using an automated switching valve Navarette et al. ${ }^{[59]}$ reciprocated the fracturing fluid from one accumulator and its tubing set to the other one by alternatively pressurizing the backside of the fracturing fluid using oil. They located their fluid loss cell in between (Figure 30). They designed their system in a way that they could bypass any of the aforementioned shear histories by switching valves (Figure 30).

McGowen \& Vittha ${ }^{[61,62]}$ preconditioned the fracturing fluid while testing through fluid loss and API conductivity cells by placing a high-shear flow loop (HSFL) at room temperature, simulating tubing shear conditions, and two low-shear flow loops (LSFL), simulating the shear fracturing fluid is exposed to in the fracture before reaching a certain point which is represented by the core. Figure 31 shows a picture of the general setup including the shear loops. Shear loops were placed immediately after a static mixer which was used to crosslink the fluid uniformly. The static mixer was placed after the high pressure pump and crosslinker injection point. Fluid leaving the static mixer enters HSFL which is a $1 / 4$ in. diameter stainless steel (SS) tubing at ambient temperature with the length varied between $140 \mathrm{ft}, 340 \mathrm{ft}, 540 \mathrm{ft}, 740 \mathrm{ft}$, and $940 \mathrm{ft}$ which represents the residence times between 0.6 and 4.1 minutes at $1.31 \mathrm{~L} / \mathrm{min}\left(1900 \mathrm{~s}^{-1}\right)$. The LSFL, which is a loop of $1 / 2$ in. or $1 / 4$ in., is located immediately after the HSFL immersed within a large 400-gal oil bath simulating the rapid shear and temperature changes applied to the fracturing fluid after reaching the fracture. Valving was used to change the length of $1 / 2$ in. diameter loop between $39 \mathrm{ft}, 78 \mathrm{ft}, 155 \mathrm{ft}$, and $311 \mathrm{ft}$ and the length of $3 / 4$ in. loop between $30 \mathrm{ft}$, $60 \mathrm{ft}, 90 \mathrm{ft}$, or $120 \mathrm{ft}$. resulting in available residence times at $56 \mathrm{~s}^{-1}$ of $2.7-22$ minutes for the $1 / 2$ in. diameter loop and 1.4-5.5 minutes for the $3 / 4$ in. loop. A positive displacement pump after the HSFL reduced the rates if needed.

Comparing the static and dynamic fluid loss data of pre-conditioned fracturing fluids, McGowen \& Vitthal ${ }^{[61,62]}$ indicated no significant effect of fluid pre-conditioning on total fluid loss volume under static conditions for HPG and Borate crosslinked HPG fluids. Higher spurt loss volumes were observed in the static case though. They indicated significant changes in the dynamic fluid loss coefficient when changing the LSFL and temperature of preconditioning for one of their crosslinked shear sensitive fracturing fluids. 


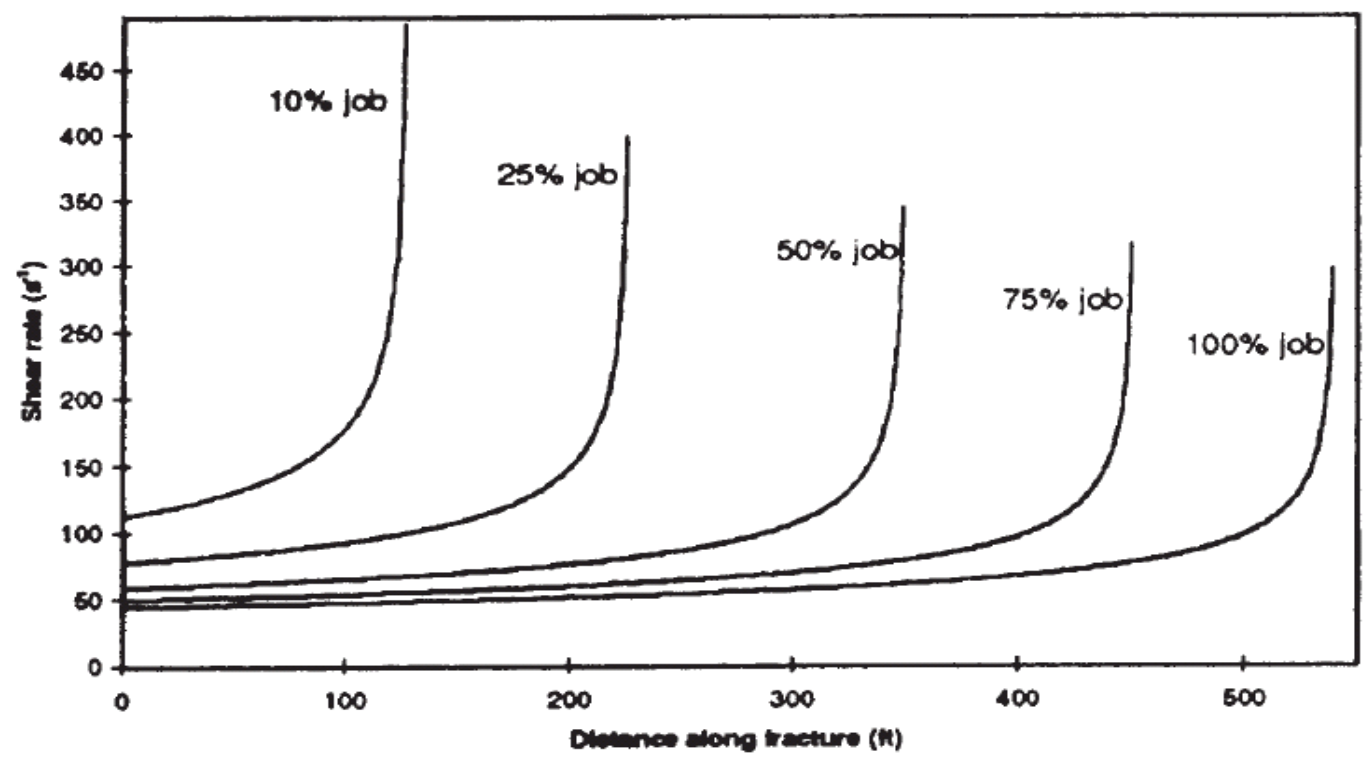

Figure 26 Shear rate profile during a hydraulic fracturing job, fracture height $=300 \mathrm{ft}$, injection rate $=40$ $\mathrm{BBL} / \mathrm{min}$, pump time $=145 \mathrm{~min}^{[59]}$

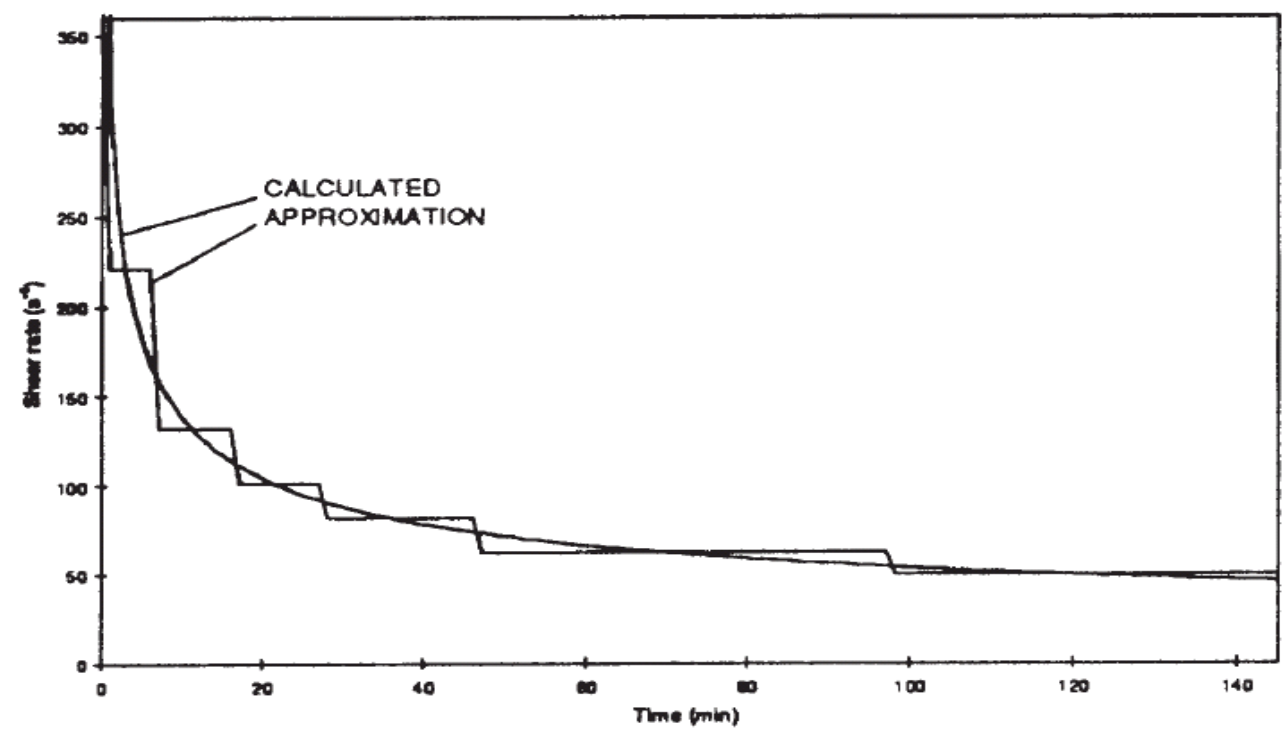

Figure 27 Shear rate history over a rock segment $50 \mathrm{ft}$ away from the wellbore ${ }^{[59]}$ 


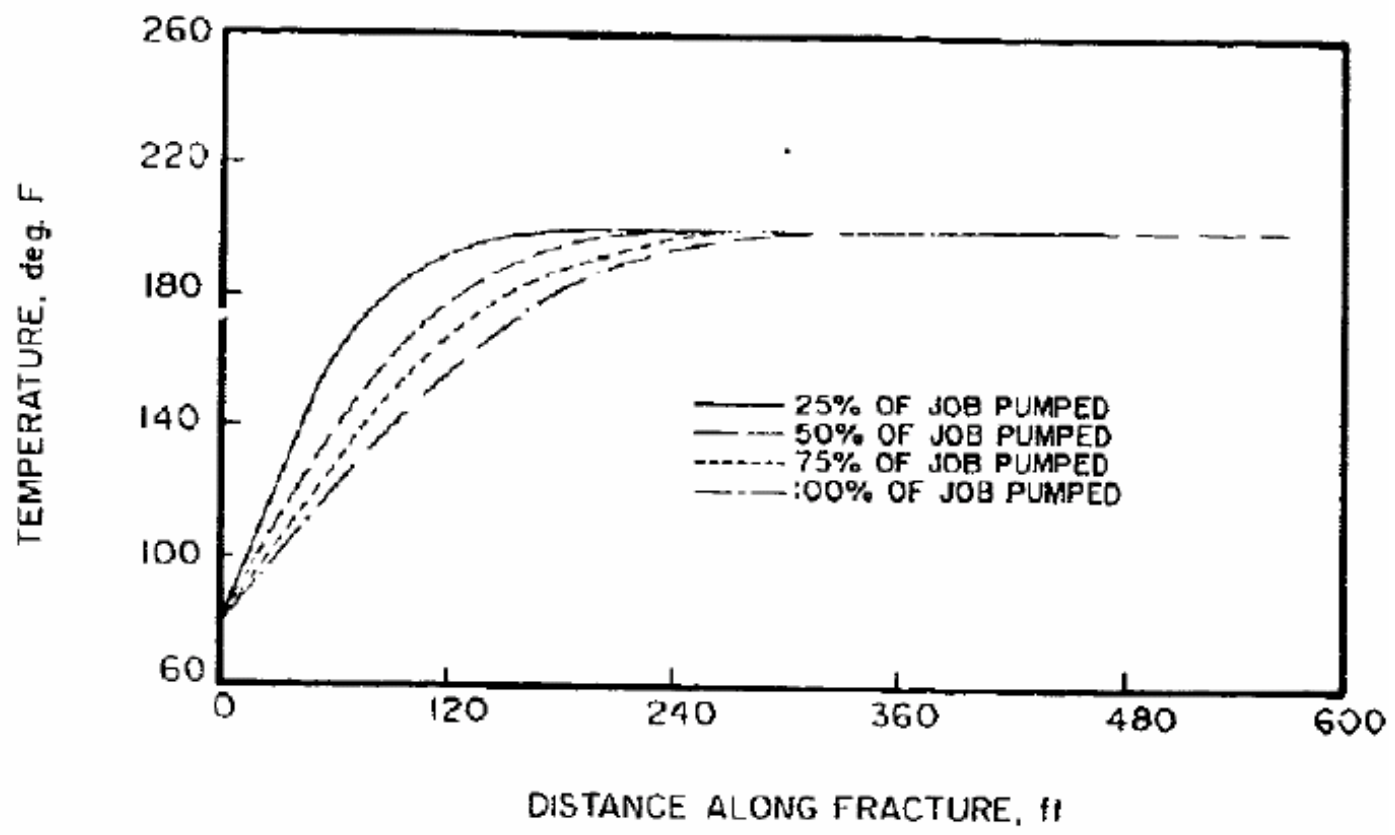

Figure 28 Temperature profile during a hydraulic fracturing job ${ }^{[63]}$

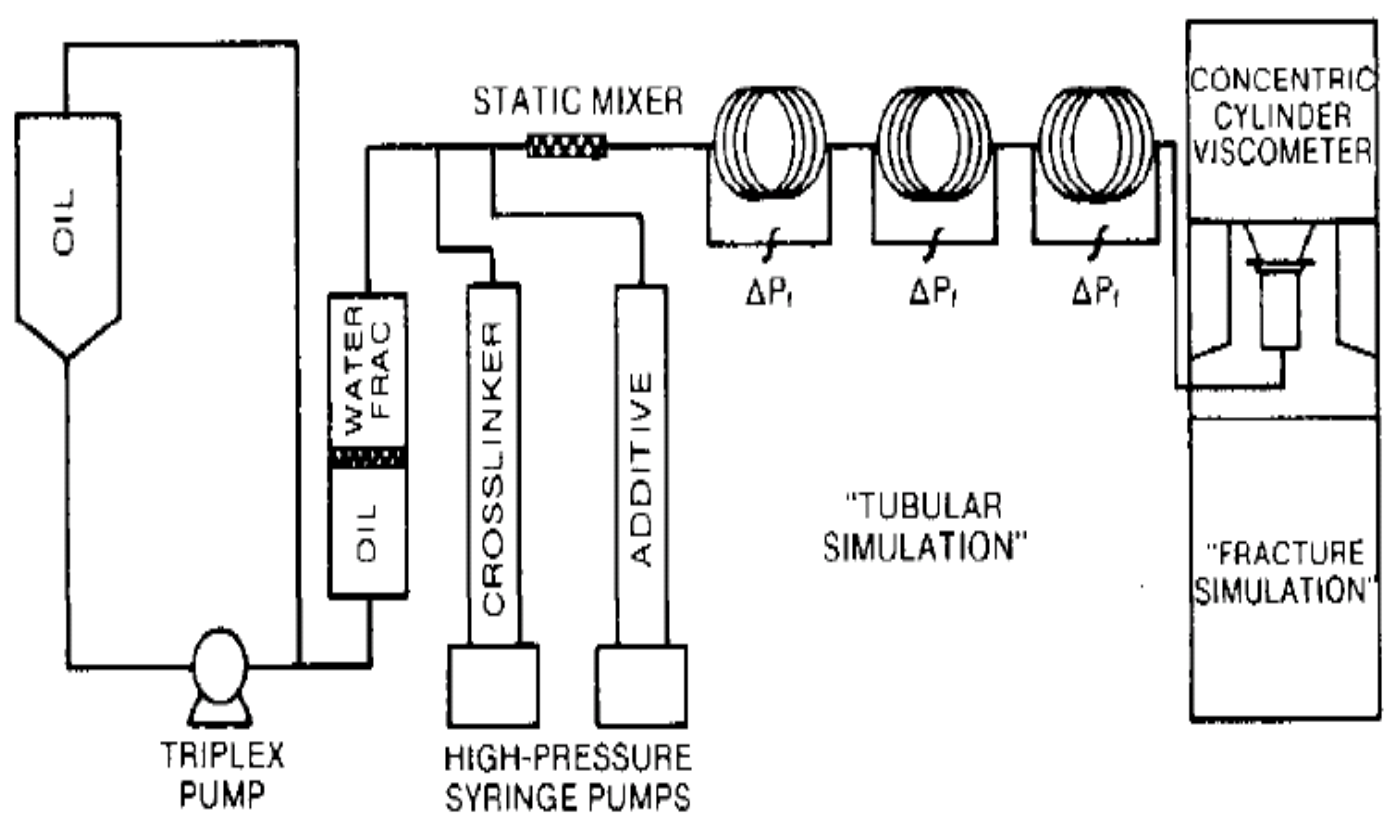

Figure 29 Shear history simulator of Craigie ${ }^{[60]}$ 


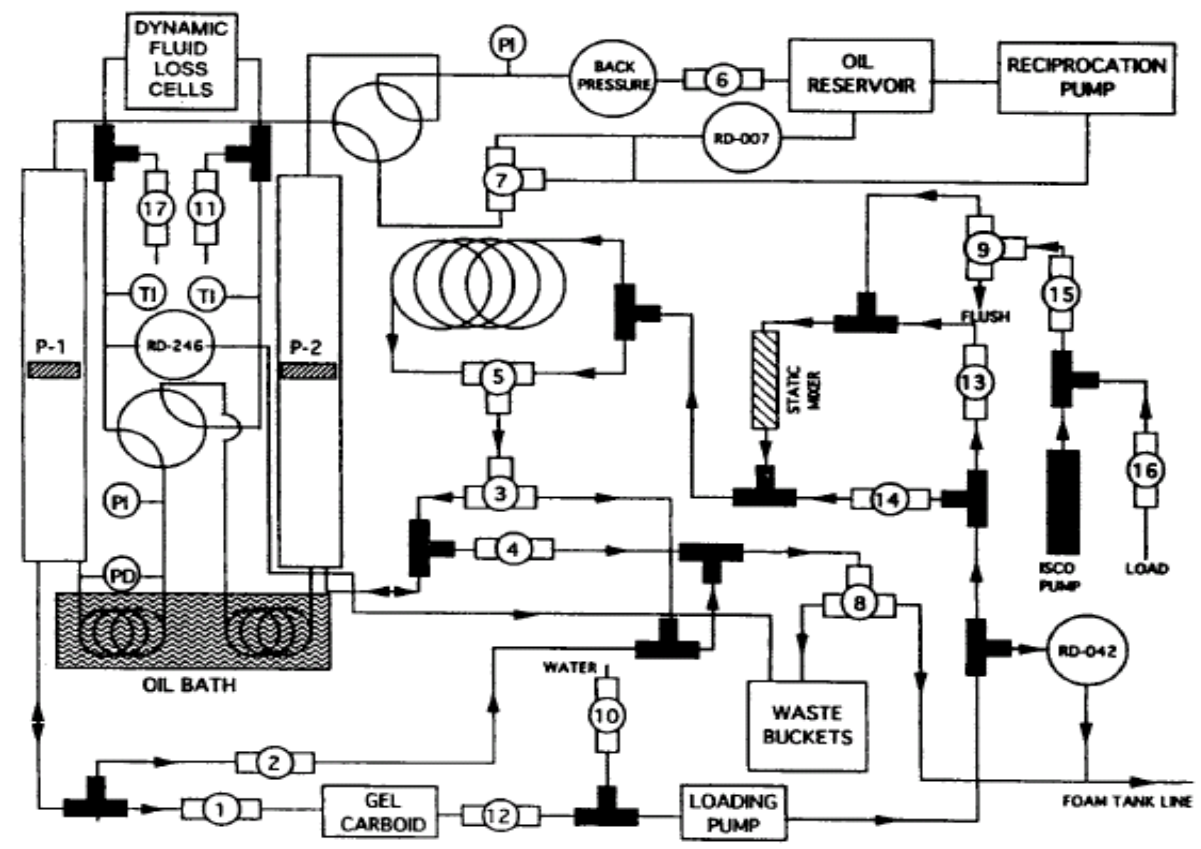

Figure 30 Flow diagram of reciprocating Schlumberger Dowell dynamic fluid loss cell published by Navarette et al. ${ }^{[59]}$

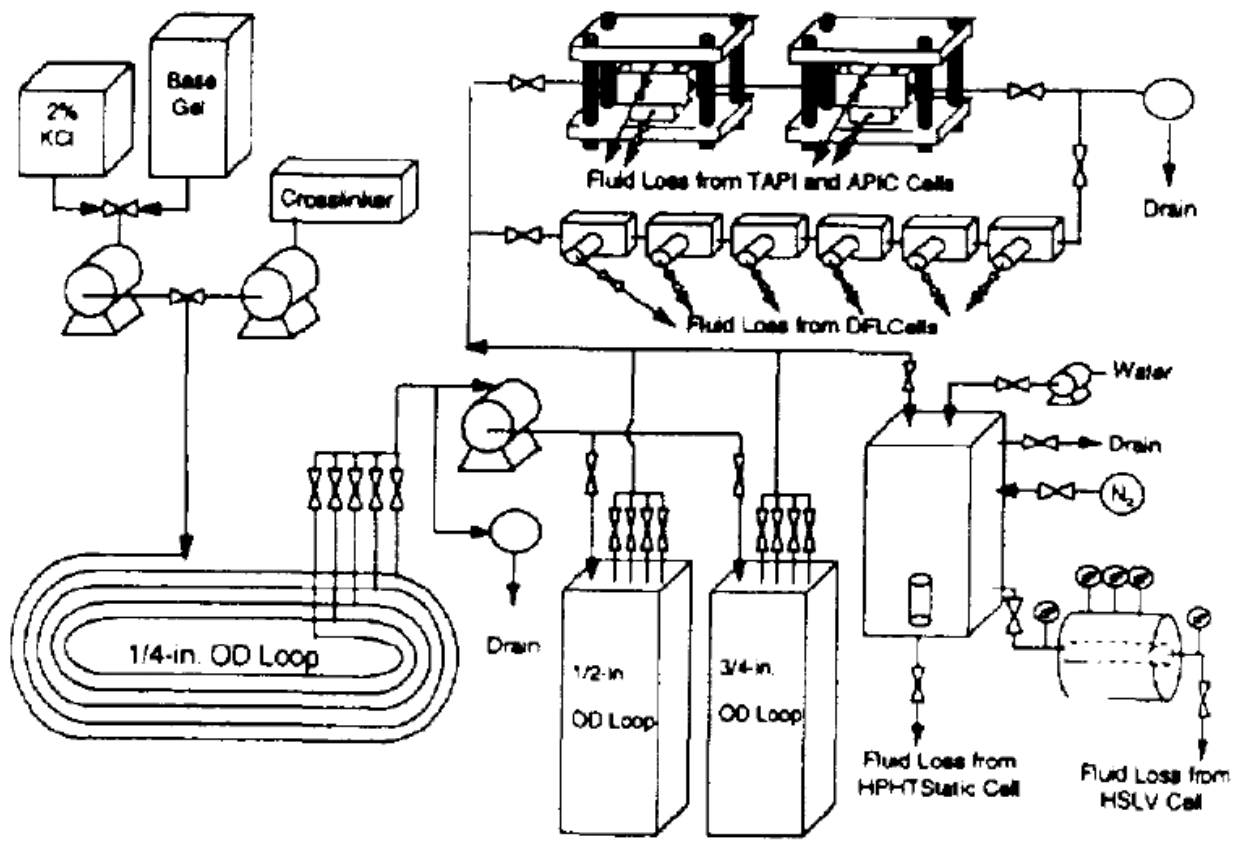

Figure 31 Schematic picture of the laboratory setup used by McGowen and Vitthal ${ }^{[61]}$ 


\subsection{Fluid Loss during the Formation of Filter Cake}

\subsubsection{Classical Carter Model for Filtration}

Filtration of fluids on the surface of formation rock causes invasion of filtrate from fracturing fluids into the reservoir while generating filter cake. Carter ${ }^{[64]}$ presented a linear relation between the leakoff volume and the square root of exposure time of rock to fracturing fluid. Howard and Fast $^{[64]}$ published Equation 23 based on Carter's derivation ${ }^{[64]}$ calculating the total area of one face of the fracture in which $\mathrm{C}$ is the total fluid loss constant.

$A=\left[Q_{i} W / 4 \pi C^{2}\right]\left[e^{x} \bullet \operatorname{erfc}(x)\right]$

Equation 23

Where, $x=2 C \sqrt{\pi t} / W$, A is the total area of one face of fracture $\left(\mathrm{ft}^{2}\right), \mathrm{Q}_{\mathrm{i}}$ is the injection rate $\left(\mathrm{ft}^{3} / \mathrm{min}\right), \mathrm{W}$ is the constant fracture width ( $\mathrm{ft}$ ), and $\mathrm{t}$ is the total pumping time ( $\mathrm{min}$ ).

Carter ${ }^{[64]}$ presented Equation 24 as a representative of the leakoff velocity, $u_{L}$, at a point on the fracture wall.

$u_{L}=\frac{C}{\sqrt{t-t_{S p}}}$

Equation 24

Three types of linear flow mechanisms were indicated by Howard and Fast ${ }^{[64]}$ for which Equation 24 is applicable:

1. Viscosity and relative permeability effects.

2. Viscosity and compressibility effects of reservoir fluid.

3. Wall building effects.

Williams ${ }^{[65]}$ and Settari ${ }^{[66]}$ defined Equation 24 for three different time periods during the leakoff:

1. Displacement and compressibility of reservoir fluid (initial period): leakoff is quick during this time and filter cake has not formed yet. Fluid leakoff is controlled by the formation resistance to flow (Spurt time). 
2. Invasion of the formation by filtrate or fracturing fluid (decreasing leakoff rate): the external filter cake builds during this period

3. Buildup of filter cakes either externally, in low permeability rocks, or internally, in rocks with higher permeability. The filter cake stops buildingbecause the high velocity fluid in the fracture prevents further polymer deposition (dynamic leakoff).

Filter Cake: At a constant pressure, the rate of filtration is proportional to the square root of time assuming that: ${ }^{[1,30]}$

- The amount of cake deposited is proportional to the volume of fluid $\mathrm{V}_{\mathrm{L}}$ passed through a unit surface area $\left(V_{L}=\alpha L_{\text {cake }}\right)$.

- Cake permeability is independent of its thickness.

- Pressure drop across the cake is constant.

$\begin{array}{lr}u_{L}=\frac{d V_{L}}{d t}=\frac{k_{\text {cake }}}{\mu_{\text {fil }}} \frac{\alpha \Delta p_{\text {cake }}}{V_{L}} & \text { Equation } 25\end{array}$

By integrating for $\mathrm{V}_{\mathrm{L}}$ :

$u_{L}=\frac{C_{w}}{\sqrt{t}}$

Equation 26

where the fluid loss coefficient through the wall filter cake is:

$C_{w}=\sqrt{\frac{k_{\text {cake }} \alpha \Delta p_{\text {cake }}}{2 \mu_{\text {fil }}}}$

Equation 27

and $\mu_{\text {fil }}$ is the filtrate viscosity.

Carter $^{[64]}$ determined fluid loss volume by integrating Equation 26 as:

$V_{L}=2 C_{w} \sqrt{t}+S_{p}$

Equation 28 
Where $\mathrm{S}_{\mathrm{p}}$ is the volume leaked off without forming a filter cake and can be interpreted as integration constant.

A more accurate form of Carter's equation ${ }^{[62]}$ can be written as:

$V_{L}=2 C_{w} \sqrt{t-t_{S p}}+S_{p}$

Equation 29

Pressurizing the polymer solution against a porous media one might be able to measure the wall building coefficient $\left(\mathrm{C}_{\mathrm{w}}\right)$ by plotting the filtrate volume vs. $\sqrt{t}$ using Equation 30.

$$
C_{w}=\frac{m}{A}
$$

Equation 30

where $\mathrm{m}$ is the slope of volume vs. $\mathrm{t}^{1 / 2}$ plot $\left(\mathrm{ft}^{3} / \mathrm{min}^{1 / 2}\right)$ and $\mathrm{A}$ is the area of core used in laboratory test $\left(\mathrm{ft}^{2}\right)$. The spurt volume can be calculated in Equation 28 and Equation 29 thus:

$t_{S p}=\left(\frac{V_{s p}}{2 C_{v c}}\right)$

Equation 31

In which $\mathrm{C}_{\mathrm{vc}}$ is the overall reservoir resistance coefficient defined during the spurt time (no filter cake) as in Equation 32. Figure 32 presents a typical fluid loss curve based on the classical Carter model.

$C_{v c}=\frac{2 C_{v} C_{c}}{C_{v}+\left(C_{v}^{2}+C_{c}^{2}\right)^{0.5}}$

Equation 32 


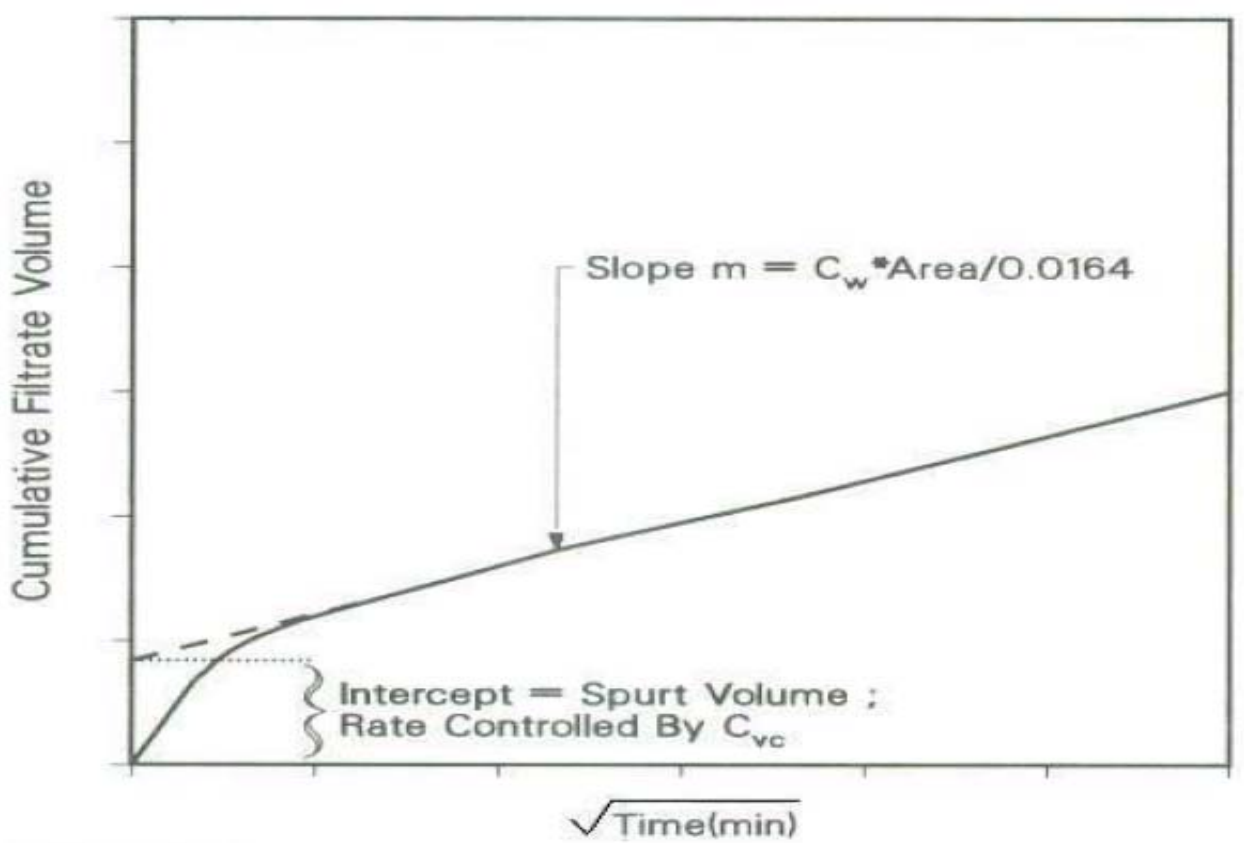

Figure 32 Classical Carter filtration model (Glenn, and Penny) ${ }^{[30]}$

Filtrate Zone: Is the zone invaded by the filtrate from fracturing fluids assuming that: ${ }^{[1,64,65]}$

- Pressure drop across the zone is constant

- Filtrate fully displaces the mobile phase(s) within the formation resulting in piston-like displacement and $100 \%$ filtrate saturation

- The fluid and rock are incompressible

$u_{L}=\frac{d V_{L}}{d t}=\frac{k_{f i l}}{\mu_{f i l}} \frac{\Delta p_{v}}{V_{v}}$

Equation 33

Where $k_{f i l}$ is the permeability related to the filtrate and $\mathrm{L}_{\mathrm{v}}$ is the length of the invaded zone.

Integrating Equation 33, $L_{v}=V_{L} / \phi=\frac{1}{\phi} \int u_{L} d t$, the leakoff velocity is represented as:

$$
u_{L}=\frac{C_{v}}{\sqrt{t}}
$$

Equation 34

Where: 
$C_{v}=\sqrt{\frac{k_{f i} \phi \Delta p_{v}}{2 \mu_{f i l}}}$

Equation 35

and the leakoff volume at any time is:

$V_{L}=2 C_{v} \sqrt{t}$

Equation 36

$\mathrm{k}_{\mathrm{fil}}$ reflects the relative permeability of the formation to flow of the filtrate in Equation 35

Reservoir Zone: Pressure is required to displace the reservoir fluid away from the fracture face. Assuming: ${ }^{[1,64,65]}$

- Constant $\Delta p_{c}$ between the filtrate/reservoir interface and the far-field reservoir

- Compressible fluid with constant total compressibility $\mathrm{c}_{\mathrm{t}}$

- Relatively slow movement of the front of the invading zone

- An infinite reservoir

Analytical solution of Collins ${ }^{[67]}$ is presented for the leakoff velocity inside the reservoir as:

$u_{L}=\frac{C_{c}}{\sqrt{t}}$

Equation 37

Where the compressibility control leakoff coefficient is:

$C_{c}=\sqrt{\frac{k_{r} c_{t} \phi}{\pi \mu_{r}}} \Delta p_{c}$

Equation 38

Combined Coefficients: In practice all these mechanisms occur simultaneously. Velocities in filtrate zone and filter cake must be equal and the sum of the pressure drops must be equal to the total pressure drop between the reservoir pressure and the fracturing pressure: 


$$
\begin{aligned}
& \frac{C_{t}}{\sqrt{t}}=\sqrt{\frac{k_{\text {cake }} \alpha \Delta p_{\text {cake }}}{2 \mu_{\text {fil }}\left(t-t_{\text {sp }}\right)}}=\sqrt{\frac{k_{\text {fil }} \phi \Delta p_{v}}{2 \mu_{\text {fil }} t}}=\sqrt{\frac{k_{r} \phi c_{t}}{\pi \mu_{r} t}} \Delta p_{c} \\
& \Delta p_{\text {total }}=\Delta p_{\text {cake }}+\Delta p_{v}+\Delta p_{c}
\end{aligned}
$$

Equation 39

Equation 40

Assuming that spurt time and volume can be neglected, Williams ${ }^{[65]}$ yielded the total leakoff coefficient as:

$$
C_{t}=C_{w c v}=\frac{2 C_{c} C_{v} C_{w}}{C_{v} C_{w}+\sqrt{C_{w}^{2} C_{v}^{2}+4 C_{c}^{2}\left(C_{v}^{2}+C_{w}^{2}\right)}}
$$

This assumption is only valid if the cake permeability is independent of pressure.

\subsubsection{Static Fluid Loss Tests}

The formation of filter cake in a static manner has been studied by filtering the solution while it is flowing perpendicular to the rock surface. In static fluid loss tests, after placing the premixed gelled fluid with additives, fluid temperature is typically raised to a specific temperature, then a differential pressure is applied across the core. ${ }^{[1]}$ Differential pressure is usually supplied by $\mathrm{N}_{2}$. Leakoff volume is monitored vs. time after differential pressure is applied. A plot of filtrate volume vs. $\mathrm{t}^{1 / 2}$ gives enough information to model the filtration process. The test is typically terminated when steady state leak-off rate is achieved. However, longer times can be applied for treatments with long pump times. ${ }^{[30]}$ In order to minimize the variations in results Glenn and Penny ${ }^{[30]}$ recommended to pay enough attention to mix the fluids based on a correct and repeatable procedure, apply the differential pressure after the temperature is stable and just before opening the leakoff valve and keeping the core length constant for different tests. A schematic picture of a static fluid loss cell, published by Constien et al. ${ }^{[1]}$ is shown in Figure 33.

Permeability and thickness of the core must be chosen in a way that more than $95 \%$ of the pressure differential gets adsorbed by the filter cake and not by the core. ${ }^{[30,62]}$ For example a one inch core which generates reasonable results for medium permeability values causes increase in the slope of fluid loss curve at early times for low permeability cores resulting in negative 
calculated spurt volumes. McGowen and Vitthal ${ }^{[62]}$, stating that $\mathrm{C}_{\mathrm{w}}$ should not be changing significantly with core permeability, published the following equation to calculate the minimum core thickness required for a fluid loss test.

$T_{\min }=1.6 \frac{S_{p}}{\phi} R_{p}$

Equation 42

In which $R_{p}$ is the retention coefficient defined for the filtrate over the specified rock type with values between 0.5 and 1 . This equation basically states that spurt loss values greater than the pore volume of the core may be overestimated.

Using filter paper instead of core is not recommended, however, three pieces of Whatman 50 hardened filter papers is recommended for pressure differential of $1000 \mathrm{psi}$ if one chooses to use papers. ${ }^{[62]}$

API recommended practices (RP) $39^{[68]}$ was used extensively as a standard procedure for running static fluid loss tests. The type of core or filter cell had not been standardized in API RP 39. Modified Baroid high pressure high temperature cell (Figure 34) with a core size of 15/16 $\times 1$ in. and Halliburton cell (Figure 35) that accepts cores with $3 / 4$ in. or $15 / 16$ in. diameter and up to 4 in. long were used instead.

McGowen and Vitthal ${ }^{[61,62]}$ used two types of static fluid loss cells during their studies: 1) High pressure, high temperature static fluid loss cell of Halliburton shown in Figure 36, and 2) Hassler sleeve static fluid loss cell

Asadi et al. ${ }^{[69]}$ provided a standard procedure for measuring fluid loss of fluids used in stimulation and gravel packing under static conditions. After giving guidelines on similarity of procedure for preparing the base fluid, $\mathrm{pH}$, type of containers and mixers, time of mixing, and additives added to the fluid in order to reach repeatability, they presented two typical static fluid loss apparatus with $175 \mathrm{~mL}$ and $500 \mathrm{~mL}$ capacities capable of using filter paper, synthetic or natural core as porous medium. A schematic picture of their apparatus is shown in Figure 37. In Figure 37 the numbered sections are as follows:

1. pressurizing valve

2. fluid-loss cell

3. heating source 
4. sample fluid

5. porous medium

6. filtrate valve

7. back pressure receiver, optional

8. filtrate collector

Figure 38 shows the disassembled schematic of $175 \mathrm{~mL}$ cell presented by Asadi et al. ${ }^{[69]}$ The numbered parts are listed as follows:

1. O-ring seal

2. stem/valve

3. top cap

4. O-ring seal

5. backup ring

6. cell body

7. set screw

8. filter-paper (three 8 micron cellulosic papers) assembly or synthetic core $\left(2.5^{\prime \prime} \times 0.25^{\prime \prime}\right)$ ceramic disk.

9. bottom cap

10. seal mechanism

11. natural core $\left(1^{\prime \prime} \times 1^{\prime \prime}\right)$

Cores, synthetic or natural, are recommended to be pre-saturated with the base fluid or synthetic formation fluid $\left(2 \% \mathrm{KCl}\right.$ or $\left.4 \% \mathrm{NH}_{4} \mathrm{Cl}\right)$. 


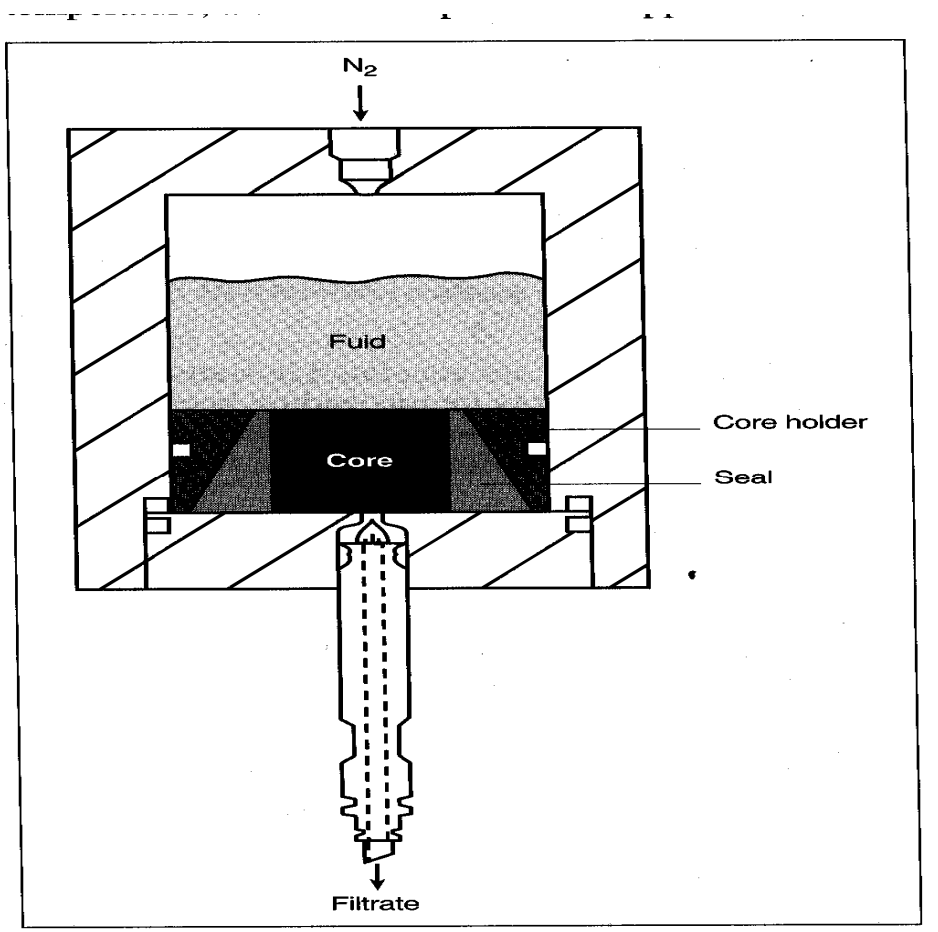

Figure 33 Schematic picture of a static fluid loss cell (Constien et al.) ${ }^{[1]}$

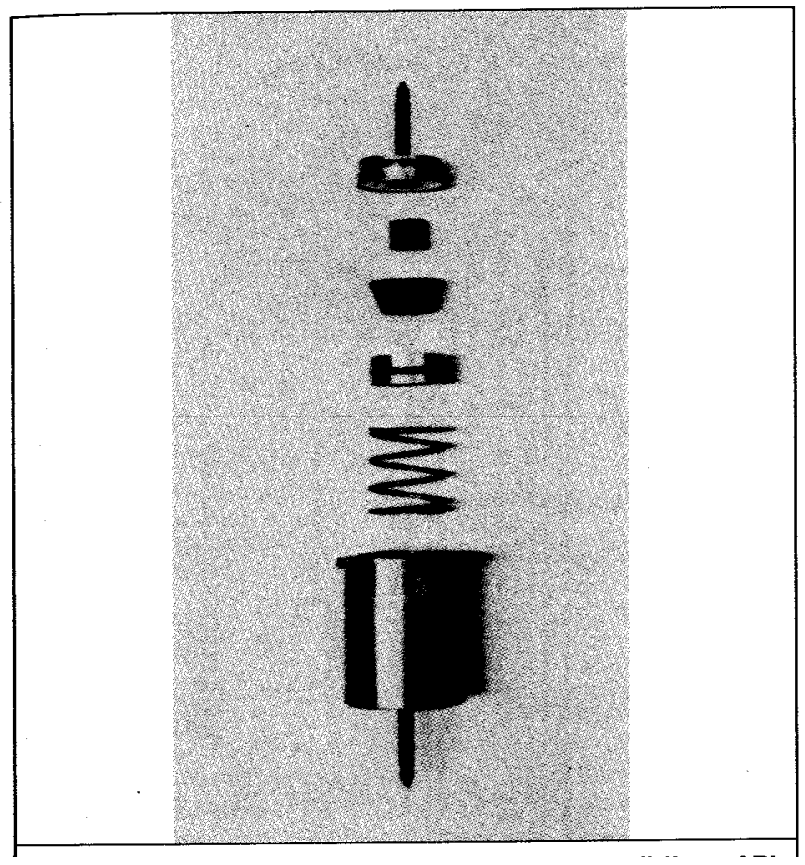

Figure 34 Modified Baroid high-pressure static fluid loss cell (From API RP 39) ${ }^{[68]}$ 


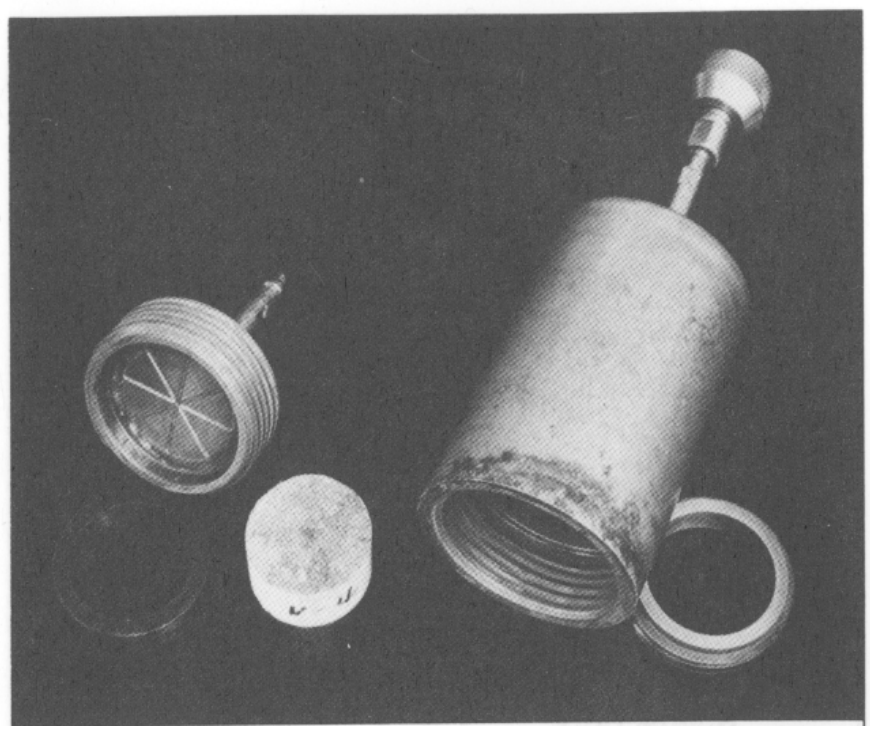

Figure 35 Halliburton high pressure static fluid loss cell (From API RP 39) ${ }^{[68]}$

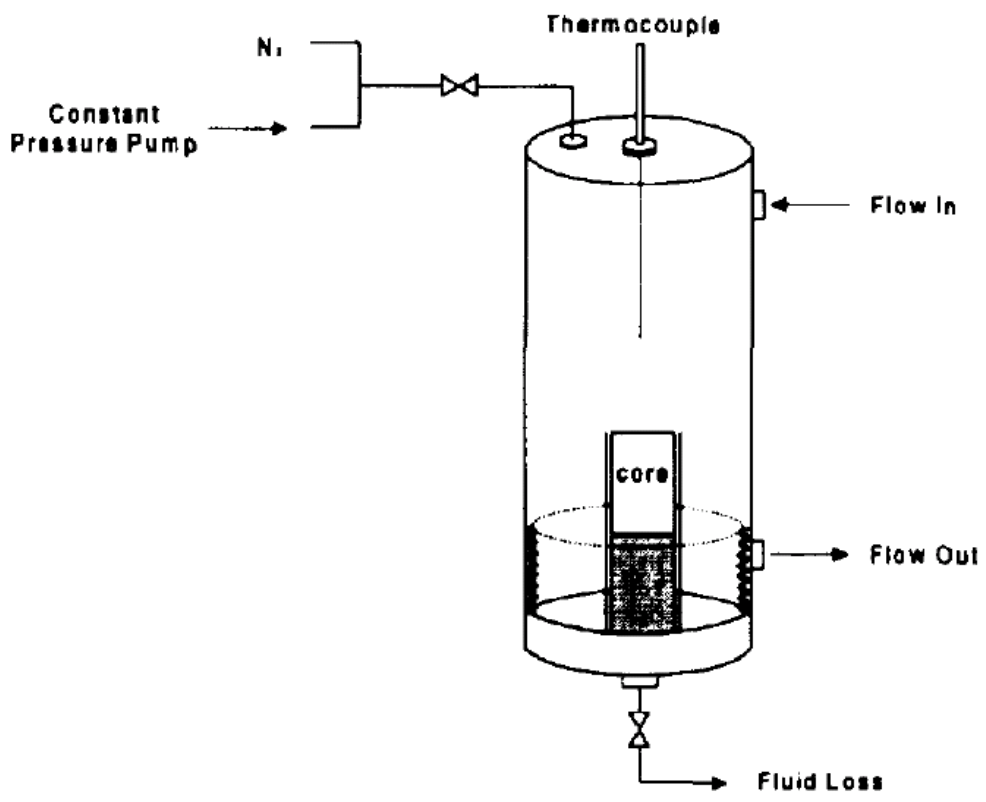

Figure 36 Schematic figure of high pressure high temperature cell used by McGowen and Vitthal ${ }^{[61,62]}$ 


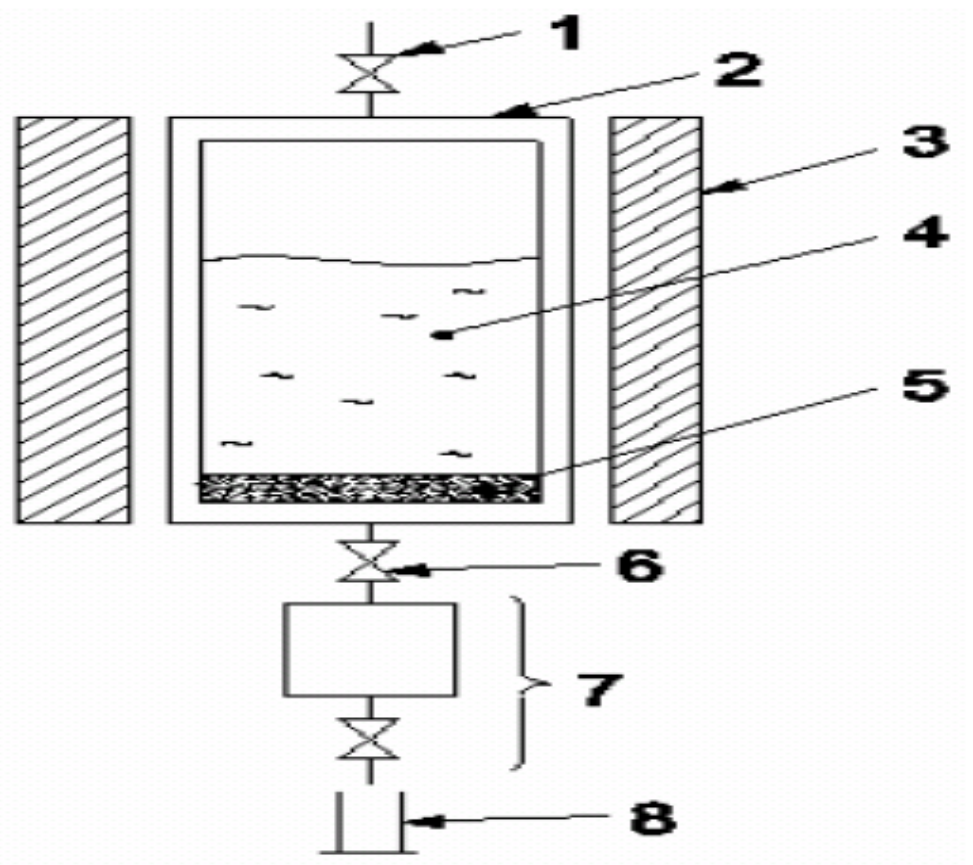

Figure 37 Schematic picture of static fluid loss cell proposed by Asadi et al. ${ }^{[69]}$ See page 64 for key.

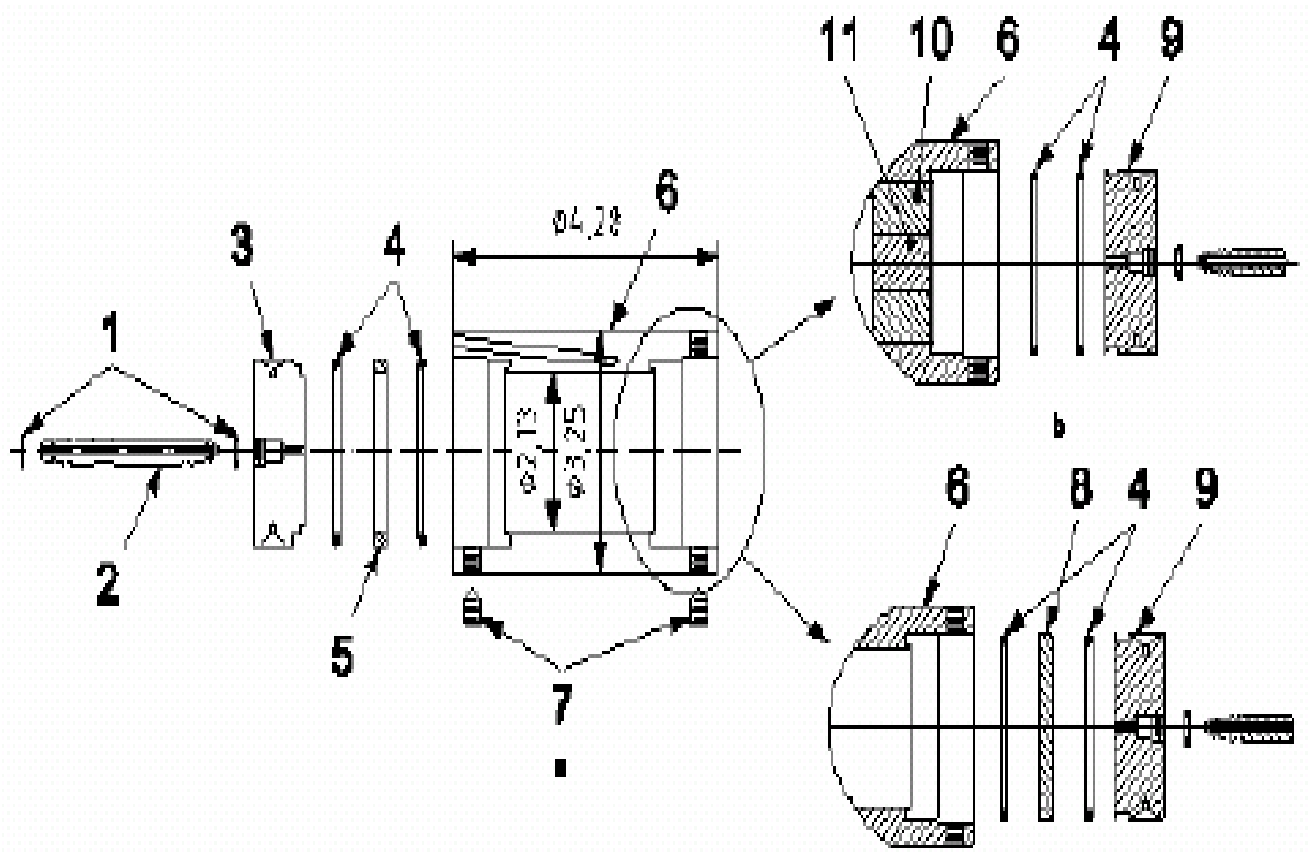

Figure 38 Schematic of a disassembled $175 \mathrm{~mL}$ static fluid loss cell presented by Asadi et al. ${ }^{[69]}$ See page 64 for key. 


\subsubsection{Dynamic Fluid Loss}

Effects of shear rate on the viscosity and formation of filter cake are not considered in static fluid loss tests. Roodhart, ${ }^{[70]}$ Navarette et al. ${ }^{[59]}$ and McGowen et al. ${ }^{[61,62]}$ demonstrated three stages for filter-cake buildup during a fracturing job when fracturing fluid flows perpendicular to the direction in which filter cake forms:

1. Initiation of the filter cake (spurt loss).

2. Filter cake buildup: leakoff volume is proportional to the square root of time.

3. Filter cake growth limitation by erosion: Filter cake thickness is controlled by the equilibrium between the shear stress caused by fluid flowing along the fracture and the pressure drop across the cake (Figure 39). Filter cake thickness stays constant after this equilibrium and filtrate rate simply follows Darcy's law.

Prud'homme and Wang ${ }^{[71]}$ presented the above-mentioned phenomena mathematically as follows:

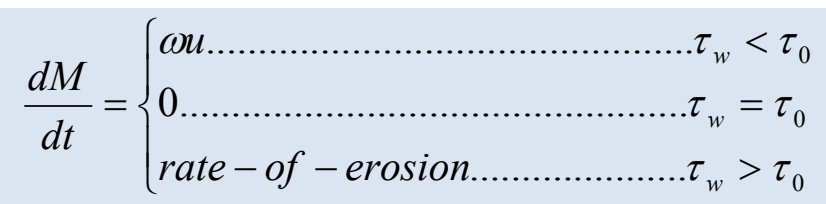

In which:

$\omega=$ Mass fraction of solids in the fluid phase

$\mathrm{M}=$ Mass over area of the filter cake

$\tau_{0}=$ Yield stress of filter cake

$\mathrm{u}=$ Velocity of solvent through the filter cake

The first stage listed above is simulated by the static fluid loss experiments. However, the third stage caused by flow of fracturing fluids along the filter cake can only be modeled under dynamic conditions. The equilibrium time reportedly occurs earlier for higher shears and fluid viscosities. $^{[61]}$

Roodhart $^{[70]}$ and McGowen et al. ${ }^{[61,62]}$ modeled the dynamic fluid loss process as in Equation 44 
with two different fluid loss coefficients after the spurt time. The static fluid loss coefficient models the formation of filter cake and the dynamic fluid loss coefficient models the fluid loss during the time after equilibrium.

$V=V_{s p}+\left.2 C_{w} \sqrt{t}\right|_{t_{s p}} ^{t_{e}}+\left.v_{n} t\right|_{t_{e}} ^{\infty}$

Equation 44

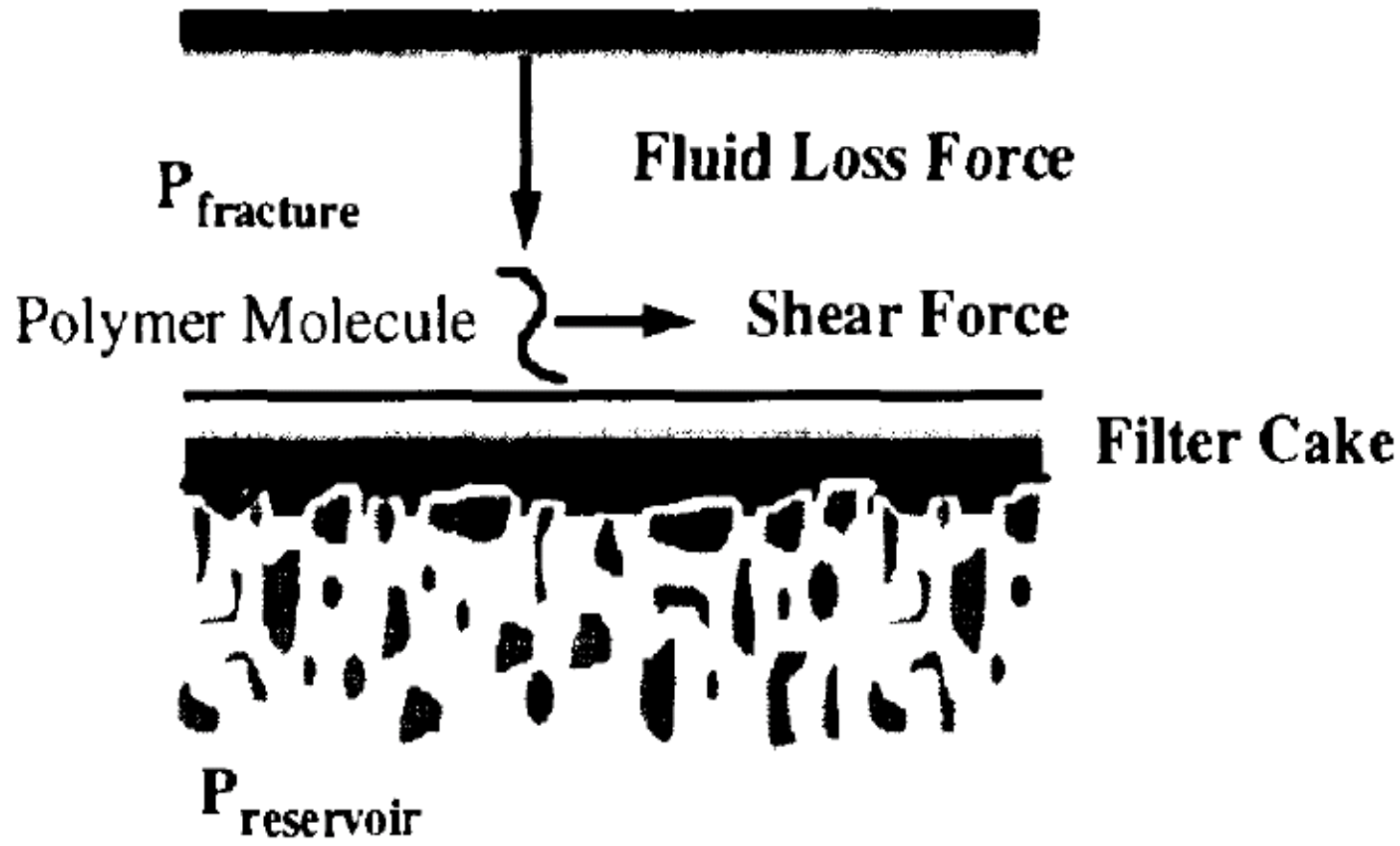

Figure 39 Schematic picture of dynamic fluid loss process, Vitthal et al. ${ }^{[61]}$

Glenn at al. ${ }^{[30]}$ classified the laboratory models designed by different researchers to measure the fluid loss dynamically into four groups:

1. Hollow cores: Fluid leaks off radially while flowing inside a cylindrical hollow core or in an annulus between the core and an impermeable wall around it. Cylindrical coordinate of this setup causes unnecessary complication.

2. Fluid stirring above a core wafer: This method makes the flow geometry complex while it is not a good representative of flow in a fracture.

3. Fluid impinging on a core wafer and spreading away radially: This method also makes the flow geometry complex while it is not a good representative of flow in a fracture. 
4. Slot flow through a rectangular geometry bounded by two cores or one core and a nonpermeable wall.

The last geometry was shown to be more representative of the real fracturing conditions and has been used by different researchers like Roodhart, ${ }^{[70]}$ Navarette et al. ${ }^{[59]}$ and McGowen et al. ${ }^{[61,62]}$ successfully.

Navarette et al. ${ }^{[59]}$ designed a reciprocating dynamic fluid loss simulator which simulates the fracture condition for fracturing fluid that has already experienced the shear of pipelines and the traveled distance inside a fracture (Figure 40). Fracturing fluid is accumulated and reciprocated between two cylindrical accumulators after being crosslinked and sheared in shear loops. The dynamic fluid loss cell is located in the middle of two $3 / 8$ in. tubing coils connecting the two accumulators. Proper valves switched to keep the flow direction on the core surface always constant. Figure 30 shows the schematic of dynamic fluid loss cells, made of SS 316 and capable of operating up to 3500 psi, used by Navarette et al. ${ }^{[59]}$ A 2.205 in. long, 1 inch wide with a 1/8 inch gap slot is designed to simulate the shear that fracturing fluid undergoes during a fracturing job. The cell is mounted in a cylindrical heater with a thermocouple which controls the temperature.

Higher filtrate volumes during a dynamic test comparing to the static tests done in the same conditions lead Navarette et al. ${ }^{[59]}$ to conclude that static fluid loss cells underestimate the fluid loss volume. They also concluded that fluid loss additives are more effective at higher permeabilities and less effective in higher shear rates. Navarette et al. ${ }^{[59]}$ also indicated that in high permeability rocks internal filter cake is the dominant control mechanism for fluid loss. 

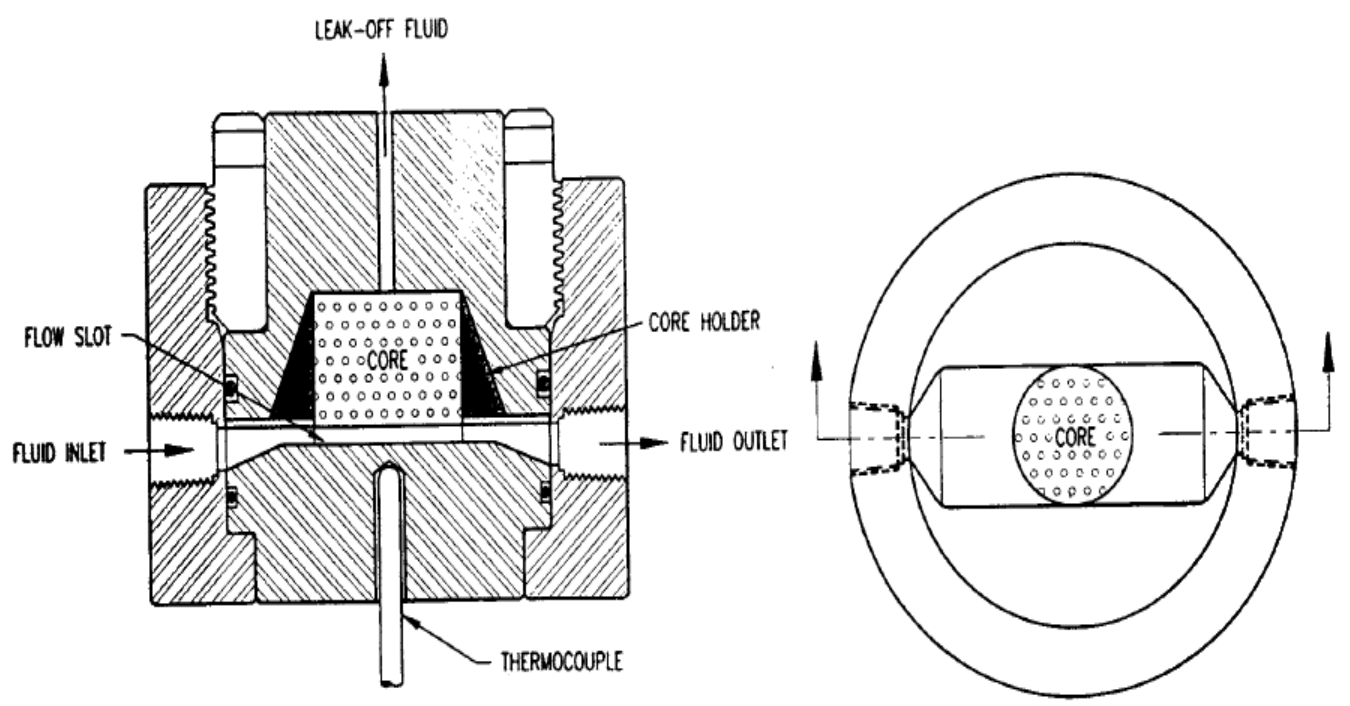

Figure 40 Schematic picture of dynamic fluid loss cells used by Navarette et al. ${ }^{[59]}$

McGowen and Vitthal ${ }^{[61,62]}$ used a dynamic fluid loss cell which is shown in Figure 31 and Figure 41. Their system is designed for 1.5 in. diameter cores with thicknesses variable from 0.5 in. to $4 \mathrm{in}$. They ran the tests for at least 90 minutes. They reported 2 to 3 times greater filtration volume for the dynamic tests comparing to the static tests. They noted that the static period is almost similar for both dynamic and static tests and the main difference occurs during the postequilibrium period. They also reported that leakoff velocity is expected to reach a constant value $\left(v_{n}\right)$ for the times greater than the equilibrium time while it is a function of time during the filter cake build up period $\left(\frac{C_{w}}{\sqrt{t}}\right)$.

Vitthal and McGowen ${ }^{[61,62]}$ demonstrated that filter cakes made by polymeric fluids are compressible. They presented Equation 45 and Equation 46 to show dependency of static and dynamic fluid loss coefficients to pressure. Where $\alpha$ varies from 0.0 for a completely compressible filter cake to 0.5 for a completely incompressible filter cake.

$C_{w} \propto \Delta P^{\alpha}$

Equation 45

$v_{n} \propto \Delta P^{2 \alpha}$

Equation 46 
Shear Effects Studied by Vitthal and McGowen: ${ }^{[61,62]}$ Applying different shear rate values on the fluid inside the slot (fracture simulator) Vitthal and McGowen ${ }^{[61,62]}$ reported that fluid loss behavior of linear fluids is not sensitive to shear while increasing the shear increases the total leak off for crosslinked fluids. They also reported that fluid loss rates in high permeability cores do not show shear sensitivity because of formation of an internal instead of an external filter cake.

Plotting the velocity during the leak off for different permeabilities and pressures Vitthal and McGowen ${ }^{[61,62]}$ reported that velocity during the equilibrium phase is not constant and decreases slowly with time. This indicates that a true equilibrium is not reached. They ascribed this to filter cake consolidation or growth at a very slow rate.

They also reported $25 \mathrm{~s}^{-1}$ as a limit below which the leak off rate follows only $\mathrm{t}^{1 / 2}$ behavior. This criteria was amended to $40 \mathrm{~s}^{-1}$ by Penny et al. ${ }^{[72]}$

Permeability Effects Studied by Vitthal and McGowen: Studying fluid loss caused by linear and crosslinked gels, Vitthal and McGowen ${ }^{[61,62]}$ indicated that major influence of permeability occurs during the initial filter cake buildup period. They reported that even though linear gels form an external filter cake in permeabilities between 1 and $20 \mathrm{mD}$ some of the polymer leaks off through the core reducing the permeability.

Running both static and dynamic fluid loss tests for borate and titanate crosslinked guar they demonstrated that permeability only affects the spurt volume and very little change was observed in $\mathrm{C}_{\mathrm{w}}$ when changing the permeability. Spurt loss has an increasing linear trend with permeability. Higher leak off volumes were reported in low permeability cores compared to the high permeability cores because of the sensitivity of leak off to shear in low permeability cores.

Differential Pressure Effects Studied by Vitthal and McGowen: ${ }^{[61, ~ 62] ~ A p p l y i n g ~ d i f f e r e n t ~}$ pressures using the static fluid loss cell and using linear HPG they observed a straight line of fluid loss versus square root of time as a result of internal pore plugging followed by a second line as a result of external filter cake building. They reported that the total fluid loss is increasing as a result of increase in the slope of the first line increasing the total driving force $(k \Delta P)$.

Using borate and titanate crosslinked HPG and differential pressures up to 10000 psi for static tests and 2000 psi for dynamic tests, Vitthal and McGowen ${ }^{[61,62]}$ showed that the (HPG+borate) system shows no dependency of fluid loss coefficients on differential pressure. Static fluid loss coefficient was shown to change with $\Delta \mathrm{P}$ based on Equation 45 when $\alpha=0.17$ for the 
(HPG+titanate) fluids. However, the spurt loss increases with differential pressure for both fluids. Spurt loss values are higher for titanate crosslinked fluids.

Vitthal and McGowen ${ }^{[61,62]}$ indicated that increasing the pressure drop after the formation of filter cake has no significant effect on fluid loss.

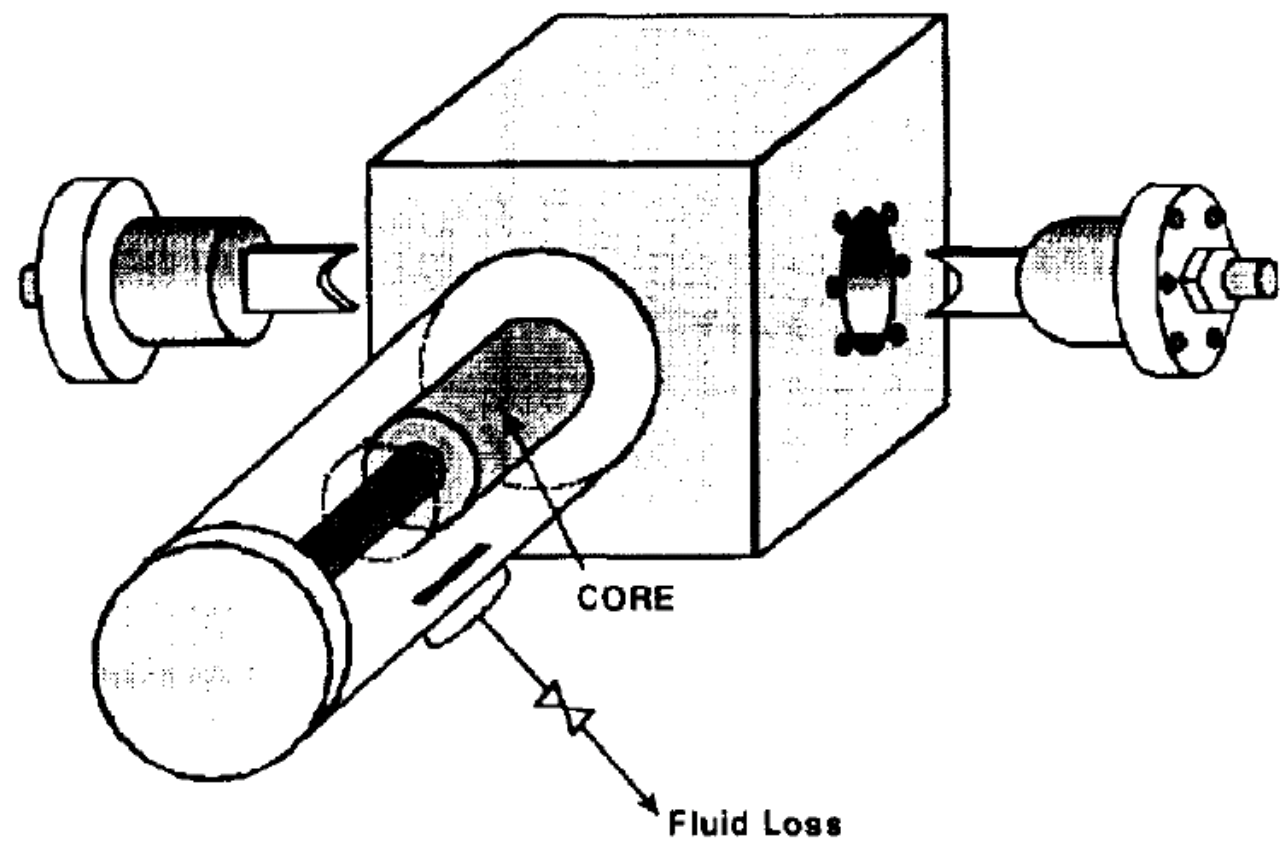

Figure 41 Dynamic fluid loss cell of McGowen and Vitthal ${ }^{[61,62]}$

\subsection{Laboratory Measurement of Fracture Conductivity}

Conductivity cells were designed to determine retained permeability or conductivity of the proppant pack under closure stress and temperatures similar to that of a real hydraulically generated fracture. Conductivity cells were used mainly to simulate the permeability reduction as a result of crushed proppants, proppant embedment on the rock surface, and residues remain after the reaction of breakers with fracturing fluids. Conductivity cells were also used to study the non-Darcy behavior of flow at high flow rates especially when gas is the produced hydrocarbon. 
The term "fracture conductivity" has been used because of difficulties encountered in measurement of fracture width. This problem led researchers to calculate the multiplication of fracture permeability and its width using Darcy's law and name it fracture conductivity. ${ }^{[73]}$

$k_{f} w=\frac{q \mu L_{f}}{h \Delta P}$

Equation 47

Several setups have been recommended by different laboratories to measure the conductivity of a proppant pack. Two main categories ${ }^{[74]}$ of such setups are linear and radial flow conductivity cells. Radial flow conductivity cells have not been approved by the industry because of their geometry which causes pressure distributions related to the radius of setup. This causes inaccurate measurement of conductivity. The most approved family of conductivity cells are the family of so-called Mobil ${ }^{[74]}$ or Cooke ${ }^{[75,76]}$ conductivity cells which were proposed by API ${ }^{[77]}$ as a standard procedure and modified by different laboratories later. The rest of this section is focused on this latter family of conductivity cells.

API Conductivity Cell ${ }^{[77]}$ : API recommended a setup for evaluating the short term proppant pack conductivity. Figure $\mathbf{4 2}$ and Figure 43 show the schematic picture of the API conductivity cell and the flow paths of its unit. This unit is designed mainly to measure the closure stress under which proppants crush and the permeability of proppant pack reduces. Proppants are located between two pistons and no core wafer is used in this setup. The test unit is designed to be a linear flow with 10 square inch surface area in contact wid proppants and fluids.

A hydraulic load frame capable of developing 150,000 pounds force with loading rate of 5,000 $\mathrm{lbf} / \mathrm{min}$ is recommended. This force can apply $15000 \mathrm{psi}$ pressure over the core which equates to almost 20,000 ft depth of reservoir assuming the closure stress gradient of $0.75 \mathrm{psi} / \mathrm{ft}$.

Constant flow rate pumps, e.g. chromatographic pumps, are recommended to keep the flow rate constant in a range of 1 to $10 \mathrm{~mL} / \mathrm{min}$ with pressure fluctuations less than $\%$. Applying high flow rates must be avoided to prevent non-Darcy effects. API recommended using deionized water as the test fluid. However, other fluids can be used so long as limitations due to viscosity and salinity are taken into account.

Differential pressure transducers with a range of 0-1 psi are reported to be satisfactory. However, more sensitive transducers can be used if small pressure gradients are expected along the bed. 
Back pressure regulators capable of keeping the downstream pressure 50 psi more than the vapor pressure of the test fluid are needed to prevent a gas phase from plugging the pores. Test fluid must also be degassed and have known viscosity and density at the test temperature. Deoxygenation and saturating with dissolved silica is recommended by $\mathrm{API}^{[77]}$ and other researchers. ${ }^{[78,79]}$

Pack width must be measured at the ends of the pack using appropriate instruments with accuracy of 0.001 inch. Proppants with volume equivalent to 0.25 inch pack width (unstressed) or concentration (mass per surface area of unit cell) of $2 \mathrm{lb}_{\mathrm{m}} / \mathrm{ft}^{2}$ are recommended. Ambient temperature $\left(75^{\circ} \mathrm{F}\right)$ is recommended by API for test cell and proppant pack while it is possible to design a cell for high temperatures. Use of a load measuring device is recommended over the use of hydraulic gauges to measure the closure stress.

Applying different closure stresses across the test unit, conductivity of the fracture is calculated after measuring the width, pressure gradient, and flow rate at each stress level. Some time is usually needed for the proppant bed to be under stress and reach a semi-steady state condition. This time can be recognized by the noise produced by the proppants while they crush. Knowing the typical volume/area of injected fracturing fluid inside a real fracture, the same amount could be added to the proppant before it is loaded. The fluid will be cleaned up partially/totally using the test fluid. 


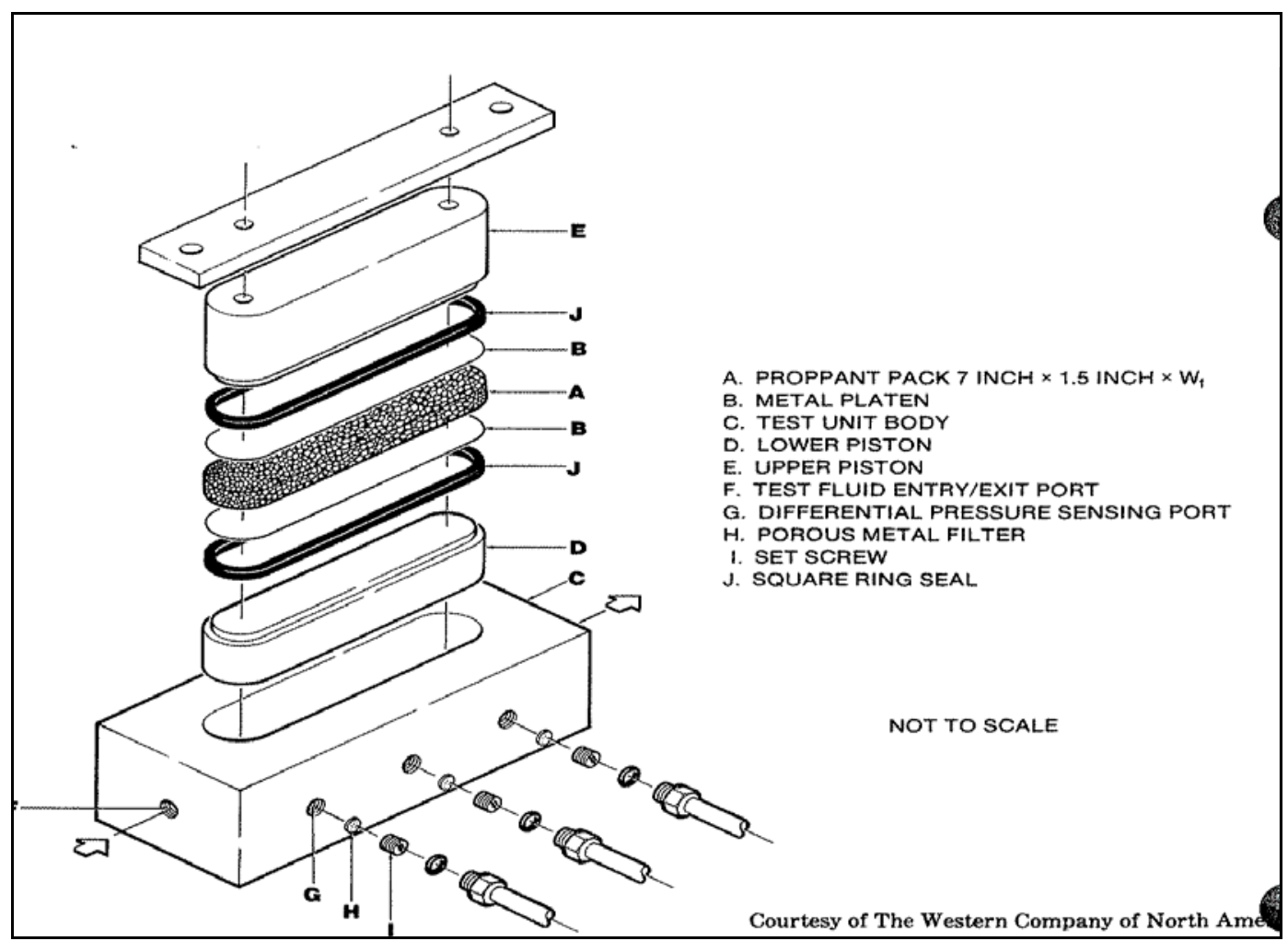

Figure 42 Disassembled API conductivity cell $^{[77]}$

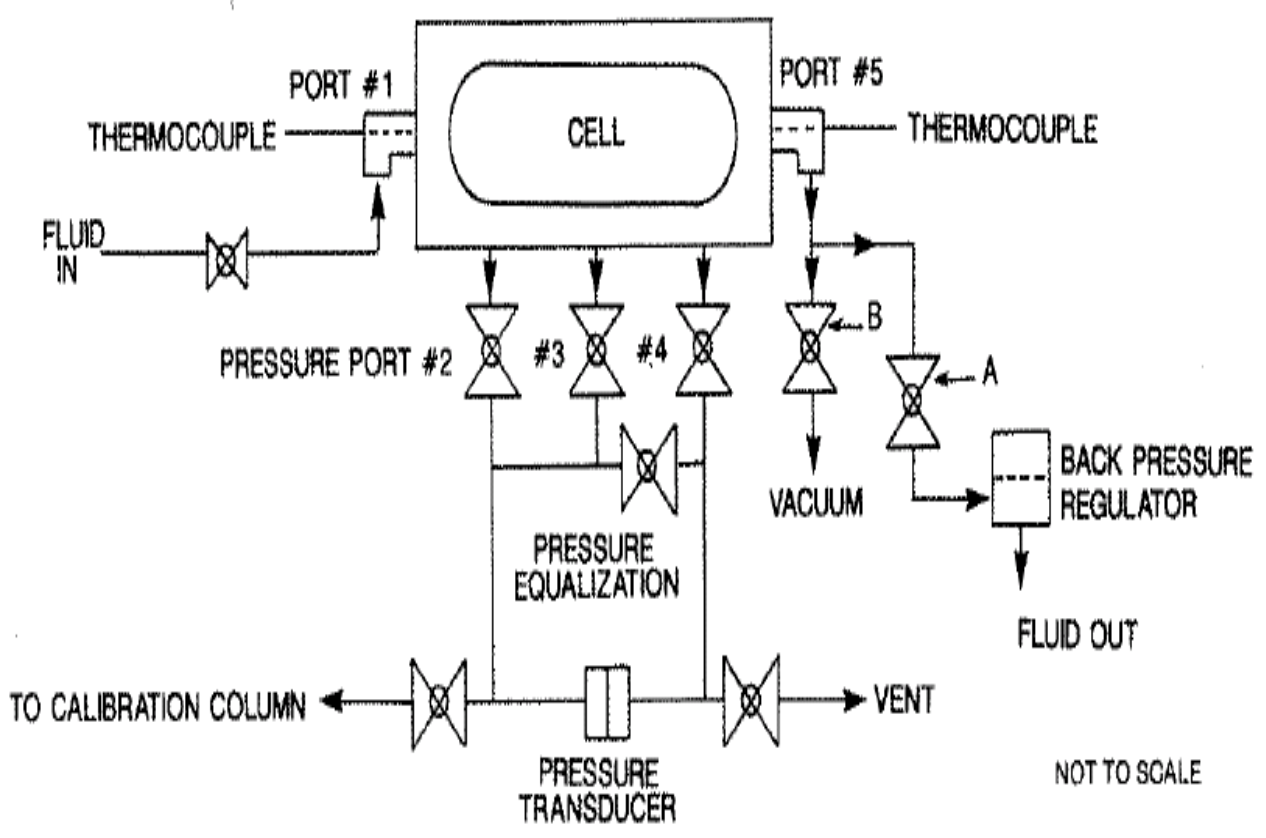

Figure 43 Schematic of flow paths through the API conductivity cell ${ }^{[77]}$ 
Modified Conductivity cell of Much and Penny ${ }^{[79]}$ and Penny ${ }^{[78]}$ : Much and Penny ${ }^{[79]}$ and Penny ${ }^{[78]}$ presented a modified version of the API conductivity cell and made it capable of accepting two core slabs and measuring fluid loss dynamically. Figure 44 and Figure 45 show their modification. This setup was capable of collecting the fluid leak off by making three 1/8 in. holes in the piston sides connected by small grooves allowing free flow of the filtrate fluid.

The fluid distribution when entering the cell and fluid gathering when leaving the cell were also modified to follow the shape of the core and make a smooth transition from 1 in. pipe to the $1 \frac{1 / 2}{2}$ in. by $1 / 3$ in. slot and vice versa.

After deoxygenating the fluid and saturating with dissolved silica they added $2 \% \mathrm{KCl}$ to dehydrate the fluid and blended the fluid with proppant and crosslinker (titanate). They used three duplex pumps to pump the solution providing $1 / 3$ to $1 \mathrm{gal} / \mathrm{min}$ rates and 1000 psi pressure. The fluid was sent through a wellbore shear simulator (1000-1500 s $\mathrm{s}^{-1}$ for 5 minutes) and a formation shear simulator (30-40 $\mathrm{s}^{-1}$ for 5 minutes) before being sent to the cell with shear rate of $30-40 \mathrm{~s}^{-1}$ between two $3 / 8$ in. Ohio sandstone slabs saturated with $2 \% \mathrm{KCl}$. They scheduled their tests similar to a real fracturing job (Table 9) under 1000 psi differential pressure. Core slabs were held apart using 1/3 in. spacers during the run while 3000 psi closure stress was applied. Leak off volumes were gathered and recorded during the test.

After the injection the "pipe-to-slot" flow parts were replaced with the inserts containing a 1/8 in. hole with a filter screen to confine proppants during the closure. During the leak off time when 1000 psi closure stress was applied, Penny ${ }^{[78]}$ increased the temperature from test temperature $\left(120-200^{\circ} \mathrm{F}\right)$ to $\left(175-300^{\circ} \mathrm{F}\right)$. Once the leak off was completed he shut-in the cell for $12 \mathrm{~h}$ then he started flowing back using $2 \% \mathrm{KCl}$ through the cores and pack starting with 1000 psi closure stress. He increased the stress to 4000-10000 psi over a 4-12 h period. After flowing 2\% $\mathrm{KCl}$ from both pack and cores for total of $24 \mathrm{~h}$, Penny ${ }^{[78]}$ flowed $2 \% \mathrm{KCl}$ only from the pack at a rate of $2 \mathrm{~mL} / \mathrm{min}$ for the rest of test.

Using titanate as a crosslinker for HPG, Much and Penny ${ }^{[79]}$ and Penny ${ }^{[78]}$ measured the retained conductivity of proppant packs after forming the filter cake dynamically and placing the proppants. They reported that increasing the closure stress and temperature consistently, as representative of formation conditions, conductivity decreases significantly. Penny ${ }^{[78]}$ indicated that polymer in the center of the pack remains at the original concentration while the filter cake forms with 5-7 times more concentrations of polymer. 
Table 9 Treatment schedule of Penny ${ }^{[78]}$

\begin{tabular}{|l|l|l|}
\hline Stage & Fluid & Time (min.) \\
\hline 1 & $2 \% \mathrm{KCl}$ & 10 \\
\hline 2 & Prepad (base polymer) & 10 \\
\hline 3 & Pad (gelled polymer) & 180 \\
\hline 4 & 1 ppg proppant & 20 \\
\hline 5 & 3 ppg proppant & 20 \\
\hline 6 & 5 ppg proppant & 20 \\
\hline 7 & Slurry to pack cell to $2 \mathrm{lb} / \mathrm{ft}^{2}$ concentraion. & $\cdots .$. \\
\hline
\end{tabular}
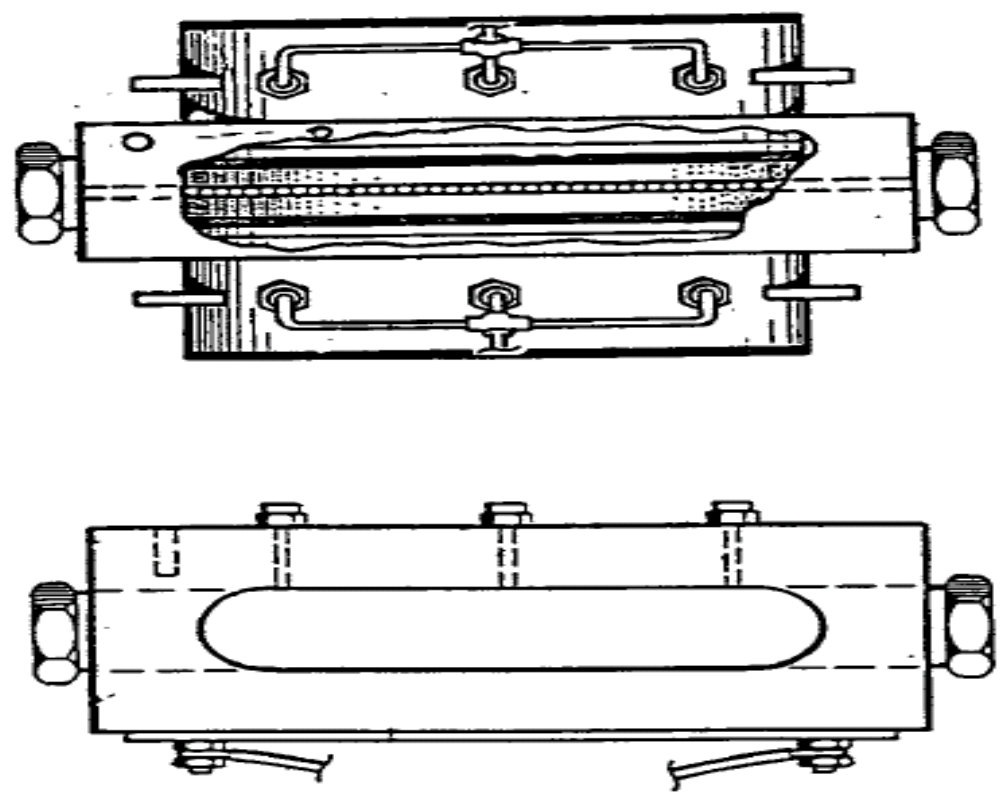

Figure 44 Body of conductivity cell published by Penny ${ }^{[78]}$ 


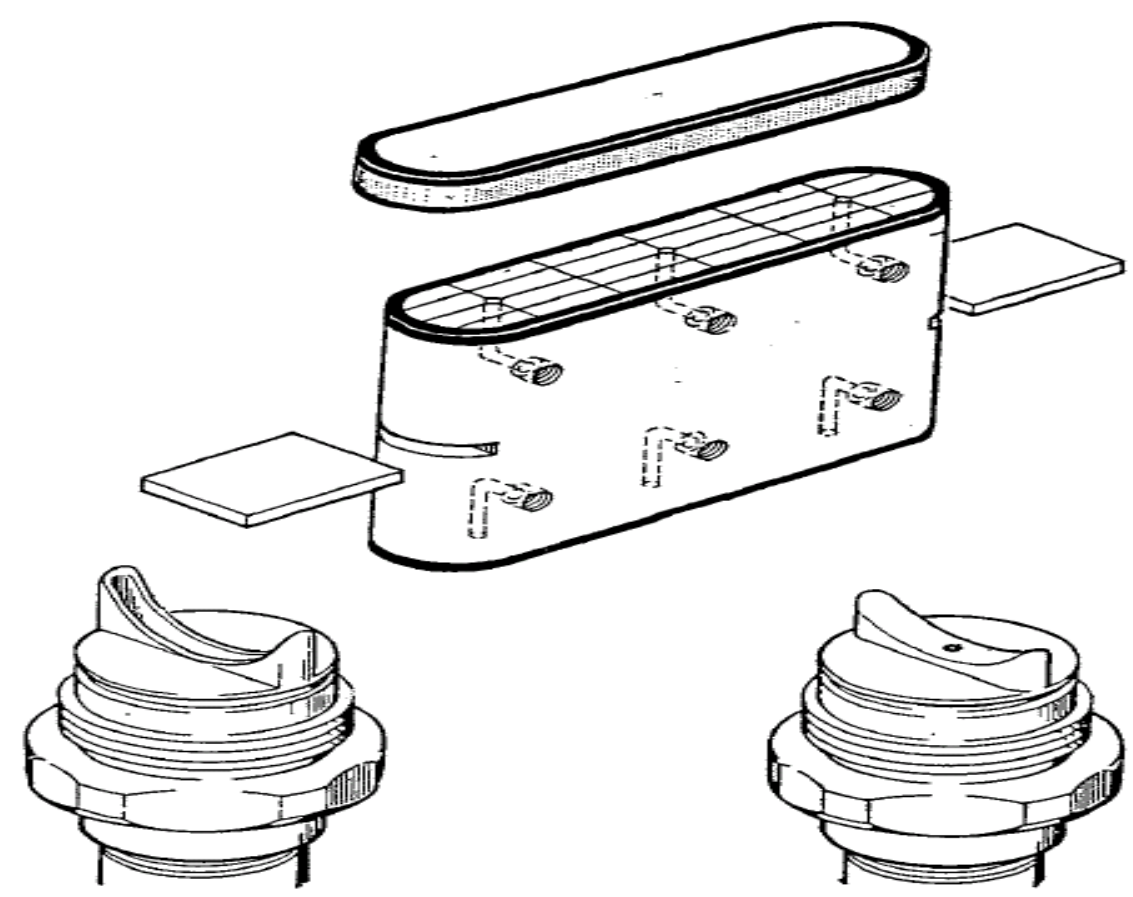

Figure 45 Modified core slab and piston of Penny to allow fluid leak off ${ }^{[78]}$

McGowen and Vitthal ${ }^{[62]}$ compared the results of a typical dynamic fluid loss (DFL) test with data measured using an API cell and a transition API (TAPI) cell similar to the one used by Penny ${ }^{[78]}$. They reported that DFL test shows slightly higher leak off volume comparing to the API and TAPI cells. They indicated that the cause of this difference in the shape of filter cake is the drag effects of the walls on the fluid in the API shape cells. This effect is smaller in DFL since the slot size and the core area near the slot walls are smaller (Figure 46). 


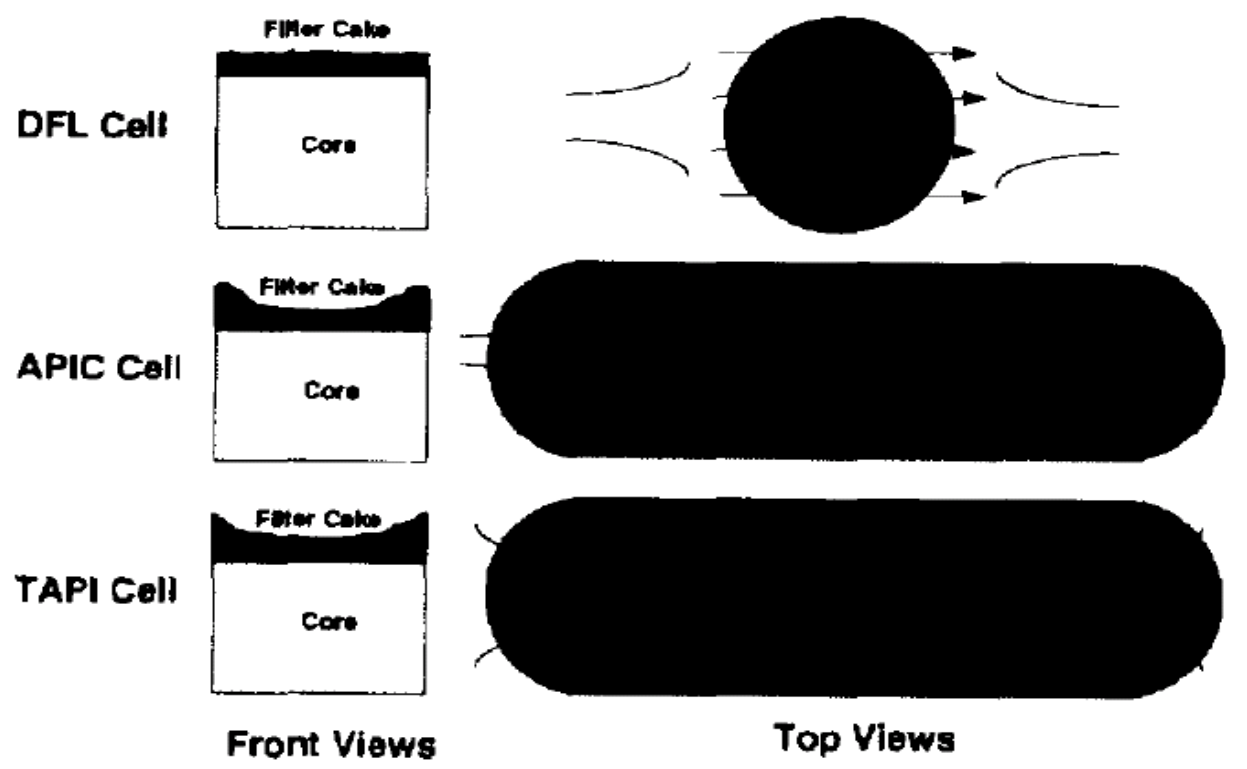

Figure 46 Flow effects on filter cake using different dynamic fluid loss cells, McGowen and Vitthal ${ }^{[62]}$

Marpaung et al. ${ }^{[80]}$, Chen et al. ${ }^{[81]}$ and Zou et al ${ }^{[82]}$ presented a dynamic conductivity cell similar to that of Much and Penny ${ }^{[79]}$ and Penny ${ }^{[78]}$ to compare the results traditionally presented using static conductivity cells with the dynamic results using wet gas to clean up the broken fracturing fluids from the proppant pack. Using the setups presented in Figure 47 and Figure 48 for fluid loss and cleanup of fluid they reported that the measured conductivity using the static conductivity cells is much higher than that measured using the dynamic cells. Even though they indicated that the cell was filled with the same amount of proppant as the dynamic case to have same concentrations, they did not report what volume of fluid they used to run the fluid loss. They claimed the cause of this difference in the conductivities to be the result of higher leakoff volumes during the dynamic runs creating more volume of filter cake, thus causing more damage. They also reported that increasing the cleanup rates increased the conductivity of proppant packs significantly. 


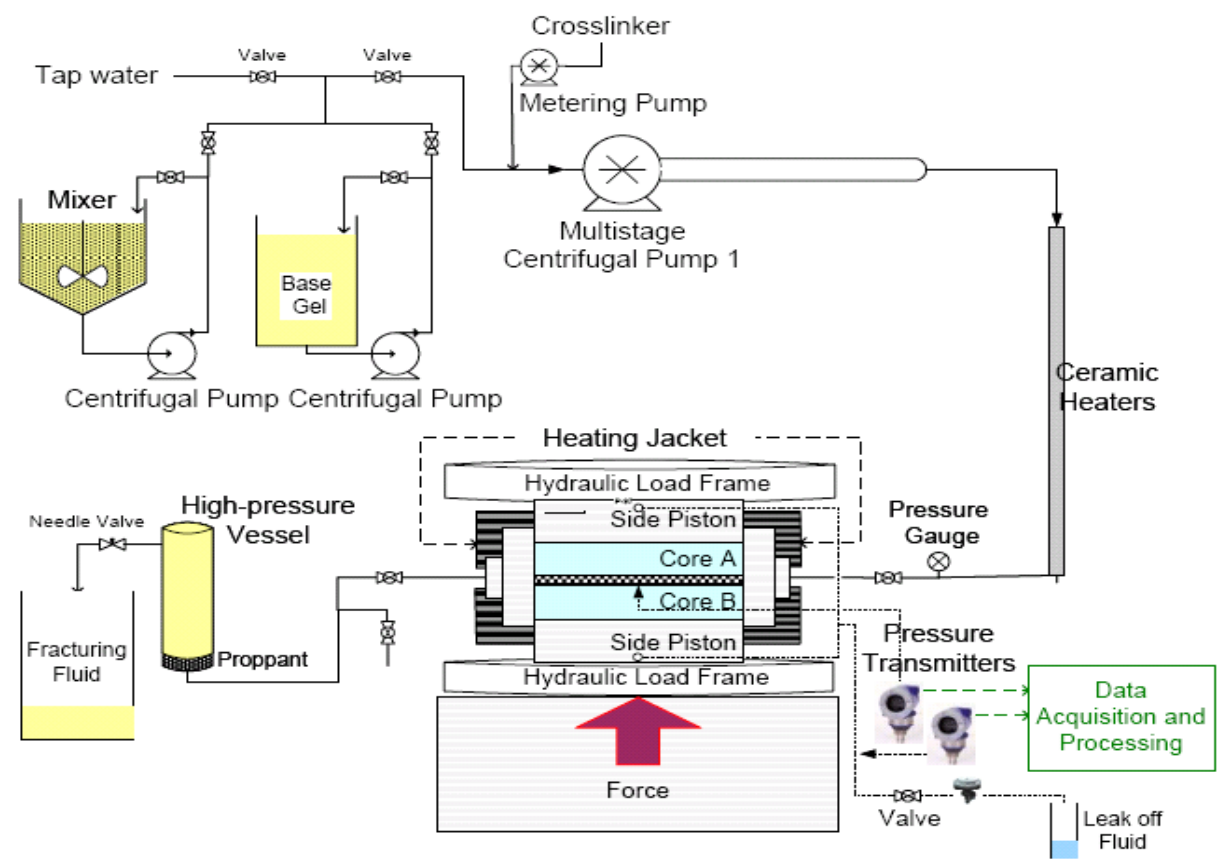

Figure 47 Schematic picture of pumping setup for dynamic conductivity cell (Marpaung et al.) ${ }^{[80]}$

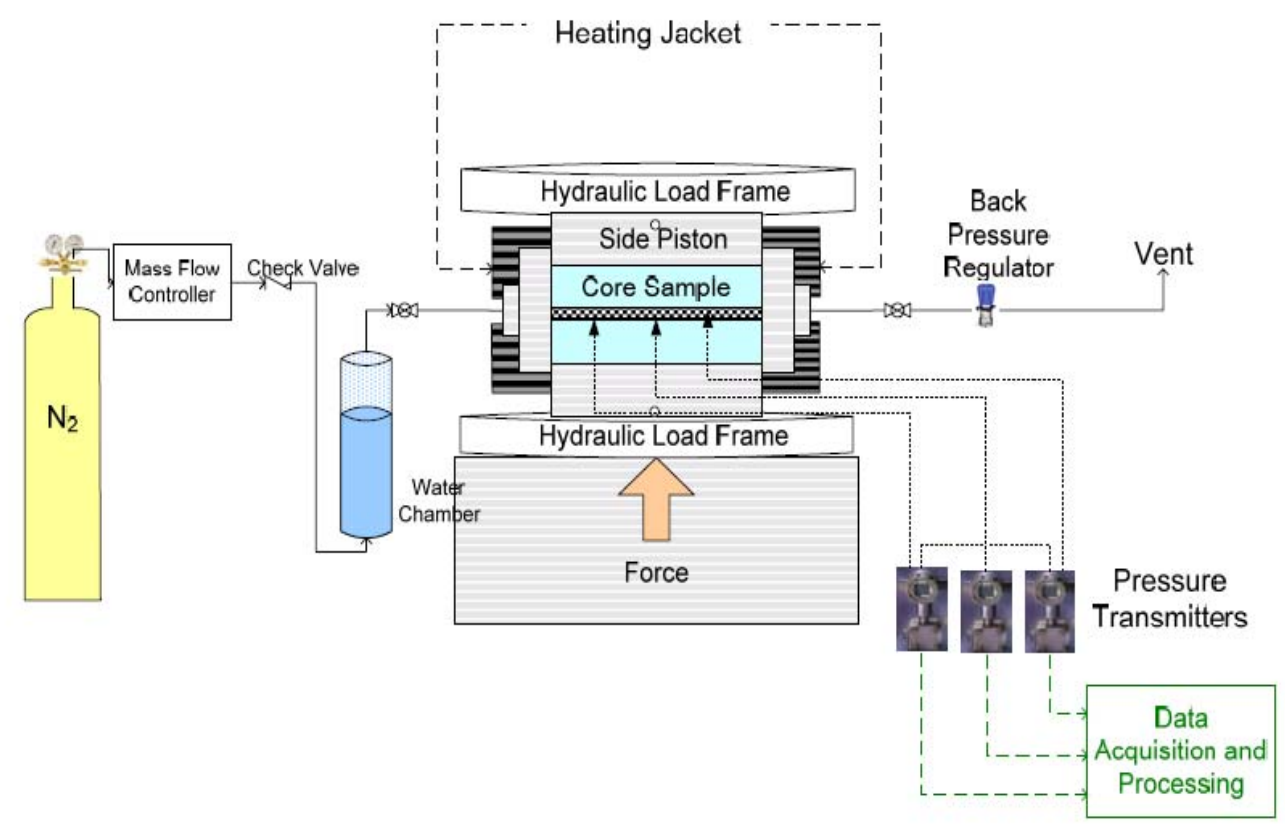

Figure 48 Schematic picture of conductivity measurement (Marpaung et al.) ${ }^{[80]}$ 


\subsection{Fracture Cleanup}

Hydraulic fractures usually fail to produce the designed conductivity. Conductivity of a fracture is affected by factors like incomplete cleanup of gels, non-Darcy effects, multiphase flow effects, and distribution, crush and embedment of proppants. Palisch et al ${ }^{[8]}$ reported gel damage as a significant factor caused by one of the following mechanisms:

Gel residue distributed along the fracture reducing porosity and permeability of the proppant pack (Figure 49).

$>$ Width loss due to filter cake (Figure 50). Ratio of filter cake over the fracture width plays a critical role in fluid resistance against any applied pressure difference in a proppant pack. $^{[3,4]}$

D Loss in length of fracture because of unbroken fluids bypassed near the tip. This problem occurs especially in low permeability reservoirs with long fractures (Figure 51). It is a function of yield stress caused by concentrated fracturing fluid and dimensionless conductivity of fracture defined in the following equation. ${ }^{[7]}$

$C_{f d}=\frac{k_{f} w}{k L}$

Equation 48

It is well known that gels are pseudo-plastic, power law fluids. Al-Fariss et al ${ }^{[83]}$ proved that gels follow Herchel-Bulkly model under shear. Herschel-Bulkley fluids need a minimum stress before they flow (yield stress) and behave according to power-law model after they start flowing.

$\tau=\tau_{0}+K^{\prime} \dot{\gamma}^{n^{\prime}}$

Equation 49

Where $\tau_{0}$ is the yield stress, $K^{\prime}$ is the fluid consistency index, $\dot{\gamma}$ is the shear rate, and $n^{\prime}$ is the fluid behavior index.

The amount of damage caused by polymers depends on polymer concentration, and type and concentration of breakers. ${ }^{[76]}$ Polymer concentrations were decreased significantly after crosslinking the guar molecules using different metal ions started. However, concentrations 
below the critical overlap concentration of guar are not suitable as long as strongly crosslinked fluids are required.

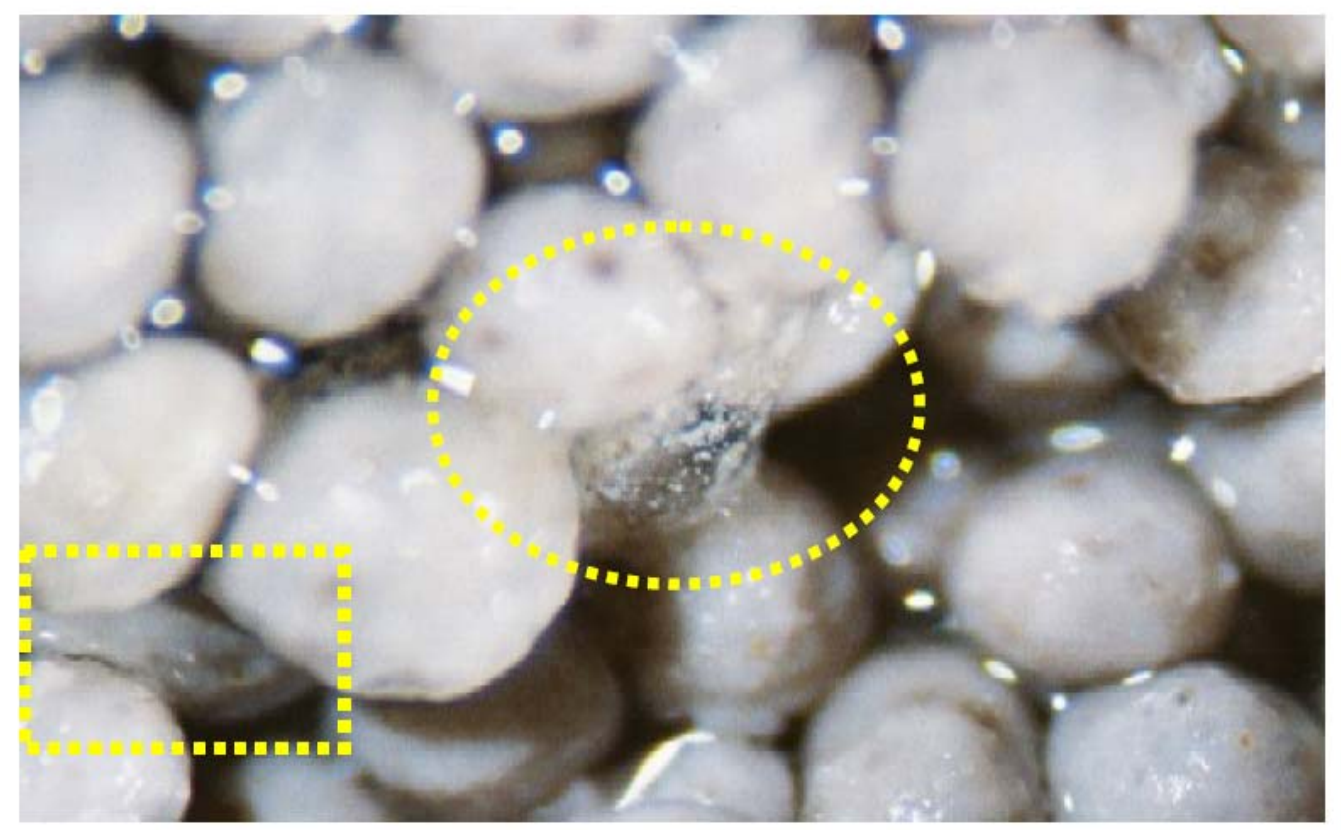

Figure 49 Residual gel damage remain after breaking a Zr X-linked CMHPG (35 ppt) in a long term conductivity cell (Palisch et al.) ${ }^{[8]}$

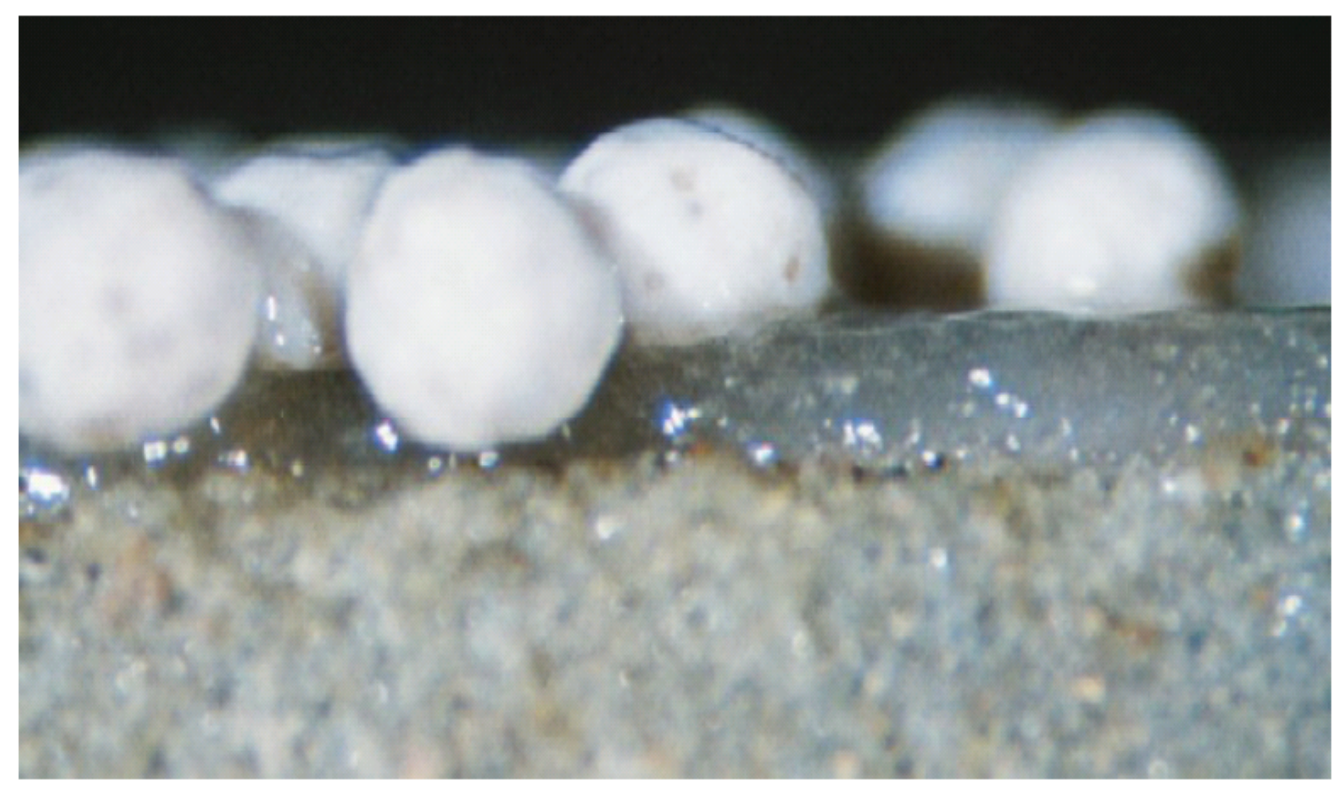

Figure 50 Filter cake build up of 35 ppt Zr X-linked CMHPG (Palisch et al.) ${ }^{[8]}$ 


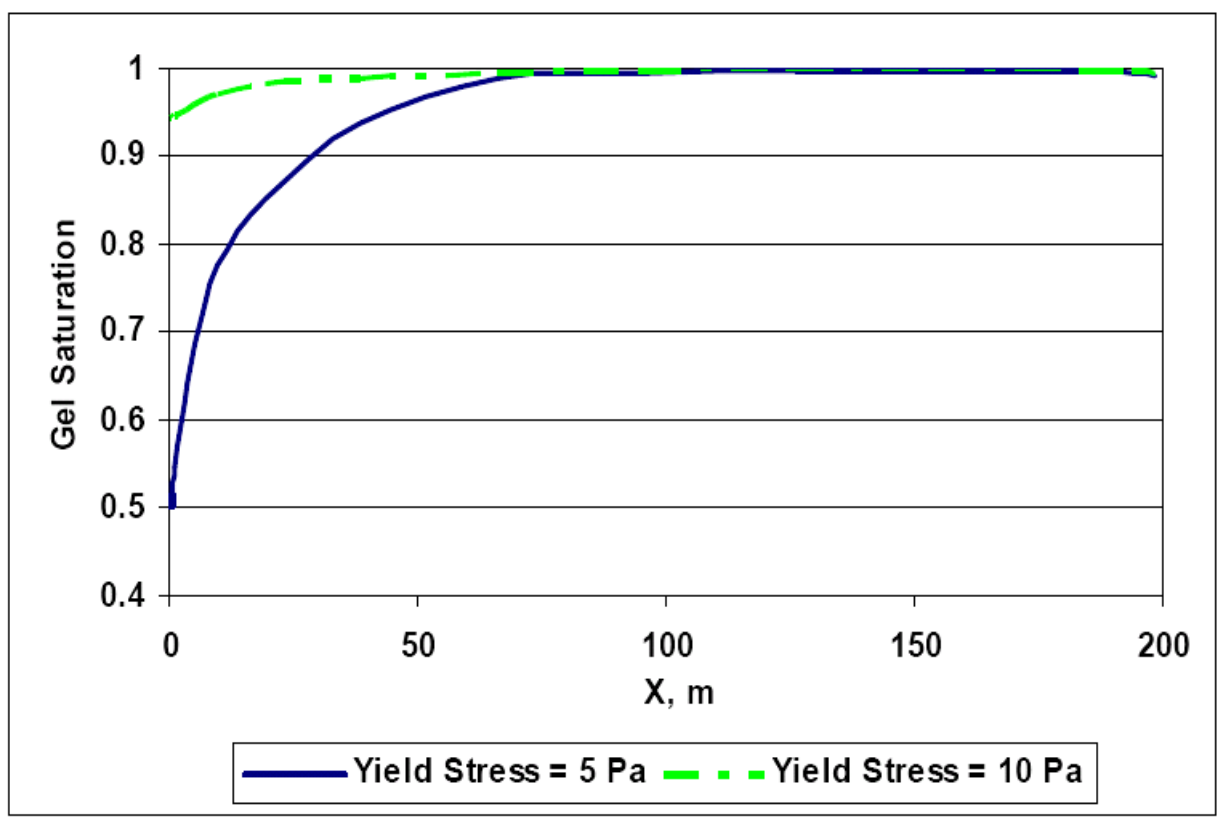

Figure 51 Simulated incomplete cleanup of fracture with a Herschel-Bulkley fluid as fracturing fluid in a tight gas formation (Barati et al.) ${ }^{[7]}$

Hawkins, ${ }^{[84]}$ using a static API cell which was modified to take the fluid loss statically and generate filter cake, indicated that HPG crosslinked with borate causes less damage to the permeability of the proppant pack comparing to the uncrosslinked, and titanate or zirconate corsslinked HPG. He used ammonium persulfate as breaker and reported up to 25-fold concentration increases for the filter cake formed during a fracturing job compared to the injected fluid.

Ayoub et al. ${ }^{[3,4]}$, using a modified static fluid loss cell capable of measuring the concentration midway between the two cores and translating the yield stress of fluids into flow initiation gradient (FIG), reported the following important results:

$>$ Increasing the concentration of polymer increases the FIG for a constant proppant concentration.

Increasing the breaker decreases the FIG for constant polymer and proppant concentrations. This effect is much more significant at larger widths of proppant pack, i.e. lower average polymer concentrations or higher proppant concentrations. 
Polymer concentrates only in the filter cakes and polymer concentration in the middle of fracture is close to the injected concentrations unless the two filter cakes join. The latter case causes significant FIG and yield stress even when significant concentrations of breaker were added.

Ayoub et al. ${ }^{[4]}$ conducted a separate study measuring the FIG and conductivity of proppants filled with filtered borate crosslinked guar and broken using encapsulated oxidizers. They concluded that the yield stress caused by filter cakes decreases significantly when breaker is delivered directly to the filter cake instead of distributing randomly in the slurry. Note that yield stress and FIG were calculated along the proppant pack. They also reported better conductivity for the runs with encapsulated breakers located in the filter cake.

Ayoub et al. ${ }^{[4]}$ presented the setup shown in Figure 52 to study the flow initiation pressure across the filter cake and vertical to the core surface. In this apparatus, after placing the proppant mixed with fluid, excess fluid was placed on top of the core and filter cake was made by leaking off the fluid and measuring the filtrate vs. time. After forming the filter cake, keeping the proppant pack under the pressure and letting the breaker act the flow initiation pressure was measured by flushing back from the core to the proppant pack. They generated results consistent with the data measured along the pack. However, comparison failed since the true thickness of filter cake was unknown. 


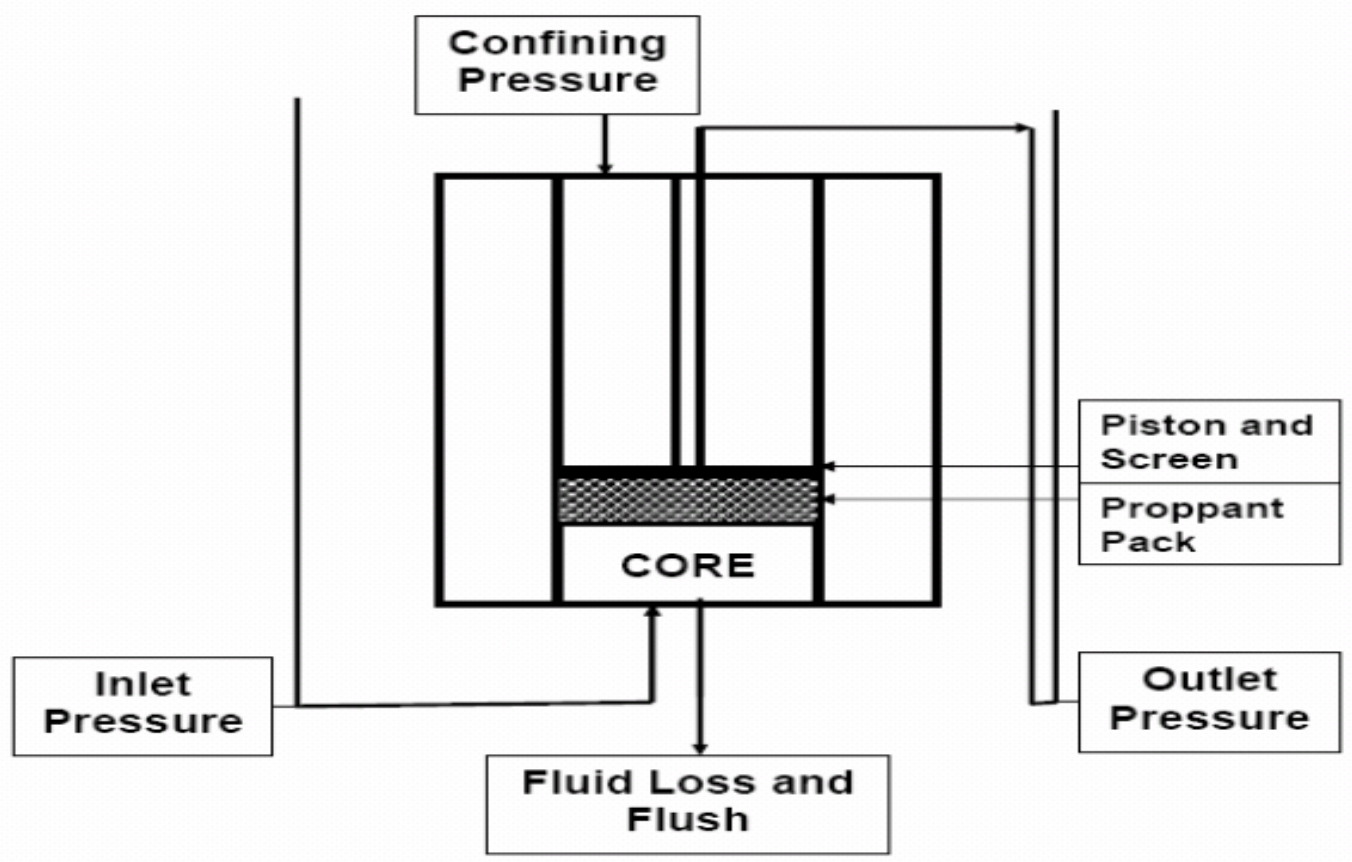

Figure 52 Experimental apparatus for measuring flow initiation pressure across the filter cake (Ayoub et al.) $[4]$ 


\subsection{Summary}

Even though combinations of enzymes and encapsulated oxidizers have a reasonable performance for breaking the crosslinked guar and its filter cake in a proppant pack, gel damage is still one of the main mechanisms reducing the permeability of hydraulic fractures. There is still need for a more uniformly distributed breaker which is designed to target the filter cake and release after the shut-in time. This breaker must be designed to stay in the filter cake and act when it is required. ${ }^{[3,4,7]}$ Viscous filtrates into the high permeability reservoirs can damage the formation by making an internal filter cake in addition to the externally formed filter cake. Breakers with delayed activation time that can invade the formation with the leaked off fluid and act after the well is shut seem necessary to clean up the formation. The fracturing fluid is required to show high viscosity as soon as it reaches the reservoir.

Polyelectrolyte nanoparticles are reportedly capable of carrying charged chemicals and release them at a specific target after a designed period of time. ${ }^{[10-14]}$ Combining this technology with knowledge of breakers for fracturing fluids provides the impetus for the following research. Taking advantage of high surface area and delayed release, polyelectrolyte nanoparticles can theoretically direct high concentrations of breakers into the filter cake without being filtrated into the reservoir. The breaker will be released within a controlled time period to remove the filter cake.

Fracturing fluids are required to have specific rheological properties after being prepared over the bench. Viscoelastic parameters must be checked before injecting the fracturing fluids. Injecting the fracturing fluid through the shear loop exposes the fluid to the typical experienced shear during an injection job. Fracturing fluids must survive during the fluid loss and the breaker must act on the filter cake within an expected time period resulting in high fracture conductivity. 


\section{Materials and Experimental Procedures}

This chapter describes the materials and experimental procedures used during the course of this research. The first section introduces the materials used. Equipment used in this research and experimental procedures are introduced in the next section.

\subsection{Materials}

\subsubsection{Enzymes}

Two enzymes with two different temperature optima were used during the course of this research. Pectinase from Aspergillus aculaceatus (Sigma-Aldrich, St. Louis, MO, Catalogue \# P2611) was used for $25{ }^{\circ} \mathrm{C}$ and in a few cases of $40{ }^{\circ} \mathrm{C}$ applications. ${ }^{[85]}$ Before use, $3 \mathrm{~mL}$ pectinase solution as received was diluted by adding $9 \mathrm{~mL}(25 \% \mathrm{w} / \mathrm{w})$ phosphate buffer $(\mathrm{pH} 7)$. It was then dialyzed against $1 \mathrm{~L}$ of phosphate buffer in a Slide-A-Lyzer Dialysis Cassette (MWCO 3500) for 8 hours. Standard activity of pectinase reported by supplier was 28,472 $\mathrm{U} / \mathrm{mL}$.

A commercial enzyme used in the hydraulic fracturing industry, Econo Gelbreak-EL2X (Economy Polymers and Chemicals, Houston, TX, Lot No. L0901415) was used for $40{ }^{\circ} \mathrm{C}$ applications. The enzyme was used as provided. ${ }^{[86]}$ The activity of EL2X enzyme at $\mathrm{pH} 7$ and room temperature was reported to be $7000 \mathrm{U} / \mathrm{mL}$. Cleaving mechanism of guar using enzymes is presented in Figure 53.

\subsubsection{Guar Products}

Guar, a polysaccharide comprising a $(1 \rightarrow 4)$-linked $\beta$-D-mannose backbone with $(1 \rightarrow 6)$-linked $\alpha$-D-galactose residues, can be gelled with borate ions complexing with the hydroxyl groups on the galactose (Figure 53). The ether bonds between the sugar units on the backbone can be degraded by enzymes.

Guar (Sigma-Aldrich, St. Louis, MO, Catalogue \#G4129, CAS 9000-30-0) was used for some experiments. Guar solutions (100 mL samples) were centrifuged at $9600 \mathrm{~g}$ for 1.5 hours in $25^{\circ} \mathrm{C}$ in order to separate the residue after hydration.

Hydroxypropyl guar (HPG) gum blend (Jaguar ${ }^{\circledR}$ 415, Rhodia, Paris, France, Lot No. H0904166E) was used for the majority of the experiments reported in this research. Jaguar ${ }^{\circledR} 415$ 
is a high viscosity chemically modified polysaccharide which disperses readily and then selfhydrates to yield a smooth, viscous solution. ${ }^{[87]}$

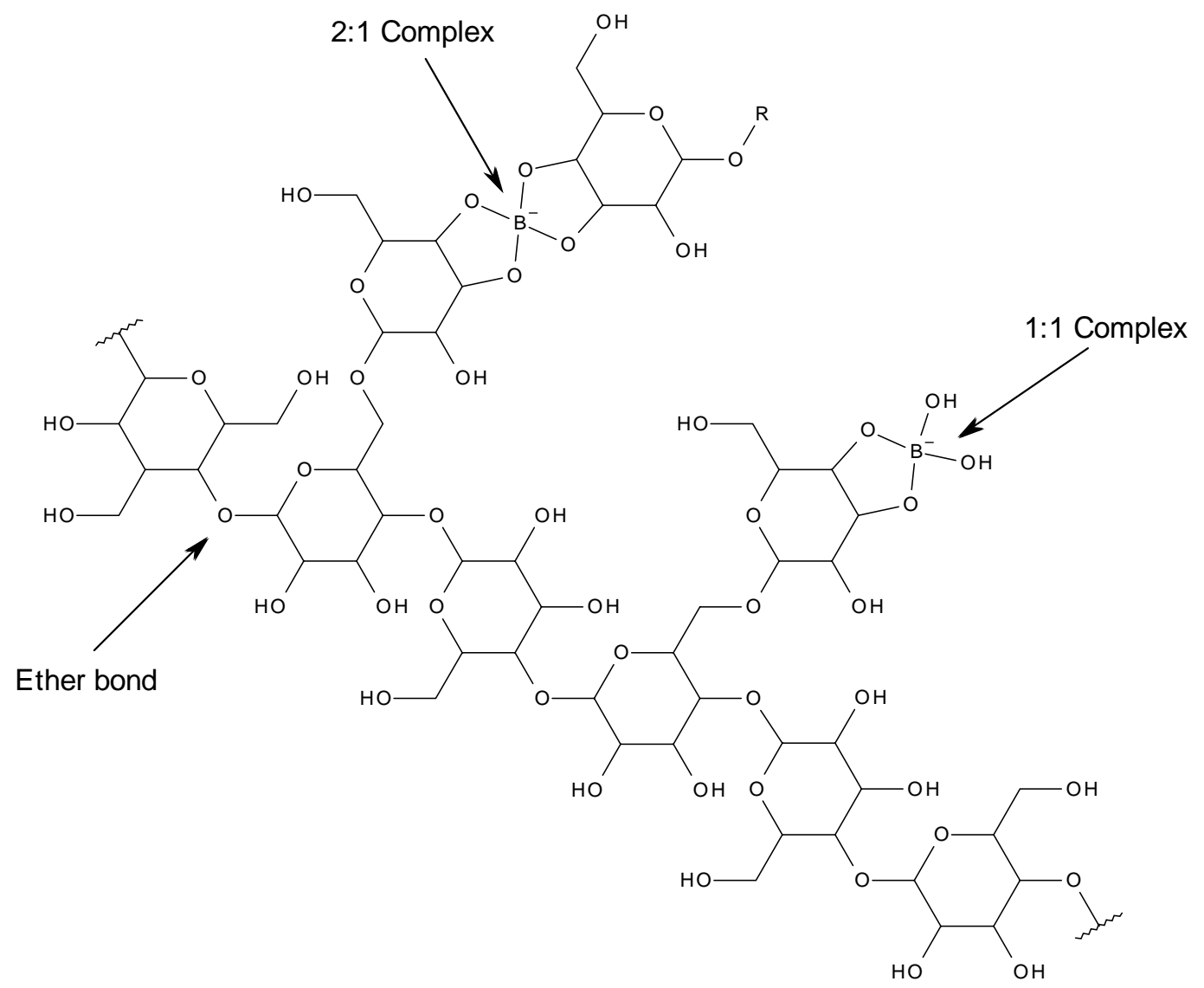

Figure 53 Chemical structures of guar showing mechanism of crosslinking by borate, and ether bond cleaved by enzymes. $R$ is another guar molecule. ${ }^{[23]}$

\subsubsection{Polyelectrolytes}

A polycation (polyethylenimine branched with $\mathrm{M}_{\mathrm{w}}=25 \mathrm{kDa}$ from SIGMA, Batch\# 06321D3) and a polyanion (dextran sulfate sodium salt with $\mathrm{M}_{\mathrm{w}}=500 \mathrm{kDa}$ from SIGMA, Lot No. 080063) were obtained from Fisher Scientific (Pittsburgh, PA). Structure of PEI and DS and their properties are 
described in the literature review section. Figure 12 and Figure 13 show the chemical structures of PEI and DS.

\subsubsection{Borate Cross-linker}

Sodium borate decahydrate (J.T. Baker Chemical Co. reagent, Phillipsburg, NJ, Lot No. 214346) was used as a source of borate ions to cross-link guar polymer and generate guar gel. Borate ions generate 1:1 or 2:1 complexes with guar chains (Figure 53). The latter makes a gel structure.

\subsubsection{PH Modifiers}

Sodium hydroxide $(1 \mathrm{M}$ or $0.1 \mathrm{M})$ and hydrochloric acid $(4 \mathrm{~N})$ were used as $\mathrm{pH}$ modifiers. They were both obtained from Fisher Scientific (Pittsburgh, PA).

\subsubsection{Stabilizer}

Sodium thiosulfate pentahydrate certified ACS (Fisher Scientific, Pittsburgh, PA, Lot No. 076679) was used as a stabilizer. Sodium thiosulfate pentahydrate stabilizes the guar solutions by working as an oxygen scavenger.

\subsubsection{SDS-PAGE Kit}

NuPAGE 4-12\% Bis-Tris Gel (1.5 mL×10 lanes), NuPAGE MES SDS running buffer $(20 \times)$, NuPAGE LDS sample buffer $(4 \times)$ and MARK12 unstained buffer $(1 \times)$ were obtained from Invitrogen (Carlsbad, CA) as a kit. Coomassie Brilliant Blue was used as staining buffer and destaining buffer was made in the lab $(2.6 \mathrm{~L} \mathrm{H} 2 \mathrm{O}+1 \mathrm{~L}$ Ethanol + $0.4 \mathrm{~L}$ Acetic Acid $)$. Analysis were performed per the instructions provided. ${ }^{[88,89]}$

\subsubsection{Core Materials}

Berea sandstone cores within the permeability ranges of $110-160 \mathrm{mD}$ and $60-90 \mathrm{mD}$ were cut from larger plugs available at TORP. Cores with higher permeability were used for $25{ }^{\circ} \mathrm{C}$ applications using pectinase while the cores with lower permeability were used for $40{ }^{\circ} \mathrm{C}$ applications using EL2X. Properties of the cores are summarized in Table 10. 


\subsubsection{Brine}

Brine was prepared by mixing potassium chloride (Fisher Scientific, Pittsburgh, PA. Lot No. 073280 ) with R.O. water in $2 \% \mathrm{w} / \mathrm{w}$ ratio. Brine had a density of $1.0105 \mathrm{~g} / \mathrm{cm}^{3}$ and viscosity of $0.95 \mathrm{cP}$ at $25^{\circ} \mathrm{C}$.

\subsubsection{Bleach for Core Restoration}

Clorox ${ }^{\circledR}$ bleach containing $6.15 \%$ sodium hypochlorite (Lot\# A56023TX-1) was diluted $3 \times$ using $2 \% \mathrm{KCl}$ to be used in restoration of cores after the fluid loss tests.

Table 10 Calculations related to porosity measurement for six different cores before the tracer test

\begin{tabular}{|c|c|c|c|c|c|c|c|c|}
\hline Core \# & Dry weight, g & $\mathrm{L}, \mathrm{cm}$ & $\mathrm{D}, \mathrm{cm}$ & $\begin{array}{c}\text { Core } \\
\text { volume, } \\
\text { mL } \\
\end{array}$ & $\begin{array}{l}\text { Saturated } \\
\text { weight, g }\end{array}$ & PV, g & PV, $\mathrm{mL}$ & Porosity, \% \\
\hline 1 & 28.15 & 2.65 & 2.53 & 13.30 & 30.52 & 2.38 & 2.35 & 17.71 \\
\hline 2 & 27.52 & 2.59 & 2.55 & 13.27 & 29.64 & 2.12 & 2.10 & 15.80 \\
\hline 3 & 29.32 & 2.74 & 2.53 & 13.75 & 31.79 & 2.46 & 2.44 & 17.73 \\
\hline 4 & 27.94 & 2.62 & 2.52 & 13.04 & 30.36 & 2.41 & 2.39 & 18.31 \\
\hline 5 & 27.57 & 2.57 & 2.53 & 12.94 & 29.84 & 2.27 & 2.25 & 17.38 \\
\hline 6 & 28.63 & 2.67 & 2.53 & 13.40 & 30.98 & 2.35 & 2.33 & 17.37 \\
\hline 7 & 26.75 & 2.49 & 2.53 & 12.54 & 28.96 & 2.21 & 2.19 & 17.45 \\
\hline 8 & 28.55 & 2.67 & 2.54 & 13.50 & 30.94 & 2.39 & 2.37 & 17.54 \\
\hline 9 & 29.07 & 2.71 & 2.53 & 13.63 & 31.51 & 2.45 & 2.42 & 17.78 \\
\hline 10 & 29.07 & 2.75 & 2.53 & 13.82 & 31.48 & 2.41 & 2.39 & 17.27 \\
\hline 11 & 28.20 & 2.80 & 2.54 & 14.22 & 31.30 & 3.10 & 3.07 & 21.58 \\
\hline 12 & 27.76 & 2.55 & 2.53 & 12.74 & 30.03 & 2.27 & 2.25 & 17.64 \\
\hline 13 & 29.04 & 2.67 & 2.53 & 13.42 & 31.95 & 2.91 & 2.88 & 21.46 \\
\hline 14 & 28.55 & 2.61 & 2.53 & 13.04 & 30.84 & 2.29 & 2.27 & 17.38 \\
\hline 15 & 29.12 & 2.70 & 2.53 & 13.58 & 31.57 & 2.45 & 2.43 & 17.86 \\
\hline 16 & 28.48 & 2.64 & 2.57 & 13.68 & 30.80 & 2.33 & 2.30 & 16.82 \\
\hline 17 & 28.37 & 2.65 & 2.53 & 13.31 & 30.79 & 2.42 & 2.39 & 17.99 \\
\hline 18 & 27.15 & 2.50 & 2.54 & 12.65 & 29.44 & 2.29 & 2.27 & 17.92 \\
\hline 19 & 27.67 & 2.55 & 2.53 & 12.85 & 29.96 & 2.29 & 2.26 & 17.63 \\
\hline 20 & 27.51 & 2.54 & 2.53 & 12.75 & 29.80 & 2.30 & 2.27 & 17.83 \\
\hline 21 & 28.59 & 2.64 & 2.53 & 13.32 & 31.03 & 2.44 & 2.41 & 18.10 \\
\hline 22 & 28.40 & 2.63 & 2.54 & 13.26 & 30.83 & 2.43 & 2.41 & 18.17 \\
\hline 23 & 27.68 & 2.59 & 2.54 & 13.13 & 30.05 & 2.36 & 2.34 & 17.80 \\
\hline 24 & 28.40 & 2.64 & 2.54 & 13.40 & 30.86 & 2.47 & 2.44 & 18.21 \\
\hline 25 & 27.17 & 2.54 & 2.53 & 12.75 & 29.54 & 2.37 & 2.35 & 18.40 \\
\hline
\end{tabular}




\subsection{Equipment and Experimental Procedures}

\subsubsection{Preparation of Polyelectrolyte Complex Nanoparticles}

Nanoparticles were made using different ratios of PEI:DS or concentration of enzymes using the method presented previously. ${ }^{[14]}$ Different ratios, concentrations and $\mathrm{pH}$ were used in order to optimize the nanoparticle systems. In a typical formulation, $1 \mathrm{~mL}$ of a $1 \% \mathrm{w} / \mathrm{w}$ aqueous solution of DS ( $\mathrm{pH}=7.8$ ) was added drop-wise or rapidly to $2 \mathrm{~mL}$ of a $1 \% \mathrm{w} / \mathrm{w}$ aqueous solution of PEI ( $\mathrm{pH}$ adjusted using $4 \mathrm{~N} \mathrm{HCl}$ ) while stirring. The solution was then stirred for 20 minutes at 600 rpm unless otherwise indicated. Enzyme was added drop-wise or rapidly as $0.1 \mathrm{~mL}$ of a $25 \%$ w/w pectinase solution either before or after the DS (Table 11). Enzyme-loaded nanoparticles were used as a breaker in polymer systems at a final concentration of $0.02 \% \mathrm{w} / \mathrm{w}$ pectinase or $0.08 \% \mathrm{w} / \mathrm{w}$ EL2X. Formulations were assigned a letter A-L, with a prime symbol (') applied to indicate where enzyme was added before the polyanion. The letter $G$ was not used to avoid confusion with the viscoelastic moduli, $\mathrm{G}^{\prime}$ and $\mathrm{G}^{\prime \prime}$. 
Table 11 Nanoparticle systems differed in the ratio of PEI, DS, and pectinase and order of addition.

\begin{tabular}{|c|c|c|c|c|c|c|}
\hline $\begin{array}{c}\text { Syste } \\
\text { m }\end{array}$ & $\begin{array}{c}1 \% \text { w/w } \\
\text { PEI(aq),m } \\
\text { L }\end{array}$ & $\begin{array}{c}1 \% \mathrm{w} / \mathrm{w} \\
\mathrm{DS}(\mathrm{aq}), \\
\mathrm{mL}\end{array}$ & $\begin{array}{c}25 \% \mathrm{w} / \mathrm{w} \\
\text { Pectinase(aq), } \\
\mathrm{mL}\end{array}$ & $\begin{array}{c}\text { Equivalent } \\
100 \% \mathrm{w} / \mathrm{w} \\
\text { EL2X, mL }\end{array}$ & $\begin{array}{c}\text { Make-up } \\
\text { water, } \\
\text { mL }\end{array}$ & $\begin{array}{l}\text { Order of } \\
\text { Addition }\end{array}$ \\
\hline $\bar{A}$ & 2 & 1 & 0.10 & 0 & 0 & $\begin{array}{l}\text { PEI, DS, } \\
\text { Pectinase }\end{array}$ \\
\hline $\overrightarrow{A^{\prime}}$ & 2 & 1 & 0.10 & 0 & 0 & $\begin{array}{c}\text { PEI, Pectinase, } \\
\text { DS }\end{array}$ \\
\hline$B$ & 3 & 1 & 0.10 & 0 & 0 & $\begin{array}{l}\text { PEI, DS, } \\
\text { Pectinase }\end{array}$ \\
\hline$B^{\prime}$ & 3 & 1 & 0.10 & 0 & 0 & $\begin{array}{c}\text { PEI, Pectinase, } \\
\text { DS }\end{array}$ \\
\hline$C$ & 4 & 1 & 0.10 & 0 & 0 & $\begin{array}{l}\text { PEI, DS, } \\
\text { Pectinase }\end{array}$ \\
\hline$C^{\prime}$ & 4 & 1 & 0.10 & 0 & 0 & $\begin{array}{c}\text { PEI, Pectinase, } \\
\text { DS }\end{array}$ \\
\hline $\bar{D}$ & 2 & 1 & 0.07 & 0 & 0.03 & $\begin{array}{l}\text { PEI, DS, } \\
\text { Pectinase }\end{array}$ \\
\hline$E$ & 3 & 1 & 0.06 & 0 & 0.04 & $\begin{array}{l}\text { PEI, DS, } \\
\text { Pectinase }\end{array}$ \\
\hline$H$ & 2 & 1 & 0 & 0.1 & 0 & PEI, DS, EL2X \\
\hline$H^{\prime}$ & 2 & 1 & 0 & 0.1 & 0 & PEI, EL2X, DS \\
\hline$I$ & 2 & 1 & 0 & 0.05 & 0.05 & PEI, DS, EL2X \\
\hline$I^{\prime}$ & 2 & 1 & 0 & 0.05 & 0.05 & PEI, EL2X, DS \\
\hline$J$ & 2 & 1 & 0 & 0.025 & 0.075 & PEI, DS, EL2X \\
\hline$J^{\prime}$ & 2 & 1 & 0 & 0.025 & 0.075 & PEI, EL2X, DS \\
\hline$K$ & 3 & 1 & 0 & 0.1 & 0 & PEI, DS, EL2X \\
\hline$K^{\prime}$ & 3 & 1 & 0 & 0.1 & 0 & PEI, EL2X, DS \\
\hline$L$ & 4 & 1 & 0 & 0.1 & 0 & PEI, DS, EL2X \\
\hline$L^{\prime}$ & 4 & 1 & 0 & 0.1 & 0 & PEI, EL2X, DS \\
\hline
\end{tabular}




\subsubsection{Size and Zeta Potential Measurement of Polyelectrolyte Complex Nanoparticles}

A ZetaPALS zeta potential analyzer (Brookhaven Instruments Corp., Long Island, NY) was used to measure the mean particle size of nanoparticles. Samples of nanoparticles were diluted approximately $40 \times$ by volume with deionized water. Recorded data were the average of three measurements by detecting light scattering at a $90^{\circ}$ angle. The zeta potential was also measured by phase analysis light scattering using the same instrument. Samples were diluted approximately $20 \times$ with $1.0 \mathrm{mM} \mathrm{KCl}$ solution. Three measurements were averaged for each sample. Zeta potential was estimated using the Smoluchowski approximation from the previously measured hydrodynamic diameter and the electrophoretic mobility of the nanoparticles.

\subsubsection{Separation of Nanoparticles}

Samples of the enzyme-loaded nanoparticles were centrifuged at $14000 \mathrm{~g}$ for 1.5 hour at $4{ }^{\circ} \mathrm{C}$. Supernatants were then decanted for activity measurements.

\subsubsection{Determination of Enzyme Activity for Nanoparticles and their Supernatants}

$2.0 \mathrm{~g}$ of $5000 \mathrm{ppm}$ guar or HPG solution was mixed with $0.5 \mathrm{~g}$ of the diluted nanoparticles or supernatants. After mixing, $1.5 \mathrm{~mL}$ of the solution was placed between the plates of a parallel plate Bohlin CS rheometer (Malvern Instruments, Malvern, England) and the viscosity of the solution was measured over time at $25{ }^{\circ} \mathrm{C}$ for pectinase and $40{ }^{\circ} \mathrm{C}$ for EL2X. The time for the viscosity of the solution to fall to $50 \%$ of its initial value $\left(\mathrm{t}_{1 / 2}\right.$, hours) was used to calculate the activity of the nanoparticles. Activity was defined as the reciprocal of $t_{1 / 2}$. Activity of the free enzyme in the supernatant was also calculated using the same method. Entrapment efficiency based on enzyme activity, $\mathrm{EE}_{\mathrm{A}}$, was calculated using the activities of the supernatant and free enzyme as in Equation 50.

$$
E E_{A}=\frac{A_{E}-A_{S}}{A_{E}} \times 100 \%
$$

Equation 50 
Where $A_{E}$ is the activity of the equivalent enzyme concentration added to the nanoparticles and $A_{S}$ is the activity of the supernatant.

\subsubsection{Determination of Enzyme Concentration using SDS-PAGE}

SDS-PAGE analysis was performed using a NuPAGE ${ }^{\circledR}$ kit, following the manufacturer's instructions. ${ }^{[88,89]}$ SDS-PAGE is a type of gel electrophoresis technique which uses the sodium dodecyl sulfate to separate proteins by size, independent of their net charge. This technique works by binding of the negatively-charged SDS molecule to hydrophobic side chains of a protein. On average, one SDS molecule binds to every two residues of a typical protein, giving it a large overall negative charge proportional to the length of protein. ${ }^{[90]}$

Samples including several concentrations of enzyme and supernatants were first diluted $(30 \mu \mathrm{L}$ of samples $+10 \mu \mathrm{L}$ of buffer). One lane was loaded with a mixture of protein markers of known molecular weights in order to calibrate the gel and determine the weight of unknown proteins. After the electrophoresis, the gel was stained, destained and optically measured. The area under the intensity-distance curve was measured for different intensity peaks. Figure 54 shows a schematic picture of SDS-PAGE experimental setup.

The following procedure was followed while performing the SDS-PAGE: ${ }^{[88,89]}$

1. Samples were diluted using the sample buffer $(30 \mu \mathrm{L}$ of samples $+10 \mu \mathrm{L}$ of buffer).

2. Diluted samples were kept in $100{ }^{\circ} \mathrm{C}$ for 10 minutes to denature secondary and tertiary structures further and a negative charge proportion to the mass of each protein was applied.

3. Samples were placed in the wells with one well filled with the markers (specific proteins with known molecular weight) in order to calibrate the gel and determine the weight of unknown proteins by comparing the distance traveled relative to the marker.

4. Tanks of the electrophoresis apparatus were filled with $20 \times$ diluted running buffer (MESSDS buffer).

5. Electric current (220 V DC) was applied for 35 minutes to migrate the negatively charged proteins toward anode depending on the size. Short proteins penetrate more rapidly.

6. Samples were stained using Coomassie Brilliant Blue for 4-5 hours. The dye bound with the proteins at this time. 
7. The excess dye incorporated in the gel was removed by destaining buffer $\left(2.6 \mathrm{~L} \mathrm{H}_{2} \mathrm{O}+\right.$ $1 \mathrm{~L}$ Ethanol $+0.4 \mathrm{~L}$ Acetic Acid). Proteins were detected as blue bands on a clear background at this stage.

8. Gels were scanned to measure the concentration of the proteins.

Calibration curves of peak area against enzyme concentration were generated to determine the concentration of enzyme in the supernatants. The entrapment of enzyme in the nanoparticles based on concentration, $\mathrm{EE}_{\mathrm{C}}$, was calculated using Equation $\mathbf{5 1}$

$$
E E_{C}=\frac{C_{E}-C_{S}}{C_{E}} \times 100 \%
$$

Equation 51

Where $C_{E}$ is the enzyme concentration added to the nanoparticle system and $C_{S}$ is the concentration of enzyme in the supernatant.

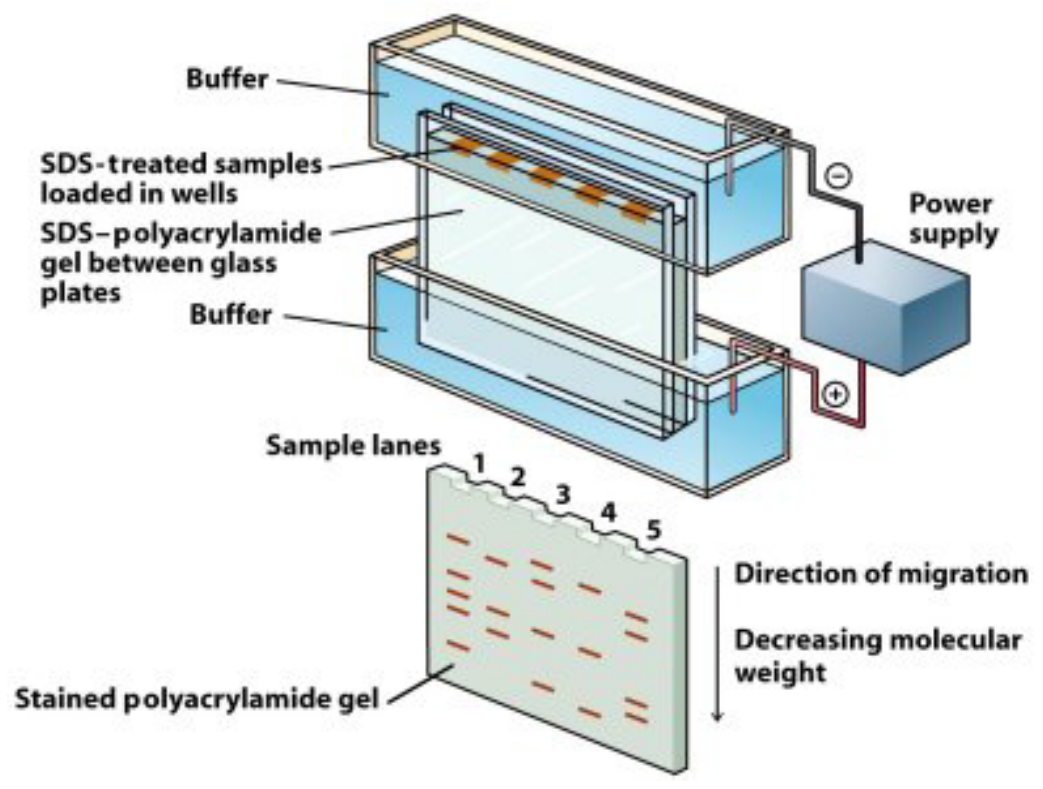

Figure 54 SDS-PAGE apparatus ${ }^{[28]}$

\subsubsection{Preparation of Guar and HPG Solutions}

Sufficient mass of polymer to create a $5000 \mathrm{ppm}$ guar or HPG solution was added slowly to the shoulder of a vortex of a vigorously $(800 \mathrm{rpm})$ stirred solution of $2 \% \mathrm{KCl}$ and $1.35 \mathrm{~g} / \mathrm{L}$ of sodium thiosulfate in a 1-liter beaker. The solution was stirred for 5 minutes after the addition of 
guar. Then the stirring rate was reduced to $600 \mathrm{rpm}$ for another hour and allowed to become hydrated for another $24 \mathrm{~h}$ at $200 \mathrm{rpm}$. Guar solutions were centrifuged at $9600 \mathrm{~g}$ for 1.5 hours at $25^{\circ} \mathrm{C}$ in order to separate the residue. ${ }^{[56]}$

\subsubsection{Preparation of Borate-Crosslinked Guar/HPG}

$24 \mathrm{~mL}$ of the $5000 \mathrm{ppm}$ HPG or guar was mixed with $7.5 \mathrm{~mL}$ of diluted enzyme-loaded nanoparticles, an aqueous solution with equivalent concentration of enzyme (concentration $=$ $0.1 \% \mathrm{w} / \mathrm{w}$ pectinase or $0.4 \% \mathrm{w} / \mathrm{w}$ EL2X) or R.O. water with adjusted $\mathrm{pH} 8$ for the control cases. $7.5 \mathrm{~mL}$ of a $1000 \mathrm{ppm}$ borax aqueous solution for the $25{ }^{\circ} \mathrm{C}$ applications or $2000 \mathrm{ppm}$ borax aqueous solution for $40{ }^{\circ} \mathrm{C}$ applications was added to the mixture and the $\mathrm{pH}$ was adjusted using $0.1 \mathrm{M} \mathrm{NaOH}$. R.O. water with adjusted $\mathrm{pH}$ was used for the control polymeric solution cases. Gel samples were incubated on a table shaker (LAB-LINE $3520 \mathrm{JR}$, Melrose, IL) at $150 \mathrm{rpm}$ at $25{ }^{\circ} \mathrm{C}$ or $40{ }^{\circ} \mathrm{C}$ depending on the enzyme used.

\subsubsection{Measurement of Viscosity and Viscoelastic Moduli}

Guar/HPG Solution: A Bohlin CS10 rheometer equipped with a 4/40 cone and plate geometry was used to measure the viscosity of guar and HPG solutions under continuous shear. All the viscometric assays were performed under a shear rate of $90 \mathrm{~s}^{-1}$, except for the shear sensitivity analysis, in which viscosities were also measured at 180 and $270 \mathrm{~s}^{-1}$.

Gelled Guar/HPG: A digital cone-and-plate viscometer (DVII+ Pro, SP-40 $0.8^{\circ}$ cone, Brookfield Engineering, Middleboro, MA) was used to monitor the viscosity of the gelled guar or HPG at $0.6 \mathrm{rpm}\left(4.5 \mathrm{~s}^{-1}\right)$ versus time. Table 12 shows the shear rate calculated for different RPM values using this geometry of viscometer. This technique is convenient due to the closure surrounding the platens that retain the gel in place, and is a method of choice in industry. It is included here to allow comparison to existing data. However, viscosity is not strictly a property of gels and so in addition, a Bohlin CS10 rheometer was used to measure the elastic $\left(\mathrm{G}^{\prime}\right)$ and viscous $\left(\mathrm{G}^{\prime \prime}\right)$ moduli for $30 \mathrm{~mL}$ gel samples over time at a frequency of $0.5 \mathrm{~Hz}$, strain of $0.1 \mathrm{~Pa}$, and initial stress of $0.1 \mathrm{~Pa}$ in "auto-stress" mode. The double-gap configuration of Couette geometry was used to measure $\mathrm{G}^{\prime}$ and $\mathrm{G}^{\prime \prime}$. 
Table 12 Shear rate calculated for different RPM values of Brookfield viscometer fitted with SP-40 spindle

\begin{tabular}{|c|c|}
\hline RPM & Shear rate, $\mathbf{s}^{-1}$ \\
\hline 0.6 & 4.5 \\
\hline 1.5 & 11.25 \\
\hline 3 & 22.5 \\
\hline 6 & 45 \\
\hline 12 & 90 \\
\hline 30 & 225 \\
\hline 60 & 450 \\
\hline
\end{tabular}

\subsubsection{Shear Loop Experiments}

A $1031 \mathrm{ft}$ shear loop was available in TORP and was modified to include a 4-way and inline mixer to allow injection of HPG, breaker and borax. Five valves were installed along the loop to make the user capable of taking samples. The stainless steel tube with ID of 0.0566 in was divided into five sections with sections 1 and 2 being $205 \mathrm{ft}$, section 3 being $206 \mathrm{ft}$, section 4 being $207 \mathrm{ft}$, and section five being $208 \mathrm{ft}$ long. ${ }^{[91]}$ The volume of the shear loop itself was 510 $\mathrm{mL}$. Calcuated volume for the first three sections was $304.8 \mathrm{~mL}$ and the tubing volume before the fluid reaches the shear loop was $5.15 \mathrm{~mL}$. Figure 55 shows a schematic of the shear loop. The shear loop was placed in a temperature controlled air bath. Three Constametric pumps capable of injecting fluids at up to $10 \mathrm{~mL} / \mathrm{min}$ were used to inject $5000 \mathrm{ppm}$ HPG solution, borax and breaker/nanoparticles. Figure 56 shows a picture of the setup used for the experiments available in TORP labs. Table 13 shows the mixing rates of different aqueous solutions.

Shear rates and the residence time calculated for three sections (616 ft) of shear loop typically used during the tests are reported in Table 14. A total flow rate of $3.26 \mathrm{~mL} / \mathrm{min}$ results in $186 \mathrm{~s}^{-1}$ shear rate. 
Table 13 Mixing flow rates used for different shear loop experiments

\begin{tabular}{|c|c|c|c|c|c|}
\hline & $\begin{array}{c}5000 \text { ppm } \\
\text { HPG solution }\end{array}$ & $\begin{array}{c}\text { Aqueous borax } \\
\text { solution (1000 } \\
\text { or } 2000 \text { ppm) }\end{array}$ & $\begin{array}{c}\text { Diluted } \\
\text { nanoparticles } \\
\text { (loaded or } \\
\text { control) }\end{array}$ & $\begin{array}{c}\text { Diluted } \\
\text { enzyme } \\
\text { solutions } \\
(0.1 \% \text { w/w } \\
\text { pectinase } \\
\text { or } 0.4 \% \\
\text { w/w EL2X) }\end{array}$ & $\begin{array}{c}\text { R.O. Water, } \\
\text { pH adjusted to } \\
8 \text { using } 0.1 \mathrm{M} \\
\mathrm{NaOH}\end{array}$ \\
\hline HPG solutions & 2 & 0 & 0 & 0 & 1.25 \\
\hline HPG gel & 2 & 0.65 & 0 & 0 & 0.65 \\
\hline $\begin{array}{c}\text { HPG gel + } \\
\text { Nanoparticles } \\
\text { (controlled or } \\
\text { loaded) }\end{array}$ & 2 & 0.65 & 0.65 & 0 & 0 \\
\hline $\begin{array}{c}\text { HPG gel + } \\
\text { diluted enzyme } \\
\text { solutions }(0.1 \% \\
\text { w/w pectinase or } \\
0.4 \% \text { w/w } \\
\text { EL2X) }\end{array}$ & 2 & 0.65 & 0 & 0.65 & 0 \\
\hline
\end{tabular}

Table 14 Shear calculations for three sections of shear loop

\begin{tabular}{|c|c|c|}
\hline Shear Rate, $\mathbf{s}^{-1}$ & Q,mL/min & $\begin{array}{c}\text { Injection Time for } \\
\text { one void volume, } \\
\text { min }\end{array}$ \\
\hline 100 & 1.75 & 177.60 \\
\hline 186 & 3.26 & 95.48 \\
\hline 200 & 3.50 & 88.80 \\
\hline 300 & 5.25 & 59.20 \\
\hline 400 & 7.00 & 44.40 \\
\hline 500 & 8.75 & 35.52 \\
\hline 600 & 10.50 & 29.60 \\
\hline 700 & 12.25 & 25.37 \\
\hline 800 & 14.00 & 22.20 \\
\hline 900 & 15.75 & 19.73 \\
\hline 1000 & 17.50 & 17.76 \\
\hline & & \\
\hline
\end{tabular}




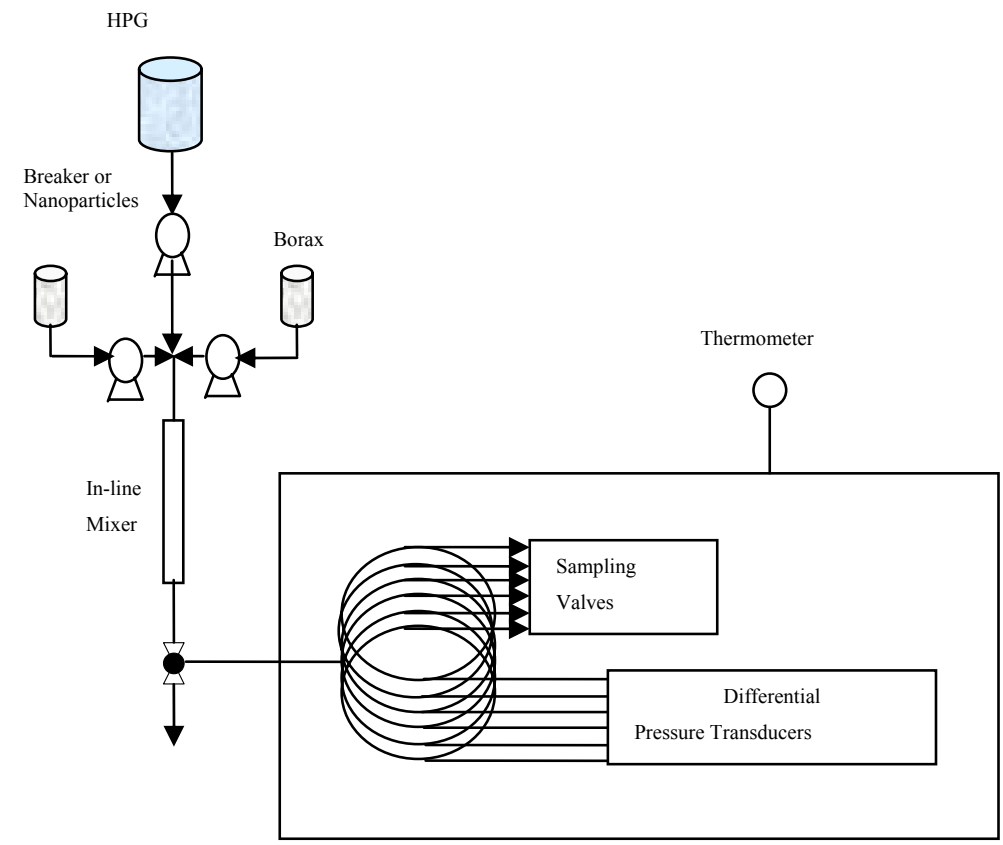

Figure 55 Schematic picture of the shear loop

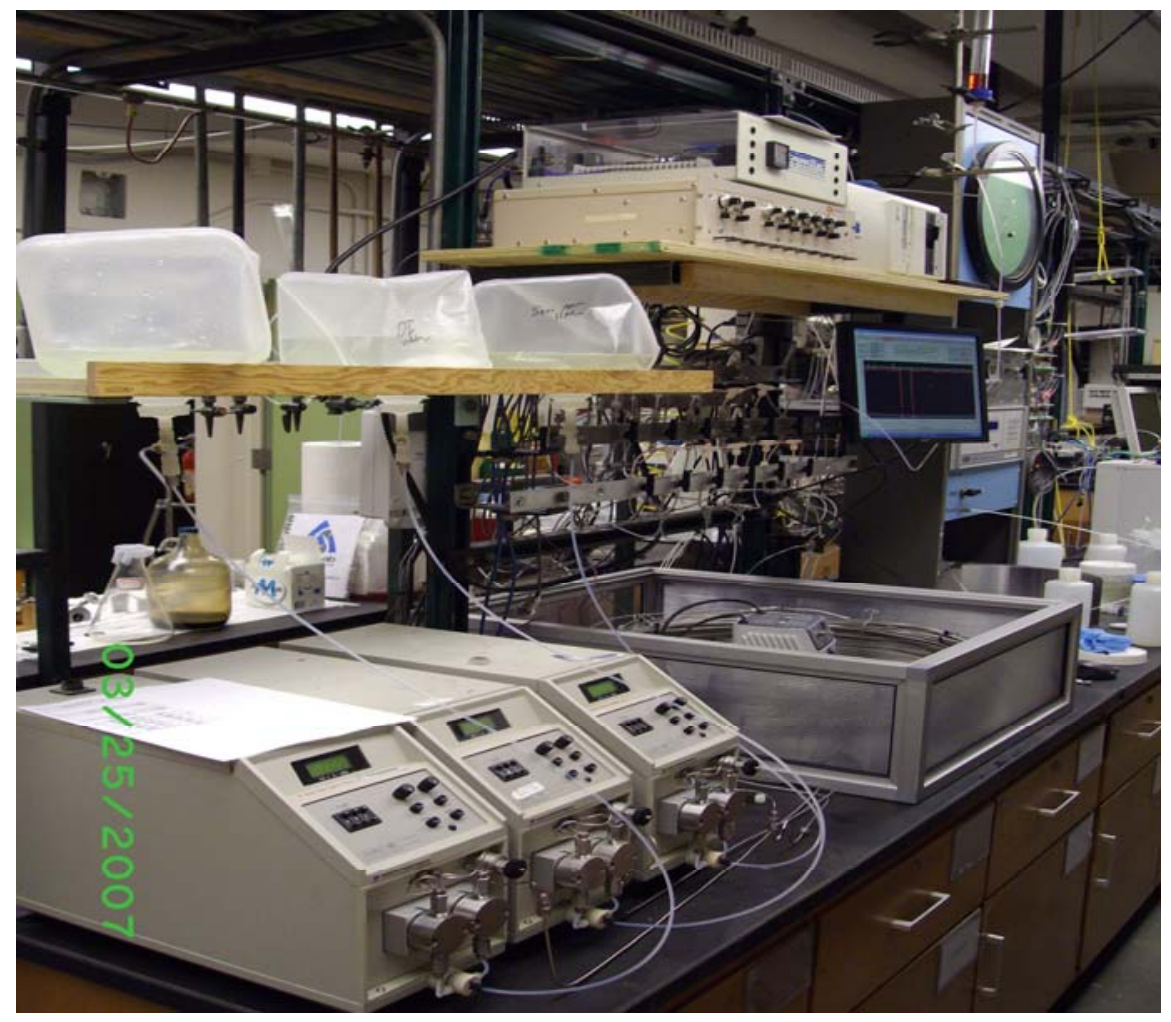

Figure 56 Shear loop set up including three Constametric pumps, temperature controlled air bath, differential pressure transducers and the computer with Labview software used to monitor and save the data over time 


\subsubsection{Core Characterization}

Core Saturation and Porosity Measurement: After measuring the length and diameters of cores they were dried in a $73{ }^{\circ} \mathrm{C}$ oven for at least 48 hours till they reached a constant weight. Using a Hassler-type core holder connected to a vacuum pump, cores were evacuated for 1 hour under $500 \mathrm{psi}$ confining pressure. The valve to the vacuum pump was closed and inlet valve was opened to allow the inhibition of $2 \% \mathrm{KCl}$, for clean cores, or $2 \%$ bleach in $2 \% \mathrm{KCl}$ when the cores were being restored. Inlet lines were filled with the saturation fluid before opening the inlet valve. Figure 57 is a schematic of the core saturation setup. Given the weight of cores saturated with fluids of known density, porosity was calculated using the following equation (Table 10). When the cores were restored after the tracer test or after the fluid loss tests they were dried and saturated again with either 2\% $\mathrm{KCl}$ or $(2 \%$ bleach in $2 \% \mathrm{KCl})$ respectively. In Equation 52:

$\mathrm{PV}=$ Pore volume, $\mathrm{cm}^{3}$

$\mathrm{W}_{\mathrm{D}}=$ Dry weight of core, $\mathrm{g}$

$\mathrm{W}_{\mathrm{S}}=$ Saturated weight of core, $\mathrm{g}$

$\rho=$ Density of saturating fluid, $\mathrm{g} / \mathrm{cm}^{3}$

$P V=\frac{\left[W_{D}-W_{S}\right]}{\rho}$

Equation 52

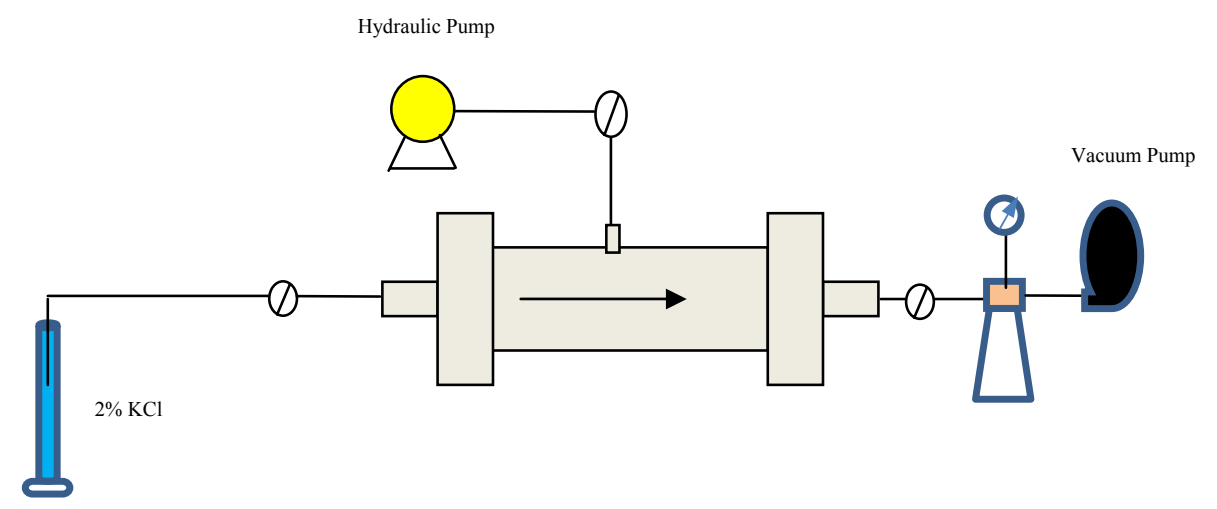


Permeability Measurement: Figure 58 shows the apparatus used to measure the permeability of the cores and conduct tracer tests to find the homogeneity and check the porosity of the cores. An ISCO pump filled with SOLTROL-130 was connected to two transfer cylinders filled with SOLTROL- 130 and $2 \% \mathrm{KCl}$ or $\left(2 \% \mathrm{KCl}+1 \% \mathrm{KNO}_{3}\right)$ as conservative tracer. Valving required for switching between the two fluids is provided. A Hassler-type core holder supported by a hydraulic pump, in order to apply 750 psi confining pressure, was connected to the transfer cylinders and the output was connected to UV-vis. A differential pressure transmitter (Honeywell) connected to the inlet and outlet of the core and the tubing connecting the transmitter to the flow line was filled with SOLTROL-130. The whole setup was at room temperature. Permeability measurement and tracer tests were run for the cores saturated in the previous section. Permeability measurement for the cores used for fluid loss tests were also done using this setup.

Pressure drop, temperature of cabinet, viscosity, calculated permeability using Darcy's law and UV absorbance were recorded during each test using the LabView (National Instruments Corporation) software. A $\log$ file including the mentioned data was also generated by the software for each test.

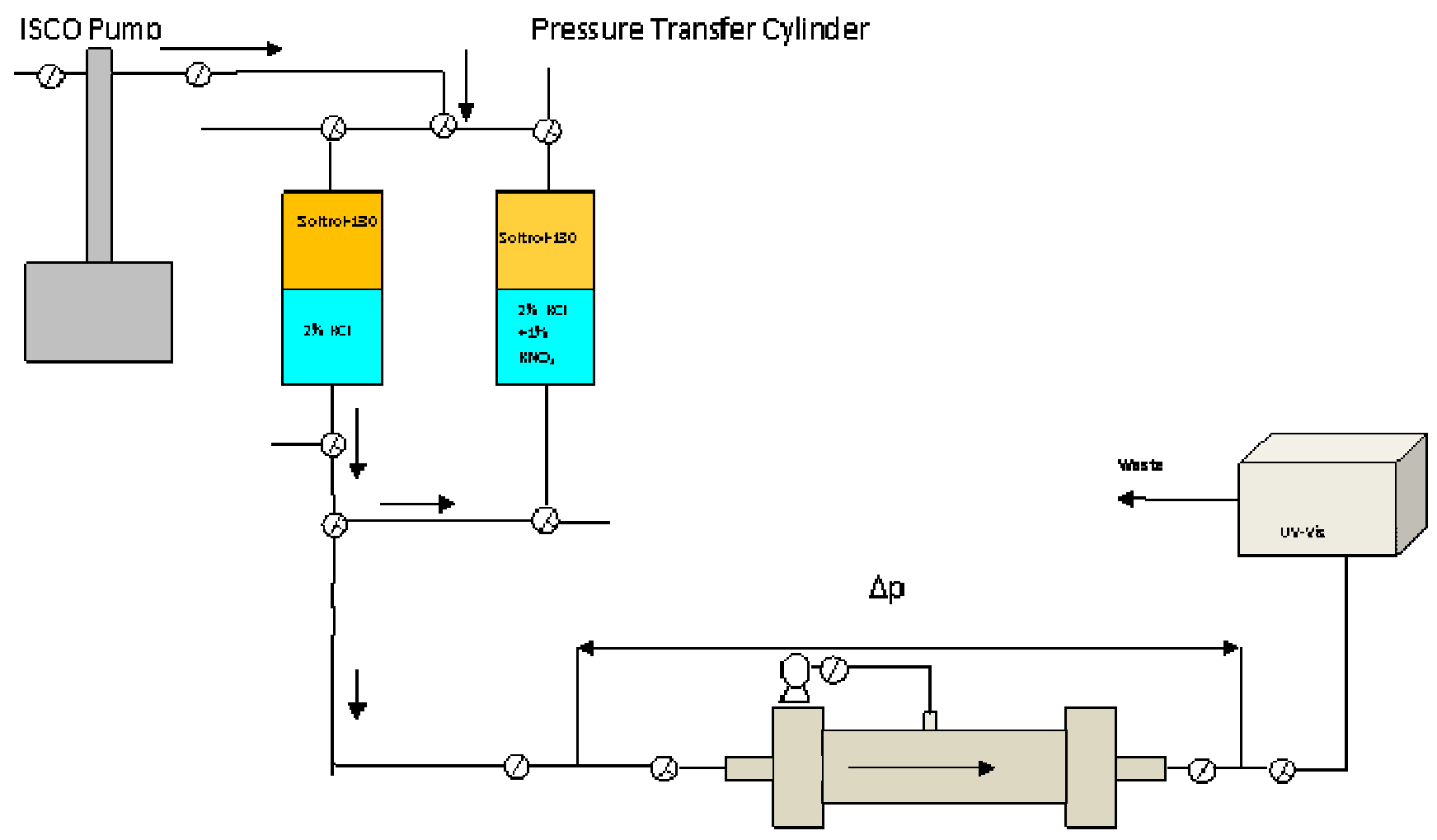

Figure 58 Schematic of the permeability measurement and tracer test setup 
Tracer Tests: Tracer tests were performed to assure the homogeneity of the cores used for the fluid loss experiments. Pore volume of the cores was also calculated from the tracer test results to confirm the pore volume measured by weighing the cores.

During displacement of a fluid with another miscible fluid in a porous medium, mixing occurs in the transition zone. Tracers can be used as the "mixed" chemical. Tracer is a dissolved component that is neither adsorbed nor generated in the core. Mixing and spreading of tracer in the transition zone is known as dispersion. Normalized concentration of the displacing fluid increases from zero to one, while concentration of the displaced fluid decreases from one to zero. Peclet number defined as in Equation 54 is a measure of dispersion shown in Figure 59. The higher the Peclet number the smaller the mixing zone. A symmetric S-shaped curve is representative of an ideal homogenous core. When $C$ (Equation 55) at the outlet reaches 0.5, the dimensionless time (Equation 53) represents the pore volume of core.

A long tail of tracer curve caused by heterogeneity and blind-ended pores that exist in every core results in the breakthrough occurrence at $\mathrm{C}$ values different than 0.5 . For such cases pore volume can be calculated using an equal area method. In this case instead of using the time to reach $\mathrm{C}=0.5$ the time at which the area calculated under the concentration versus time curve from one side is equal to the time above the curve from the other side is used. ${ }^{[22]}$ Subtracting the starting time from this time and knowing the flow rate, pore volume can be calculated. Note that any dead volume must be subtracted from the calculated pore volume.

During a typical tracer test cores are flooded using the base fluid $(2 \% \mathrm{KCl}$ in this case) and then the fluid is switched to the base fluid with a known concentration of $\operatorname{tracer}\left(1 \% \mathrm{KNO}_{3}\right.$ in $2 \% \mathrm{KCl}$ in this case). Tracer concentration is measured at the outlet versus time. In the case of $\mathrm{KNO}_{3}$, $\mathrm{UV}$-vis is used to measure the concentration of tracer via UV absorbance at $302 \mathrm{~nm}$ wavelength. Constant flow rate of $1 \mathrm{~mL} / \mathrm{min}$ was used for the tracer tests. Both pressure drop and UV absorbance were recorded versus time. Figure 58 shows a schematic of the permeability measurement and tracer test setup. 
Where

$\mathrm{C}=$ Normalized tracer concentration

$\mathrm{C}_{\mathrm{i}}=$ Injected concentration of tracer

$\mathrm{C}_{0}=$ Initial concentration of tracer in the porous medium

$\mathrm{u}_{\mathrm{x}}=$ Darcy velocity of fluid, $\mathrm{cm} / \mathrm{min}$.

$\mathrm{t}=$ Injection time, minutes

$\mathrm{L}=$ Core length, $\mathrm{cm}$

$\mathrm{q}=$ Injection flow rate, $\mathrm{cm}^{3} / \mathrm{min}$.

$\mathrm{D}_{\mathrm{L}}=$ Dispersion coefficient in the direction of flow, $\mathrm{cm}^{2} / \mathrm{min}$. 


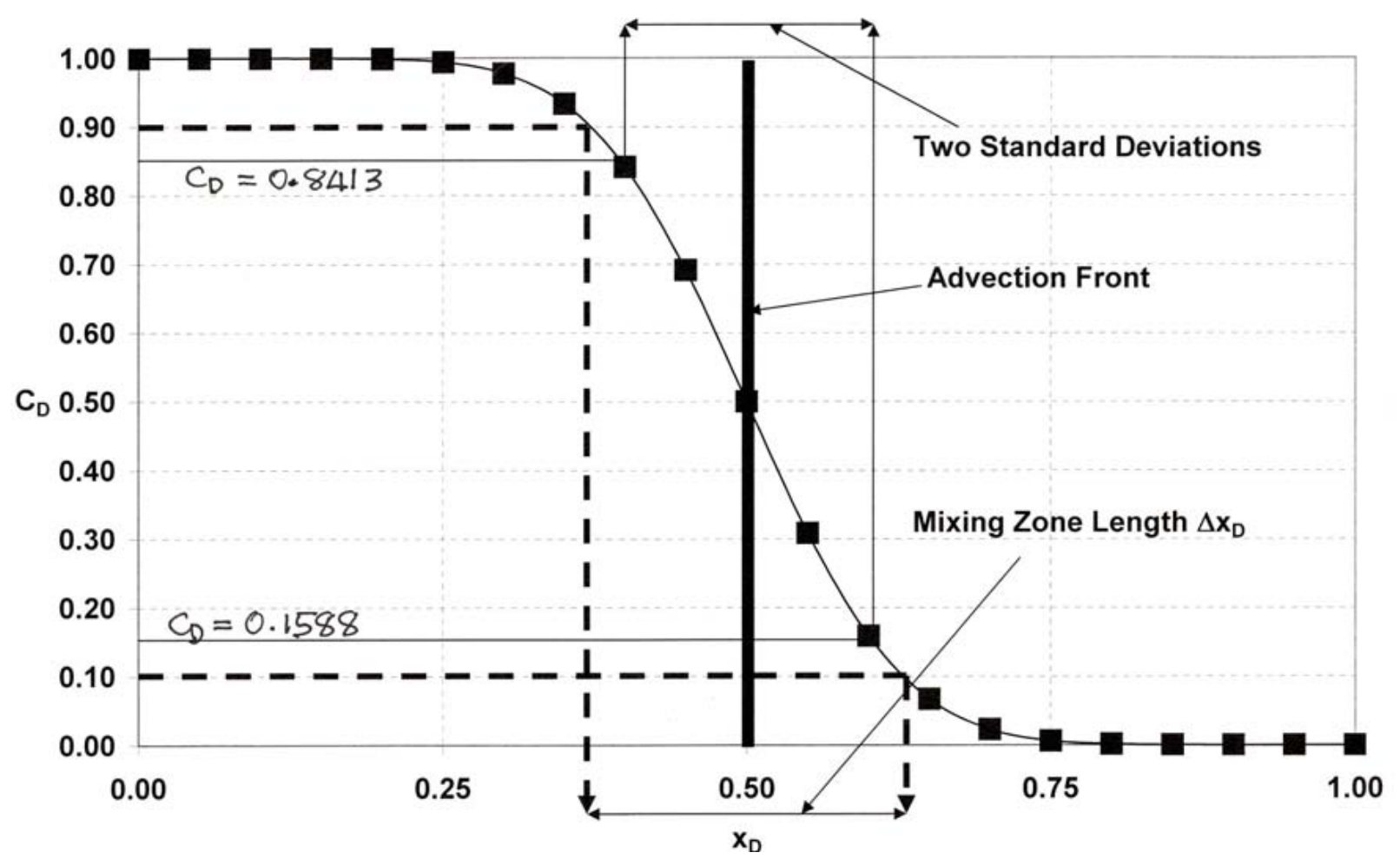

Figure 59 Solution of the convection-dispersion equation for a Peclet Number of 100 at $t_{D}=0.50$ pore volume injected $^{[03]} \mathrm{X}_{\mathrm{D}}$ is the dimensionless distance.

\subsubsection{Static Fluid Loss Tests}

The static fluid loss cell has been used traditionally to measure the fluid loss caused by fracturing or drilling fluids under a predefined pressure drop and temperature. Static fluid loss cells followed by a cleanup procedure were performed as the last phase of this work. Fracturing fluids with enzymes, enzyme-loaded nanoparticles or control nanoparticle systems with no enzyme were used. After forming the filter cake and plotting the graph of filtrate volume versus square root of time, resistivity of filter cake was measured by replacing the fluid on top of the filter cake with $2 \% \mathrm{KCl}$ and running the test until cleanup was achieved.

Flow Chart and Setup: A static fluid loss cell was obtained from Schlumberger. The cell can handle three different ends used to filter through paper, ceramic disks or core plugs. The filter paper is typically used in a mud filter press. Ceramic disks are also employed for mud filtration studies. Finally, cores were introduced with the design of the endcap setup different for different ends. 
These cells use six grub screws to secure the endcaps to the cell body. Leakage is prevented by O-ring seals plus a rubber core holder in place which prevent any possible overpass of the core. Because the screws are the only thing holding the endcap on, one must always be sure to cool cell and relieve all pressure before loosening the grub screws. The cell must not be operated above 1000 psi.

The fluid loss cell was set up according to the procedure provided by Schlumberger. The flow schematic of the setup is shown in Figure 60. A nitrogen supply provides the pressure applied for the fluid loss and the back pressure required preventing any vaporization of water in the outlet. Pressure is recorded at the inlet and the outlet and temperature is recorded at the inlet and heat jacket. The fluid loss cell is placed inside a cabinet equipped with valves used to set the inlet and backpressure (Figure 61).

The following procedure is the one recommended by Schlumberger for running the fluid loss test. $^{[94]}$

1. Bottom endcap was assembled. The endcap can be a filter paper end, disk end or core plug end.

2. Bottom endcap was inserted into cell with either 1 O-ring or 2 O-rings. The endcap outer surface was verified to be flush with the top of the cell when the grub screws were in place. If not, it was assumed that the number of O-rings is incorrect for that setup. Endcap was secured with grub screws. The screws were verified for not being deformed before being used. All six screws were tighten

3. The cell was placed on a corner of a sink. Note that this method could result in the cell falling over. A wooden holder can be made to accommodate the bottom endcap but allow the bottom endcap piping to pass through the hole in the wooden block. A block of wood can be countersunk out to a diameter slightly larger than the cell to hold the cell with its endcap extending through a smaller hole drilled in the bottom. Wearing steel toed shoes and safety glasses when handling the cell is obligatory.

4. After securing the bottom endcap, the cell was inverted and filled with the desired fluid. Some room was left for fluid expansion.

5. The top endcap was attached with O-rings and the six grub screws were tightened.

6. The cell with endcaps attached was placed into heater jacket. The inlet nitrogen was attached to the upper quick connect. With a syringe filled with the base fluid, the bottom 
exit assembly was flushed with $2 \% \mathrm{KCl}$ and quick connect was attached. This ensures that the first drop of liquid coming out should be seen on the balance as liquid will displace liquid rather than air.

7. The inlet pressure regulator was increased to desired pressure. If back pressure is used, its pressure was increased as well.

8. Heating was begun. Note that the thermocouple can be placed into the heater for preheating and into the cell for more accurate temperature monitoring during the test (Figure 63). The heater was connected to a temperature controller.

9. When the fluid reached temperature, the balance was tared, the outlet valve was opened and data collection was begun. Fluid was collected on a balance and the weight versus time was recorded using suitable data acquisition equipment. The test must be stopped before the cell is empty of liquid.

10. The cell and endcaps were cleaned thoroughly before reuse.

11. The cells received by TORP were pressure tested with the corresponding endcaps. Schlumberger typically conduct a visual inspection for signs of cracking, pitting or corrosion each year and a hydrostatic pressure test to 1.5 times the allowable working pressure every five years. This long stainless cell (PV-334) was placed into service on November 1, 1999 and was last hydraulically tested to 2250 psi on February 18, 2002. It had not been in use since 2004 . 


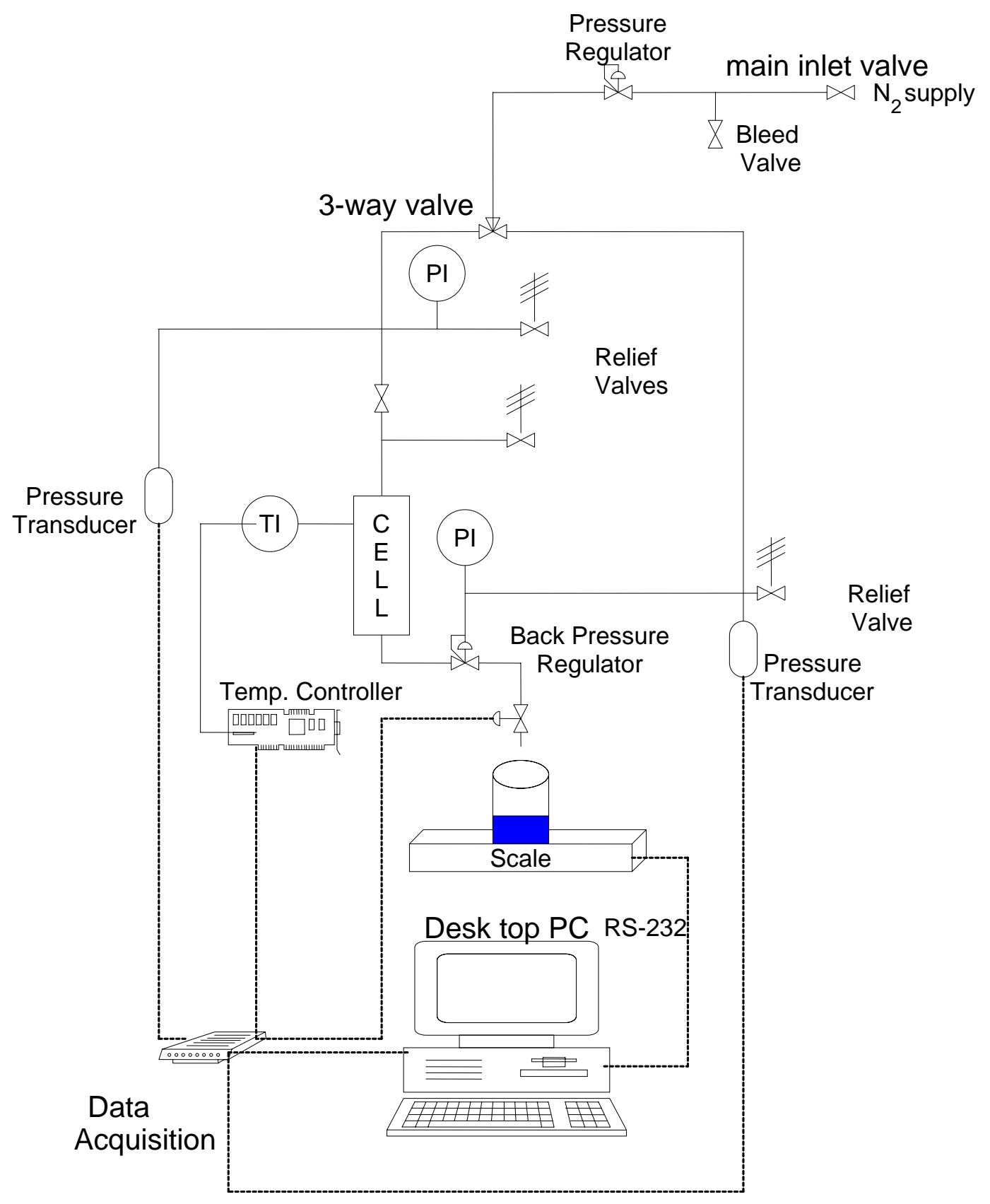

Figure 60 Fluid loss cell flow schematic (Drawn by Richard Hutchins) ${ }^{[94]}$ 


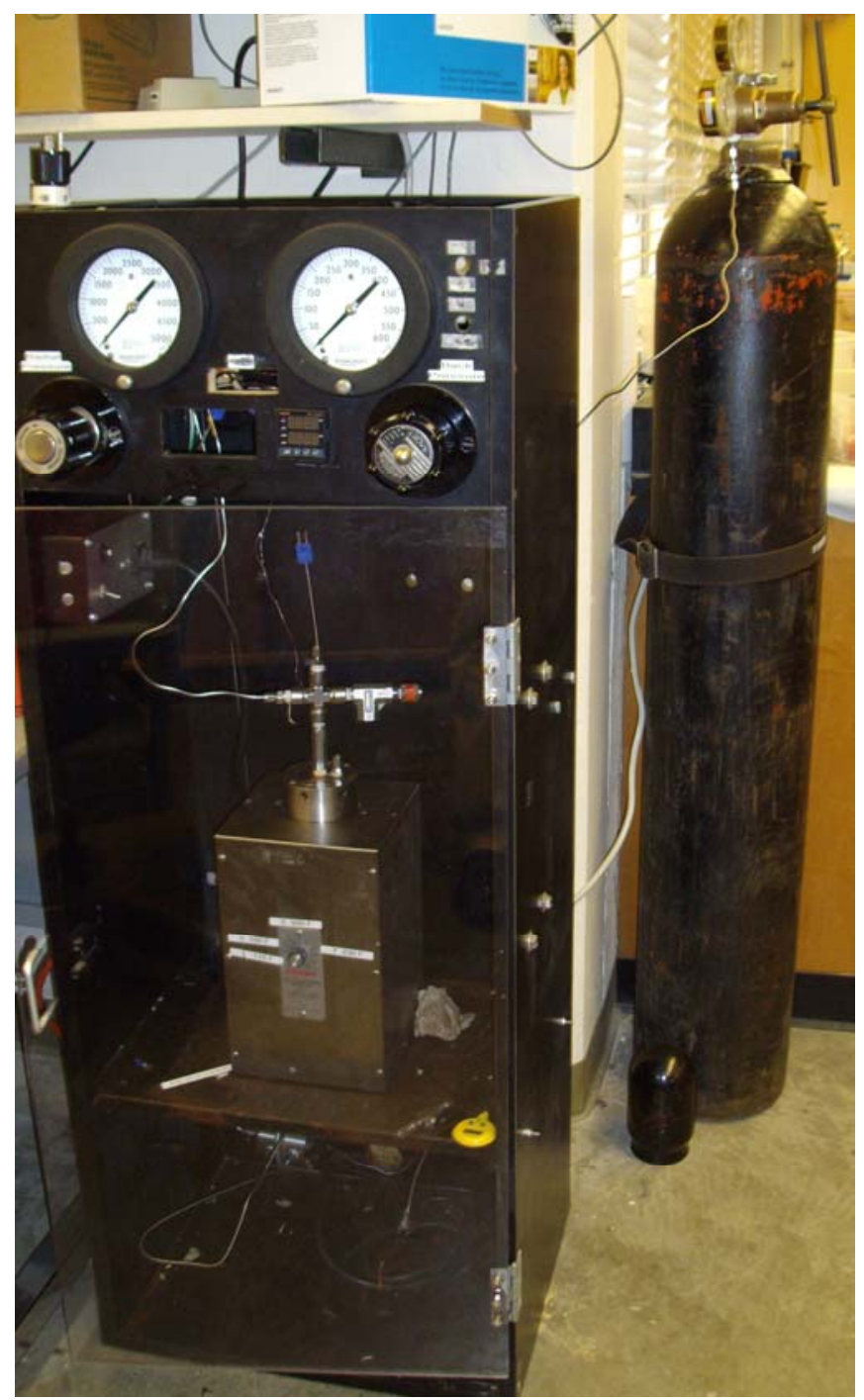

Figure 61 Fluid loss cell placed inside a pressure control cabinet supplied with a nitrogen tank 


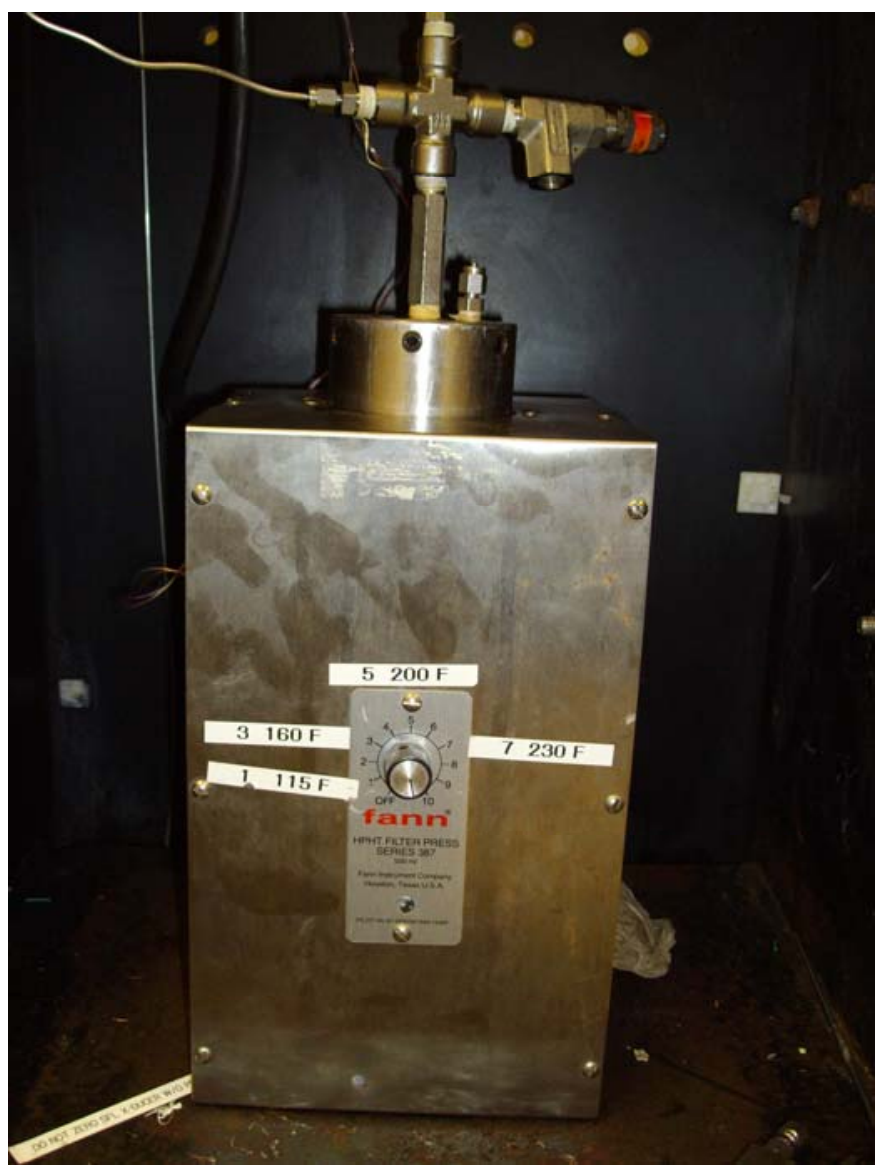

Figure 62 Cell heater with thermocouple inserted inside for preheating. A thermocouple is placed inside the heating jacket to control its temperature. 


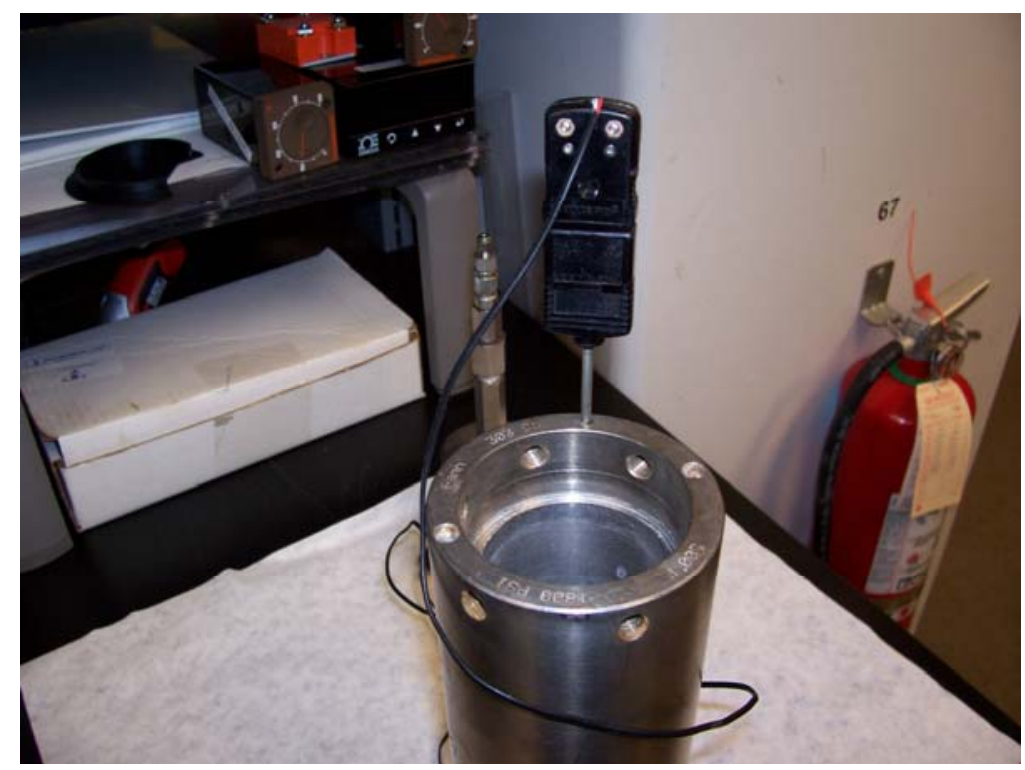

Figure 63 Fluid loss cell with thermocouple inserted (photo by Richard Hutchins, used by permission) ${ }^{[94]}$

Assembly of different endcaps is shown in Figure 64. Different endcaps are used for different applications. In order to use a core in the fluid loss cell rubber sleeve with plastic collet, a stainless retainer, a tapered stainless receptacle and an endcap having a tapered cone for sealing inside the rubber sleeve were used. The one-inch by one-inch core was inserted into the rubber sleeve. One end of the rubber sleeve was attached onto the endcap with tapered cone. The core was pushed against the tapered cone. The thin stainless retainer was screwed using small screws into the endcap to secure the sleeve against the tapered cone. The plastic collet was inserted, large diameter first, over the rubber sleeve. The large stainless shaped-receptacle was placed onto the collet and secured into the small retainer using the long screws. Screws were evenly tightened. The core was recessed to accommodate the filter cake as seen in the assembled endcap (Figure 65). 


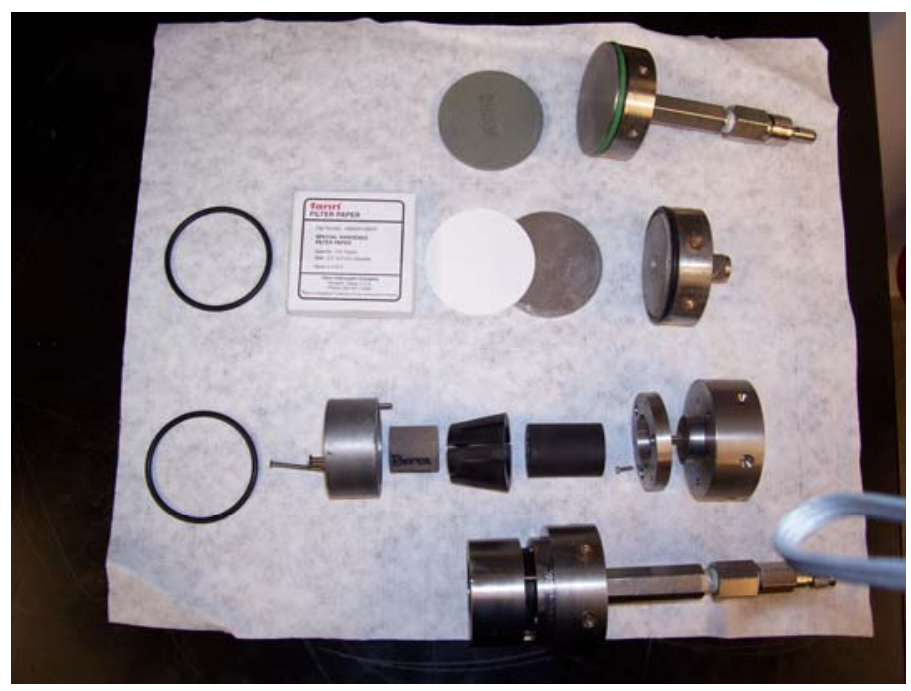

Figure 64 Various endcap assemblies for short cell. The assembled core endcap is shown at the bottom of the picture $^{[94]}$ The top two are the endcap used for disk or filter paper supported by a disk. The third and fourth from the top show the assembly of the setup for cores. (photo by Richard Hutchins, used by permission) ${ }^{[94]}$

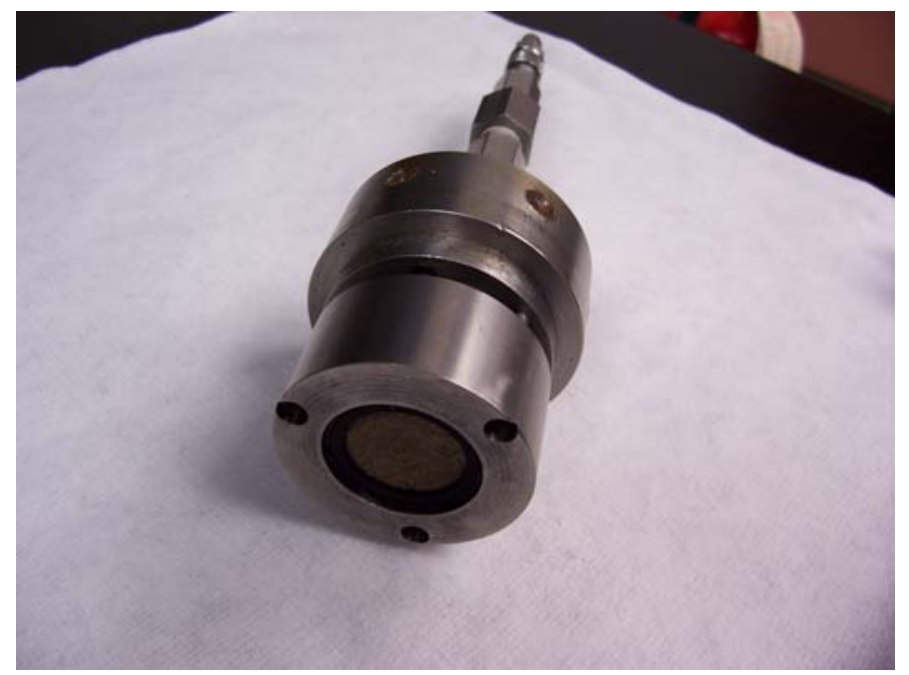

Figure 65 Assembled core endcap with core in place

Procedure for running Static Fluid Loss Tests in Order to Study the Effectiveness of Nanoparticles in Degrading Filter Cake and Preventing Fluid Loss

The following procedure was performed for running tests in order to study the effectiveness of filter cake degradation by the enzyme-loaded nanoparticles (Figure 66). 
Four cores with similar properties (porosity, permeability and tracer tests) were chosen in order to run fluid loss tests using HPG gel, HPG gel mixed with enzyme, HPG gel mixed with control nanoparticles and HPG gel mixed with enzyme-loaded nanoparticles. Porosity and permeability of each core were measured before running the fluid loss tests. Fluid loss tests were then run for 120 minutes using $200 \mathrm{~mL}$ of different fluids. At the end the cell still had some of the fluid remaining on top of the filter cake. The fluid stayed in the cell under no pressure and at test temperature $\left(25^{\circ} \mathrm{C}\right.$ or $\left.40^{\circ} \mathrm{C}\right)$ for 12 hours.

The fluid on top of the filter cake was then replaced with $250 \mathrm{~mL}$ of $2 \% \mathrm{KCl}$ and the pressure (100 psi) was applied for another 2-24 hours till the cleanup slope reached a constant value. The fluid loss during this period is representative of how effective the filter cake was degraded.

After the $2 \% \mathrm{KCl}$ flow, the filter cake was scraped off and the permeability of the core was measured again. Difference in the permeability of the core before and after the test is a measure of formation damage caused by invasion of gel or nanoparticles. This was compared between different fluids. Fluid loss from different fluids during the fluid loss tests was also compared to study the effect of nanoparticles on the fluid loss volume. These tests were repeated for two different enzymes, pectinase and EL2X at two different temperatures of $25^{\circ} \mathrm{C}$ and $40{ }^{\circ} \mathrm{C}$. 


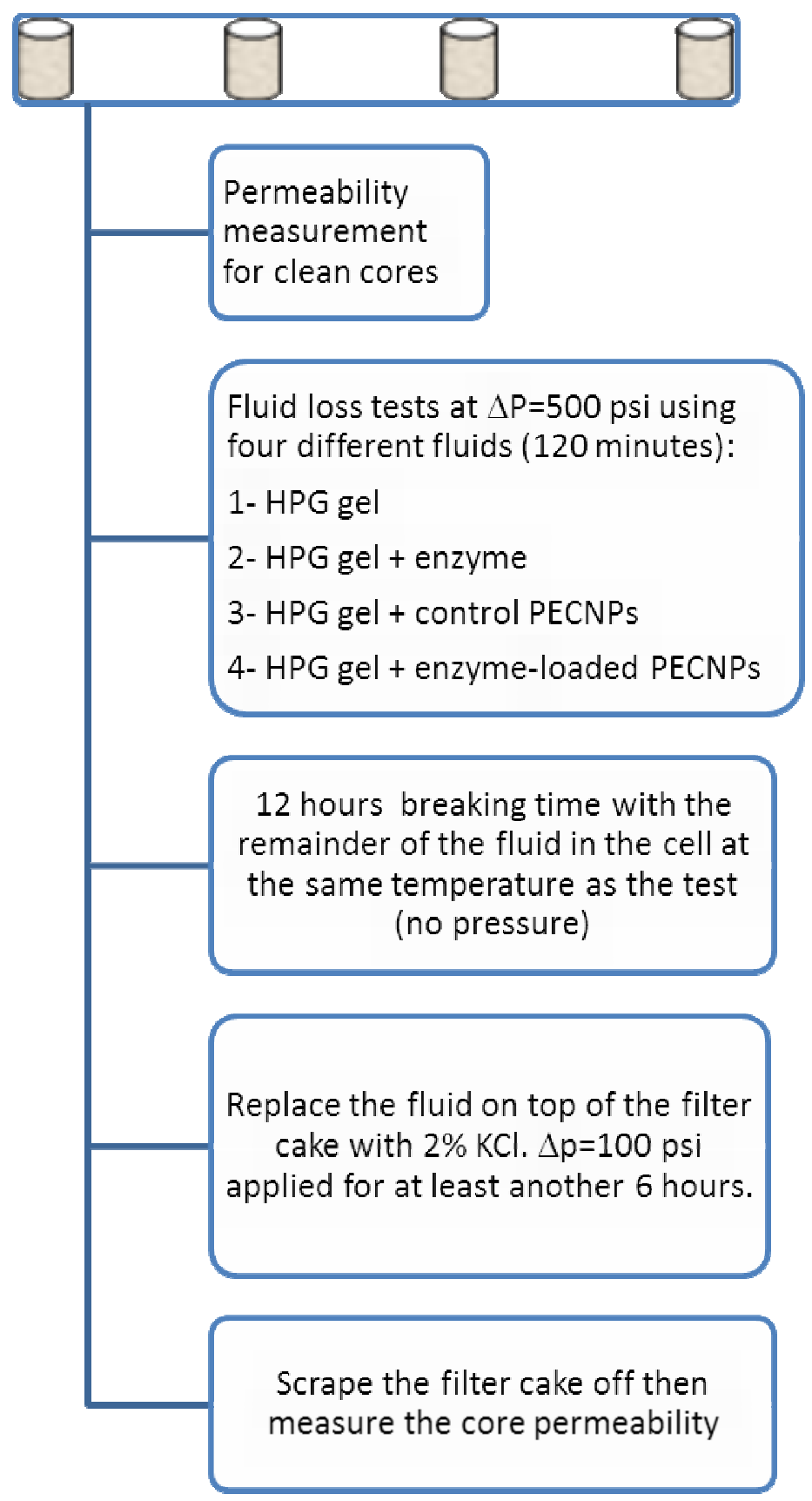

Figure 66 Procedure for the fluid loss tests

\subsubsection{Filtration of Polymeric Solutions or Gelled HPG}

A filtration setup was developed to study the measurement of fluid loss and formation of filter cake using different fluids (Figure 67). The filtration column (with $47 \mathrm{~mm}$ MFS filter holder) was connected to nitrogen pressure. A $0.45 \mu \mathrm{m}$ cellulosic filter paper (47 mm Micro Sep 
membrane filters) was used with a fiberglass pre-filter paper (2.4-6 $\mu \mathrm{m})$ used as a support under it. This was done to prevent the filter paper from breaking under pressure. The effluent was collected in a bottle placed on top of a balance. The balance was connected to a data acquisition system to record the fluid loss data. The data was plotted in real time.

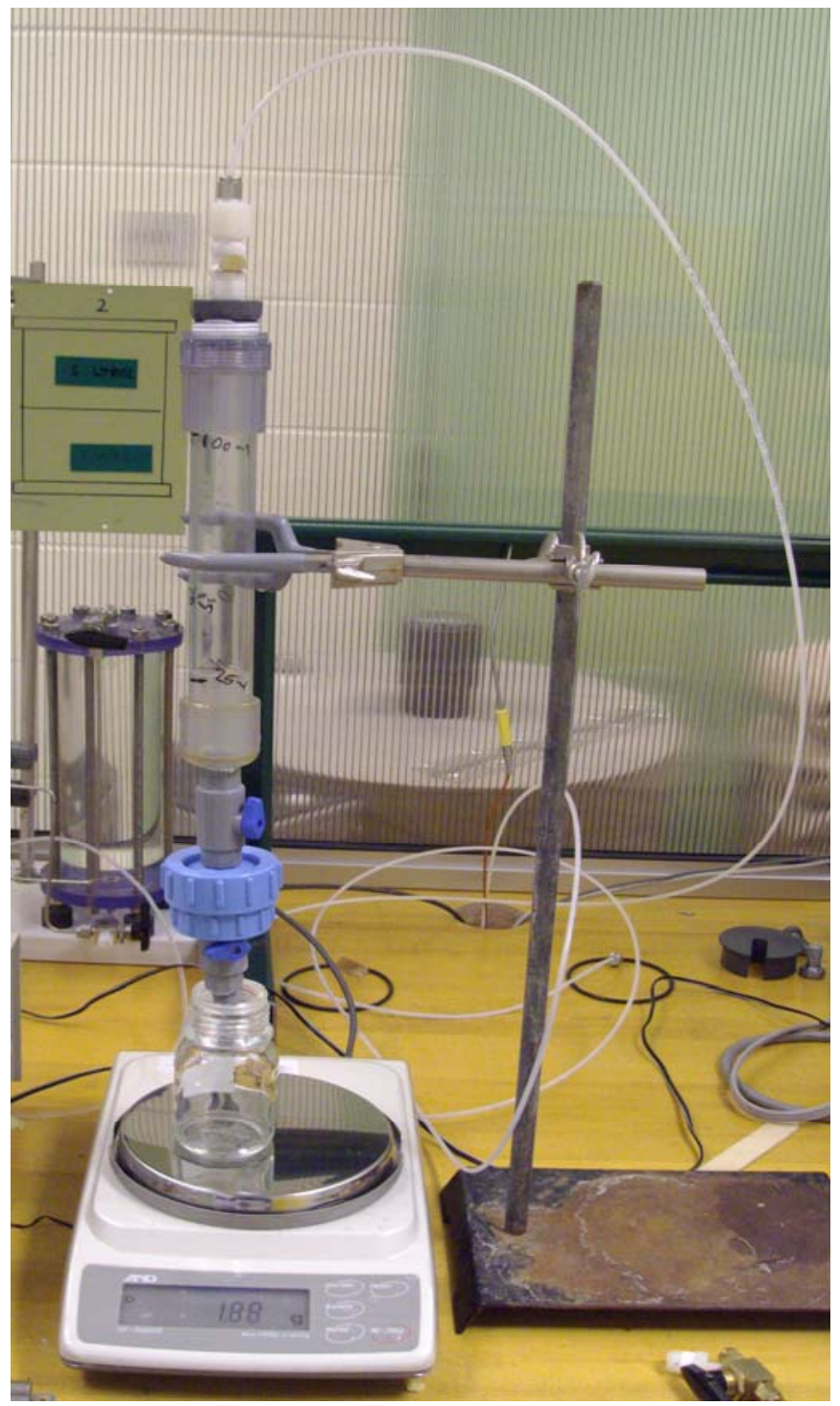

Figure 67 picture of the filtration column 


\subsubsection{Core Restoration after Tracer and Fluid Loss Tests}

Tracer tests using $1 \% \mathrm{KNO}_{3}$ in $2 \% \mathrm{KCl}$ damaged the permeability of the cores. It was possible to restore the permeability of cores, by drying at $73{ }^{\circ} \mathrm{C}$ until they reach a constant weight and then resaturating with $2 \% \mathrm{KCl}$.

In order to reuse the cores after fluid loss tests, cores were dried at $73{ }^{\circ} \mathrm{C}$ until they reach a constant weight and then saturated with $2 \%$ bleach in $2 \% \mathrm{KCl}$. Cores were then flooded with $2 \%$ $\mathrm{KCl}$ and the effluent was checked for chlorine. Flooding the cores continued until the effluent $\mathrm{Cl}$ concentration was below the detection limit of the chlorine test kit. Permeability of the cores was then measured to assure restoration.

\subsubsection{Chlorine Measurement in Effluent During Core Cleanup after Restoration}

SenSafe ${ }^{\mathrm{TM}}$ Free Chlorine water check kit (Industrial Test Systems, Inc., Rock Hill, SC) was used to estimate the chlorine content in the outlet of core cleanup after core restoration. Figure $\mathbf{6 8}$ shows the concentration range of chlorine shown by different colors.

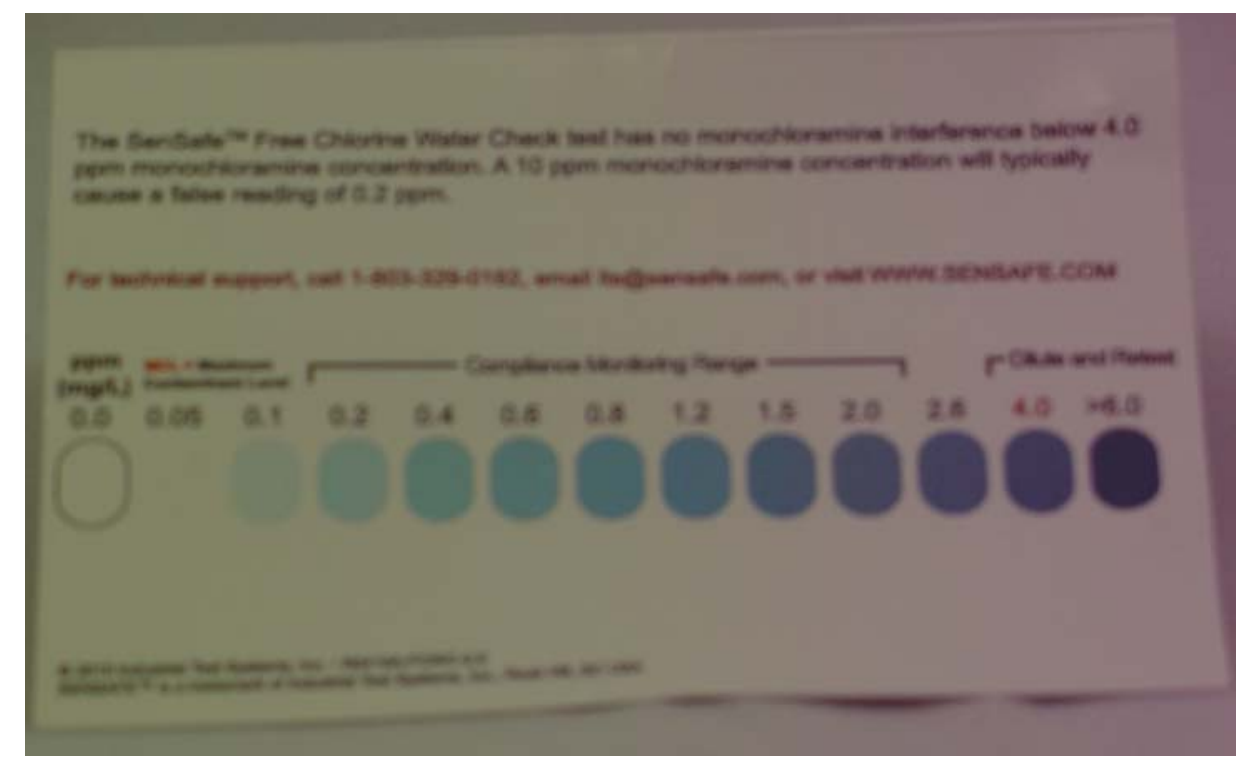

Figure 68 Chlorine test kit, ppm concentrations shown by different colors 


\subsubsection{Measurement of pH for Solutions and Gelled Polymers}

Twin $\mathrm{pH}$ meter (HORIBA B-213) was used to measure the $\mathrm{pH}$ of small samples (between 0.1 $\mathrm{mL}$ and $40 \mathrm{~mL}$ ). However, Accumet (AB15) basic $\mathrm{pH}$ meter (Fisher Scientific) fitted with a glass $\mathrm{pH}$ electrode was used for large samples (typically HPG polymer solutions and HPG gel samples).

\subsubsection{Total Organic Carbon and Nitrogen Measurement}

An Apollo $9000^{\mathrm{TM}}$ combustion TOC/TON analyzer (Teledyne Instruments) was used to measure the total organic carbon and nitrogen of the fluids used during the course of this research. Multi element calibration standard (Perkin Elmer Life and Analytical Sciences, Shelton, CT, Lot No. 18-195JB), Nitrogen standard (Lot No. 1007207), Carbon standard organic (Lot NO. 4911001) and carbon standard inorganic (Lot No.4911001) all from Ricca Chemical Company (Arlington, TX) were used as standards for the analysis.

The Apollo $9000^{\mathrm{TM}}$ measures the total nitrogen and carbon by converting all forms of nitrogen to $\mathrm{NO}$ and all forms of carbon to $\mathrm{CO}_{2}$. After being converted, $\mathrm{NO}$ and $\mathrm{CO}_{2}$ are swept into the nondispersive infrared detector to measure the concentration of $\mathrm{CO}_{2}$ in the sample gas. Next, excited $\mathrm{NO}_{2}\left(\mathrm{NO}_{2}{ }^{*}\right)$ is formed by mixing with ozone. $\mathrm{NO}_{2}{ }^{*}$ gives off extra energy as light while returning to its original state. The light signal is then converted to an electronic signal. The amount of light detected is proportional to the amount of NO in the sample gas ${ }^{[95]}$. Plotting the area under the curve of detected voltage $(\mathrm{mV})$ vs. time generates the calibration curve. Samples were measured and their carbon or nitrogen concentration was found using the generated calibration curves. 


\section{Results and Discussion}

Experimental work performed during the course of this research and discussion of results are presented in this chapter. Section 4.1 covers the studies performed to prepare a guar or HPG solution or borate cross-linked guar or HPG. Section 4.2 presents the experimental work done to characterize the enzyme and measure the activity and concentration of enzymes. In section 4.3 the results related to preparation and optimization of enzyme-loaded nanoparticles are presented. Section 4.4 shows the results related to degradation of borate cross-linked guar/HPG using enzymes and enzyme-loaded nanoparticles. Results of experimental work related to the protective effect of nanoparticles on enzymes are demonstrated in section 4.5. Section 4.6 covers the results of experiments performed to study the effect of simulated wellbore shear on enzymeloaded nanoparticles. Filter-cake formation and cleanup results are discussed in section 4.7. In a different application, section 4.8 presents a proof of concept for the application of enzymeloaded nanoparticles in slick-water fracturing jobs.

\subsection{Preparation of Guar/HPG Solutions and Borate Cross-linked Guar/HPG}

During the course of this research, guar polymer was used for some of the $25{ }^{\circ} \mathrm{C}$ applications while HPG was used for both $25{ }^{\circ} \mathrm{C}$ and $40{ }^{\circ} \mathrm{C}$ applications. Use of different polymers will be described for different experiments.

Guar and HPG solutions were prepared in a solution of $2 \% \mathrm{KCl}$ and $1.35 \mathrm{~g} / \mathrm{L}$ of sodium thiosulfate. Guar/HPG was added to the shoulder of a vortex of a vigorously stirred solution. The polymer solution was stirred at high rate $(800 \mathrm{rpm}$ in a 1-liter beaker or $600 \mathrm{rpm}$ in a $0.5 \mathrm{~L}$ beaker) for 5 minutes. Mixing rate was then reduced to $600 \mathrm{rpm}$ for the $1 \mathrm{~L}$ beaker or to $500 \mathrm{rpm}$ for the $500 \mathrm{~mL}$ beaker and the fluid was stirred for another 1 hour. Finally, the solution was hydrated for another 24 hours at $200 \mathrm{rpm}$ after $0.1 \mathrm{~mL}$ of $1 \mathrm{M} \mathrm{NaOH}$ per $500 \mathrm{~mL}$ of solution was added to adjust $\mathrm{pH}$. Guar solutions were centrifuged at $9600 \mathrm{~g}$ for 1.5 hours in $25^{\circ} \mathrm{C}$ in order to separate the residue after hydration. ${ }^{[96]}$

\section{1-1 Centrifuged Guar Solutions}

Guar solutions were first centrifuged in order to separate the residue. Density of the solutions decreased slightly after the centrifuge. Residues were also dried in a $40{ }^{\circ} \mathrm{C}$ oven to measure the 
polymer weight separated from the intended $5000 \mathrm{ppm}$ solution. The final weight of dried residue was checked with the difference in the density of solution before and after being centrifuged. Reproducibility of the process was assured by making six different samples of 5000 ppm guar in $2 \% \mathrm{KCl}$. Weight of residues was observed versus time while they were sealed in the centrifuge tube and kept in a $40{ }^{\circ} \mathrm{C}$ oven (Table 15). Guar solutions showed around 3.7-5\% w/w of wet residues and around $0.11-0.15 \% \mathrm{w} / \mathrm{w}$ of dried residues.

Table 15 Weight percent of residues taken from 5000 ppm guar solution versus time

\begin{tabular}{|c|c|c|c|c||}
\hline \multirow{2}{*}{ Sample\# } & \multicolumn{4}{|c|}{ Weight \% of residue } \\
\cline { 2 - 5 } & $\mathbf{0 ~ h}$ & $\mathbf{2 4} \mathbf{~ h}$ & $\mathbf{7 2} \mathbf{~ h}$ & $\mathbf{1 6 8} \mathbf{~ h}$ \\
\hline 1 & 3.69 & 1.52 & 0.13 & 0.12 \\
\hline 2 & 4.38 & 2.03 & 0.14 & 0.11 \\
\hline 3 & 4.05 & 1.53 & 0.14 & 0.13 \\
\hline 4 & 4.08 & 1.72 & 0.16 & 0.14 \\
\hline 5 & 4.38 & 2.12 & 0.16 & 0.15 \\
\hline 6 & 4.92 & 2.77 & 0.33 & 0.15 \\
\hline
\end{tabular}

The effect of hydration time on viscosity of guar solution after centrifuging was also studied. A sample of $5000 \mathrm{ppm}$ guar in $2 \% \mathrm{KCl}$ was centrifuged after 1 hour of vigorous stirring while another sample was centrifuged after 24 hours of hydration. 24-hour hydration of guar after the vigorous stirring for 1 hour increased the viscosity of the final solution significantly. However, it did not change the rate of hydrolysis versus time (Figure 69).

Guar solutions prepared in $2 \% \mathrm{KCl}$ showed a reproducible viscosity curve under different shear rates. For example samples made on three different days gave essentially the same viscosity versus shear curves (Figure 70). Fitting the linear part of the curves using a power-law model gave an average flow behavior index $\left(\mathrm{n}^{\prime}\right)$ of 0.34 (with standard deviation of 0.01 ) and average flow consistency index ( $\mathrm{K}^{\prime}$ ) of $160.67 \mathrm{mPa} . \mathrm{s}$ (with standard deviation of 8.7). 


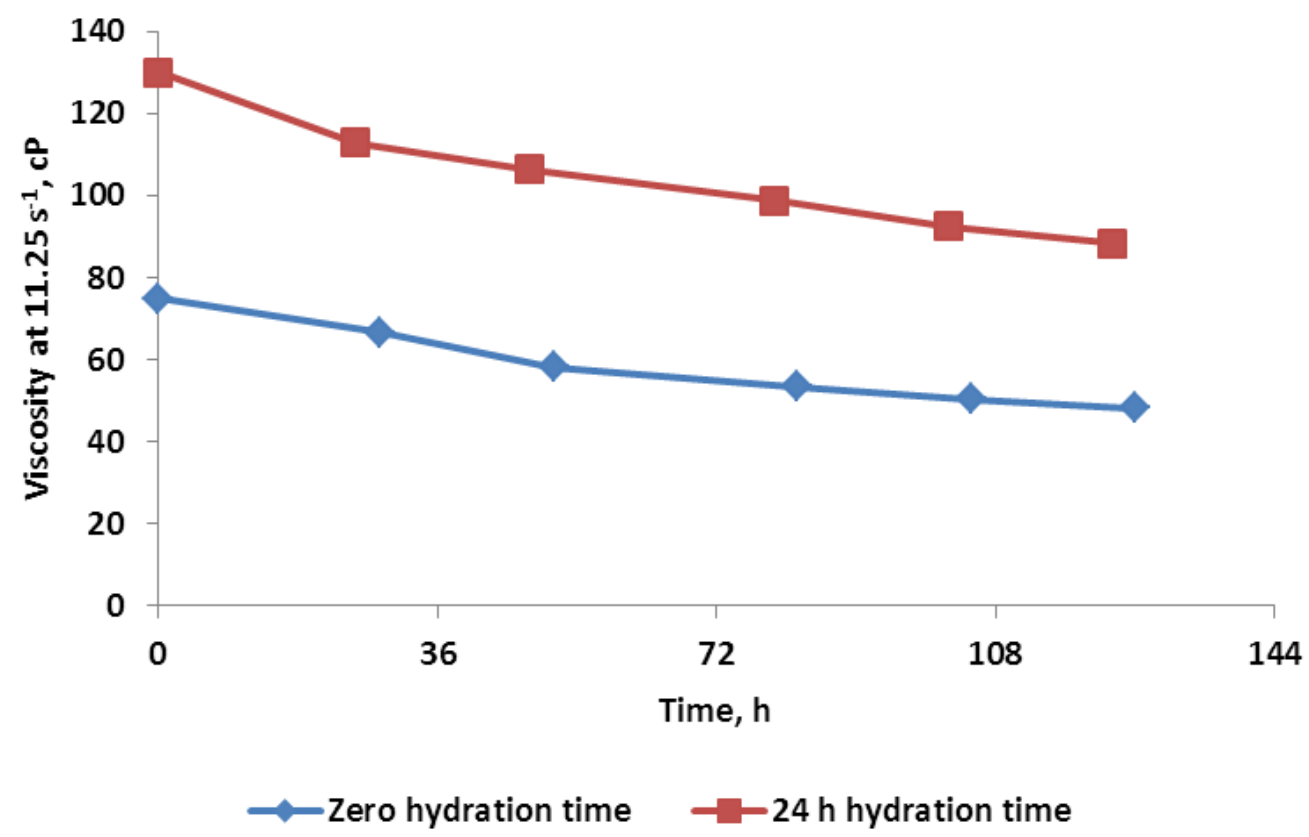

Figure 69 Effect of hydration time on guar viscosity. Viscosity values were measured at $11.25 \mathrm{~s}^{-1}$ using Brookfields viscometer.

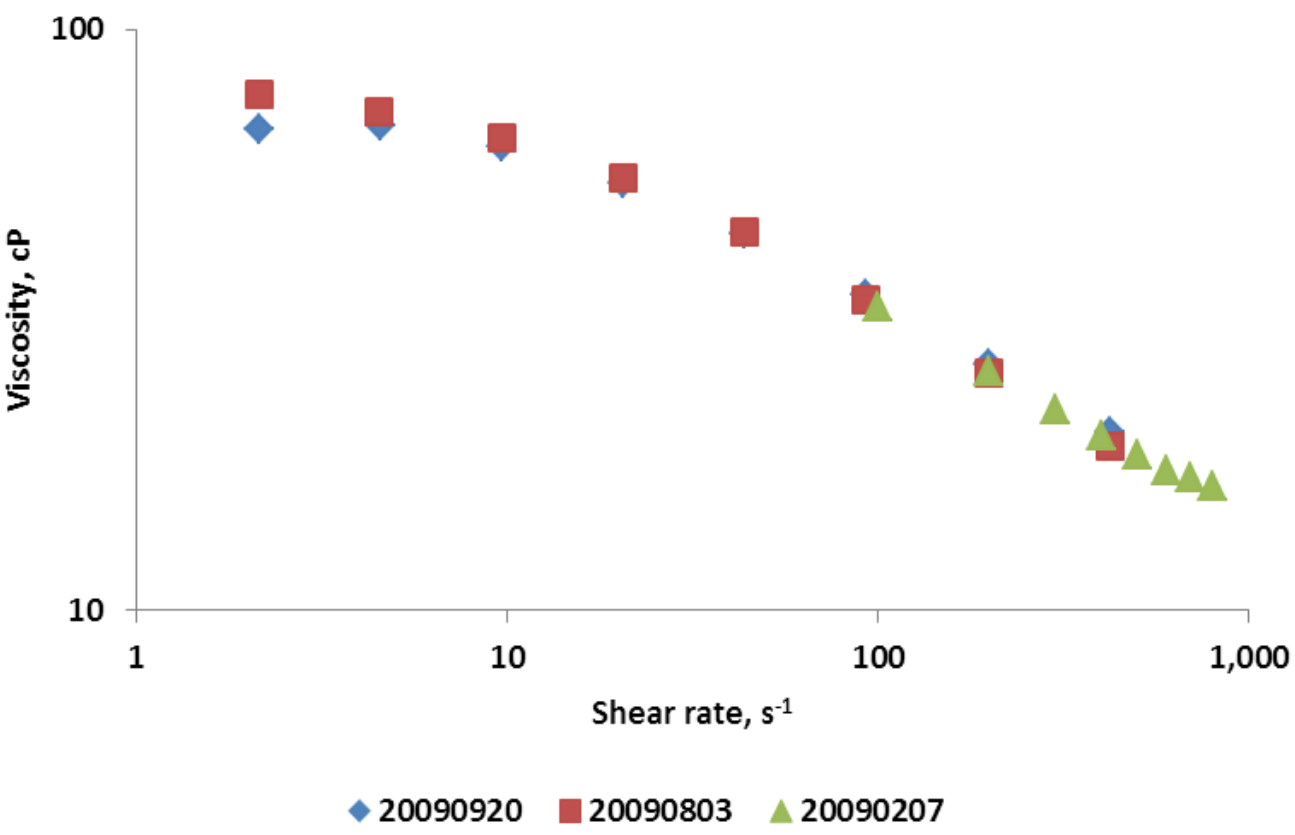

Figure 70 Viscosity versus shear rate for three different $5000 \mathrm{ppm}$ guar solutions in $2 \% \mathrm{KCl}$ 


\section{1-2 Hydroxypropyl Guar (HPG) Solutions}

HPG solutions prepared in $2 \% \mathrm{KCl}$ and $1.35 \mathrm{~g} / \mathrm{L}$ of sodium thiosulfate showed similar viscosity at different $\mathrm{pH}$ values within the 6.5-9.1 range. In one study viscosity (measured at $90 \mathrm{~s}^{-1}$ ) of four HPG samples with different $\mathrm{pH}$ values adjusted using $1 \mathrm{M} \mathrm{NaOH}$ showed similar trends versus temperature. Results are shown in Figure 71 and Table 16.

The viscosity of HPG solutions with $\mathrm{pH}$ values of $6.5,7.5,8.5$ and 9.1 over a range of shear rates was measured at different temperatures. Flow behavior index $\left(\mathrm{n}^{\prime}\right)$ and flow consistency index $\left(\mathrm{K}^{\prime}\right)$ were measured versus temperature (shown in Figure $\mathbf{7 2}$ and Figure 73). Both indices decreased with increasing temperature and no significant change was observed with $\mathrm{pH}$. Table 17 shows the decreasing trend of average indices versus temperature for all the samples measured during the course of this research.

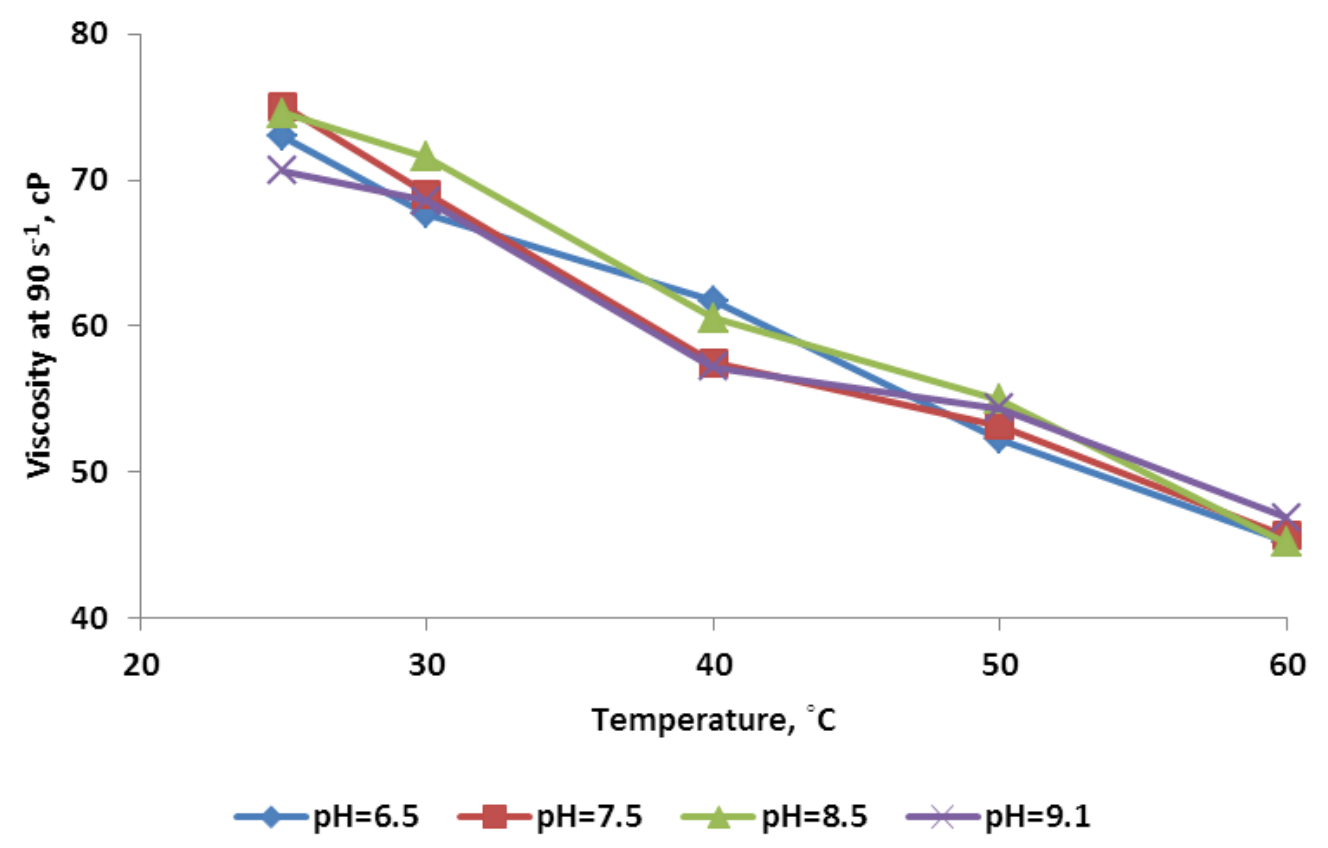

Figure 71 Viscosity $\left(90 \mathrm{~s}^{-1}\right)$ versus temperature measured for HPG solutions with different $\mathrm{pH}$ values 
Table 16 Viscosity $\left(90 \mathrm{~s}^{-1}\right)$ versus temperature measured for HPG solutions with different $\mathrm{pH}$ values

\begin{tabular}{||c|c|c|c|c|}
\hline \multirow{2}{*}{$\mathbf{T},{ }^{\circ} \mathbf{C}$} & \multicolumn{4}{|c|}{ Viscosity, $\mathbf{c P}$} \\
\cline { 2 - 5 } & $\mathbf{p H = 6 . 5}$ & $\mathbf{p H = 7 . 5}$ & $\mathbf{p H = 8 . 5}$ & $\mathbf{p H = 9 . 1}$ \\
\hline 25 & 72.97 & 75.02 & 74.57 & 70.65 \\
\hline 30 & 67.71 & 69.05 & 71.59 & 68.55 \\
\hline 40 & 61.72 & 57.49 & 60.58 & 57.20 \\
\hline 50 & 52.27 & 53.18 & 54.96 & 54.38 \\
\hline 60 & 45.26 & 45.66 & 45.21 & 46.92 \\
\hline
\end{tabular}

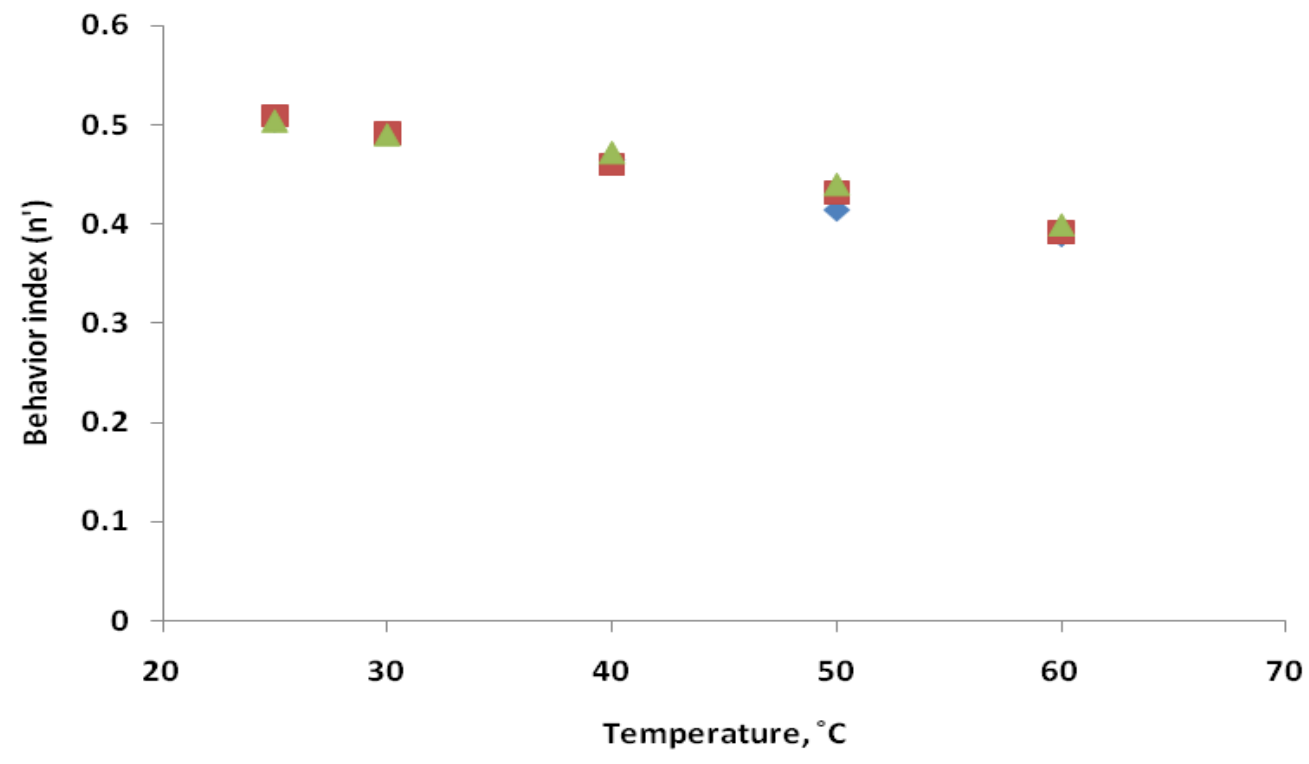

$\rightarrow \mathrm{pH}=8.5 \square \mathrm{pH}=7.5 \quad \triangle \mathrm{pH}=6.5$

Figure 72 Flow behavior index calculated for HPG solutions with three different pH values versus temperature 


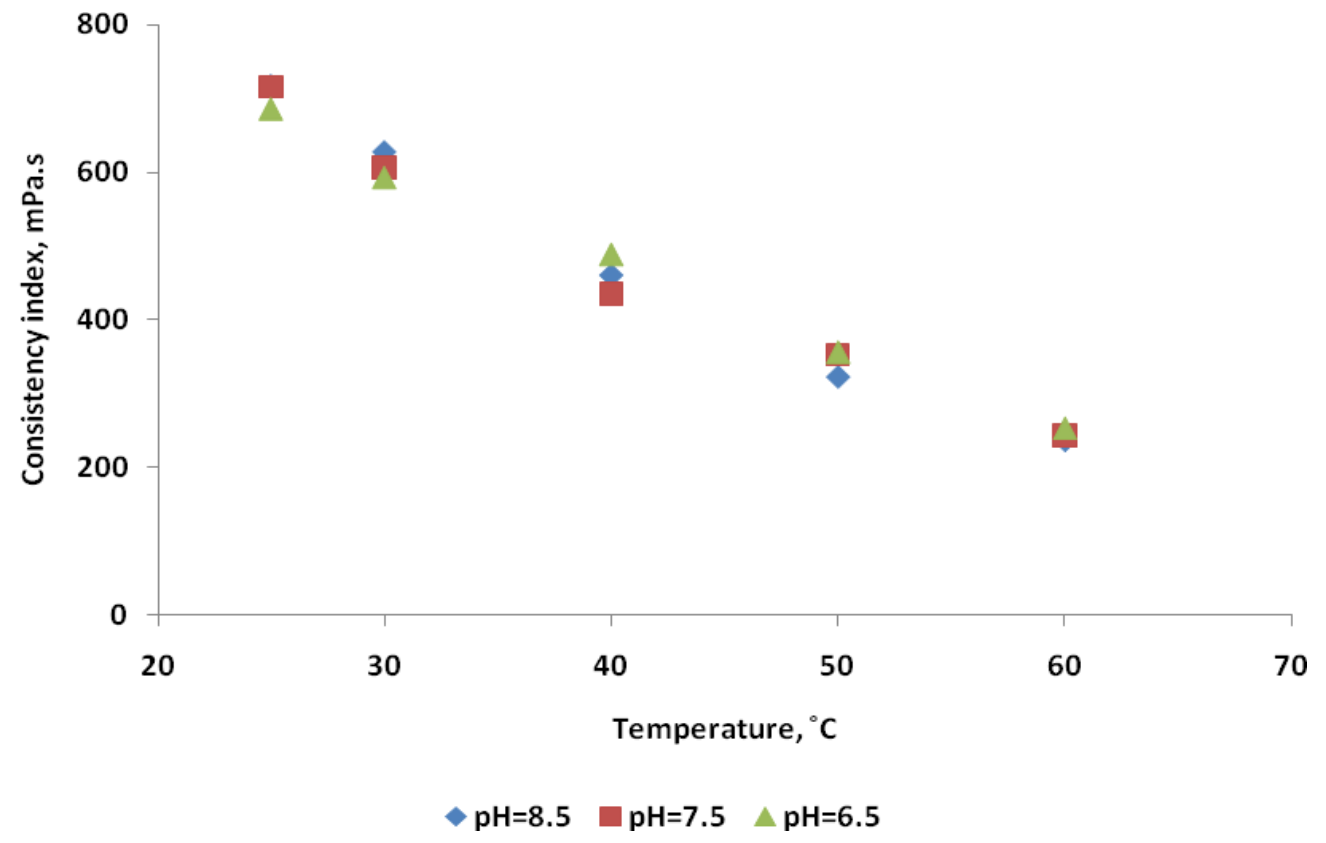

Figure 73 Flow consistency index calculated for HPG solutions with three different pH values versus temperature

Table 17 Average flow behavior $\left(n^{\prime}\right)$ and flow consistency $\left(K^{\prime}\right)$ indices versus temperature measured for different samples

\begin{tabular}{|c|c|c|c|c|c|}
\hline $\mathbf{T}^{\circ}{ }^{\circ} \mathbf{C}$ & $\begin{array}{c}\text { Mean } \\
\mathbf{n}^{\prime}\end{array}$ & $\begin{array}{c}\text { Standard deviation } \\
\text { of } \mathbf{n}^{\prime}\end{array}$ & Mean $\mathbf{K}^{\prime}, \mathbf{m P a . s}$ & $\begin{array}{c}\text { Standard deviation } \\
\text { of } \mathbf{K}^{\prime}\end{array}$ & $\begin{array}{c}\text { Number of } \\
\text { samples }\end{array}$ \\
\hline 25 & 0.50 & 0.02 & 667.41 & 62.90 & 7 \\
\hline 30 & 0.49 & 0.00 & 608.87 & 17.51 & 3 \\
\hline 40 & 0.46 & 0.02 & 444.13 & 65.87 & 12 \\
\hline 50 & 0.43 & 0.01 & 339.85 & 17.00 & 4 \\
\hline 60 & 0.39 & 0.01 & 238.20 & 13.99 & 4 \\
\hline
\end{tabular}

\section{1-3 Borate Cross-linked Guar/HPG}

Guar and HPG solutions were cross-linked using different concentrations of borax in R.O. water.

Gel properties were studied for different borax concentrations added to HPG solution at two temperatures of $25{ }^{\circ} \mathrm{C}$ and $40{ }^{\circ} \mathrm{C}$ (Figure 74 and Figure 75). $7.5 \mathrm{~mL}$ of borax with concentrations of 1000, 2000, 4000 or 6000 were added to $24 \mathrm{~mL}$ of 5000 ppm HPG solution (in $2 \% \mathrm{KCl}$ ) mixed with $7.5 \mathrm{~mL}$ of R.O. water ( $\mathrm{pH} 8$ ). With increasing concentration of borate ions, 
elastic moduli increases linearly till it reaches a saturation point after which the slope changes to a lower value. This behavior occurred at $25^{\circ} \mathrm{C}$ while the maximum borax concentration of 6000 ppm was still in the linear range at $40{ }^{\circ} \mathrm{C}$. This is caused by less borate ions being released at higher temperature. Saturation of sites capable of making complexes with borate ions causes the change in slope at high borate ion concentrations.

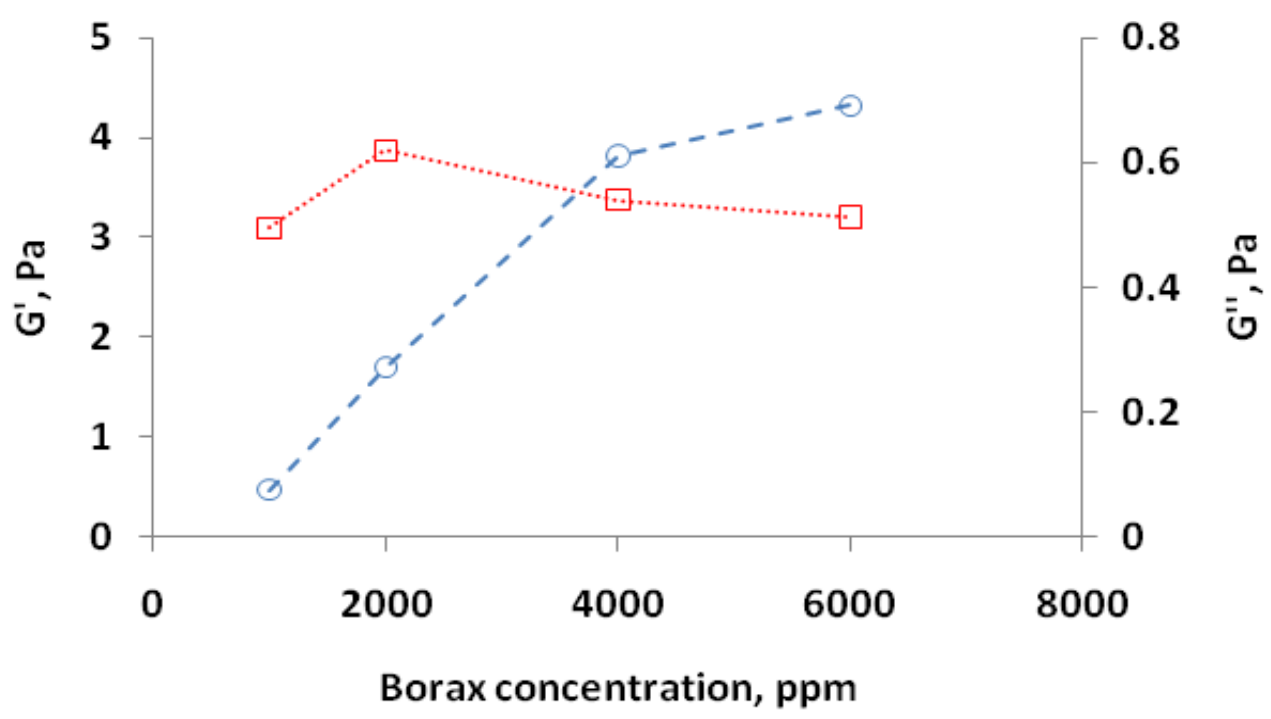

Figure 74 Viscoelastic parameters measured for borate-crosslinked HPG versus different concentrations of borax added at $25^{\circ} \mathrm{C}$ and $0.5 \mathrm{~Hz}$ 


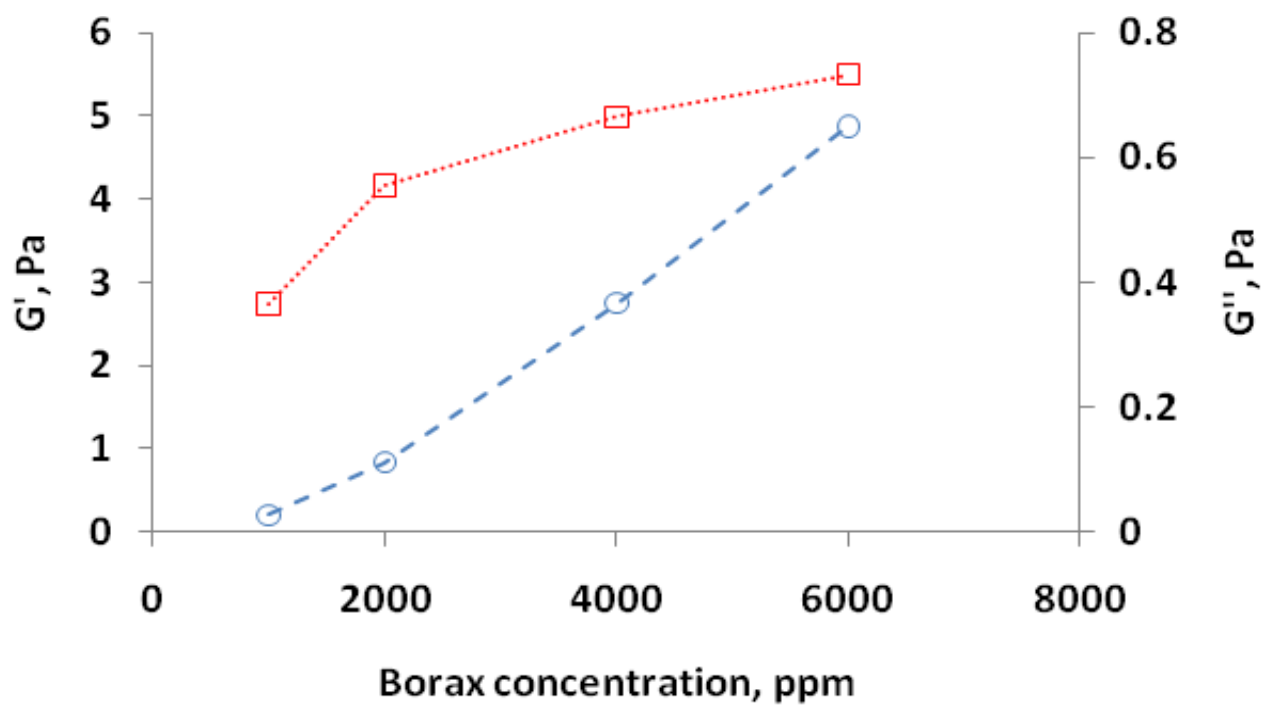

$--G-G^{\prime} \quad \cdots \cdots+\cdots \cdot G^{\prime \prime}$

Figure 75 Viscoelastic parameters measured for borate-crosslinked HPG versus different concentrations of borax added at $40{ }^{\circ} \mathrm{C}$ and $0.5 \mathrm{~Hz}$

\subsection{Characterization and Activity Measurement of Two Enzymes Used In this Study}

Pectinase from Aspergillus aculaceatus and a commercial enzyme used in the fracturing industry (EL2X) were examined and used in this research. A method was sought to measure the concentration and/or activity of the enzymes in presence of polyelectrolytes (PEI and DS). Measuring the concentration and/or activity of enzymes in the supernatant of PEC nanoparticles is required in order to calculate their entrapment efficiencies. Ultraviolet-visible (UV-vis) spectrometry, sodium dodecyl sulfate polyacrylamide gel electrophoresis (SDS-PAGE) and viscometric assay were all used to measure the entrapment efficiency of nanoparticles. Some properties of enzymes measured using such methods are explained in the following sections.

\section{2-1 Ultraviolet-visible (UV-vis) Spectrometry}

Ultraviolet-visible (UV-vis) spectroscopy to measure the concentration of enzymes in presence of polyelectrolytes was tried. Measuring the absorbance over a range of wavelengths for PEI, DS, and pectinase, it was observed that DS and high concentrations of PEI both show absorbance 
at $280 \mathrm{~nm}$ where enzymes have a peak (Figure 76, Figure 77 and Figure 78). The peaks observed at $280 \mathrm{~nm}$ for enzymes are not very significant thus not very accurate for the low concentrations seen in the supernatants. Therefore, measuring the concentration of enzymes in presence of polyelectrolytes using this method was not possible. Figure $\mathbf{7 8}$ shows the UV absorbance spectrum for supernatant of nanoparticle system $A$ after being diluted eight times. UV absorbance at $280 \mathrm{~nm}$ shows higher absorbance than $0.1 \% \mathrm{w} / \mathrm{w}$ pectinase which is the pectinase loaded in $8 \times$ diluted nanoparticle system. This is caused by the absorbance of free PEI and/or DS.

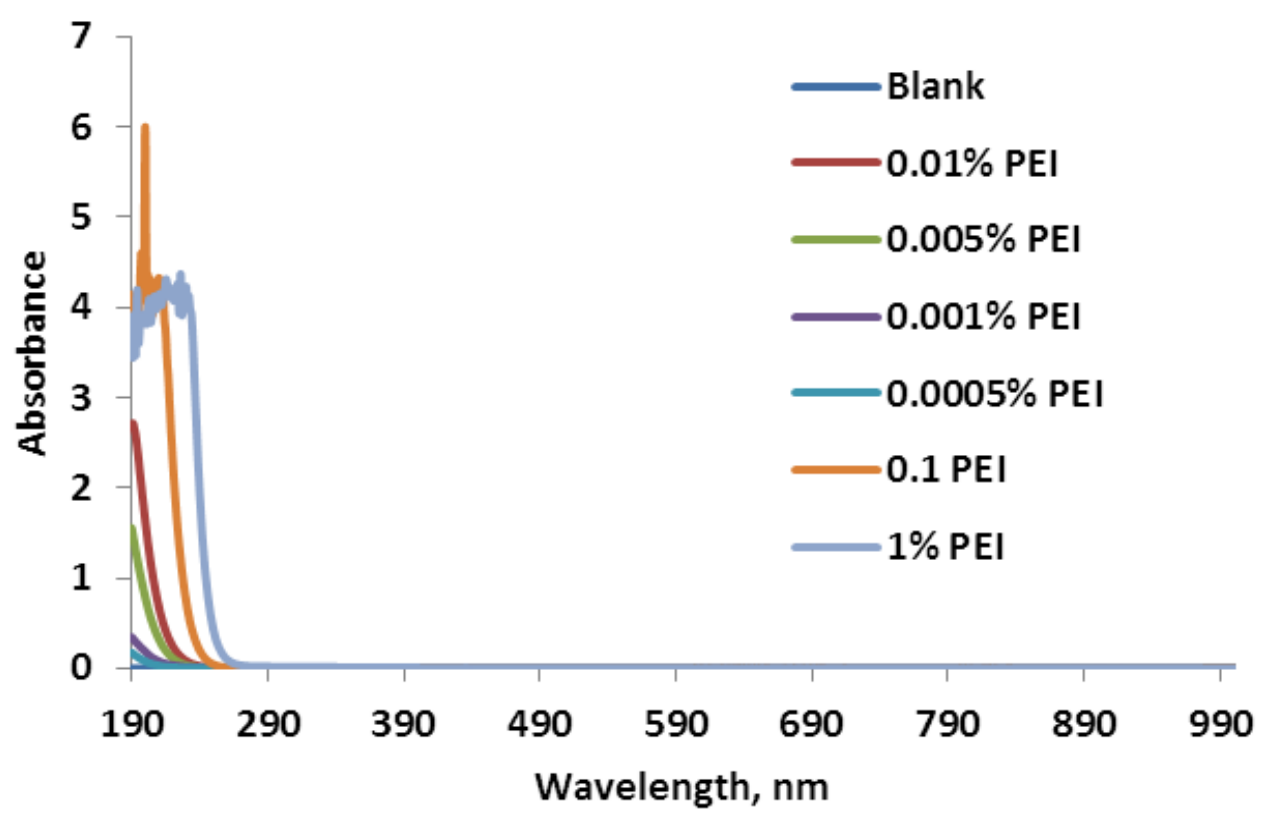

Figure 76 UV absorbance spectrum of PEI 


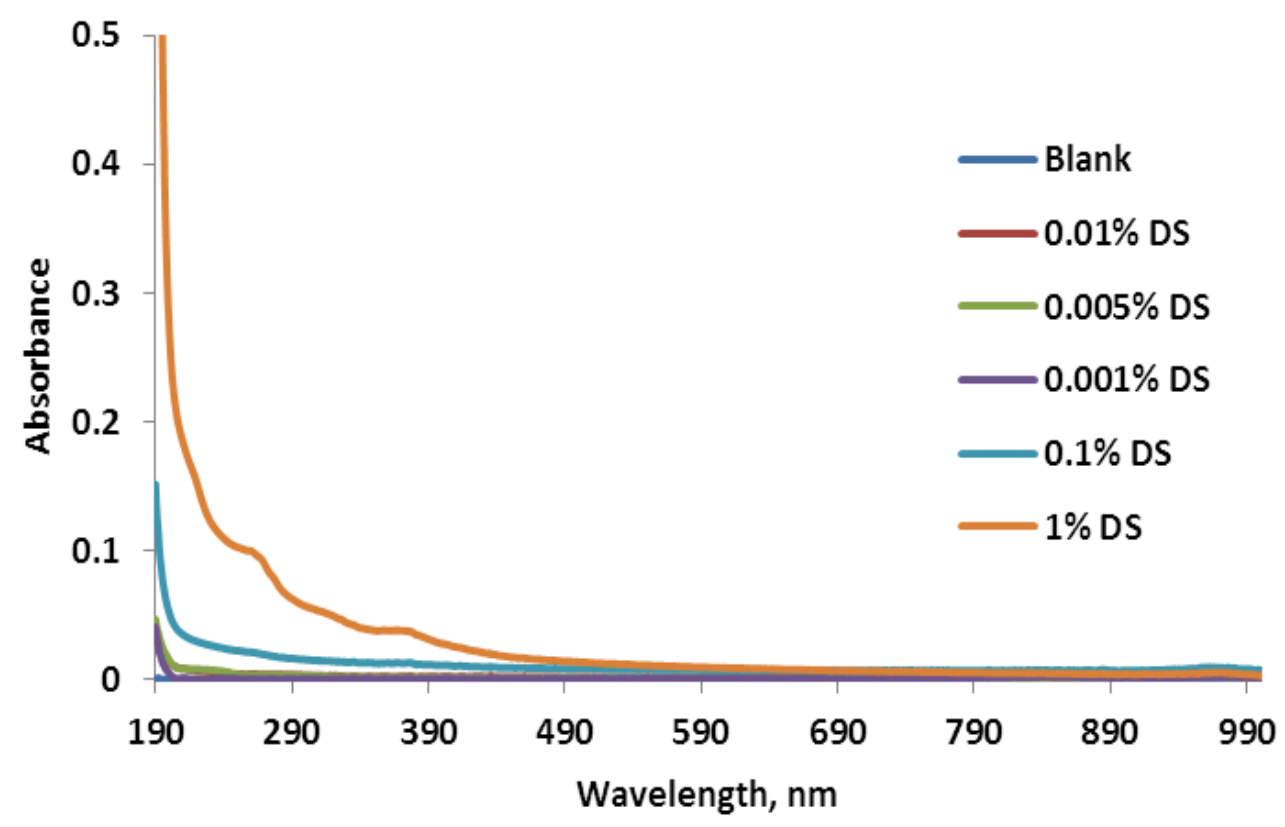

Figure 77 UV absorbance spectrum of DS
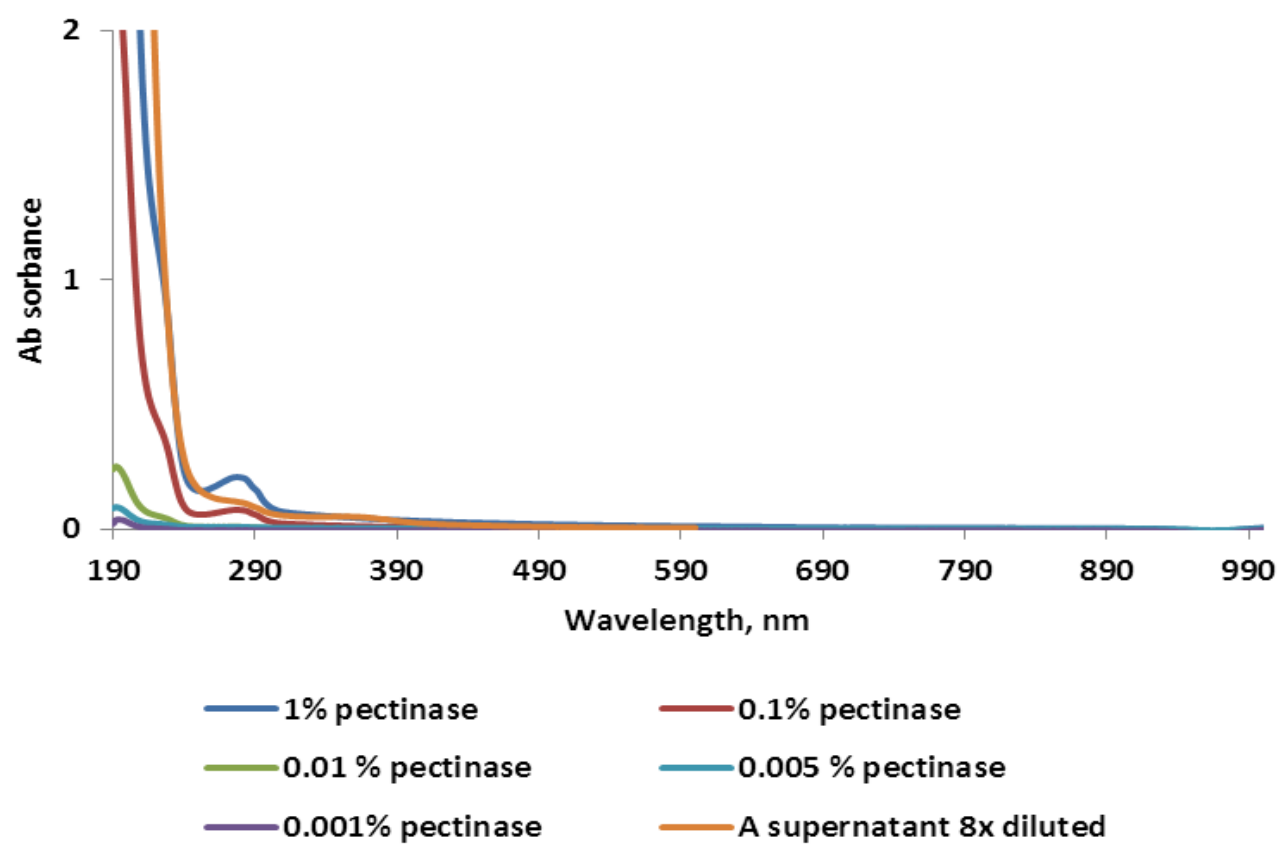

Figure $78 \mathrm{UV}$ absorbance spectrum of pectinase 


\section{2-2 Sodium Dodecyl Sulfate Polyacrylamide Gel Electrophoresis (SDS- PAGE)}

In order to measure the concentration of enzyme remaining in the supernatant of nanoparticles after being centrifuged, and considering the existence of polyelectrolytes, separation based on electrophoretic mobility was used. SDS-PAGE analysis was performed using a NuPAGE ${ }^{\circledR}$ kit, following the instructions reported in the previous chapter. SDS-PAGE analysis was performed for different concentrations of enzymes and for supernatant separated from the nanoparticles loaded with enzymes.

SDS-PAGE was used to measure the molecular weight of the pectinase and to compare PEI, DS, pectinase and supernatants from centrifuged nanoparticle samples. (Figure 79, lane contents reported in Table 18). A marker containing proteins of a range of known molecular weight was run in Lane 1. Lane 5 shows the molecular weight distribution of pectinase. Distinct peaks were seen at $116 \mathrm{kDa}$ and $66 \mathrm{kDa}$. PEI travelled (Lane 2) off the end of the gel suggesting a low MW. Lane 10 is the supernatant of the 2:1:0.1 nanoparticles (PECNP system $A$ ), in which low concentrations of pectinase were observed. Note that Lane 3 shows no peaks since the NuPAGE 4-12\% Bis-Tris Gel accepts proteins with maximum $\mathrm{M}_{\mathrm{W}}$ of $200 \mathrm{kDa}$ while $\mathrm{M}_{\mathrm{W}}$ of DS is 500 $\mathrm{kDa}$. Molecular weight distribution of pectinase was found to be in the range of 3.5-116.3 kDa. 


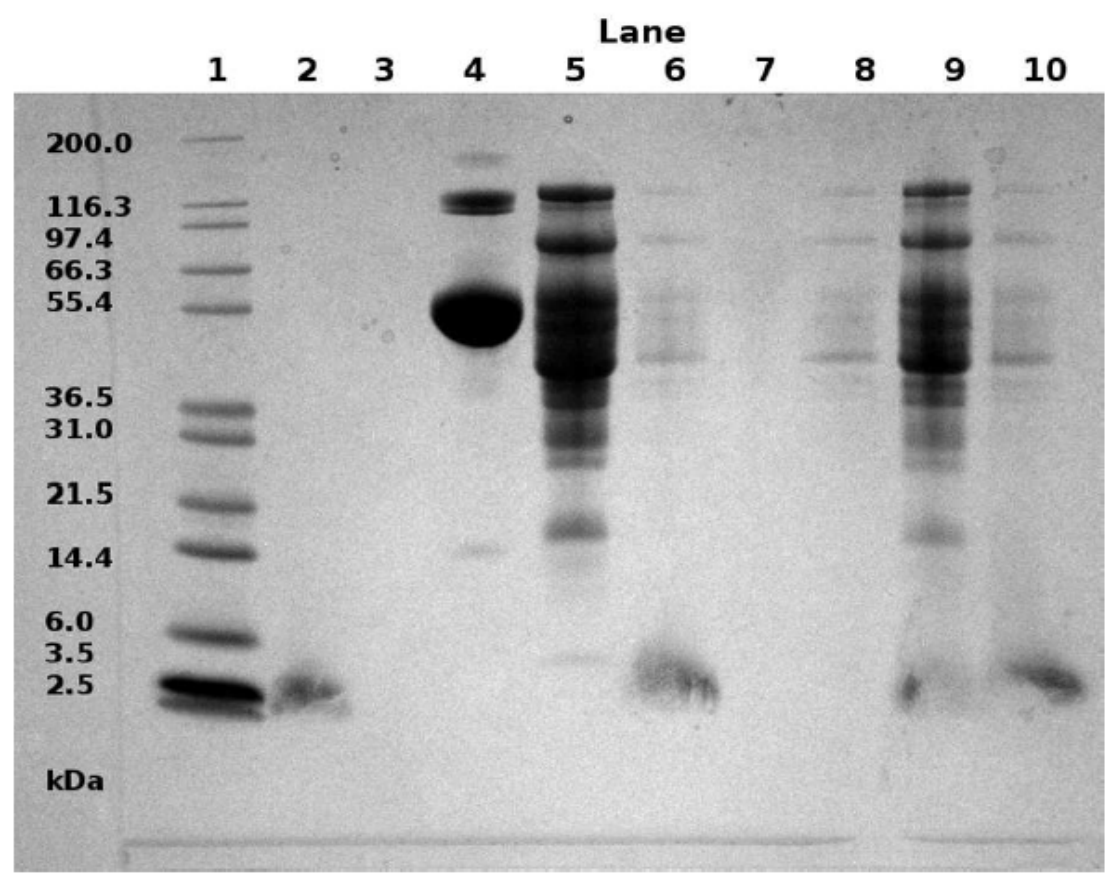

Figure 79 SDS-PAGE gel. Contents of each lane are described in Table 18. Numbers on left are molecular weight of marker proteins in Lane 1.

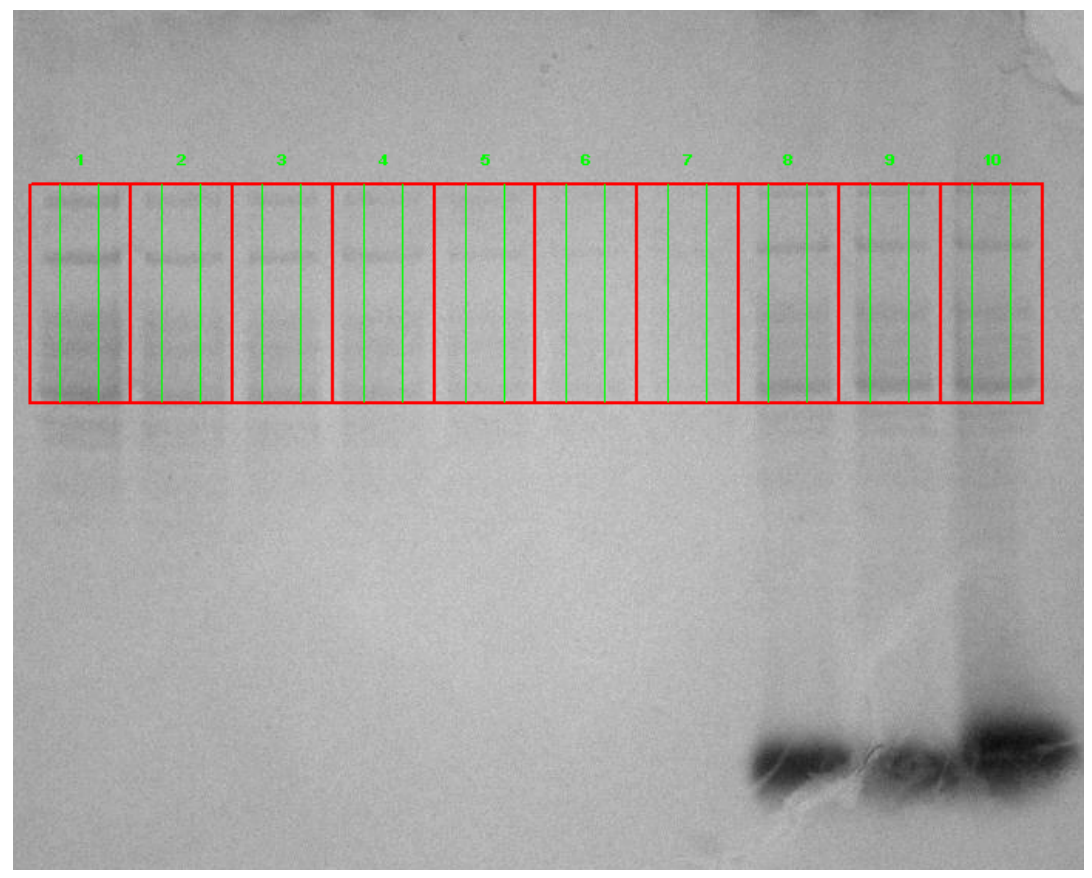

Figure 80 SDS-PAGE run for different concentrations of pectinase reported in Table 19 (lanes 1-7), the supernatant of PEC nanoparticle system A (Lanes 8-9) and the supernatant of PEC nanoparticle system B (Lane 10) 
Table 18 Samples $(\mathrm{mL})$ in SDS-PAGE lanes shown in Figure 79

\begin{tabular}{|c|c|c|c|c|c|c|c|c|c|c|}
\hline Lane & $\begin{array}{c}1 \\
\text { MW } \\
\text { Marke } \\
\text { r }\end{array}$ & $\begin{array}{c}2 \\
\text { Diluted } \\
\text { PEI }\end{array}$ & $\begin{array}{c}3 \\
\text { Diluted } \\
\text { DS }\end{array}$ & $\begin{array}{c}4 \\
\text { BS } \\
\text { A }\end{array}$ & $\begin{array}{c}5 \\
\text { Pectinase }\end{array}$ & $\begin{array}{c}6 \\
\text { Diluted } \\
\text { PEI + } \\
\text { pectinase }\end{array}$ & $\begin{array}{c}7 \\
\text { Diluted } \\
\text { DS + } \\
\text { pectinase }\end{array}$ & $\begin{array}{c}8 \\
\text { Diluted } \\
\text { pectinase }\end{array}$ & $\begin{array}{c}9 \\
50: 50 \\
\text { PEI:pectinas } \\
\text { e }\end{array}$ & $\begin{array}{c}10 \\
\text { Supernatant } \\
\text { (A) }\end{array}$ \\
\hline $\begin{array}{c}\text { MW } \\
\text { marker }\end{array}$ & 3.1 & - & - & - & - & - & - & - & - & - \\
\hline $\begin{array}{l}25 \% \mathrm{w} / \mathrm{w} \\
\text { pectinase }\end{array}$ & - & - & - & - & 3.1 & 0.1 & 0.1 & 0.1 & 1.55 & 0.1 \\
\hline $\begin{array}{c}1 \% \mathrm{w} / \mathrm{w} \\
\mathrm{PEI}\end{array}$ & - & 2.0 & - & - & - & 2.0 & - & - & 1.55 & 2.0 \\
\hline $\begin{array}{c}1 \% \mathrm{w} / \mathrm{w} \\
\mathrm{DS}\end{array}$ & - & - & 1.0 & - & - & - & 1.0 & - & - & 1.0 \\
\hline BSA & - & - & - & 3.1 & - & - & - & - & - & - \\
\hline D.I. Water & - & 1.1 & 2.1 & - & - & 1.0 & 2.0 & 3.0 & - & - \\
\hline
\end{tabular}

SDS-PAGE was also performed for different pectinase concentrations (Figure 80) and the area of the most distinct absorbance peak $(\sim 116 \mathrm{kDa})$ of pectinase was plotted versus pectinase concentration to yield a calibration curve (Figure 81). The area under the intensity-distance peak for the supernatant of the nanoparticle systems was correlated to the concentration of enzyme in order to calculate the entrapment efficiency of the PECNP systems based on concentration on entrapped enzyme. The last three lanes of Figure $\mathbf{8 0}$ are the supernatant of PECNP systems $A$ and $B$ reported in Table 19. Measured area under the intensity-length curve for the supernatants of systems A and B shown in lanes 8 and 10 of Table 19 are shown in Figure 81.

Table 19 Samples in SDS-PAGE lanes shown in Figure 80

\begin{tabular}{|c|c|c|c|c|c|c|c|c|c|}
\hline Lane\#1 & Lane\#2 & Lane\#3 & Lane\#4 & Lane\#5 & Lane\#6 & Lane\#7 & $\begin{array}{c}\text { Lane\# } \\
8\end{array}$ & Lane\#9 & Lane\#10 \\
\hline $\begin{array}{l}1 \% \mathrm{w} / \mathrm{w} \\
\text { pectinase }\end{array}$ & $\begin{array}{l}0.8 \% \mathrm{w} / \mathrm{w} \\
\text { pectinase }\end{array}$ & $\begin{array}{l}0.6 \% \mathrm{w} / \mathrm{w} \\
\text { pectinase }\end{array}$ & $\begin{array}{l}0.5 \% \mathrm{w} / \mathrm{w} \\
\text { pectinase }\end{array}$ & $\begin{array}{l}0.4 \% \mathrm{w} / \mathrm{w} \\
\text { pectinase }\end{array}$ & $\begin{array}{l}0.2 \% \mathrm{w} / \mathrm{w} \\
\text { pectinase }\end{array}$ & $\begin{array}{l}0.1 \% \mathrm{w} / \mathrm{w} \\
\text { pectinase }\end{array}$ & $A$ sup. & $A$ sup. & $B$ sup. \\
\hline
\end{tabular}




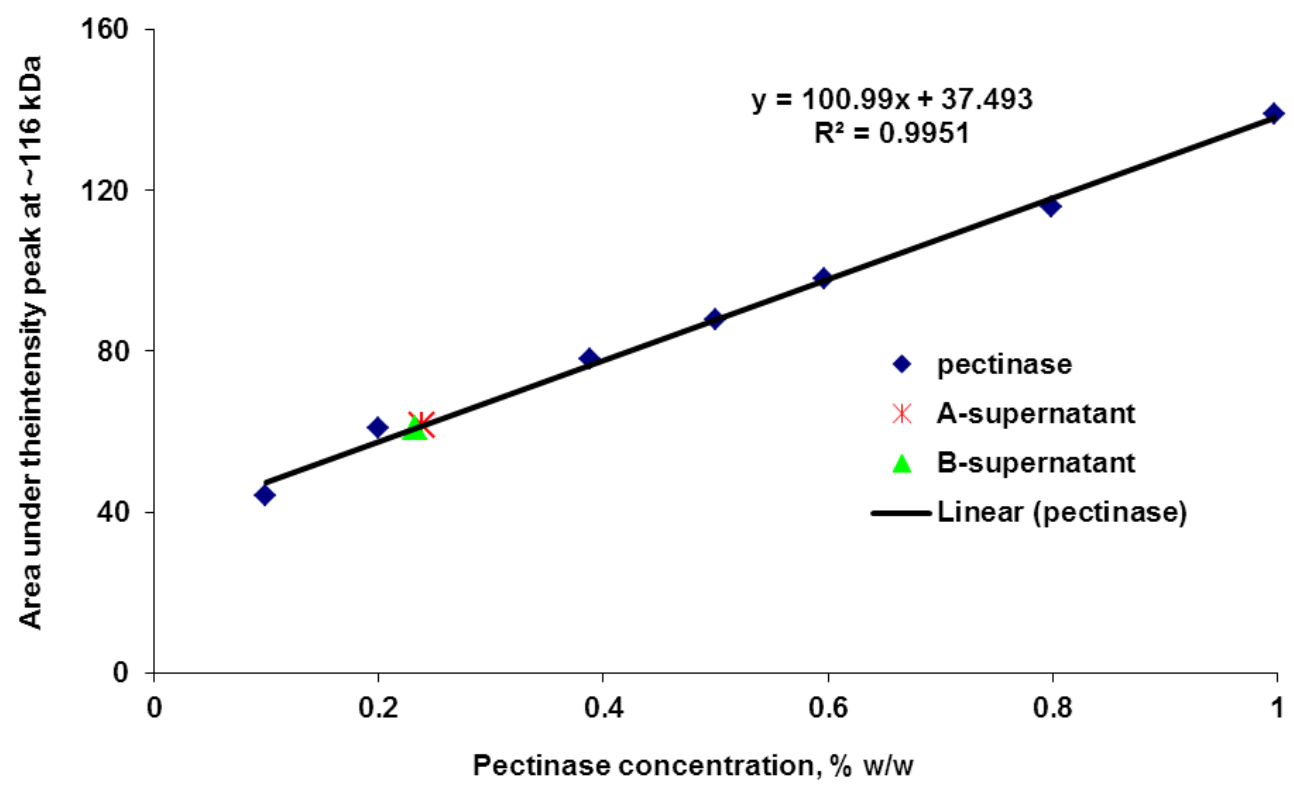

Figure 81 Calibration curve for the area under the intensity peak at $116 \mathrm{kDa}$ versus the concentration of pectinase. Supernatants of PECNP systems $A$ and $B$ reported in Lanes 8 and 10 of Table 19 are also shown.

SDS-PAGE was also performed for EL2X and supernatant of $H^{\prime}$ PEC nanoparticle system (Figure 82). The only observable concentration of EL2X after scanning the gel was the $100 \%$ EL2X loaded in lane 1. Diluted samples of EL2X were not visible using this method. Supernatants of three $H^{\prime}$ PECNP samples loaded in the last three lanes showed no peaks in the molecular weight range of EL2X. Molecular weight distribution of EL2X was found to be in the range of 31-97.4 $\mathrm{kDa}$.

Table 20 Samples in SDS-PAGE lanes shown in Figure 82

\begin{tabular}{|c|c|c|c|c|c|c|c|c|c|}
\hline Lane\#1 & Lane\#2 & Lane\#3 & Lane\#4 & Lane\#5 & Lane\#6 & Lane\#7 & $\begin{array}{c}\text { Lane\# } \\
\mathbf{8}\end{array}$ & $\begin{array}{c}\text { Lane\# } \\
\mathbf{9}\end{array}$ & $\begin{array}{c}\text { Lane\#10 } \\
\end{array}$ \\
& & & & & & & & & \\
\hline $100 \% \mathrm{w} / \mathrm{w}$ & $3.20 \% \mathrm{w} / \mathrm{w}$ & $2.56 \% \mathrm{w} / \mathrm{w}$ & $1.92 \% \mathrm{w} / \mathrm{w}$ & $1.28 \% \mathrm{w} / \mathrm{w}$ & $0.64 \% \mathrm{w} / \mathrm{w}$ & $0.32 \% \mathrm{w} / \mathrm{w}$ & \\
$\mathrm{EL} 2 \mathrm{X}$ & $\mathrm{EL} 2 \mathrm{X}$ & $\mathrm{EL} 2 \mathrm{X}$ & $\mathrm{EL} 2 \mathrm{X}$ & $\mathrm{EL} 2 \mathrm{X}$ & $\mathrm{EL} 2 \mathrm{X}$ & $\mathrm{EL} 2 \mathrm{X}$ & $H^{\prime}$ sup. & $H^{\prime}$ sup. & $H^{\prime}$ sup. \\
\hline
\end{tabular}




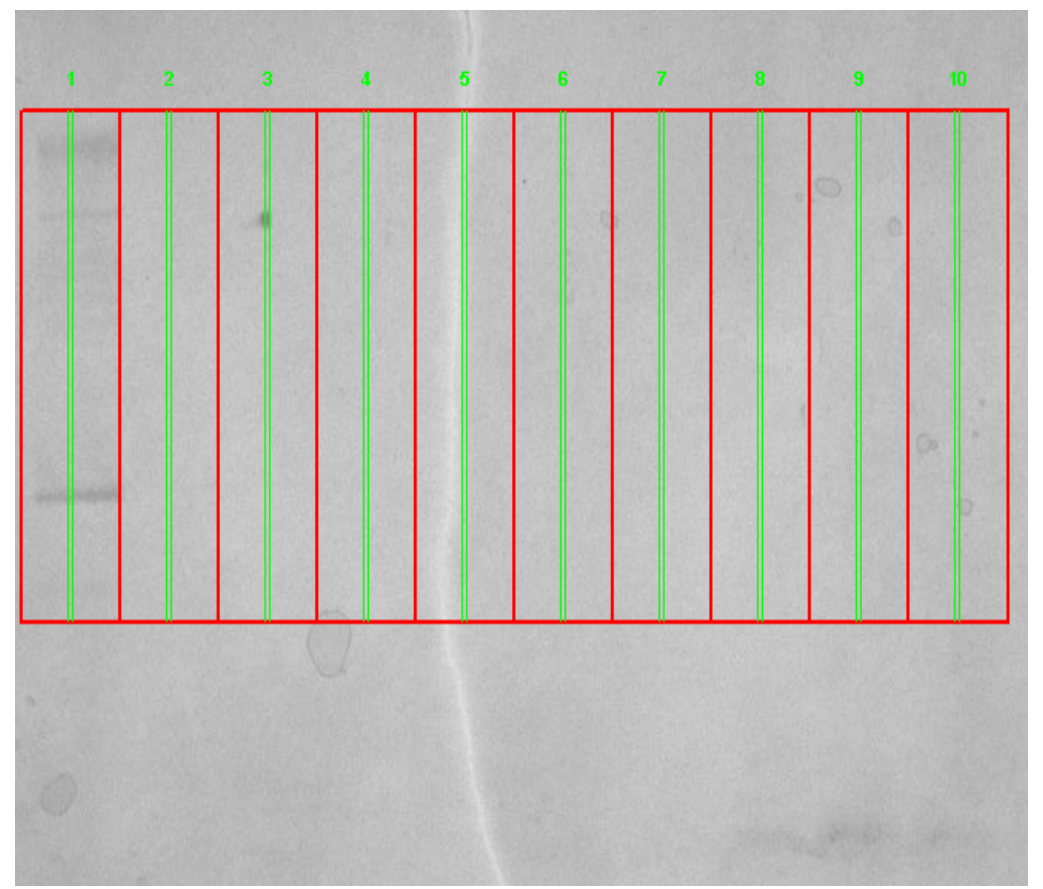

Figure 82 SDS-PAGE run for different concentrations of EL2X reported in Table 20 (lanes 1-7) and the supernatant of PEC nanoparticle system $H^{\prime}$ (Lanes 8-10)

\section{2-3 Viscometric Assay}

The time for the viscosity of the solution to fall to $50 \%$ of its initial value ( $t_{1 / 2}$, hours) was used to calculate the activity of the nanoparticles. Activity was defined as the reciprocal of $t_{1 / 2}$. Using this method, calibration curves were generated for both enzymes at the $\mathrm{pH}$ values of interest where the viscometric assay was performed. Final concentrations of pectinase and EL2X in the final solution used for the viscometric assays of supernatant separated from nanoparticles were $0.02 \% \mathrm{w} / \mathrm{w}$ and $0.08 \% \mathrm{w} / \mathrm{w}$ respectively.

Calibration curves were generated for both enzymes at $25{ }^{\circ} \mathrm{C}$ and $\mathrm{pH}$ of 8.4 by measuring the viscosity of HPG solutions mixed with different concentrations of enzyme (Figure 83). A plot of activity, defined as the reciprocal of $\mathrm{t}_{1 / 2}$, versus concentration of pectinase is shown in Figure 84. Concentrations used during the course of this study were all low enough to be in the linear region of the activity versus concentrations curve. Activity versus concentration reaches a plateau at high concentrations of enzyme. 
A calibration curve for different concentrations of EL2X at $25{ }^{\circ} \mathrm{C}$ and $\mathrm{pH}$ of 8.4 is also shown in Figure 85. The lower slope of EL2X compared to pectinase shows lower activity of this enzyme at this temperature and $\mathrm{pH}$.

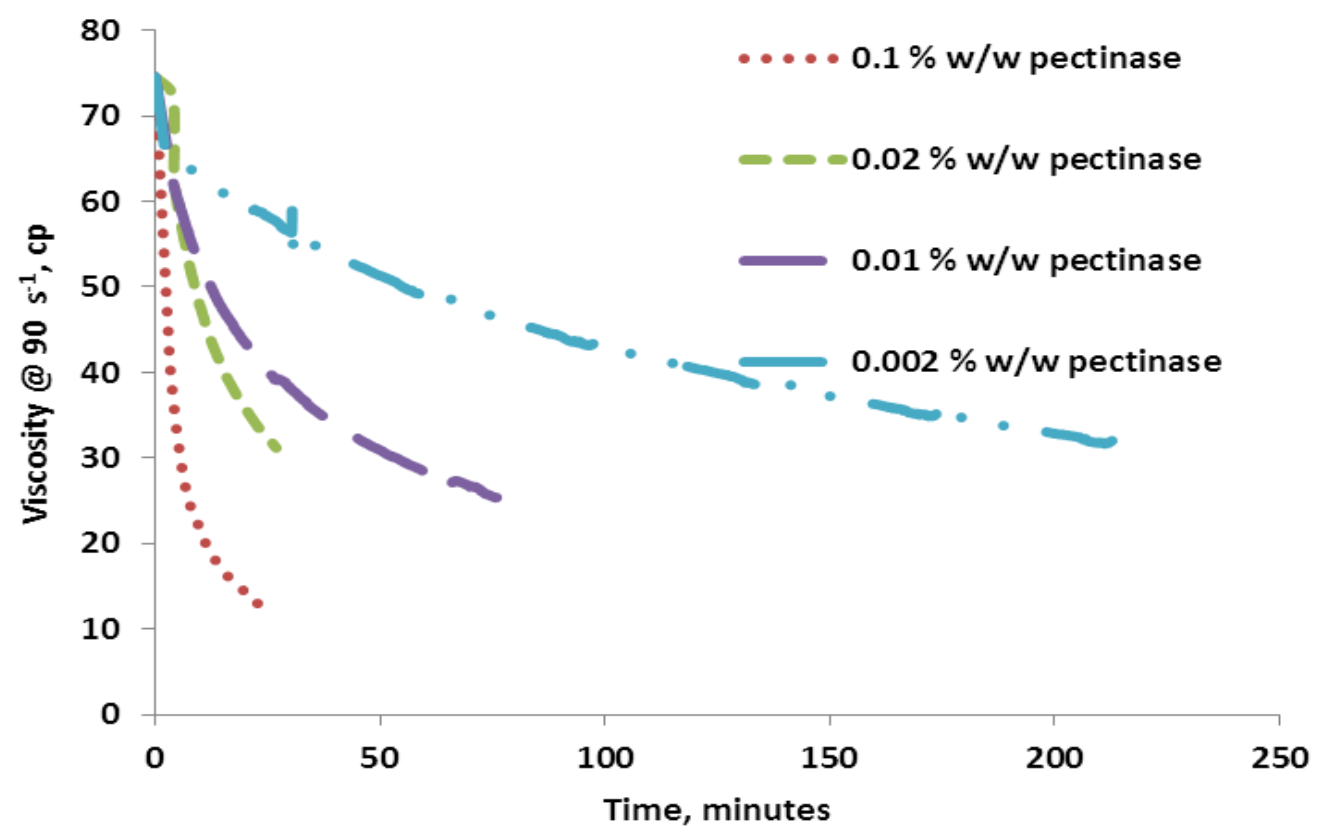

Figure 83 Viscometric assay performed for different concentrations of pectinase mixed with HPG solutions at pH of 8.4 and $25^{\circ} \mathrm{C}$ 


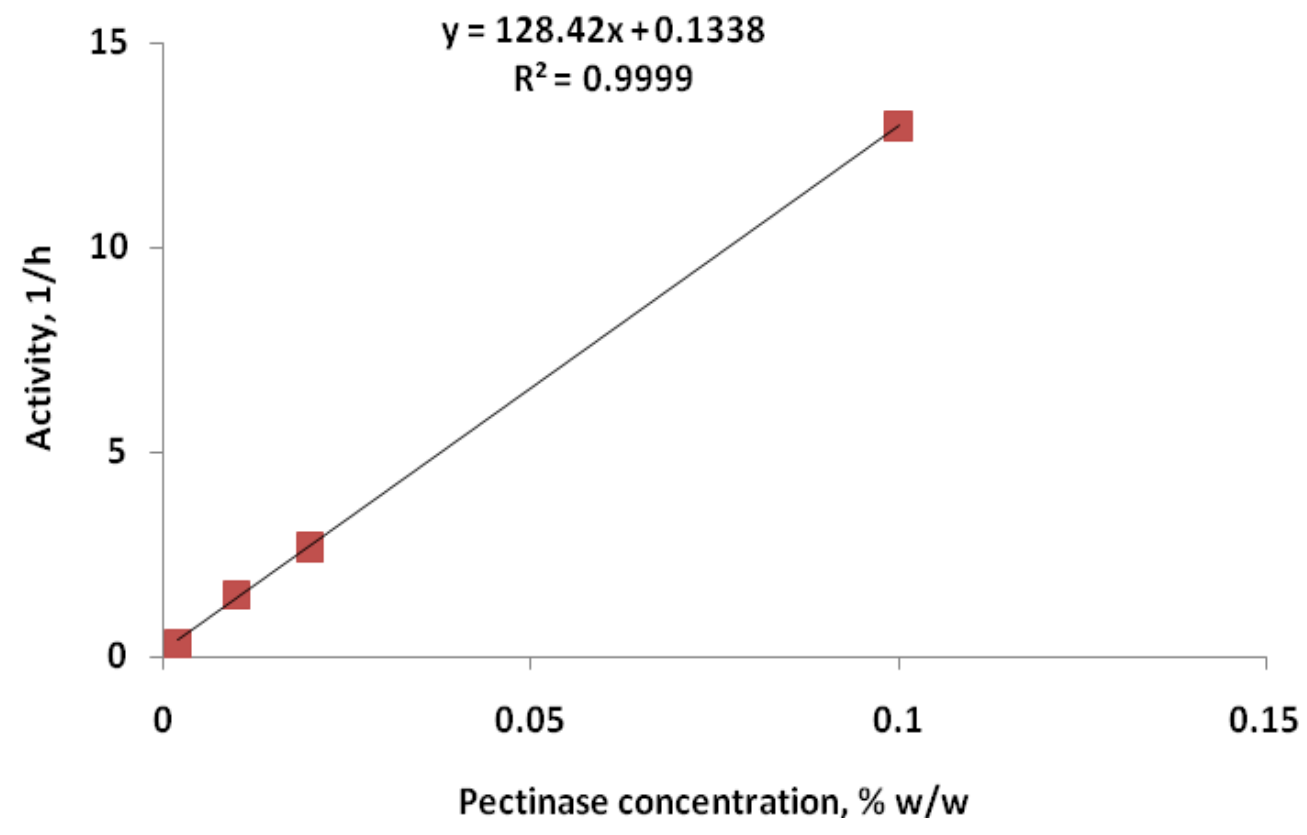

Figure 84 Calibration curve measured for different concentrations of pectinase mixed with HPG solutions at pH of 8.4 and $25^{\circ} \mathrm{C}$

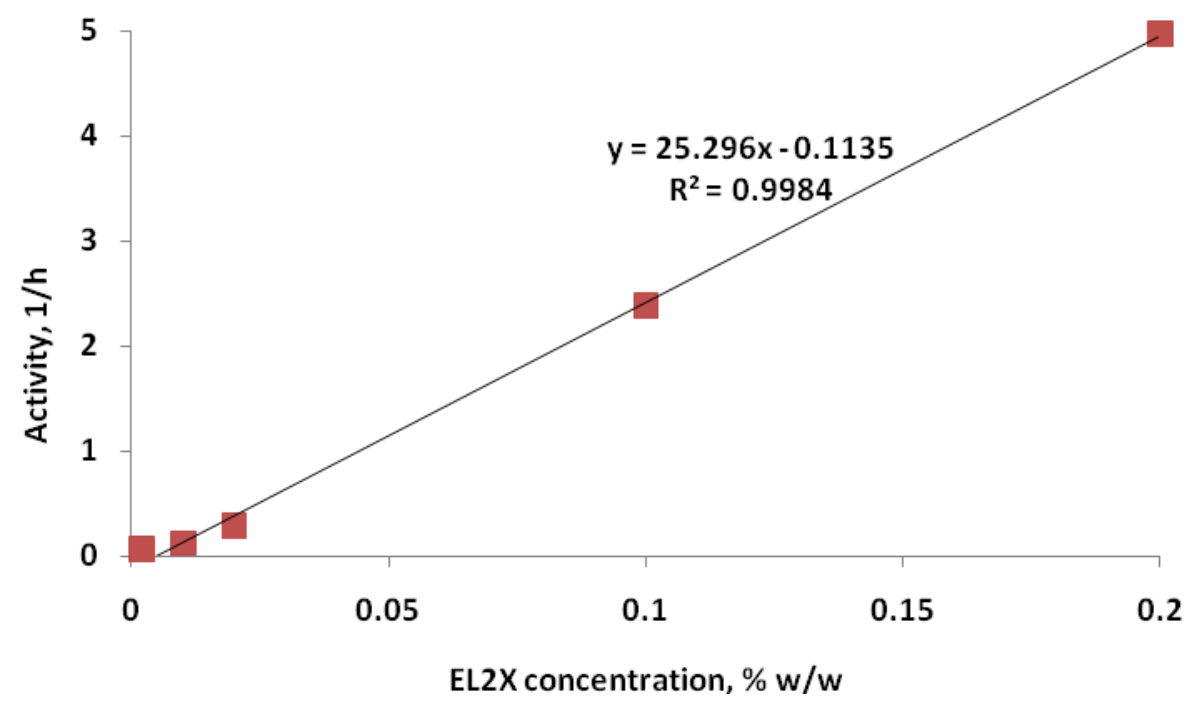

Figure 85 Calibration curve measured for different concentrations of EL2X mixed with HPG solutions at pH of 8.4 and $25^{\circ} \mathrm{C}$ 
Viscometric assay was also performed using HPG solutions with final pectinase concentration of $0.02 \% \mathrm{w} / \mathrm{w}$ and final EL2X concentration of $0.08 \% \mathrm{w} / \mathrm{w}$ at different $\mathrm{pH}$ and temperatures. Figure 86 and Figure 87 show the activity versus temperature plot for different $\mathrm{pH}$ values for pectinase. The data related to these plots are shown in Table 21. Pectinase is denatured at some temperature and $\mathrm{pH}$ values. For example, at $\mathrm{pH}$ values above 9 and $40{ }^{\circ} \mathrm{C}$ pectinase shows no activity. Bell-shaped plots were seen for activity of pectinase versus temperature at $\mathrm{pH} 8.5$ and 9.1 while the enzyme is still active at high temperatures at $\mathrm{pH}$ of 7.5 and 6.5.

Activity versus temperature and pH of EL2X are shown in Figure 88, Figure 89 and Table 22. This enzyme is denatured at $\mathrm{pH}$ of 9 and temperature of $60{ }^{\circ} \mathrm{C}$.

Figure 90 shows activity of different concentrations of pectinase on HPG solutions at pH 9.1 and $40{ }^{\circ} \mathrm{C}$ where the enzyme was demonstrated to be denatured (Figure 86). Pectinase showed some activity before being totally denatured. Increasing the concentration of pectinase decreased the final viscosity of HPG solution but not the denaturation time of enzyme (Figure 90).

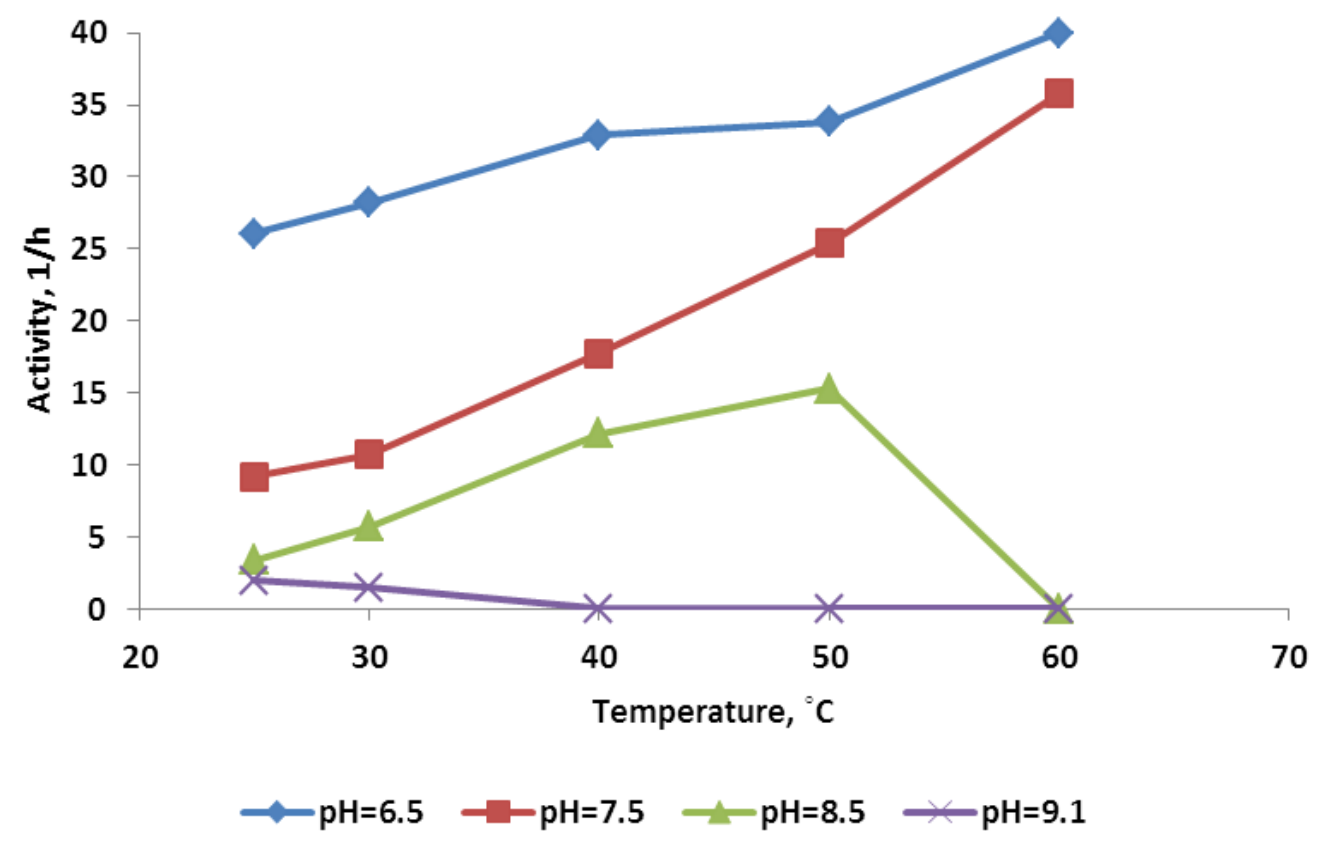

Figure 86 Activity versus temperature of pectinase for different $\mathrm{pH}$ values. Final concentration of pectinase in the HPG solutions is $0.02 \% \mathrm{w} / \mathrm{w}$. 


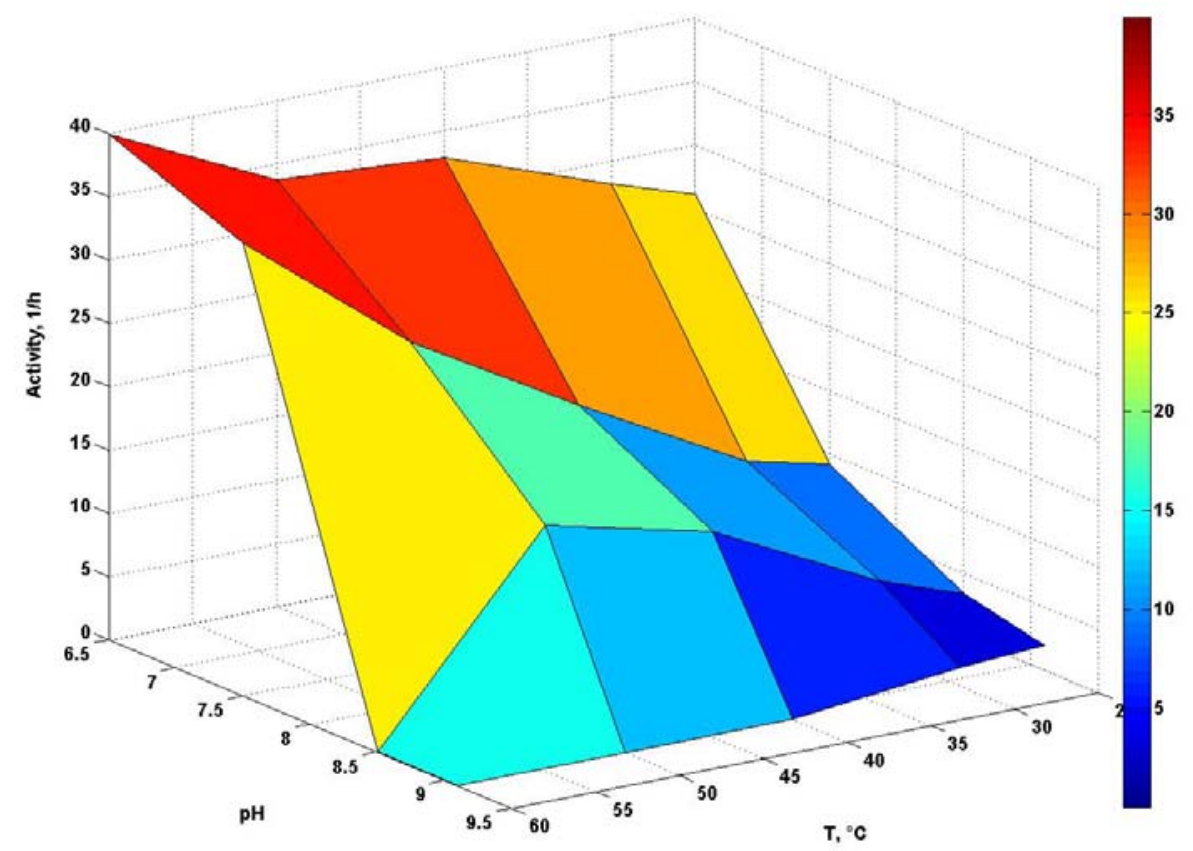

Figure 87 Activity versus temperature and pH of pectinase. Final concentration of pectinase in the HPG solutions is $0.02 \% \mathrm{w} / \mathrm{w}$.

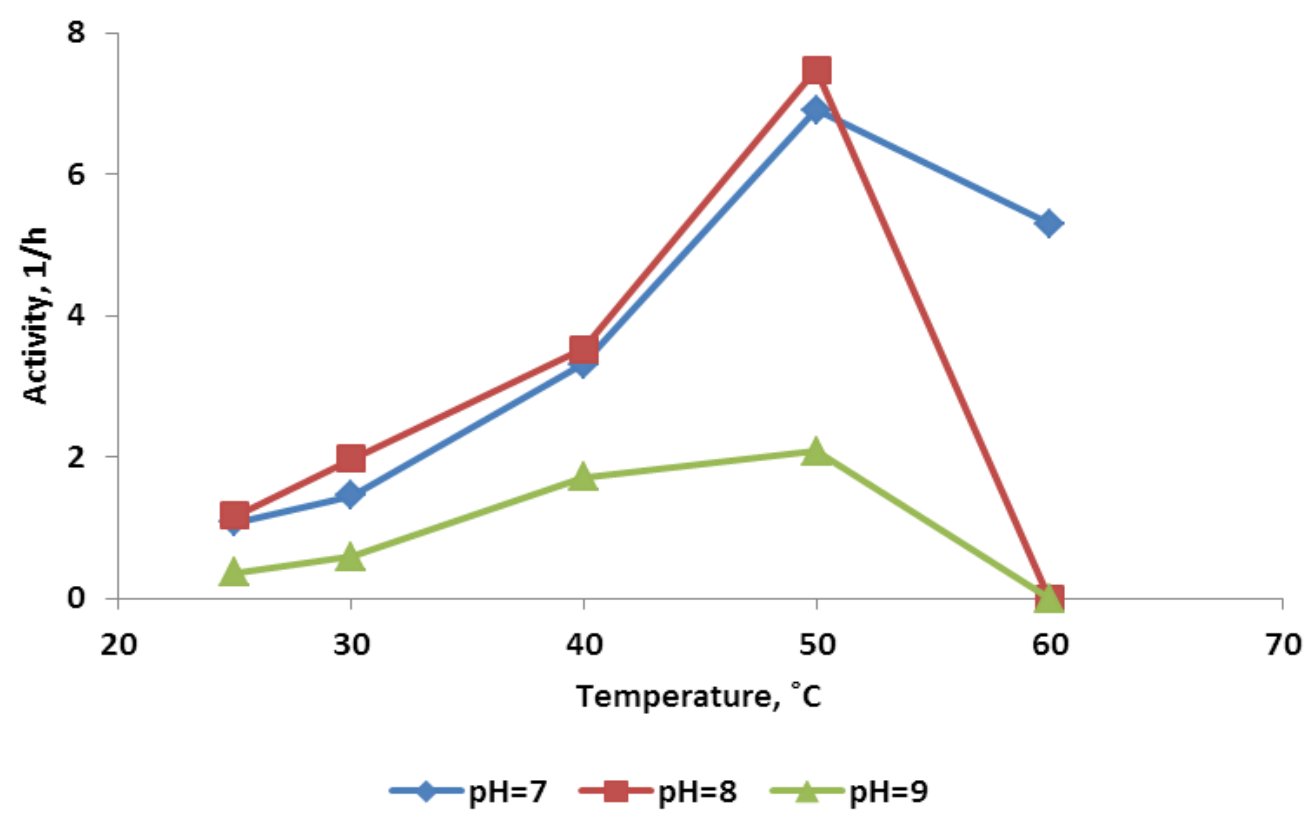

Figure 88 Activity versus temperature of EL2X for different pH values. Final concentration of EL2X in the HPG solutions is $0.08 \%$ w/w. 


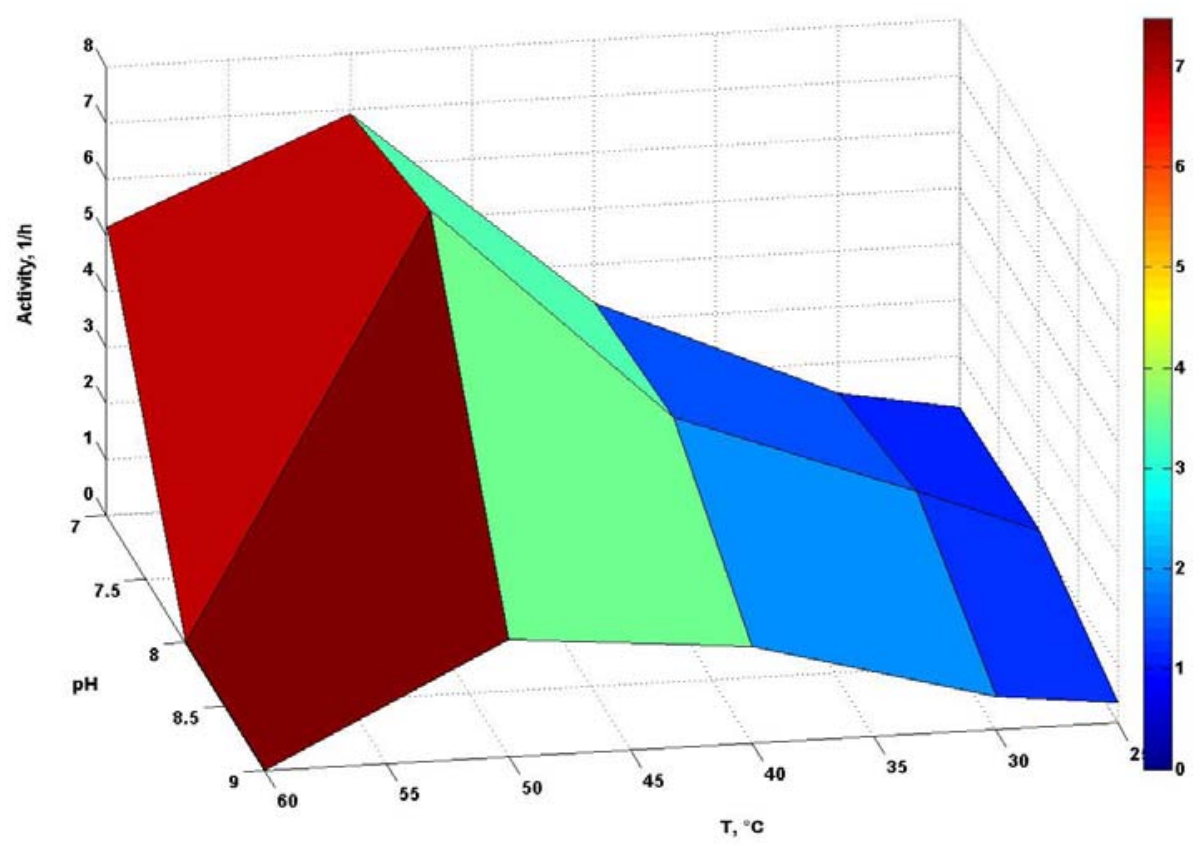

Figure 89 Activity versus temperature and pH of EL2X. Final concentration of EL2X in the HPG solutions is $0.08 \% \mathrm{w} / \mathrm{w}$.

Table 21 Activity versus temperature of pectinase for different $\mathrm{pH}$ values. Final concentration of pectinase in the HPG solutions is $0.02 \% \mathrm{w} / \mathrm{w}$.

\begin{tabular}{|c|c|c|c|c|}
\hline \multirow{2}{*}{ T,${ }^{\circ} \mathbf{C}$} & \multicolumn{4}{|c|}{ Activity, 1/h } \\
\cline { 2 - 5 } & $\mathbf{p H = 6 . 5}$ & $\mathbf{p H}=7.5$ & $\mathbf{p H}=\mathbf{8 . 5}$ & $\mathbf{p H = 9 . 1}$ \\
\hline 25 & 26.03 & 9.19 & 3.39 & 1.95 \\
\hline 30 & 28.17 & 10.68 & 5.70 & 1.48 \\
\hline 40 & 32.87 & 17.72 & 12.18 & 0 \\
\hline 50 & 33.79 & 25.39 & 15.28 & 0 \\
\hline 60 & 39.98 & 35.78 & 0 & 0 \\
\hline
\end{tabular}


Table 22 Activity versus temperature of EL2X for different pH values. Final concentration of EL2X in the HPG solutions is $0.08 \% \mathrm{w} / \mathrm{w}$.

\begin{tabular}{|c|c|c|c|}
\hline \multirow{2}{*}{$\mathbf{T},{ }^{\circ} \mathbf{C}$} & \multicolumn{3}{|c|}{ Activity, 1/h } \\
\cline { 2 - 4 } & $\mathbf{p H}=7$ & $\mathbf{p H}=\mathbf{8}$ & $\mathbf{p H}=\mathbf{9}$ \\
\hline 25 & 1.08 & 1.17 & 0.37 \\
\hline 30 & 1.45 & 1.98 & 0.60 \\
\hline 40 & 3.31 & 3.53 & 1.72 \\
\hline 50 & 6.91 & 7.48 & 2.08 \\
\hline 60 & 5.30 & 0 & 0 \\
\hline
\end{tabular}

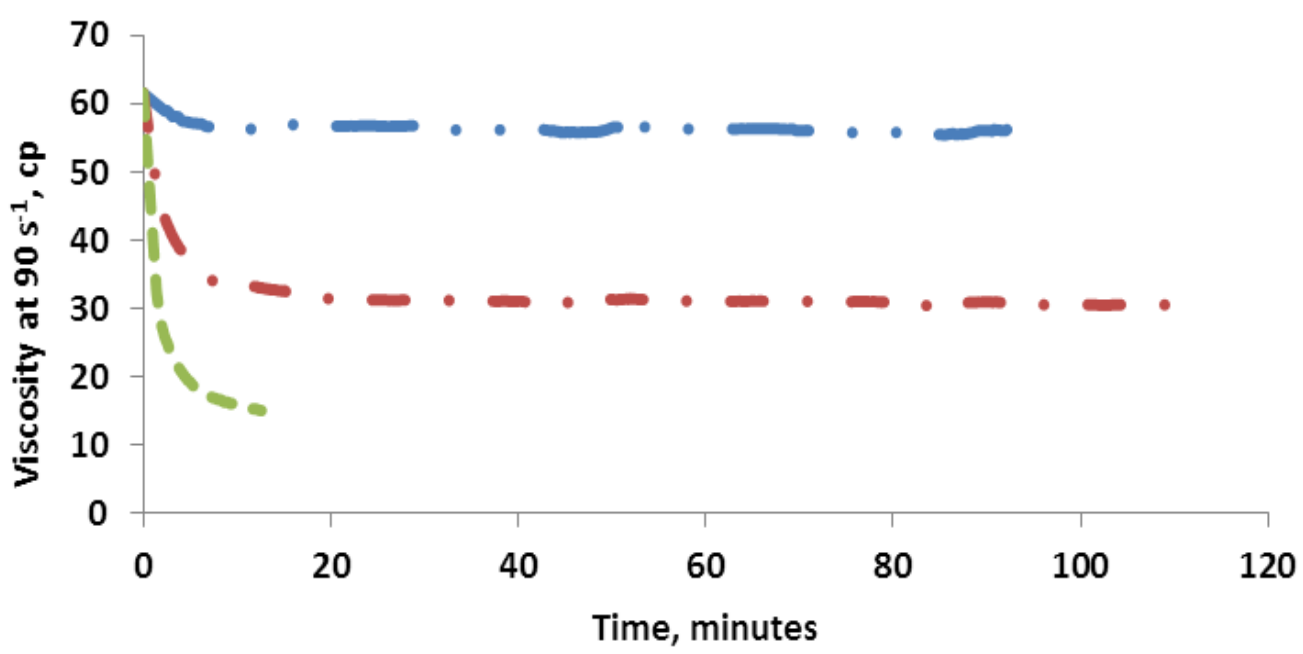

$0.02 \% \mathrm{w} / \mathrm{w}$ pectinase $-\cdot 0.1 \% \mathrm{w} / \mathrm{w}$ pectinase $-0.2 \% \mathrm{w} / \mathrm{w}$ pectinase

Figure 90 Denaturation of different concentrations of pectinase at $\mathrm{pH}$ of 9.1 and $40{ }^{\circ} \mathrm{C}$. Final concentrations of pectinase in the solution are used as labels.

In summary, both SDS-PAGE and viscometric assay were found to be suitable methods for measuring the entrapment efficiency of enzymes using PEC nanoparitcles. Denaturation of both 
enzymes was studied and temperature and $\mathrm{pH}$ environment at which both enzymes are denatured were characterized.

\subsection{Preparation and Optimization of Enzyme-Loaded Nanoparticles}

PEC nanoparticles prepared using different ratios of PEI over DS and concentrations of enzymes were studied for reproducibility, stability and shear sensitivity. Entrapment efficiencies (EE) were then measured and maximized for nanoparticles loaded with both enzymes.

\section{3-1 Preparation and Optimization of Enzyme-Loaded PEC Nanoparticles}

Pectinase-Loaded PEC Nanoparticles: Nanoparticles were prepared with different formulations which varied in PEI:DS ratio and in the order of addition as shown in Table 23. Precipitation occurred when PEC nanoparticles with PEI:DS ratio of 1:1 were prepared. Repeatability of the formulation procedure was tested by preparing 10 samples of PECNP system $A$ under identical conditions and measuring their sizes over time (Table 24). Size and zeta potential were measured periodically for another set of nanoparticles to demonstrate the stability of the nanoparticles over time (Table 25). The size and charge of the nanoparticles decreased with increasing PEI over DS ratio. No significant change was observed in size and charge of these nanoparticles versus time. This finding is in line with what Tiyaboonchai reported when loading a different chemical on PEI-DS nanoparticles. ${ }^{[10]}$

Nanoparticles were diluted to reach a specified enzyme concentration $(0.1 \% \mathrm{w} / \mathrm{w}$ pectinase $)$. In addition, $1 \mathrm{~mL}$ samples of undiluted nanoparticles were centrifuged and the supernatants were diluted by the same dilution factor ( $8 \times$ for systems $A$ and $A^{\prime} ; 6 \times$ for systems $B$ and $B^{\prime}$ ). Viscometric assay was performed for nanoparticle suspensions, supernatants and enzymes with concentrations equivalent to those of diluted nanoparticles.

Table 26 shows EE of nanoparticles with different ratios of PEI:DS loaded with pectinase. Nanoparticle systems with a 2:1 PEI:DS ratio (Systems $A$ and $A$ ) showed the highest EE of the systems prepared using $0.1 \mathrm{~mL}$ of pectinase. Entrapment efficiency $\left(\mathrm{EE}_{\mathrm{A}}\right)$ of the nanoparticles decreased with increasing PEI:DS ratio in the range studied. Nanoparticles which were prepared by adding pectinase to the PEI before addition of DS (Systems $A^{\prime}$ and $B$ ) showed higher $\mathrm{EE}_{\mathrm{A}}$ compared to the nanoparticles prepared by addition of pectinase to pre-formed PEI-DS nanoparticles (systems $A$ and $B$ ). 
To increase $\mathrm{EE}_{\mathrm{A}}$, the amount of pectinase added to the nanoparticles was reduced from $0.1 \mathrm{~mL}$ to $0.07 \mathrm{~mL}$ and $0.06 \mathrm{~mL}$ of $25 \% \mathrm{w} / \mathrm{w}$ pectinase for $A$ and $B$ nanoparticles. Nanoparticles prepared with less pectinase (Systems $D$ and $E$ ) showed improved $\mathrm{EE}_{\mathrm{A}}$ of $88 \%$ and $81 \%$ respectively. The concentration of pectinase in the supernatant of $A$ and $B$ PECNPs, measured by SDS-PAGE, was also used to calculate $\mathrm{EE}_{\mathrm{C}}$ of the nanoparticles (Table 27 and Figure 81). Calculated entrapment efficiencies were verified by measuring the activity of the enzyme in the supernatant. Entrapment efficiencies determined from concentration $\left(\mathrm{EE}_{\mathrm{C}}\right)$ were slightly higher than those calculated from enzyme activity $\left(\mathrm{EE}_{\mathrm{A}}\right)$.

In order to study the release of enzymes from the PEC nanoparticles compared to the activity of the enzyme not entrapped (i.e. free enzyme in the supernatant) PEC nanoparticle systems $A$ and $D$ and their supernatants were added to centrifuged samples of $5000 \mathrm{ppm}$ guar in $2 \% \mathrm{KCl}$. Viscosity of the mixtures was measured over time and compared to the viscosity reduction curves for mixtures of guar with different concentrations of pectinase (Figure 91). Higher viscosity of guar solutions mixed with the supernatant separated from PECNP systems compared to the guar solution mixed with only PEC nanoparticles is indicative of release of pectinase from the PEC nanoparticle system. 
Table 23 Nanoparticle systems differed in the ratio of PEI, DS, and pectinase and order of addition.

\begin{tabular}{|c|c|c|c|c|c|c|}
\hline $\begin{array}{c}\text { Syste } \\
\text { m }\end{array}$ & $\begin{array}{c}\text { 1\% w/w } \\
\text { PEI(aq), } \\
\text { mL }\end{array}$ & $\begin{array}{c}1 \% \text { w/w } \\
\text { DS(aq), } \\
\text { mL }\end{array}$ & $\begin{array}{c}25 \% \text { w/w } \\
\text { Pectinase(aq), } \\
\text { mL }\end{array}$ & $\begin{array}{l}\text { Equivalent } \\
100 \% \mathrm{w} / \mathrm{w} \\
\text { EL2X, mL }\end{array}$ & $\begin{array}{l}\text { Make-up } \\
\text { water, mL }\end{array}$ & $\begin{array}{l}\text { Order of } \\
\text { Addition }\end{array}$ \\
\hline$A$ & 2 & 1 & 0.10 & 0 & 0 & $\begin{array}{l}\text { PEI, DS, } \\
\text { Pectinase }\end{array}$ \\
\hline$A^{\prime}$ & 2 & 1 & 0.10 & 0 & 0 & $\begin{array}{c}\text { PEI, Pectinase, } \\
\text { DS }\end{array}$ \\
\hline$B$ & 3 & 1 & 0.10 & 0 & 0 & $\begin{array}{c}\text { PEI, DS, } \\
\text { Pectinase }\end{array}$ \\
\hline$B^{\prime}$ & 3 & 1 & 0.10 & 0 & 0 & $\begin{array}{c}\text { PEI, Pectinase, } \\
\text { DS }\end{array}$ \\
\hline$C$ & 4 & 1 & 0.10 & 0 & 0 & $\begin{array}{l}\text { PEI, DS, } \\
\text { Pectinase }\end{array}$ \\
\hline$C^{\prime}$ & 4 & 1 & 0.10 & 0 & 0 & $\begin{array}{c}\text { PEI, Pectinase, } \\
\text { DS }\end{array}$ \\
\hline$D$ & 2 & 1 & 0.07 & 0 & 0.03 & $\begin{array}{l}\text { PEI, DS, } \\
\text { Pectinase }\end{array}$ \\
\hline E & 3 & 1 & 0.06 & 0 & 0.04 & $\begin{array}{l}\text { PEI, DS, } \\
\text { Pectinase }\end{array}$ \\
\hline$H$ & 2 & 1 & 0 & 0.1 & 0 & PEI, DS, EL2X \\
\hline$H^{\prime}$ & 2 & 1 & 0 & 0.1 & 0 & PEI, EL2X, DS \\
\hline$I$ & 2 & 1 & 0 & 0.05 & 0.05 & PEI, DS, EL2X \\
\hline$I^{\prime}$ & 2 & 1 & 0 & 0.05 & 0.05 & PEI, EL2X, DS \\
\hline$J$ & 2 & 1 & 0 & 0.025 & 0.075 & PEI, DS, EL2X \\
\hline$J^{\prime}$ & 2 & 1 & 0 & 0.025 & 0.075 & PEI, EL2X, DS \\
\hline$K$ & 3 & 1 & 0 & 0.1 & 0 & PEI, DS, EL2X \\
\hline$K^{\prime}$ & 3 & 1 & 0 & 0.1 & 0 & PEI, EL2X, DS \\
\hline$L$ & 4 & 1 & 0 & 0.1 & 0 & PEI, DS, EL2X \\
\hline$L^{\prime}$ & 4 & 1 & 0 & 0.1 & 0 & PEI, EL2X, DS \\
\hline
\end{tabular}


Table 24 Mean diameter vs. time for 10 samples of PEC nanoparticles $A$

\begin{tabular}{|c|c|c|}
\hline Time, $\mathbf{h}$ & Mean Diameter, $\mathbf{n m}$ & Standard Deviation \\
\hline 0 & 461 & 10.2 \\
\hline 1 & 473 & 13.8 \\
\hline 2 & 463 & 16.9 \\
\hline 5 & 473 & 16.1 \\
\hline 13 & 472 & 12.4 \\
\hline 24 & 460 & 15.1 \\
\hline
\end{tabular}

Table 25 Mean particle diameter and zeta potential $(\zeta)$ vs. time for nanoparticles, $\mathbf{p H = 8 . 7}$

\begin{tabular}{|c|c|c|c|c|}
\hline \multirow{2}{*}{$\begin{array}{c}\text { Nanoparticle } \\
\text { System }\end{array}$} & \multicolumn{2}{|c|}{$\mathbf{8}$ hours } & \multicolumn{2}{c|}{ 32 hours } \\
\cline { 2 - 5 } & Diameter, $\mathbf{n m}$ & $\zeta, \mathbf{m V}$ & Diameter, $\mathbf{n m}$ & $\zeta, \mathbf{m V}$ \\
\hline$A$ & 433 & 29.4 & 408 & 35.0 \\
\hline$A^{\prime}$ & 435 & 36.3 & 424 & 34.9 \\
\hline$B$ & 370 & 28.3 & 362 & 29.6 \\
\hline$B^{\prime}$ & 313 & 28.2 & 292 & 24.3 \\
\hline$C$ & 250 & 27.4 & 238 & 17.5 \\
\hline$C^{\prime}$ & 239 & 18.7 & 235 & 10.4 \\
\hline
\end{tabular}


Table 26 Activity and entrapment efficiency of pectinase-loaded nanoparticles and their supernatants using viscometric assay $\left(\mathrm{T}=25^{\circ} \mathrm{C}, \mathrm{pH}=8.8\right)$. Centrifuged $5000 \mathrm{ppm}$ guar prepared in de-ionized water was used for this assay.

\begin{tabular}{|c|c|c|}
\hline Nanoparticle system & $\begin{array}{c}\text { Activity of } \\
\text { supernatant, } \mathbf{1} / \mathbf{h}\end{array}$ & $\mathbf{E E}_{\mathbf{A}}, \mathbf{\%}$ \\
\hline$B$ & 1.11 & 60 \\
\hline$A$ & 1.00 & 65 \\
\hline$B^{\prime}$ & 0.75 & 73 \\
\hline$A^{\prime}$ & 0.70 & 75 \\
\hline$D$ & 0.86 & 88 \\
\hline$E$ & 1.00 & 81 \\
\hline
\end{tabular}

Table 27 Entrapment efficiencies and enzyme concentrations for different supernatants using the first intensity peak

\begin{tabular}{|c|c|c|c|}
\hline $\begin{array}{c}\text { Polyelectrolyte Complex } \\
\text { Nanoparticle System }\end{array}$ & $\begin{array}{c}\text { Pectinase } \\
\text { concentration in } \\
\text { nanoparticle } \\
\text { suspension, } \% \mathbf{w} / \mathbf{w}\end{array}$ & $\begin{array}{c}\text { Pectinase } \\
\text { concentration in } \\
\text { supernatant, \% w/w }\end{array}$ & $\begin{array}{c}\text { Entrapment } \\
\text { efficiency }\left(\mathbf{E E}_{\mathbf{C}}\right)\end{array}$ \\
$\mathbf{\%}$ \\
\hline$A$ & 0.8 & 0.22 & 72 \\
\hline$B$ & 0.6 & 0.23 & 62 \\
\hline
\end{tabular}




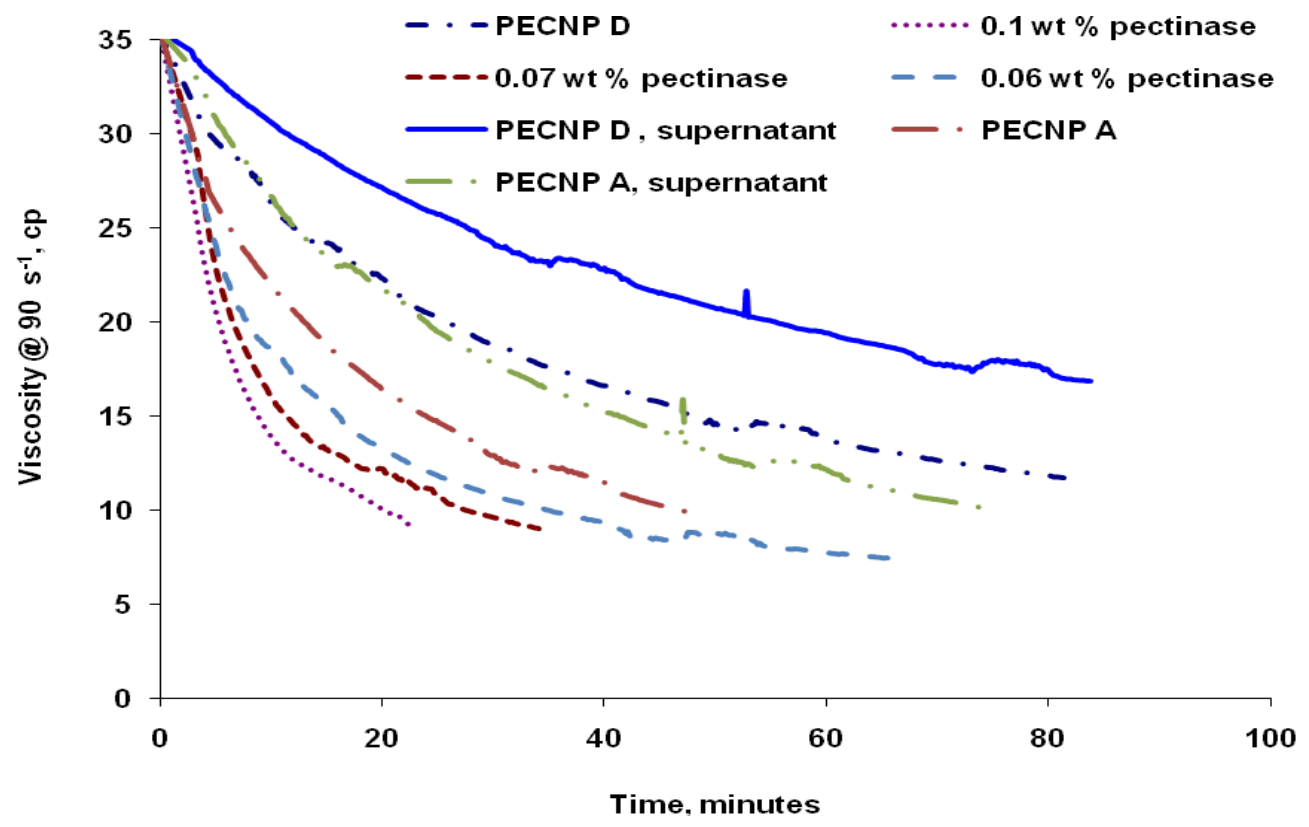

Figure 91 Viscometric assay for guar solutions mixed with PECNP D, supernatant of PECNP D or different concentrations of pectinase at $25 \mathrm{C}$ and $\mathrm{pH} 8.7$

Triplicates of several formulations varying in enzyme concentration and in the order of reagent addition were prepared as shown in Table 23. Size and zeta potential were measured in a range of $\mathrm{pH}$ and are shown in Figure 92. Zeta potential of the pectinase-loaded nanoparticles (PECNP $A^{\prime}$ ) decreased with increasing $\mathrm{pH}$ in the range tested, while particle size showed a maximum near $\mathrm{pH} 7$, then decreased with further increase in $\mathrm{pH}$. Note that $\mathrm{pH}$ of nanoparticles was varied by changing the $\mathrm{pH}$ of $1 \% \mathrm{w} / \mathrm{w}$ PEI solution. Four $1 \% \mathrm{w} / \mathrm{w}$ PEI samples used during the preparation of four PEC nanoparticles with different $\mathrm{pH}$ values had $\mathrm{pH}$ values of $6,7.5,8$ and 8.7. Adjustment of $\mathrm{pH}$ was done by adding $4 \mathrm{~N} \mathrm{HCl}$ to the originally prepared $1 \% \mathrm{w} / \mathrm{w}$ PEI solution. R.O. water was added to the $1 \% \mathrm{w} / \mathrm{w}$ PEI solutions with $\mathrm{pH}$ above 6 to adjust the final PEI concentration (0.98 wt \%). Figure 93 shows pH of $1 \%$ PEI solution versus volume of $4 \mathrm{~N}$ added $\mathrm{HCl}$.

Nanoparticles were diluted to reach a final enzyme concentration of $0.1 \% \mathrm{w} / \mathrm{w}$ for pectinase. In addition, $1 \mathrm{~mL}$ samples of undiluted nanoparticles were centrifuged and the supernatants were diluted by the same dilution factor as the nanoparticles. Viscometric assay was performed for nanoparticle suspensions, supernatants and enzymes with concentrations equivalent to those of 
diluted nanoparticles. Entrapment efficiency of the pectinase-loaded nanoparticles (PECNP $A^{\prime}$ ) showed a maximum of $91 \%$ at $\mathrm{pH} 8.5$ (Figure 94).

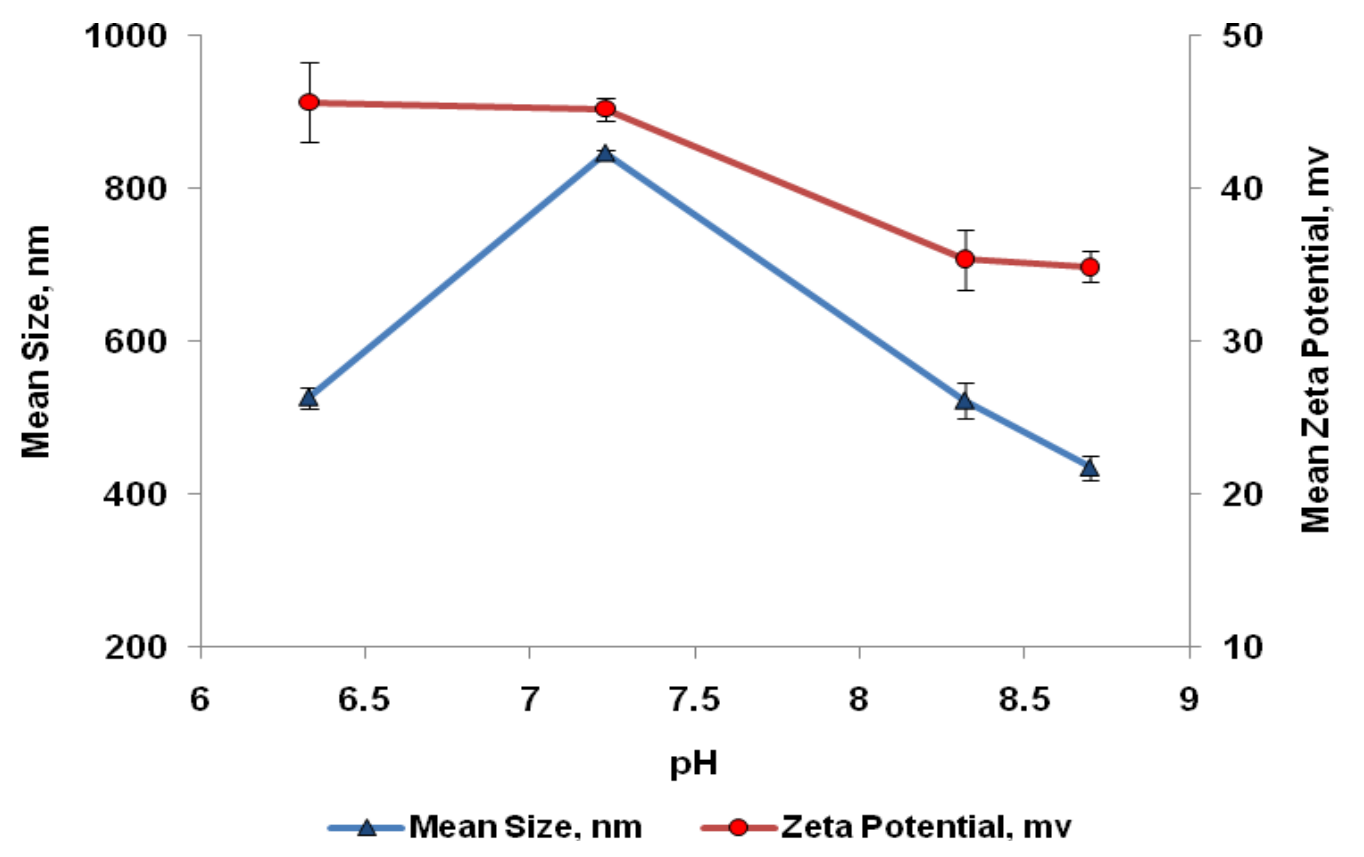

Figure 92 Mean size and zeta potential versus $\mathrm{pH}$ for pectinase-loaded PECNPs $(A)$.

Error bars $=1 \mathrm{SE}, \mathrm{n}=3$. 


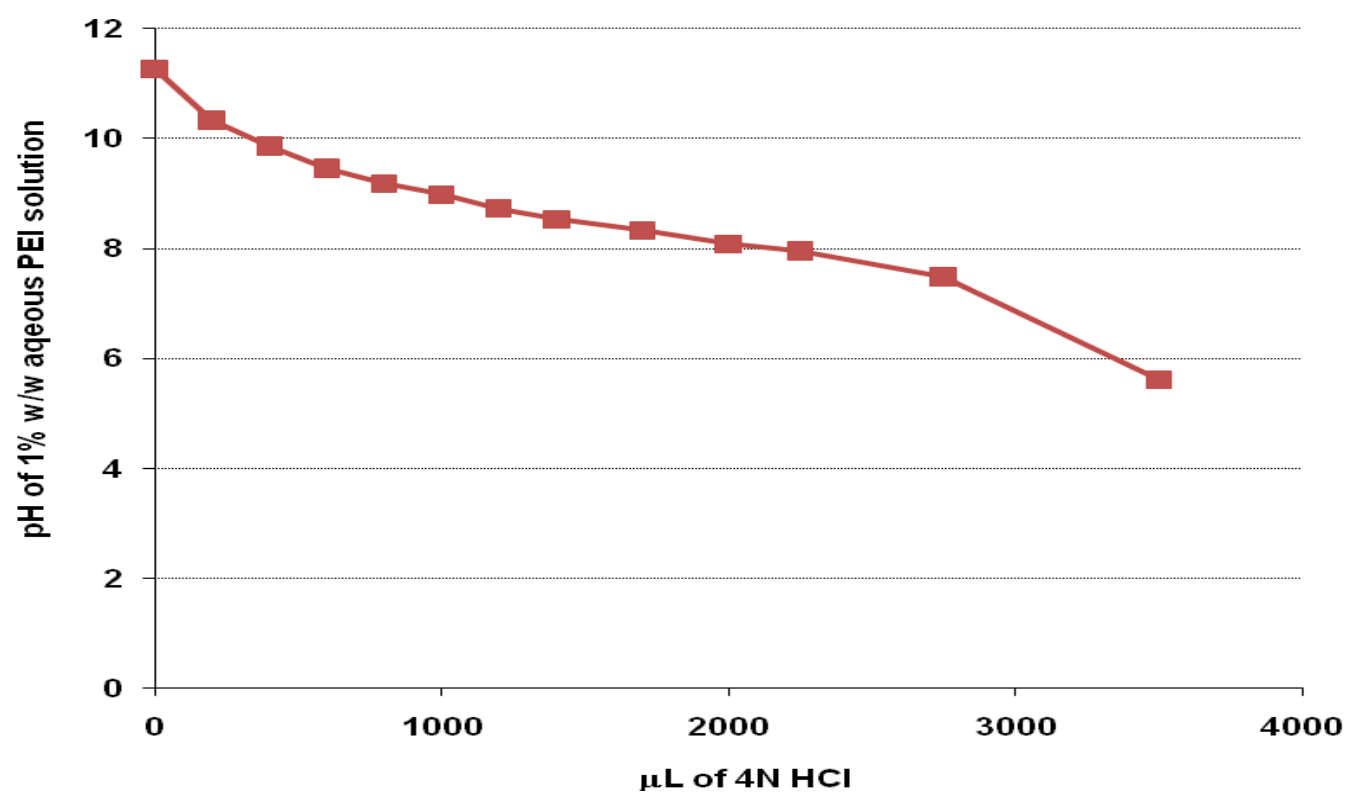

Figure $93 \mathrm{pH}$ of $1 \% \mathrm{w} / \mathrm{w}$ PEI (1 L) versus volume of $4 \mathrm{~N} \mathrm{HC}$ added at $25^{\circ} \mathrm{C}$

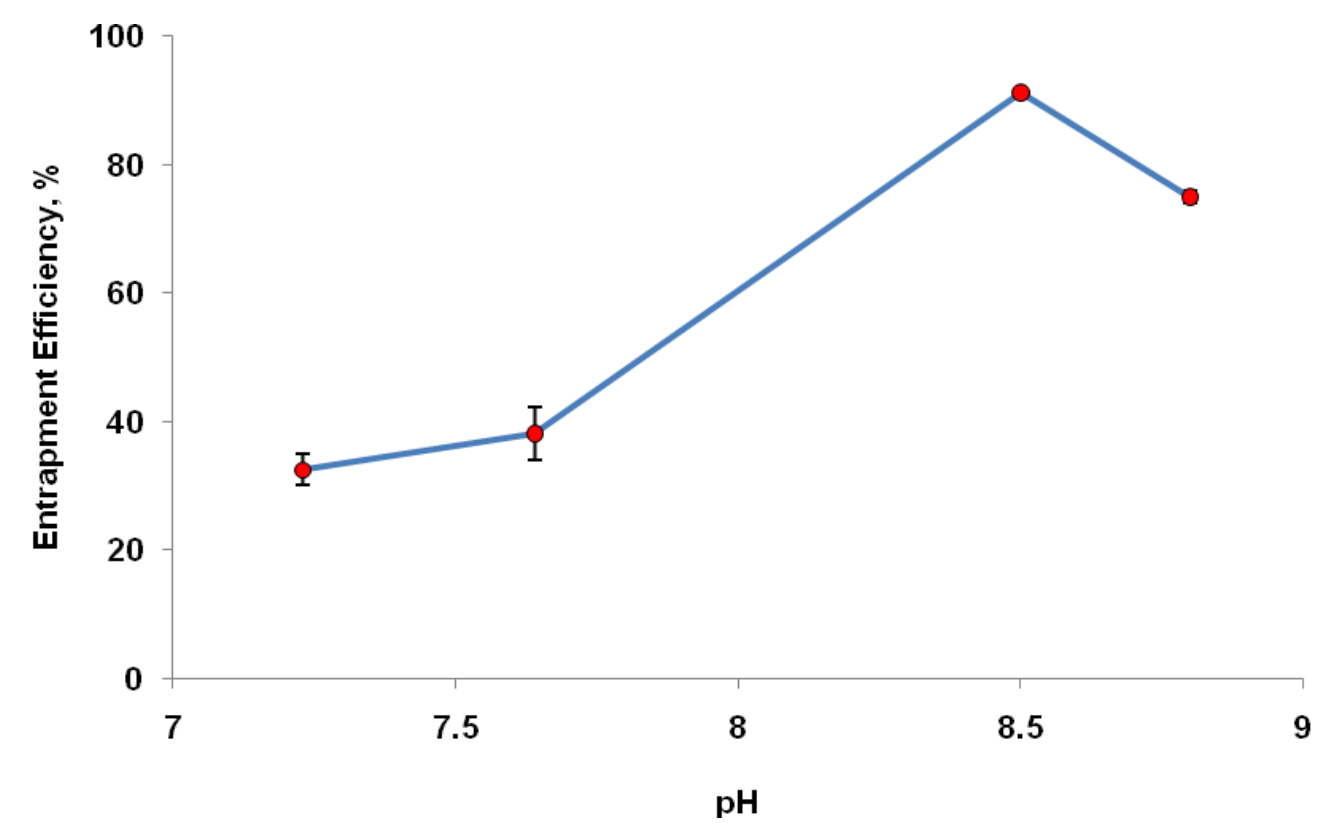

Figure 94 Entrapment efficiency $\left(\mathrm{EE}_{\mathrm{A}}\right)$ vs. $\mathrm{pH}$ for pectinase $\left(\mathrm{A}^{\prime}\right)$ loaded PECNPs at $25^{\circ} \mathrm{C}$.

Error bars $=1 \mathrm{SD}, \mathbf{n}=\mathbf{3}$ 
EL2X-Loaded PEC Nanoparticles: Nanoparticles were prepared with different formulations, varying in PEI:DS ratio, order of addition and concentration of enzyme as shown in Table 23. Triplicates of several formulations varying in enzyme concentration and in the order of reagent addition were prepared. Size and zeta potential were measured in a range of $\mathrm{pH}$ (6.5-9.4) and are shown in Figure 95. Both particle size and zeta potential of the EL2X-loaded PECNPs decreased with increasing $\mathrm{pH}$. EL2X-loaded PEC nanoparticle systems with $\mathrm{pH} 10$ and 11 were also prepared, but precipitation was observed for these systems after addition of DS.

Nanoparticles were diluted to reach a final enzyme concentration of $0.4 \%$ for EL2X. In addition, $1 \mathrm{~mL}$ samples of undiluted nanoparticles were centrifuged and the supernatants were diluted by the same dilution factor as the nanoparticles. Viscometric assay was performed for nanoparticle suspensions, supernatants and enzymes with concentrations equivalent to those of diluted nanoparticles. EL2X systems showed maximum EE of $46 \%$ when $0.1 \mathrm{~mL}$ of $100 \%$ EL2X was added (PECNP $\left.H^{\prime}\right)$. By decreasing the concentration of EL2X to $50 \%\left(I^{\prime}\right)$ and $25 \%\left(J^{\prime}\right), \mathrm{EE}_{\mathrm{A}}$ was slightly improved to about 54\% and 61\% respectively (Figure 96).

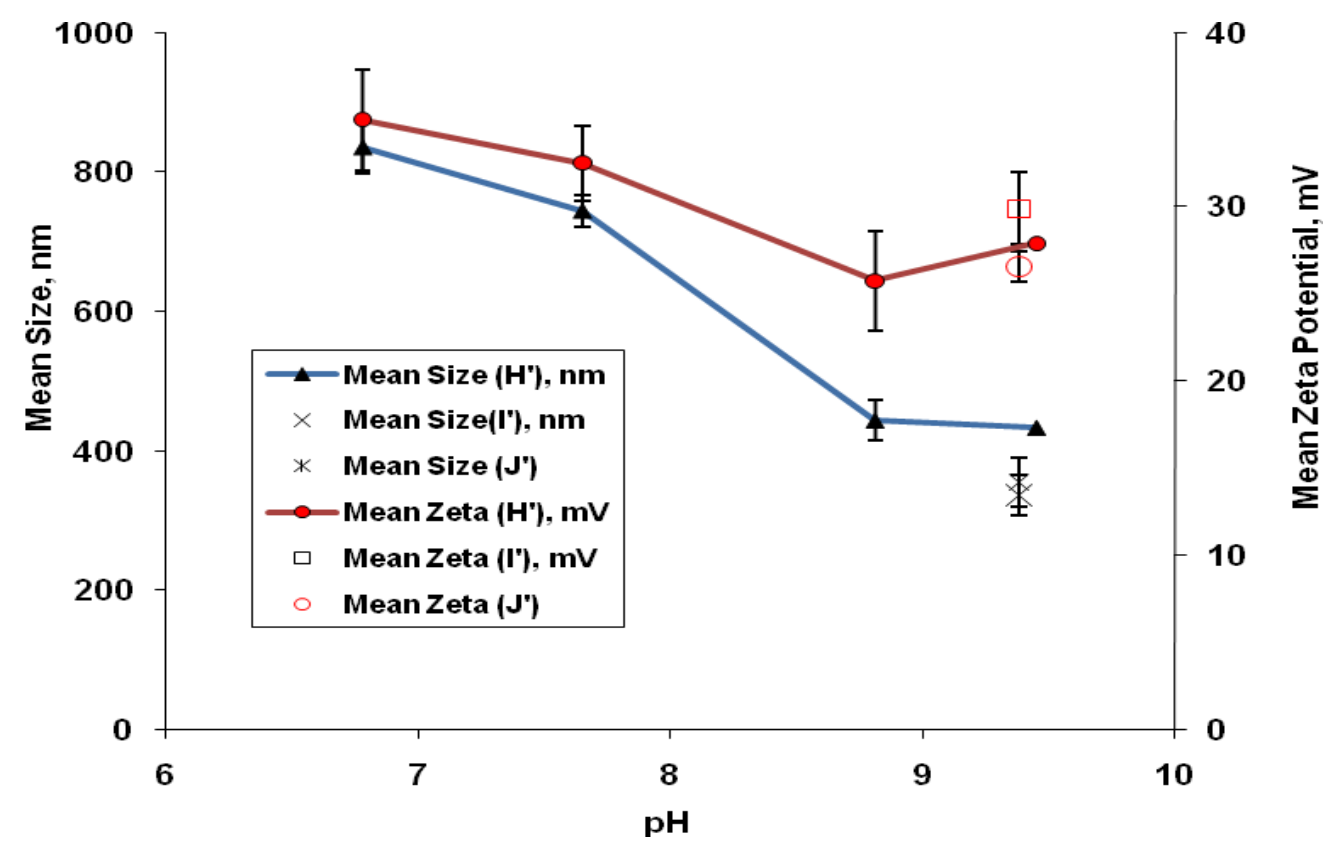

Figure 95 Mean size and zeta potential versus pH for EL2X-loaded PECNPs $\left(H^{\prime}, I^{\prime}, J^{\prime}\right)$. Error bars $=1 \mathrm{SE}, \mathrm{n}$ $=3$. 


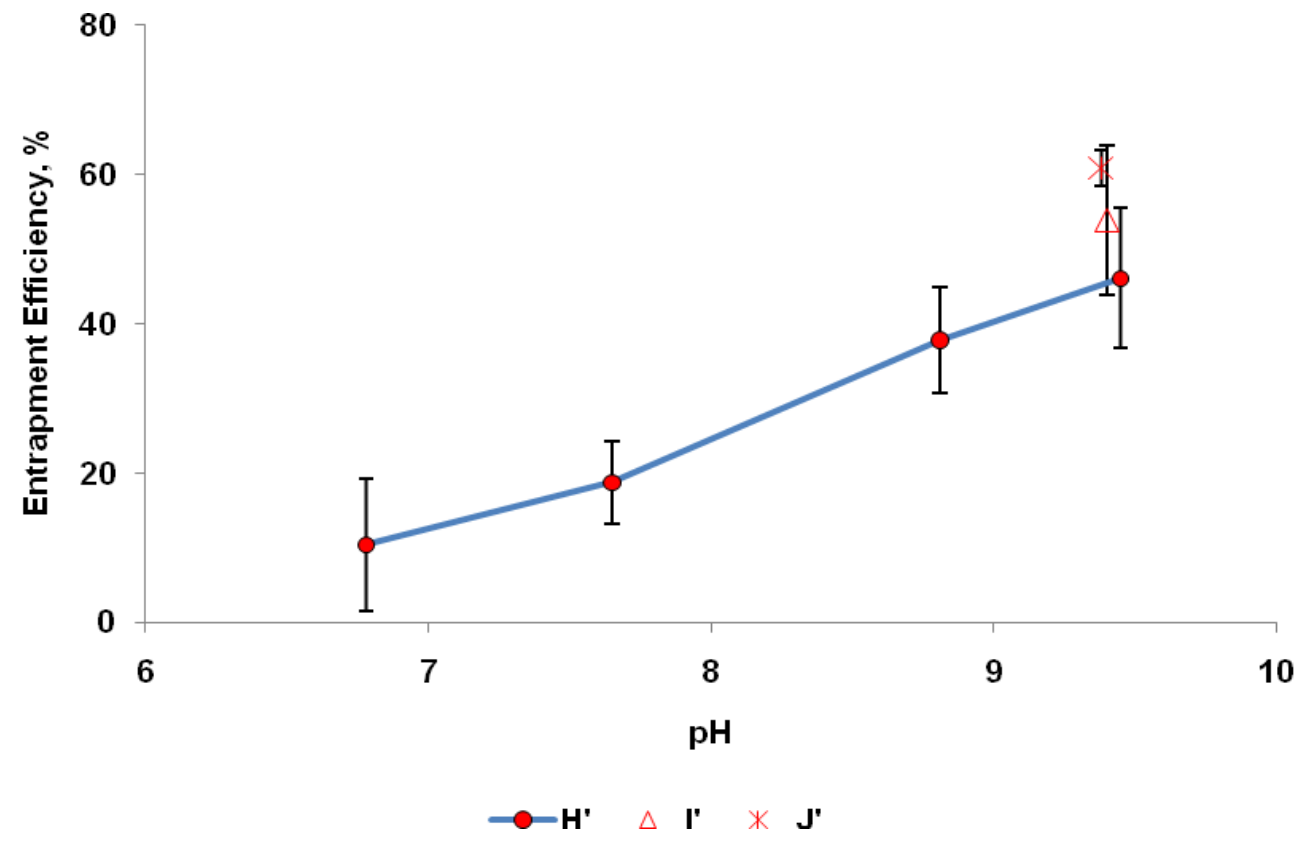

Figure 96 Entrapment efficiency $\left(\mathrm{EE}_{\mathrm{A}}\right)$ vs. $\mathrm{pH}$ for EL2X-loaded PECNPs at $40{ }^{\circ} \mathrm{C}$. Error bars $=1 \mathrm{SD}, \mathrm{n}=3$

\section{3-2 Effect of Shear During Particle Preparation and Effect of Applied Shear on Enzyme-Loaded Nanoparticles}

Effect of Shear during the Preparation of Pectinase-Loaded Nanoparticles: Batches of nanoparticle system $A$ were prepared while stirring at 300,600 and $900 \mathrm{rpm}$ at $25^{\circ} \mathrm{C}$ and $\mathrm{pH} 8.8$. There was no relationship between preparation shear and size, zeta potential or entrapment efficiency of the nanoparticles within this range (Table 28).

During the preparation process it was discovered that fast addition of DS and enzyme results in smaller nanoparticles compared to the drop-wise method of adding chemicals. This was proven after samples $A^{\prime}(\mathrm{pH} 8.38)$ and $H^{\prime}(\mathrm{pH}$ 9.4) were prepared by adding enzyme and DS drop-wise to the stirring 1\% PEI solution. Particle size for these samples was $658 \mathrm{~nm}$ and $521 \mathrm{~nm}$ respectively. These sizes were respectively $200 \mathrm{~nm}$ and $100 \mathrm{~nm}$ higher than the size of same systems prepared by fast addition of chemicals. Subsequently all the nanoparticles in this research were made by fast addition of chemicals unless otherwise mentioned. 
Table 28 Effect of preparation shear on size and zeta potential of PECNP $A^{\prime}$ system at pH 8.8 and $25^{\circ} \mathrm{C}$

\begin{tabular}{|c|c|c|c|c|c|c|}
\hline RPM & Mean Size, nm & $\begin{array}{c}\text { Size Std. } \\
\text { Dev. }\end{array}$ & $\begin{array}{c}\text { Mean Zeta. } \\
\mathbf{m V}\end{array}$ & $\begin{array}{c}\text { Zeta Std. } \\
\text { Dev. }\end{array}$ & $\begin{array}{c}\text { Mean EE } \\
\text { A },\end{array}$ & $\begin{array}{c}\text { EE }_{\mathbf{A}} \text { Std. } \\
\text { Dev. }\end{array}$ \\
\hline 300 & 488.93 & 39.54 & 51.05 & 2.50 & 77.37 & 3.7 \\
\hline 600 & 481.17 & 21.30 & 53.66 & 1.20 & 79.91 & 2.42 \\
\hline 900 & 461.83 & 41.01 & 52.08 & 1.50 & 79.69 & 2.71 \\
\hline
\end{tabular}

Effect of Applied Shear on Activity of Enzyme-Loaded Nanoparticles: Viscometric assays were performed on samples from the nanoparticle system $A$, prepared while stirring at $600 \mathrm{rpm}$. Activity of the nanoparticles was determined from the time required for viscosity of a $5000 \mathrm{ppm}$ HPG solution in $2 \% \mathrm{KCl}$ to decrease to $50 \%$ of its starting value. Figure 97 shows that when the shear applied during the experiment increased, the activity of the nanoparticles decreased. However, the activity of the pectinase also decreased with the same trend, indicating that any additional release of pectinase from the nanoparticles caused by increase in the shear is insignificant. Shear rates were chosen to be broadly representative of the shear rates encountered by the gel in the field. ${ }^{[1]}$ A similar effect of shear was observed on activity of $0.4 \%$ w/w EL2X. However, EL2X-loaded PECNP system $H$ was slightly sensitive to shear (Figure 98).

The decreasing trend of enzyme activity with shear demonstrated here is in line with previous research. ${ }^{[97]}$ Unbounding of enzyme from substrate due to agitation and distortion of 3D structure of enzyme caused by shear were reported as main causes of this activity loss. ${ }^{[97]}$ 


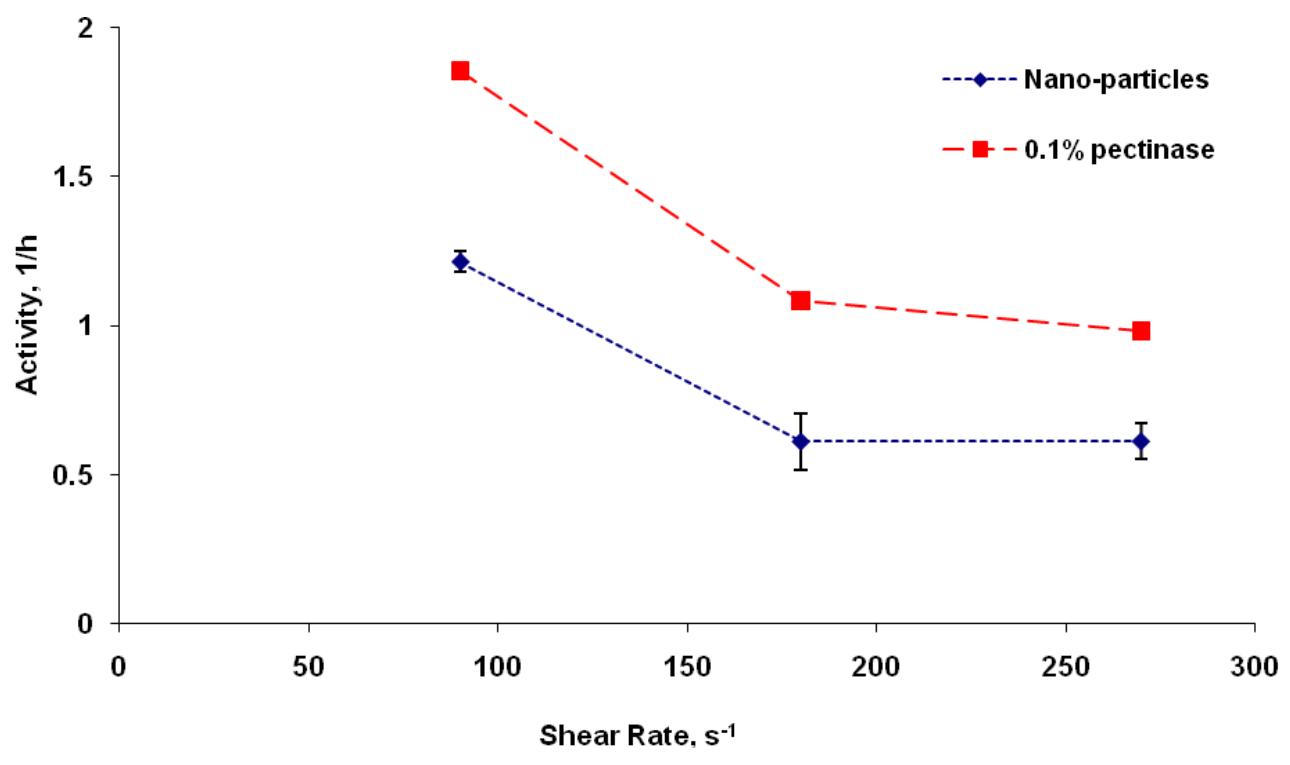

Figure 97 Comparison of effect of shear on the activity of $0.1 \%$ pectinase solution and pectinase-loaded nanoparticles (PECNP System A) mixed with HPG solution at $25^{\circ} \mathrm{C}$ and $\mathrm{pH}$ 8.8. Activity $=1 /$ time taken to degrade 5000 ppm HPG solution to $50 \%$ of its initial viscosity. Error bars $=1$ SD, $n=3$

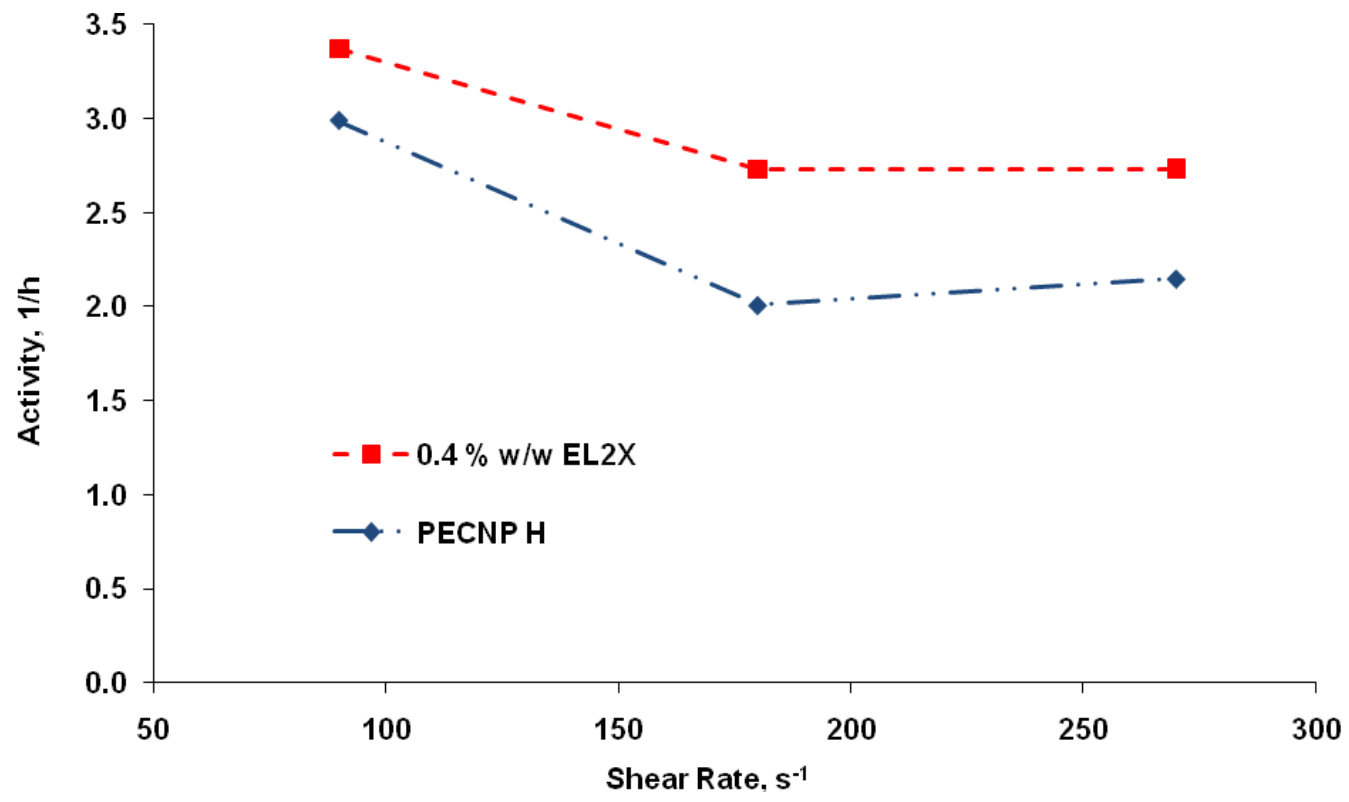

Figure 98 Comparison of effect of shear on the activity of 0.4\% EL2X solution and EL2X-loaded nanoparticles (PECNP System $H$ ) mixed with HPG solution at $40{ }^{\circ} \mathrm{C}$ and pH 9. Activity $=1 /$ time taken to degrade 5000 ppm HPG solution to $50 \%$ of its initial viscosity. 


\subsection{Degradation of Borate Cross-linked Guar/HPG Using Enzymes and Enzyme-Loaded Nanoparticles}

Degradation of borate cross-linked guar and HPG gels are studied in this section. Measurement of both viscosity and viscoelastic moduli was performed to study controlled release of both enzymes using the PECNP systems introduced in previous sections.

\section{4-1 Degradation of Borate Cross-Linked Guar/HPG Using Pectinase and Pectinase-Loaded Nanoparticles}

A $5000 \mathrm{ppm}$ solution of guar in $2 \% \mathrm{KCl}$ was used to prepare borate $(1000 \mathrm{ppm})$ cross-linked gels. Diluted nanoparticles with different ratios of PEI:DS were mixed with the guar before addition of borate. Final concentrations of guar, borate and enzyme were the same in all the preparations (Table 29). The resulting gels were incubated on a table shaker at $150 \mathrm{rpm}$ and $25^{\circ} \mathrm{C}$ and samples were removed at intervals for viscosity measurement at a shear rate of $4.5 \mathrm{~s}^{-1}$. Figure 99 shows the viscosity of gelled guar containing pectinase entrapped nanoparticles using different ratios of PEI:DS. Nanoparticles with pectinase added before addition of DS (Systems $A^{\prime}$ and $B^{\prime}$ ) showed a later degradation time compared to the nanoparticles where pectinase was added after addition of DS (Systems $A$ and $B$ ).

While viscosity is the most commonly measured property during field application, it is difficult to measure viscosity of a gel. In order to further characterize the degradation process, a rheometric study was performed. Pectinase-loaded nanoparticles (final pectinase concentration $0.02 \%$ ) with 2:1 ratios of PEI:DS (Systems $A$ ) were mixed with 5000 ppm HPG solution in 2\% KCl. 2000 ppm borax was then added to make crosslinked HPG. Gels were shaken on a table shaker at $150 \mathrm{rpm}$ and $25{ }^{\circ} \mathrm{C}$; samples were removed at intervals and viscoelastic moduli were measured using a Bohlin rheometer. A frequency sweep was performed at different times and viscous and elastic moduli were plotted versus time for a frequency of $0.5 \mathrm{~Hz}$ (Figure 100). Delay in degradation of both moduli was observed. Both $\mathrm{G}^{\prime}$ and $\mathrm{G}^{\prime \prime}$ reached values lower than those of an equivalent HPG solution in $10 \mathrm{~h}$, indicating a significant delay in activity of enzyme. The point at which plots of $\mathrm{G}^{\prime}$ and $\mathrm{G}^{\prime \prime}$ cross (i.e. the time at which the elastic modulus becomes smaller than the viscous modulus) was shifted towards longer times for the systems degraded with nanoparticles compared to the systems degraded with enzyme represents a delay in transition from gel to solution. Faster decline of $\mathrm{G}^{\prime}$ compared to $\mathrm{G}^{\prime \prime}$ is typical for enzymatic 
degradation of guar solutions since the gel structure is attacked first, followed by the degradation of the guar back bone. ${ }^{[32]}$

Note that viscosity measurements (Figure 99) showed that gels containing PEC nanoparticles reach the same viscosity values as those degraded using unentrapped pectinase given enough time. The decreasing rate of viscoelastic moduli confirms this result (Figure 100).

Reproducibility of the degradation of viscoelastic parameters for borate-cross linked guar gels were assured by making three samples of gels mixed with PECNP $A$ system and three samples of gel mixed with 0.1 wt \% pectinase (Figure 101 and Figure 102).

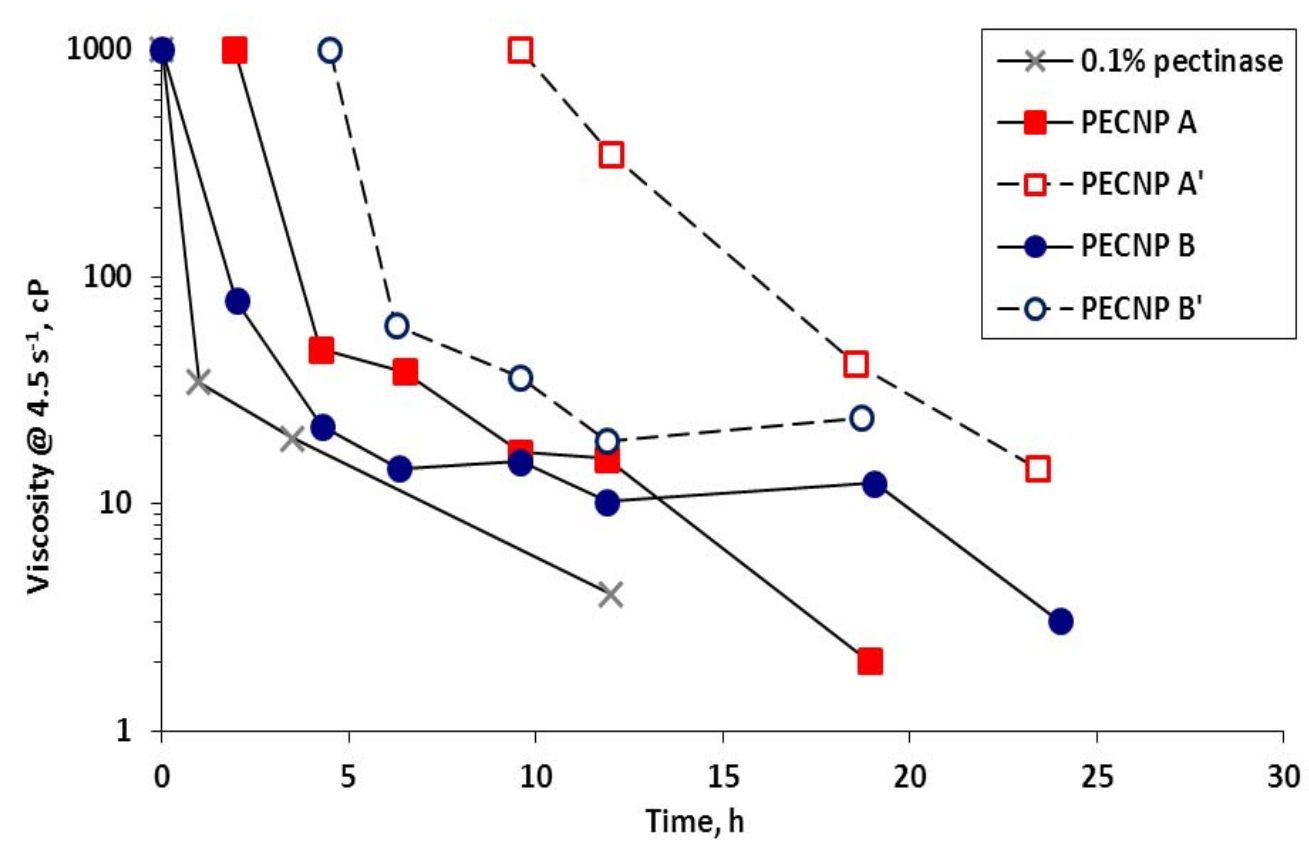

Figure 99 Viscosity of borate $(1000 \mathrm{ppm})$ cross-linked guar $(5000 \mathrm{ppm})$ gel mixed with $0.1 \%$ pectinase or pectinase-loaded PEC nanoparticles vs. time at $25{ }^{\circ} \mathrm{C}$ and $\mathrm{pH}$ 9. N.B. the viscometer is only able to measure viscosity up to $1028 \mathrm{cP}$ so graph indicates the latest measurement for which viscosity exceeded this value. 
Table 29 Final concentrations of HPG/guar, borax and enzymes in the solutions for all gel degradation studies reported in this research

\begin{tabular}{|l|l|l|l|l||}
\hline & $\begin{array}{l}\text { Guar/HPG, } \\
\text { ppm }\end{array}$ & $\begin{array}{l}\text { Borax, } \\
\text { ppm }\end{array}$ & $\begin{array}{l}\text { pectinase, wt } \\
\%\end{array}$ & $\begin{array}{l}\text { EL2X, } \\
\text { wt \% }\end{array}$ \\
\hline $\begin{array}{l}\text { Stock solutoins for pectinase-loaded } \\
\text { systems }\end{array}$ & 5000 & 1000 & 0.1 & 0 \\
\hline Stock solutoins for EL2X-loaded systems & 5000 & 2000 & 0 & 0.4 \\
\hline Final solution for pectinase-loaded systems & 3077 & 192 & 0.02 & 0 \\
\hline Final solution for EL2X-loaded systems & 3077 & 385 & 0 & 0.08 \\
\hline
\end{tabular}

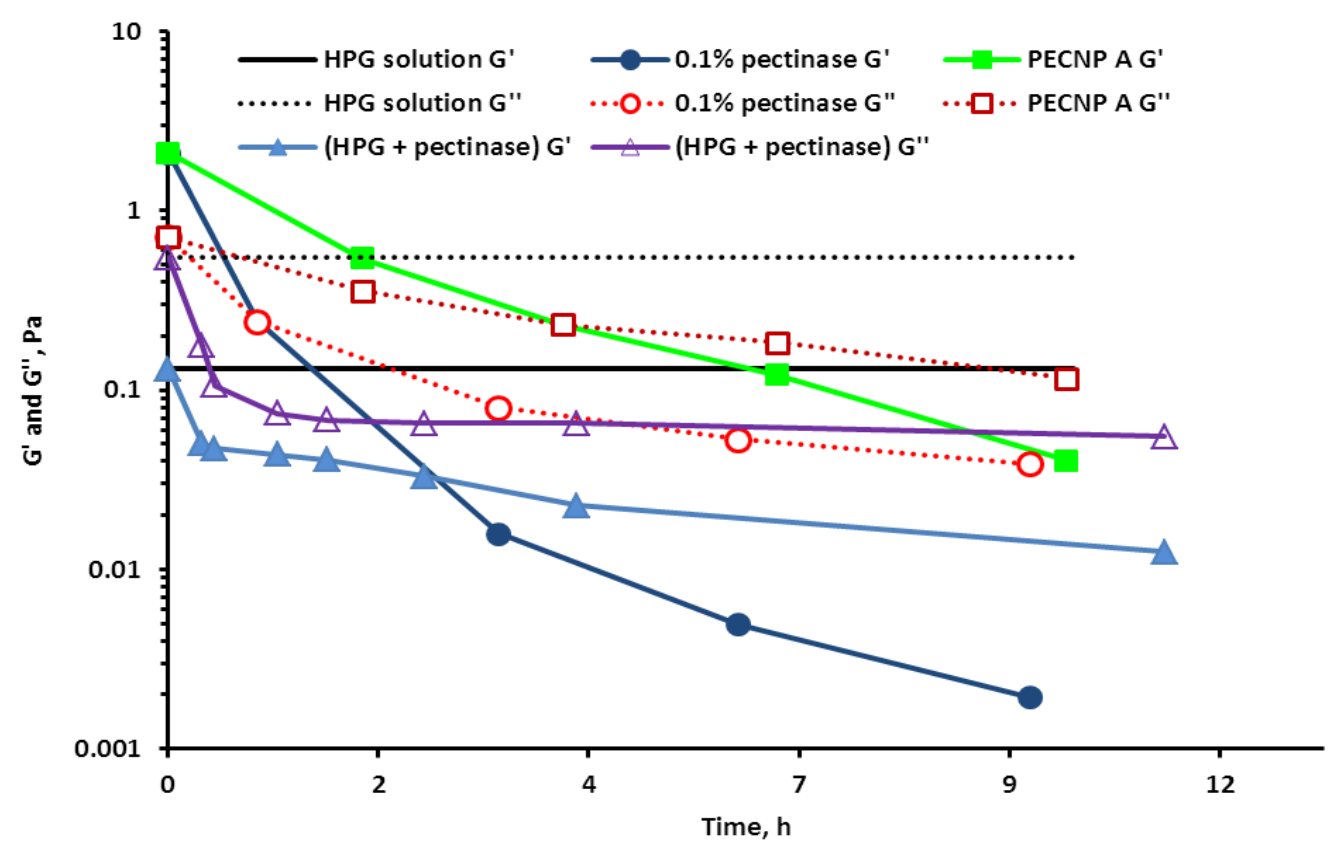

Figure $100 \mathrm{G}^{\prime}$ and $\mathrm{G}^{\prime \prime}$ vs. time for $5000 \mathrm{ppm}$ HPG solution, and borate (2000 ppm)-crosslinked HPG gel degraded using either $0.1 \%$ pectinase or nanoparticle-entrapped pectinase (PEC nanoparticle System $A$ ) at $25^{\circ} \mathrm{C}$ and $\mathrm{pH} 9$. 


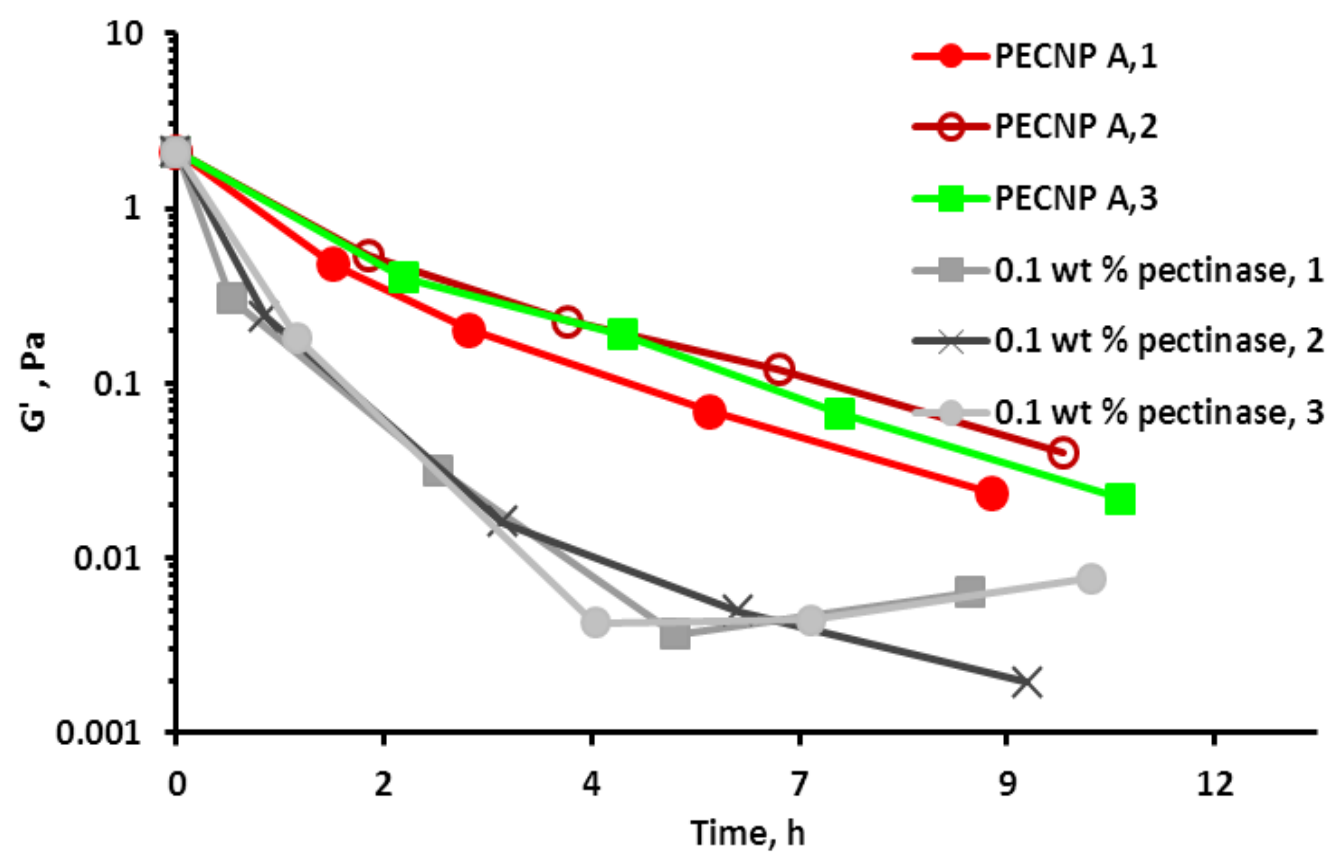

Figure 101 Reproducible degradation of elastic modulus versus time for $5000 \mathrm{ppm}$ HPG solution, and borate (2000 ppm)-crosslinked HPG gel degraded using either $0.1 \%$ pectinase or nanoparticle-entrapped pectinase (PECNP System $A$ ) at $25^{\circ} \mathrm{C}$ and pH 9.

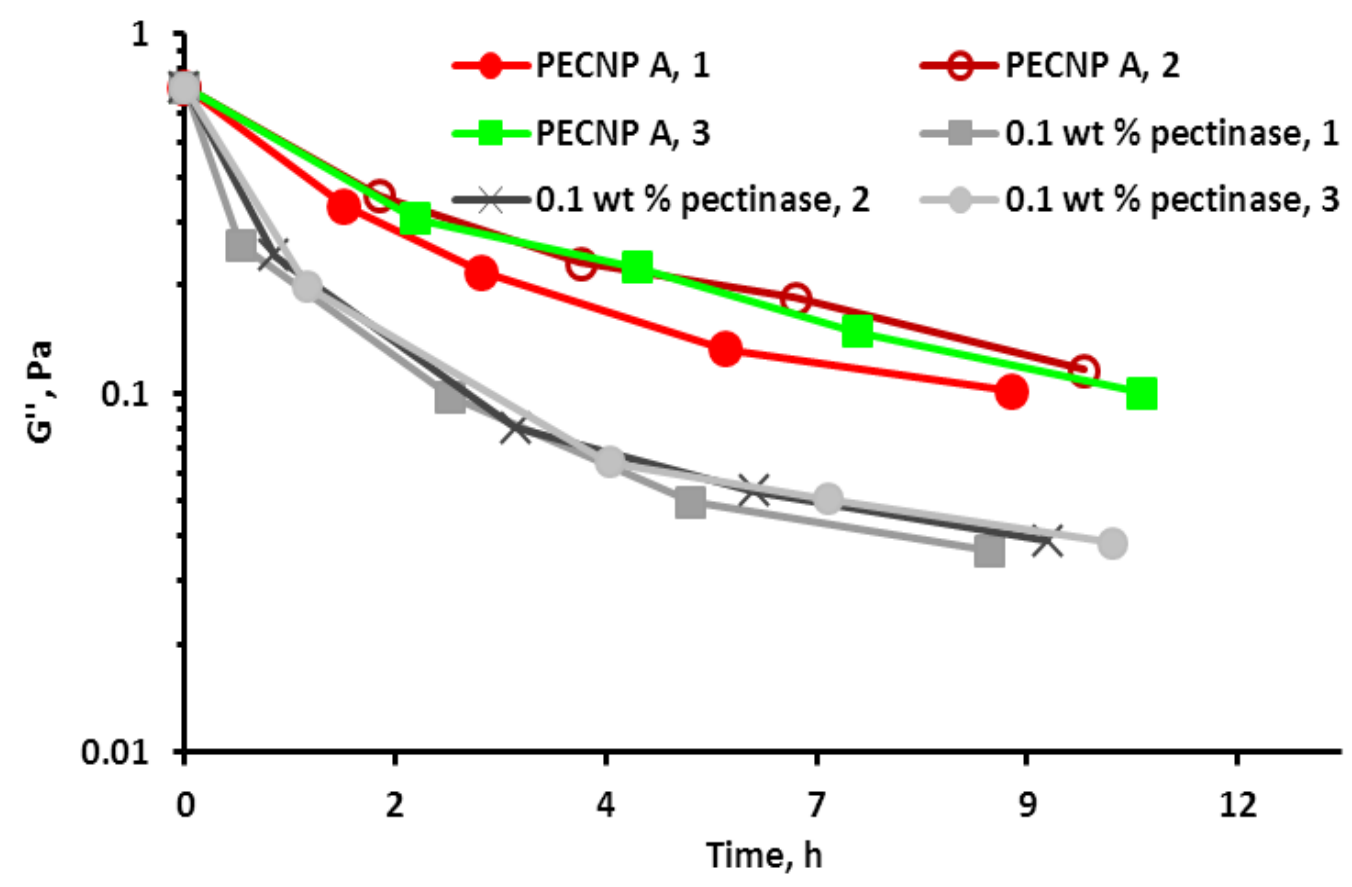

Figure 102 Reproducible degradation of viscous modulus versus time for $5000 \mathrm{ppm}$ HPG solution, and borate (2000 ppm)-crosslinked HPG gel degraded using either $0.1 \%$ pectinase or nanoparticle-entrapped pectinase (PECNP System $A$ ) at $25^{\circ} \mathrm{C}$ and $\mathrm{pH} 9$. 


\section{4-2 Degradation of Borate Cross-Linked HPG Using EL2X and EL2X- Loaded PEC Nanoparticles}

In this section delayed release of EL2X from the PEC system (prepared at) $\mathrm{pH} 9.4(\mathrm{EE}=54 \%)$ and $40{ }^{\circ} \mathrm{C}$ is shown. Viscosity is commonly measured during field application since it is a relatively simple and fast assay.

Before the $\mathrm{EE}_{\mathrm{A}}$ of EL2X-loaded nanoparticles was maximized, particles with different ratios of PEI over DS (PECNP systems $H, H^{\prime}, K, K^{\prime}, L$ and $L^{\prime}$ ) were mixed with 5000 ppm HPG solution in $2 \% \mathrm{KCl}$ and $2000 \mathrm{ppm}$ borax cross-linker. A sample of gel mixed with $0.4 \mathrm{wt} \%$ EL2X solution was also prepared. The resulting gels were incubated on a table shaker at $150 \mathrm{rpm}$ and $40{ }^{\circ} \mathrm{C}$ and samples were removed at intervals for viscosity measurement at $40{ }^{\circ} \mathrm{C}$ and shear rate of $4.5 \mathrm{~s}^{-1}$. Figure 103 shows the viscosity of gelled guar containing EL2X entrapped nanoparticles using different ratios of PEI: DS. Nanoparticles with EL2X added before addition of DS (Systems $H^{\prime}, K^{\prime}$ and $L^{\prime}$ ) showed delayed degradation compared to the nanoparticles where EL2X was added after addition of DS (Systems $H, K$ and $L$ ).

In order to further characterize the degradation process, a rheometric study was also performed. EL2X-loaded nanoparticles with final EL2X concentration of $0.4 \% \mathrm{w} / \mathrm{w}$ were mixed with $5000 \mathrm{ppm} \mathrm{HPG}$ solution in $2 \% \mathrm{KCl}$ and $2000 \mathrm{ppm}$ borax cross-linker was added. The mixture gelled immediately upon addition of the borax at room temperature. Gels with enzymes and control gels without enzyme added were shaken at $150 \mathrm{rpm}$ and $40{ }^{\circ} \mathrm{C}$; samples were removed at intervals and viscoelastic moduli were measured using a Bohlin rheometer. Before maximizing the $\mathrm{EE}_{\mathrm{A}}$ of nanoparticles viscoelastic moduli were measured for PECNP systems $H, K$ and $L$. Viscous $\left(\mathrm{G}^{\prime \prime}\right)$ and elastic $\left(\mathrm{G}^{\prime}\right)$ moduli were plotted versus time for a frequency of $0.5 \mathrm{~Hz}($ Figure 104 and Figure 105). Delay in degradation of elastic modulus was observed; G' reached values equal to or lower than those of an equivalent HPG gel degraded with unentrapped enzyme in $6 \mathrm{~h}$. No significant delay in reduction of viscous modulus was observed.

Viscoelastic moduli were also measured for PECNP system $I^{\prime}$ (Figure 106). Delay in degradation of both moduli was observed. $\mathrm{G}^{\prime}$ and $\mathrm{G}^{\prime \prime}$ reached values equal to or lower than those of an equivalent HPG gel degraded with unentrapped enzyme in $7 \mathrm{~h}$ and $11 \mathrm{~h}$ respectively, indicating a significant delay in activity of enzyme (by 3 and $7 \mathrm{~h}$ ). Plots of both $\mathrm{G}^{\prime}$ and $\mathrm{G}^{\prime \prime}$ were shifted towards longer delays for the systems degraded with nanoparticles compared to the 
systems degraded with unentrapped enzyme, indicating a delay in transition from gel to a low viscosity solution. Faster decline of $\mathrm{G}^{\prime}$ compared to $\mathrm{G}^{\prime \prime}$ is also typical for enzymatic degradation of guar solutions since the gel structure is attacked first, followed by the degradation of the guar back bone. ${ }^{[32]}$ Reproducibility of gel degradation using PECNP system $I^{\prime}$ and $0.4 \mathrm{wt} \%$ EL2X is shown in Figure 107 and Figure 108.

In a different study, one control HPG gel sample and a HPG gel sample mixed with control PECNP system $H$ were made and were shaken at $150 \mathrm{rpm}$ and $40^{\circ} \mathrm{C}$. Samples were removed at intervals and viscoelastic moduli were measured using a Bohlin rheometer. Both viscoelastic moduli decreased slightly in a 24 hour period. HPG gel sample mixed with control nanoparticles decreased faster than the control HPG gel system with no nanoparticles. Decrease in moduli is not as significant as the reduction caused by enzymes or nanoparticles loaded with enzymes (Figure 109). Adding PEI and DS to HPG gel separately, viscoelastic parameters of HPG gel slightly decreased (Figure 110 and Figure 111).

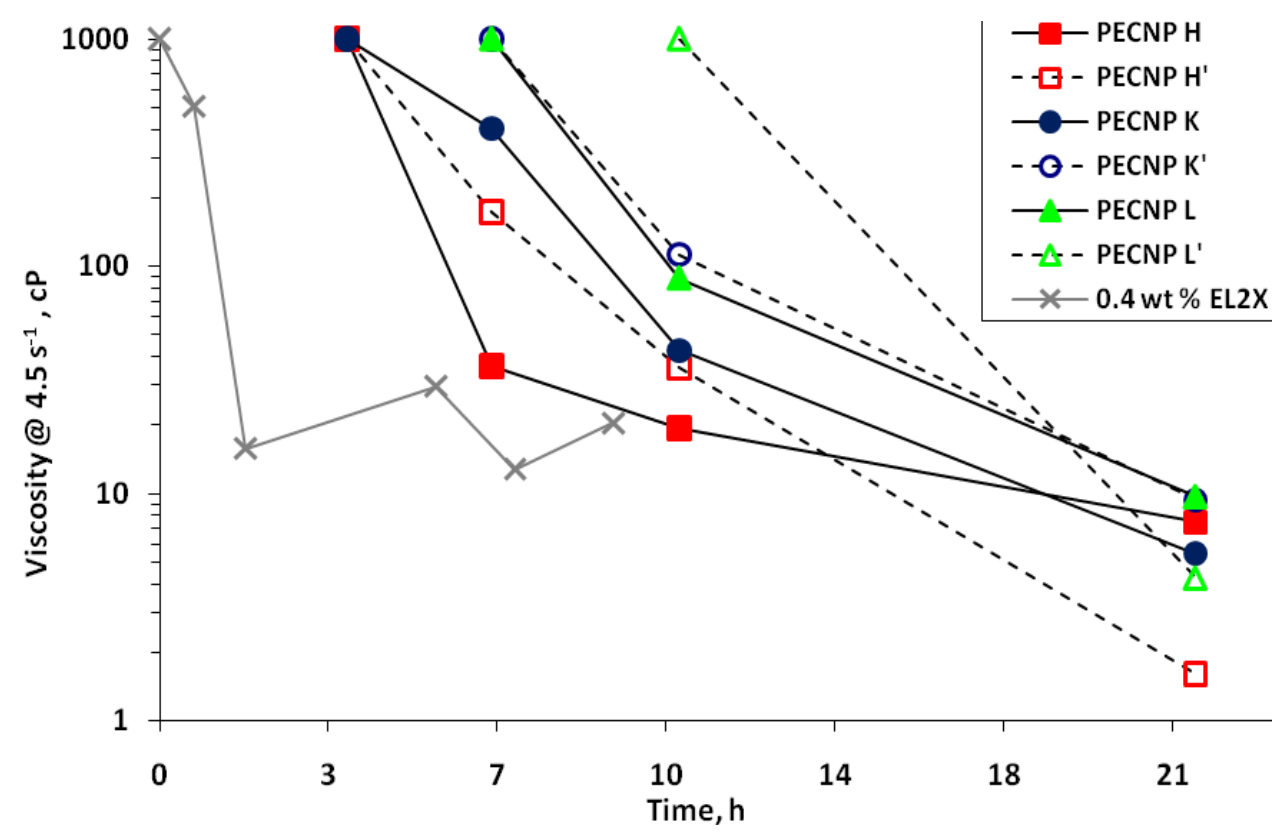

Figure 103 Viscosity of borate (2000 ppm) cross-linked HPG (5000 ppm) gel mixed with $0.4 \%$ EL2X or EL2X-loaded PEC nanoparticles vs. time at $40{ }^{\circ} \mathrm{C}$ and $\mathrm{pH}$ 9.2. N.B. the viscometer is only able to measure viscosity up to $1028 \mathrm{cP}$ so graph indicates the latest 


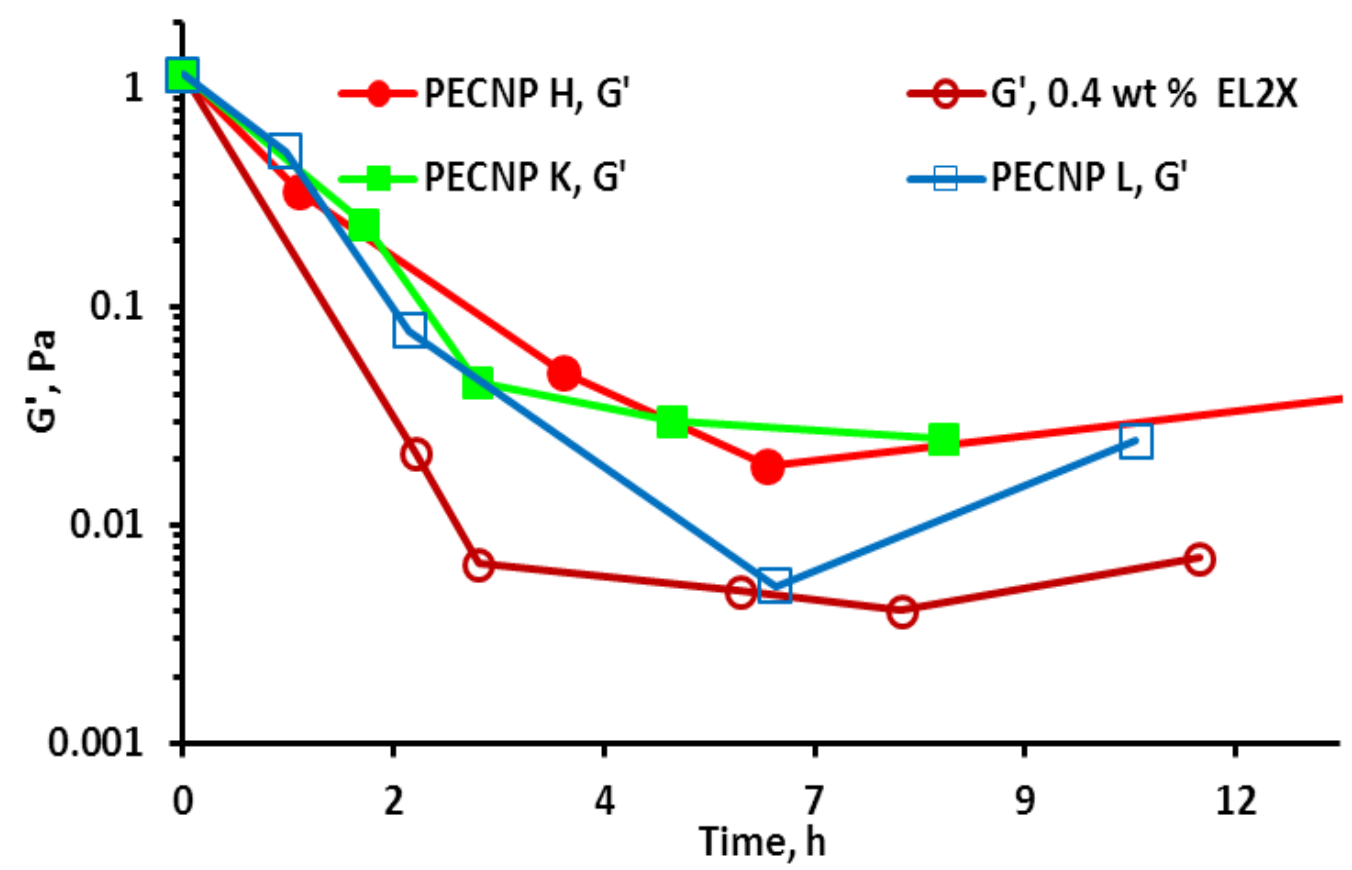

Figure $104 \mathrm{G}^{\prime}$ vs. time for borate (2000 ppm)-crosslinked HPG gel degraded using either $0.4 \mathrm{wt} \%$ EL2X or nanoparticle-entrapped EL2X (PECNP systems $H, K$ and $L$ ) at $40^{\circ} \mathrm{C}$ and $\mathrm{pH} 9.2$.

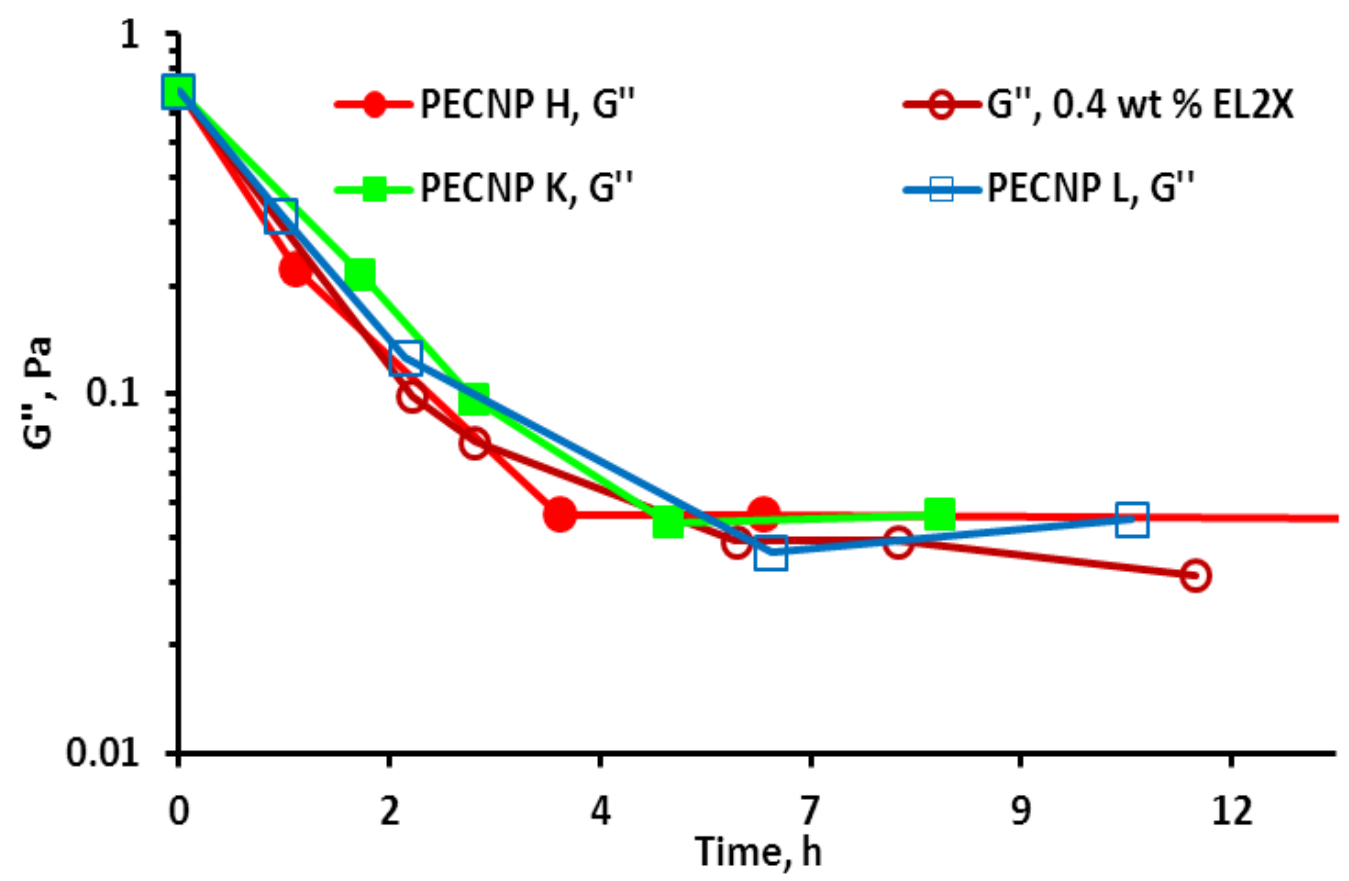

Figure $105 \mathrm{G}^{\prime \prime}$ vs. time for borate (2000 ppm)-crosslinked HPG gel degraded using either $0.4 \mathrm{wt} \%$ EL2X or nanoparticle-entrapped EL2X (PECNP systems $H, K$ and $L$ ) at $40{ }^{\circ} \mathrm{C}$ and $\mathrm{pH} 9.2$. 


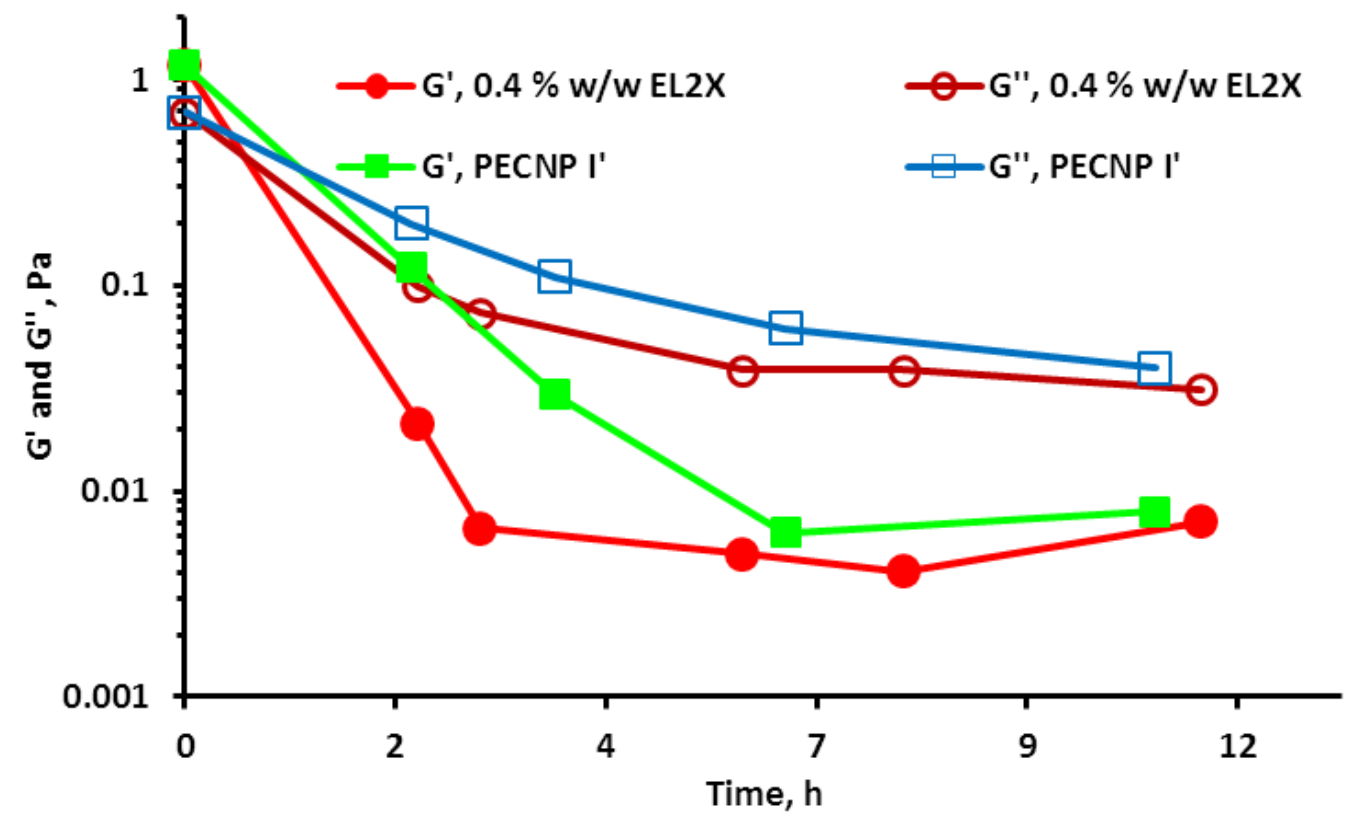

Figure $106 \mathrm{G}^{\prime}$ and $\mathrm{G}^{\prime \prime}$ vs. time for borate (2000 ppm)-crosslinked HPG gel degraded using either $0.4 \mathrm{wt} \%$ EL2X or nanoparticle-entrapped EL2X (PECNP system I') at $40^{\circ} \mathrm{C}$ and pH 9.2.

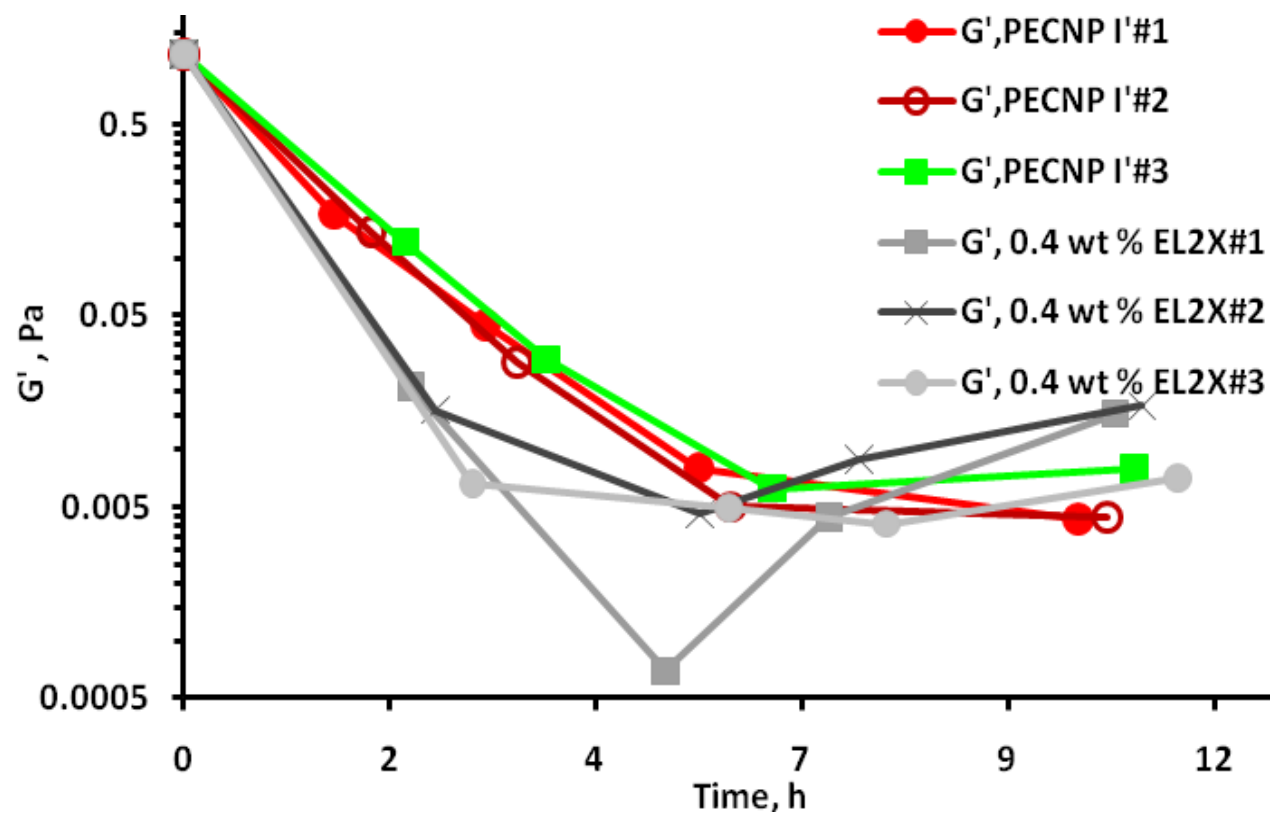

Figure 107 Reproducible degradation of elastic modulus versus time for $5000 \mathrm{ppm}$ HPG solution, and borate (2000 ppm)-crosslinked HPG gel degraded using either 0.4 wt \% EL2X or PECNP System $I^{\prime}$ at $40{ }^{\circ} \mathrm{C}$ and $\mathrm{pH}$ 9.2. 


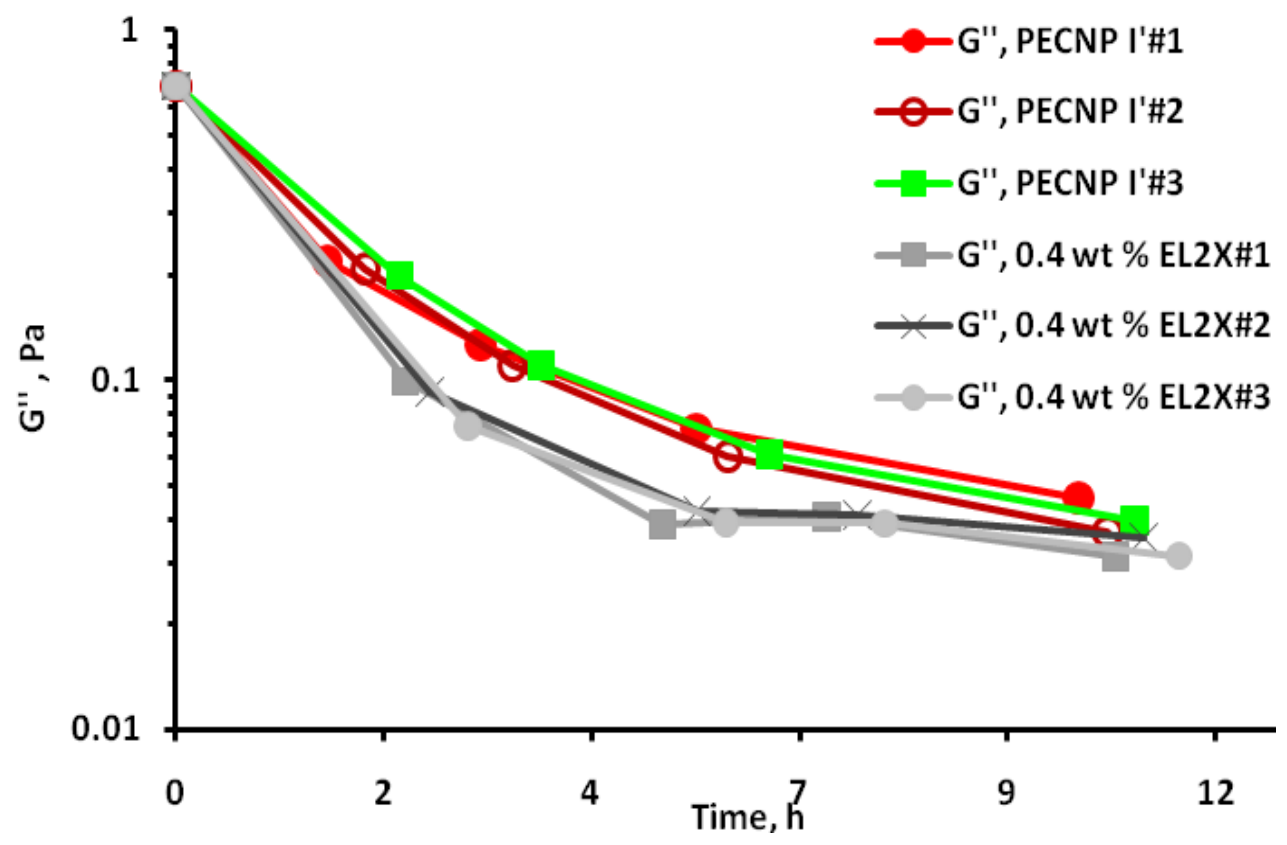

Figure 108 Reproducible degradation of viscous modulus versus time for 5000 ppm HPG solution, and borate (2000 ppm)-crosslinked HPG gel degraded using either 0.4 wt \% EL2X or PECNP System I' at $40{ }^{\circ} \mathrm{C}$ and pH 9.2.

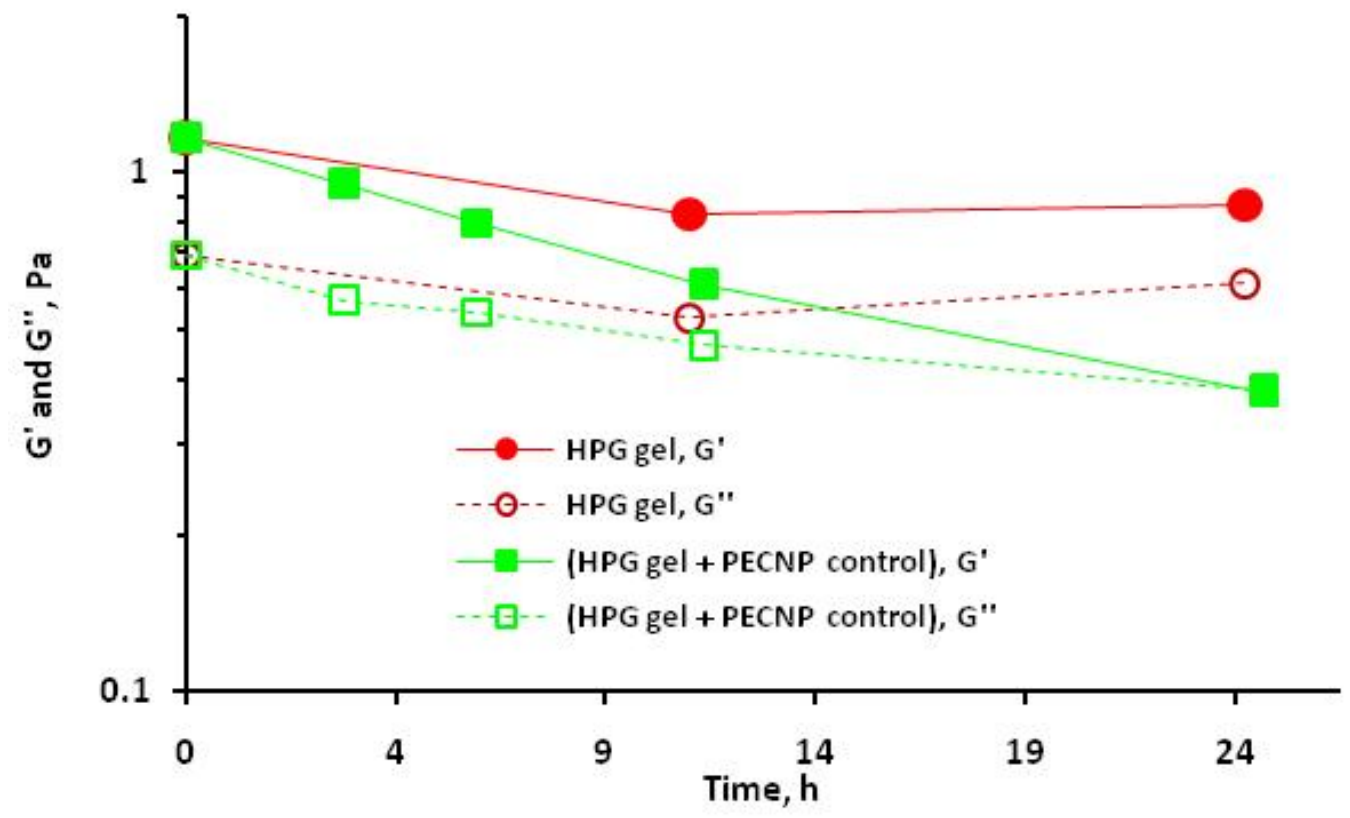

Figure $109 \mathrm{G}^{\prime}$ and $\mathrm{G}^{\prime \prime}$ vs. time for borate $(2000 \mathrm{ppm})$-crosslinked HPG gel and HPG gel mixed with control nanoparticles system $\mathrm{H}$ at $40^{\circ} \mathrm{C}$ and $\mathrm{pH} 9.2$. 


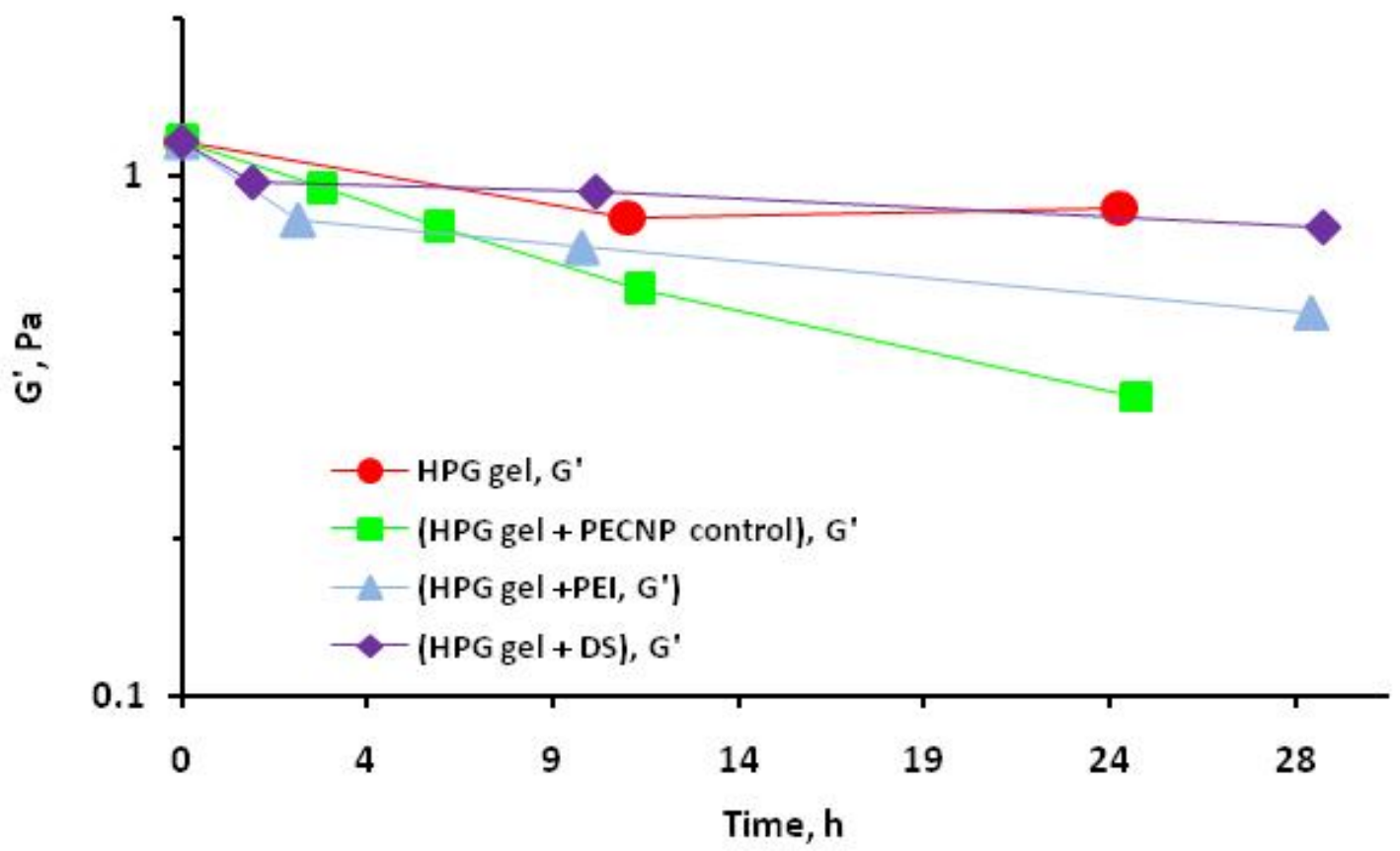

Figure $110 \mathrm{G}^{\prime}$ vs. time for borate (2000 ppm)-crosslinked HPG gel, HPG gel mixed with control PEC nanoparticles, HPG gel mixed with PEI and HPG gel mixed with DS at $40{ }^{\circ} \mathrm{C}$ and pH 9.2.

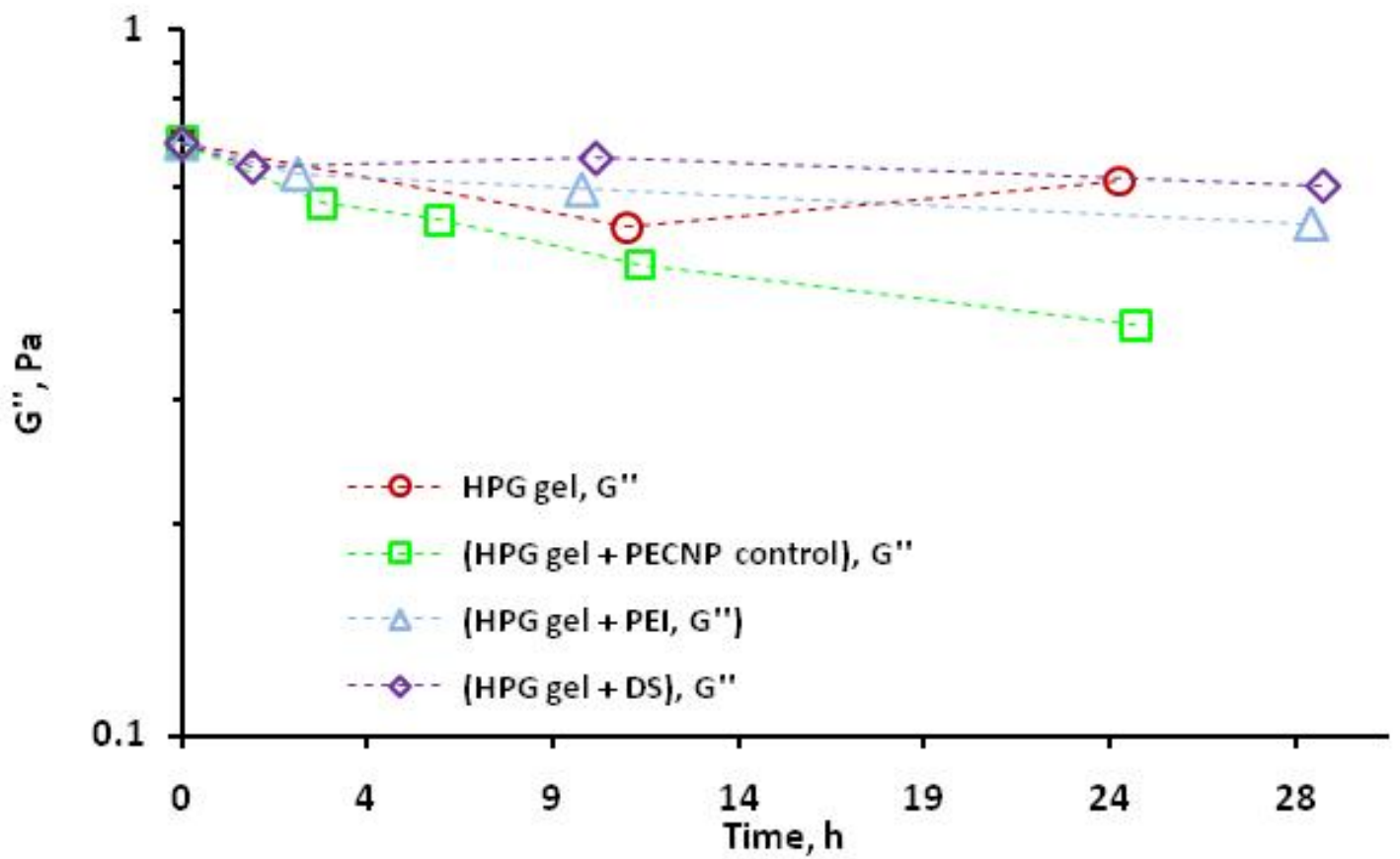

Figure $111 \mathrm{G}^{\prime \prime}$ vs. time for borate (2000 ppm)-crosslinked HPG gel, HPG gel mixed with control PEC nanoparticles, HPG gel mixed with PEI and HPG gel mixed with DS at $40{ }^{\circ} \mathrm{C}$ and pH 9.2. 
Injection of higher enzyme concentrations has the potential to break fracturing fluids and their filter-cake more efficiently. ${ }^{[1,3,6,7]}$ Breakers are required to break the fracturing fluid and formed filter-cakes typically in a 6-24 hour period of time, depending on the size of the fracturing job and depth of the reservoir. However, if efficient fracturing and proppant transport are to be achieved, premature degradation of fracturing fluids during the injection time must be avoided. Reversible trapping of enzymes in polyelectrolyte complex nanoparticles by a combination of transient electrostatic binding and steric interactions (electrosteric interactions) ${ }^{[36,38]}$ delays the activity of the enzyme. This may allow the injection of higher enzyme concentrations.

\subsection{Protective Effect of Nanoparticles on Enzymes}

Enzyme breakers have many advantages over chemical oxidizers: they are cheap, are not consumed during their catalytic reaction with guar, react only with the polymer, are environmentally benign, easy to handle and do not damage wellhead equipment. ${ }^{[1,9]}$ However, denaturation of enzymes at temperature and $\mathrm{pH}$ environments inhospitable to native enzyme is a distinct disadvantage. ${ }^{[9,38]}$ The ability to protect enzymes under such conditions has potential to extend the application of enzymes in fracturing fluids to previously unsuitable reservoir conditions.

Although studying the protective effect of PEC nanoparticles was not initially part of the scope of this research, it was discovered during the course of research that PECNPs are capable of protecting enzymes at temperature and $\mathrm{pH}$ environments inhospitable to native enzyme. Temperature and $\mathrm{pH}$ conditions at which both enzymes denature have been presented in this chapter previously (Figure 86 and Figure 88). Two critical points at which pectinase and EL2X are denatured were chosen. PEC nanopaticles loaded with same concentration of enzymes were then applied at the same $\mathrm{pH}$ and temperature in order to study their protective effect.

To test for this ability, viscometric assays were performed for pectinase and EL2X enzymes at $\mathrm{pH}$ values of 9.1 and 9.75 and temperatures of $40{ }^{\circ} \mathrm{C}$ and $50{ }^{\circ} \mathrm{C}$ respectively. Both enzymes were denatured under these conditions. However, they retain their activities under similar conditions after being entrapped by the PEC nanoparticles. This was repeated three times and plots of viscosity versus time are shown in Figure 112 and Figure 113. It would appear that entrapment of enzymes within the nanoparticles confers protection from the $\mathrm{pH}$ of the bulk solution, 
presumably by a local buffering effect by the PEI, which dominates the $\mathrm{pH}$ of the nanoparticle preparations (8.4 for PECNP $A^{\prime}$ and 9.4 for PECNP $I^{\prime}$ ). This effect also shows the release of enzymes from PEC nanoparticle system. If the viscosity degradation of HPg solution was caused only by the free enzyme in the supernatant that enzyme would be denatured thus the viscosity would reach a plateau. However, continued degradation of HPG solutions versus time using the PECNP A' system proves the release of enzymes from PEC nanoparticles.

PEC nanoparticle system $A^{\prime}$ was made three times with average size of $439 \pm 35.2 \mathrm{~nm}$, average zeta potential of $44.3 \pm 3.2 \mathrm{mV}$ and $\mathrm{pH}$ of 8.4. PEC nanoparticle system $H^{\prime}$ was also made three times with average size of $381.4 \pm 21.5 \mathrm{~nm}$, average zeta potential of $20.6 \pm 2.4 \mathrm{mV}$ and $\mathrm{pH}$ of 9.4.

Crosslinking of guar/HPG using borate ions occurs only at $\mathrm{pH}$ values above 8.5 and $\mathrm{pH}$ must be increased for higher temperature applications to generate enough borate ions. Therefore, protective effect at such conditions, shown by PEC nanoparticles, on both enzymes is of particular interest.

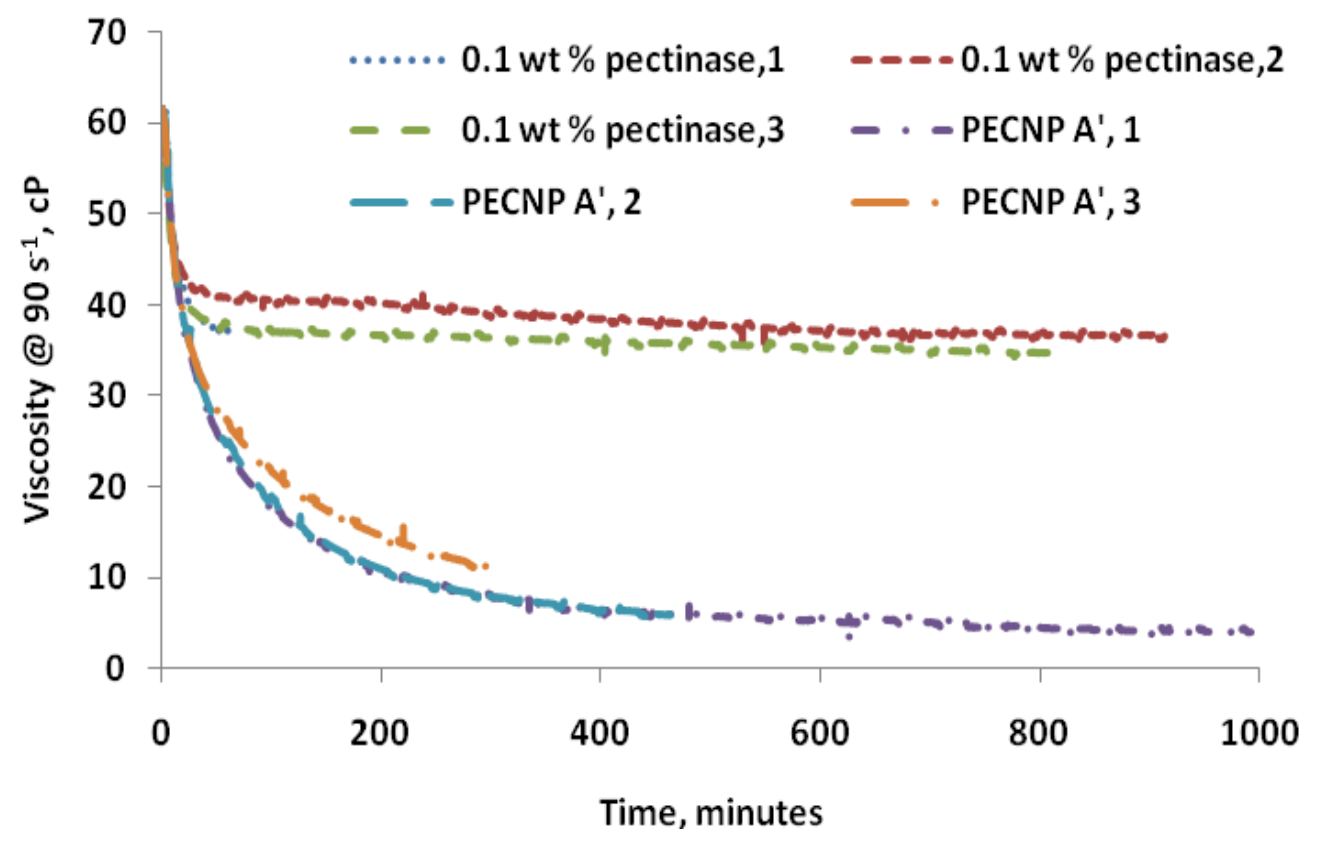

Figure 112 Entrapment of enzymes in PEC nanoparticles $A^{\prime}$ protects activity of pectinase $\left(\mathrm{pH} \mathrm{9.1,40}{ }^{\circ} \mathrm{C}\right)$ 


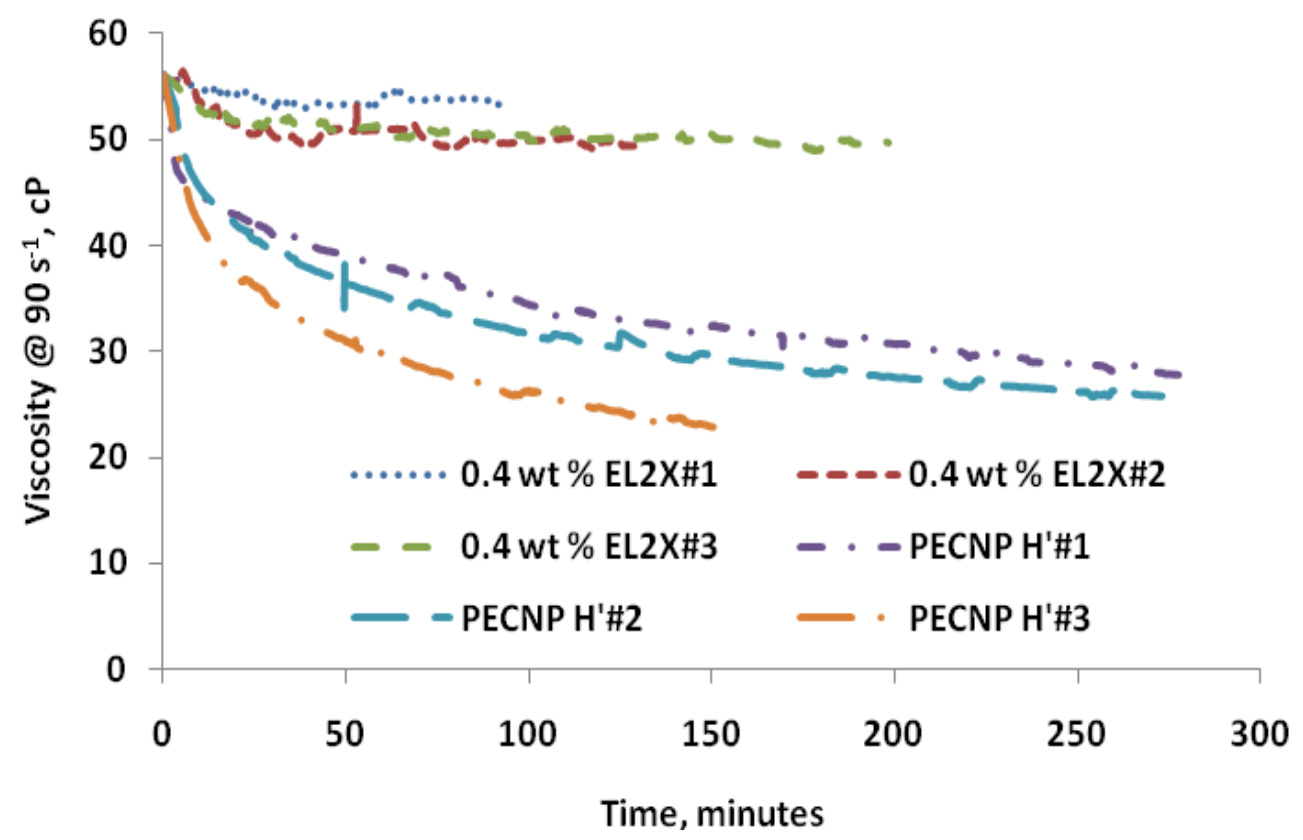

Figure 113 Entrapment of enzymes in PEC nanoparticles $H^{\prime}$ protects activity of EL2X $\left(\mathrm{pH} \mathrm{9.75,50}{ }^{\circ} \mathrm{C}\right)$.

\subsection{Effect of Simulated Wellbore Shear on Enzyme-loaded Nanoparticles: Shear Loop Experiments}

Fracturing fluids experience a period of shear before reaching the sand surface. Shear history in the pipelines was simulated using the shear loop introduced in the previous chapter. This section includes the results of applying shear on HPG gel mixed with enzymes and enzyme-loaded nanoparticles.

\section{6-1 Effect of Simulated Wellbore Shear on Pectinase-Loaded Nanoparticles}

Crosslinked HPG (5000 ppm) using 1000 ppm borax mixed with either R.O. water (control), 0.1 $\% \mathrm{w} / \mathrm{w}$ pectinase or PEC nanoparticle system $A$ was injected at a constant flow rate, $\mathrm{pH}$ of 9 and $25{ }^{\circ} \mathrm{C}$ using the procedure explained in the previous chapter. Only the first three sections of the shear loop were used for this experiment.

Figure 114, Figure 115 and Figure 116 show the pressure drops required for different fluid systems to flow through different sections of the shear loop. Pressure drop across each section was recorded versus time. Pressure drop caused by HPG gel was constant across different 
sections showing no shear sensitivity of gel properties. Pressure drop across the sections for samples with only pectinase and pectinase-loaded nanoparticles was smaller than the pressure drop for the HPG gel. Pressure drop across the sections decreased for both samples with enzyme. However, rate of decrease was significantly slower for the PECNP system $A$ compared to the sample mixed with $0.1 \% \mathrm{w} / \mathrm{w}$ pectinase. For example, by the time the fluid containing PECNP $A$ reaches the third section the pressure drop for its flow is 3.5 times lower than the HPG gel system while the pressure drop for the fluid containing $0.1 \% \mathrm{w} / \mathrm{w}$ pectinase is 19.3 times lower. After injecting the fluids through all three sections, samples were taken from each section, starting from the last section. Viscoelastic parameters were measured for each sample (Figure 117 and Figure 118). Comparison between the viscous and elastic moduli of different samples showed that both moduli measured for PECNP system $A$ and $0.1 \mathrm{wt} \%$ pectinase decreased with similar slope versus time. However, both moduli showed significantly lower values for the system with only pectinase compared to the system with pectinase-loaded nanoparticles (Table 30).

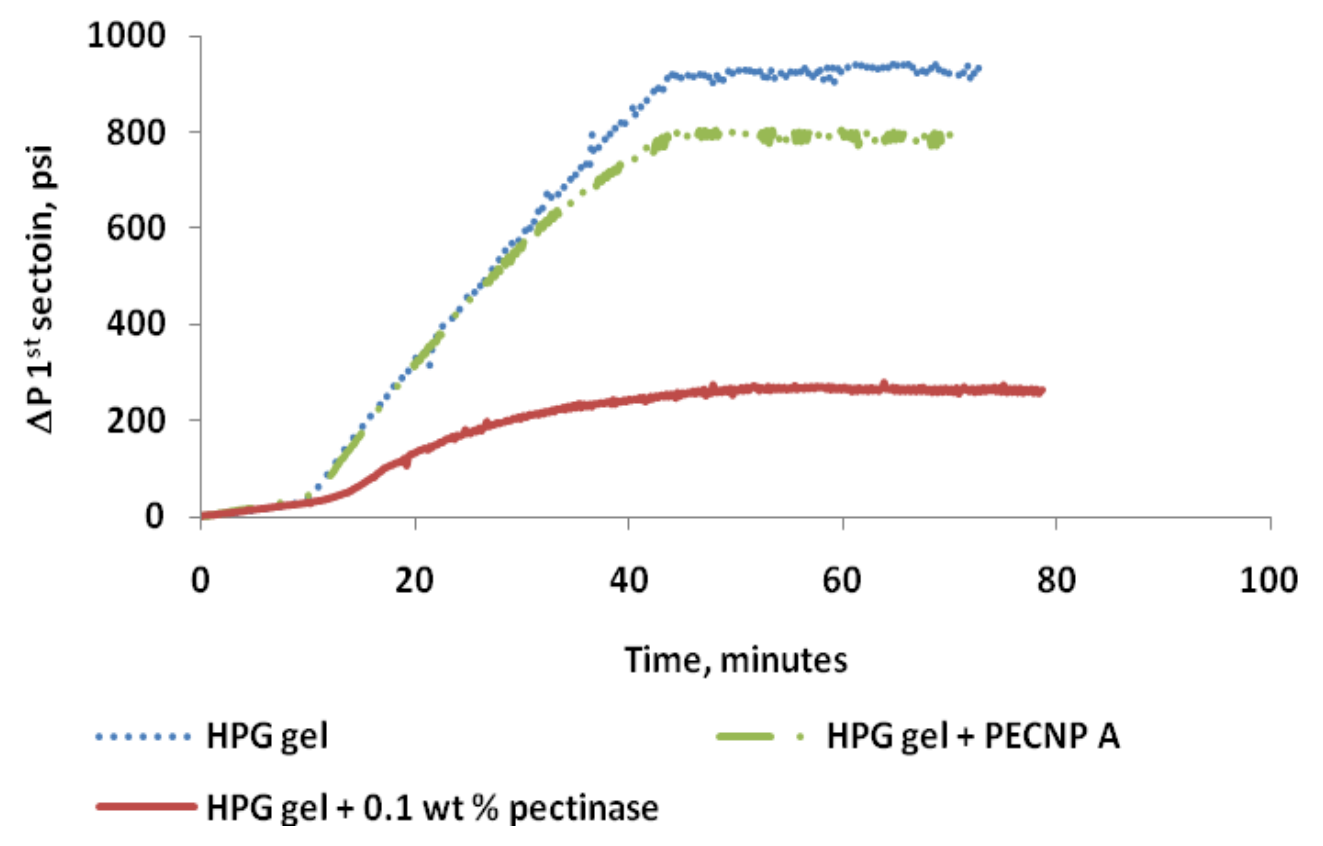

Figure 114 Pressure drop across the first section of shear loop for HPG gel, (HPG gel + PECNP $A$ ) and (HPG gel $+0.1 \% \mathrm{w} / \mathrm{w}$ pectinase) at $\mathrm{pH} 9$ and $25{ }^{\circ} \mathrm{C}$ 


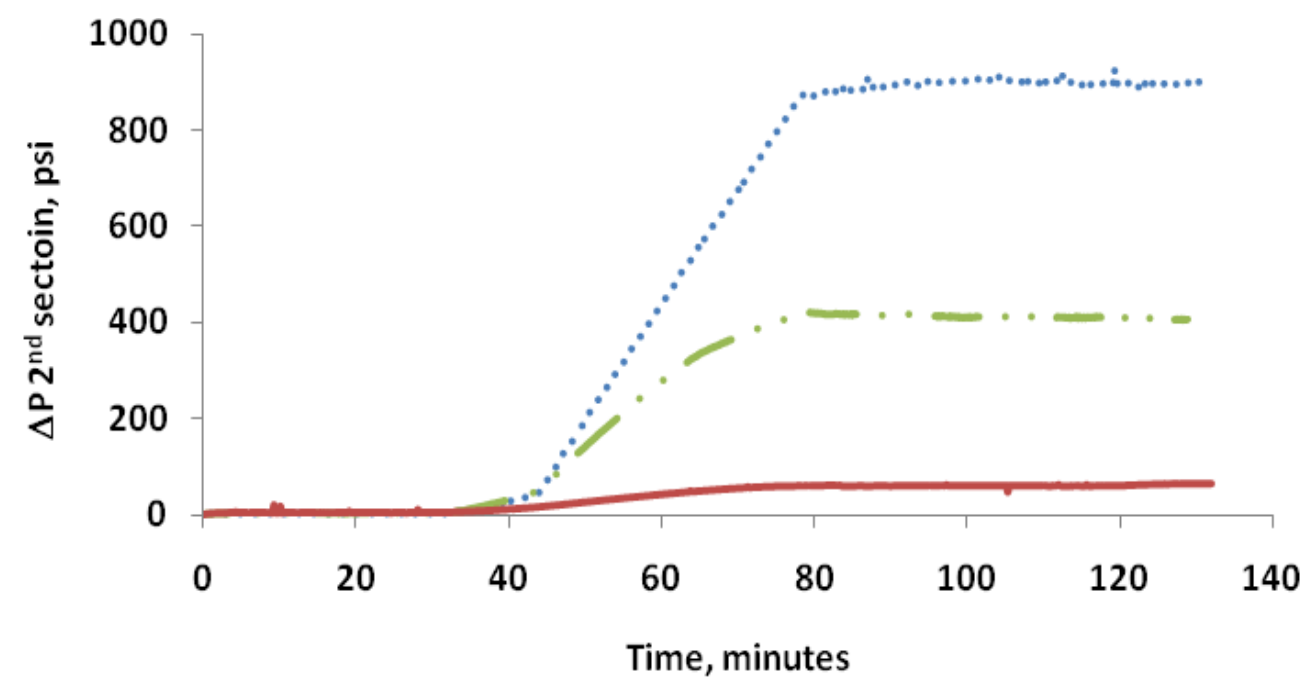

HPG gel

- HPG gel + PECNP A

HPG gel + 0.1 wt $\%$ pectinase

Figure 115 Pressure drop across the second section of shear loop for HPG gel, (HPG gel + PECNP $A$ ) and (HPG gel $+0.1 \% \mathrm{w} / \mathrm{w}$ pectinase) at $\mathrm{pH} 9$ and $25{ }^{\circ} \mathrm{C}$
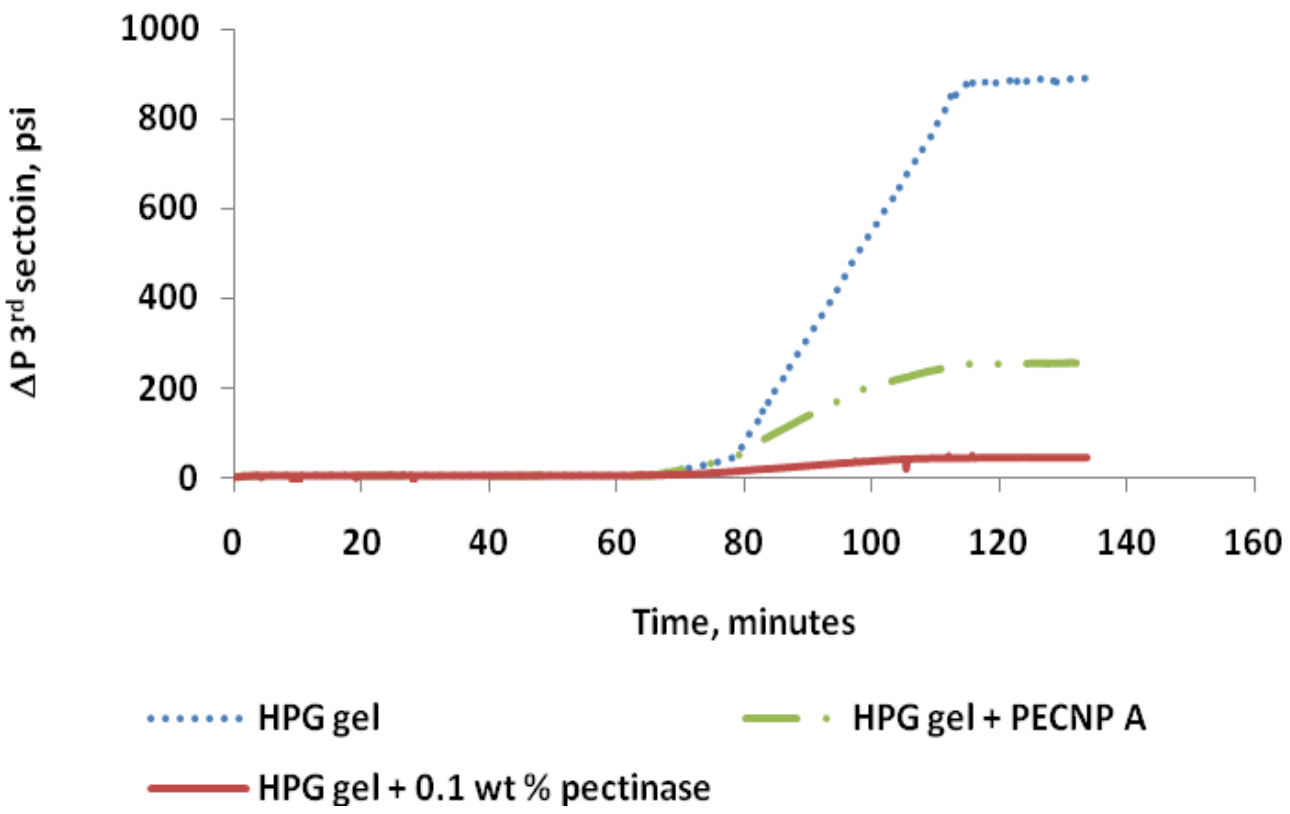

Figure 116 Pressure drop across the third section of shear loop for HPG gel, (HPG gel + PECNP $A$ ) and (HPG gel $+0.1 \% \mathrm{w} / \mathrm{w}$ pectinase) at $\mathrm{pH} 9$ and $25^{\circ} \mathrm{C}$ 


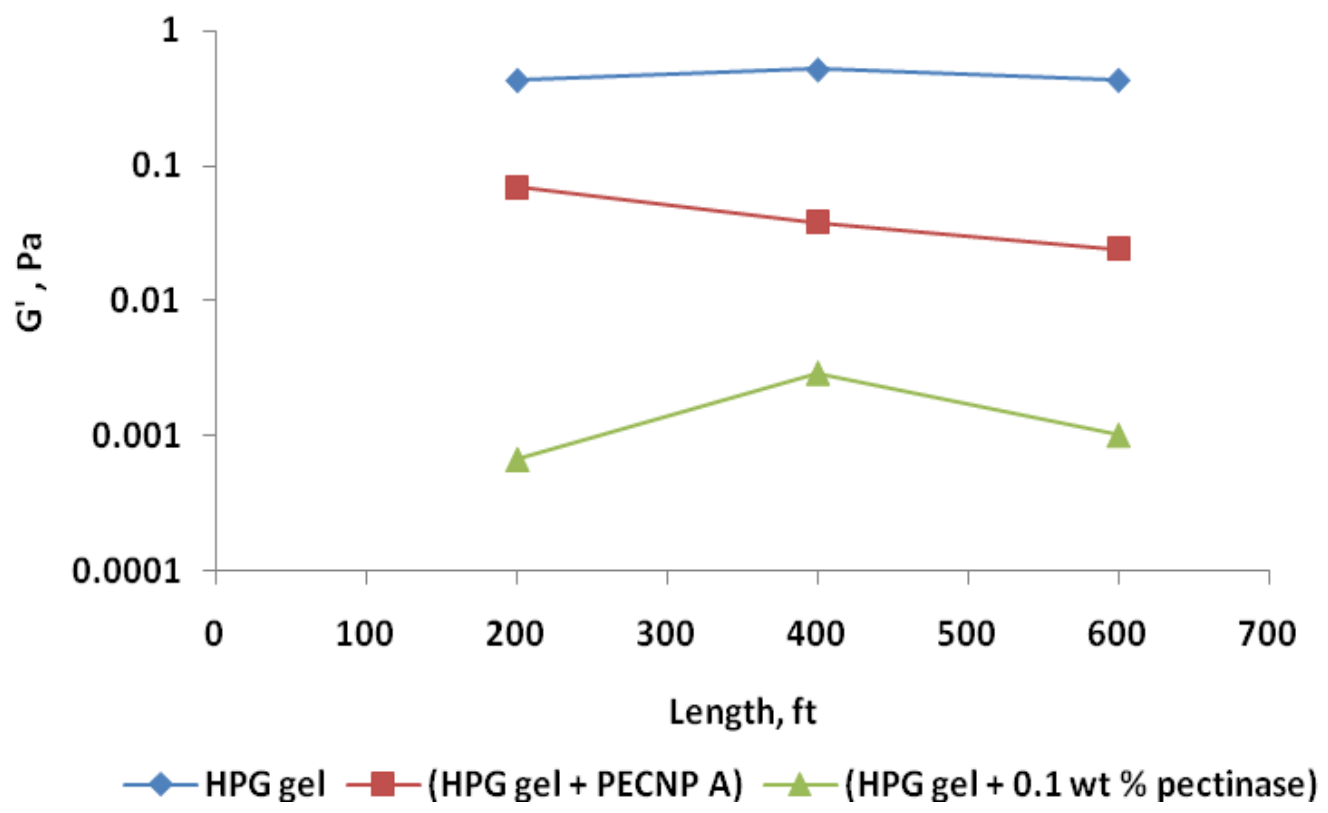

Figure 117 Elastic modulus measured at $0.5 \mathrm{~Hz}$ for samples taken from different sections of shear loop for HPG gel, (HPG gel + PECNP $A$ ) and (HPG gel $+0.1 \%$ w/w pectinase) at pH 9 and $25^{\circ} \mathrm{C}$

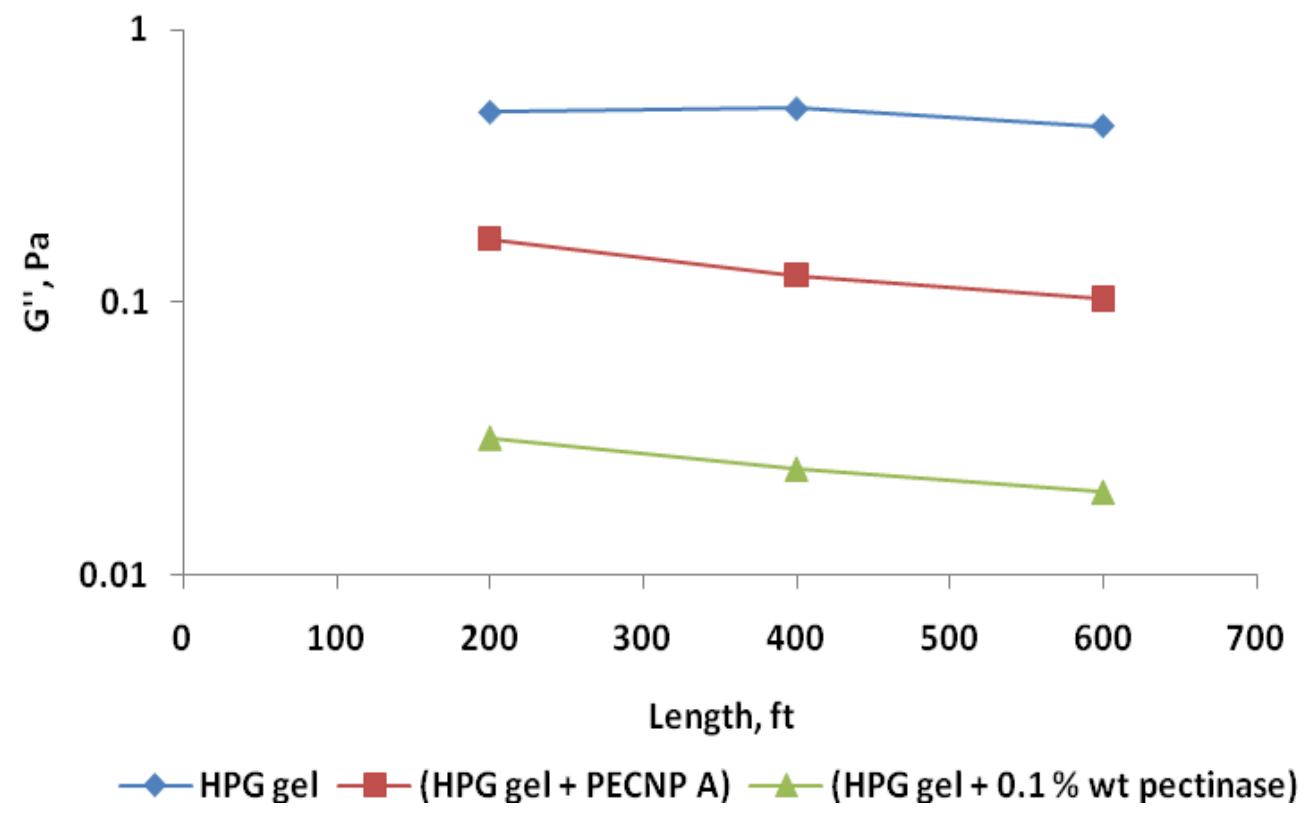

Figure 118 Viscous modulus measured at $0.5 \mathrm{~Hz}$ for samples taken from different sections of shear loop for HPG gel, (HPG gel + PECNP $A)$ and (HPG gel $+0.1 \%$ w/w pectinase) at pH 9 and $25^{\circ} \mathrm{C}$ 
Table 30 Percentage of viscoelastic moduli for HPG gel with PECNP $A$ and $0.1 \%$ w/w pectinase over that of HPG gel at pH 9 and $25^{\circ} \mathrm{C}$

\begin{tabular}{|c|c|c|c|c|}
\hline Section \# & $\begin{array}{c}\mathbf{G}^{\prime}\left(\text { (PECNP A) } / \mathbf{G}^{\prime}\right. \\
\text { (HPG gel) }\end{array}$ & $\begin{array}{c}\mathbf{G}^{\prime}(0.1 \% \text { w/w } \\
\text { pectinase }) / \mathbf{G}^{\prime}{ }_{(\mathrm{HPG} \text { gel) }}\end{array}$ & $\begin{array}{c}G^{\prime \prime} \text { (PECNP } \\
\text { A) / } \mathbf{G}^{\prime \prime}{ }_{(\text {HPG gel) }}\end{array}$ & $\begin{array}{c}\mathbf{G}^{\prime \prime}(0.1 \% \text { w/w } \\
\text { pectinase) } / \mathbf{G}^{\prime \prime}{ }_{(\mathrm{HPG} \text { gel) }}\end{array}$ \\
\hline 1 & 16.08 & 0.15 & 34.33 & 6.40 \\
\hline 2 & 7.40 & 0.56 & 24.54 & 4.79 \\
\hline 3 & 5.58 & 0.23 & 23.37 & 4.58 \\
\hline
\end{tabular}

\section{6-2 Effect of Simulated Wellbore Shear on EL2X-loaded Nanoparticles}

Before optimizing the entrapment efficiency of EL2X-loaded nanoparticles PECNP system $H$ was applied to break HPG gel prepared using 4000 ppm borax under shear. This system was compared with the control HPG gel system and a HPG gel system mixed with 0.4 wt \% EL2X. Fluids were prepared at $9.2 \mathrm{pH}$ and tests were run at $40{ }^{\circ} \mathrm{C}$. Pressure drops for each section using different fluids are reported in Table 31. Although higher pressure drops were reported for the system mixed with PECNP $H$ along the first section, pressure drops of this sytem were similar to those reported for (HPG gel $+0.4 \mathrm{wt} \%$ EL2X). This shows that this sytem is sensitive to shear history to which it is exposed to. This result is suppored by viscoelastic parameters measured for samples taken at the end of each section (Figure 119 and Figure 120). Note that section zero is the valve installed immediately after the inline mixer. Typically, viscoelastic parameters are slightly higher for section one compared to the gel not exposed to shear. HPG gel pressure drop was only measured along the first section since total pressure drop would reach the maximum allowable pressure drop for the Validyne pressure transducers. Pressure drops reported for HPG gel at sections 2 and 3 are just the repeats of pressure drop in the first section since no shear sensitivity of HPG gel was seen previously. 
Table 31 Pressure drops of HPG gel (4000 ppm borax), (HPG gel + PECNP H) and (HPG gel + 0.4 wt \% EL2X) across the shear loop at $\mathrm{pH} 9.2$ and $40{ }^{\circ} \mathrm{C}$. HPG gel was prepared using $4000 \mathrm{ppm}$ borax.

\begin{tabular}{|c|c|c|c||}
\hline Section \# & $\begin{array}{c}\text { HPG } \\
\text { gel }\end{array}$ & $\begin{array}{c}\text { HPG gel + } \\
\text { PECNP H }\end{array}$ & $\begin{array}{c}\text { HPG gel + 0.4 wt \% } \\
\text { EL2X }\end{array}$ \\
\hline $\mathbf{1}$ & 2100 & 1035 & 839 \\
\hline $\mathbf{2}$ & 2100 & 510 & 508 \\
\hline $\mathbf{3}$ & 2100 & 327 & 326 \\
\hline
\end{tabular}

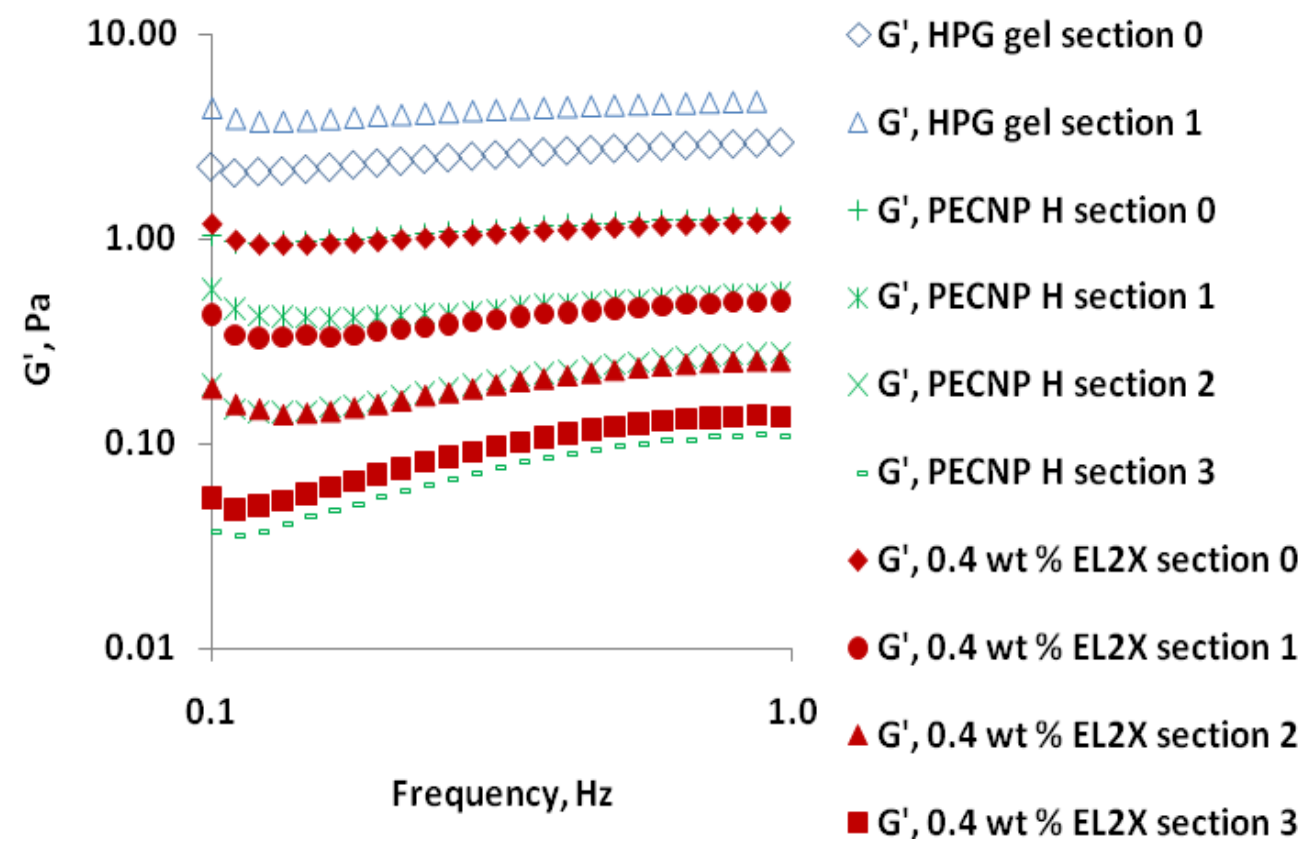

Figure 119 Elastic modulus measured for HPG gel, (HPG gel + PECNP H) and (HPG gel + 0.4 wt \% EL2X) at the end of different sections of shear loop $\left(\mathrm{pH}=9.2\right.$ and $\left.\mathrm{T}=40{ }^{\circ} \mathrm{C}\right)$. HPG gel was prepared using $4000 \mathrm{ppm}$ borax. 


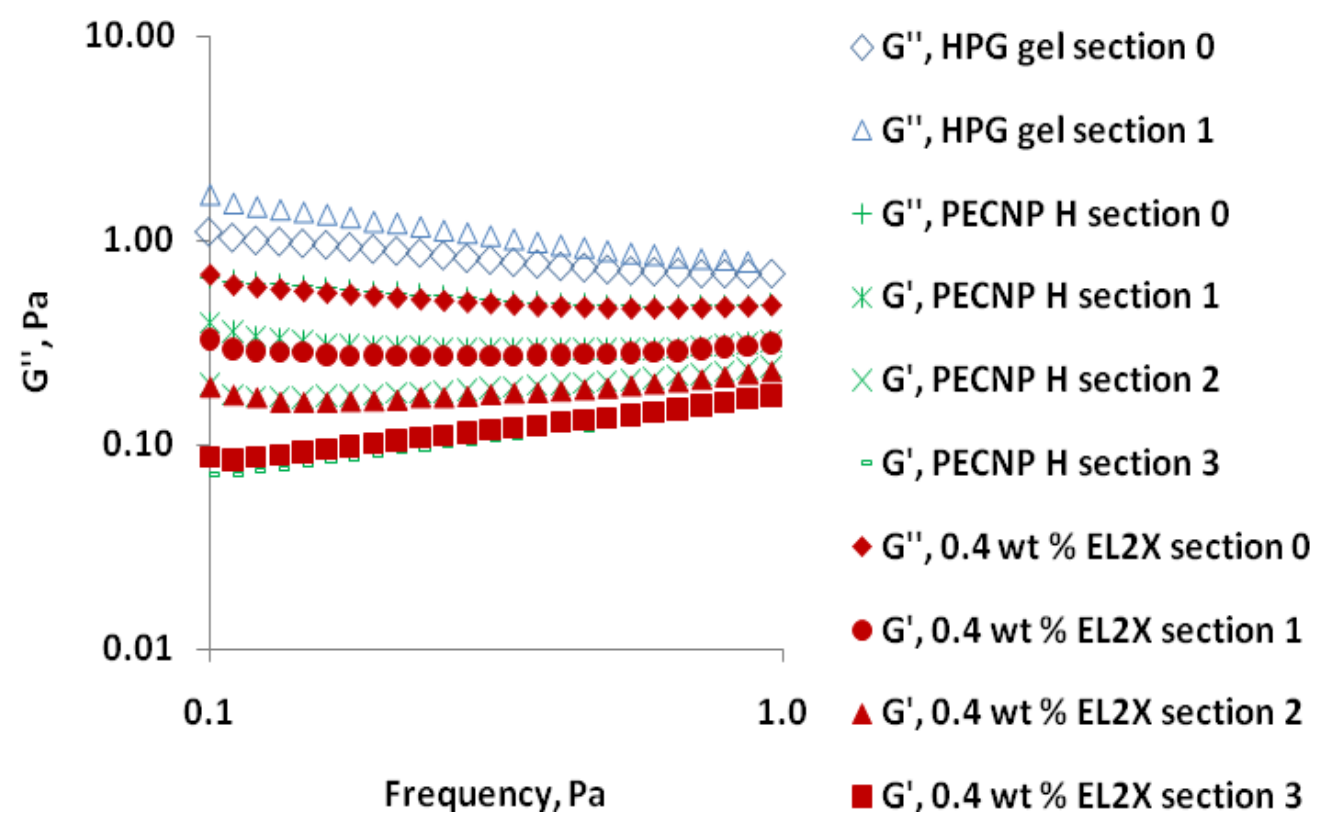

Figure 120 Viscous modulus measured for HPG gel, (HPG gel + PECNP H) and (HPG gel + 0.4 wt \% EL2X) at the end of different sections of shear loop $\left(\mathrm{pH}=9.2\right.$ and $\left.\mathrm{T}=40{ }^{\circ} \mathrm{C}\right)$. HPG gel was prepared using $4000 \mathrm{ppm}$ borax.

After optimizing the EL2X-loaded PECNP systems PECNP $I^{\prime}$ was applied to break HPG gel prepared using $2000 \mathrm{ppm}$ borax under shear. This system was compared with the control HPG gel system and an HPG gel system mixed with $0.4 \mathrm{wt} \%$ EL2X. Fluids were prepared at $\mathrm{pH} 9.2$ and tests were run at $40{ }^{\circ} \mathrm{C}$. Pressure drops for each section using different fluids are reported in Table 32, Figure 121, Figure 122 and Figure 123. Similar pressure drops along the first two sections were seen for the gel mixed with $0.4 \mathrm{wt} \%$ EL2X and the gel mixed with PECNP $I^{\prime}$. Slightly lower pressure drop was seen for the (HPG gel + 0.4 wt \% EL2X) system compared to the (HPG gel+ PECNP $I^{\prime}$ ) along the last section.

None of the EL2X-loaded nanoparticles $H$ or $I^{\prime}$ survived under the shear history applied in the shear loop.Considering the shear insenstivity of nanoparticles mixed with HPG polymer solutions shown in this chapter previously, this sensitivity of the activity of nanoparticles to shear when mixed with HPG gels can be the result of continous shear applied on gel structure causing the gel structure to be broken. Low entrapment efficiency of PEC nanoparticles or higher pressures experienced by the fracturing fluid flowing through the shear loop may also contribute to shear sensitivity of nanoparticles. 
Table 32 Pressure drops of HPG gel (2000 ppm borax), (HPG gel + PECNP I) and (HPG gel + 0.4 wt \% EL2X) across the shear loop at $9.2 \mathrm{pH}$ and $40{ }^{\circ} \mathrm{C}$. HPG gel was prepared using $2000 \mathrm{ppm}$ borax.

\begin{tabular}{|c|c|c|c||}
\hline Section \# & $\begin{array}{c}\text { HPG } \\
\text { gel }\end{array}$ & $\begin{array}{c}\text { HPG gel + } \\
\text { PECNP } \boldsymbol{I}^{\prime}\end{array}$ & $\begin{array}{c}\text { HPG gel + 0.4 wt \% } \\
\text { EL2X }\end{array}$ \\
\hline $\mathbf{1}$ & 1188 & 762 & 768 \\
\hline $\mathbf{2}$ & 1175 & 620 & 601 \\
\hline $\mathbf{3}$ & 1175 & 533 & 486 \\
\hline
\end{tabular}

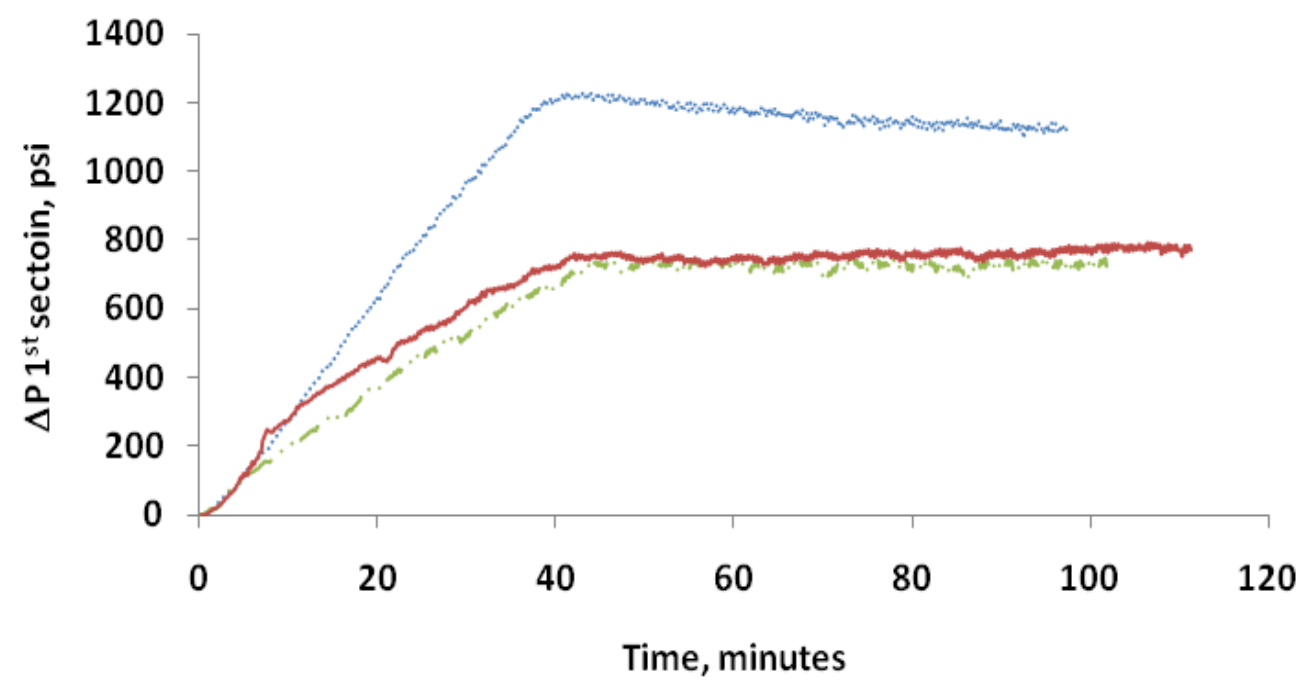

HPG gel - - - HPG gel + PECNPI' - HPG gel + 0.4 wt \% EL2X

Figure 121 Pressure drop across the first section of shear loop for HPG gel, (HPG gel + PECNP I') and (HPG gel $+0.4 \% \mathrm{w} / \mathrm{w} \mathrm{EL2X)} \mathrm{at} \mathrm{pH} 9.2$ and $40{ }^{\circ} \mathrm{C}$ 


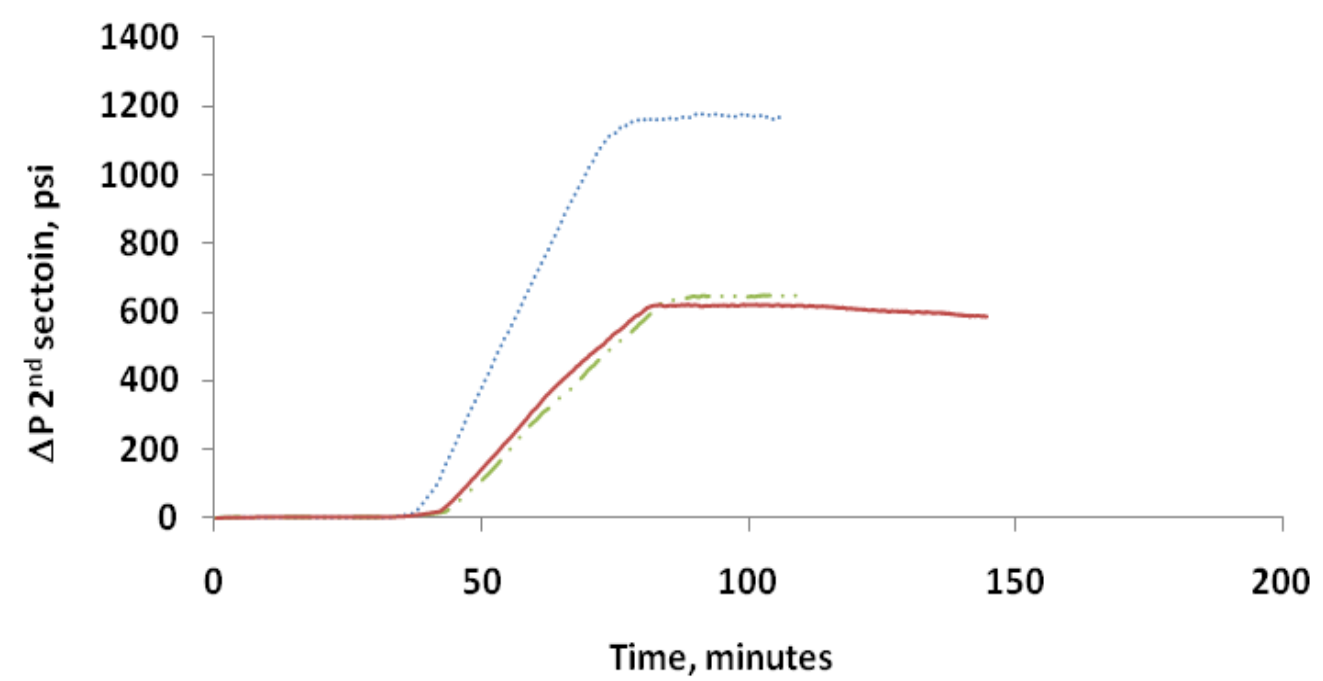

HPg gel $-\cdots-$ HPG gel + PECNP I' - HPG gel + $0.4 \mathrm{wt} \%$ EL2X

Figure 122 Pressure drop across the second section of shear loop for HPG gel, (HPG gel + PECNP I') and (HPG gel $+0.4 \%$ w/w EL2X) at pH 9.2 and $40{ }^{\circ} \mathrm{C}$

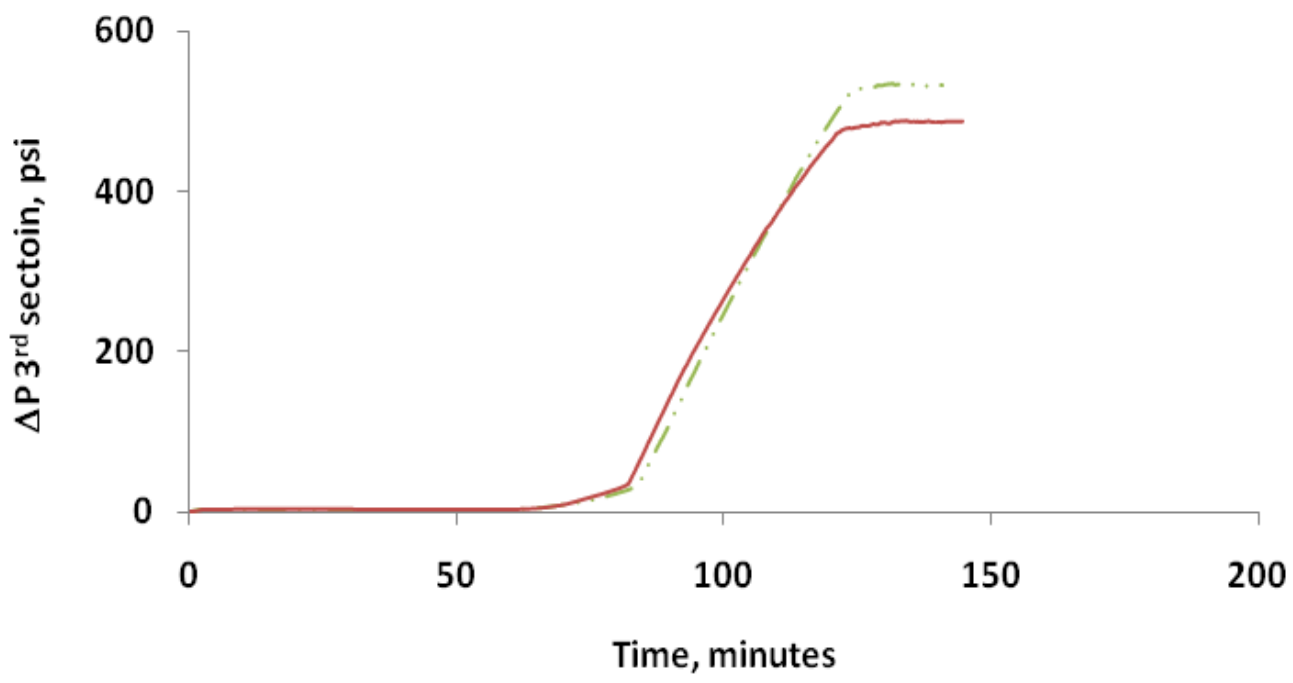

$$
-\cdots-\text { HPG gel + PECNPI' - } \quad \text { HPG gel + } 0.4 \text { wt \% EL2X }
$$

Figure 123 Pressure drop across the third section of shear loop for (HPG gel + PECNP I') and (HPG gel + 0.4 $\%$ w/w EL2X) at pH 9.2 and $40{ }^{\circ} \mathrm{C}$ 


\subsection{Filter-cake Formation and Cleanup Using Static Fluid-Loss Tests}

In this section characterizations of cores used in fluid-loss tests, permeability measurements before and after the fluid-loss tests, fluid-loss results and filter-cake cleanup results are presented.

\section{7-1 Permeability Measurement and Tracer Tests for Cores Used in Fluid- Loss Tests}

In order to use Berea sandstone cores for fluid-loss tests, $1 " \times 1 "$ cores were cut. Cores were selected and dried at $73{ }^{\circ} \mathrm{C}$ for 48 hours till they reached a constant weight. Permeability of cores was measured by flooding with $2 \% \mathrm{w} / \mathrm{w} \mathrm{KCl}$. Some cores showed a reduction in their permeability versus time. Such cores were discarded since a constant permeability is required for fluid-loss tests. Two sets of cores were chosen with the permeability ranges of $60-90 \mathrm{mD}$ and 110-150 mD. Permeability measurements before fluid-loss tests are shown in Appendix A.

Table 33 shows the permeability values measured for different cores used for fluid loss tests at $25{ }^{\circ} \mathrm{C}$. Red cells are the cores that were discarded after permeability measurements since their permeability did not reach a stable value.

Tracer tests were also run for cores within the $110-150 \mathrm{mD}$ range to assure the homogeneity of the cores. Figure 124 shows an example of permeability measurement and tracer test for core\#6. It was observed that permeability of the cores decreased significantly after tracer tests and this was not restored upon flooding with $2 \% \mathrm{KCl}$. In order to restore the cores after tracer tests, they were dried again and resaturated with $2 \% \mathrm{w} / \mathrm{w} \mathrm{KCl}$. Figure 125 shows the permeability measurement for the same core after resaturation. Permeability measurement of the cores after resaturation is also reported in Table $\mathbf{3 3}$ and the rest of the measurements are shown in Appendix A. Cores with stable permeability in the range of $102-162 \mathrm{mD}$ after being resaturated were used in fluid-loss tests.

UV absorbance of effluent fluid (at $302 \mathrm{~nm}$ ) was measured for different cores while switching the injection from $2 \% \mathrm{KCl}$ to a $1 \% \mathrm{KNO}_{3}$ in $2 \% \mathrm{KCl}$ solution. Injection was switched back after the absorbance reached a plateau. Tracer injection and cleanup curves, using which porosity values were calculated are shown in Figure 126 and Figure 127. Similar tracer curves for different cores showed that similar homogenous cores were used. 
In order to repeat the fluid-loss tests some cores were washed under vacuum using $2 \% \mathrm{w} / \mathrm{w}$ bleach in $2 \% \mathrm{w} / \mathrm{w} \mathrm{KCl}$. Cores were washed after the cleanup using $2 \% \mathrm{w} / \mathrm{w} \mathrm{KCl}$ and removal of bleach was confirmed by checking the chlorine concentration in the effluent using a SenSafe ${ }^{\mathrm{TM}}$ Free Chlorine water check kit (Industrial Test Systems, Inc., Rock Hill, SC). Permeability was measured for the cores after being washed using bleach. Figure 128 shows an example of these permeability measurements for core \#6. The rest of such measurements are shown in Appendix A. Cores 1, 4, 5, 6, 9 and 10 showed stable permeability values in the range of $117-155 \mathrm{mD}$ after restoration.

Table 33 Permeability and porosity measurements for cores used at $25^{\circ} \mathrm{C}$. Porosity using tracer test and porosity and permeability after restoration is also reported.

\begin{tabular}{|c|c|c|c|c|c|c|c|c||}
\hline $\begin{array}{c}\text { Core } \\
\#\end{array}$ & $\begin{array}{c}\mathbf{k} \\
\mathbf{m} \mathbf{m}) \\
\text { before } \\
\text { tracer }\end{array}$ & $\begin{array}{c}\mathbf{k}(\mathbf{m D}) \\
\mathbf{a f t e r} \\
\text { tracer }\end{array}$ & $\begin{array}{c}\mathbf{k}(\mathbf{m D}) \\
\mathbf{a f t e r} \\
\text { resaturation }\end{array}$ & $\begin{array}{c}\text { Porosity } \\
\mathbf{1 s t} \mathbf{\%}\end{array}$ & $\begin{array}{c}\text { Porosity } \\
\mathbf{2 n d} \mathbf{\%}\end{array}$ & $\begin{array}{c}\text { Tracer } \\
\text { porosity } \\
\mathbf{\%}\end{array}$ & $\begin{array}{c}\text { Restored } \\
\text { porosity, } \\
\mathbf{\%}\end{array}$ & $\begin{array}{c}\text { Restored } \\
\mathbf{k}(\mathbf{m D})\end{array}$ \\
\hline 1 & 141 & 105 & 154 & 17.71 & 17.90 & 16.40 & 18.12 & 153 \\
\hline 2 & 162 & 13.18 & 31.42 & 15.80 & 17.68 & ND & ND & ND \\
\hline 3 & 123 & 88 & 120 & 17.73 & 17.87 & 15.84 & ND & ND \\
\hline 4 & 141 & 121 & 153 & 18.31 & 17.96 & 17.51 & 18.04 & 117 \\
\hline 5 & 145 & 126 & 162 & 17.38 & 17.81 & 17.65 & 18.04 & 155 \\
\hline 6 & 133 & 102 & 143 & 17.37 & 17.63 & 16.11 & 17.91 & 127 \\
\hline 7 & 112 & 31 & ND & 17.45 & ND & ND & ND & ND \\
\hline 8 & 133 & 82 & 102 & 17.54 & 16.89 & 16.34 & ND & ND \\
\hline 9 & 139 & 96 & 138 & 17.78 & 16.91 & 17.09 & 18.34 & 154 \\
\hline 10 & 146 & 101 & 129 & 17.27 & 17.18 & 16.99 & 18.11 & 131 \\
\hline
\end{tabular}




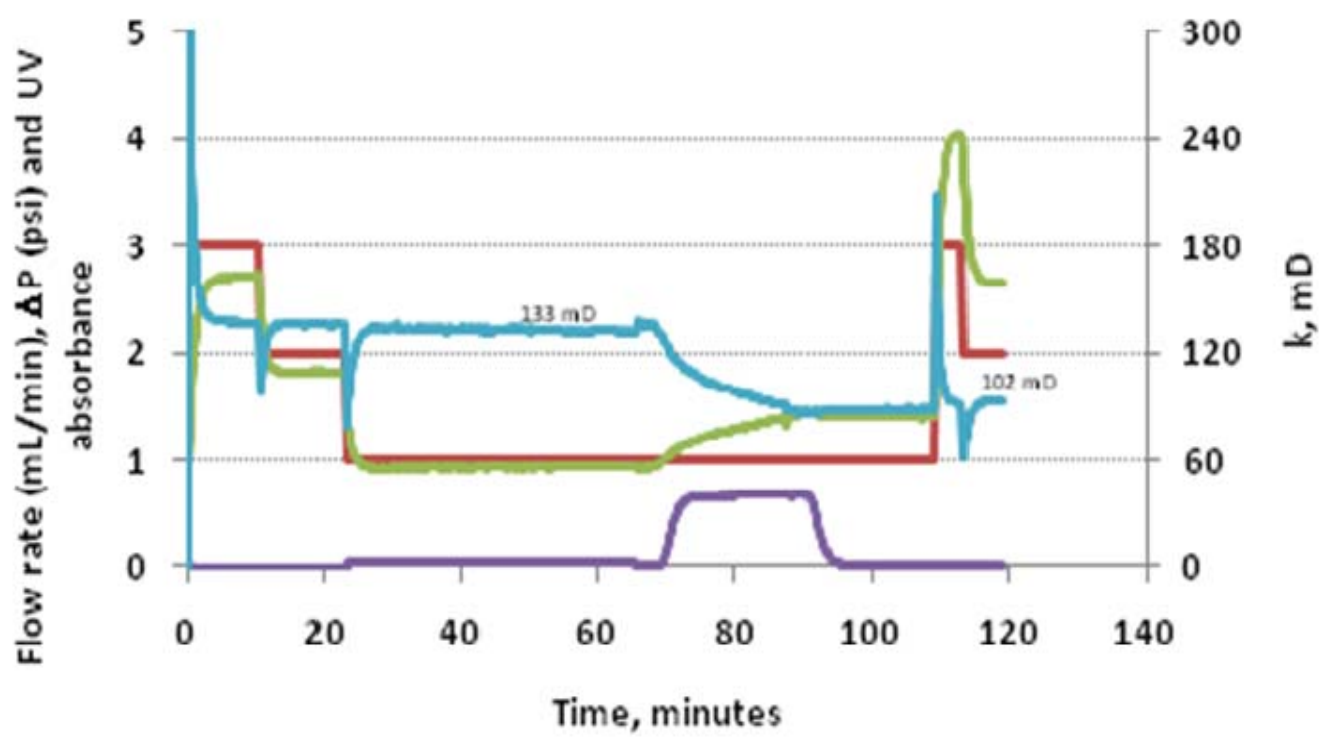

Flow rate, $\mathrm{mL} / \mathrm{min}=\mathrm{Delta} P, \mathrm{psi}=\mathrm{UV} \mathrm{mD}$

Figure 124 Flow rate, pressure drop, UV absorbance and permeability of core \#6 before fluid-loss tests at 25 ${ }^{\circ} \mathbf{C}$

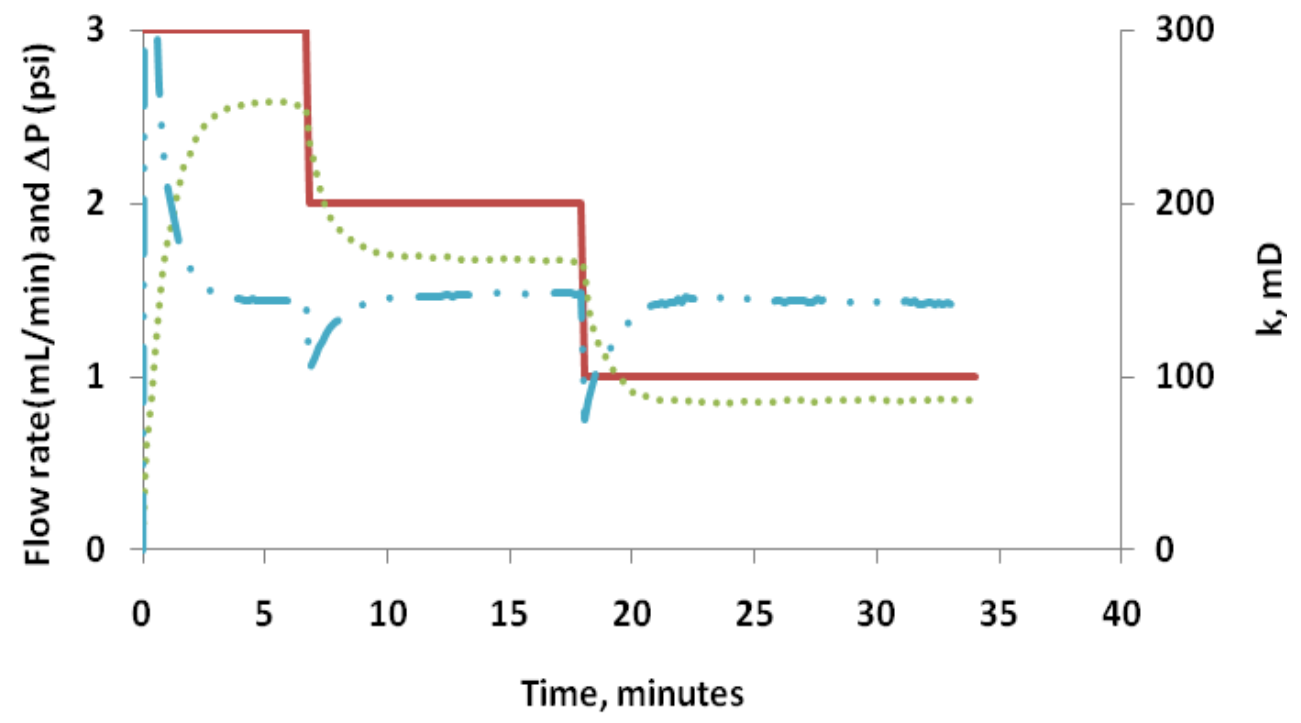

Flow rate, $\mathrm{mL} / \mathrm{min} \quad \ldots . .$. Delta $\mathrm{P}, \mathrm{psi} \quad-\mathrm{k}, \mathrm{mD}$

Figure 125 Flow rate, pressure drop and permeability versus time for core\# 6 resaturated after tracer test 


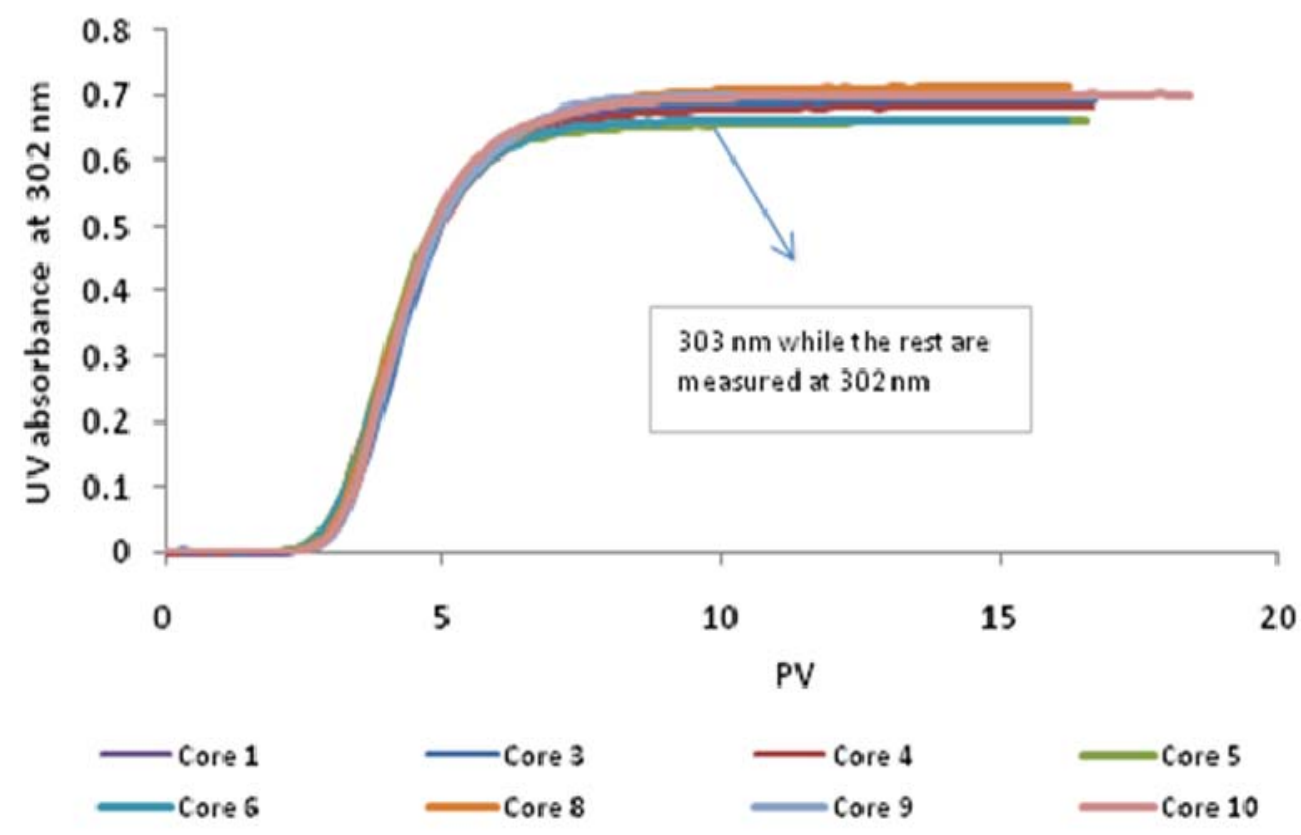

Figure 126 Tracer injection UV absorbance versus time curves for different cores used in fluid-loss tests. UV absorbance was measured at $302 \mathrm{~nm}$ while tracer was injected and cleaned up with $1 \mathrm{~mL} / \mathrm{minutes}$ flow rate.

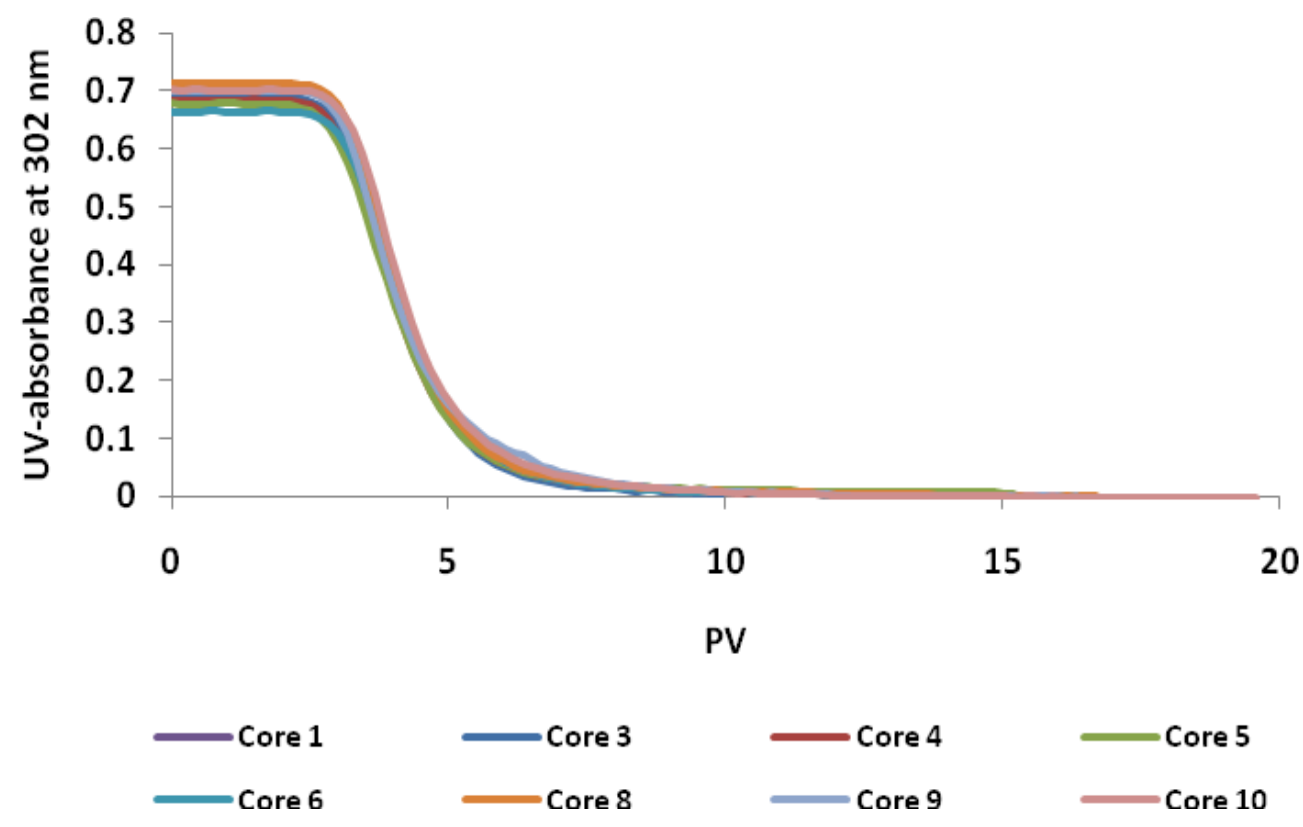

Figure 127 Tracer cleanup UV absorbance versus time curves for different cores used in fluid-loss tests. UV absorbance was measured at $302 \mathrm{~nm}$ while $2 \% \mathrm{w} / \mathrm{w} \mathrm{KCl}$ was injected and cleaned up with $1 \mathrm{~mL} / \mathrm{minutes}$ flow rate. 


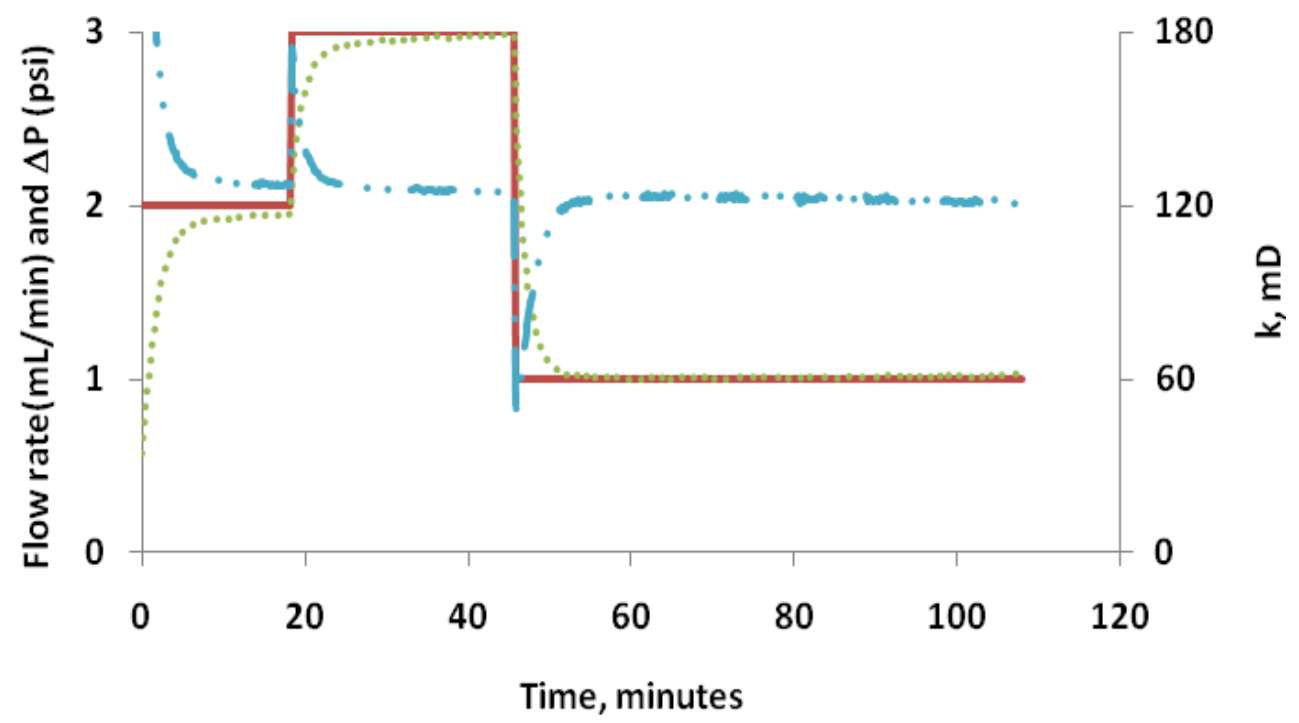

Flow rate, $\mathrm{mL} / \mathrm{min} \quad \ldots \ldots$ Delta $\mathrm{P}$, psi $\quad \cdots, \mathrm{mD}$

Figure 128 Flow rate, pressure drop and permeability versus time for core\# 6 after restoration

Table 34 shows the permeability values measured for different cores used at $40{ }^{\circ} \mathrm{C}$ (within the range 52-98 $\mathrm{mD}$ ). Red cells are the cores that were discarded after permeability measurement or tracer test since their permeability did not reach a stable value or reduced significantly after the tracer test. Figure 129 shows the permeability measurement for one of the cores (core\# 17).

Tracer tests were run for cores 12 and 13 to assure the homogeneity of the cores. Figure 130 shows an example of permeability measurement and tracer test for core\#12. A more severe reduction in permeability of the cores occurred for this set compared to the previous set of cores used at $25{ }^{\circ} \mathrm{C}$. In order to restore the cores after tracer tests they were dried again and saturated with $2 \% \mathrm{w} / \mathrm{w} \mathrm{KCl}$, but their permeability was not recovered nor was it stable versus time (Figure 131). Consequently, a tracer test was not run for the rest of the cores from this group. Figure 129 shows the permeability measurement for a representative core. The rest of the measurements and tracer tests are shown in Appendix A. 
Table 34 Permeability and porosity measurements for cores used at $40{ }^{\circ} \mathrm{C}$. Permeability after resaturation is also reported.

\begin{tabular}{|c|c|c|c|c|}
\hline Core \# & $\mathbf{k}(\mathbf{m D})$ before tracer & $\mathbf{k}(\mathbf{m D})$ after tracer & $\mathbf{k}(\mathbf{m D})$ after resaturation & Porosity, $\%$ \\
\hline 12 & 65 & 9.61 & 45 & 17.64 \\
\hline 13 & 56 & 12 & 44 & 21.00 \\
\hline 14 & 52 & ND & ND & 17.38 \\
\hline 15 & 80 & ND & ND & 17.86 \\
\hline 16 & 58.5 & ND & ND & 16.82 \\
\hline 17 & 75.4 & ND & ND & 17.99 \\
\hline 18 & 65.5 & ND & ND & 17.92 \\
\hline 19 & 76 & ND & ND & 17.63 \\
\hline 20 & 74.6 & ND & ND & 17.83 \\
\hline 21 & 82 & ND & ND & 18.10 \\
\hline 22 & 82.2 & ND & ND & 18.17 \\
\hline 23 & 88.2 & ND & ND & 17.80 \\
\hline 24 & 88 & ND & & 18.21 \\
\hline
\end{tabular}

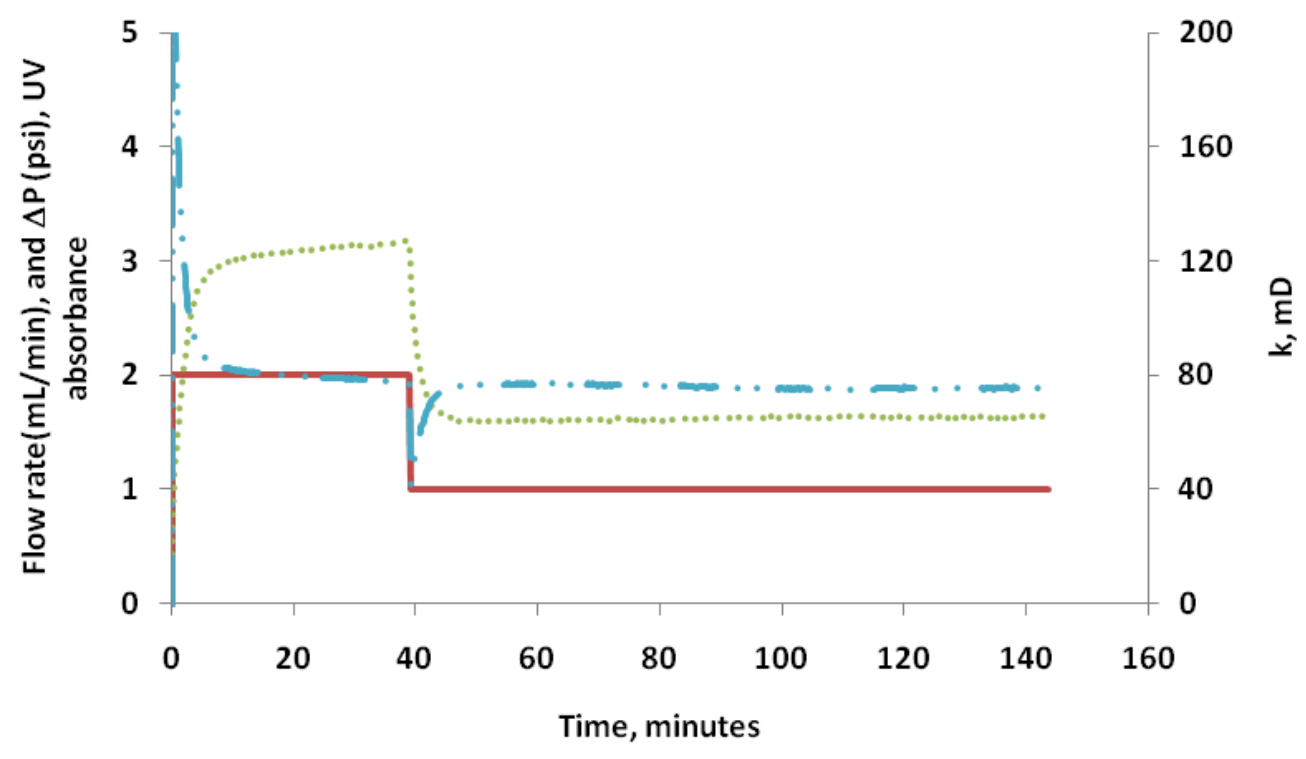

Flow rate, $\mathrm{mL} / \mathrm{min}$

Delta P, psi $\quad \cdot k, \mathrm{mD}$

Figure 129 Flow rate, pressure drop and permeability of core\#17 before fluid-loss tests at $40{ }^{\circ} \mathrm{C}$. 


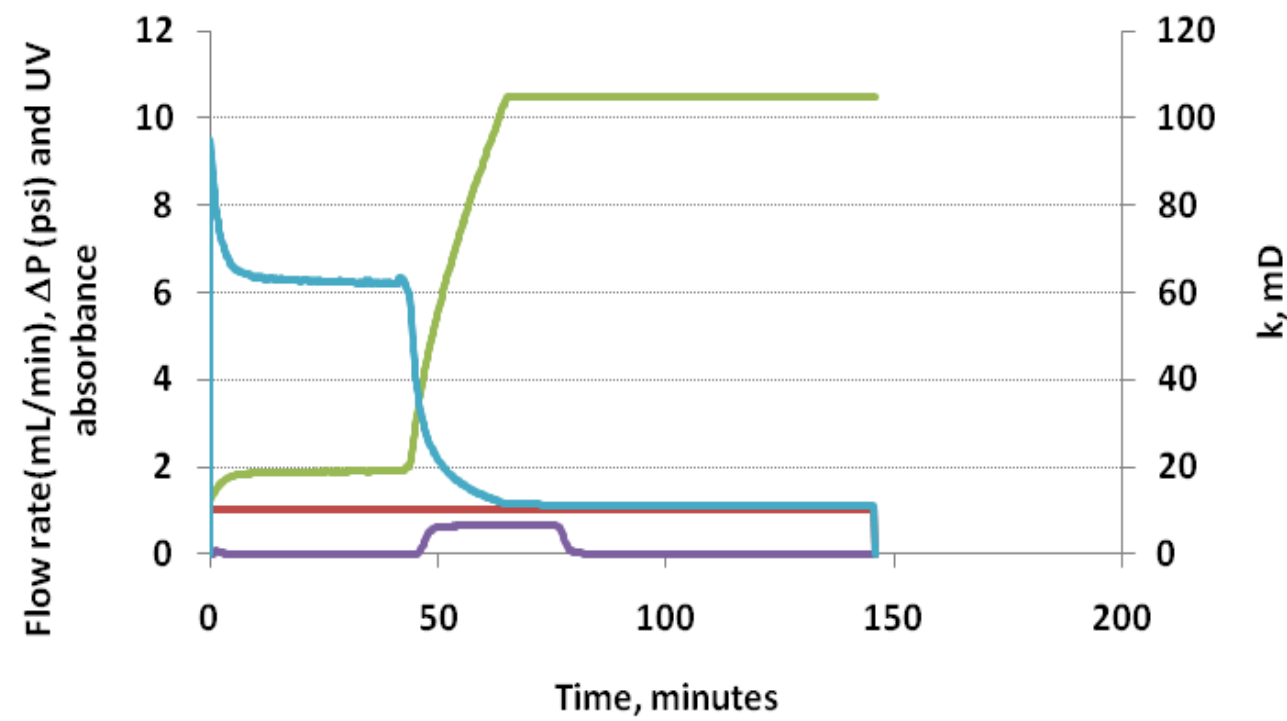

Flow rate, $\mathrm{mL} / \mathrm{min} \longrightarrow$ Delta $\mathrm{P}, \mathrm{psi} \longrightarrow \mathrm{UV}=\mathrm{k}, \mathrm{mD}$

Figure 130 Flow rate, pressure drop, UV absorbance and permeability of core\#12 before fluid-loss tests at 40 ${ }^{\circ} \mathrm{C}$. Pressure drop was not recorded above $10 \mathrm{psi}$ thus permeability was not calculated since the pressure transmitters were set for this range.

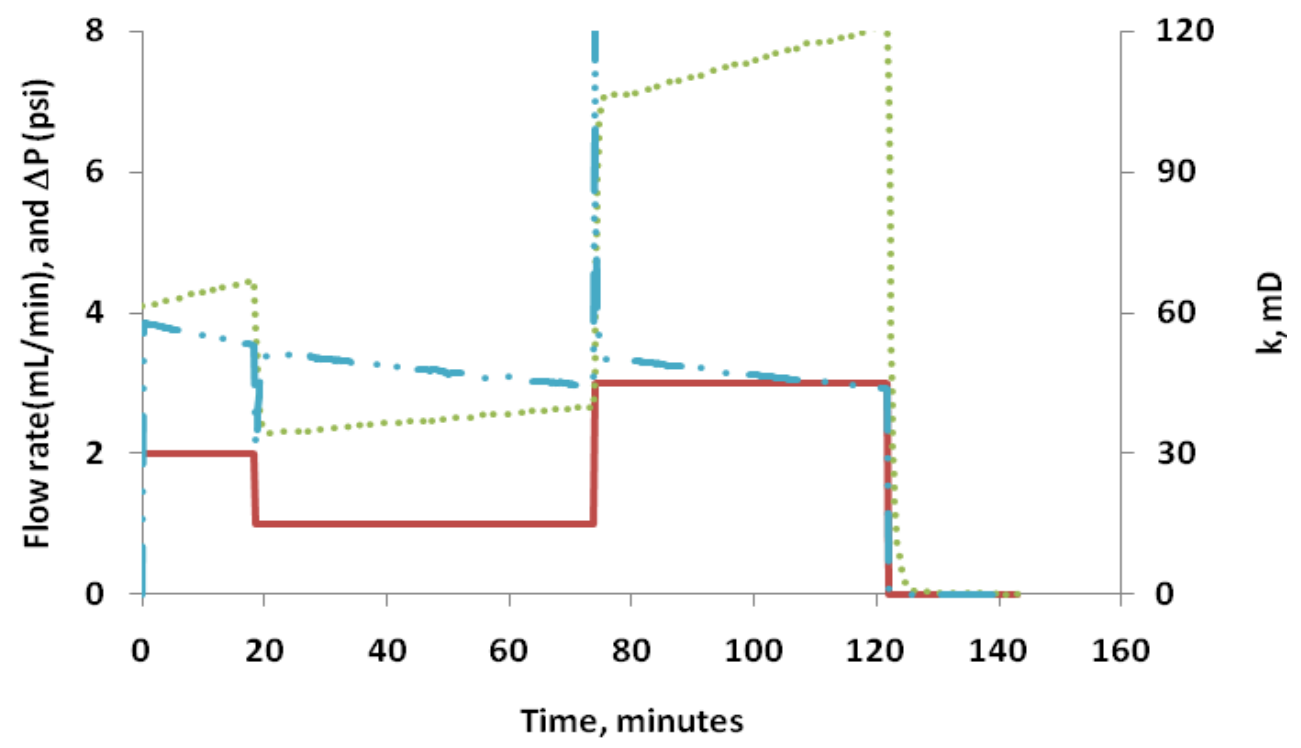

Flow rate, $\mathrm{mL} / \mathrm{min} \quad \ldots$. Delta $\mathrm{P}$, psi $\quad$ k, $\mathrm{mD}$

Figure 131 Flow rate, pressure drop and permeability versus time for core\# 12 after resaturation. Core was dried and resaturated after the tracer test was performed. 


\section{7-2 Fluid-Loss Tests and Filter-Cake Cleanup}

Cores with permeability in the range of $117-155 \mathrm{mD}$ were used for fluid-loss tests run at $25^{\circ} \mathrm{C}$ using HPG gels made with 192 ppm final borax concentration as a control system, or mixed with pectinase-loaded PEC nanoparticles, pectinase or control PEC nanoparticles. Final concentration of HPG and pectinase after mixing was $3077 \mathrm{ppm}$ and $0.02 \% \mathrm{w} / \mathrm{w}$ for all systems respectively. Cores with permeability in the range of $52-98 \mathrm{mD}$ were used for tests run at $40{ }^{\circ} \mathrm{C}$ using $\mathrm{HPG}$ gels made with 385 ppm final borax concentration as a control system or mixed with EL2Xloaded PEC nanoparticles, EL2X or control PEC nanoparticles. Final concentration of HPG and EL2X after mixing was $3077 \mathrm{ppm}$ and $0.08 \% \mathrm{w} / \mathrm{w}$ for all systems respectively.

First, a fluid-loss test was performed under a specific temperature and 500 psi pressure condition for each core. Then the fluid-loss setup was shut in overnight $(12 \mathrm{~h})$ with no pressure applied but at the same temperature as for the fluid-loss test. The excess fluid was decanted off, then replaced with $2 \% \mathrm{w} / \mathrm{w} \mathrm{KCl}$ and 100 psi pressure was applied while measuring the fluid-loss. This last part of the procedure was termed "filter-cake cleanup".

Fluid-Loss and Filter-Cake Cleanup Tests Run at $25{ }^{\circ} \mathrm{C}$ for Pectinase-Loaded PEC Nanoparticles: Cores listed in Table $\mathbf{3 3}$ were used, either fresh or restored, for fluid-loss tests run at $25{ }^{\circ} \mathrm{C}$ using HPG gels made with $1000 \mathrm{ppm}$ borax as a control system or mixed with pectinase-loaded PEC nanoparticle system A, 0.1 wt \% pectinase or control PEC nanoparticles. $200 \mathrm{~mL}$ samples with final $\mathrm{pH}$ of 9 were prepared and shaken at $150 \mathrm{rpm}$ and $25^{\circ} \mathrm{C}$ for 1 hour. Samples were then put in the fluid-loss cell and 500 psi pressure was applied at $25{ }^{\circ} \mathrm{C}$ while fluid-loss was collected in a beaker set on a balance. Data were recorded and plotted using Labview software.

Table 35 reports the cores, spurt volume, fluid-loss coefficient, permeability of the core before and after, viscosity of filtrate and density of filtrate for each fluid-loss test. The only result that can be extracted from this table and Figure 132 is that both pectinase-loaded PEC nanoparticles and pectinase systems showed significantly more fluid-loss than the control HPG samples or HPG samples mixed with control PEC nanoparticles. However, fluid-loss coefficients were similar for all fluid-loss tests.

Filter-cake cleanup results, shown in Figure 133, demonstrate that both nanoparticle systems $A$ and $A^{\prime}$ and the HPG gel samples mixed with pectinase showed a significantly better cleanup compared to the HPG gel systems or HPG gel systems mixed with control nanoparticles. 
Cleanup slope was calculated and compared with each other (before the test was finished) for the different samples when they showed a stable and constant slope (Table 36). The highest cleanup slopes were observed for the PEC nanoparticles followed by samples with only pectinase as a breaker. These systems showed significantly higher slopes compared to the control HPG system or HPG system mixed with control nanoparticles. Calculated slope for $2 \% \mathrm{KCl}$ fluid under similar pressure difference and using Darcy's law is $121.7 \mathrm{~mL} /$ minutes for a core with permeability of $150 \mathrm{mD}$.

Higher retained permeability values were measured for the fluid-loss test using HPG gel or HPG gel mixed with control PEC nanoparticles compared to the systems mixed with pectinase-loaded PEC nanoparticles or only pectinase. No distinction can be made between the last two systems. This was interpreted to be the result of more invasion of polymer into the core for the systems containing enzyme since higher degradation of polymer occurs in those systems (Table 35).

Table 35 shows no difference between the viscosity and density of filtrates for different fluidloss tests.

Properties of the nanoparticles used in fluid-loss tests are shown in Table 37. PEC nanoparticles were prepared at $\mathrm{pH} 8.7$.

Filtrates from four different tests were analyzed for total organic and inorganic carbon and total nitrogen (Table 38). Both organic and inorganic carbon showed significantly higher concentrations for the tests run using pectinase-loaded nanoparticles or $0.1 \% \mathrm{w} / \mathrm{w}$ pectinase compared to the fluid-loss tests using no enzyme. This is caused by more significant degradation of HPG in those tests caused by enzyme. 
Table 35 Fluid-loss parameters for different fluids run at $25^{\circ} \mathrm{C}$

\begin{tabular}{|c|c|c|c|c|c|c|c|c|c|}
\hline & Core & $\begin{array}{c}\text { Spurt } \\
\text { Volume, } \\
\text { mL }\end{array}$ & $\underset{\mathrm{mL} / \mathrm{t}^{1 / 2}}{\mathrm{C}_{\mathrm{w}}}$ & $\begin{array}{l}\text { Filter- } \\
\text { cake, } g\end{array}$ & $\begin{array}{l}\text { Permeability } \\
\text { before, } \mathrm{mD}\end{array}$ & $\begin{array}{l}\text { Permeability } \\
\text { after, } \mathrm{mD}\end{array}$ & $\begin{array}{c}\text { Retained } \\
\text { permeability, } \\
\%\end{array}$ & $\begin{array}{l}\text { Viscosity } \\
\text { of filtrate } \\
\text { at } 60 \\
\text { rpm, cP }\end{array}$ & $\begin{array}{c}\begin{array}{c}\text { Density } \\
\text { of }\end{array} \\
\text { filtrate, } \\
\mathrm{g} / \mathrm{cm}^{3}\end{array}$ \\
\hline HPG gel & 10 & 0.23 & 0.54 & 0.29 & 129 & 60 & 46.5 & 1.11 & 1.01 \\
\hline HPG gel & 8 & 2.54 & 0.61 & 0.28 & 102 & 43 & 42.2 & 1.08 & 1.01 \\
\hline HPG gel & $\begin{array}{l}10, \\
\text { Rest. }\end{array}$ & 2.36 & 0.59 & 0.19 & 131 & 60 & 45.8 & 1.27 & 1.01 \\
\hline $\begin{array}{l}\text { PECNP } \\
\text { control }\end{array}$ & 9 & 2.51 & 0.57 & 0.39 & 138 & 62 & 44.9 & 1.13 & 1.01 \\
\hline $\begin{array}{l}\text { PECNP } \\
\text { control }\end{array}$ & 6, Rest. & 2.33 & 0.53 & 0.28 & 127 & 40 & 31.5 & 1.50 & 1.01 \\
\hline $\begin{array}{l}\text { PECNP } \\
\text { A }\end{array}$ & 1 & 11.91 & 0.53 & 0.14 & 154 & 54 & 35.1 & 1.92 & 1.01 \\
\hline $\begin{array}{l}\text { PECNP } \\
\text { A }\end{array}$ & 9, Rest. & 7.28 & 0.49 & 0.11 & 154 & 44 & 28.6 & 1.31 & 1.01 \\
\hline $\begin{array}{l}\text { PECNP } \\
\text { A }\end{array}$ & 4, Rest. & 7.48 & 0.58 & 0.32 & 117 & 55 & 47.0 & 1.67 & 1.01 \\
\hline $\begin{array}{l}\text { PECNP } \\
\text { A }^{\prime}\end{array}$ & 6 & 7.22 & 0.58 & 0.18 & 143 & 30 & 21.0 & 1.30 & 1.01 \\
\hline $\begin{array}{l}\text { PECNP } \\
\text { A }^{\prime}\end{array}$ & 5, Rest. & 5.46 & 0.55 & 0.31 & 155 & 46.5 & 30.0 & 1.30 & 1.01 \\
\hline $\begin{array}{l}0.1 \% \\
\text { pectinase }\end{array}$ & 3 & 7.99 & 0.54 & 0.15 & 120 & 28 & 23.3 & 1.54 & 1.01 \\
\hline $\begin{array}{l}0.1 \% \\
\text { pectinase }\end{array}$ & 5 & 8.34 & 0.62 & 0.17 & 162 & 46.7 & 28.8 & 1.79 & 1.01 \\
\hline $\begin{array}{l}0.1 \% \\
\text { pectinase }\end{array}$ & 1, Rest. & 8.8 & 0.58 & 0.14 & 153 & 55 & 36.0 & 1.64 & 1.01 \\
\hline
\end{tabular}




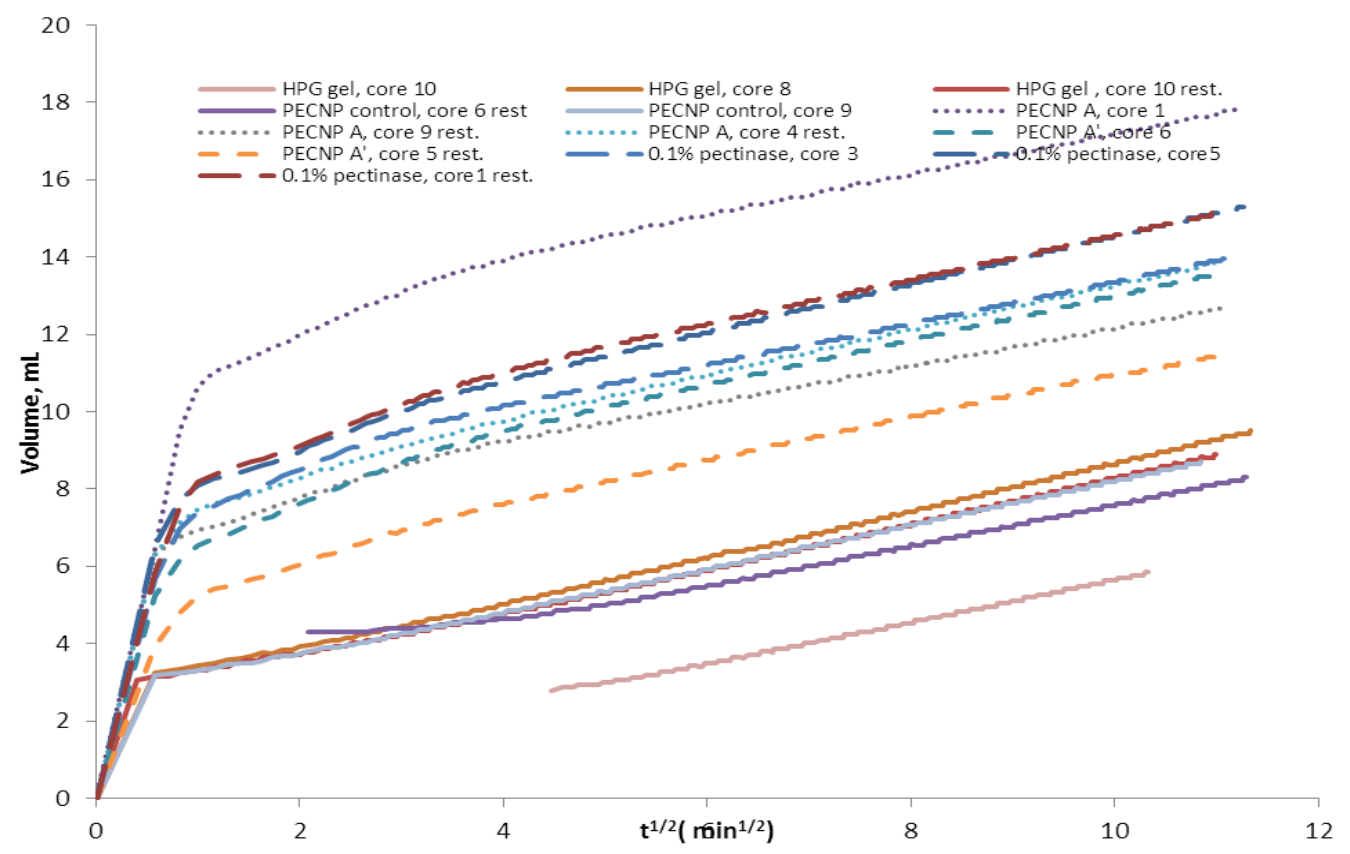

Figure 132 Fluid-loss versus $\mathbf{t}^{1 / 2}$ for HPG gel, (HPG gel + PECNP control), (HPG gel +PECNP $A$ ) and (HPG gel $+0.1 \%$ w/w pectinase) at $25^{\circ} \mathrm{C}$

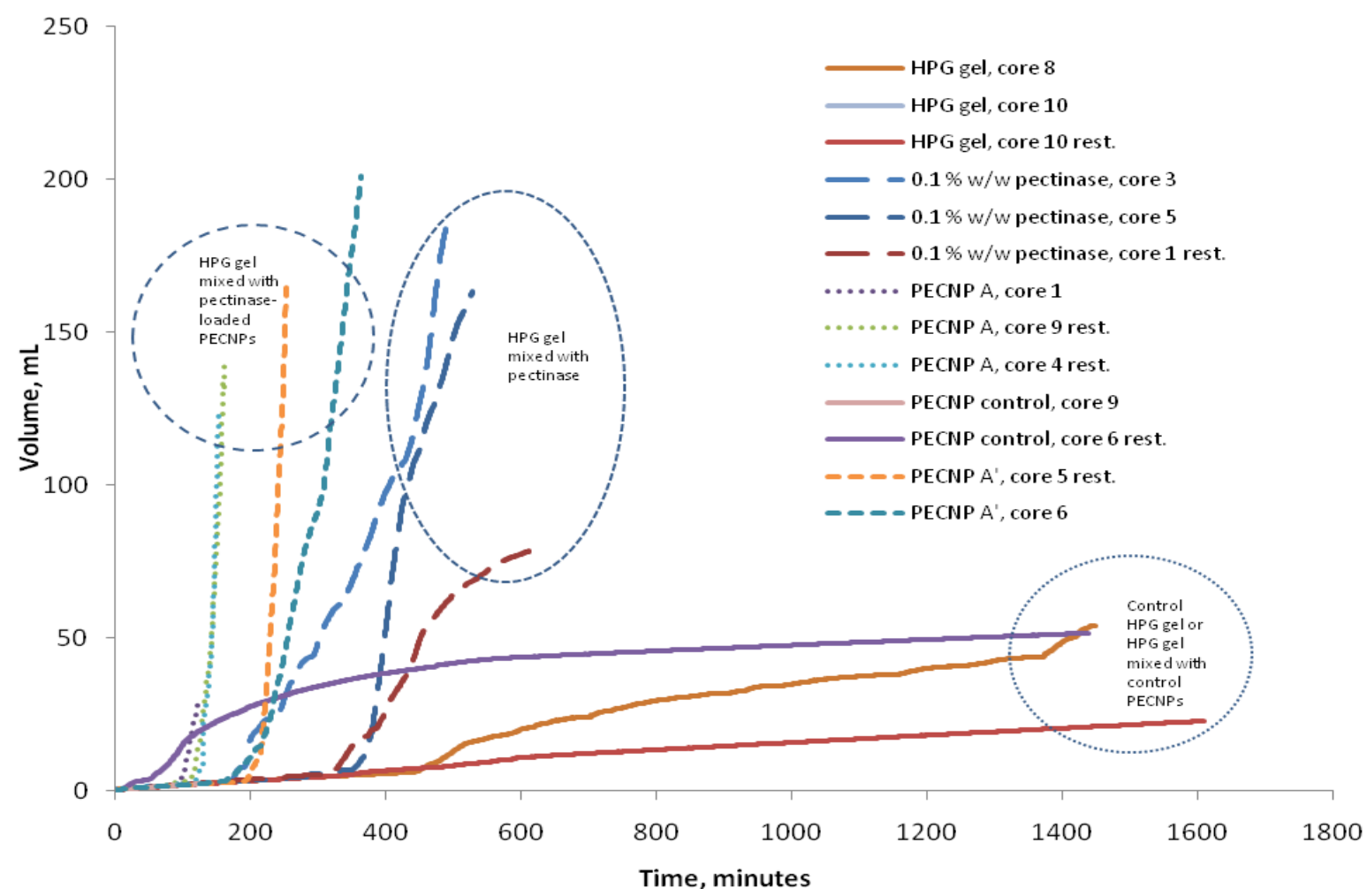

Figure 133 Filter-cake cleanup versus time for HPG gel, (HPG gel + PECNP control), (HPG gel +PECNP $A$ ) and (HPG gel $+0.1 \%$ w/w pectinase) at $25^{\circ} \mathrm{C}$ 
Table 36 Cleanup slope calculated for different fluid-loss tests reported in Table 35

\begin{tabular}{|c|c|c||}
\hline & Core \# & $\begin{array}{c}\text { Cleanup slope, } \\
\text { mL/min }\end{array}$ \\
\hline HPG gel & 10 & 0.01 \\
\hline HPG gel & 8 & 0.14 \\
\hline HPG gel & 10, restored & 0.01 \\
\hline PECNP control & 9 & 0.01 \\
\hline PECNP control & 6, restored & 0.01 \\
\hline PECNP A & 1 & 1.16 \\
\hline PECNP A & 9, restored & 3.87 \\
\hline PECNP A & 4, restored & 8.19 \\
\hline PECNP A' & 6 & 1.82 \\
\hline PECNP A & 5, restored & 4.89 \\
\hline 0.1\% pectinase & 3 & 1.50 \\
\hline 0.1\% pectinase & 5 & 1.69 \\
\hline 0.1\% pectinase & 1, restored & 0.41 \\
\hline
\end{tabular}

Table 37 Size measurement for PEC nanoparticles used in fluid-loss tests reported in Table 35 (pH=8.7).

\begin{tabular}{|c|c|c|c|}
\hline $\begin{array}{c}\text { Nanoparticle systems diluted } \\
\text { 40 } \times\end{array}$ & $\begin{array}{c}\text { Mean size, } \\
\text { nm }\end{array}$ & $\begin{array}{c}\text { Std. } \\
\text { Error }\end{array}$ & $\begin{array}{c}\text { Average } \\
\text { count rate, } \\
\text { kcps }\end{array}$ \\
\hline PECNP control for core9 & 427.3 & 2.9 & 446.6 \\
\hline PECNP A, core 1 & 422.3 & 6.0 & 384.1 \\
\hline PECNP A', core 6 & 473.0 & 5.9 & 555.7 \\
\hline PECNP A, core 9 rest. & 414.7 & 5.5 & 411.2 \\
\hline $\begin{array}{c}\text { PECNP control for core 6 } \\
\text { rest. }\end{array}$ & 423.3 & 7.0 & 458.1 \\
\hline PECNP A, core 4 rest. & 425.5 & 5.4 & 505.2 \\
\hline PECNP A', core 5 rest. & 429.9 & 3.3 & 387.6 \\
\hline
\end{tabular}


Table 38 TOC and ICP measured by Dr. Karen Peltier for filtrates remain after the fluid-loss tests

\begin{tabular}{|c|c|c|c|c|c|}
\hline & Fe (mg/L) & $\begin{array}{c}\text { Total } \\
\text { Carbon } \\
(\mathbf{m g} / \mathrm{L})\end{array}$ & $\begin{array}{c}\text { Total Nitrogen } \\
(\mathbf{m g} / \mathrm{L})\end{array}$ & $\begin{array}{c}\text { Inorganic } \\
\text { Carbon } \\
\text { (mg/L) }\end{array}$ & $\begin{array}{c}\text { Total Organic } \\
\text { Carbon by } \\
\text { Difference (mg/L) }\end{array}$ \\
\hline RO water & 0 & 0 & 0 & 0 & 0 \\
\hline HPG gel_core10 & 0 & 49 & 1.5 & 0 & 49 \\
\hline $\begin{array}{c}\text { HPG gel +PECNP } \\
\text { control_core 9 }\end{array}$ & 1.4 & 145 & 6.4 & 26 & 119 \\
\hline $\begin{array}{c}\text { HPG gel +PECNP } \\
\text { A_core 1 }\end{array}$ & 2.5 & 446 & 8.3 & 8.7 & 423 \\
\hline $\begin{array}{c}\text { HPG gel+0.1\% } \\
\text { pectinase_core 4 }\end{array}$ & 1.6 & 653 & 6.4 & 23 & 645 \\
\hline
\end{tabular}

Fluid-Loss and Filter Cleanup Tests Run at $40{ }^{\circ} \mathrm{C}$ for EL2X-Loaded PEC Nanoparticles: :

Cores listed in Table 34 were used for fluid-loss tests run at $40{ }^{\circ} \mathrm{C}$ using HPG gels made with 2000 ppm borax as a control system or mixed with EL2X-loaded PEC nanoparticle system $I^{\prime}, 0.4$ wt \% EL2X or control PEC nanoparticles. $200 \mathrm{~mL}$ samples with final $\mathrm{pH}$ of 9.2 were prepared and were shaken at $150 \mathrm{rpm}$ at $40{ }^{\circ} \mathrm{C}$ cabinet for 15 minutes. Samples were then put in the fluidloss cell and 500 psi pressure was applied until the temperature at the top of the cell reached 40 ${ }^{\circ} \mathrm{C}$ (45 minutes). The fluid-loss test was then started while filtrate was collected in a beaker on a balance. Data was saved and plotted using Labview software.

Table 39 reports the core, spurt volume, fluid-loss coefficient, and permeability of the core before and after, viscosity of filtrate and density of filtrate for each fluid-loss test. This table and Figure 134 indicate that all samples showed very similar fluid-loss trends. The case with $0.8 \%$ w/w EL2X (enzyme concentration twice the other samples) showed higher fluid-loss since it was more degraded during the first hour. Unlike the tests run at $25^{\circ} \mathrm{C}$, no difference was observed between the fluid-loss data of HPG gels with no enzyme and the samples with enzyme.

Filter-cake cleanup results, shown in Figure 135, demonstrate that nanoparticle system $I^{\prime}$ and the HPG gel samples mixed with EL2X showed a significantly better cleanup compared to the HPG gel systems or HPG gel systems mixed with control nanoparticles. This is in line with filter-cake cleanup results measured for pectinase-loaded PEC nanoparticles. Cleanup slope was calculated for different samples when they show a stable and constant slope (before the test is finished) and compared with each other (Table 39). The largest cleanup slopes were observed for the PEC nanoparticles and the samples with only EL2X as a breaker. These systems showed significantly larger slopes compared to the control HPG system or HPG system mixed with control 
nanoparticles. Calculated slope for $2 \% \mathrm{KCl}$ fluid using Darcy's law is $64.9 \mathrm{~mL} / \mathrm{minutes}$ for core with permeability of $80 \mathrm{mD}$. Cleanup slope is obviously a function of core permeability since the flow direction is towards the core surface.

No distinction can be made between the retained permeability of cores after the fluid-loss test and cleanup using different fluid systems (Table 39). Moreover, no difference between the viscosity and density of filtrates for different fluid-loss tests was observed.

Properties of the nanoparticles used in fluid-loss tests are shown in Table 40. PEC nanoparticles were measured at $\mathrm{pH} 9.4$.

Table 39 Fluid-loss parameters for different fluids run at $40{ }^{\circ} \mathrm{C}$

\begin{tabular}{|c|c|c|c|c|c|c|c|c|c|c|}
\hline & Core\# & $\begin{array}{c}\text { Spurt } \\
\text { Volume, } \\
\text { mL }\end{array}$ & $\underset{\mathrm{mL} / \mathrm{t} 1 / 2}{\mathrm{Cw}}$ & $\begin{array}{l}\text { Filter- } \\
\text { cake } \\
\text { scraped, } \\
\text { g }\end{array}$ & $\begin{array}{c}\text { K(before) } \\
\text { mD }\end{array}$ & $\begin{array}{c}\text { K(after) } \\
\text { mD }\end{array}$ & $\begin{array}{c}\text { Retained } \\
\text { permeability, } \\
\%\end{array}$ & $\begin{array}{c}\text { Viscosity } \\
\text { of } \\
\text { filtrate } \\
\text { at } 60 \\
\text { rpm, cp } \\
\end{array}$ & $\begin{array}{c}\text { Density } \\
\text { of } \\
\text { filtrate, } \\
\mathrm{g} / \mathrm{cm} 3\end{array}$ & $\begin{array}{c}\text { Cleanup } \\
\text { slope, } \\
\text { mL/ } \\
\text { minutes }\end{array}$ \\
\hline HPG gel & 15 & 2.72 & 0.76 & 0.26 & 80 & 39 & 48.7 & 0.98 & 1.01 & 0.01 \\
\hline $\begin{array}{l}\text { PECNP } \\
\text { control }\end{array}$ & 18 & 3.92 & 0.6 & 0.77 & 65.5 & 33 & 50.4 & 1.23 & 1.01 & 0.01 \\
\hline $0.8 \%$ EL2X & 19 & 6.32 & 0.66 & 0.04 & 76 & 43 & 56.6 & 0.92 & 1.01 & 4.83 \\
\hline $0.4 \%$ EL2X & 21 & 3.47 & 0.81 & 0.32 & 82 & 36 & 43.9 & 1.04 & 1.01 & 0.66 \\
\hline $0.4 \%$ EL2X & 22 & 3.51 & 0.69 & 0.27 & 82.2 & 45 & 54.7 & 0.95 & 1.01 & 0.30 \\
\hline PECNP I' & 23 & 2.47 & 0.61 & 0.25 & 88.2 & 35.3 & 40.0 & 0.96 & 1.01 & 0.80 \\
\hline PECNP I' & 24 & 3.66 & 0.56 & 0.19 & 88 & 43 & 48.9 & 0.96 & 1.01 & 0.4 \\
\hline
\end{tabular}




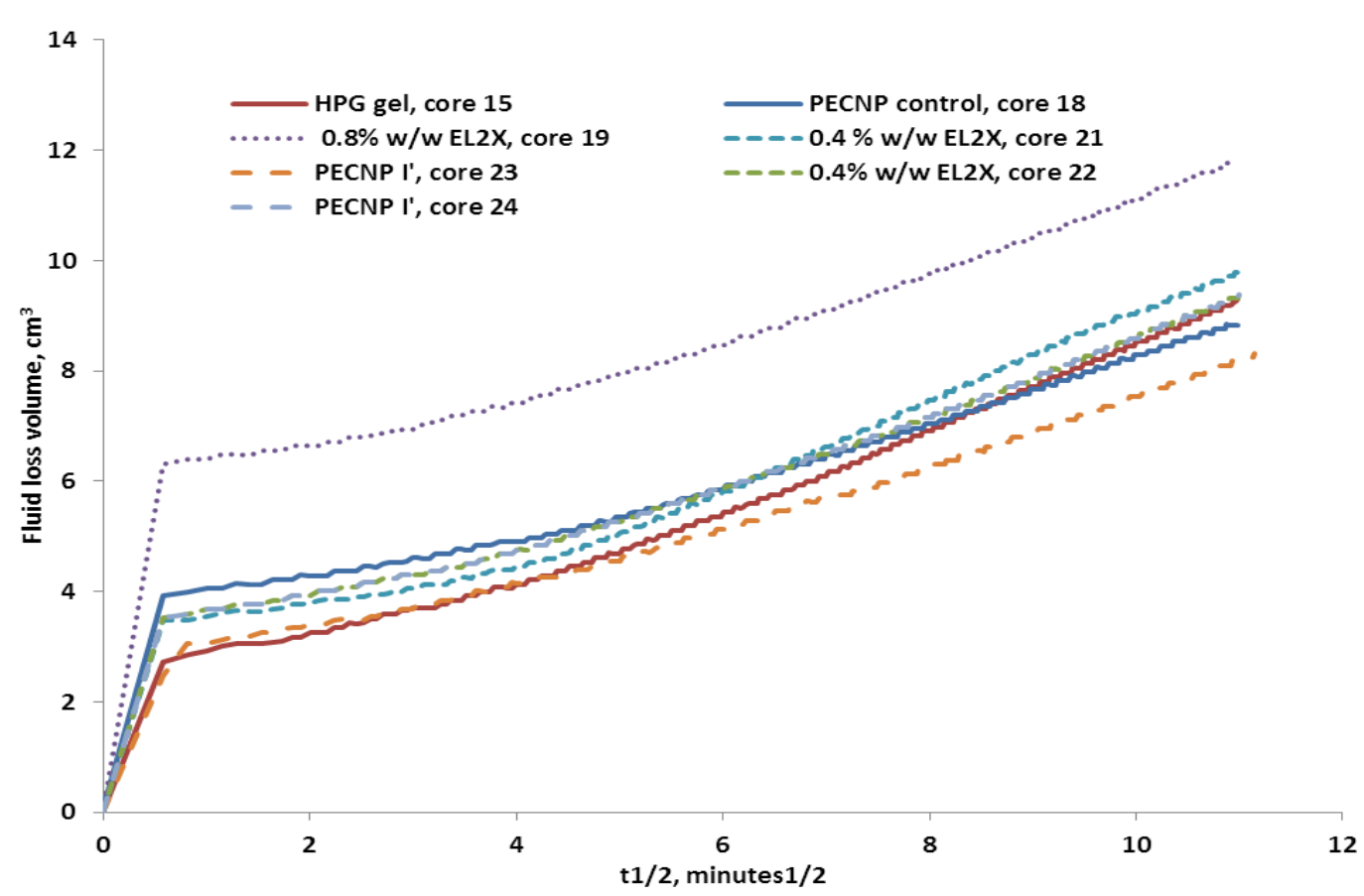

Figure 134 Fluid-loss versus $\mathrm{t}^{1 / 2}$ for HPG gel, (HPG gel + PECNP control), (HPG gel +PECNP I') and (HPG gel $+0.4 \% \mathrm{w} / \mathrm{w}$ EL2X) at $40{ }^{\circ} \mathrm{C}$

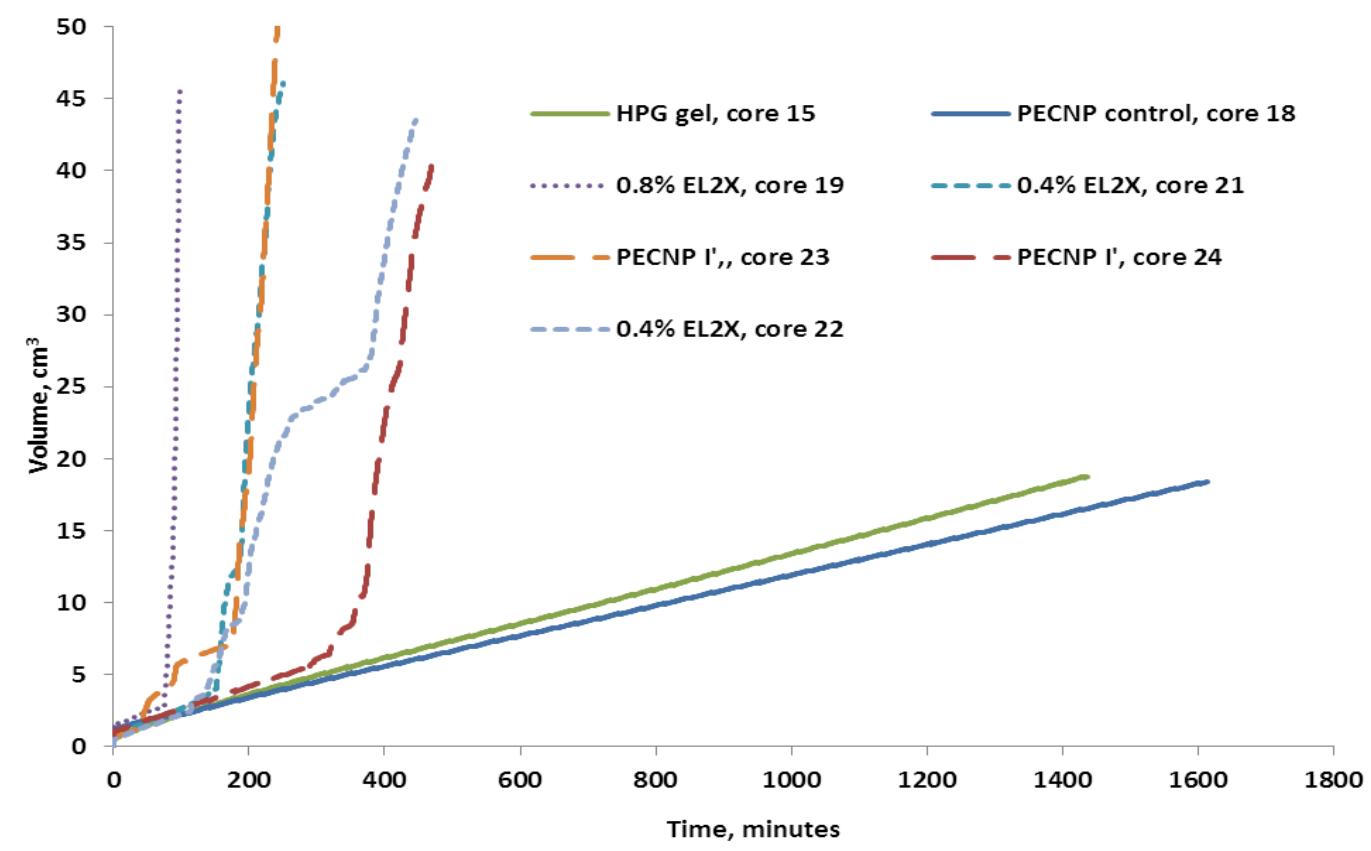

Figure 135 Filter-cake cleanup versus time for HPG gel, (HPG gel + PECNP control), (HPG gel +PECNP I') and (HPG gel $+0.4 \% \mathrm{w} / \mathrm{w} \mathrm{EL2X)}$ at $40{ }^{\circ} \mathrm{C}$ 
Table 40 Size measurement for PEC nanoparticles used in fluid-loss tests reported in Table 39 (pH=9.4).

\begin{tabular}{|c|c|c|c|c|}
\hline & Mean size, nm & $\begin{array}{c}\text { Std. } \\
\text { Error }\end{array}$ & Average count rate, kcps & $\begin{array}{c}\text { \# of } \\
\text { distribution }\end{array}$ \\
\hline PECNP control, core 18 & 317.4 & 8.6 & 410 & 1 \\
\hline PECNP I', core 17 & 361.7 & 6.3 & 418 & 1 \\
\hline PECNP I', core 23 & 404.3 & 5.5 & 410.6 & 2 \\
\hline PECNP I', core 24 & 378.1 & 16.6 & 483 & 2 \\
\hline
\end{tabular}

\section{7-3 Permeability of Cores After the Fluid-Loss Tests}

At the end of each fluid-loss test, filter-cake was scraped off the core surface and permeability was measured for each core in the opposite direction of the fluid loss. Pressure transmitters were set for maximum pressure drop of 10 psi. When the flow rates were small enough to have pressure drops in that range, a cleanup trend was observed for pressure drop, and thus permeability of cores versus time. Pressure drops with high values were recorded first, but they decreased versus time at a constant flow rate. In some cases the permeability increased with increasing flow rate. Flow rates were not increased to values above $3 \mathrm{~mL} / \mathrm{min}$ since that was the maximum flow rate used while measuring the permeability values before the fluid-loss tests. In some cases, pressure drops exceeded the maximum measurable values (10 psi). As a default, all pressure drops above 10 psi were saved as 10 psi. Figure 136 and Figure 137 show the permeability measurement for cores 4 (restored) and 24 after the fluid-loss cleanup tests respectively. The rest of the measurements are shown in Appendix B. Cleanup of cores from degraded HPG gel is shown for all cores. 


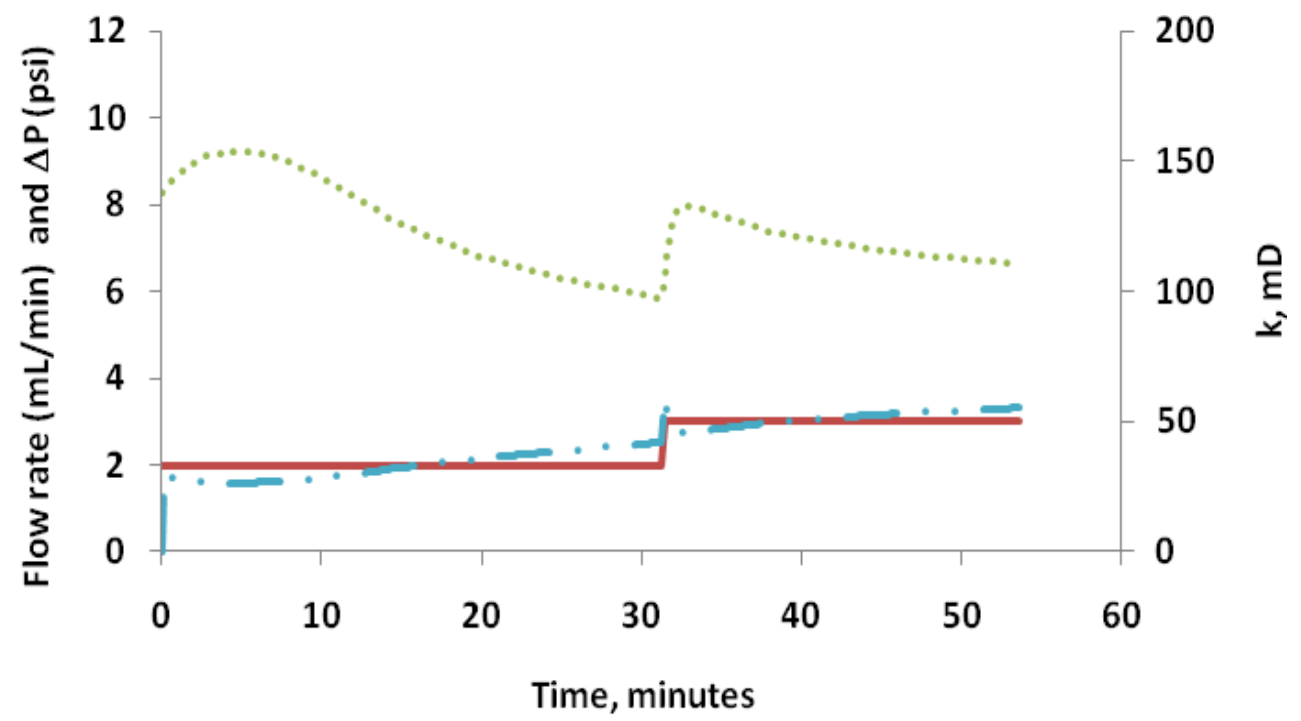

Flow rate, $\mathrm{mL} / \mathrm{min} \quad \ldots$. Delta $\mathrm{P}$, psi $\quad \mathrm{k}, \mathrm{mD}$

Figure 136 Permeability measurement for core 4 (restored) after the fluid-loss and cleanup tests reported in

Table 35

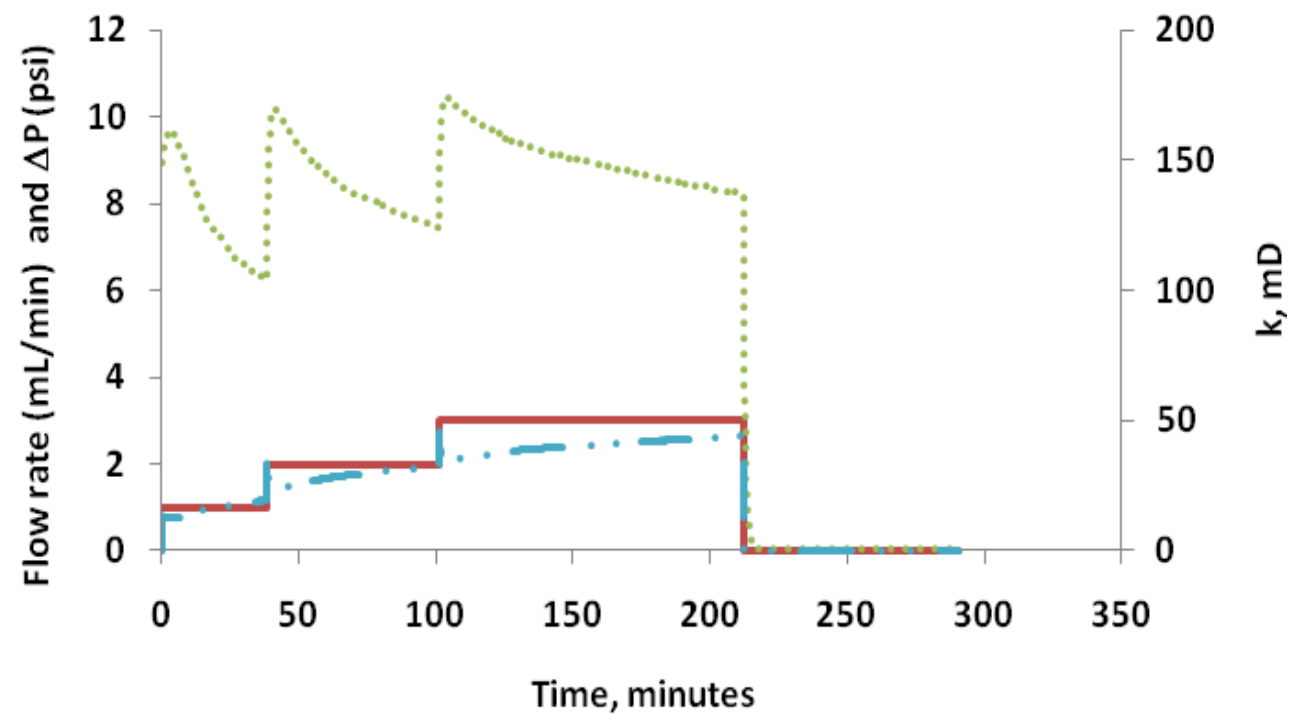

Flow rate, $\mathrm{mL} / \mathrm{min} \quad \ldots .$. Delta $\mathrm{P}$, psi $\quad \mathrm{k}, \mathrm{mD}$

Figure 137 Permeability measurement for core 24 after the fluid-loss and cleanup tests reported in Table 39 


\subsection{Effect of Enzyme-Loaded Nanoparticles on Low Concentration HPG For Slick-water Applications}

In this section PECNP systems $A^{\prime}$ and $I^{\prime}$ were used to break low concentration HPG solutions prepared in $2 \% \mathrm{KCl}$. This application is of interest for slick-water fracturing jobs when there is no need for crosslinkers. Delaying the degradation of HPG solutions would significantly improve the capability of fluids to carry proppants.

\section{8-1 Pectinase-Loaded PEC Nanoparticles Used for Breaking Low Concentration HPG}

Four different samples with final concentration of 1000 ppm HPG were prepared as listed in Table 41. The two PEC nanoparticle systems, one control and one loaded with pectinase, were prepared and their sizes are reported in Table 42. 5000 ppm HPG stock solution was diluted to 1250 ppm with R.O. water. All samples were kept in a $25{ }^{\circ} \mathrm{C}$ cabinet while being stirred at 400 rpm. Viscosity was measured for different samples versus time using a Brookfield viscometer (Figure 138). Higher viscosity of the HPG fluid mixed with pectinase-loaded nanoparticles compared to the HPG systems mixed with only pectinase was observed. Increasing the viscosity of low concentration HPG, used as slick-water, decreases the proppant settling velocity. Furthermore, PEC nanoparticles carry enzymes to break the polymeric solution. This is of specific interest in fracturing fluids used for unconventional reservoirs. 
Table 41 Mixing ratios for different HPG samples mixed with R.O. water, control PECNPs and PECNP $A^{\prime}$ at $25^{\circ} \mathrm{C}$.

\begin{tabular}{|c|c|c|c|c|c|}
\hline & $\begin{array}{c}1250 \\
\text { ppm } \\
\text { HPG, } \\
\text { mL }\end{array}$ & $\begin{array}{c}\text { R.O. } \\
\text { water } \\
(\mathrm{pH}=8), \\
\mathrm{mL}\end{array}$ & $\begin{array}{c}\text { Diluted } \\
\text { Control PEC } \\
\text { NPs, mL }\end{array}$ & $\begin{array}{c}\text { Diluted } \\
\text { PECNP } A^{\prime}, \\
\text { mL }\end{array}$ & $\begin{array}{c}0.1 \% \\
\text { w/w } \\
\text { pectinase, } \\
\text { mL }\end{array}$ \\
\hline HPG & 16 & 4 & & & \\
\hline $\begin{array}{c}\text { HPG \& PECNP control, } \\
\qquad \times\end{array}$ & 16 & & 4 & & \\
\hline HPG \& PECNP $A^{\prime}, 8 \times$ & 16 & 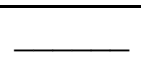 & - & 4 & \\
\hline $\begin{array}{c}\text { HPG \& } 0.1 \% \text { w/w } \\
\text { pectinase }\end{array}$ & 16 & & & & 4 \\
\hline
\end{tabular}

Table 42 Particle size report for pectinase-loaded nanoparticles used for slick-water applications

\begin{tabular}{|l|l|l|l|l||}
\hline & Mean size, \\
& nm & $\begin{array}{l}\text { Std. } \\
\text { error }\end{array}$ & $\begin{array}{l}\text { Average } \\
\text { count rate, } \\
\text { kcps }\end{array}$ & $\begin{array}{l}\text { \# Of } \\
\text { distributions }\end{array}$ \\
\hline Control PECNP System & 452.2 & 7.8 & 444.3 & 2 \\
\hline PECNP A' System & 463.4 & 4.8 & 424.1 & 1 \\
\hline
\end{tabular}




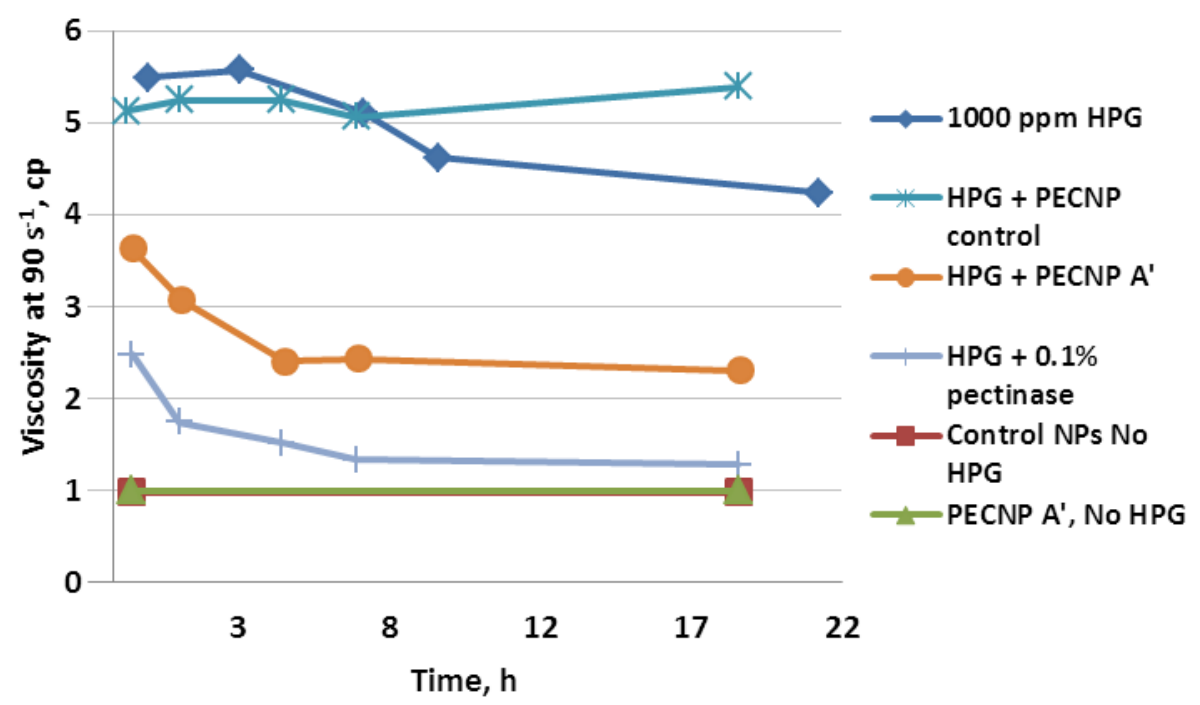

Figure 138 Viscosity at $90 \mathrm{~s}^{-1}$ versus time for $1000 \mathrm{ppm}$ HPG samples mixed with pectinase, PECNP $A^{\prime}$ and controlled PECNPs diluted $8 \times$ compared with a control HPG system at $25^{\circ} \mathrm{C}$.

\section{8-2 EL2X-Loaded PEC Nanoparticles Used for Breaking Low Concentration HPG}

Four different samples with final concentration of $1200 \mathrm{ppm}$ HPG were prepared as listed on Table 43. Higher concentrations of HPG were used since experiments were done at higher temperature for this enzyme (EL2X). The two PEC nanoparticle systems, one controlled and one loaded with EL2X, were prepared and their sizes are reported in Table 44. 5000 ppm HPG stock solution was diluted to $1500 \mathrm{ppm}$ with R.O. water.. All samples were kept at $40{ }^{\circ} \mathrm{C}$ while being stirred at $400 \mathrm{rpm}$. Viscosity was measured for different samples versus time using a Brookfield viscometer (Figure 139). Higher viscosity of the HPG fluid mixed with EL2X-loaded nanoparticles compared to the HPG systems mixed with EL2X itself was observed. Increasing the viscosity of low concentration HPG, used as slick-water, decreases the proppant settling velocity. For example a doubling in the viscosity decreases the proppant settling velocity by a factor of two. Furthermore, PEC nanoparticles carry enzymes to break the polymeric solution. This is similar to viscosity measurements for pectinase-loaded PEC nanoparticles. This is of particular interest in fracturing fluids used for unconventional reservoirs. 
Table 43 Mixing ratios for different HPG samples mixed with R.O. water, control PECNPs and PECNP $I^{\prime}$ at $40{ }^{\circ} \mathrm{C}$.

\begin{tabular}{|c|c|c|c|c|c|}
\hline & $\begin{array}{c}1500 \\
\text { ppm } \\
\text { HPG, } \\
\text { mL }\end{array}$ & $\begin{array}{c}\text { R.O. } \\
\text { water } \\
(\mathrm{pH}=8), \\
\mathrm{mL}\end{array}$ & $\begin{array}{c}\text { Control } \\
\text { PECNP, mL }\end{array}$ & $\begin{array}{l}\text { PECNP I', } \\
\text { mL }\end{array}$ & $\begin{array}{c}0.4 \% \\
\text { w/wEL2X }\end{array}$ \\
\hline 1200 ppm HPG & 16 & 4 & & & \\
\hline $\begin{array}{c}\text { HPG \& PECNP control, } \\
4 \times \text { diluted }\end{array}$ & 16 & & 4 & & \\
\hline $\begin{array}{c}\text { HPG \& PECNP I', } \\
4 \times \text { diluted }\end{array}$ & 16 & & & 4 & \\
\hline HPG \& $0.4 \%$ w/w EL2X & 16 & & 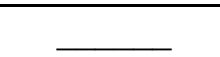 & & 4 \\
\hline
\end{tabular}

Table 44 Particle size report for EL2X-loaded nanoparticles used for slick water applications

\begin{tabular}{|c|c|c|c|c|}
\hline & $\begin{array}{c}\text { Mean size, } \\
\mathbf{n m}\end{array}$ & $\begin{array}{c}\text { Std. } \\
\text { Error }\end{array}$ & $\begin{array}{c}\text { Average } \\
\text { count rate, } \\
\mathbf{k c p s}\end{array}$ & $\begin{array}{c}\text { \# Of peaks } \\
\text { in } \\
\text { distribution }\end{array}$ \\
\hline PECNP Control & 186.3 & 3 & 530 & 1 \\
\hline PECNP $\boldsymbol{I}^{\prime}$ & 226.1 & 0.9 & 385.2 & 1 \\
\hline
\end{tabular}




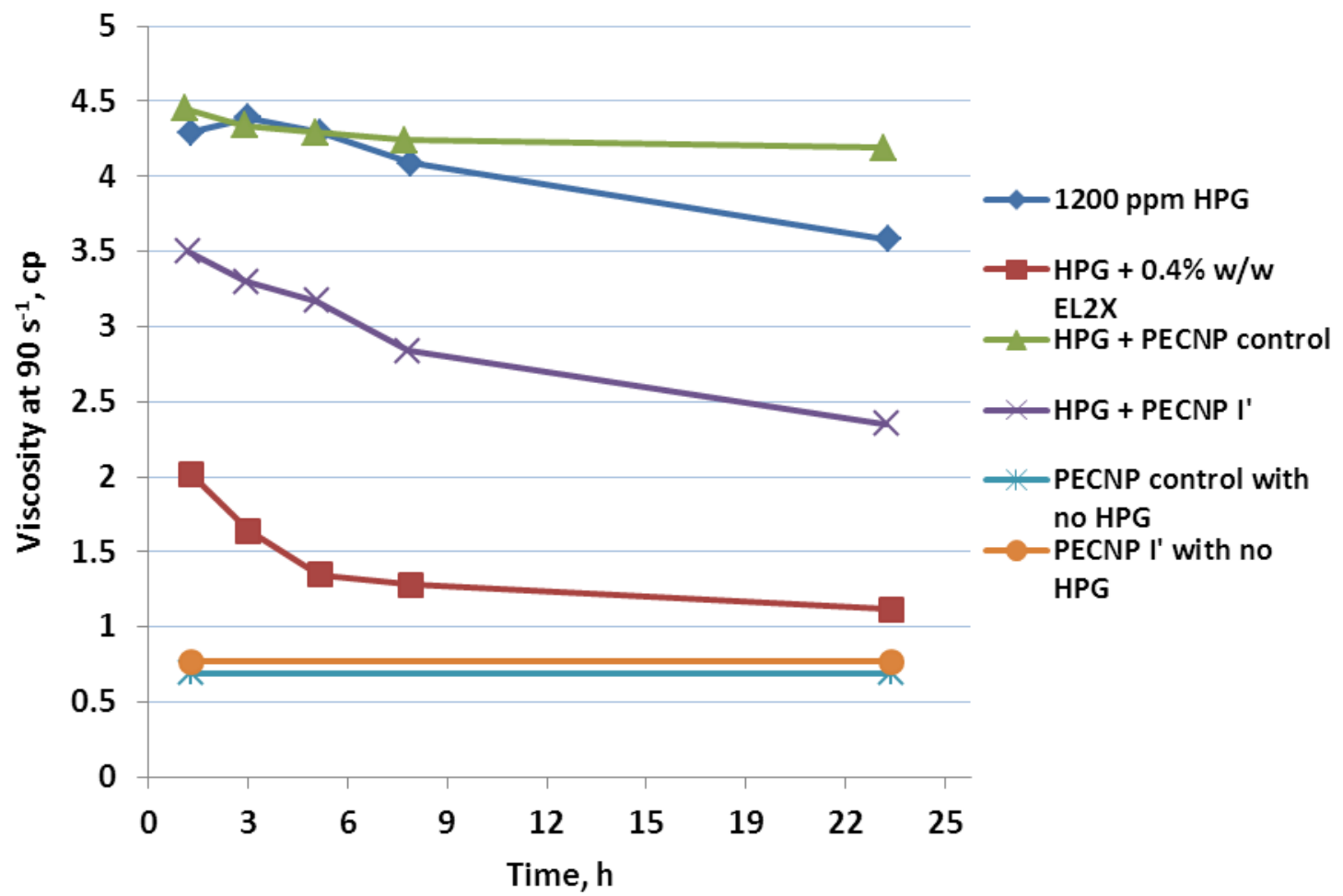

Figure 139 Viscosity at $90 \mathrm{~s}^{-1}$ versus time for $1200 \mathrm{ppm}$ HPG samples mixed with EL2X, PECNP I' and controlled PECNPs diluted $4 \times$ compared with a control HPG system at $40^{\circ} \mathrm{C}$. 


\subsection{Summary of Results}

The following is a summary of the results and main findings discussed in this chapter:

- Viscosity of guar solutions was maximized by giving them sufficient hydration time. Reproducible viscosity versus temperature and $\mathrm{pH}$ trends were observed for polymeric solutions. Power-law parameters were correlated versus temperature for HPG solutions while $\mathrm{pH}$ in the range of 6.5-9.1 showed no significant effect.

- Critical borax concentration was determined for borate crosslinked HPG solutions at $25^{\circ} \mathrm{C}$ and $40{ }^{\circ} \mathrm{C}$. This was determined by measuring both viscous and elastic moduli of gels versus borax concentration at each temperature. A critical concentration above which complexing sites were saturated with borate ions was observed at $25{ }^{\circ} \mathrm{C}$. This critical borax concentration was not observed at $40{ }^{\circ} \mathrm{C}$ since more borax is required for higher temperatures to generate equivalent concentration of borate ions. ${ }^{[25,26]}$

- SDS-PAGE was used successfully to generate a linear calibration curve between the intensity of protein peaks penetrated into the SDS-PAGE gel and concentration of enzyme. Using the same technique, the concentration of enzyme in the supernatants was found for two pectinase-loaded nanoparticles.

- Activity of the enzymes in a solution in the presence of polyelectrolytes was measured using a viscometric assay. In this technique the reciprocal of the time required for the enzyme solution to decrease the viscosity of a HPG or guar solution to $50 \%$ of its initial value was interpreted as activity of enzyme. Generating a linear calibration curve between the concentration and activity of enzyme and considering that all the enzyme concentrations used during this research were in the linear range, concentration of enzyme in the supernatant of enzyme-loaded PEC nanoparticles was determined. Entrapment efficiencies calculated using this method were in close agreement with the ones calculated using SDS-PAGE. Therefore, this method was used for the rest of EE measurements during the course of this research since it was significantly cheaper and easier to run and all the instruments were available at TORP.

- Using viscometric assay to measure the activity of enzymes and supernatants separated from PEC nanoparticles and preparing different nanoparticles systems loaded with two 
different enzymes (pectinase and EL2X), PEC nanoparticle systems with maximized entrapment efficiencies were determined over a range of $\mathrm{pH}$.

- Neither preparation shear nor applied shear showed any effect on the activity of enzymeloaded nanoparticles when used to degrade HPG solutions. However, rate of addition of DS and enzyme to $1 \% \mathrm{w} / \mathrm{w}$ PEI while preparing the PEC nanoparticles had a significant effect. Fast addition of chemicals resulted in significantly smaller PEC nanoparticles compared to the drop-wise method of adding chemicals.

- PEC nanoparticle systems entrapping different enzymes were used successfully to delay degradation of both viscous and elastic moduli for HPG gel samples at $25{ }^{\circ} \mathrm{C}$ and $40{ }^{\circ} \mathrm{C}$. Nanoparticles with enzyme added before addition of DS showed later degradation time compared to the nanoparticles where enzyme was added after addition of DS. This effect is important since it prevents premature degradation during injection of high enzyme concentrations. It was also shown that viscosity and viscoelastic moduli of gelled HPG reach similar or smaller values as compared to the HPG gel degraded using enzyme only.

- PEC nanoparticle systems $A^{\prime}$ and $I^{\prime}$ were used successfully to protect enzymes (pectinase and EL2X) against $\mathrm{pH}$ and temperature conditions at which both enzymes were denatured. After determining $\mathrm{pH}$ and temperature conditions at which both enzymes denature, enzymes entrapped by PEC nanoparticle systems were applied to degrade HPG solutions at similar conditions. Both enzyme-loaded PEC nanoparticles degraded HPG solutions successfully. Protecting enzymes at extreme $\mathrm{pH}$ and temperature conditions is important since denaturation is the main limitation of using enzymes for most fracturing jobs. ${ }^{[1,9]}$

- Although pectinase loaded PEC nanoparticle system $A$ showed no sensitivity to shear applied in the shear loop when mixed with HPG gel, EL2X-loaded nanoparticle systems $H$ and $I^{\prime}$ both showed sensitivity to shear applied in the shear loop. None of the latter systems showed higher pressure gradients compared to the enzyme system itself. Considering that EL2X-loaded PEC nanoparticles showed insensitivity to shear while degrading HPG solution, this behavior needs further study.

- Performing cleanup test after forming filter-cakes for borate crosslinked HPG samples mixed with enzymes or enzyme loaded nanoparticles and comparing them with the control HPG gel system or HPG gel system mixed with control PEC nanoparticles, 
significant cleanup for the cases with enzyme or enzymes entrapped by PEC nanoparticles was observed.

- Mixing of low concentration HPG solutions (slick-water) with enzyme-loaded nanoparticles increased viscosity of such fluid significantly compared to the HPG solutions mixed with only enzyme. This increase in viscosity decreases the proppant settling velocity while enzyme exists in the environment to degrade the polymer. This is significant for low or extra low permeability reservoirs where a small reduction in permeability of sand pack or rock causes significant damage to the production of the well. $^{[23]}$ 


\section{Conclusions}

\subsection{Preparation of Guar/HPG Solutions and Borate Cross-linked Guar/HPG}

- Sufficient hydration time is required for guar solutions to reach their maximum stable viscosity. However, this hydration time does not affect the rate of hydrolysis of solutions in water.

- Both consistency and behavior index of HPG solution decrease with temperature.

- There is no significant effect of $\mathrm{pH}$ in the range of 6.5-9.1 on viscosity of HPG solution in $2 \% \mathrm{KCl}$.

- There is a critical concentration of borate ions above which complexing sites are saturated with borate ions for borate crosslinked HPG. This critical final concentration was $770 \mathrm{ppm}$ for gel samples measured at $25{ }^{\circ} \mathrm{C}$ while no critical concentration was observed for gel samples studied at $40{ }^{\circ} \mathrm{C}$ within the studied concentrations range.

\subsection{Characterization and Activity Measurement of Pectinase and EL2X}

- SDS-PAGE and viscometric assays are both effective in measuring the concentration of enzymes in presence of polyelectrolytes.

- The time required for the pectinase to become denatured (5 minutes for pectinase at $\mathrm{pH}$ of 9.1 and $40{ }^{\circ} \mathrm{C}$ ) is independent of the concentration of enzymes at denaturation $\mathrm{pH}$ and temperature conditions.

\subsection{Preparation and Optimization of Enzyme-Loaded PEC} Nanoparticles

- Stable enzyme-loaded PEC nanoparticles (200-700 nm diameter) can be prepared with different formulations which vary in PEI: DS ratio, order of addition and concentration of enzyme.

- Entrapment efficiency of enzyme-loaded nanoparticles can be optimized successfully over a range of $\mathrm{pH}$. $\mathrm{EE}_{\mathrm{A}}$ was maximized to reach $92 \%$ for pectinase-loaded PEC nanoparticles and $61 \%$ for EL2X-loaded PEC nanoparticles. 
- Preparation stirring rate has no effect on the size of pectinase-loaded PEC nanoparticles. However, fast addition of chemicals while preparing the PEC nanoparticles results in smaller nanoparticle size compared to the nanoparticles prepared by drop-wise addition of chemicals.

- PEC nanoparticles mixed with HPG solution are largely insensitive to the shear forces likely to be encountered in the field.

\subsection{Degradation of Borate Cross-linked Guar/HPG Using Enzymes and Enzyme-Loaded Nanoparticles}

- Nanoparticle-entrapped enzyme completely break borate-crosslinked guar and HPG gels, with the breaking being delayed significantly compared to unentrapped enzymes at the same concentration. The delayed release of the enzyme allows the loaded particles to be mixed with the gelant before gelation occurs. This, along with the small size of the particles means that the enzyme is distributed homogeneously through the gel, which may result in a more complete breakage of the gel and hence higher post-treatment hydraulic fracture conductivity.

- Control PEC nanoparticles with no enzyme also degrade HPG gel but with a significantly slower rate compared to the enzyme-loaded PEC nanoparticles.

\subsection{Protective Effect of Nanoparticles on Enzymes}

- Entrapment of enzymes within the nanoparticles confers protection from the $\mathrm{pH}$ of the bulk solution. This is promising for the application of enzymes where $\mathrm{pH}$ and temperature conditions inhospitable to native enzyme are encountered.

\subsection{Effect of Simulated Wellbore Shear on Enzyme-loaded Nanoparticles: Shear Loop Experiments}

- Pectinase-loaded PEC nanoparticles show little shear sensitivity when mixed with HPG gel compared to the HPG gel mixed with only pectinase.

- EL2X-loaded PEC nanoparticles show faster release when injected into the shear loop compared to the samples made on the bench. This may be caused by the difference pressure and shear conditions under which the shear loop tests were performed compared 
to the tests on the bench, lower EE of this nanoparticle system compared to the pectinaseloaded PEC nanoparticles or weaker structure of this system against shear. Measuring the viscoelastic parameters at high pressures is recommended in order to investigate this phenomenon.

\subsection{Filter-Cake Formation and Cleanup Using Static Fluid-Loss Tests}

- Enzyme-loaded PEC nanoparticles clean up the filter-cake to the same degree as the enzyme alone, at least within the scope of the fluid-loss cell. This result must be supported by the conductivity cell results.

\subsection{Enzyme-Loaded PEC Nanoparticles Used for Breaking Low Concentration HPG}

- Enzyme-loaded PEC nanoparticles show significantly slower reduction in viscosity of HPG solution over time compared to the HPG systems mixed with enzyme itself. This may improve the applicability of low concentration HPG, used as slick-water, by decreasing the proppant settling velocity. 


\section{Recommendations for Future Work}

\subsection{Use of Guar Specific and High Temperature Enzymes}

Use of breaker products made of only one type of enzyme designed for fracturing fluid applications at high or extremely high temperature and $\mathrm{pH}$ is recommended. Although, the commercial enzyme used in this study was designed for higher temperature and $\mathrm{pH}$ values compared to pectinase, no knowledge of its components was available. Based on research reported in the literature review, commercial products are typically made of more than one type of enzyme. This may be one reason for lower entrapment efficiencies observed for this enzyme compared to pectinase. Entrapping $\beta$-mannanase would be a good start. Next entrapping high temperature enzymes used in the fracturing industry is recommended. Alternatively, the protective effect of PEC nanoparticles can be applied for different enzymes to broaden their active $\mathrm{pH}$ and temperature range.

\subsection{Improving Enzyme-Loaded Polyelectrolyte Complex Nanoparticles}

All the enzyme-loaded PEC nanoparticles prepared in this research were prepared in R.O. water. However, they were applied to guar/HPG solutions or HPG gels prepared in $2 \% \mathrm{KCl}$. Preparing PEC nanoparticles in different concentrations of $\mathrm{KCl}$ or other types of brine is recommended to study effect of salts on their stability and entrapment efficiency.

PEC nanoparticles used during the course of this research are in the 300-500 $\mathrm{nm}$ range. Using multilayer enzyme-loaded PEC nanoparticles and studying the stability and entrapment efficiency of such nanoparticles is recommended. This technique may result in higher entrapment efficiency, specifically for the EL2X-loaded PEC nanoparticles. In addition, preparing nanoparticles with larger size is possible using such technique. On the other side, one may study preparing smaller PEC nanoparticles with different preparation techniques.

Using other polyelectrolytes may improve the entrapment efficiency issue with EL2X-loaded PEC nanoparticles as well.

\subsection{Rheology Tests}

All rheology tests reported in this research are performed under atmospheric pressure. It has been reported that viscosity of borate crosslinked guar is a strong function of pressure and viscosity 
decreases with increasing the pressure. ${ }^{[98]}$ Studying the rheological properties of PEC nanoparticles under typical pressure applied during a fracturing job or after the injection is recommended. This may give some insight about the shear sensitivity of EL2X-loaded nanoparticles mixed with HPG gel while they showed little shear sensitivity when mixed with HPG solution.

\subsection{Protective Effect of Nanoparticles on Enzymes}

Applying the PEC nanoparticles loaded with different enzymes at environments with higher temperature and $\mathrm{pH}$ and studying the effect of $\mathrm{pH}$ at which the nanoparticles were prepared on protective effect of nanoparticles is recommended.

\subsection{Shear Loop Tests}

Using pressure transducers capable of measuring pressure drops higher than 2000 psi and changing the oil-filled tubing connecting the transducers to the shear loop to a tubing tolerant to higher pressures one may increase the injection rate and hence shear rate. This will simulate the high injection rate fracturing jobs applied in the field.

Using higher concentrations of borax for application of EL2X-loaded PEC nanoparticles is recommended to determine if EL2X-loaded PEC nanoparticles are sensitive to shear when a stronger gel is used.

\subsection{Filter Cake Cleanup}

A fluid loss cell was used to run filter cake cleanup tests due to lack of access to such conductivity cell. Use of a conductivity cell to form filter cake either dynamically or statically and breaking the filter cake using enzyme-loaded PEC nanoparticles is recommended. Comparing such results with the cleanup caused by enzymes with no nanoparticles will give more insight about the fracture cleanup performance of PEC nanoparticles. 


\section{Nomenclature and Abbreviations}

$\alpha$ : light absorption coefficient $(1 / \mathrm{cm})$ or conversion factor coefficient

$\gamma:$ shear rate $\left(\mathrm{s}^{-1}\right)$

$\varepsilon:$ dielectric constant

$\zeta$ : zeta potential, $(\mathrm{mV})$ when measured and $(\mathrm{V})$ when used in the equations

$\eta$ : viscosity of suspension (cP)

$[\eta]$ : Intrinsic viscosity (dimensionless)

$\eta_{0}:$ zero shear viscosity of suspension (cP)

$\eta_{\mathrm{r}}:$ relative viscosity $(\mathrm{cP})$

$\eta_{\mathrm{sp}}:$ specific viscosity

$\lambda$ : wavelength $(\mathrm{nm})$

$\mu$ : viscosity of fluid (cP)

$\mu_{\mathrm{a}}$ : apparent viscosity of fluid (cP)

$\mu_{\mathrm{E}}:$ electrophoretic mobility $\left(\mathrm{cm}^{2} / \mathrm{s} / \mathrm{V}\right)$

$\mu_{\mathrm{i}}$ : viscosity of fluid at initial time (cP)

$\mu_{\mathrm{fil}}$ : viscosity of filtrate fluid during fluid loss (cP)

$\mu_{\mathrm{r}}$ : viscosity of reservoir fluid (cP)

$\mu_{\mathrm{t}}$ : viscosity of fluid at a given time (cP)

$\mu_{\mathrm{w}}:$ viscosity of water (cP)

$\mathrm{v}:$ reaction rate $(\mathrm{mol} / \mathrm{g} / \mathrm{s})$

$\rho$ : density of saturating fluid $\left(\mathrm{g} / \mathrm{cm}^{3}\right)$

$\rho_{\mathrm{p}}$ : density of proppants $\left(\mathrm{g} / \mathrm{cm}^{3}\right.$ or $\left.\mathrm{kg} / \mathrm{m}^{3}\right)$

$\rho_{\mathrm{s}}$ : density of fluids $\left(\mathrm{g} / \mathrm{cm}^{3}\right.$ or $\left.\mathrm{kg} / \mathrm{m}^{3}\right)$

$\tau_{0}:$ yield stress $(\mathrm{Pa})$

$\tau_{\mathrm{w}}$ : shear stress at the wall of tubing $(\mathrm{Pa})$

$\phi:$ rock porosity $(\%)$

$\psi$ : electric potential at distance $\mathrm{x}(\mathrm{V})$

$\psi_{0}:$ surface electric potential (V) 
$\omega$ : Mass fraction of solids in the fluid phase

A : molar absorptivity $(\mathrm{L} / \mathrm{mol} / \mathrm{cm})$ or total area of one face of fracture $\left(\mathrm{ft}^{2}\right)$

$A_{E}$ : activity of the equivalent enzyme concentration added to the nanoparticles $(1 / h)$

AmB : Amphotericin B

$A_{S}$ : activity of the supernatants separated from the nanoparticles $(1 / h)$

API : American Petroleum Institute

BPM : barrel per minute

$\mathrm{C}$ : normalized concentration or leakoff coefficient $\left(\mathrm{ft} / \mathrm{min}^{1 / 2}\right)$

$\mathrm{C}_{\mathrm{c}}$ : reservoir fluid loss coefficient $\left(\mathrm{ft} / \mathrm{min}^{1 / 2}\right.$ )

$\mathrm{C}_{\mathrm{E}}$ : concentration of the equivalent enzyme added to the nanoparticles

$\mathrm{C}_{\mathrm{i}}$ : injected normalized concentration of tracer

$\mathrm{C}_{\mathrm{S}}$ : concentration of the supernatants separated from the nanoparticles

$c_{t}$ : total compressibility of reservoir fluid $\left(\mathrm{psi}^{-1}\right)$

$\mathrm{C}_{\mathrm{v}}$ : filtrate zone fluid loss coefficient $\left(\mathrm{ft} / \mathrm{min}^{1 / 2}\right)$

$\mathrm{C}_{\mathrm{vc}}$ : overall reservoir resistance coefficient $\left(\mathrm{ft} / \mathrm{min}^{1 / 2}\right.$ )

$\mathrm{C}_{\mathrm{w}}$ : wall building coefficient $\left(\mathrm{ft} / \mathrm{min}^{1 / 2}\right)$

$\mathrm{C}_{0}$ : initial normalized concentration of tracer in the porous medium

$\mathrm{C}^{*}$ : critical overlap concentration

CMHPG : carboxymethyl-hydroxypropyl guar

CMHEC : carboxymethylhydroxyethylcellulose

$\mathrm{d}:$ proppant diameter $(\mathrm{cm})$

$\mathrm{D}$ : tubing diameter, $\mathrm{cm}$

DC : direct current

DF : dilution factor

DFL : dynamic fluid loss

$\mathrm{D}_{\mathrm{L}}$ : dispersion coefficient in the direction of flow $\left(\mathrm{cm}^{2} / \mathrm{min}\right)$

Delta P: pressure difference across the cores while measuring their permeability (psi)

DS : dextran sulfate

E : enzyme

[E] : enzyme concentration

EB : encapsulated breaker 
EDL : electric double layer $(\mathrm{cm})$

$\mathrm{EE}_{\mathrm{A}}$ : entrapment efficiency based on enzyme activity (\%)

$\mathrm{EE}_{\mathrm{C}}$ : entrapment efficiency based on enzyme concentration (\%)

FSHS : fracture shear history simulator

$\mathrm{G}^{\prime}$ : elastic modulus $(\mathrm{Pa})$

$\mathrm{G}^{\prime \prime}$ : viscous modulus $(\mathrm{Pa})$

$\mathrm{g}:$ acceleration due to gravity $\left(\mathrm{m} / \mathrm{s}^{2}\right)$

$\mathrm{h}$ : fracture thickness ( $\mathrm{ft}$ )

HPAM : hydrolyzed polyacrylamide

HSFL : high shear fluid loss

$\mathrm{k}$ : reciprocal EDL or permeability $(\mathrm{mD})$ depend on the application

$\mathrm{k}_{\text {cake }}$ : filter cake permeability $(\mathrm{mD})$

$\mathrm{k}_{\mathrm{f}}$ : fracture permeability $(\mathrm{mD})$

$\mathrm{k}_{\text {fil }}$ : permeability of rock in filtrate invaded zone $(\mathrm{mD})$

$\mathrm{k}_{\mathrm{r}}$ : reservoir permeability $(\mathrm{mD})$

$\mathrm{k}_{2}$ : reaction rate constant, for order zero reaction $(\mathrm{mol} / \mathrm{cm} / \mathrm{s})$

$\mathrm{K}^{\prime}$ : consistency index non-Newtonian fluid $\left(\mathrm{kPa} \bullet \mathrm{sn}^{\prime}\right)$

$1:$ light absorption distance $(\mathrm{cm})$

$\mathrm{L}$ : core or tubing length $(\mathrm{cm})$

$\mathrm{L}_{\mathrm{f}}$ : fracture length $(\mathrm{ft})$

LSFL : low shear fluid loss

$\mathrm{m}$ : slope of spurt volume versus $\mathrm{t}^{1 / 2}$ plot

$\mathrm{M}$ : mass over area of the filter cake $\left(\mathrm{g} / \mathrm{ft}^{2}\right)$ or mole

$\mathrm{M}_{\mathrm{i}}$ : molecular weight

$\mathrm{M}_{\mathrm{n}}$ : number average molecular weight

$\mathrm{M}_{\mathrm{w}}$ : weight average molecular weight

$\mathrm{M}_{\mathrm{v}}$ : viscosity-average molecular weight

MWCO : molecular-weight cutoff

$\mathrm{n}^{\prime}$ : behavior index of a non-Newtonian fluid

$\mathrm{N}_{\mathrm{i}}$ : number of molecules

NMR : nuclear magnetic resonance 
PDI : polydispersity index

PEC : polyelectrolyte complex

PECNP : polyelectrolyte complex nanoparticle

PEI : polyethylenimine

pptg : pounds per thousand gallons

$\Delta \mathrm{P}_{\mathrm{f}}$ : pressure drop as a result of friction (psi)

$\Delta \mathrm{P}:$ pressure drop across the fracture (psi)

$\Delta \mathrm{p}_{\text {cake }}$ : pressure drop across the filter cake (psi)

$\Delta \mathrm{p}_{\mathrm{v}}$ : pressure drop across the filtrate zone (psi)

$\Delta \mathrm{p}_{\mathrm{c}}$ : pressure drop across the reservoir perpendicular to fracture surface (psi)

$\mathrm{PV}$ : pore volume $\left(\mathrm{cm}^{3}\right)$

$\mathrm{Q}$ : flow rate in a pipe $(\mathrm{L} / \mathrm{s})$

$\mathrm{Q}_{\mathrm{i}}$ : injection rate $\left(\mathrm{ft}^{3} / \mathrm{min}\right) \mathrm{x}$ : distance from a charged surface or along a core

$\mathrm{q}:$ Injection flow rate $\left(\mathrm{cm}^{3} / \mathrm{min}\right)$

$\mathrm{r}$ : tubing radius $(\mathrm{cm})$

$\mathrm{r}_{1}$ : radius of inside tubing in an annular flow $(\mathrm{cm})$

$\mathrm{r}_{2}$ : radius of outside tubing in an annular flow $(\mathrm{cm})$

$R_{p}$ : retention coefficient defined for the filtrate over the specified rock type

R.O. : reverse osmosis

S : substrate

$\mathrm{S}_{\mathrm{p}}:$ spurt volume $\left(\mathrm{ft}^{3}\right)$

SDS-PAGE : sodium dodecyl sulfate- polyeachrylamide gel electrophoresis

$\mathrm{t}:$ injection time (minutes)

$\mathrm{T}$ : temperature $\left({ }^{\circ} \mathrm{C}\right)$ or light transmissivity

$\mathrm{T}_{\min }$ : minimum thickness of cores used for fluid loss tests (inch)

$\mathrm{t}$ : time (minutes or hours)

$t_{1 / 2}$ : time required a polymeric solution to reach $50 \%$ of its initial viscosity (h)

$\mathrm{t}_{\mathrm{Sp}}$ : spurt volume time ( $\left.\mathrm{min}\right)$

TOC : total organic carbon

TON : total organic nitrogen

TORP : Tertiary Oil Recovery Project 
TSHS : tubing shear history simulator

$\mathrm{u}_{\mathrm{L}}$ : leakoff velocity $(\mathrm{ft} / \mathrm{min})$

$\mathrm{u}_{\mathrm{x}}$ : Darcy velocity of fluid ( $\left.\mathrm{cm} / \mathrm{min}\right)$

$\mathrm{V}_{\mathrm{L}}$ : leakoff volume $\left(\mathrm{ft}^{3}\right)$

$\mathrm{v}_{\mathrm{S}}$ : Proppant settling velocity $(\mathrm{m} / \mathrm{s})$

$\mathrm{W}$ : fracture width ( $\mathrm{ft})$

$\mathrm{w} / \mathrm{w}:$ weight ratio

$\mathrm{W}_{\mathrm{D}}$ : dry weight of core $(\mathrm{g})$

$\mathrm{W}_{\mathrm{S}}$ : saturated weight of core $(\mathrm{g})$

$\mathrm{x}_{\mathrm{D}}$ : dimensionless distance along a core

ZetaPALS : zeta potential analysis using phase analysis light scattering 


\section{References}

1. Economides, M. J.; Nolte, K. G., Reservoir Stimulation. 3 ed.; John Wiley \& Sons, Inc.: 2000 .

2. Palisch, T. T.; Vincent, M. C.; Handren, P. J. Slickwater Fracturing- Food for Thought, SPE/ATCE Denver, Colorado, September 21-24, 2008; Denver, Colorado, 2008.

3. Ayoub, J. A.; Hutchins, R. E.; Van der Bas, F.; Cobianco, S.; Emiliani, C. N.; Glover, M.; Kohler, M.; Marino, S.; Nitters, G.; Norman, D.; Turk, G., New Results Improve Fracture Cleanup Characterization and Damage Mitigation. SPE Production \& Operations 2006, 24, (3), 374-380.

4. Ayoub, J. A.; Hutchins, R. E.; Van der Bas, F.; Cobianco, S.; Emiliani, C. N.; Glover, M.; Kohler, M.; Marino, S.; Nitters, G.; Norman, D.; Turk, G. New Findings in Fracture Cleanup Change Industry Perceptions, SPE 98746, 2008; 2008.

5. Samuel, M.; Mohsen, A. H.; Ejan, A. B.; Ooi, Y. S.; Ashraf, S.; Nasr-El-Din, H. A. Novel Enzyme Stabilizers for Applications at High Temperatures, SPE Annual Technical Conference and Exhibition, New Orleans, Lousiana, 4-7 October, 2009; New Orleans, Lousiana, 2009. 6. Ayoub, J. A.; Hutchins, R. E.; Van der Bas, F.; Cobianco, S.; Emiliani, C. N.; Glover, M.; Kohler, M.; Marino, S.; Nitters, G.; Norman, D.; Turk, G. New Findings in Fracture Cleanup Change Industry Perceptions, International Symposium and Exhibition on Formation Damage Control, Lafayette, Louisiana U.S.A., 15-17 February 2006; Lafayette, Louisiana U.S.A., 2006.

7. Barati, R.; Hutchins, R. E.; Friedel, T.; Ayoub, J. A.; Dessinges, M.; England, K. W., Impact of Yield Stress and Fracture Face Damage on Production Using a Three-Phase, 2D Model. SPE Production and Operations 2009, 24, (2), 336-345.

8. Palisch, T.; Duenckel, R.; Bazan, L.; Heidt, H. J.; Turk, G. Determining Realistic Fracture Conductivity and Understanding Its Impact on Well Performance - Theory and Field Examples, Hydraulic Fracturing Technology Conference, College Station, TX, 28-31 January 2007.

9. Armstrong, C. D.; Stevens, R. F.; Le, H.; Stephenson, C.; Qu, Q. The Next Generation of Regenerative Catalytic Breakers for Use in Alkaline and High-Temperature Fracturing Fluids, SPE International Symposium and Exhibition on Formation Damage Control, Lafayette LA, 1012 Feb., 2010. 
10. Tiyaboonchai, W. Development of a New Nanoparticle Delivery Vehicle Based on an Aqueous Polymer System: Polyethylenimine and Dextran Sulfate. The University of Kansas, Lawrence, 2003.

11. Waree Tiyaboonchai, J., Robert C. Sims, and C. Russel Middaugh, Insulin Containing Polyethylenimine-Dextran Sulfate Nanoparticles. International Journal of Pharmaceutics 2003, 225, 139-151.

12. Waree Tiyaboonchai, J. W., and C. Russel Middaugh, Formulation and Characterization of DNA-Polyethylenimine-Dextran Sulfate Nano-Particles. European Journal of Pharmaceutical Sciences 2003, 19, 191-202.

13. Berkland, C.; Cordova, M.; Liang, J.-T.; Willhite, G. P. Polyelectrolyte Complexes for Oil and Gas Applications. WO 2008/030758 A1, 13 March 2008.

14. Cordova, M.; Cheng, M.; Trejo, J.; Johnson, S. J.; Willhite, G. P.; Liang, J.-T.; Berkland, C., Delayed Hpam Gelation Via Transient Sequestration of Chromium in Polyelectrolyte Complex Nanoparticles. Macromolecules 2008, 41, (12), 4398-4404.

15. Seright, R. S. Conformance Improvements Using Gels; New Mexico Petroleum Recovery Research Cent: 2002.

16. May, E. A.; Britt, L. K.; Nolte, K. G. The Effect of Yield Stress on Fracture Fluid Cleanup, SPE Annual Technical Conference and Exhibition, San Antonio, Texas, 5-8 October 1997.

17. Friedel, T. Numerical Simulation of Production from Tight-Gas Reservoirs by Advanced Stimulation Technologies. Freiberg University 2004.

18. Schein, G. The Application and Technology of Slickwater Fracturing, 2005; SPE

Distinguished Lecture, 2005.

19. Palisch T.T., Vincent, M. C. and Handren, P. G. Slickwater Fracturing, Food for Thought, SPE ATCE Denver, Colorado, 21-24 September 2008; SPE.

20. Robert, M.; Tjon-Joe-Pin Enzyme Breaker for Galactomannan Based Fracturing Fluid. US Patent 5201370, April 13, 1993.

21. Weaver, J.; Schmelzl, E.; Jamieson, M.; Schiffner, G. In New Fluid Technology Allows Fracturing without Internal Breakers, SPE Gas Technology Symposium, Calgary, Alberta, Canada, Apri 30- May 2, 2002. 
22. Brannon, H. D.; Pulsinelli, R. J., Breaker Concentrations Required to Improve the Permeability of Proppant Packs Damaged by Concentrated Linear and Borate-Crosslinked Fracturing Fluids. SPE Production Engineering 1992, 7, (4), 338-342.

23. Barati, R.; Johnson, S. J.; McCool, C. S.; Green, D. W.; Willhite, G. P.; Liang, J.-T., Fracturing Fluid Cleanup by Controlled Release of Enzymes from Polyelectrolyte Complex Nanoparticles. Applied Polymer Science Peer-reviewed: 2010, In Press.

24. Rae, P. and Lullo, G. d.In Fracturing Fluids and Breaker Systems - a Review of the State-of-the-Art, SPE Eastern Regional Meeting, Columbus, Ohio, 23-25 October 1996.

25. Harris, P. C., Chemistry and Rheology of Borate-Crosslinked Fluids at Temperatures to 300 F. JPT 1993, 45, (3), 264-269.

26. Harris, P. C.; Heath, S. J., Rheological Properties of Low-Gel-Loading Borate Fracture Gels. SPE Production \& Facilities 1998, 13, (4), 230-235.

27. Rae, P.; Lullo, G. D. In Fracturing Fluids and Breaker Systems - a Review of the Stateof-the-Art, SPE Eastern Regional Meeting, Columbus, Ohio, 23-25 October 1996.

28. Horton, R.H.; Moran, L. A.; Ochs, R. S.; Rawn, J. D. and K. G. Scrimgeour, Principles of Biochemistry. 2 Ed; Prentice Hall: 1996.

29. Brannon, H. D.; Tjon-Joe-Pin, R. M. In Biotechnological Breakthrough Improve Performance of Moderate to High-Temperature Fracturing Applications, SPE 69th Annual Technical Conference and Exhibition New Orleans, LA, 25-28 September 1994.

30. Glenn S. Penny, and Conway, M.W., Fluid Leakoff. In SPE Monograph Volume 12 : Recent Advances in Hydraulic Fracturing, Ed. 1987.

31. Hutchins, R. E., Stimulation Fluids Adviser at Schlumberger. Sugarland, 2008.

32. Cheng, Y.; Prud'homme, R. K., Enzymatic Degradation of Guar and Substituted Guar. Biomacromolecules 2000, 1, (4), 782-788.

33. Elbel, J. L.; Thomas, R. L. In The Use of Viscosity Stabilizers in High Temperature Fracturing, SPE Rocky Mountain Regional Meeting, Casper Wyoming, May 14-16, 1980.

34. Walker, M. L.; Shuchart, C. E.; Yaritz, J. G.; Norman, L. R. In Effects of Oxygen on Fracturing Fluids, SPE International Symposium on Oilfield Chemistry, San Antonio, Feb. 14$17,1995$.

35. University of Oklahoma State, Physics Courses. 2006. 
36. Joachim Koetz and Kosmella, Sobine, Polyelectrolytes and Nanoparticles. Springet Laboratory: 2007.

37. Schramm, L. L., Suspensions, Fundamentals and Applications in the Petroleum Industry. The American Chemical Society: 1996.

38. Hartig, S. H.; Greene, R. R.; Dikov, M. M.; Prokop, A.; Davidson, M., Multifunctional Nanoparticulate Polyelectrolyte Complexes. Pharmaceutical Research 2007, 24, (12), 23532369.

39. Marbet, Y. http://en.wikipedia.org/wiki/File:KinEnzymo(en).svg

40. Bruno G. De Geest. Nick N. Sanders, G. B. S., Joseph Demeester and Stefaan C. De Smedt, Release Mechanisms for Polyelectrolye Capsules. Chemical Society Reviews 2007, 36, 636-649.

41. Webster, L.; Huglin, M.B. and Robb, I. D., Complex Formation between Polyelectrolytes in Dilute Aqueous Solution. Polymer 1997, 38, (6), 1373-1380.

42. Moghimi, S. M., Nanotoxicology of Synthetic Gene Transfer Vectors : Polyethylenimine and Polyfectin-Mediated Membrane Damage and Apoptosis in Human Cell Lines. In Nanomaterials for Medical Diagnosis and Therapy, Kumar, C., Ed. Wiley-VCH: 2006.

43. Knight, W., Lecture Notes : "Fundamentals of Biochemistry". University of Montana: 2005.

44. Bell, T. A.; Etchells, J. L. A Method for Testing Cucumber Salt-Stock Brine for Softening Activity; Department of Horticulture: 1955.

45. Roberts, D. V., Enzyme Kinetics. Cambridge University Press: 1977.

46. Walas, S. M., Reaction Kinetics for Chemical Engineers. Butterworths: 1989.

47. Cheng, Y.; Brown, K. M. and R.K. Prudhomme, Characterization and Intermolecular Interactions of Hydroxypropyl Guar Solutions. Biomacromolecules 2002, 3, 456-461.

48. Reddy, T.T. and Tamishetti, S., Free Radical Degradation of Guar Gum. Polymer Degradation and Stability 2004, (86), 455-459.

49. Mutlu, M.; Sariglu, k.; Demir, N.; Ercan, M.; Acar, J., The Use of Commercial Pectinase in Fruit Juice Industry. Parti: Viscometric Determination of Enzyme Activity. Journal of Food Engineering 1999, 41, 147-150.

50. SIGMA Enzymatic Assay of Pectinase; 1997.

51. SIGMA Enzymatic Assay of Pectin Lyase 1997. 
52. Instruments, T. Apollo 9000tm with Total Nitrogen 2008.

53. Anonymous, Spectroscopy Lecture Notes. North Eastern University.

54. Terrill, R. H., Chemistry 155 Lab Notes San Jose State University: 2009.

55. Akash Tayal and S.A. Khan, Degradation of Water-Soluble Polymers: Molecular Weight Changes and Chains Scission Characteristics. Macromolecules 1999, 3, 9488-9493

56. Akash Tayal, Kelly, R. M. and Khan, S. A., Rheology and Molecular Weight Changes During Enzymatic Degradation of Water-Soluble Polymer. Macromolecules 1999, 32, 294-300.

57. Akash Tayal, Pai, V. B. and Khan, S. A., Rheology and Microstructural Changes During Enzymatic Degradation of a Guar-Borax Hydrogel. Macromolecules 1999, 32, 5567-5574

58. Akash Tayal, Kelly, R. M. and Khan, S. A.,Viscosity Reduction of Hydraulic Fracturing Fluids through Enzymatic Hydrolysis. SPE Journal 1997, 2.

59. Navarette, R.C.; Cawiezel, K. E. and V.G. Constien, Dynamic Fluid Loss in Hydraulic Fracturing under Realistic Shear Conditions in High-Permeability Rocks. SPE Production and Facilities 1996.

60. Craigie, L. J. In A New Method for Determining the Rheology of Crosslinked Fracturing Fluids Using Shear History Simulation, SPE/DOE Low Permeability Gas Reservoirs Symposium, Denver, Colorado, 14-16 March, 1983.

61. Vitthal, S. and McGowen, J. M. In Fracturing Fluid Leakoff under Dynamic Conditions, Part 2: Effect of Shear Rate, Permeability, and Pressure, SPE Annual TEchnical Conference, Denver, U.S.A, 6-9 Oct, 1996.

62. McGowen, J.M. and S. Vitthal In Fracturing-Fluid Leakoff under Dynamic Conditions, Part1: Development of a Realistic Laboratory Testing Procedure, SPE ATC, Denver, U.S.A, 6-9 Oct., 1996.

63. Worlow, D.W. and Holditch, S. A. In Rheological Measurements of a Crosslinked Fracture Fluid under Conditions Expected During a Fracture Treatment, SPE Joint Rocky Mountain Regional/Low Permeability Reservoirs Symposium and Exhibition, Denver, CO, March 6-8, 1989.

64. Howard, G. C. and Fast, C. R. Optimum Fluid Characteristics for Fracturing Extension. Drilling and Production Practice 1957, 261-270

65. Williams, B. B., Fluid Loss from Hydraulically Induced Fractures. Journal of Petroleum Technology 1970, 22, (7), 882-888 
66. Settari, A., A New General Model of Fluid Loss in Hydraulic Fracturing. Society of Petroleum Engineers Journal 1985, 25, (4), 491-504

67. Collins, R. E., Flow of Fluids through Porous Materials. Van Nostrand Reinhold New York, 1961.

68. Institute, A. P. Recommended Practice Standard Procedure for Evaluation of Fracturing Fluids; 1983.

69. Asadi, M.; Penny, G. S.; Ainley, B.R.; Archacki, D.J. ; Bas van der, F.; Bern, P.A.; Brannon, H.D.; Cobianco, S.; Ghalambor, A.; McElfresh, P.M.; Milton-Tayler, D.; Parker, M.A.and S. Shah In A New Technical Standard Procedure to Measure Stimulation and GravelPack Fluid Leakoff under Static Conditions, European Formation Damage Conference Scheveningen, Netherlands, 30 May-1 June 2007.

70. Roodhart, L. P., Fracturing Fluids: Fluid-Loss Measurement under Dynamic Conditions. Society of Petroleum Engineers Journal 1985, 25, (5), 629-648

71. Pud'homme, R. K.; Wang, J. K. In Filter-Cake Formation of Fracturing Fluids, SPE International Symposium of Oilfield Chemistry Neworleans,LA, USA, March 2-5, 1993; Neworleans,LA, USA, 1993.

72. Penny, G. S.; Conway, M. W.; Lee, W. S., Control and Modeling of Fluid Leak-Off During Hydraulic Fracturing. JPT 1985, 37, (6), 1071-81.

73. Anderson, R. W.; Cooke Jr., C. E.; Wendroff, C. L., Propping Agents and Fracture Conductivity. In Spe Monograph Volume 12 : “Recent Advances in Hydraulic Fracturing” SPE: 1987.

74. Anderson, R. W.; Cooke, Jr., C.E. and Wendroff, C. L., Propping Agents and Fracture Conductivity. In Spe Monograph Volume 12 : “Recent Advances in Hydraulic Fracturing” SPE: 1987.

75. Cooke, C. E. J., Conductivity of Fracture Proppants in Multiple Layers. JPT 1973, 25, (9), 1101-07

76. Cooke, C. E. J., Effect of Fracturing Fluids on Fracture Conductivity. JPT 1975, 27, (10), $1273-82$

77. API RP-61, API Recommended Practices for Evaluating Short Term Proppant Pack Conductivity; Washington D.C, Oct. 1, 1989. 
78. Penny, G. S. In An Evaluation of the Effects of Environmental Conditions and Fracturing Fluids Upon the Long-Term Conductivity of Proppants, 62nd SPE ATCE Dallas, TX, Sep. 2730, 1987.

79. Much, M. G.; Penny, G. S. In Long-Term Performance of Proppants under Simulated Reservoir Conditions, SPE/DOE Low Permeablity Reservoir Symposium Denver, Colorado, May 18-19 1987.

80. Marpaung, F.; Chen, F.; Pongthunya, P.; Zhu, D.; Hill, A. D. In Measurement of Gel Cleanup in a Propped Fracture with Dynamic Fracture Conductivity Experiments, SPE ATCE, Denver, Colorado, USA, 2008; Denver, Colorado, USA, 2008; pp 21-24 September.

81. Chen, F.; Pongthunya, P.; Zhu, D.; Limthongchai, P.; Qgueri, O.; Wang, Y.; Holditch, S. A.; Hill, A. D.; Zhu, D. Advanced Hydraulic Fracturing Technology for Unconventional Tight Gas Reservoirs; 2007.

82. Zou, C. Development and Testing of and Advanced Acid Fracture Conductivity Apparatus. Texas A\&M University, 2006.

83. Al-Fariss, T.; King, S. U.; Pinder, K. L. Flow of Shear-Thinning Liquid with Yield Stress through Porous Media.

84. Hawkins, G. W. In Laboratory Study of Proppant-Pack Permeability Reduction Caused by Fracturing Fluids Concentrated During Closure 63rd SPE ATCE Houston, TX, Oct. 2-5, 1988.

85. SIGMA-ALDRICH, Msds for Pectinase from Aspergillus Aculeatus. 2010.

86. Economy Polymers and Chemicals Product Data Sheet for Econo Gelbreak-El2x; 2000.

87. Rhodia Product Data Sheet for Hydroxypropyl Guar Jaguar ${ }^{\circledR} 415$ Houston, TX, 2008.

88. Invitrogen, Nupage 4-12\% Bis-Tris Gel Quick Reference Card. In.

89. Invitrogen, Nupage Technical Guide. 2003.

90. PIERCE, I. b. Coomassie Plus- the Better Bradfordtm Assay Kit.

91. McCool, C. S.; Li, X.; Willhite, G. P., Flow of a Polyacrylamide/Chromium Acetate System in a Long Conduit. SPE Journal 2009, 14, (1), 54-66.

92. Green, D. W.; Willhite, G. P., Enhanced Oil Recovery. SPE Textbook: 1998.

93. Peters, E. J., Advanced Petrophysics Lecture Notes. In 2001.

94. Hutchins, R. E., Notes on the Schlumberger Static Fluid Loss Apparatus In 2010.

95. TELEDYNE TEKMAR, Apollo 9000 Total Organic Carbon (Toc) Analyzer ${ }^{\mathrm{TM}}$. In. 
96. Tayal, A.; Kelly, R. M.; Khan, S. A., Rheology and Molecular Weight Changes During Enzymatic Degradation of Water-Soluble Polymer. Macromolecules 1999, 32, (2), 294-300.

97. Cao, Y.; Tan, H., The Effect of Shear Field on the Hydrolysis of Cellulose. Journal of Macromolecular Science 2004, B43, (6), 1115-1121.

98. Parris, M. D.; MacKay, B. A.; Rathke, J. W.; Klingler, R. J.; Gerald, R. E., Influence of Pressure on Boron Cross-Linked Polymer Gels. Macromolecules 2008, 41, (21), 8181-8186. 


\section{Appendix A}

In this section permeability and tracer test measurements done for cores used in fluid loss tests, permeability measurement for cores after resaturation and permeability measurement for cores after being restored using bleach are shown.

\section{A-1 Permeability Measurements and Tracer Tests for Cores Used in}

\section{Fluid Loss Tests}

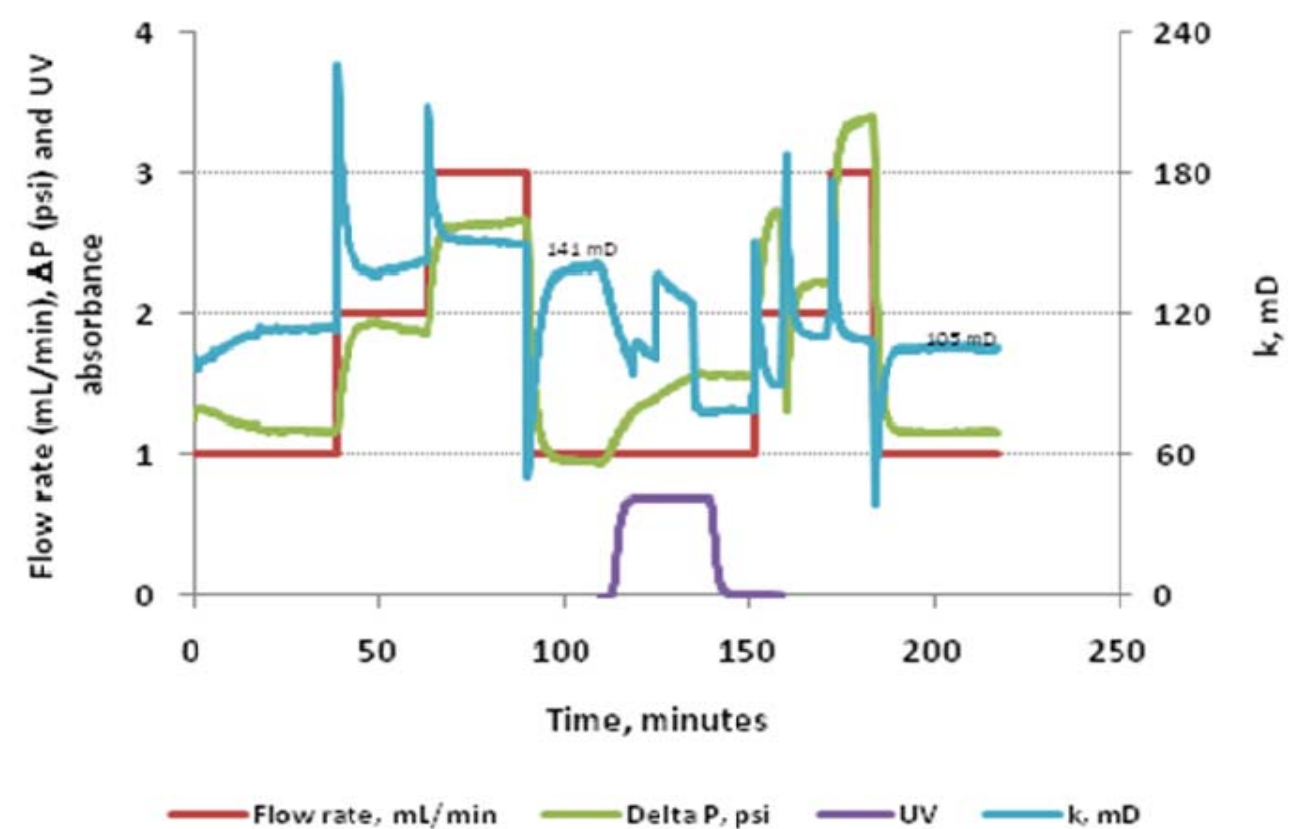

Figure 140 Flow rate, pressure drop, UV absorbance and permeability of core\#1 before fluid loss tests at 25 ${ }^{\circ} \mathrm{C}$ including tracer test results. 


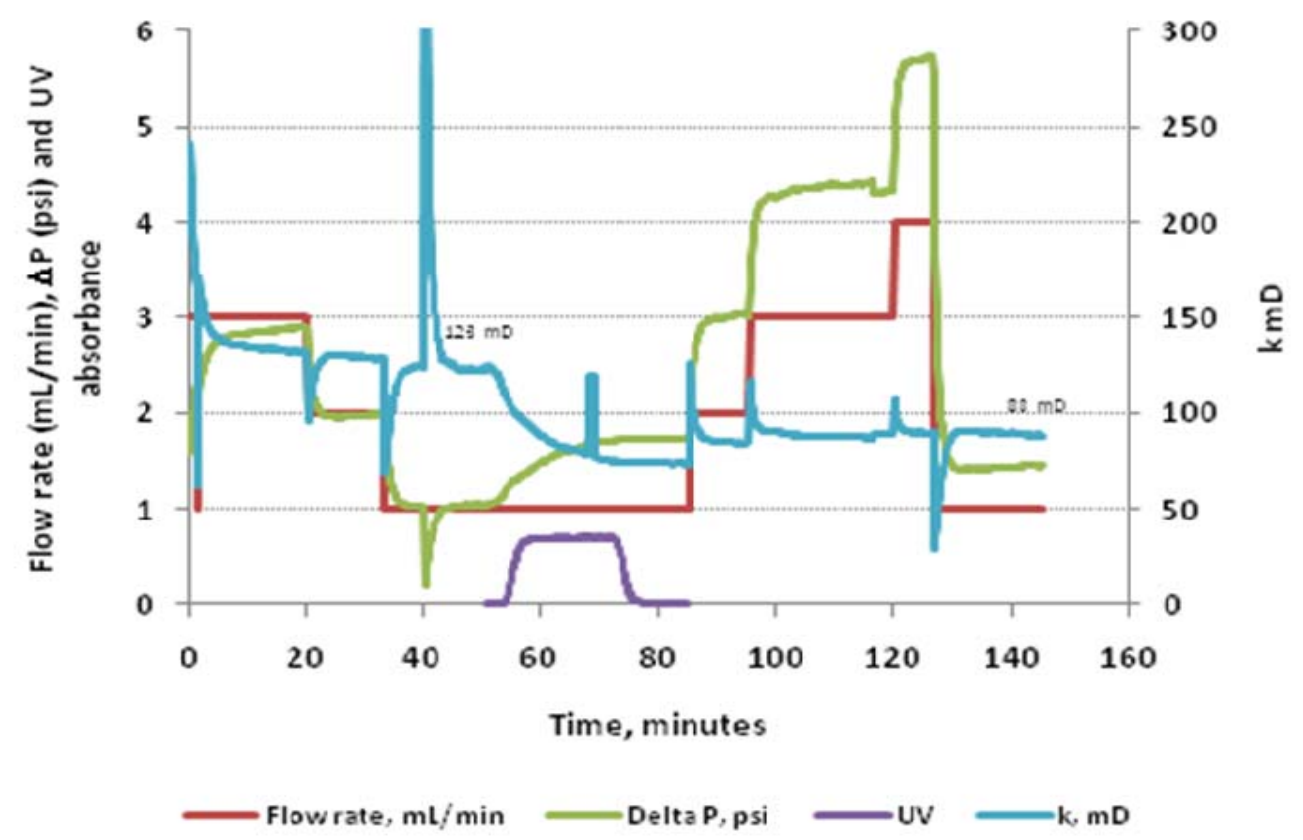

Figure 141 Flow rate, pressure drop, UV absorbance and permeability of core \#3 before fluid loss tests at 25 ${ }^{\circ} \mathrm{C}$ including tracer test results.

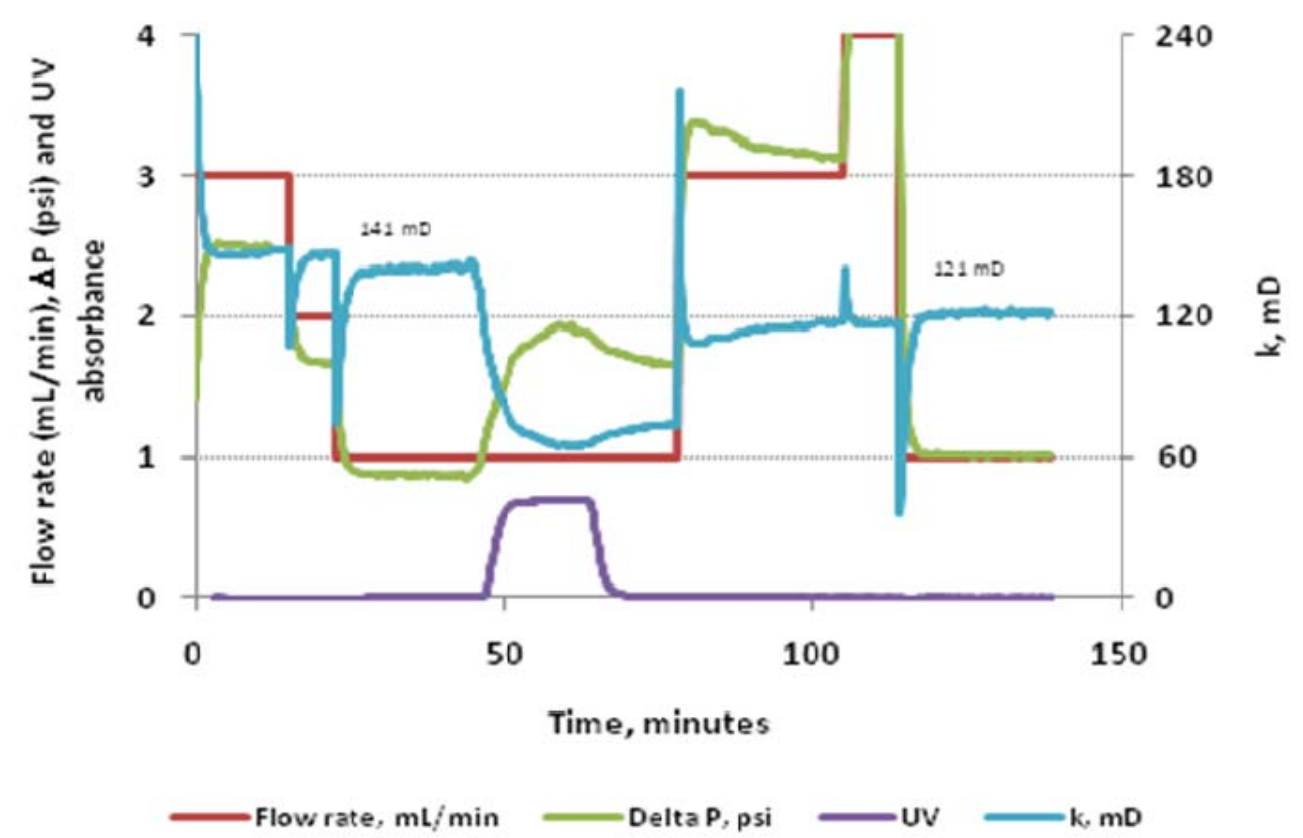

Figure 142 Flow rate, pressure drop, UV absorbance and permeability of core\#4 before fluid loss tests at 25 ${ }^{\circ} \mathrm{C}$ including tracer test results. 


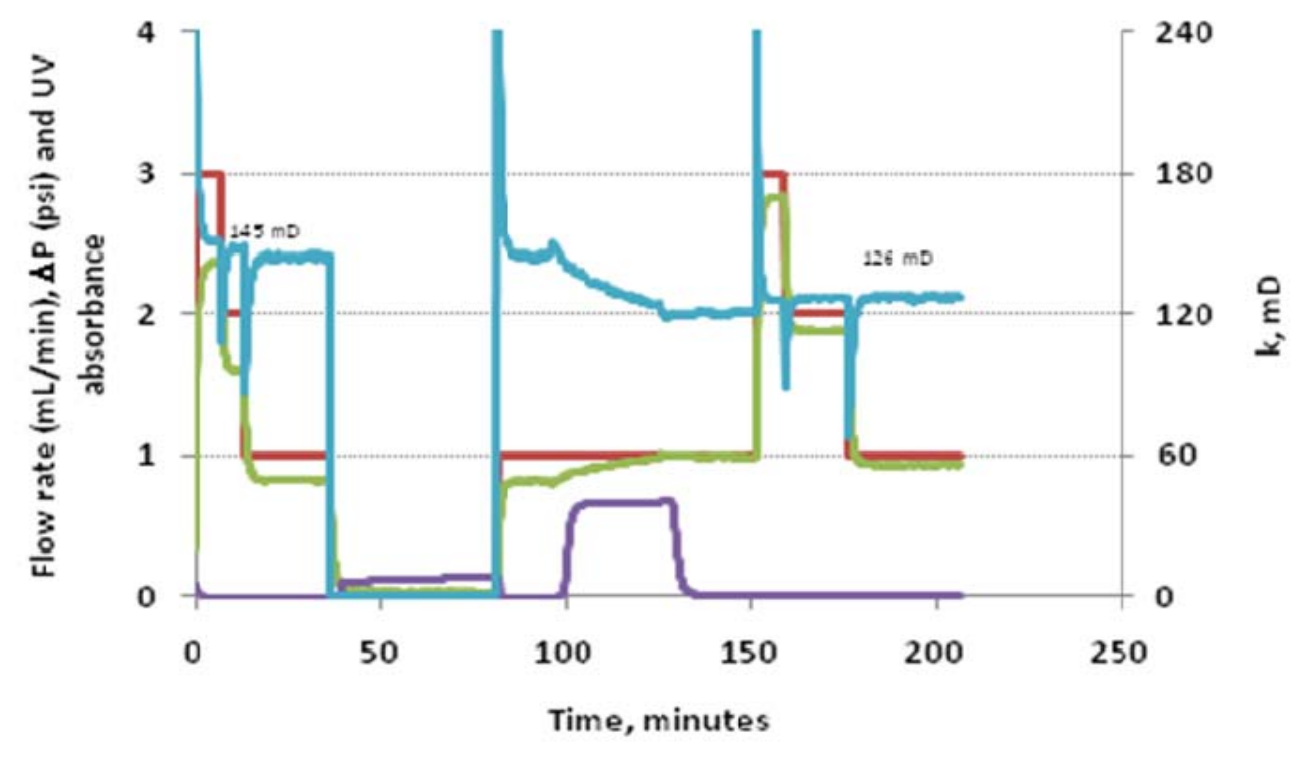

Flow rate, $\mathrm{mL} / \mathrm{min}$ Delta $\mathrm{P}$, psi $\longrightarrow \mathrm{mV}$

Figure 143 Flow rate, pressure drop, UV absorbance and permeability of core\#5 before fluid loss tests at 25 ${ }^{\circ} \mathrm{C}$ including tracer test results.

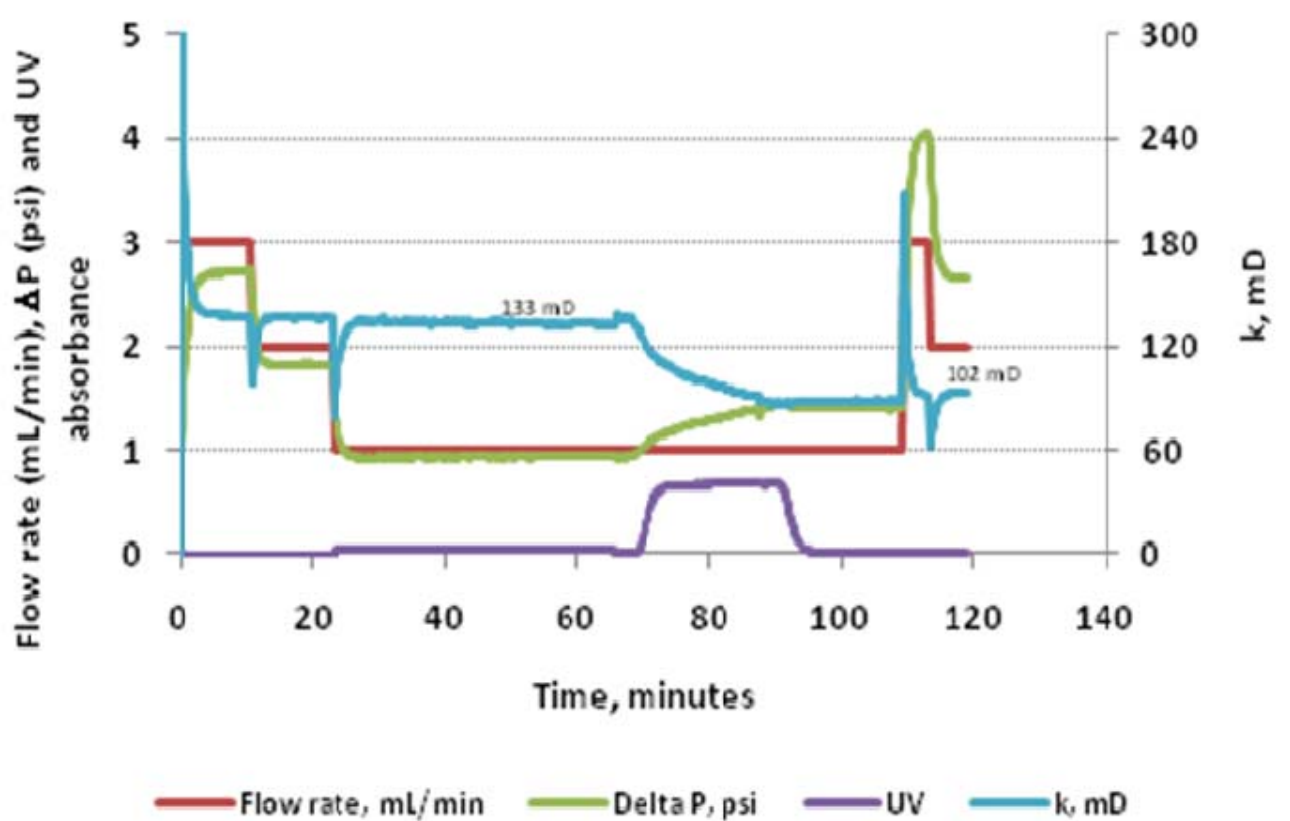

Figure 144 Flow rate, pressure drop, UV absorbance and permeability of core\#6 before fluid loss tests at 25 ${ }^{\circ} \mathrm{C}$ including tracer test results. 


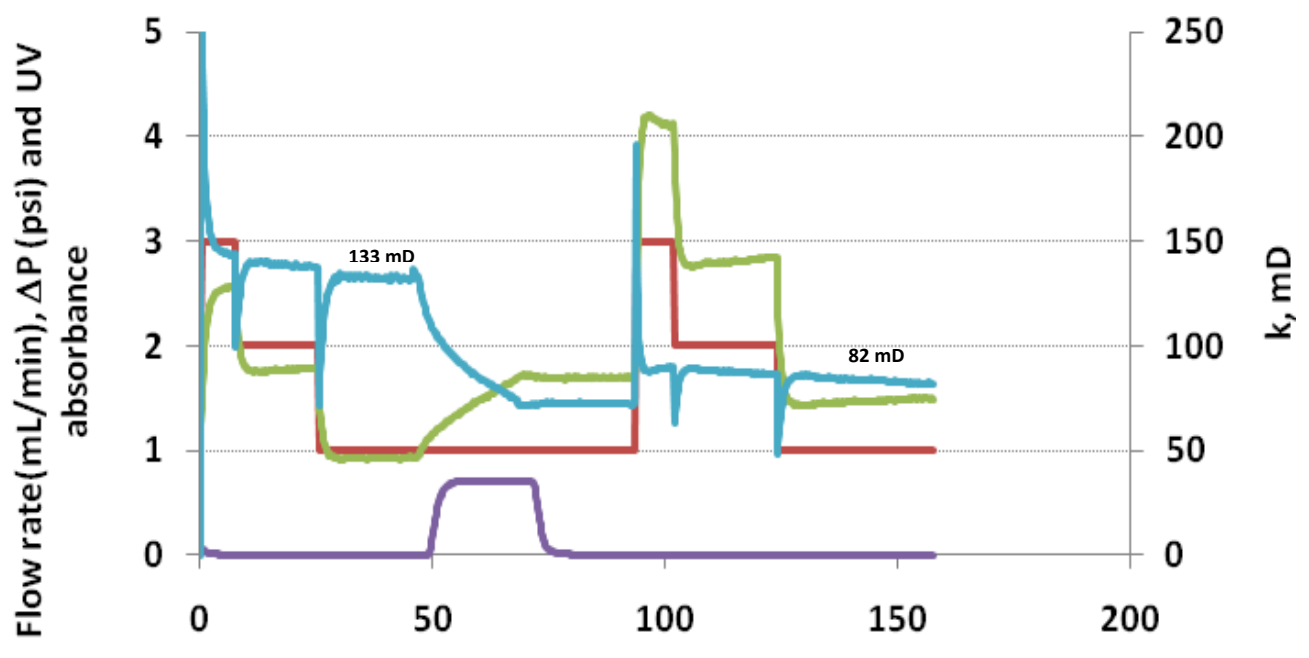

Time, minutes

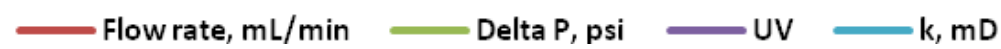

Figure 145 Flow rate, pressure drop, UV absorbance and permeability of core $\# 8$ before fluid loss tests at 25 ${ }^{\circ} \mathrm{C}$ including tracer test results.

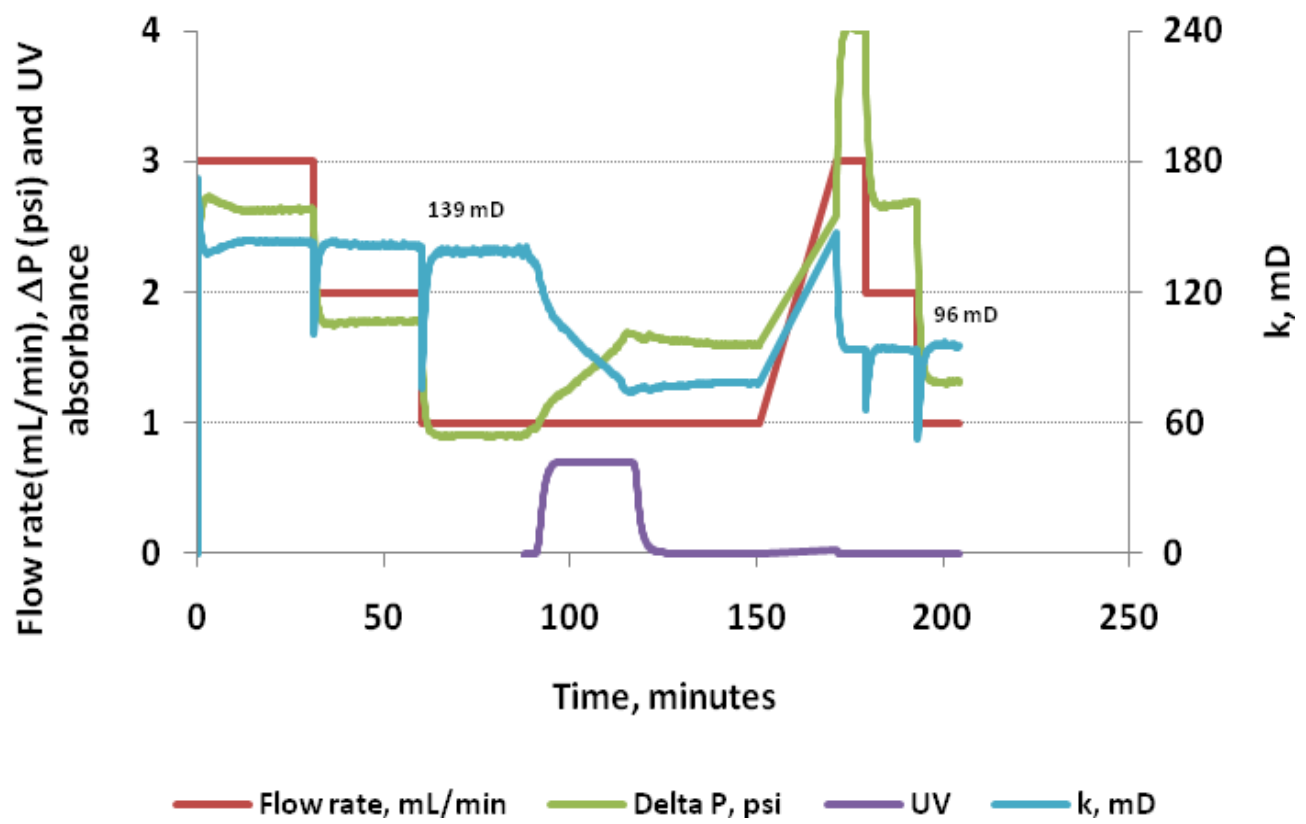

Figure 146 Flow rate, pressure drop, UV absorbance and permeability of core\#9 before fluid loss tests at 25 ${ }^{\circ} \mathrm{C}$ including tracer test results. 


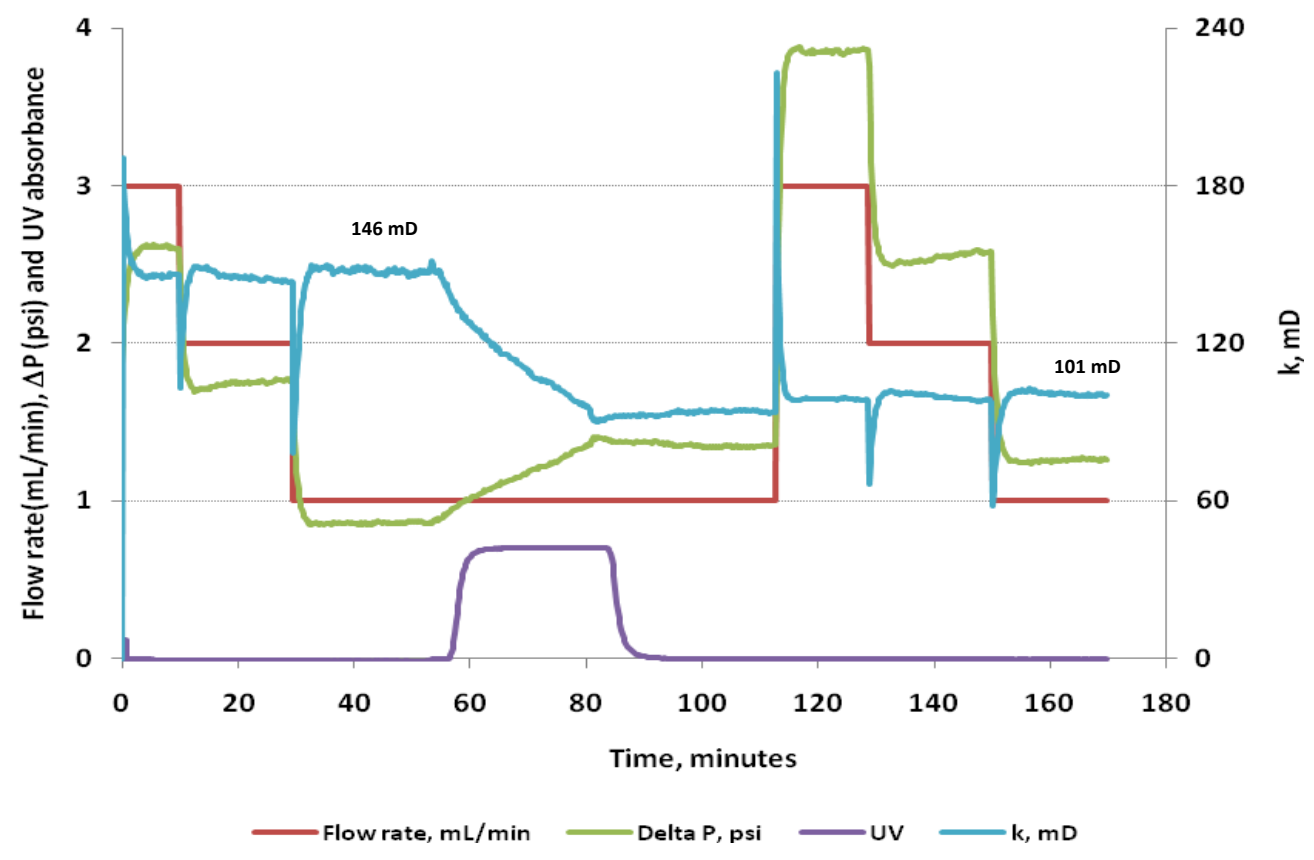

Figure 147 Flow rate, pressure drop, UV absorbance and permeability of core\#10 before fluid loss tests at 25 ${ }^{\circ} \mathrm{C}$ including tracer test results.

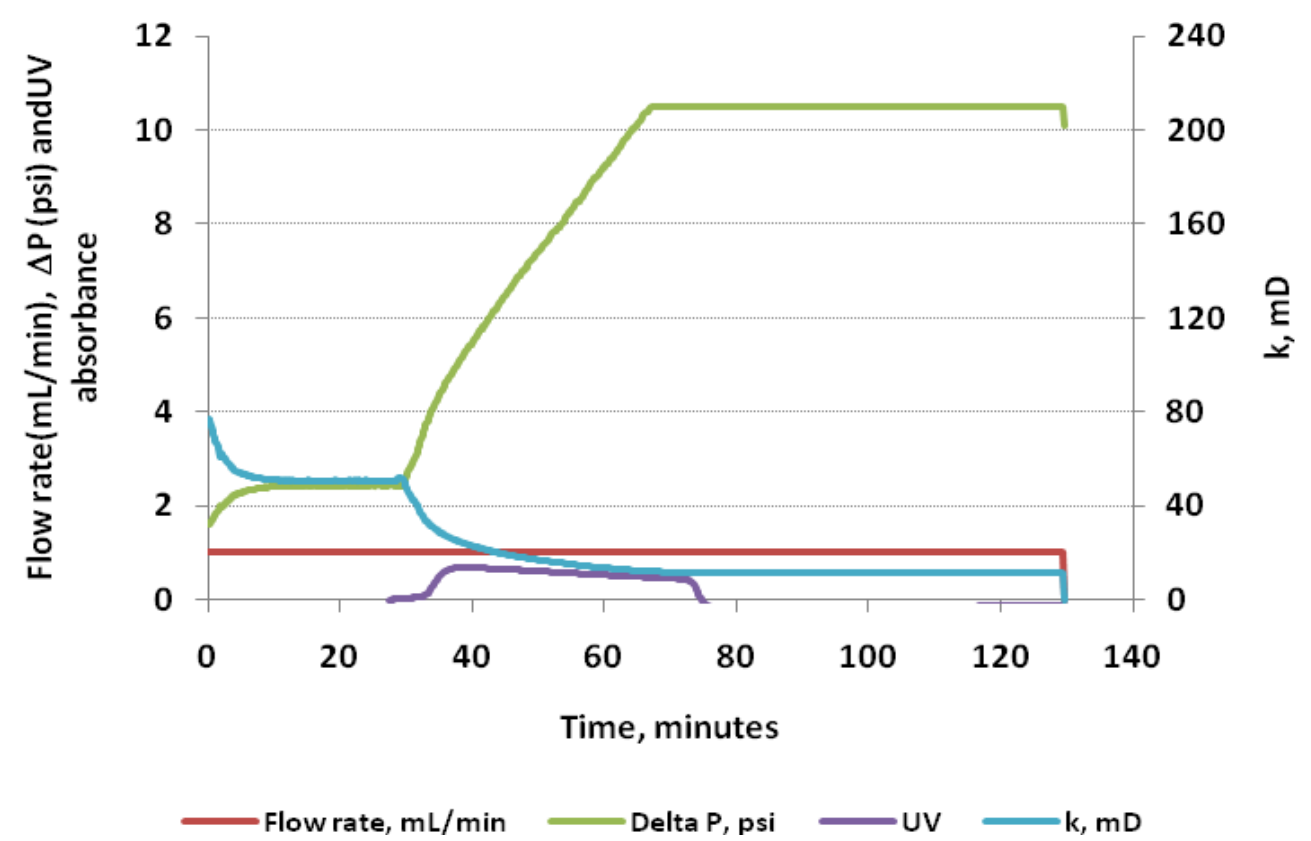

Figure 148 Flow rate, pressure drop, UV absorbance and permeability of core\#13 before fluid loss tests at 40 ${ }^{\circ} \mathrm{C}$ including tracer test results. 


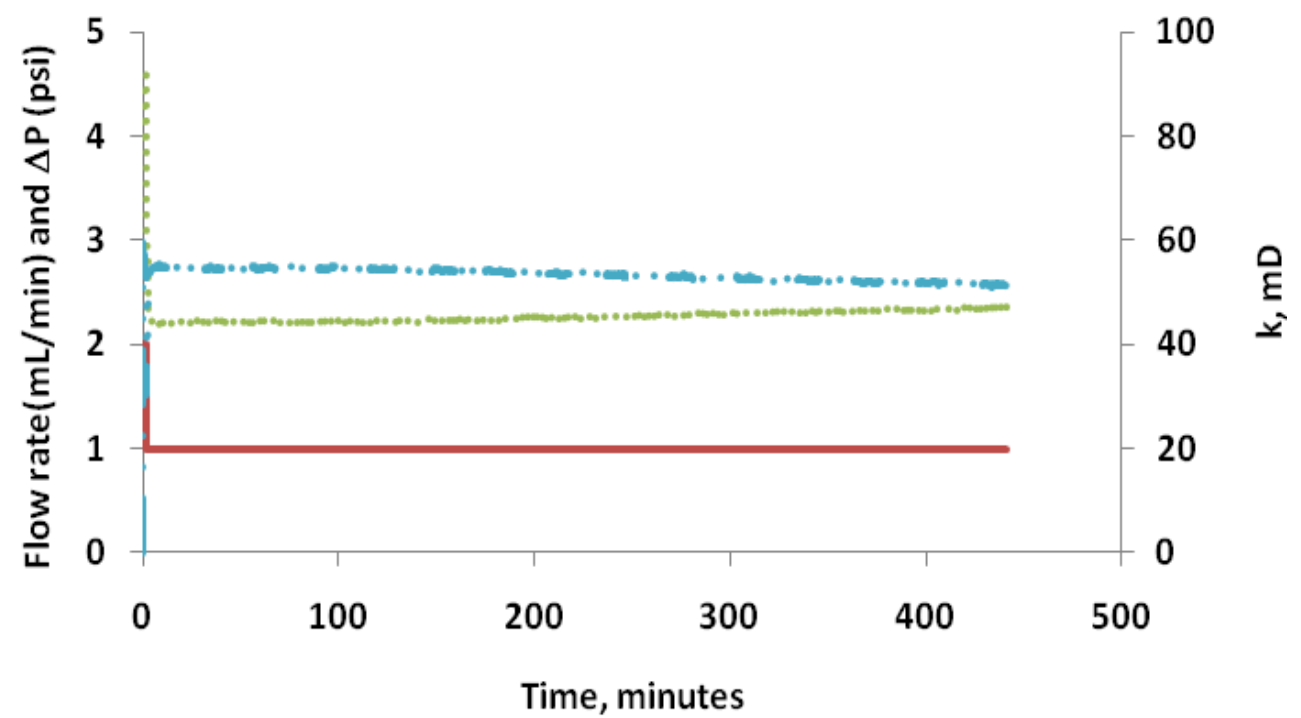

Flow rate, $\mathrm{mL} / \mathrm{min} \quad \ldots \ldots$ Delta $\mathrm{P}$, psi $\quad \cdots, \mathrm{mD}$

Figure 149 Flow rate, pressure drop and permeability of core\#14 before fluid loss tests at $40{ }^{\circ} \mathrm{C}$.

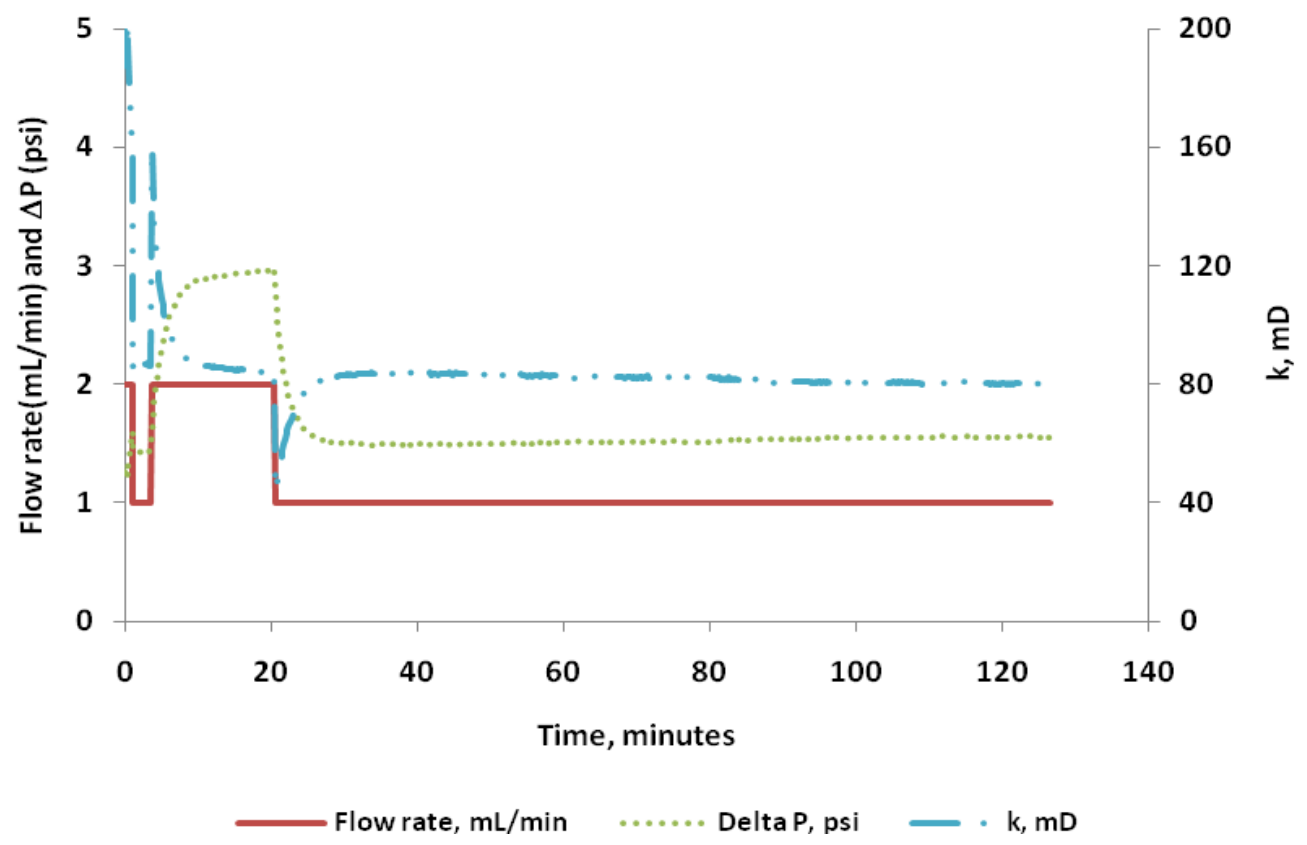

Figure 150 Flow rate, pressure drop and permeability of core\#15 before fluid loss tests at $40{ }^{\circ} \mathrm{C}$. 


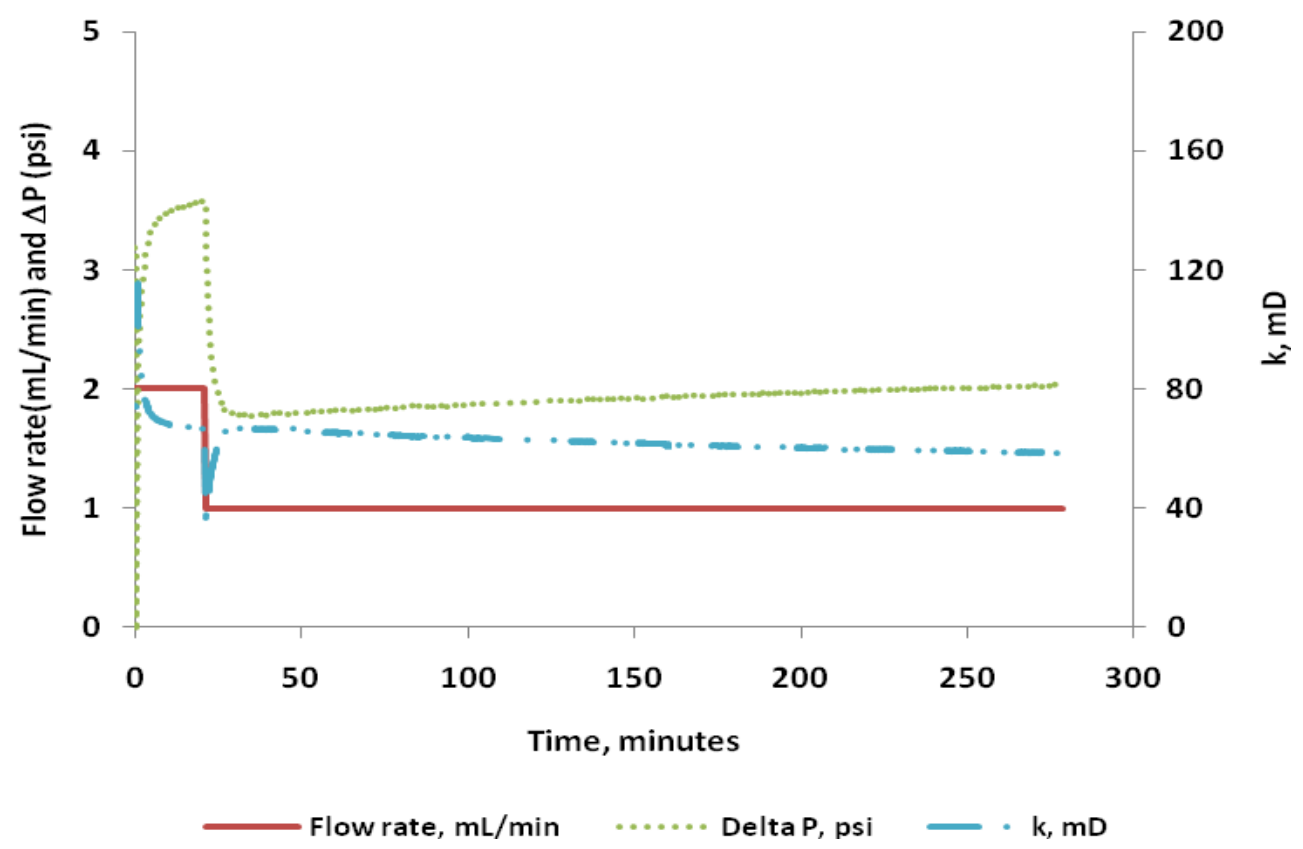

Figure 151 Flow rate, pressure drop and permeability of core\#16 before fluid loss tests at $40{ }^{\circ} \mathrm{C}$.

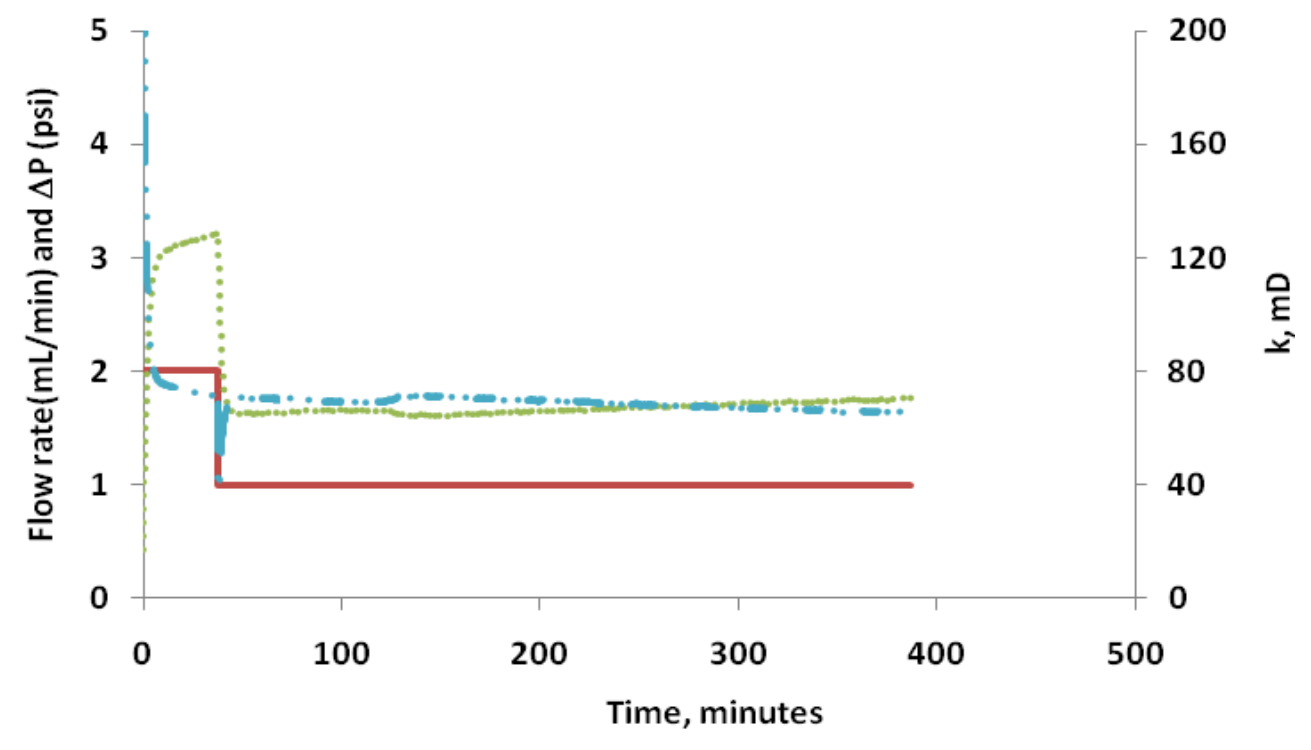

Flow rate, $\mathrm{mL} / \mathrm{min} \quad \ldots . .$. Delta $\mathrm{P}, \mathrm{psi} \quad-\mathrm{k}, \mathrm{mD}$

Figure 152 Flow rate, pressure drop and permeability of core\#18 before fluid loss tests at $40{ }^{\circ} \mathrm{C}$. 


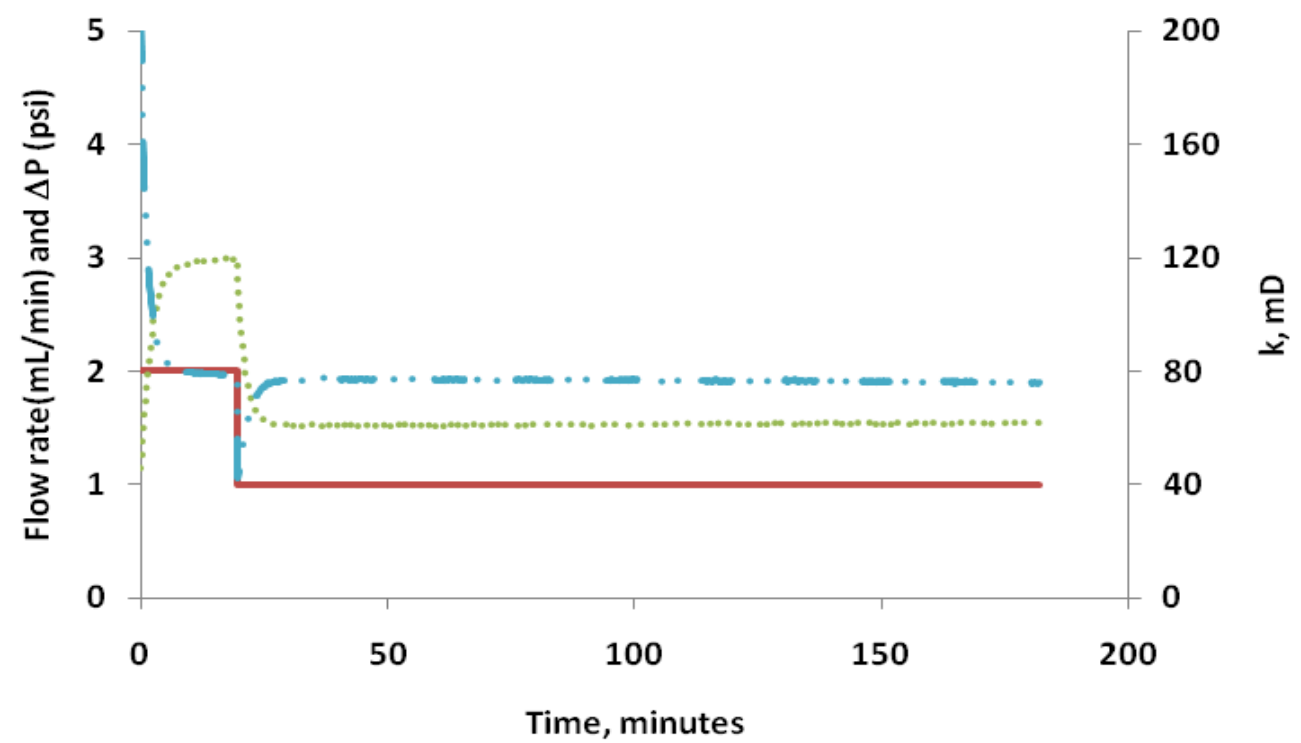

Flow rate, $\mathrm{mL} / \mathrm{min} \quad \ldots .$. Delta $\mathrm{P}$, psi $\quad \cdots, \mathrm{mD}$

Figure 153 Flow rate, pressure drop and permeability of core\#19 before fluid loss tests at $40{ }^{\circ} \mathrm{C}$.

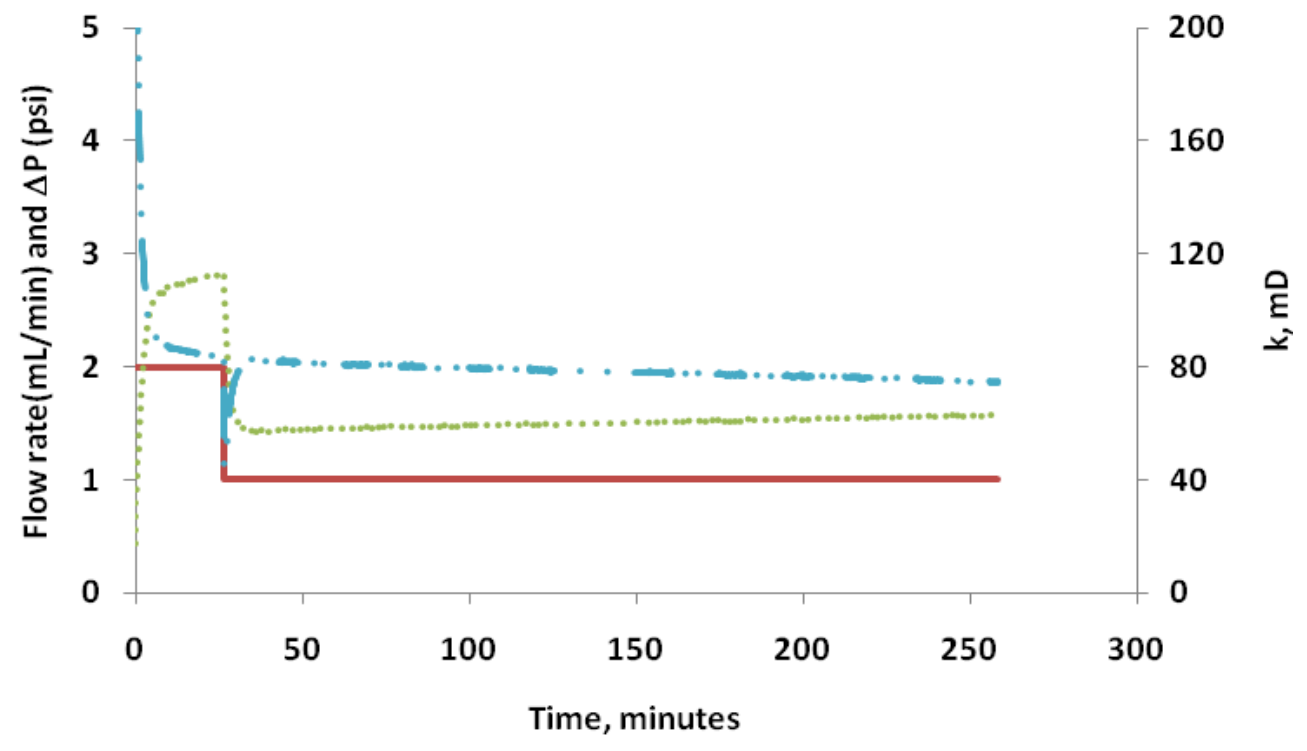

Flow rate, $\mathrm{mL} / \mathrm{min} \quad \ldots$ Delta $\mathrm{P}$, psi $\quad \cdots \mathrm{k}, \mathrm{mD}$

Figure 154 Flow rate, pressure drop and permeability of core 20 before fluid loss tests at $40{ }^{\circ} \mathrm{C}$. 


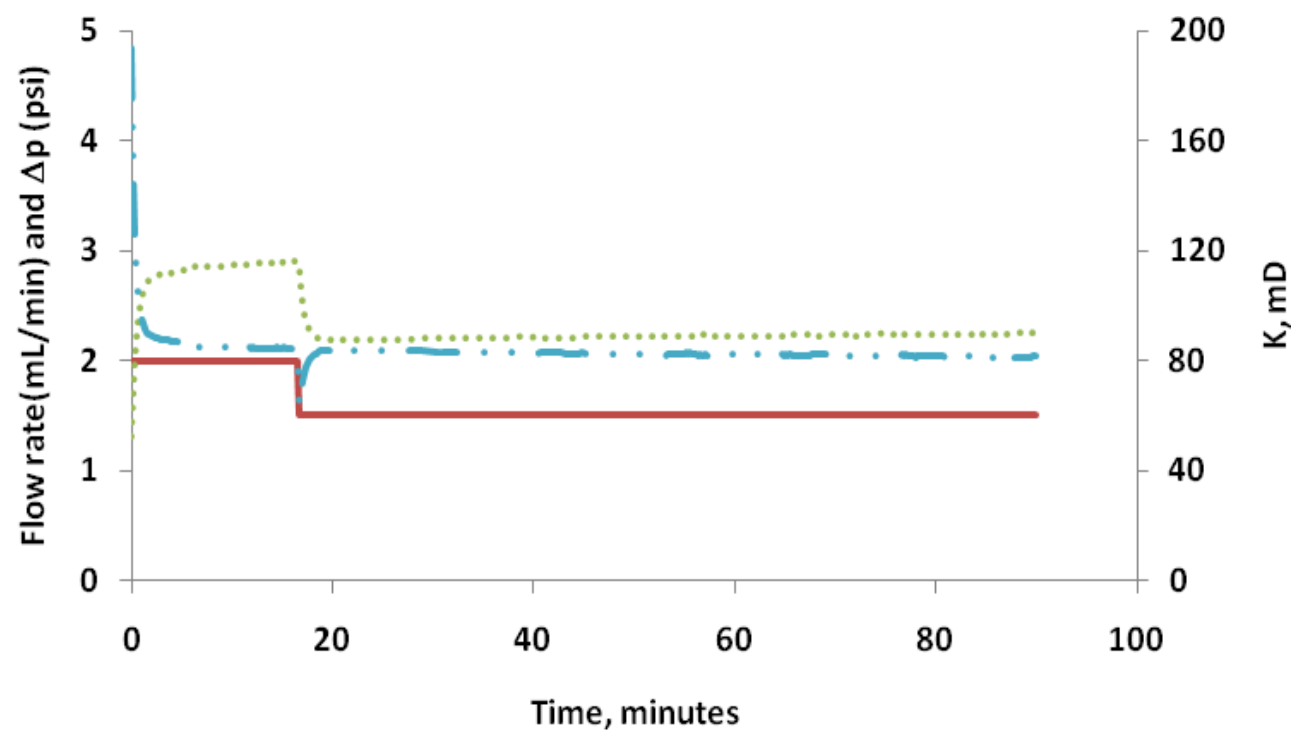

Flow rate, $\mathrm{mL} / \mathrm{min} \quad \ldots$. Delta $\mathrm{P}$, psi $\quad \mathrm{k}, \mathrm{mD}$

Figure 155 Flow rate, pressure drop and permeability of core $\# 21$ before fluid loss tests at $40{ }^{\circ} \mathrm{C}$.

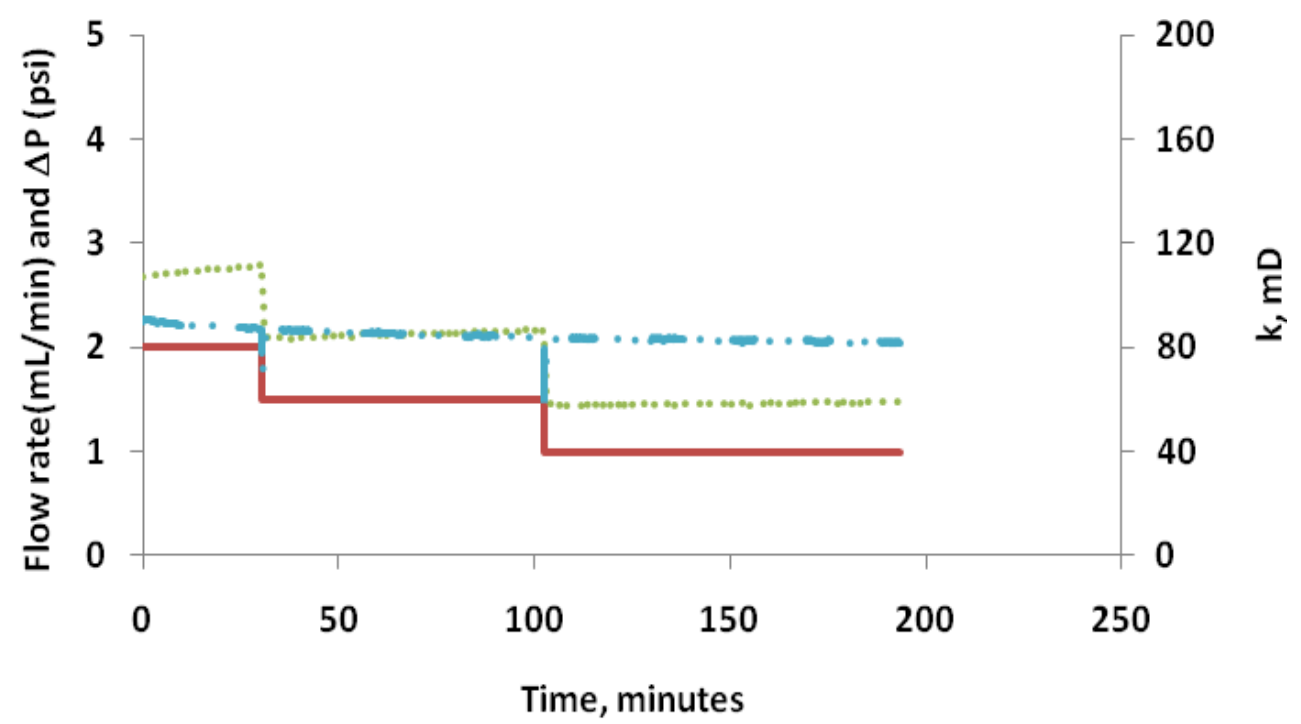

Flow rate, $\mathrm{mL} / \mathrm{min} \quad \ldots . .$. Delta $\mathrm{P}$, psi $\quad$ - $\mathrm{k}, \mathrm{mD}$

Figure 156 Flow rate, pressure drop and permeability of core\#22 before fluid loss tests at $40{ }^{\circ} \mathrm{C}$. 


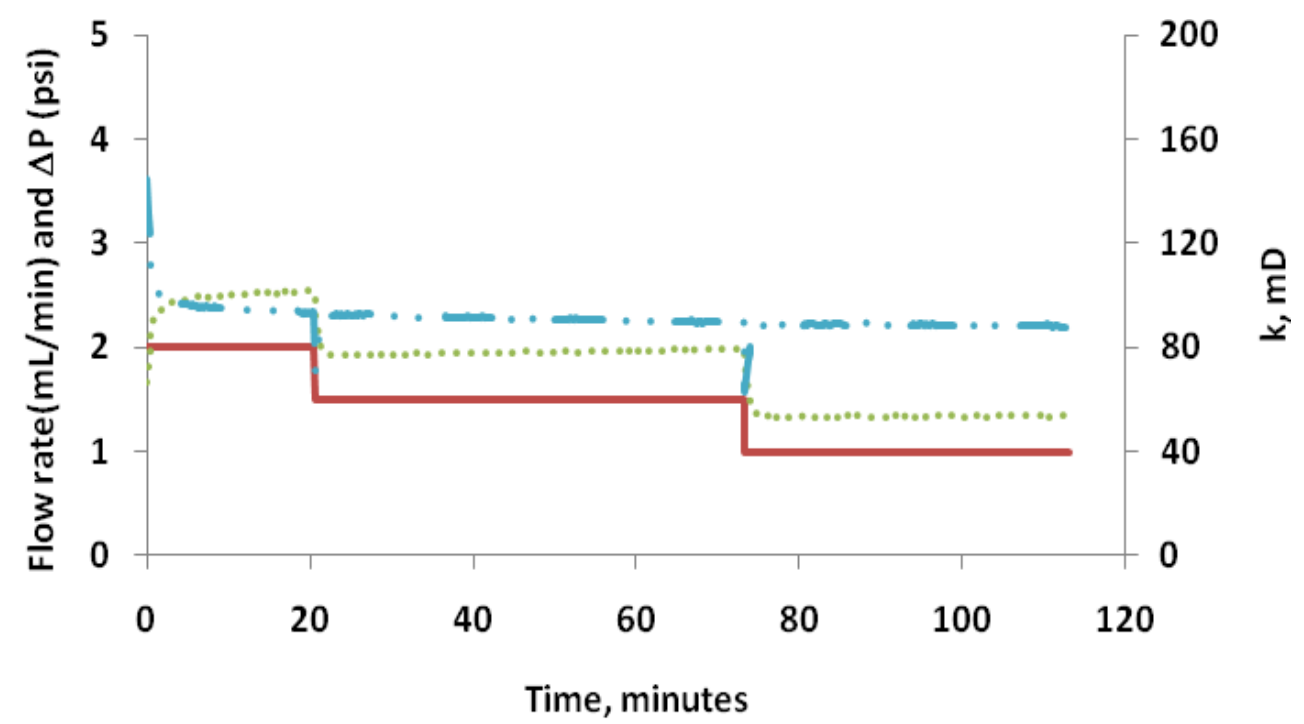

Flow rate, $\mathrm{mL} / \mathrm{min} \quad \ldots$. Delta $\mathrm{P}$, psi $\quad \mathrm{k}, \mathrm{mD}$

Figure 157 Flow rate, pressure drop and permeability of core $\# 23$ before fluid loss tests at $40{ }^{\circ} \mathrm{C}$.

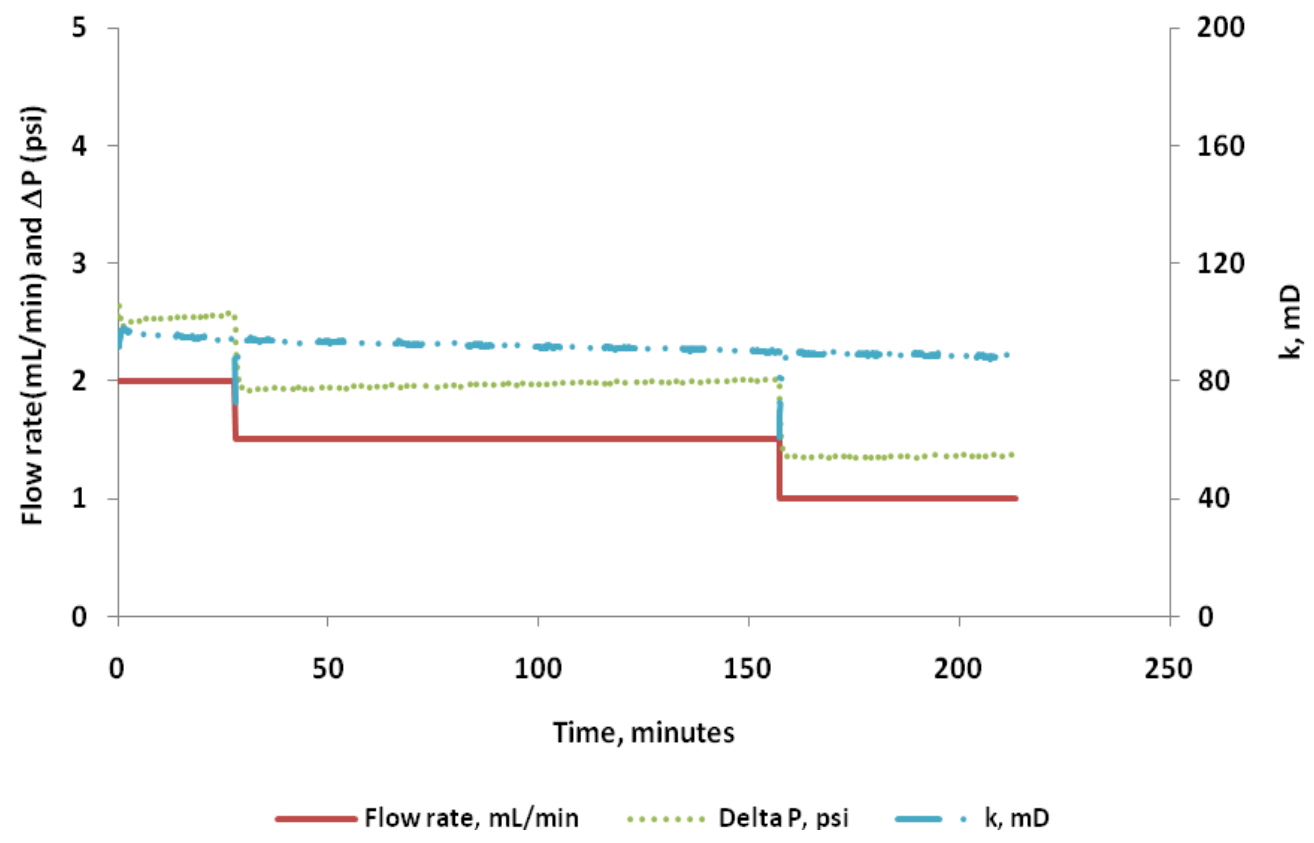

Figure 158 Flow rate, pressure drop and permeability of core $\# 24$ before fluid loss tests at $40{ }^{\circ} \mathrm{C}$. 


\section{A-2 Permeability Measurements for Cores Resaturated After Tracer Tests and Before Being Used in Fluid Loss Tests}

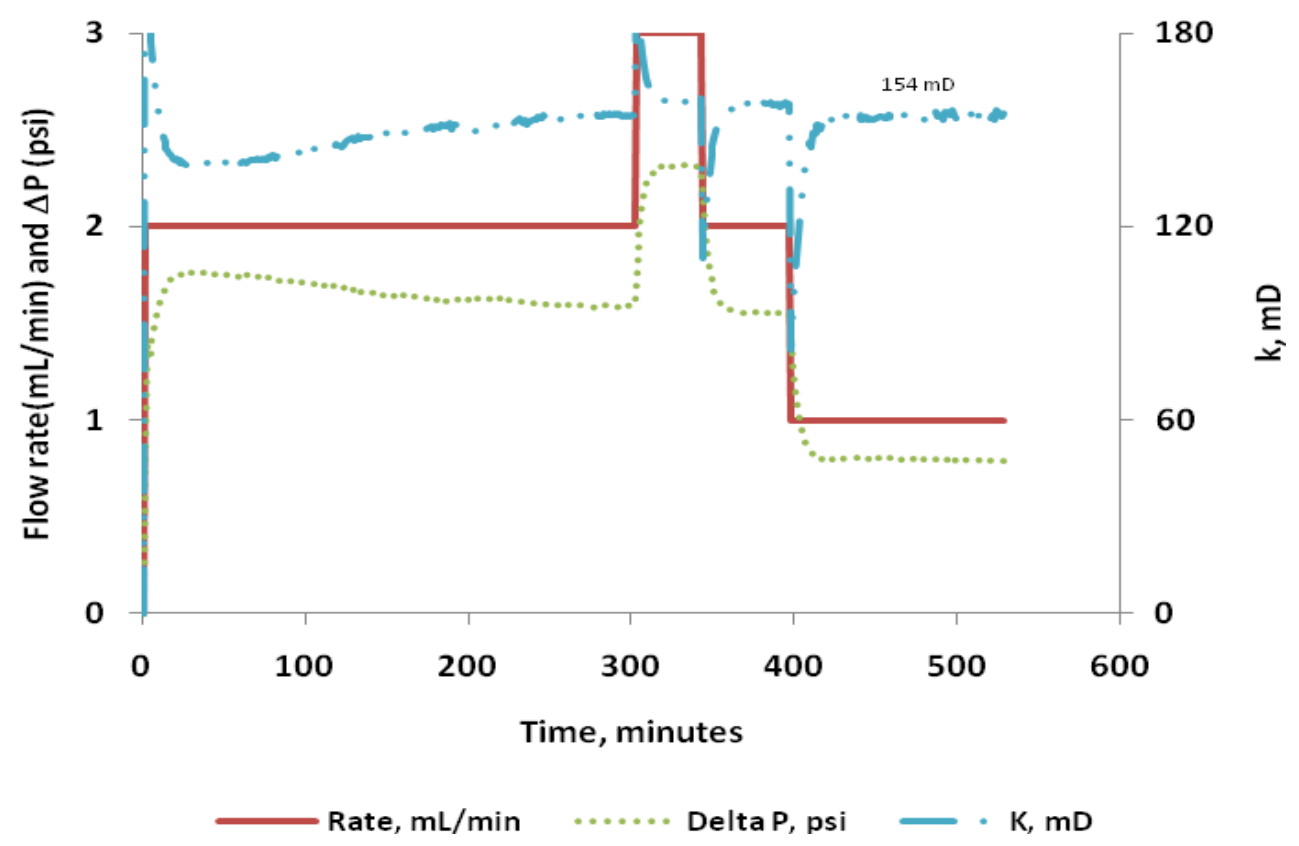

Figure 159 Flow rate, pressure drop and permeability of core\#1 resaturated after tracer test and before fluid loss tests at $25^{\circ} \mathrm{C}$. 


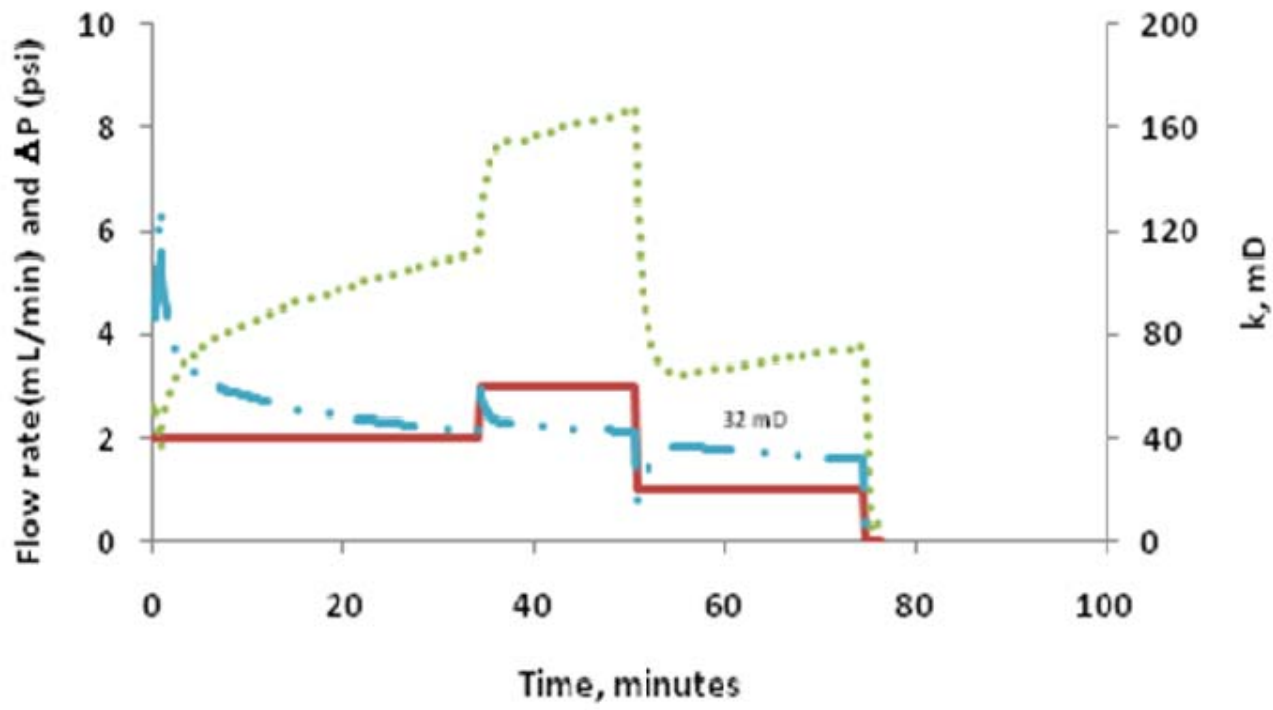

Flow rate, $\mathrm{mL} / \mathrm{min} \quad \ldots . .$. Delta $\mathrm{P}, \mathrm{psi} \quad \mathrm{k}, \mathrm{mD}$

Figure 160 Flow rate, pressure drop and permeability of core $\# 2$ resaturated after tracer test and before fluid loss tests at $25^{\circ} \mathrm{C}$.

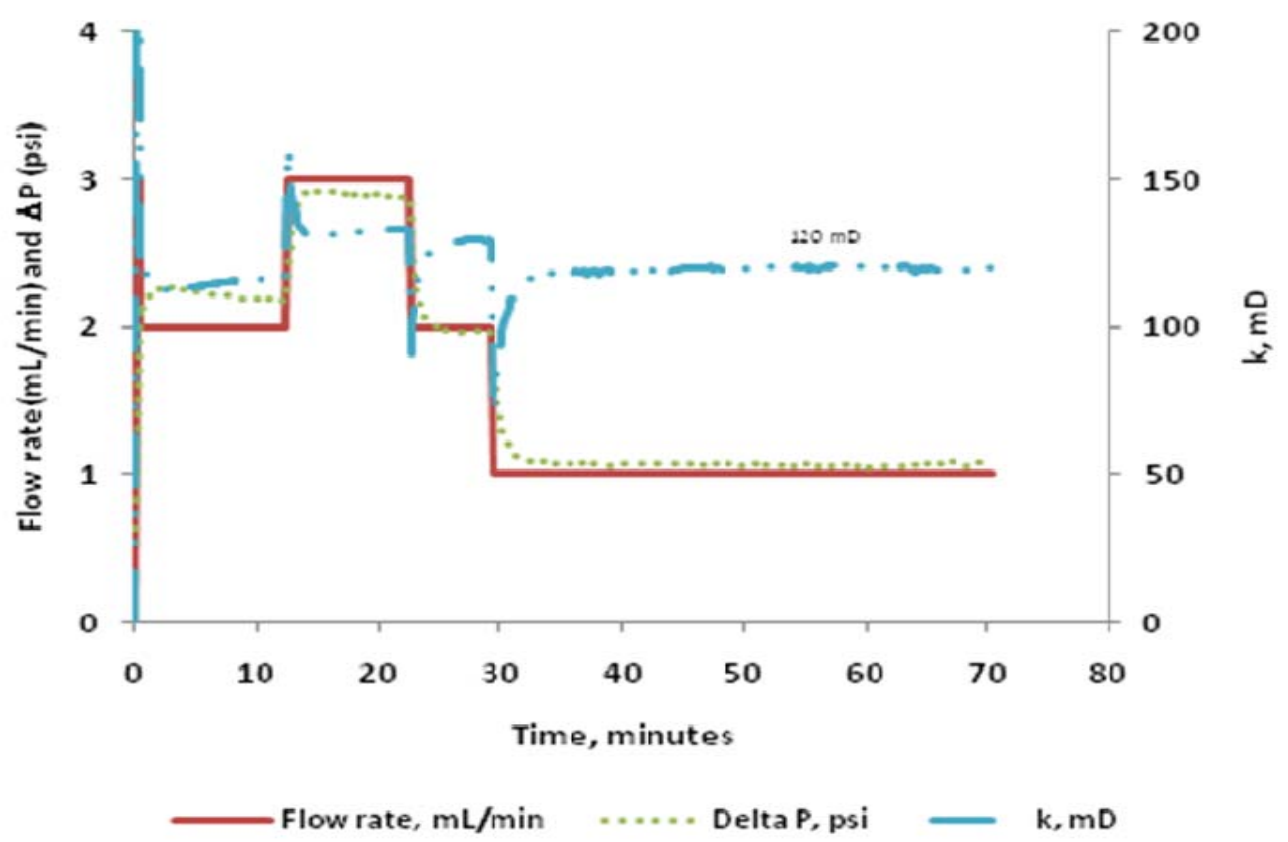

Figure 161 Flow rate, pressure drop and permeability of core\#3 resaturated after tracer test and before fluid loss tests at $25^{\circ} \mathrm{C}$. 


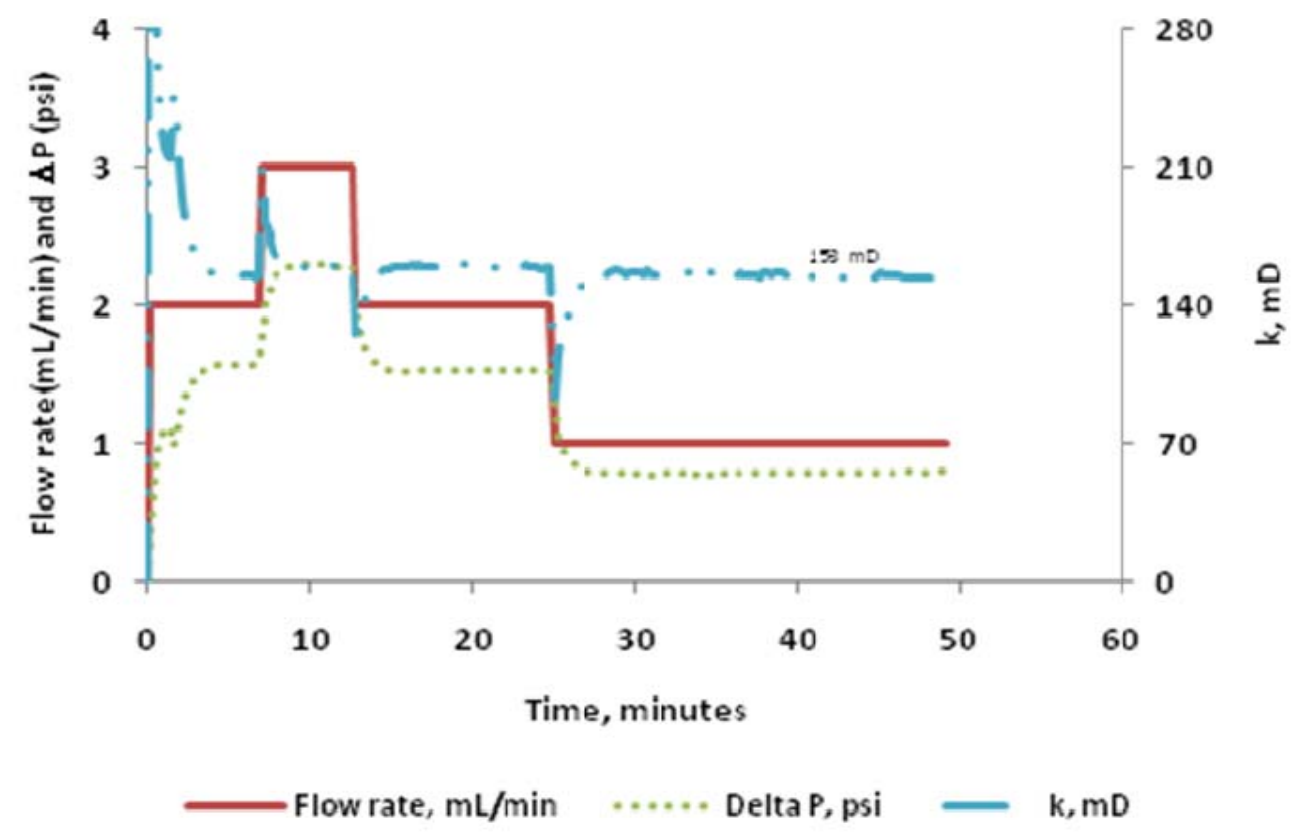

Figure 162 Flow rate, pressure drop and permeability of core\#4 resaturated after tracer test and before fluid loss tests at $25^{\circ} \mathrm{C}$.

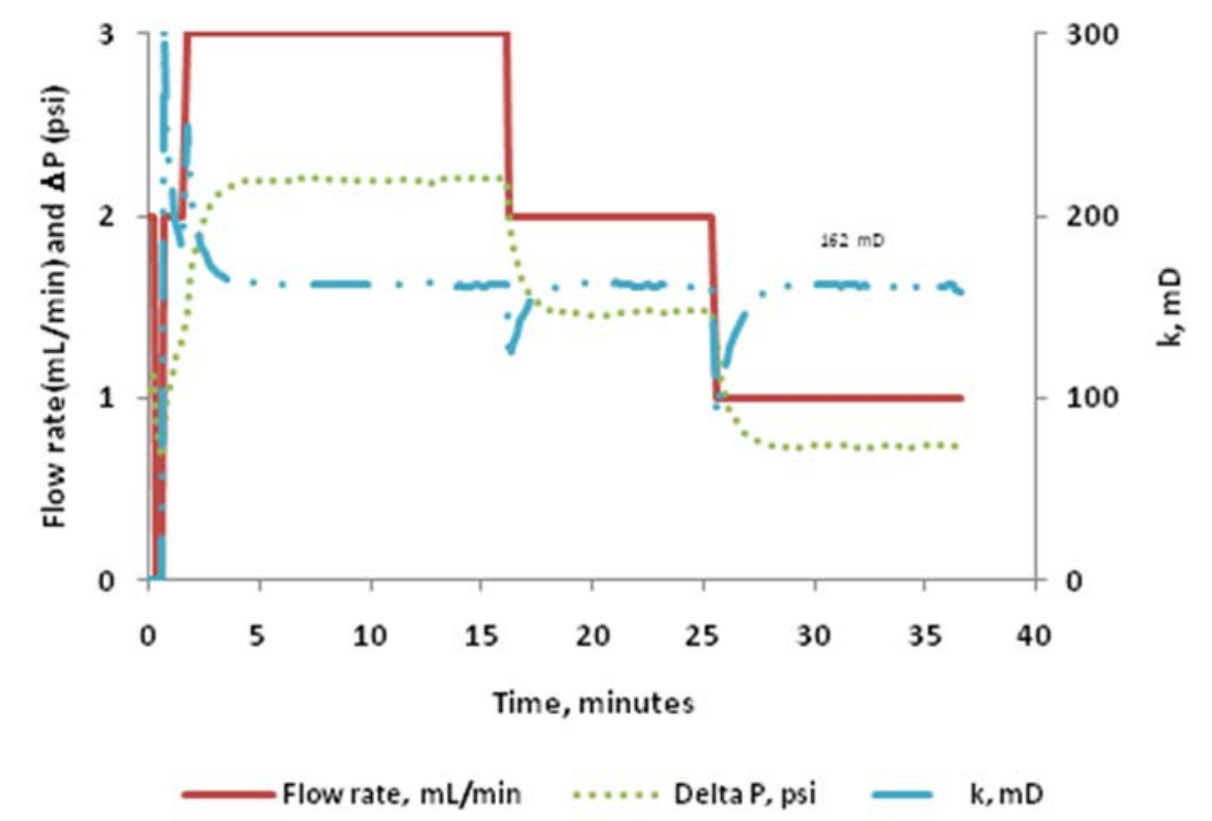

Figure 163 Flow rate, pressure drop and permeability of core $\# 5$ resaturated after tracer test and before fluid loss tests at $25^{\circ} \mathrm{C}$. 


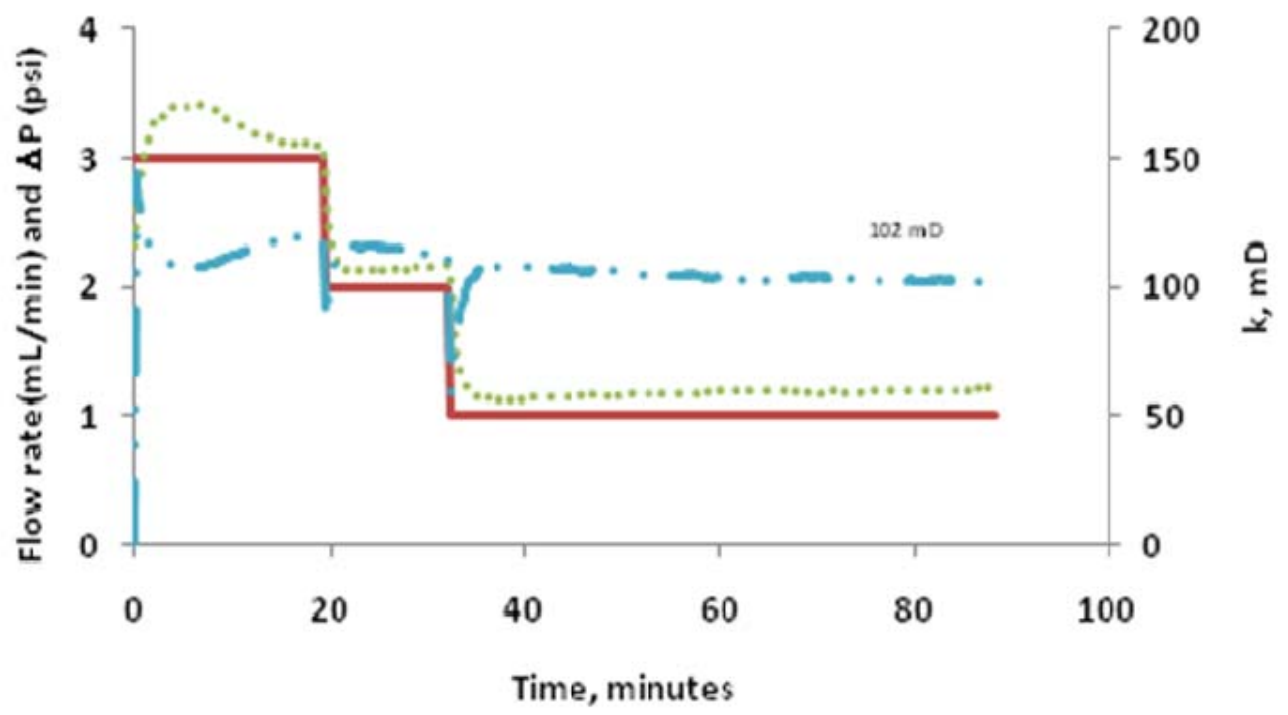

Flow rate, $\mathrm{mL} / \mathrm{min} \quad \ldots .$. Delta $\mathrm{P}, \mathrm{psi} \quad \mathrm{k}, \mathrm{mD}$

Figure 164 Flow rate, pressure drop and permeability of core $\# 8$ resaturated after tracer test and before fluid loss tests at $25^{\circ} \mathrm{C}$.

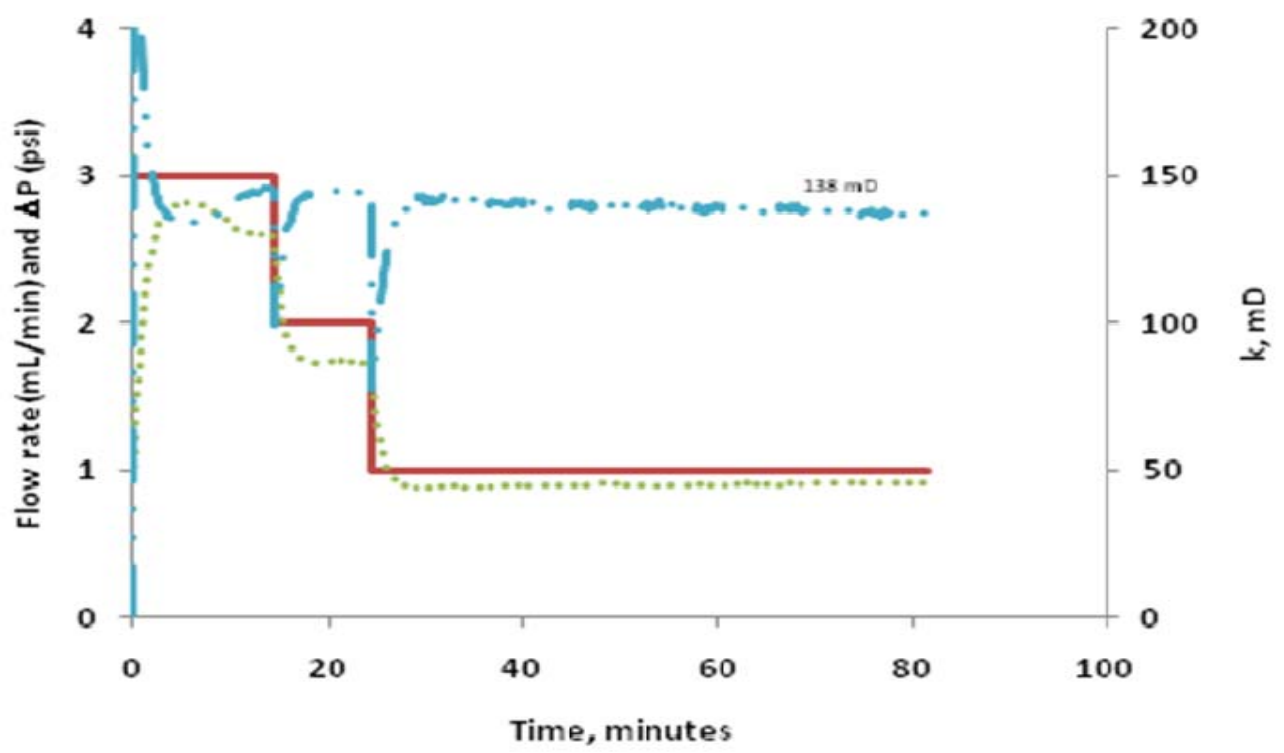

Flow rate, $\mathrm{mL} / \mathrm{min} \quad \ldots . .$. Delta $\mathrm{P}$, psi $\quad \mathrm{k}, \mathrm{mD}$

Figure 165 Flow rate, pressure drop and permeability of core\#9 resaturated after tracer test and before fluid loss tests at $25{ }^{\circ} \mathrm{C}$. 


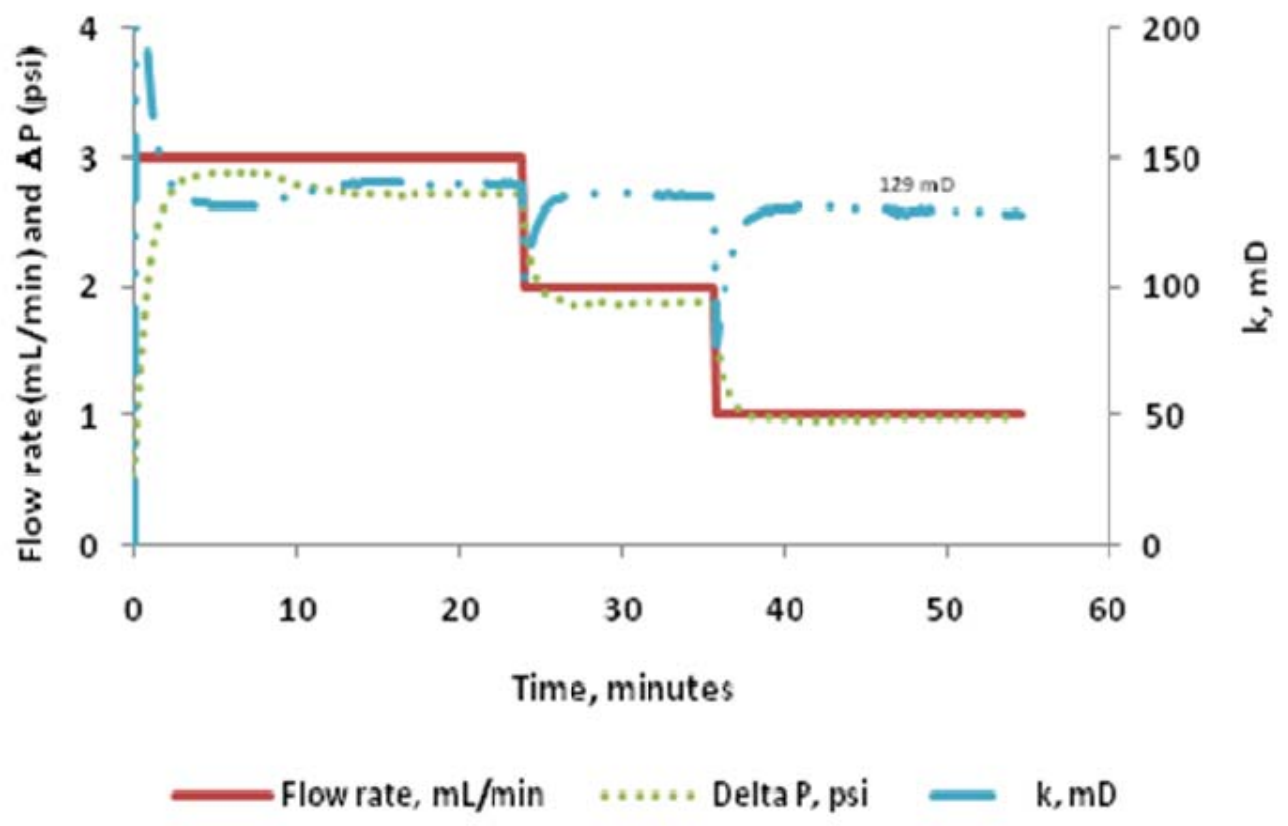

Figure 166 Flow rate, pressure drop and permeability of core\#10 resaturated after tracer test and before fluid loss tests at $25^{\circ} \mathrm{C}$.

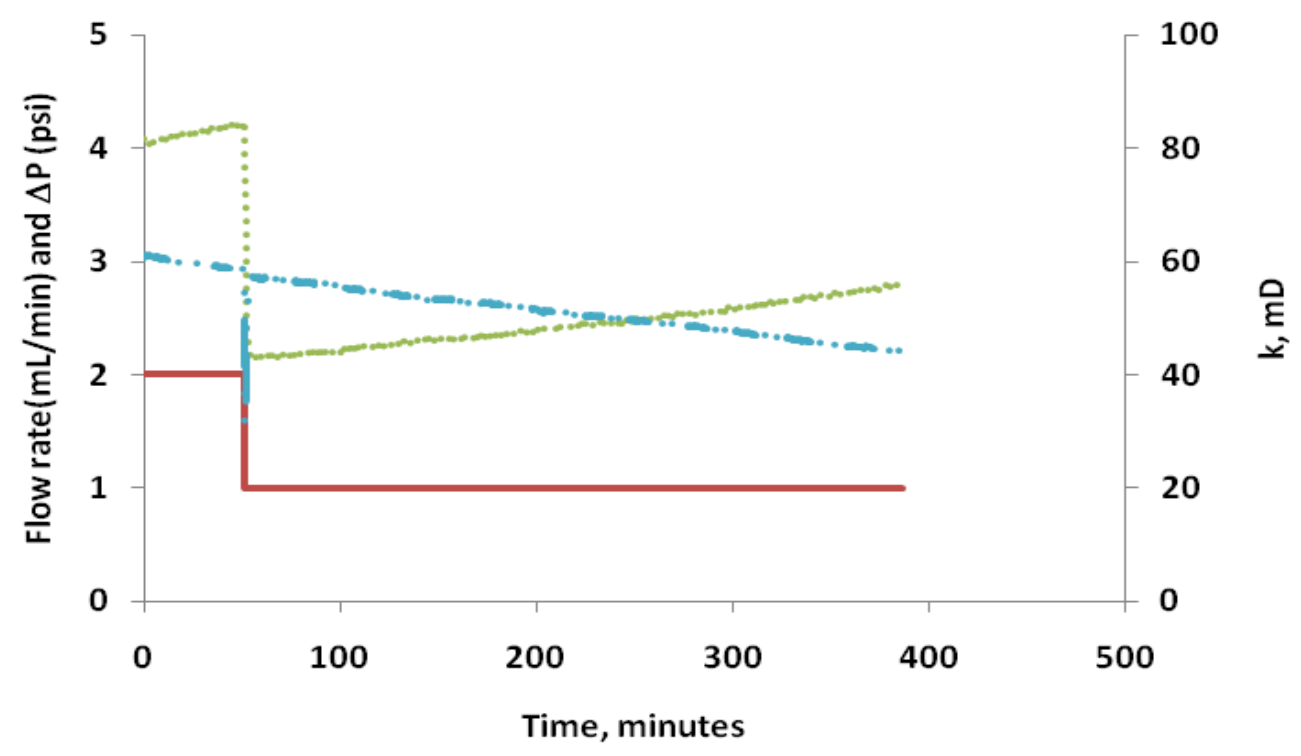

Flow rate, $\mathrm{mL} / \mathrm{min} \quad \ldots \ldots$ Delta $\mathrm{P}$, psi $\quad \cdots, \mathrm{mD}$

Figure 167 Flow rate, pressure drop and permeability of core\#13 resaturated after tracer test and before fluid loss tests at $40{ }^{\circ} \mathrm{C}$. 


\section{A-3 Permeability Measurements for Cores Restored After Fluid Loss Tests and Before Being Reused for Fluid Loss Tests}

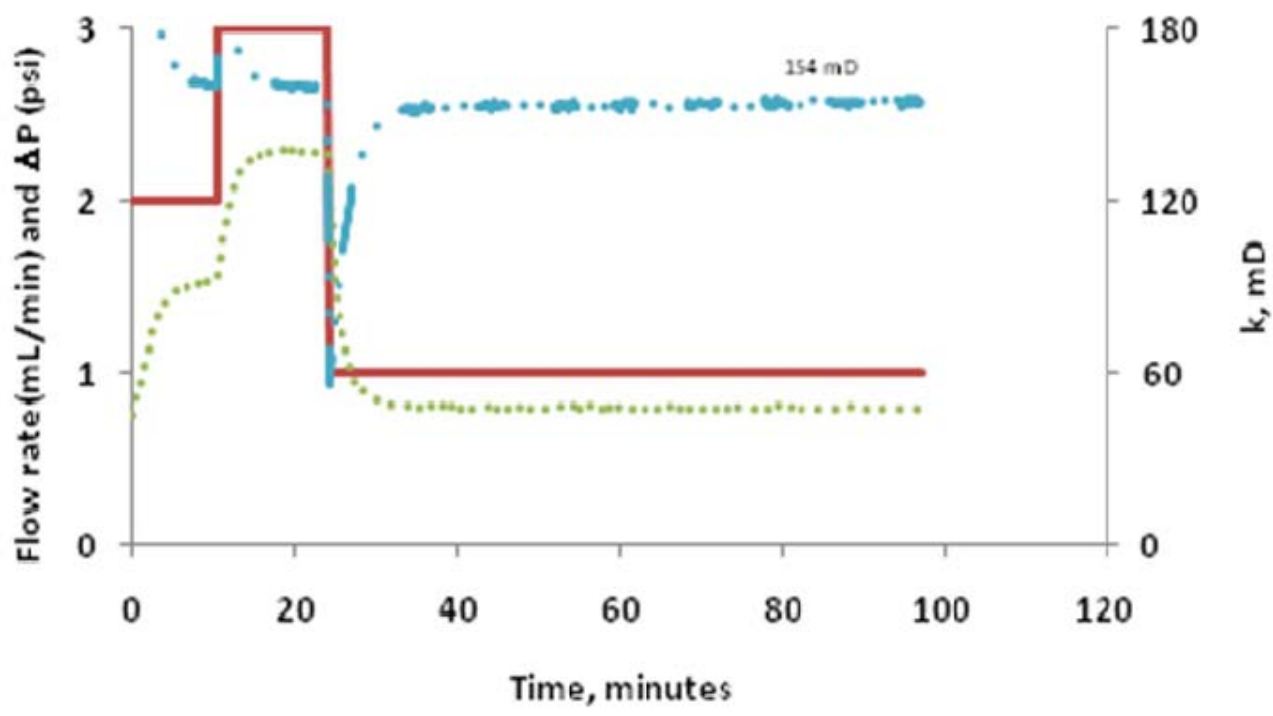

Flow rate, $\mathrm{mL} / \mathrm{min} \quad \ldots \ldots$ Delta $\mathrm{P}$, psi $\quad \mathrm{k}, \mathrm{mD}$

Figure 168 Flow rate, pressure drop and permeability of core\#1 restored after fluid loss test and before being reused for fluid loss tests at $25^{\circ} \mathrm{C}$. 


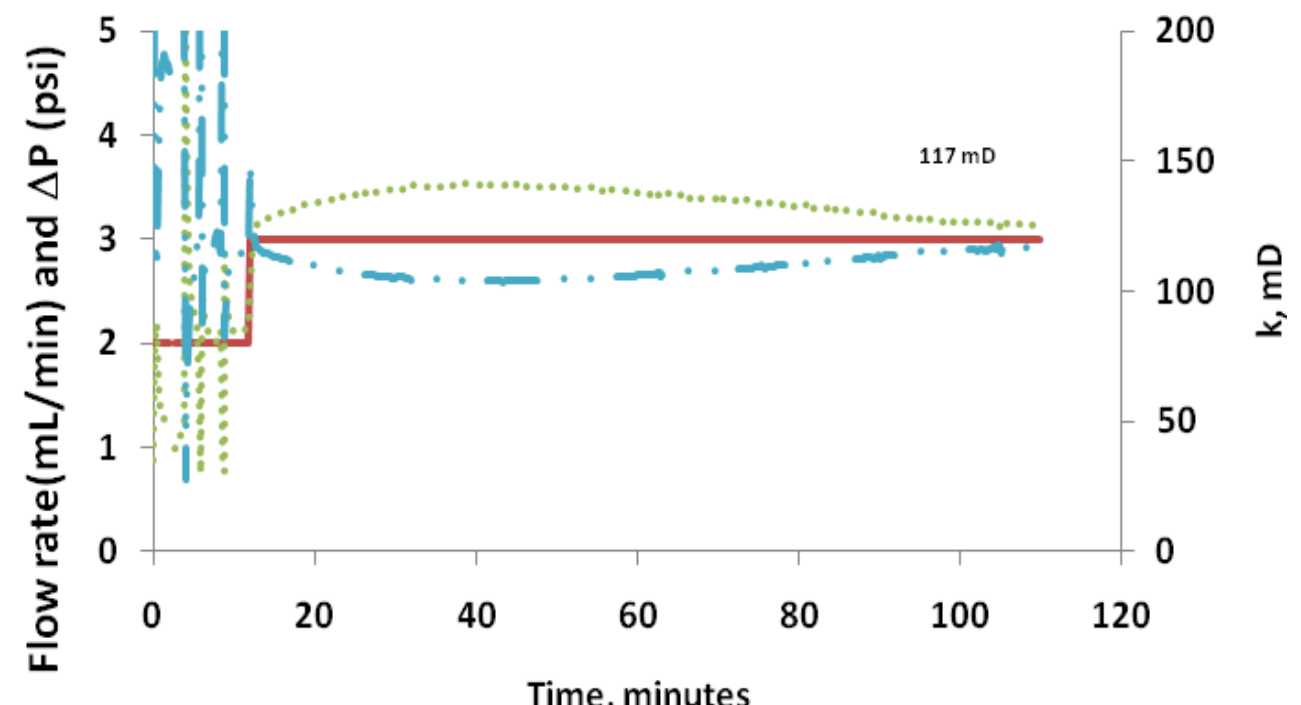

Flow rate, $\mathrm{mL} / \mathrm{min} \quad \ldots \ldots$ Delta $\mathrm{P}$, psi $\quad \mathrm{k}, \mathrm{mD}$

Figure 169 Flow rate, pressure drop and permeability of core\#4 restored after fluid loss test and before being reused for fluid loss tests at $25^{\circ} \mathrm{C}$.

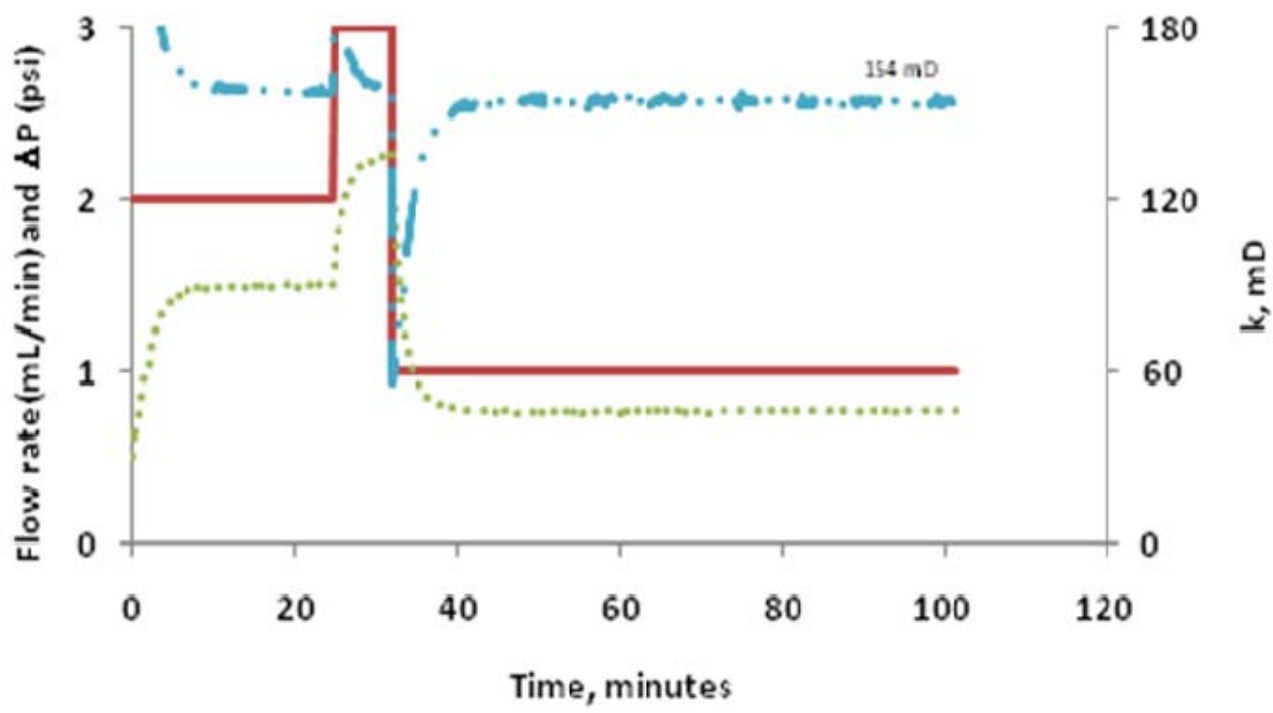

Flow rate, $\mathrm{mL} / \mathrm{min} \quad \ldots \ldots$ Delta $\mathrm{P}$, psi $\quad \mathrm{k}, \mathrm{mD}$

Figure 170 Flow rate, pressure drop and permeability of core\#5 restored after fluid loss test and before being reused for fluid loss tests at $25^{\circ} \mathrm{C}$. 


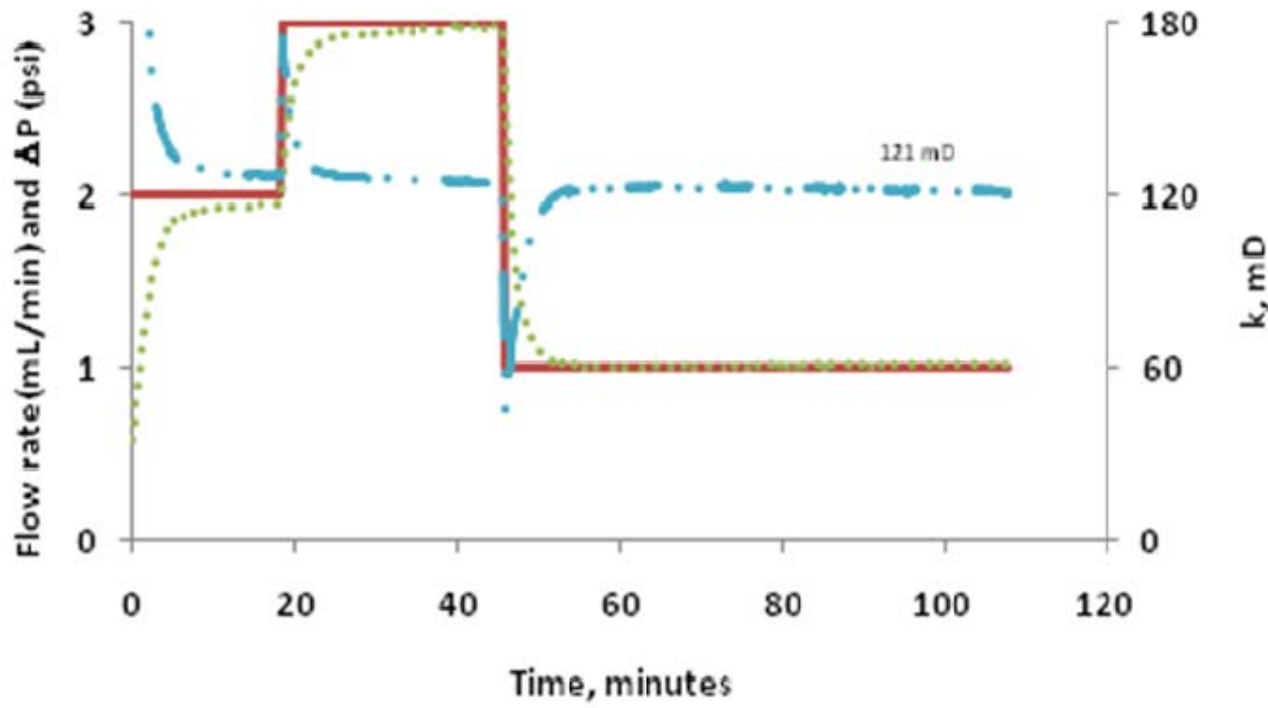

Flow rate, $\mathrm{mL} / \mathrm{min} \quad \ldots . .$. Delta $\mathrm{P}, \mathrm{psi}=\mathrm{k}, \mathrm{mD}$

Figure 171 Flow rate, pressure drop and permeability of core\#6 restored after fluid loss test and before being reused for fluid loss tests at $25{ }^{\circ} \mathrm{C}$.

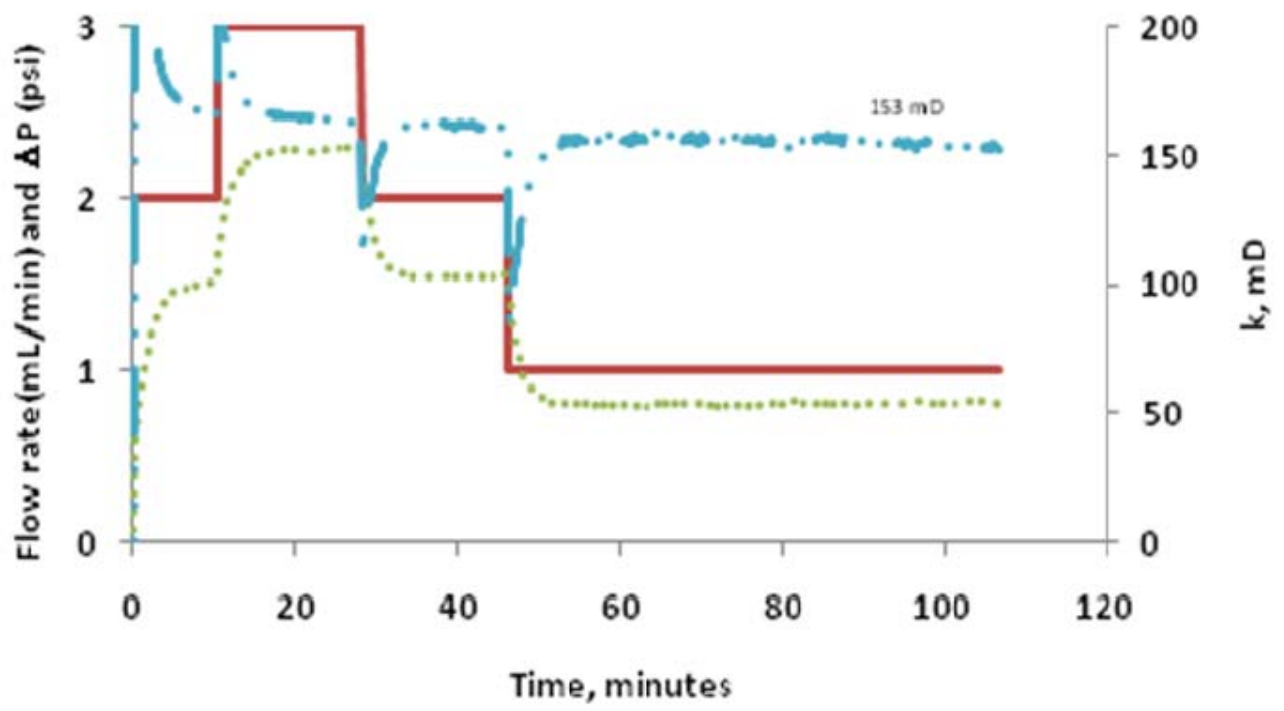

Flow rate, $\mathrm{mL} / \mathrm{min} \quad \ldots .$. Delta P, psi $\quad \mathrm{k}, \mathrm{mD}$

Figure 172 Flow rate, pressure drop and permeability of core\#9 restored after fluid loss test and before being reused for fluid loss tests at $25^{\circ} \mathrm{C}$. 


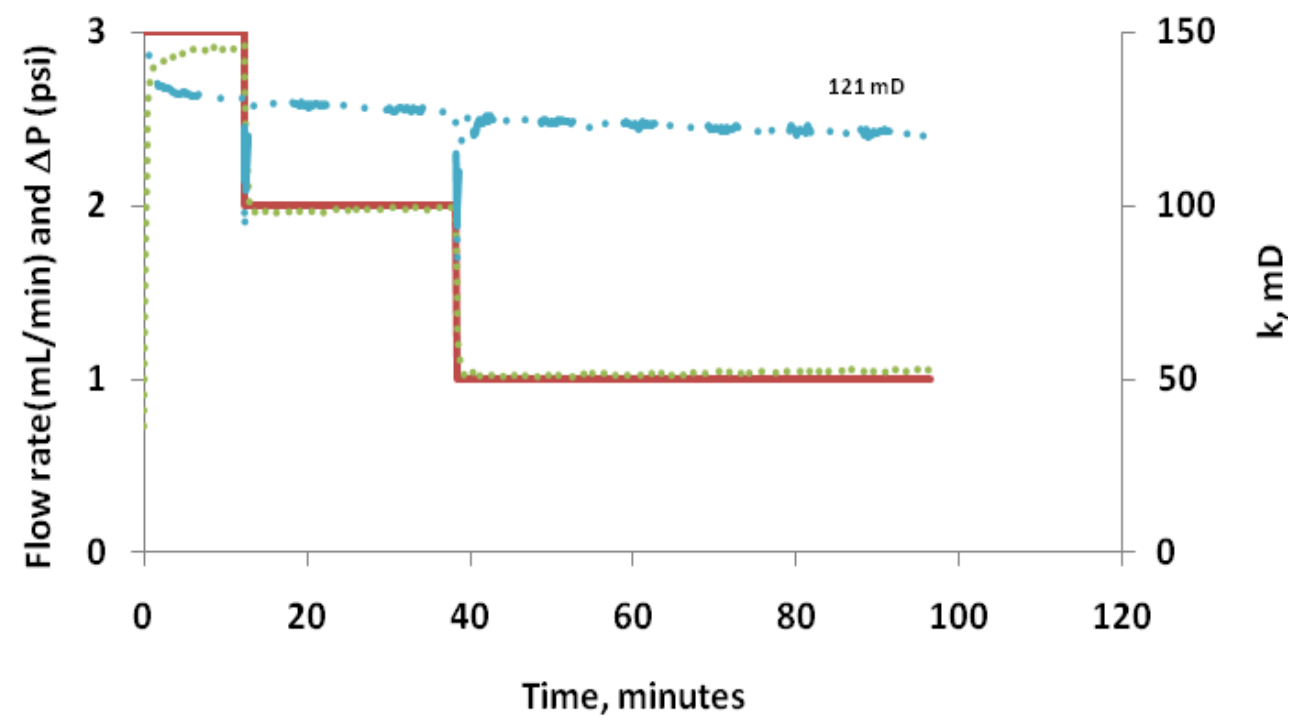

Flow rate, $\mathrm{mL} / \mathrm{min} \quad \ldots$. Delta $\mathrm{P}$, psi $\quad \mathrm{k}, \mathrm{mD}$

Figure 173 Flow rate, pressure drop and permeability of core\#10 restored after fluid loss test and before being reused for fluid loss tests at $25^{\circ} \mathrm{C}$. 


\section{Appendix B}

In this section, permeability measurements done for cores after being used in fluid loss tests are shown. Remainder of filter cake was scraped from the face of the cores and their permeability was measured after each fluid loss test. Cleanup of invaded polymers into the cores was seen in permeability measurement curves as a peak in pressure drop or a minimum in permeability.

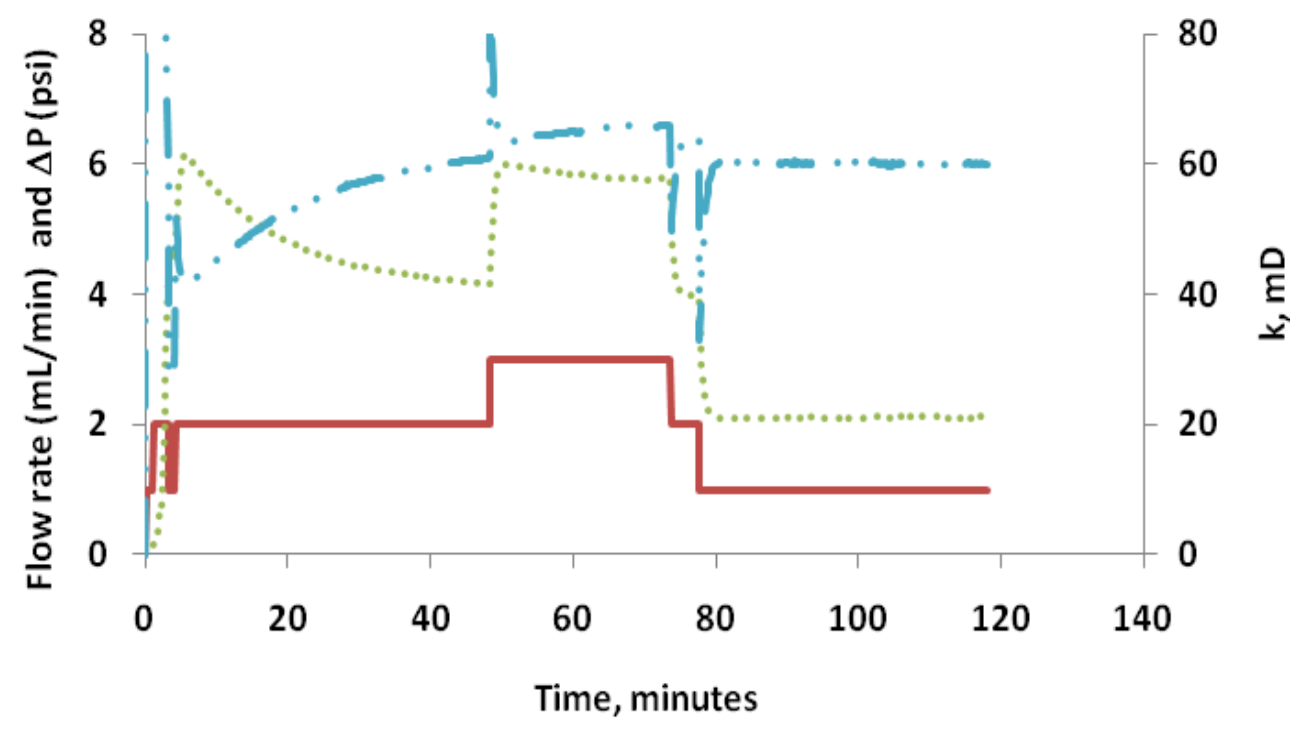

Flow rate, $\mathrm{mL} / \mathrm{min} \quad \ldots .$. Delta $\mathrm{P}, \mathrm{psi} \quad-\mathrm{k}, \mathrm{mD}$

Figure 174 Flow rate, pressure drop and permeability of core\#10 after fluid loss test at $25{ }^{\circ} \mathrm{C}$. 


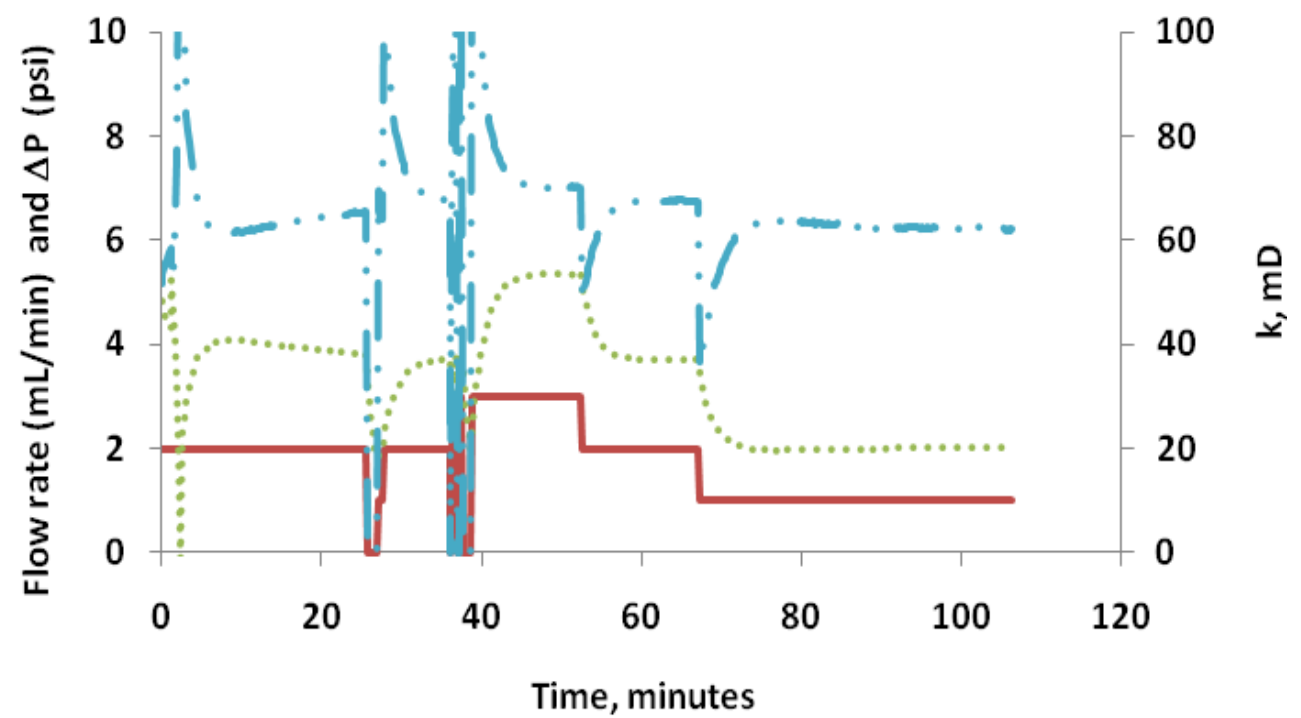

Flow rate, $\mathrm{mL} / \mathrm{min} \quad \ldots \ldots$ Delta $\mathrm{P}$, psi $\quad \mathrm{k}, \mathrm{mD}$

Figure 175 Flow rate, pressure drop and permeability of core\#9 after fluid loss test at $25^{\circ} \mathrm{C}$.

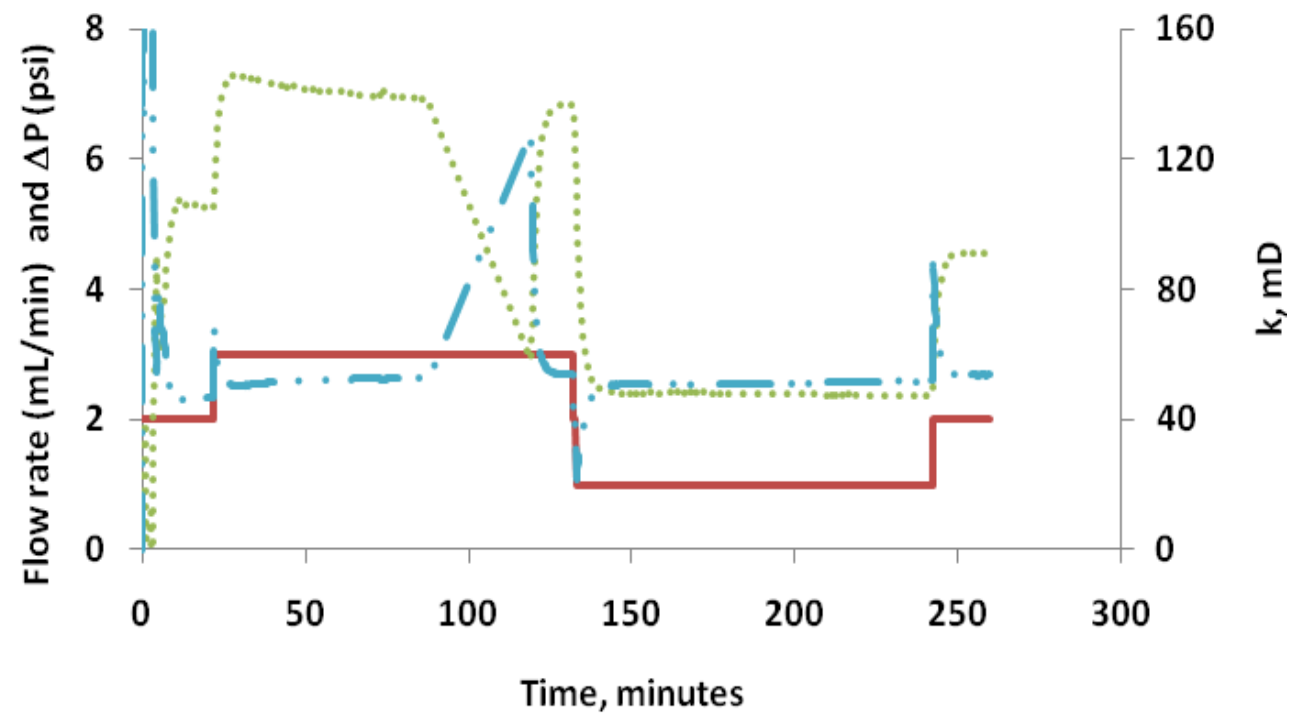

Flow rate, $\mathrm{mL} / \mathrm{min} \quad \ldots \ldots$ Delta $\mathrm{P}$, psi $\quad \mathrm{k}, \mathrm{mD}$

Figure 176 Flow rate, pressure drop and permeability of core\#1 after fluid loss test at $25{ }^{\circ} \mathrm{C}$. 


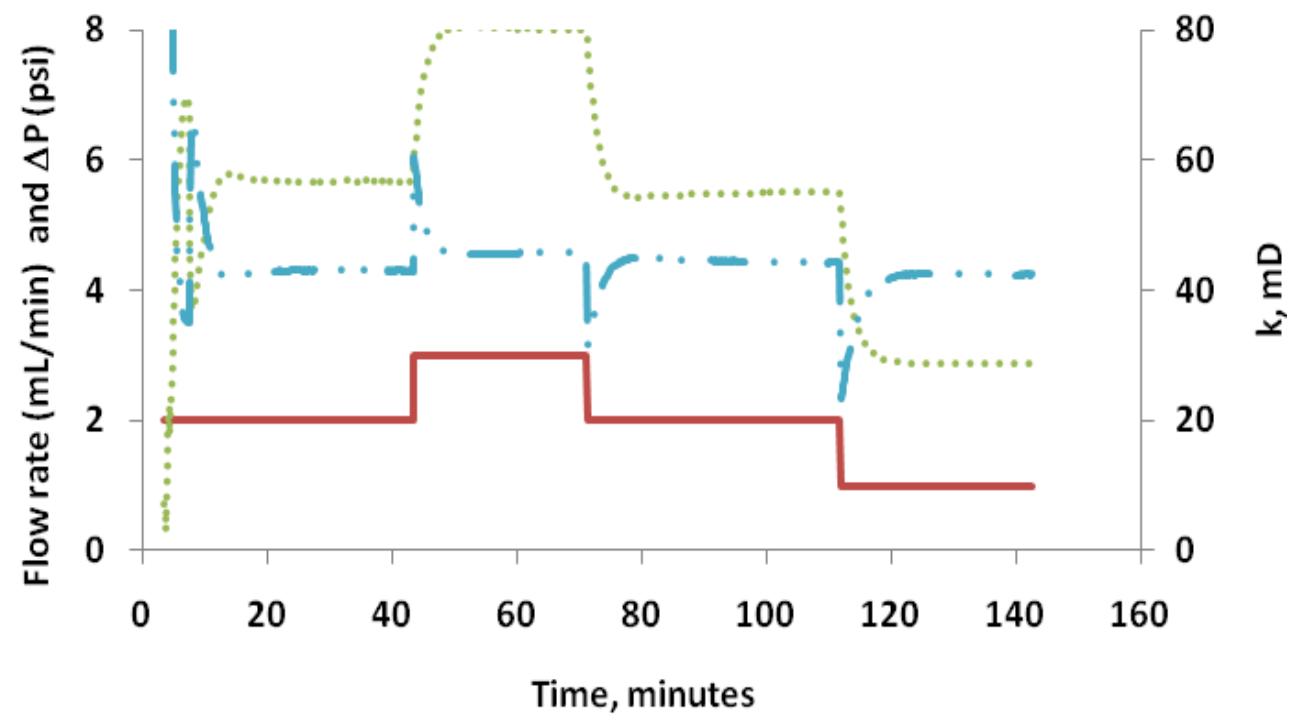

Flow rate, $\mathrm{mL} / \mathrm{min} \quad \ldots \ldots$ Delta $\mathrm{P}$, psi $\quad \mathrm{k}, \mathrm{mD}$

Figure 177 Flow rate, pressure drop and permeability of core $\# 4$ after fluid loss test at $25^{\circ} \mathrm{C}$.

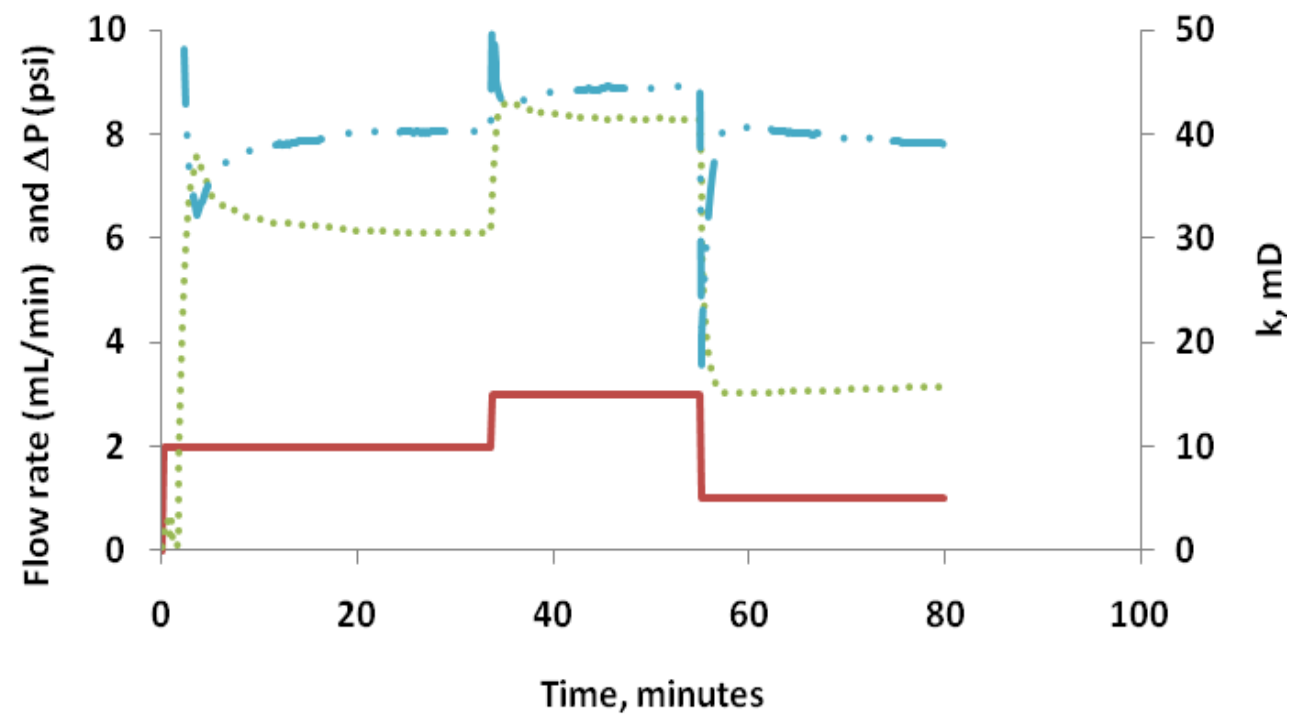

Flow rate, $\mathrm{mL} / \mathrm{min} \quad \ldots \ldots$ Delta $\mathrm{P}$, psi $\quad \mathrm{k}, \mathrm{mD}$

Figure 178 Flow rate, pressure drop and permeability of core\#8 after fluid loss test at $25^{\circ} \mathrm{C}$. 


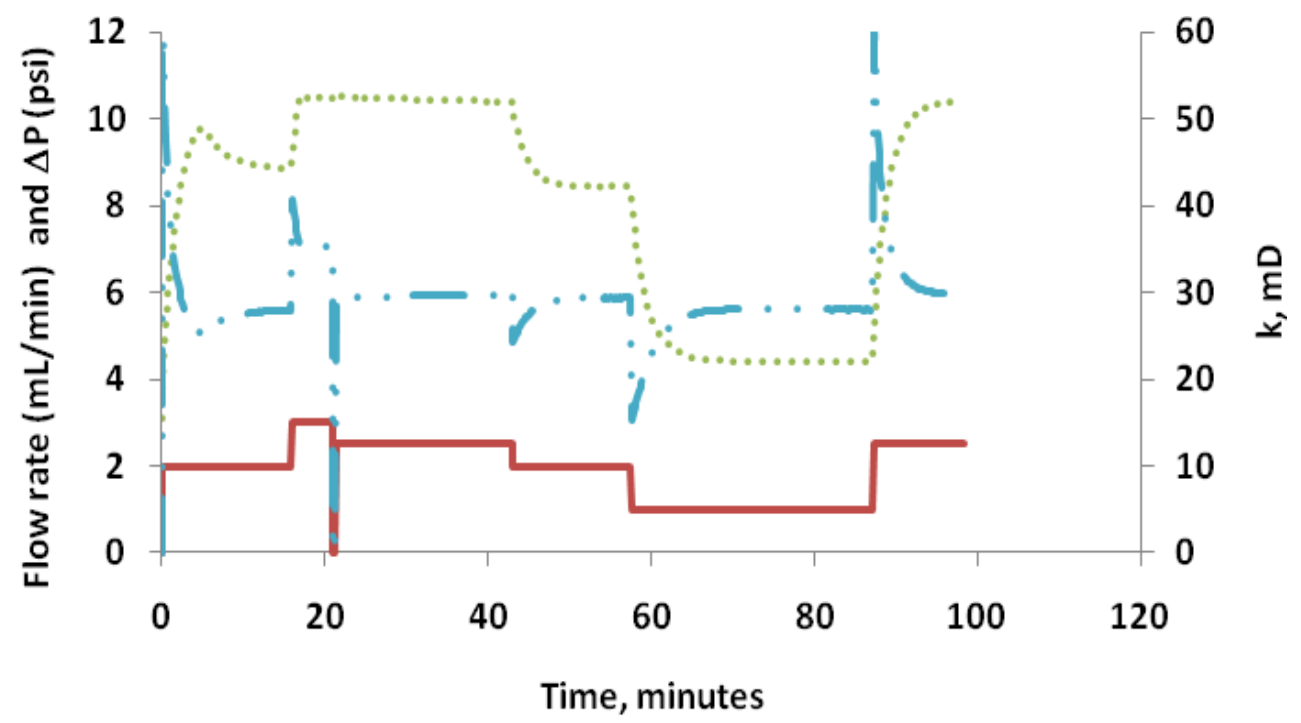

Flow rate, $\mathrm{mL} / \mathrm{min} \quad \ldots$. Delta $\mathrm{P}$, psi $\quad \mathrm{k}, \mathrm{mD}$

Figure 179 Flow rate, pressure drop and permeability of core $\# 6$ after fluid loss test at $25^{\circ} \mathrm{C}$.

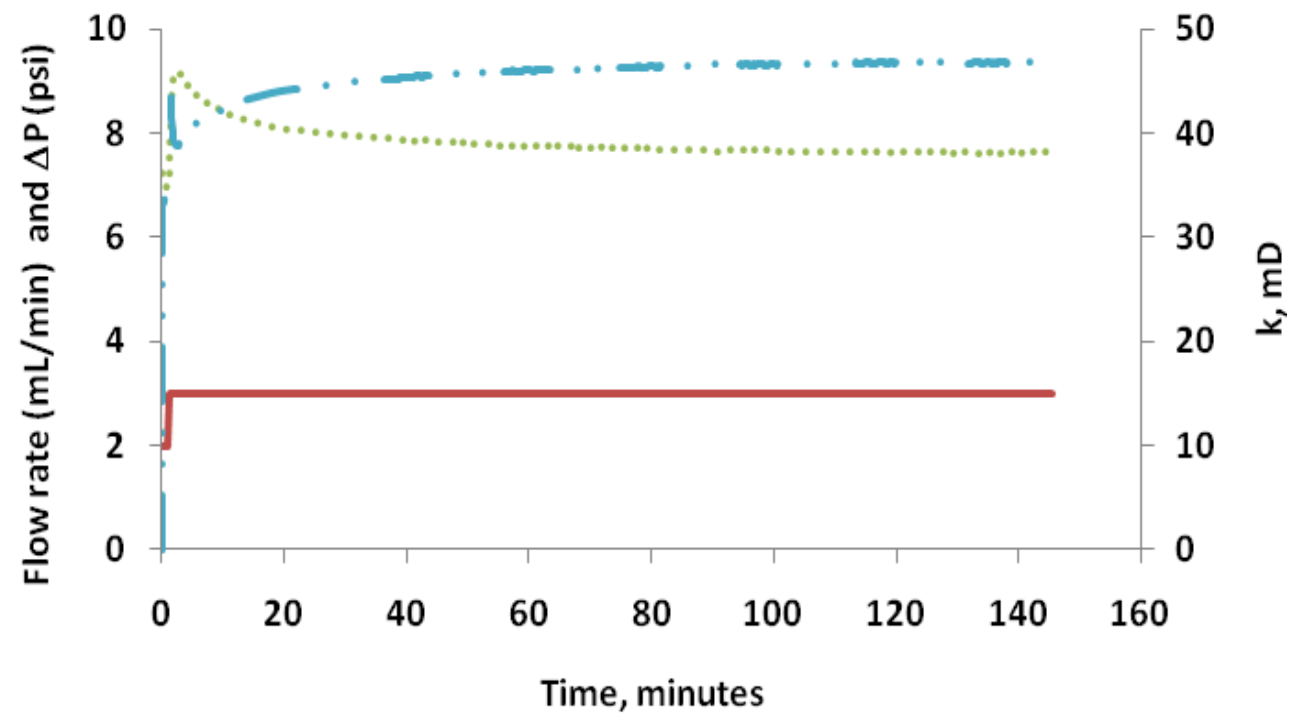

Flow rate, $\mathrm{mL} / \mathrm{min} \quad \ldots \ldots$ Delta $\mathrm{P}$, psi $\quad \mathrm{k}, \mathrm{mD}$

Figure 180 Flow rate, pressure drop and permeability of core $\# 5$ after fluid loss test at $25^{\circ} \mathrm{C}$. 


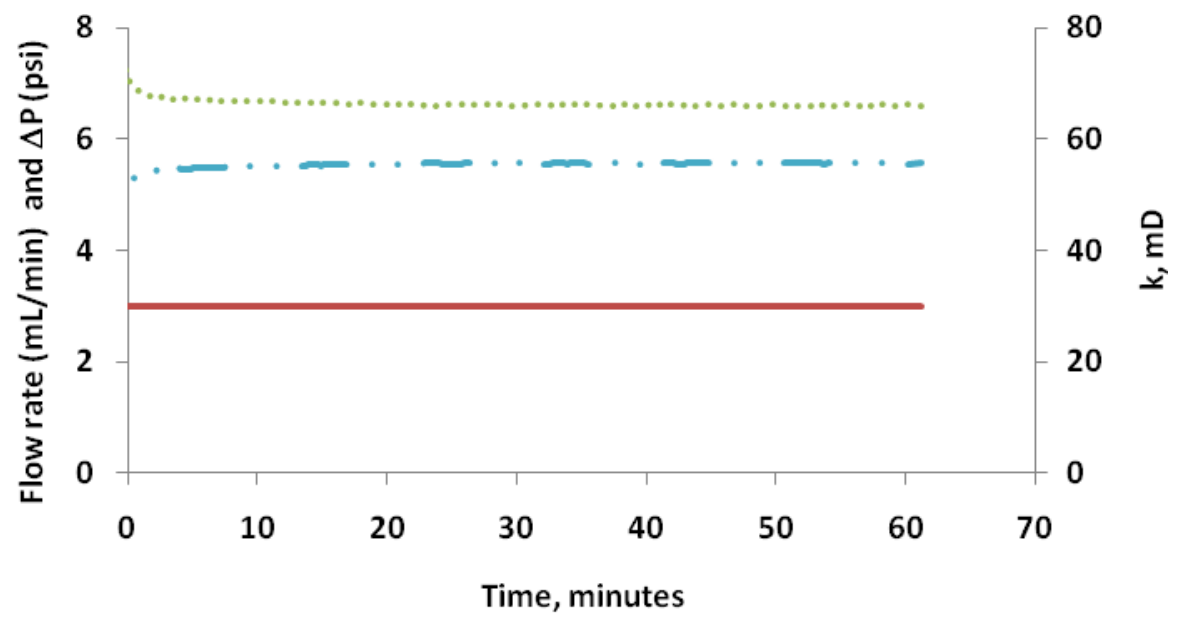

Flow rate, $\mathrm{mL} / \mathrm{min} \quad \ldots .$. Delta $\mathrm{P}$, psi $\quad \cdots \mathrm{k}, \mathrm{mD}$

Figure 181 Flow rate, pressure drop and permeability of restored core\#1 after fluid loss test at $25^{\circ} \mathrm{C}$.

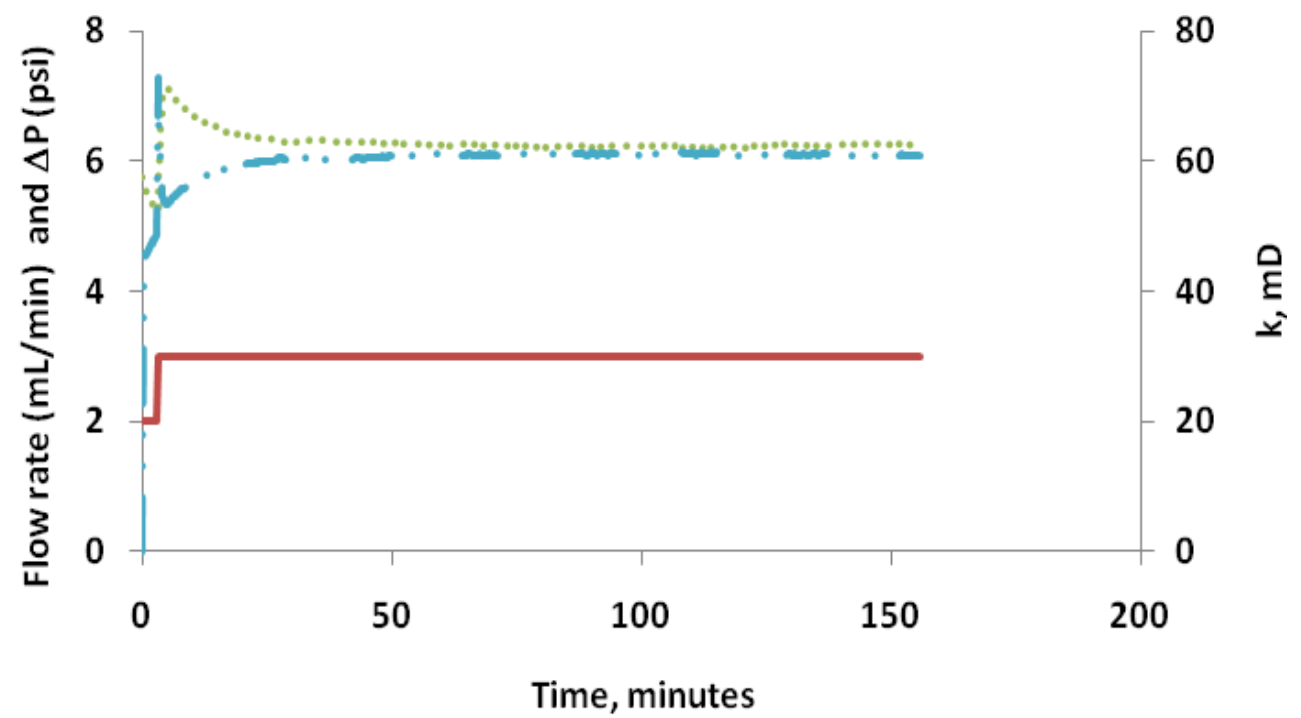

Flow rate, $\mathrm{mL} / \mathrm{min} \quad \ldots . .$. Delta $\mathrm{P}$, psi $\quad$ - $\mathrm{k}, \mathrm{mD}$

Figure 182 Flow rate, pressure drop and permeability of restored core\#10 after fluid loss test at $25{ }^{\circ} \mathrm{C}$. 


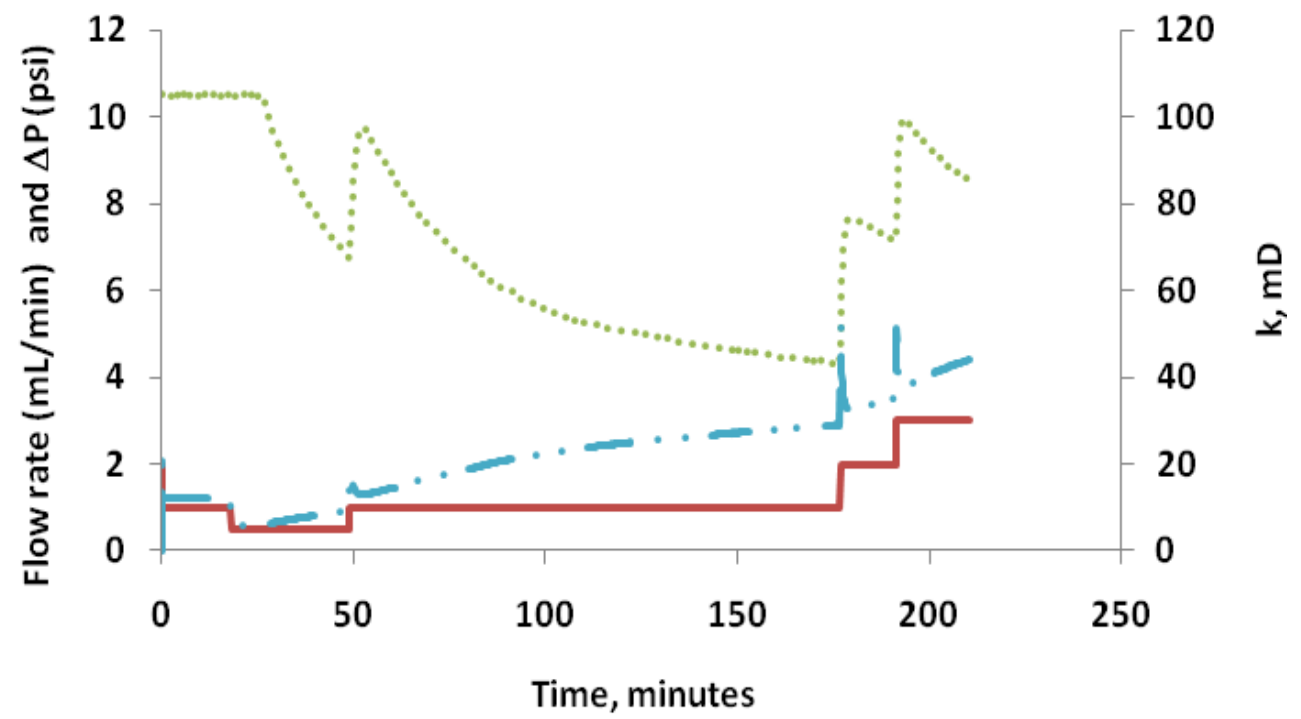

Flow rate, $\mathrm{mL} / \mathrm{min} \quad \ldots$. Delta $\mathrm{P}$, psi $\quad \mathrm{k}, \mathrm{mD}$

Figure 183 Flow rate, pressure drop and permeability of restored core $\# 9$ after fluid loss test at $25^{\circ} \mathrm{C}$.

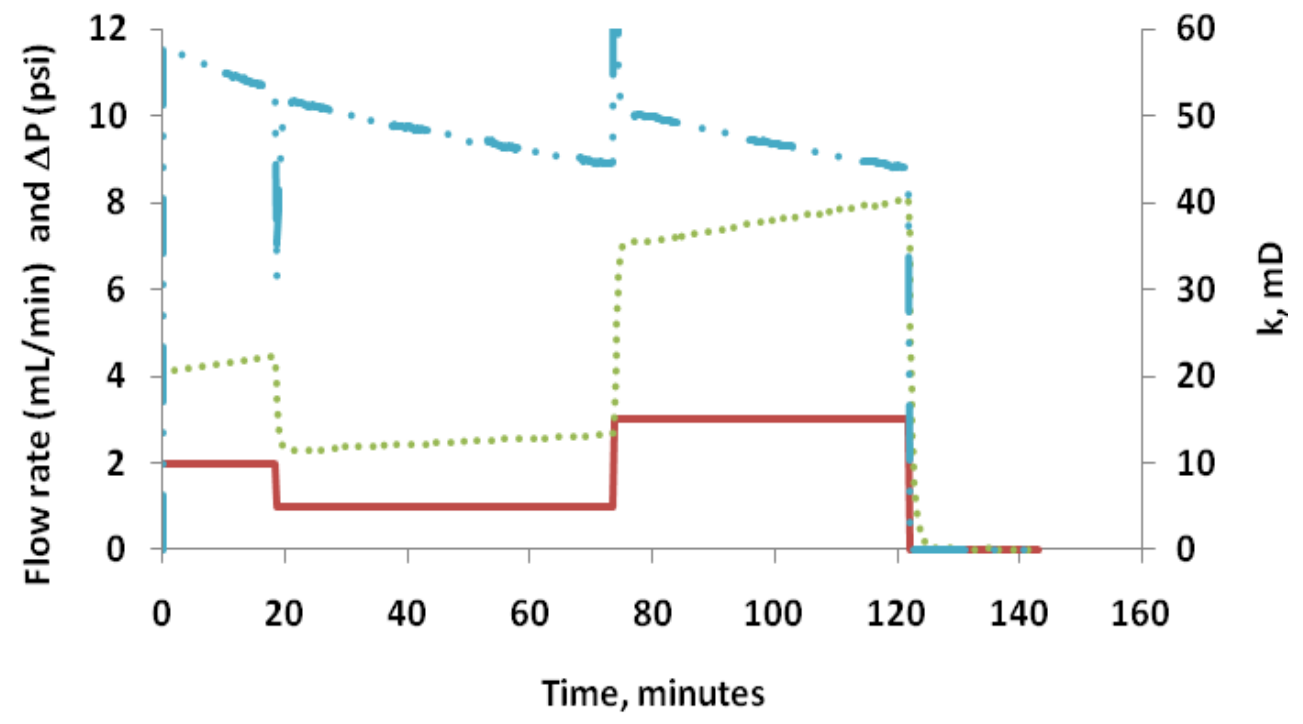

Flow rate, $\mathrm{mL} / \mathrm{min} \quad \ldots . .$. Delta $\mathrm{P}, \mathrm{psi} \quad-\mathrm{k}, \mathrm{mD}$

Figure 184 Flow rate, pressure drop and permeability of restored core $\# 6$ after fluid loss test at $25^{\circ} \mathrm{C}$. 


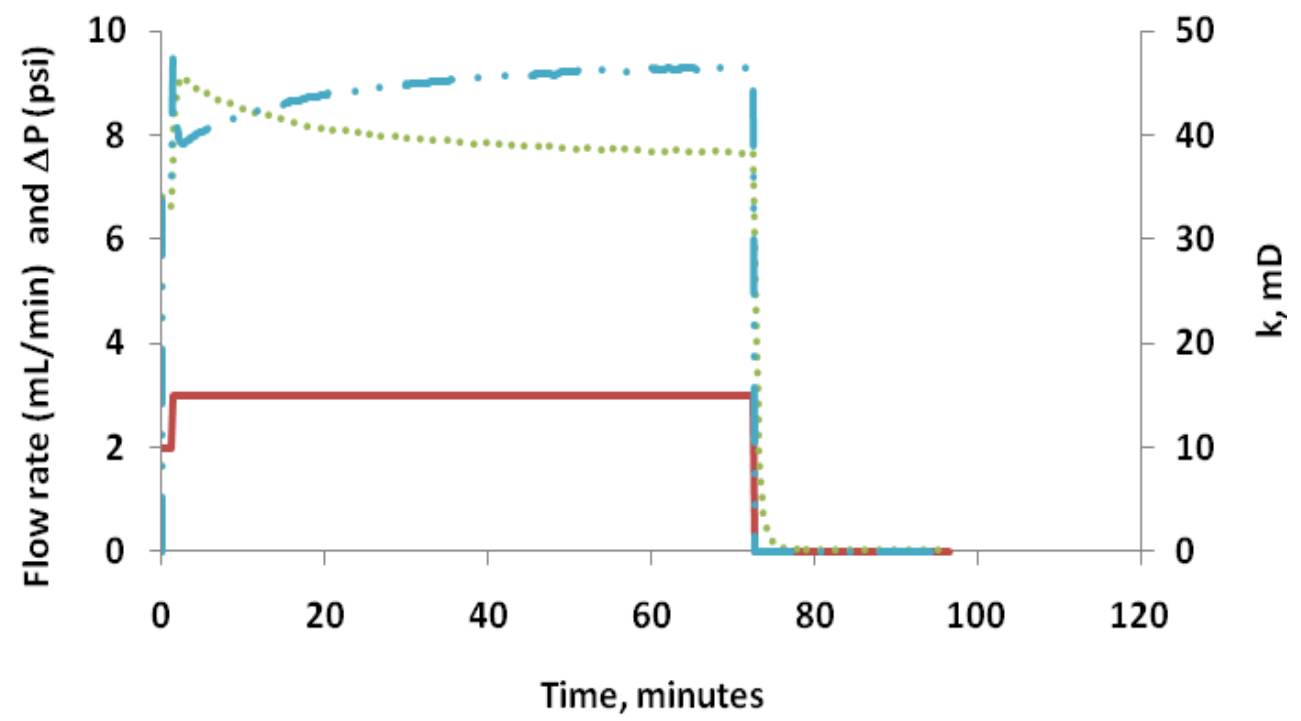

Flow rate, $\mathrm{mL} / \mathrm{min} \quad \ldots .$. Delta $\mathrm{P}$, psi $\quad \mathrm{k}, \mathrm{mD}$

Figure 185 Flow rate, pressure drop and permeability of restored core $\# 5$ after fluid loss test at $25^{\circ} \mathrm{C}$.

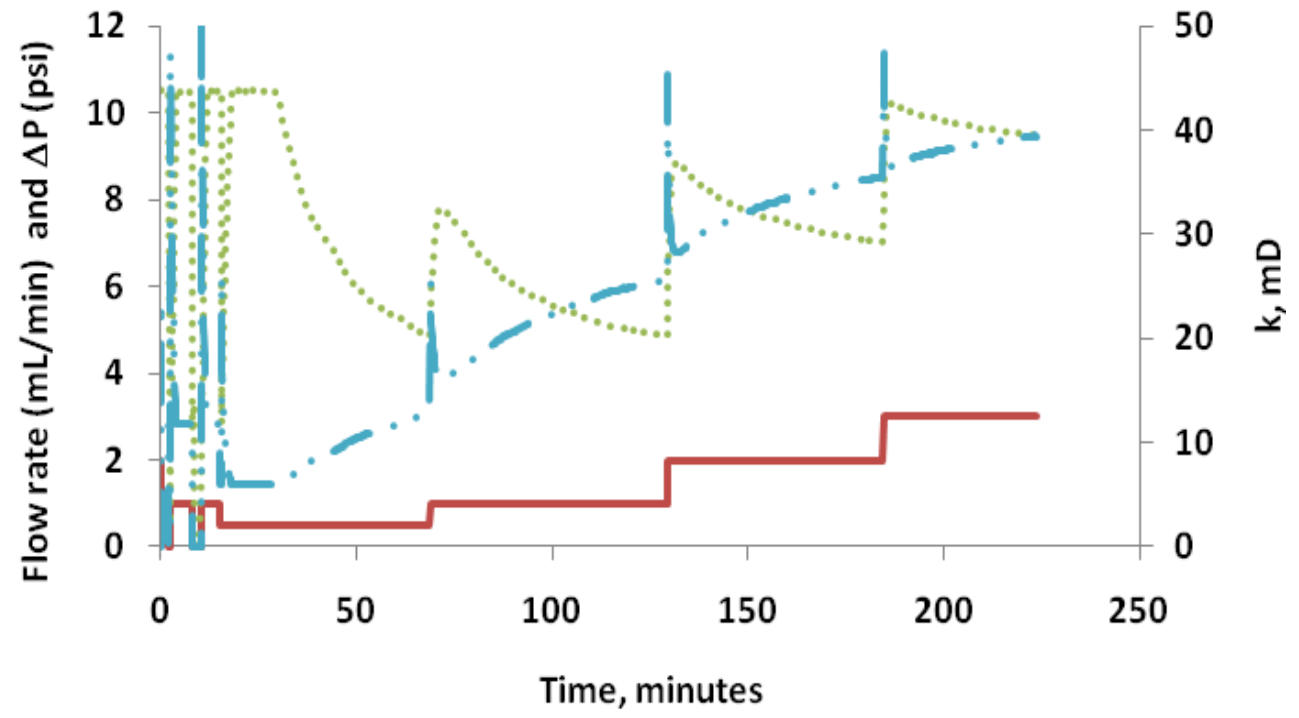

Flow rate, $\mathrm{mL} / \mathrm{min} \quad \ldots \ldots$ Delta $\mathrm{P}$, psi $\quad \cdots \mathrm{k}, \mathrm{mD}$

Figure 186 Flow rate, pressure drop and permeability of core\#15 after fluid loss test at $40{ }^{\circ} \mathrm{C}$. 


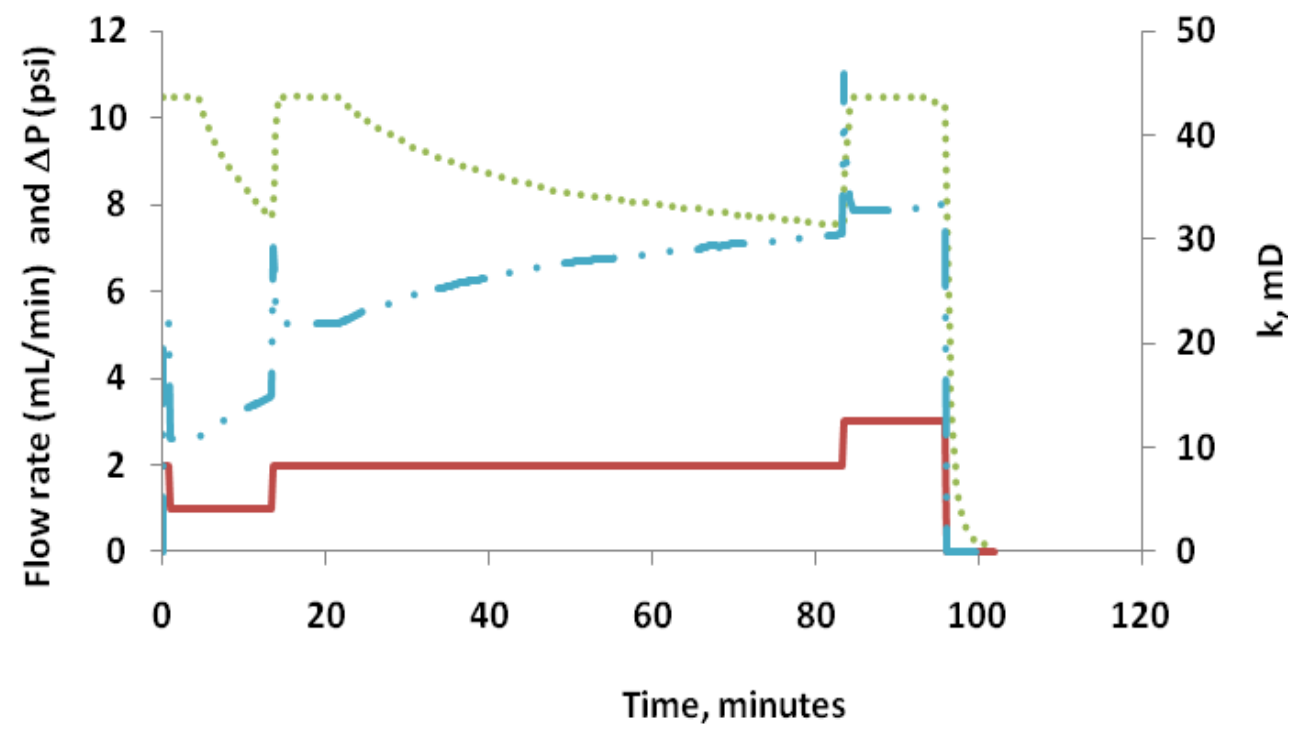

Flow rate, $\mathrm{mL} / \mathrm{min} \quad \ldots \ldots$ Delta $\mathrm{P}$, psi $\quad \cdots, \mathrm{mD}$

Figure 187 Flow rate, pressure drop and permeability of core\#18 after fluid loss test at $40{ }^{\circ} \mathrm{C}$.

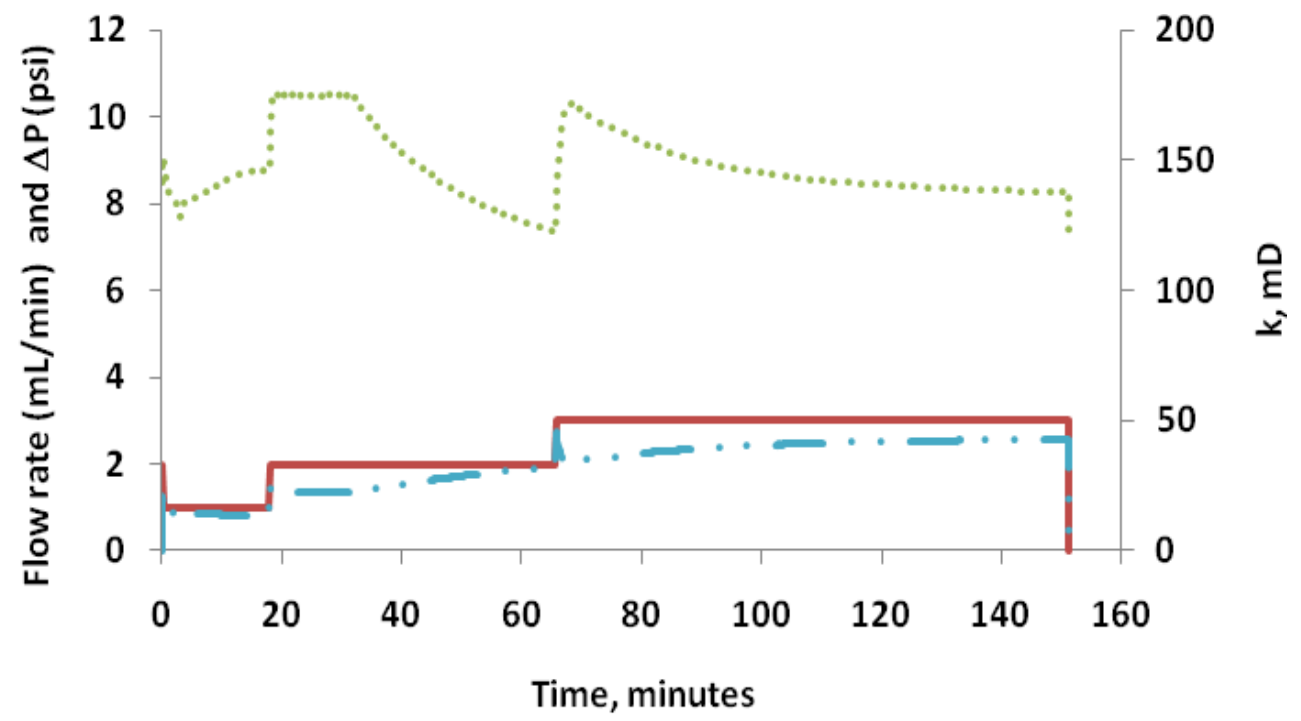

Flow rate, $\mathrm{mL} / \mathrm{min} \quad \ldots . .$. Delta $\mathrm{P}$, psi $\quad$ - $\mathrm{k}, \mathrm{mD}$

Figure 188 Flow rate, pressure drop and permeability of core\#19 after fluid loss test at $40{ }^{\circ} \mathrm{C}$. 


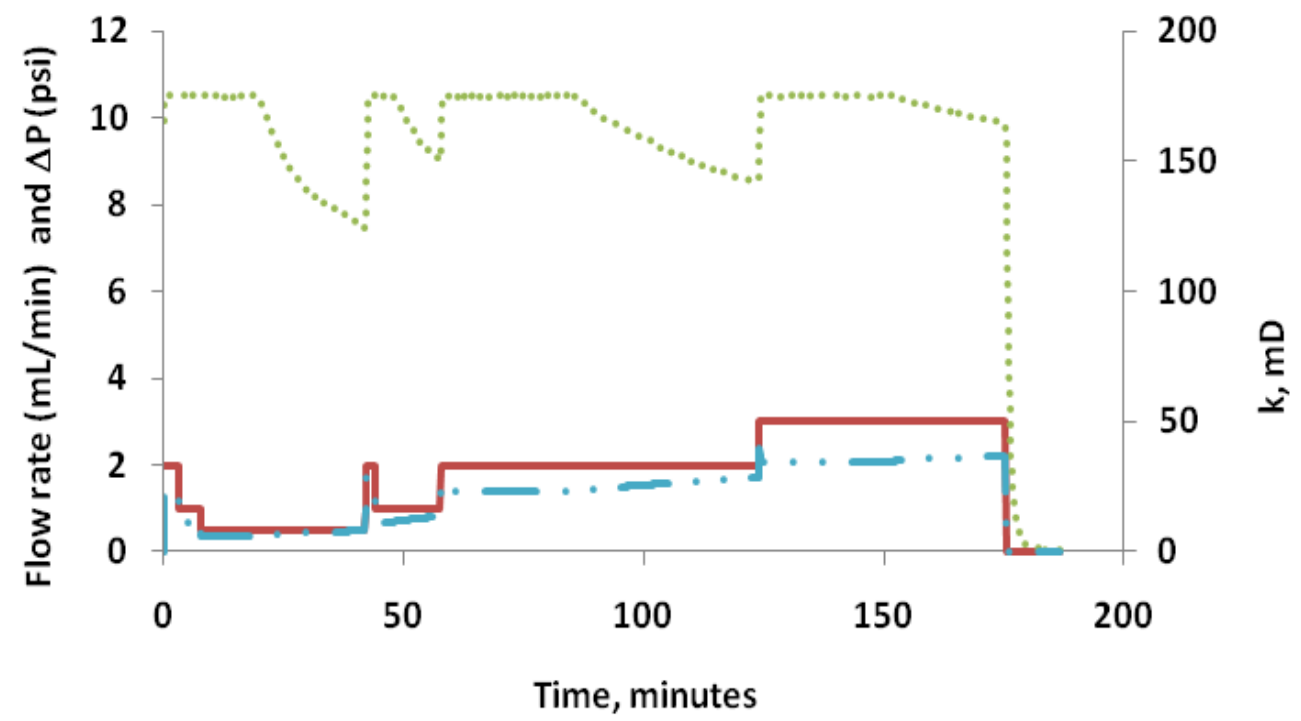

Flow rate, $\mathrm{mL} / \mathrm{min} \quad \ldots$. Delta $\mathrm{P}$, psi $\quad \mathrm{k}, \mathrm{mD}$

Figure 189 Flow rate, pressure drop and permeability of core 21 after fluid loss test at $40{ }^{\circ} \mathrm{C}$.

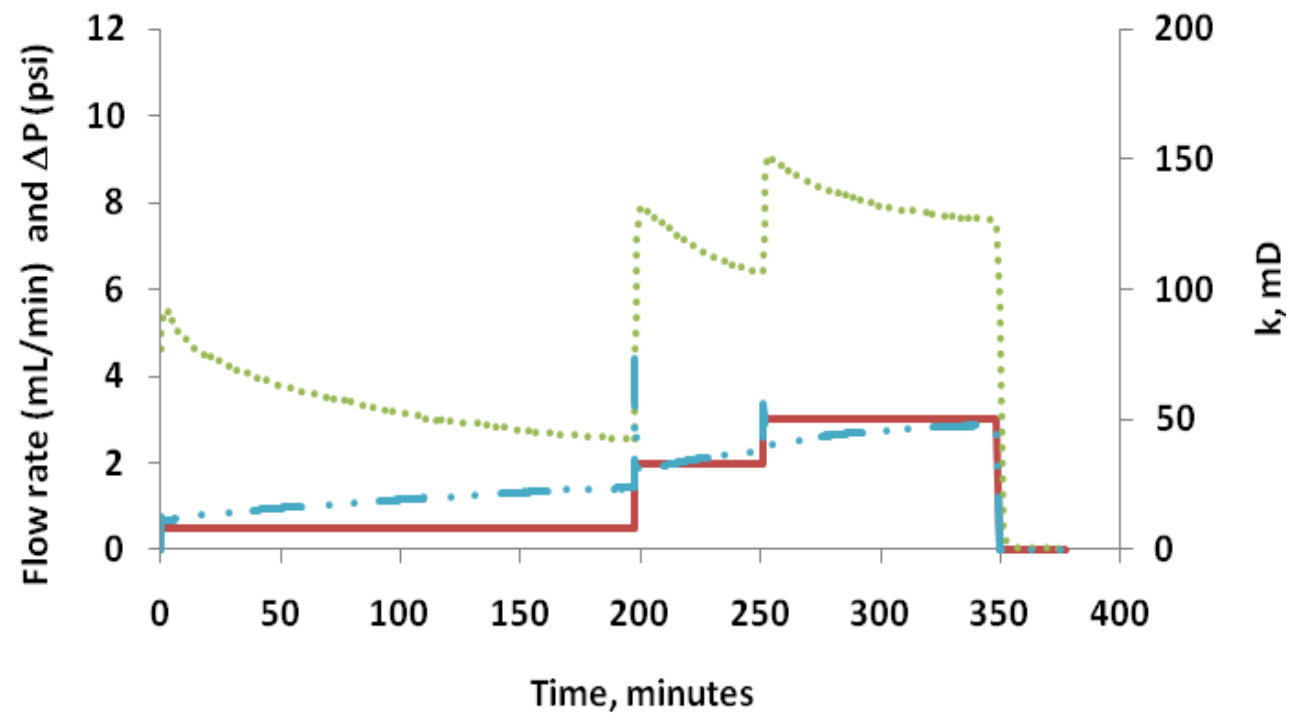

Flow rate, $\mathrm{mL} / \mathrm{min} \quad \ldots . .$. Delta $\mathrm{P}$, psi $\quad$ - $\mathrm{k}, \mathrm{mD}$

Figure 190 Flow rate, pressure drop and permeability of core\#22 after fluid loss test at $40{ }^{\circ} \mathrm{C}$. 


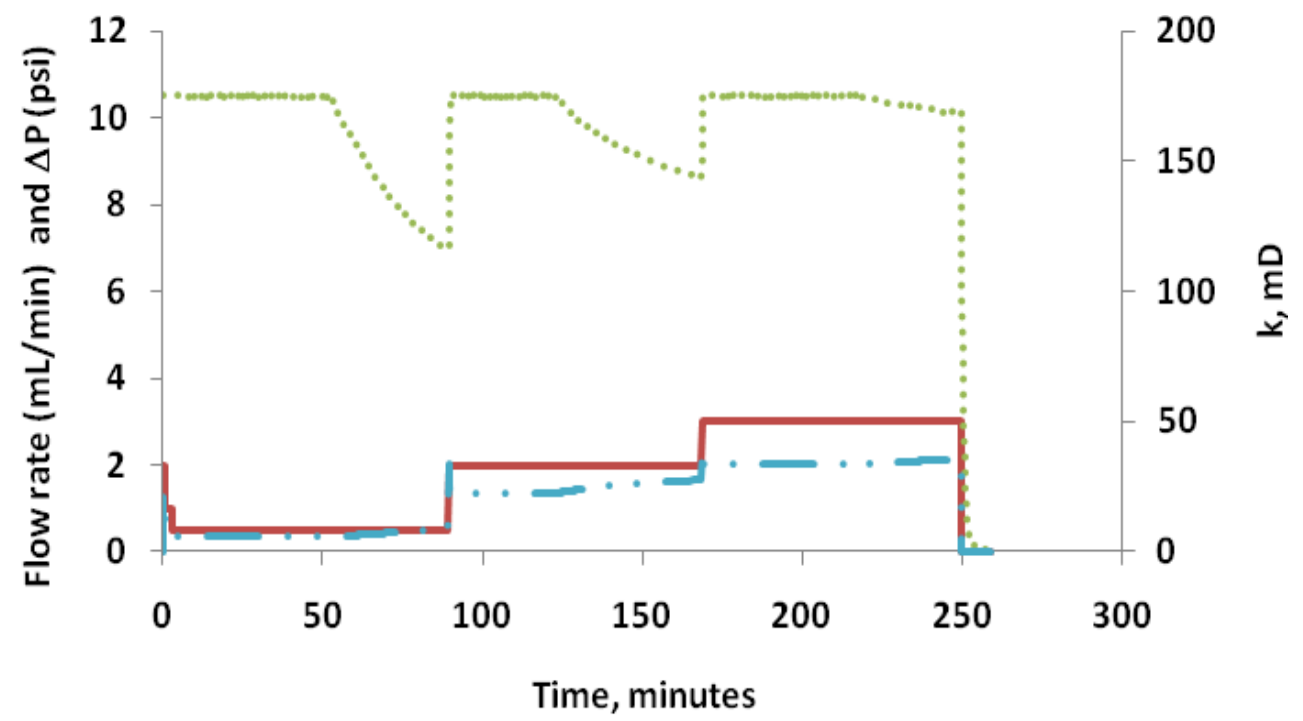

Flow rate, $\mathrm{mL} / \mathrm{min} \quad \ldots . .$. Delta $\mathrm{P}, \mathrm{psi} \quad-\mathrm{k}, \mathrm{mD}$

Figure 191 Flow rate, pressure drop and permeability of core\#23 after fluid loss test at $40{ }^{\circ} \mathrm{C}$. 